National Toxicology Program

NTP TECHNICAL REPORT ON THE TOXICOLOGY STUDIES OF

INDOLE-3-CARBINOL

(CASRN 700-06-1)

IN F344/N RATS AND

B6C3Fl/N MICE

AND TOXICOLOGY AND

CARCINOgENESIS StUdIES

OF INDOLE-3-CARBINOL IN

HaRLAN SPRAGUE DAWLEY

RATS AND B6C3FI/N MiCE (Gavage Studies)

NTP TR 584 


\title{
NTP Technical Report on the Toxicology Studies of Indole-3-carbinol (CASRN 700-06-1) in F344/N Rats and B6C3F1/N Mice and Toxicology and Carcinogenesis Studies of Indole-3-carbinol in Harlan Sprague Dawley Rats and B6C3F1/N Mice (Gavage Studies)
}

Technical Report 584

July 2017

\author{
National Toxicology Program \\ Public Health Service \\ U.S. Department of Health and Human Services
}

ISSN: 2378-8925

Research Triangle Park, North Carolina, USA 


\section{Foreword}

The National Toxicology Program (NTP) is an interagency program within the Public Health Service (PHS) of the Department of Health and Human Services (HHS) and is headquartered at the National Institute of Environmental Health Sciences of the National Institutes of Health (NIEHS/NIH). Three agencies contribute resources to the program: NIEHS/NIH, the National Institute for Occupational Safety and Health of the Centers for Disease Control and Prevention (NIOSH/CDC), and the National Center for Toxicological Research of the Food and Drug Administration (NCTR/FDA). Established in 1978, NTP is charged with coordinating toxicological testing activities, strengthening the science base in toxicology, developing and validating improved testing methods, and providing information about potentially toxic substances to health regulatory and research agencies, scientific and medical communities, and the public.

The Technical Report series began in 1976 with carcinogenesis studies conducted by the National Cancer Institute. In 1981, this bioassay program was transferred to NTP. The studies described in the Technical Report series are designed and conducted to characterize and evaluate the toxicologic potential, including carcinogenic activity, of selected substances in laboratory animals (usually two species, rats and mice). Substances selected for NTP toxicity and carcinogenicity studies are chosen primarily on the basis of human exposure, level of production, and chemical structure. The interpretive conclusions presented in NTP Technical Reports are based only on the results of these NTP studies. Extrapolation of these results to other species, including characterization of hazards and risks to humans, requires analyses beyond the intent of these reports. Selection per se is not an indicator of a substance's carcinogenic potential.

NTP conducts its studies in compliance with its laboratory health and safety guidelines and FDA Good Laboratory Practice Regulations and must meet or exceed all applicable federal, state, and local health and safety regulations. Animal care and use are in accordance with the Public Health Service Policy on Humane Care and Use of Animals. Studies are subjected to retrospective quality assurance audits before being presented for public review.

The NTP Technical Reports are available free of charge on the NTP website and cataloged in PubMed, a free resource developed and maintained by the National Library of Medicine (part of the National Institutes of Health). Data for these studies are included in NTP's Chemical Effects in Biological Systems database.

For questions about the reports and studies, please email NTP or call 984-287-3211. 


\section{Table of Contents}

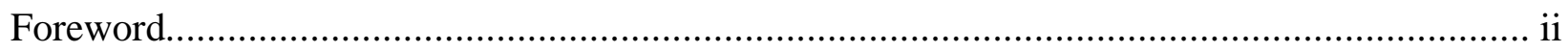

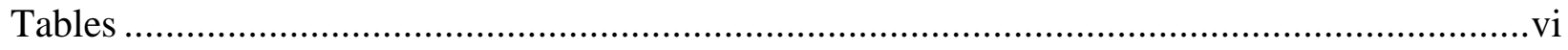

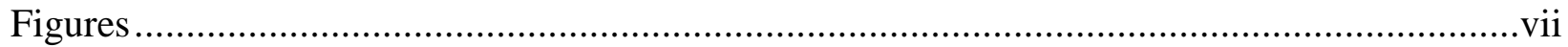

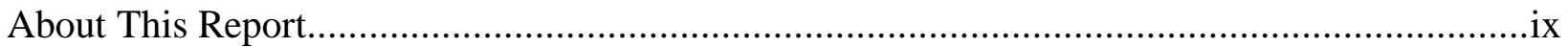

Explanation of Levels of Evidence of Carcinogenic Activity ......................................................ii

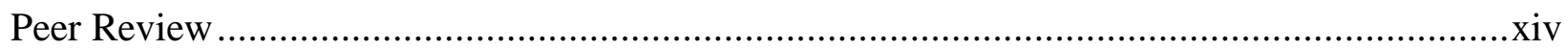

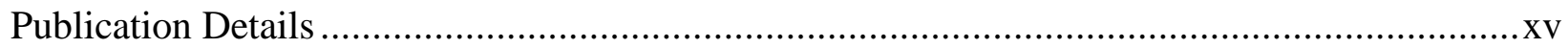

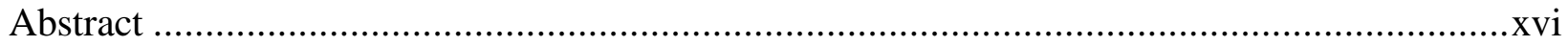

Three-month Study in F344/N Rats ..........................................................................

Three-month Study in B6C3F1/N Mice ..........................................................................

Two-year Study in Sprague Dawley Rats.............................................................................

Two-year Study in B6C3F1/N Mice ...................................................................................

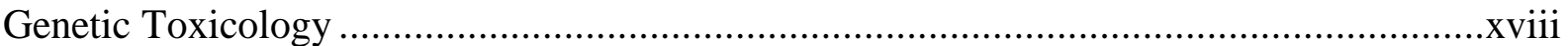

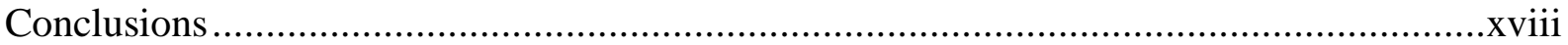

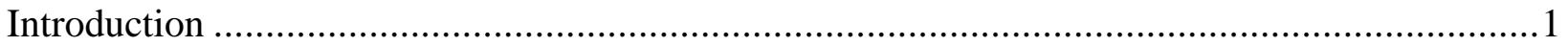

Chemical and Physical Properties ...................................................................................

Production, Use, and Human Exposure....................................................................... 1

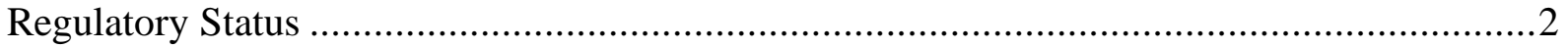

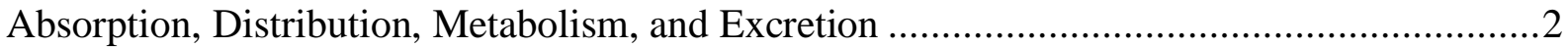

Experimental Animals ......................................................................................

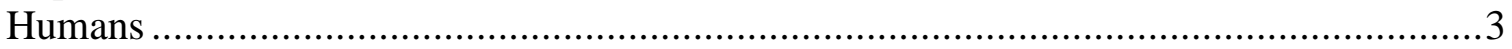

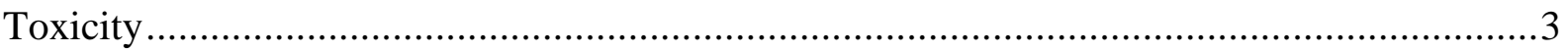

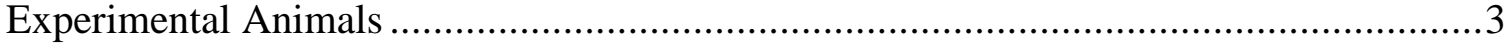

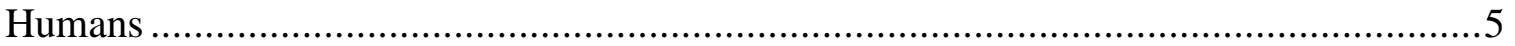

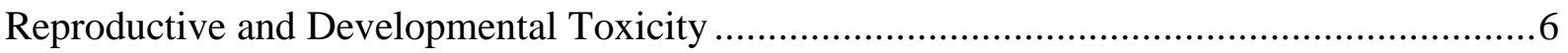

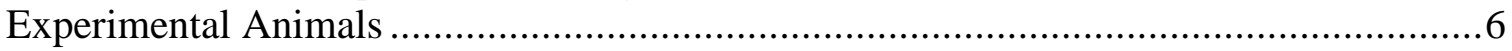

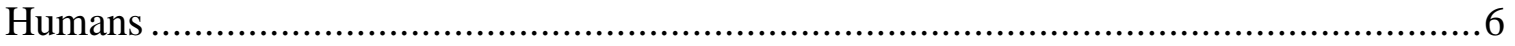

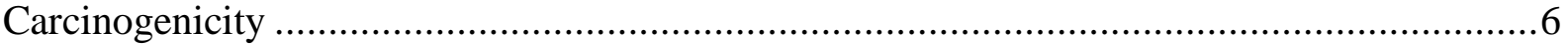

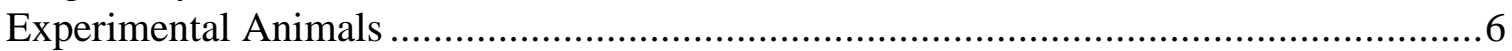

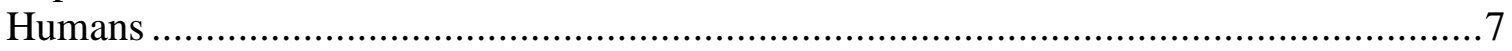

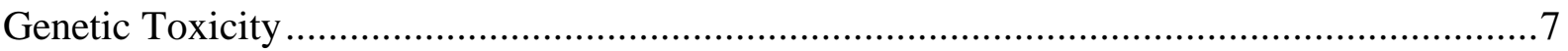

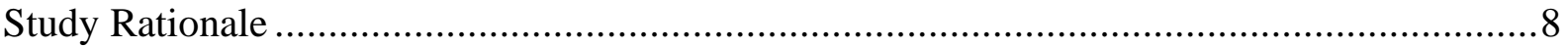

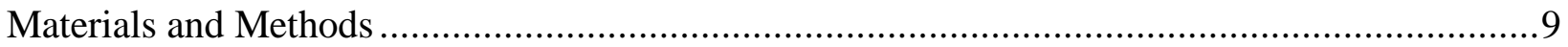

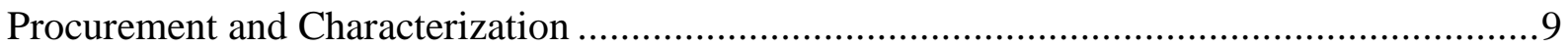

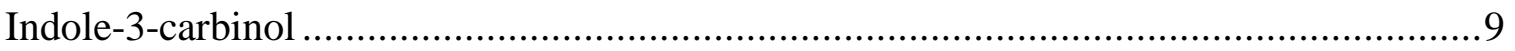

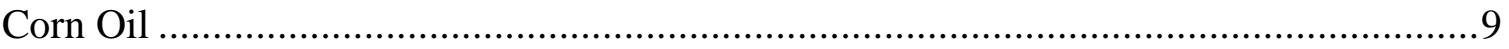

Preparation and Analysis of Dose Formulations..............................................................10

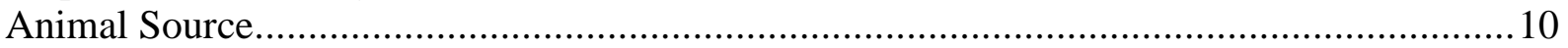

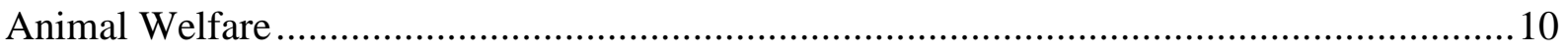

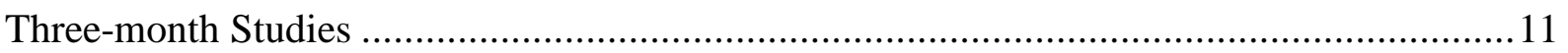




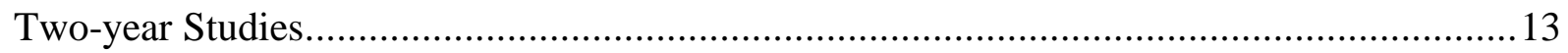

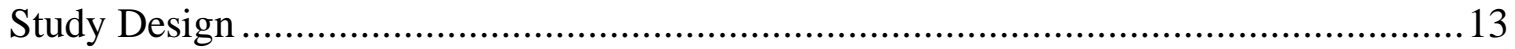

Clinical Examinations and Pathology..............................................................13

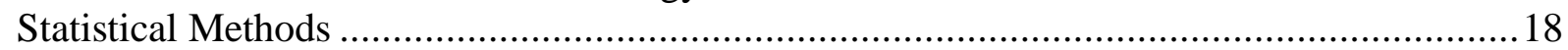

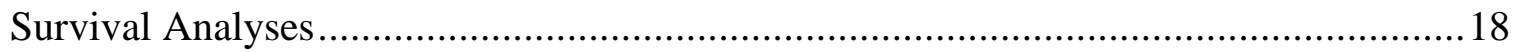

Calculation of Incidence ........................................................................................18

Analysis of Neoplasm and Nonneoplastic Lesion Incidences .........................................19

Analysis of Continuous Variables..........................................................................19

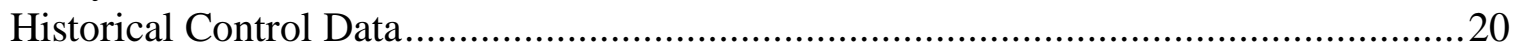

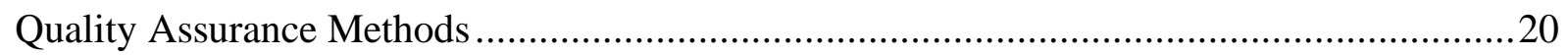

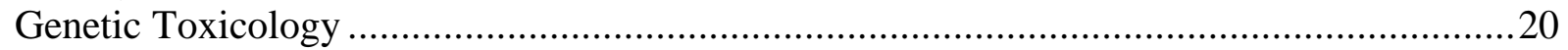

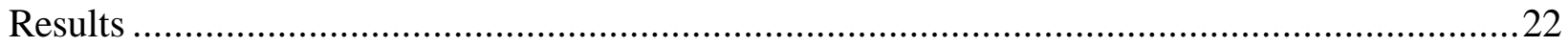

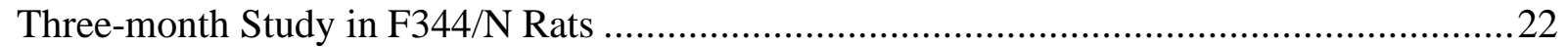

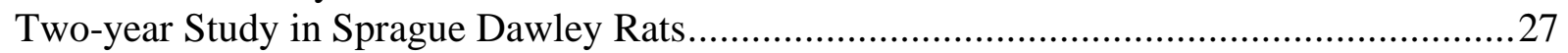

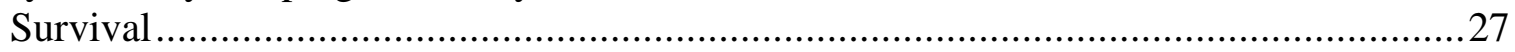

Body Weights and Clinical Findings......................................................................27

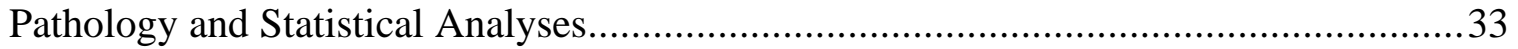

Three-month Study in Mice ..........................................................................................

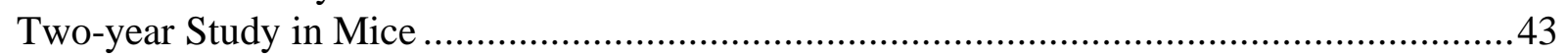

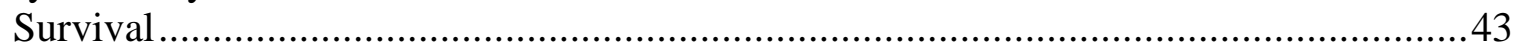

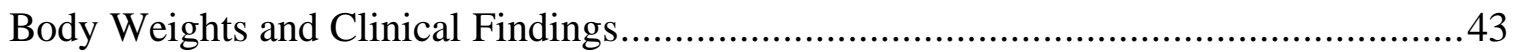

Pathology and Statistical Analyses............................................................................ 48

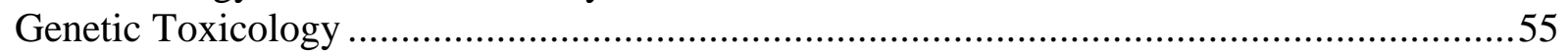

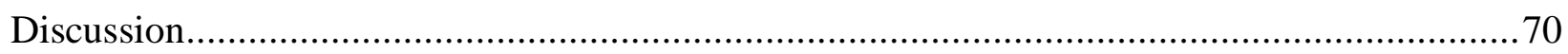

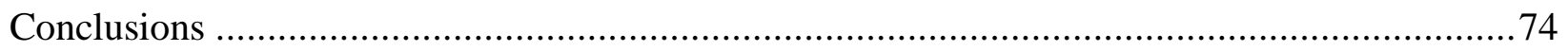

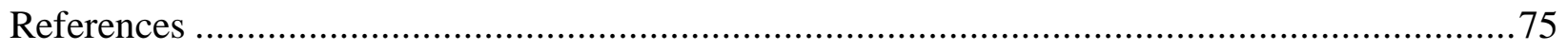

Appendix A. Summary of Lesions in Male Sprague Dawley Rats in the Two-year Gavage Study of Indole-3-carbinol.............................................................................

Appendix B. Summary of Lesions in Female Sprague Dawley Rats in the Two-year Gavage Study of Indole-3-carbinol ................................................................

Appendix C. Summary of Lesions in Male Mice in the Two-year Gavage Study of

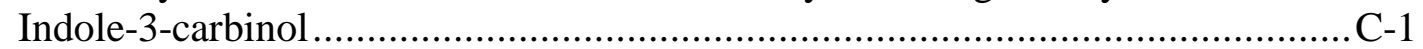

Appendix D. Summary of Lesions in Female Mice in the Two-year Gavage Study of

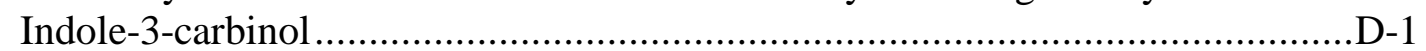

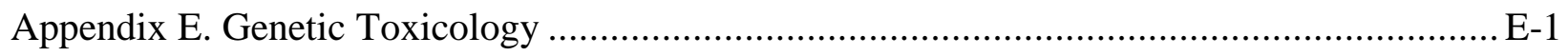

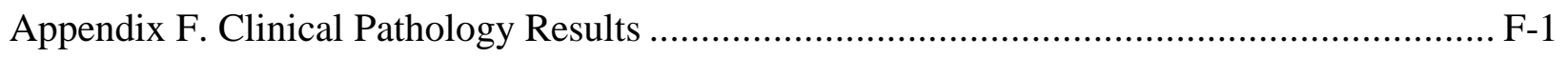

Appendix G. Organ Weights and Organ-Weight-to-Body-Weight Ratios ...................................-1

Appendix H. Reproductive Tissue Evaluations and Estrous Cycle Characterization....................H-1

Appendix I. Chemical Characterization and Dose Formulation Studies .................................... I-1 
Indole-3-carbinol, NTP TR 584

Appendix J. Ingredients, Nutrient Composition, and Contaminant Levels in NTP-2000

Rat and Mouse Ration ...........................................................................

Appendix K. Sentinel Animal Program ............................................................................

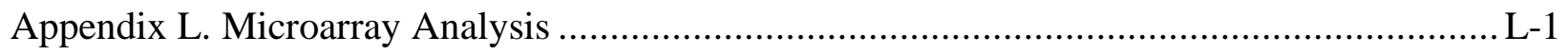

Appendix M. Summary of Peer Review Panel Comments ............................................... M-1 


\section{Tables}

Table 1. Experimental Design and Materials and Methods in the Gavage Studies of

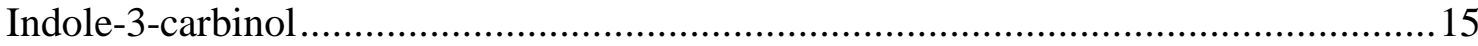

Table 2. Survival and Body Weights of F344/N Rats in the Three-month Gavage Study of

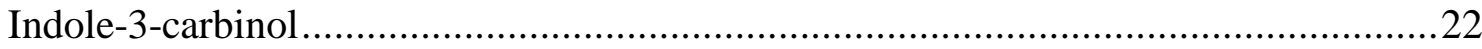

Table 3. Selected Organ Weights and Organ-Weight-to-Body-Weight Ratios for F344/N Rats in the Three-month Gavage Study of Indole-3-carbinol ....................................24

Table 4. Liver and Lung Cytochrome P450 Data for F344/N Rats in the Three-month Gavage Study of Indole-3-carbinol.

Table 5. Incidences of Selected Nonneoplastic Lesions in F344/N Rats in the Threemonth Gavage Study of Indole-3-carbinol .....................................................26

Table 6. Survival of Sprague Dawley Rats in the Two-year Gavage Study of Indole-3-carbinol

Table 8. Mean Body Weights and Survival of Female Sprague Dawley Rats in the Twoyear Gavage Study of Indole-3-carbinol

Table 9. Incidences of Neoplasms and Nonneoplastic Lesions of the Uterus in Female Sprague Dawley Rats in the Two-year Gavage Study of Indole-3-carbinol

Table 10. Incidences of Neoplasms of the Skin in Female Sprague Dawley Rats in the Two-year Gavage Study of Indole-3-carbinol

Table 11. Incidences of Selected Nonneoplastic Lesions in Sprague Dawley Rats in the Two-year Gavage Study of Indole-3-carbinol .

Table 12. Survival and Body Weights of Mice in the Three-month Gavage Study of Indole-3-carbinol ....

Table 13. Liver Weights and Liver-to Body-Weight Ratios for Mice in the Three-month Gavage Study of Indole-3-carbinol

Table 14. Liver and Lung Cytochrome P450 Data for Mice in the Three-month Gavage Study of Indole-3-carbinol

Table 15. Survival of Mice in the Two-year Gavage Study of Indole-3-carbinol.

Table 16. Mean Body Weights and Survival of Male Mice in the Two-year Gavage Study of Indole-3-carbinol.

Table 17. Mean Body Weights and Survival of Female Mice in the Two-year Gavage Study of Indole-3-carbinol

Table 18. Incidences of Neoplasms and Nonneoplastic Lesions of the Liver in Mice in the Two-year Gavage Study of Indole-3-carbinol

Table 19. Incidences of Nonneoplastic Lesions of the Glandular Stomach in Mice in the Two-year Gavage Study of Indole-3-carbinol

Table 20. Incidences of Nonneoplastic Lesions of the Nose in Mice in the Two-year Gavage Study of Indole-3-carbinol 


\section{Figures}

Figure 1. Indole-3-carbinol (CASRN 700-06-1; Chemical Formula: $\mathrm{C}_{9} \mathrm{H}_{9} \mathrm{NO}$; Molecular Weight: 147.18) .................................................................................

Figure 2. Proposed Metabolic Scheme for Indole-3-carbinol following Oral

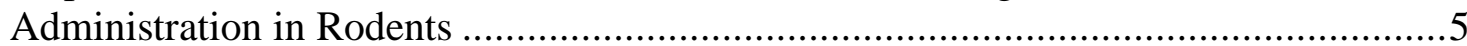

Figure 3. Growth Curves for F344/N Rats Administered Indole-3-carbinol by Gavage for

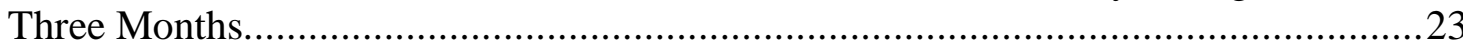

Figure 4. Kaplan-Meier Survival Curves for Sprague Dawley Rats Administered Indole-3-carbinol by Gavage for Two Years ....

Figure 5. Growth Curves for Sprague Dawley Rats Administered Indole-3-carbinol by Gavage for Two Years ................................................................................ 30

Figure 6. Growth Curves for Mice Administered Indole-3-carbinol by Gavage for Three Months

Figure 7. Kaplan-Meier Survival Curves for Mice Administered Indole-3-carbinol by Gavage for Two Years

Figure 8. Growth Curves for Mice Administered Indole-3-carbinol by Gavage for Two Years

Figure 9. Adenocarcinoma of the Endometrium in the Uterus of a Female Harlan Sprague Dawley Rat Administered 300 mg/kg Indole-3-carbinol by Gavage for Two Years (H\&E). .55

Figure 10. Higher Magnification of Figure 9 (H\&E).

Figure 11. Adenoma (Arrows) of the Endometrium in the Uterus of a Female Harlan Sprague Dawley Rat Administered 300 mg/kg Indole-3-carbinol by Gavage for Two Years (H\&E). .56

Figure 12. Higher Magnification of Figure 11 (H\&E).

Figure 13. Atypical Hyperplasia of the Endometrium in the Uterus of a Female Harlan Sprague Dawley Rat Administered 300 mg/kg Indole-3-carbinol by Gavage for Two Years (H\&E).

Figure 14. Atypical Hyperplasia of the Endometrium in the Uterus of a Female Harlan Sprague Dawley Rat Administered 300 mg/kg Indole-3-carbinol by Gavage for Two Years (H\&E).

Figure 15. Lymphatic Ectasia in the Jejunum of a Female Harlan Sprague Dawley Rat Administered 300 mg/kg Indole-3-carbinol by Gavage for Two Years (H\&E) ...........58

Figure 16. Higher Magnification of Figure 15 (H\&E)...................................................58

Figure 17. Lymphatic Ectasia in the Mesenteric Lymph Node of a Female Harlan Sprague Dawley Rat Administered 300 mg/kg Indole-3-carbinol by Gavage for Two Years (H\&E)

Figure 18. Higher Magnification of Figure 17 (H\&E)

Figure 19. Cholangiofibrosis in the Liver of a Male Harlan Sprague Dawley Rat Administered $150 \mathrm{mg} / \mathrm{kg}$ Indole-3-carbinol by Gavage for Two Years (H\&E).

Figure 20. Higher Magnification of Figure 19 (H\&E).

Figure 21. Cholangiofibrosis in the Liver of a Male Harlan Sprague Dawley Rat Administered 150 mg/kg Indole-3-carbinol by Gavage for Two Years (H\&E). 61

Figure 22. Higher Magnification of Figure 21 (H\&E). 61 
Figure 23. Eosinophilic Focus in the Liver of a Male Harlan Sprague Dawley Rat Administered 75 mg/kg Indole-3-carbinol by Gavage for Two Years (H\&E)............62

Figure 24. Clear Cell Focus (Arrows) in the Liver of a Male Harlan Sprague Dawley Rat Administered 75 mg/kg Indole-3-carbinol by Gavage for Two Years (H\&E).

Figure 25. Hepatocellular Adenoma (Arrows) in the Liver of a Male B6C3F1/N Mouse Administered $250 \mathrm{mg} / \mathrm{kg}$ Indole-3-carbinol by Gavage for Two Years (H\&E)

Figure 26. Higher Magnification of Figure 25 (H\&E).

Figure 27. Large, Well-demarcated, Nodular Hepatocellular Carcinoma (Arrows) in a Male B6C3F1/N Mouse Administered 250 mg/kg Indole-3-carbinol by

Gavage for Two Years (H\&E).

Figure 28. Higher Magnification of Figure 27 (H\&E).

Figure 29. Hepatoblastoma (Arrows) in the Liver of a Male B6C3F1/N Mouse Administered 250 mg/kg Indole-3-carbinol by Gavage for Two Years (H\&E) ..........65

Figure 30. Higher Magnification of Figure 29 (H\&E).

Figure 31. Normal Glandular Stomach Epithelium in a Vehicle Control Female B6C3F1/N Mouse in the Two-year Gavage Study of Indole-3-carbinol (H\&E) .66

Figure 32. Hyperplasia of the Glandular Epithelium of the Stomach in a Male B6C3F1/N Mouse Administered $250 \mathrm{mg} / \mathrm{kg}$ Indole-3-carbinol by Gavage for Two Years (H\&E).

Figure 33. Low Magnification of the Ethmoid Turbinates (Level III Section) in the Nose of a Female B6C3F1/N Mouse Administered $250 \mathrm{mg} / \mathrm{kg}$ Indole-3-carbinol by Gavage for Two Years (H\&E).

Figure 34. Higher Magnification of Figure 33 (H\&E).

Figure 35. Low Magnification of the Ethmoid Turbinates (Level III Section) in the Nose of a Female B6C3F1/N Mouse Administered $250 \mathrm{mg} / \mathrm{kg}$ Indole-3-carbinol by Gavage for Two Years Showing Respiratory Epithelial Metaplasia in the Olfactory Epithelium (H\&E)

Figure 36. Higher Magnification of Figure 35 (H\&E).

This report has been reformatted to meet new NTP publishing requirements; its contents has not changed. 


\section{About This Report}

National Toxicology Program ${ }^{1}$

${ }^{1}$ Division of the National Toxicology Program, National Institute of Environmental Health Sciences, Research Triangle Park, North Carolina, USA

\section{Collaborators}

M.E. Wyde, M.C. Boyle, R.A. Herbert, A. Nyska, E.T. Adams, B. Atkinson, C.R. Blystone, C.M. Briscoe, A.E. Brix, P.M. Foster, J.E. Heath, C.D. Hébert, M.R. Hejtmancik, G.D. Hill, M.J. Hooth, A.P. King-Herbert, G.E. Kissling, D.E. Malarkey, J.F. Mann, B.S. McIntyre, R.A. Miller, B.J.T. Muir, M.J. Ryan, S.L. Smith-Roe, M.D. Stout, G.S. Travlos, S. Waidyanatha, N.J. Walker, Y. Wang, K.L. Witt

Division of the National Toxicology Program, National Institute of Environmental Health Sciences, Research Triangle Park, North Carolina, USA

Evaluated and interpreted results and reported findings

M.E. Wyde, Ph.D., Study Scientist

M.C. Boyle, D.V.M., Study Pathologist

R.A. Herbert, D.V.M., Ph.D., Study Pathologist

C.R. Blystone, M.S., Ph.D.

P.M. Foster, Ph.D.

M.J. Hooth, Ph.D.

A.P. King-Herbert, D.V.M.

G.E. Kissling, Ph.D.

D.E. Malarkey, D.V.M., Ph.D.

B.S. McIntyre, Ph.D.

S.L. Smith-Roe, Ph.D.

M.D. Stout, Ph.D.

G.S. Travlos, D.V.M.

S. Waidyanatha, Ph.D.

N.J. Walker, Ph.D.

K.L. Witt, M.S.

ILS, Inc., Research Triangle Park, North Carolina, USA

Evaluated and interpreted results and reported findings

A. Nyska, D.V.M., Study Pathologist

Coordinated NTP Pathology Working Groups for 2-year rats (July 24, 2012) and 2-year mice (December 13, 2011)

G.D. Hill, D.V.M., Ph.D.

\section{Southern Research Institute, Birmingham, Alabama, USA}

Conducted 3-month studies and evaluated pathology findings

C.D. Hébert, Ph.D., Principal Investigator

J.E. Heath, D.V.M.

J.F. Mann, D.V.M. 
Battelle Columbus Operations, Columbus, Ohio, USA

Conducted 2-year studies and evaluated pathology findings

M.R. Hejtmancik, Ph.D., Principal Investigator

C.M. Briscoe, D.V.M., Ph.D.

M.J. Ryan, D.V.M., Ph.D.

Experimental Pathology Laboratories, Inc., Research Triangle Park, North Carolina, USA

Conducted pathology review

E.T. Adams, D.V.M., Ph.D.

A.E. Brix, D.V.M., Ph.D.

R.A. Miller, D.V.M., Ph.D.

Gene Logic Laboratories, Inc., Gaithersburg, Maryland, USA

Provided SMVCE analysis

B.J.T. Muir, Ph.D., Principal Investigator

B. Atkinson, M.Sc.

Y. Wang, M.S.

\section{Contributors}

Experimental Pathology Laboratories, Inc., Research Triangle Park, North Carolina, USA Supervised pathology review

M.H. Hamlin, II, D.V.M., Principal Investigator

NTP Pathology Working Group, National Institute of Environmental Health Sciences, Research Triangle Park, North Carolina, USA

Participated in NTP Pathology Working Group on 2-year rats (July 24, 2012)

A.E. Brix, D.V.M., Ph.D., Experimental Pathology Laboratories, Inc.

J.M. Cullen, D.V.M., Ph.D., North Carolina State University

S.A. Elmore, D.V.M., M.S., National Toxicology Program

R.A. Herbert, D.V.M., Ph.D., National Toxicology Program

M.J. Hoenerhoff, D.V.M., Ph.D., National Toxicology Program

D.E. Malarkey, D.V.M., Ph.D., National Toxicology Program

A. Nyska, D.V.M., ILS, Inc.

A.B. Rogers, D.V.M., Ph.D., University of North Carolina at Chapel Hill

Participated in NTP Pathology Working Group on 2-year mice (December 13, 2011)

E.T. Adams, D.V.M., Ph.D., Experimental Pathology Laboratories, Inc.

M.C. Boyle, D.V.M., National Toxicology Program

S.A. Elmore, D.V.M., M.S., National Toxicology Program

R.A. Herbert, D.V.M., Ph.D., National Toxicology Program

M.J. Hoenerhoff, D.V.M., Ph.D., National Toxicology Program

D.E. Malarkey, D.V.M., Ph.D., National Toxicology Program

R.A. Miller, D.V.M., Ph.D., Experimental Pathology Laboratories, Inc.

J.P. Nold, D.V.M., Biotechnics

A. Nyska, D.V.M., ILS, Inc. 
SRA International, Inc., Research Triangle Park, North Carolina, USA

Provided statistical analyses

R.W. Morris, Ph.D., Principal Investigator

L.J. Betz, M.S.

S.F. Harris, B.S.

Dynamac Corporation, Research Triangle Park, North Carolina, USA

Prepared quality assessment audits

S. Brecher, Ph.D., Principal Investigator

S. Iyer, B.S.

V.S. Tharakan, D.V.M.

Biotechnical Services, Inc., Little Rock, Arkansas, USA

Prepared Technical Report

S.R. Gunnels, M.A., Principal Investigator

P.A. Gideon, B.A.

L.M. Harper, B.S.

T.S. Kumpe, M.A.

D.C. Serbus, Ph.D. 


\section{Explanation of Levels of Evidence of Carcinogenic Activity}

The National Toxicology Program describes the results of individual experiments on a chemical agent and notes the strength of the evidence for conclusions regarding each study. Negative results, in which the study animals do not have a greater incidence of neoplasia than control animals, do not necessarily mean that a chemical is not a carcinogen, inasmuch as the experiments are conducted under a limited set of conditions. Positive results demonstrate that a chemical is carcinogenic for laboratory animals under the conditions of the study and indicate that exposure to the chemical has the potential for hazard to humans. Other organizations, such as the International Agency for Research on Cancer, assign a strength of evidence for conclusions based on an examination of all available evidence, including animal studies such as those conducted by NTP, epidemiologic studies, and estimates of exposure. Thus, the actual determination of risk to humans from chemicals found to be carcinogenic in laboratory animals requires a wider analysis that extends beyond the purview of these studies.

Five categories of evidence of carcinogenic activity are used in the Technical Report series to summarize the strength of evidence observed in each experiment: two categories for positive results (clear evidence and some evidence); one category for uncertain findings (equivocal evidence); one category for no observable effects (no evidence); and one category for experiments that cannot be evaluated because of major flaws (inadequate study). These categories of interpretative conclusions were first adopted in June 1983 and then revised on March 1986 for use in the Technical Report series to incorporate more specifically the concept of actual weight of evidence of carcinogenic activity. For each separate experiment (male rats, female rats, male mice, female mice), one of the following five categories is selected to describe the findings. These categories refer to the strength of the experimental evidence and not to potency or mechanism.

- Clear evidence of carcinogenic activity is demonstrated by studies that are interpreted as showing a dose-related (i) increase of malignant neoplasms, (ii) increase of a combination of malignant and benign neoplasms, or (iii) marked increase of benign neoplasms if there is an indication from this or other studies of the ability of such tumors to progress to malignancy.

- Some evidence of carcinogenic activity is demonstrated by studies that are interpreted as showing a chemical-related increased incidence of neoplasms (malignant, benign, or combined) in which the strength of the response is less than that required for clear evidence.

- Equivocal evidence of carcinogenic activity is demonstrated by studies that are interpreted as showing a marginal increase of neoplasms that may be chemical related.

- No evidence of carcinogenic activity is demonstrated by studies that are interpreted as showing no chemical-related increases in malignant or benign neoplasms

- Inadequate study of carcinogenic activity is demonstrated by studies that, because of major qualitative or quantitative limitations, cannot be interpreted as valid for showing either the presence or absence of carcinogenic activity.

For studies showing multiple chemical-related neoplastic effects that if considered individually would be assigned to different levels of evidence categories, the following convention has been 
adopted to convey completely the study results. In a study with clear evidence of carcinogenic activity at some tissue sites, other responses that alone might be deemed some evidence are indicated as "were also related" to chemical exposure. In studies with clear or some evidence of carcinogenic activity, other responses that alone might be termed equivocal evidence are indicated as "may have been” related to chemical exposure.

When a conclusion statement for a particular experiment is selected, consideration must be given to key factors that would extend the actual boundary of an individual category of evidence. Such consideration should allow for incorporation of scientific experience and current understanding of long-term carcinogenesis studies in laboratory animals, especially for those evaluations that may be on the borderline between two adjacent levels. These considerations should include:

- adequacy of the experimental design and conduct;

- occurrence of common versus uncommon neoplasia;

- progression (or lack thereof) from benign to malignant neoplasia as well as from preneoplastic to neoplastic lesions;

- some benign neoplasms have the capacity to regress but others (of the same morphologic type) progress. At present, it is impossible to identify the difference. Therefore, where progression is known to be a possibility, the most prudent course is to assume that benign neoplasms of those types have the potential to become malignant;

- combining benign and malignant tumor incidence known or thought to represent stages of progression in the same organ or tissue;

- latency in tumor induction;

- multiplicity in site-specific neoplasia;

- metastases;

- supporting information from proliferative lesions (hyperplasia) in the same site of neoplasia or other experiments (same lesion in another sex or species);

- $\quad$ presence or absence of dose relationships;

- statistical significance of the observed tumor increase;

- concurrent control tumor incidence as well as the historical control rate and variability for a specific neoplasm;

- survival-adjusted analyses and false positive or false negative concerns;

- $\quad$ structure-activity correlations; and

- in some cases, genetic toxicology. 


\section{Peer Review}

The draft NTP Technical Report on the Toxicology Studies of Indole-3-carbinol (CASRN 700-061) in F344/N Rats and B6C3F1/N Mice and Toxicology and Carcinogenesis Studies of Indole-3carbinol in Harlan Sprague Dawley Rats and B6C3F1/N Mice (Gavage Studies) was evaluated by the reviewers listed below. These reviewers served as independent scientists, not as representatives of any institution, company, or governmental agency. In this capacity, reviewers determined if the design and conditions of these NTP studies were appropriate and ensured that this NTP Technical Report presents the experimental results and conclusions fully and clearly.

\section{Peer Reviewers}

Hillary M. Carpenter, III, Ph.D., Chairperson

Minnesota Department of Health

Maplewood, Minnesota, USA

Michael W. Conner, D.V.M.

Theravance, Inc.

San Francisco, California, USA

Michelle V. Fanucchi, Ph.D.

School of Public Health

University of Alabama

Birmingham, Alabama, USA

Charles R. Mahrt, D.V.M., M.S., Ph.D.

Eli Lilly and Company

Indianapolis, Indiana, USA

Jon C. Mirsalis, Ph.D., Primary Reviewer

SRI International

Menlo Park, California, USA

Gary H. Perdew, Ph.D., Primary Reviewer

College of Agricultural Sciences

Pennsylvania State University

State College, Pennsylvania, USA

Karen Regan, D.V.M., Primary Reviewer

Regan Pathology/Toxicology Services, Inc.

Ashland, Ohio, USA 


\section{Publication Details}

Publisher: National Toxicology Program

Publishing Location: Research Triangle Park, NC

ISSN: 2378-8925

DOI: https://doi.org/10.22427/NTP-TR-584

Report Series: NTP Technical Report Series

Report Series Number: 584

Official citation: National Toxicology Program (NTP). 2017. NTP technical report on the toxicology studies of indole-3-carbinol (CASRN 700-06-1) in F344/N Rats and B6C3F1/N mice and toxicology and carcinogenesis studies of indole-3-carbinol in Harlan Sprague Dawley rats and B6C3F1/N mice (gavage studies). Research Triangle Park, NC: National Toxicology Program. Technical Report 584. 


\section{Abstract}

Indole-3-carbinol is sold as a sole ingredient in dietary supplements or as a combination nutraceutical along with a variety of herbs and/or vitamins. It is marketed for its potential ability to prevent cancer and provide other health benefits, such as detoxifying the liver and boosting the immune system. Indole-3-carbinol is a naturally formed breakdown product of glucosinolate glucobrassicin, a component found in cruciferous vegetables of the Brassica genus, including broccoli, brussels sprouts, cauliflower, cabbage, kale, kohlrabi, and turnips. Exposure to indole3-carbinol occurs through the oral route through the ingestion of Brassica vegetables or dietary supplements. Indole-3-carbinol was nominated by the National Cancer Institute for toxicity and carcinogenicity testing because of its occurrence in natural products and for its potential use as a breast cancer chemopreventive agent. Male and female F344/N rats and Harlan Sprague Dawley rats received indole-3-carbinol in corn oil by gavage for 3 months or 2 years, respectively. Male and female B6C3F1/N mice received indole-3-carbinol in corn oil gavage for 3 months or 2 years. Genetic toxicology studies were conducted in Salmonella typhimurium and Escherichia coli, rat bone marrow cells, and mouse peripheral blood erythrocytes.

\section{Three-month Study in F344/N Rats}

Groups of 10 male and 10 female core study rats were administered $0,18.75,37.5,75$, 150, or $300 \mathrm{mg}$ indole-3-carbinol/kg body weight in corn oil by gavage, 5 days per week for 14 weeks. Groups of 10 male and 10 female clinical pathology study rats were administered the same dose for 25 days. All rats survived to the end of the study. The mean body weight gain of $300 \mathrm{mg} / \mathrm{kg}$ males was significantly less than that of the vehicle controls. The absolute and relative liver weights of all dosed groups of males and females were significantly increased compared to the vehicle controls. The relative kidney weights of $75 \mathrm{mg} / \mathrm{kg}$ or greater males and all dosed groups of females were significantly increased, as were the absolute kidney weights of $75 \mathrm{mg} / \mathrm{kg}$ males and 18.75, 37.5, and $300 \mathrm{mg} / \mathrm{kg}$ females. The absolute and relative thymus weights of $75 \mathrm{mg} / \mathrm{kg}$ or greater females were significantly decreased. There were significant and dose-dependent increases in CYP1A1-associated 7-ethoxyresorufin-O-deethylase (EROD) and CYP1A2associated acetanilide-4-hydroxylase $(\mathrm{A} 4 \mathrm{H})$ activities in the liver of all dosed groups of male and females rats. Pulmonary EROD activity was significantly increased in males administered $75 \mathrm{mg} / \mathrm{kg}$ or greater and in all dosed groups of females. Indole-3-carbinol exhibited the potential to be a reproductive toxicant in female rats based on a significantly increased probability of extended diestrus and an increase in overall estrous cycle length (approximately 1 day) observed at $300 \mathrm{mg} / \mathrm{kg}$. In the small intestine, significantly increased incidences of lamina propria lipidosis and lymphatic ectasia occurred in the duodenum of 150 and $300 \mathrm{mg} / \mathrm{kg}$ males and females and in the jejunum of $75 \mathrm{mg} / \mathrm{kg}$ or greater males and females. In the mesenteric lymph node, significantly increased incidences of dilatation of the lymphatic vessels associated with lipidosis occurred in 150 and $300 \mathrm{mg} / \mathrm{kg}$ males and in $300 \mathrm{mg} / \mathrm{kg}$ females.

\section{Three-month Study in B6C3F1/N Mice}

Groups of 10 male and 10 female mice were administered 0, 15.6, 31.25, 62.5, 125, or $250 \mathrm{mg}$ indole-3-carbinol/kg body weight in corn oil by gavage, 5 days per week for 14 weeks. All mice survived to the end of the study. Mean body weights of dosed groups of males and females were similar to those of the vehicle controls. Liver weights of 125 and $250 \mathrm{mg} / \mathrm{kg}$ males and all dosed groups of females were significantly increased compared to the vehicle controls. There were significant and dose-dependent increases in A4H activities in the liver of all dosed groups of 
males, and hepatic EROD activities were significantly increased in males administered $31.25 \mathrm{mg} / \mathrm{kg}$ or greater. Hepatic A4H and EROD activities were significantly increased in 125 and $250 \mathrm{mg} / \mathrm{kg}$ females. Indole-3-carbinol exhibited the potential to be a reproductive toxicant in male and female mice based on significantly decreased sperm motility in all dosed groups of males and a significantly increased probability of extended diestrus in females administered $250 \mathrm{mg} / \mathrm{kg}$. No histopathologic lesions were observed that could be attributed to the administration of indole-3-carbinol.

\section{Two-year Study in Sprague Dawley Rats}

Groups of 50 male and 50 female rats were administered 0, 75, 150, or $300 \mathrm{mg}$ indole-3carbinol/kg body weight in corn oil by gavage, 5 days per week for 104 (males) or 105 (females) weeks. Survival of dosed groups of males and females was similar to that of the vehicle controls. Mean body weights of dosed groups of males and females were similar to those of the vehicle controls throughout the study.

In the standard evaluation of the uterus, the incidences of adenocarcinoma occurred with a positive trend and were increased in all dosed groups. Extended evaluations of the uterus were conducted and additional neoplasms were identified. In the combined standard and extended evaluations, the incidence of adenocarcinoma was significantly increased in $150 \mathrm{mg} / \mathrm{kg}$ females. In the standard evaluation, the incidence of squamous metaplasia of the endometrium was significantly increased in the $150 \mathrm{mg} / \mathrm{kg}$ group.

The incidences of fibroma or fibrosarcoma (combined) in the skin occurred with a positive trend in females. An increased incidence of fibroma in the skin was observed in $300 \mathrm{mg} / \mathrm{kg}$ females. A single incidence of fibrosarcoma occurred in vehicle control and in $300 \mathrm{mg} / \mathrm{kg}$ females.

Significantly increased incidences of lymphatic ectasia in the duodenum and jejunum of the small intestine with generally increased severities occurred in 150 and $300 \mathrm{mg} / \mathrm{kg}$ males and females.

The incidences of lymphatic ectasia in the mesenteric lymph node were significantly increased in $300 \mathrm{mg} / \mathrm{kg}$ males and females compared to the vehicle controls.

In the liver, the incidences of clear cell focus in $300 \mathrm{mg} / \mathrm{kg}$ females, eosinophilic focus in 150 and $300 \mathrm{mg} / \mathrm{kg}$ females, and bile duct cyst in $300 \mathrm{mg} / \mathrm{kg}$ males were significantly increased compared to the vehicle controls.

Gene expression studies in $300 \mathrm{mg} / \mathrm{kg}$ females suggested activation of multiple xenobiotic transcription factors in rat liver with the most pronounced activation being associated with AhR and Nrf2. Consistent with these findings was the up-regulation of genes associated with xenobiotic metabolism, which suggests the potential for indole-3-carbinol to modify drug efficacy and safety. These findings are largely similar to results from other transcriptomic studies of indole-3-carbinol.

The incidences of follicular cell hypertrophy in the thyroid gland were significantly increased in all dosed groups of males, and the severities of the lesion increased with increasing dose.

\section{Two-year Study in B6C3F1/N Mice}

Groups of 50 male and 50 female mice were administered 0, 62.5, 125, or 250 mg indole-3carbinol/kg body weight in corn oil by gavage, 5 days per week for 105 weeks. Survival of $250 \mathrm{mg} / \mathrm{kg}$ females was significantly greater than that of the vehicle controls. Mean body 
weights of dosed groups of males were similar to those of the vehicle controls throughout the study; however, those of $250 \mathrm{mg} / \mathrm{kg}$ female mice were at least $10 \%$ less than those of the vehicle controls between weeks 32 and 92 .

Incidences of hepatocellular adenoma occurred with a positive trend in males and the incidence was significantly increased in the $250 \mathrm{mg} / \mathrm{kg}$ group. The incidences of multiple hepatocellular adenoma were significantly increased in 62.5 and $250 \mathrm{mg} / \mathrm{kg}$ males. There were significantly increased incidences of single and multiple hepatocellular carcinoma in $125 \mathrm{mg} / \mathrm{kg}$ males compared to the vehicle controls. In males, the incidences of hepatoblastoma occurred with a positive trend, and the incidences of multiple hepatoblastoma increased with increasing dose. The incidences of hepatoblastoma and multiple hepatoblastoma were significantly increased in $250 \mathrm{mg} / \mathrm{kg}$ males. The combined incidences of hepatocellular adenoma, hepatocellular carcinoma, or hepatoblastoma occurred with a positive trend in males and were significantly increased in males administered 125 or $250 \mathrm{mg} / \mathrm{kg}$. The combined incidences of hepatocellular carcinoma and hepatoblastoma were significantly increased in 125 and $250 \mathrm{mg} / \mathrm{kg}$ males. The incidences of clear cell focus were significantly increased in all dosed groups of males, and the incidences of eosinophilic focus were significantly increased in 62.5 and $125 \mathrm{mg} / \mathrm{kg}$ females.

In the glandular stomach, the incidences of epithelium hyperplasia, chronic inflammation, and pigmentation were significantly increased in 125 and $250 \mathrm{mg} / \mathrm{kg}$ males and all dosed groups of females compared to the vehicle controls.

In the nose, incidences of nerve atrophy in $250 \mathrm{mg} / \mathrm{kg}$ males and females, respiratory metaplasia of the olfactory epithelium in $250 \mathrm{mg} / \mathrm{kg}$ males and 125 and $250 \mathrm{mg} / \mathrm{kg}$ females, atrophy of the olfactory epithelium in 125 and $250 \mathrm{mg} / \mathrm{kg}$ males and $250 \mathrm{mg} / \mathrm{kg}$ females, necrosis of the olfactory epithelium in $250 \mathrm{mg} / \mathrm{kg}$ males, respiratory epithelium hyaline droplet accumulation in 62.5 and $125 \mathrm{mg} / \mathrm{kg}$ males, respiratory epithelium hyperplasia in $250 \mathrm{mg} / \mathrm{kg}$ males and females, and inflammation in $250 \mathrm{mg} / \mathrm{kg}$ females were significantly greater than the vehicle control incidences.

\section{Genetic Toxicology}

Indole-3-carbinol was tested in three independent bacterial mutagenicity assays, and results were varied. Two assays yielded results that were judged to be equivocal in one or more of the tester strains (S. typhimurium strains TA97 and TA100 and E. coli strain WP2 uvrA/pKM101). Weak positive responses were seen in a third assay in S. typhimurium strain TA100, both with and without exogenous metabolic activation. In vivo, no increase in the frequency of micronucleated polychromatic erythrocytes (PCEs) was seen in bone marrow of F344/N rats given three doses of indole-3-carbinol by gavage; however, a significant decrease in the percent PCEs was seen in the bone marrow of treated rats, indicating that indole-3-carbinol (500 to 2,000 mg/kg per day) was toxic to the bone marrow. In addition to the rat study, micronucleus frequencies in normochromatic erythrocytes (NCEs) of male and female mice were assessed in peripheral blood at the end of the 3-month study; no significant increases in micronucleated NCEs were seen and no significant changes in percent PCEs occurred over the dose range tested (15.6 to $250 \mathrm{mg} / \mathrm{kg}$ per day).

\section{Conclusions}

Under the conditions of these 2-year gavage studies, there was no evidence of carcinogenic activity (see Explanation of Levels of Evidence of Carcinogenic Activity; a summary of the Peer Review Panel comments and the public discussion on this Technical Report appears in 
Appendix M) of indole-3-carbinol in male Harlan Sprague Dawley rats administered 75, 150, or $300 \mathrm{mg} / \mathrm{kg}$. There was some evidence of carcinogenic activity of indole-3-carbinol in female Harlan Sprague Dawley rats based on increased incidences of malignant uterine neoplasms (primarily adenocarcinoma). The occurrences of fibroma and fibrosarcoma in the skin may have been related to indole-3-carbinol administration. There was clear evidence of carcinogenic activity of indole-3-carbinol in male B6C3F1/N mice based on increased incidences of liver neoplasms (hepatocellular adenoma, hepatocellular carcinoma, and hepatoblastoma). There was no evidence of carcinogenic activity of indole-3-carbinol in female B6C3F1/N mice administered $62.5,125$, or $250 \mathrm{mg} / \mathrm{kg}$.

Administration of indole-3-carbinol caused increased incidences of nonneoplastic lesions in the small intestine, mesenteric lymph node, and liver of male and female rats, the thyroid gland of male rats, the uterus of female rats, and the liver, glandular stomach, and nose of male and female mice.

Synonyms: 3-(Hydroxymethyl)indole; indole-3-methanol; 3-indolemethanol; 3-indolylcarbinol; 3-indolylmethanol 
Indole-3-carbinol, NTP TR 584

Summary of the Two-year Carcinogenesis and Genetic Toxicology Studies of Indole-3-carbinol

\begin{tabular}{|c|c|c|c|c|}
\hline & $\begin{array}{c}\text { Male } \\
\text { Sprague Dawley } \\
\text { Rats }\end{array}$ & $\begin{array}{c}\text { Female } \\
\text { Sprague Dawley } \\
\text { Rats }\end{array}$ & $\begin{array}{c}\text { Male } \\
\text { B6C3F1/N Mice }\end{array}$ & $\begin{array}{c}\text { Female } \\
\text { B6C3F1/N Mice }\end{array}$ \\
\hline $\begin{array}{l}\text { Doses in Corn Oil } \\
\text { By Gavage }\end{array}$ & $\begin{array}{l}0,75,150, \text { or } \\
300 \mathrm{mg} / \mathrm{kg}\end{array}$ & $\begin{array}{l}0,75,150, \text { or } \\
300 \mathrm{mg} / \mathrm{kg}\end{array}$ & $\begin{array}{l}0,62.5,125 \text {, or } \\
250 \mathrm{mg} / \mathrm{kg}\end{array}$ & $\begin{array}{l}0,62.5,125 \text {, or } \\
250 \mathrm{mg} / \mathrm{kg}\end{array}$ \\
\hline Survival Rates & $\begin{array}{l}20 / 50,13 / 50,17 / 50 \\
12 / 50\end{array}$ & $\begin{array}{l}21 / 50,19 / 50,20 / 50 \\
30 / 50\end{array}$ & $\begin{array}{l}27 / 50,31 / 50,32 / 50 \\
32 / 50\end{array}$ & $\begin{array}{l}33 / 50,40 / 50,26 / 50 \\
45 / 50\end{array}$ \\
\hline Body Weights & $\begin{array}{l}\text { Dosed groups similar } \\
\text { to the vehicle control } \\
\text { group }\end{array}$ & $\begin{array}{l}\text { Dosed groups similar } \\
\text { to the vehicle control } \\
\text { group }\end{array}$ & $\begin{array}{l}\text { Dosed groups similar } \\
\text { to the vehicle control } \\
\text { group }\end{array}$ & $\begin{array}{l}250 \mathrm{mg} / \mathrm{kg} \text { group at } \\
\text { least } 10 \% \text { less than } \\
\text { the vehicle control } \\
\text { group between } \\
\text { weeks } 32 \text { and } 92\end{array}$ \\
\hline $\begin{array}{l}\text { Nonneoplastic } \\
\text { Effects }\end{array}$ & $\begin{array}{l}\text { Intestine small, } \\
\text { duodenum: } \\
\text { lymphatic, ectasia } \\
\text { (0/43, 0/48, 15/47, } \\
\text { 14/48) } \\
\text { Intestine small, } \\
\text { jejunum: lymphatic, } \\
\text { ectasia (0/40, 2/39, } \\
\text { 27/40, 41/42) } \\
\text { Lymph node, } \\
\text { mesenteric: } \\
\text { lymphatic, ectasia } \\
\text { (0/50, 0/50, 1/50, } \\
\text { 5/50) } \\
\text { Liver: bile duct, cyst } \\
\text { (0/50, 0/50, 2/50, } \\
\text { 5/50) } \\
\text { Thyroid gland: } \\
\text { follicular cell, } \\
\text { hypertrophy (21/50, } \\
\text { 34/46, 33/48, 36/47) }\end{array}$ & $\begin{array}{l}\text { Uterus: endometrium, } \\
\text { metaplasia, squamous } \\
\text { (standard evaluation- } \\
\text { 12/50, 18/50, 20/50, } \\
\text { 11/50) } \\
\text { Intestine small, } \\
\text { duodenum: } \\
\text { lymphatic, ectasia } \\
\text { (0/48, 0/47, 16/48, } \\
\text { 38/47) } \\
\text { Intestine small, } \\
\text { jejunum: lymphatic, } \\
\text { ectasia (0/47, 0/46, } \\
\text { 30/48, 47/48) } \\
\text { Lymph node, } \\
\text { mesenteric: } \\
\text { lymphatic, ectasia } \\
\text { (0/50, 0/50, 1/50, } \\
\text { 15/48) } \\
\text { Liver: clear cell focus } \\
\text { (6/50, 7/50, 4/50, } \\
\text { 18/48); eosinophilic } \\
\text { focus (0/50, 4/50, } \\
\text { 5/50, 6/48) }\end{array}$ & $\begin{array}{l}\text { Liver: clear cell focus } \\
\text { (7/50, 17/50, 22/49, } \\
\text { 20/50) } \\
\text { Glandular stomach: } \\
\text { epithelium, } \\
\text { hyperplasia (0/50, } \\
\text { 1/47, 22/47, 40/49); } \\
\text { inflammation, chronic } \\
\text { (1/50, 1/47, 18/47, } \\
\text { 45/49); pigmentation } \\
\text { (0/50, 1/47, 38/47, } \\
\text { 48/49) } \\
\text { Nose: nerve, atrophy } \\
\text { (0/50, 0/50, 0/50, } \\
\text { 8/50); olfactory } \\
\text { epithelium, } \\
\text { respiratory metaplasia } \\
\text { (14/50, 14/50, 20/50, } \\
\text { 27/50); olfactory } \\
\text { epithelium, atrophy } \\
\text { (3/50, 5/50, 11/50, } \\
\text { 17/50); olfactory } \\
\text { epithelium, necrosis } \\
\text { (0/50, 0/50, 0/50, } \\
\text { 6/50); respiratory } \\
\text { epithelium, } \\
\text { accumulation, hyaline } \\
\text { droplet (18/50, 34/50, } \\
\text { 30/50, 26/50); } \\
\text { respiratory } \\
\text { epithelium, } \\
\text { hyperplasia (35/50, } \\
\text { 40/50, 41/50, 45/50) }\end{array}$ & $\begin{array}{l}\text { Liver: eosinophilic } \\
\text { focus }(16 / 50,26 / 50 \text {, } \\
\text { 26/50, 21/50) } \\
\text { Glandular stomach: } \\
\text { epithelium, } \\
\text { hyperplasia (1/48, } \\
\text { 7/50, 10/49, 35/50); } \\
\text { inflammation, chronic } \\
\text { (0/48, 15/50, 29/49, } \\
\text { 47/50); pigmentation } \\
\text { (0/48, 15/50, 31/49, } \\
\text { 49/50) } \\
\text { Nose: nerve, atrophy } \\
\text { (0/50, 0/50, 1/50, } \\
\text { 50/50); olfactory } \\
\text { epithelium, } \\
\text { respiratory metaplasia } \\
\text { (7/50, 8/50, 16/50, } \\
\text { 49/50); olfactory } \\
\text { epithelium, atrophy } \\
\text { (1/50, 2/50, 3/50, } \\
\text { 45/50); respiratory } \\
\text { epithelium, } \\
\text { hyperplasia (32/50, } \\
\text { 31/50, 38/50, 50/50); } \\
\text { inflammation (4/50, } \\
\text { 1/50, 8/50, 39/50) }\end{array}$ \\
\hline
\end{tabular}


Indole-3-carbinol, NTP TR 584

\begin{tabular}{|c|c|c|c|c|}
\hline & $\begin{array}{c}\text { Male } \\
\text { Sprague Dawley } \\
\text { Rats }\end{array}$ & $\begin{array}{c}\text { Female } \\
\text { Sprague Dawley } \\
\text { Rats }\end{array}$ & $\begin{array}{c}\text { Male } \\
\text { B6C3F1/N Mice }\end{array}$ & $\begin{array}{c}\text { Female } \\
\text { B6C3F1/N Mice }\end{array}$ \\
\hline Neoplastic Effects & None & $\begin{array}{l}\text { Uterus: } \\
\text { adenocarcinoma } \\
\text { (standard evaluation- } \\
\text { 0/50, 1/50, 4/50, 4/50; } \\
\text { standard and extended } \\
\text { evaluations, } \\
\text { combined-5/50, 4/50, } \\
\text { 13/50, 10/50) }\end{array}$ & $\begin{array}{l}\text { Liver: hepatocellular } \\
\text { adenoma (26/50, } \\
\text { 32/50, 31/49, 41/50); } \\
\text { hepatocellular } \\
\text { carcinoma (12/50, } \\
\text { 11/50, 29/49, 14/50); } \\
\text { hepatoblastoma (3/50, } \\
\text { 4/50, 4/49, 14/50); } \\
\text { hepatocellular } \\
\text { adenoma, } \\
\text { hepatocellular } \\
\text { carcinoma, or } \\
\text { hepatoblastoma } \\
\text { (36/50, 36/50, 44/49, } \\
\text { 45/50) }\end{array}$ & None \\
\hline Equivocal Findings & None & $\begin{array}{l}\text { Skin: fibroma }(1 / 50 \text {, } \\
\text { 0/50, } 0 / 50,4 / 50) ; \\
\text { fibrosarcoma }(1 / 50 \text {, } \\
0 / 50,0 / 50,1 / 50) ; \\
\text { fibroma or } \\
\text { fibrosarcoma }(2 / 50 \text {, } \\
0 / 50,0 / 50,5 / 50)\end{array}$ & None & None \\
\hline $\begin{array}{l}\text { Level of Evidence of } \\
\text { Carcinogenic } \\
\text { Activity }\end{array}$ & No evidence & Some evidence & Clear evidence & No evidence \\
\hline
\end{tabular}

\section{Genetic Toxicology}

Bacterial Gene Mutations:

Negative in Salmonella typhimurium strains TA98, TA102, TA104, TA1535, and TA1537 with S9 and without S9; equivocal in strain TA97 without S9; equivocal in one study in strain TA100 with 10\% rat S9; weakly positive in a second study in strain TA100 with and without $30 \%$ hamster S9; equivocal in Escherichia coli strain WP2 uvrA/pKM101 without S9.

Micronucleated Erythrocytes

Rat Bone Marrow In Vivo:

Negative

Mouse Peripheral Blood In Vivo:

Negative 


\section{Introduction}

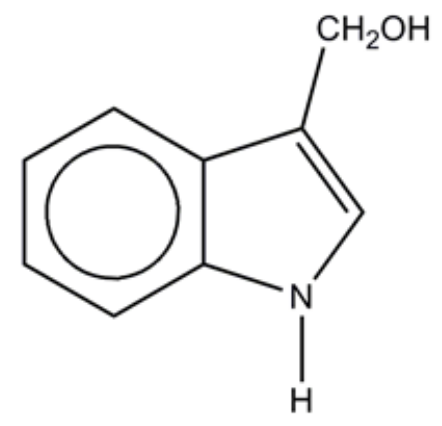

Figure 1. Indole-3-carbinol (CASRN 700-06-1; Chemical Formula: $\mathrm{C}_{9} \mathrm{H}_{9} \mathrm{NO}$; Molecular Weight: 147.18)

Synonyms: 3-(Hydroxymethyl)indole; indole-3-methanol; 3-indolemethanol; 3-indolylcarbinol; 3-indolylmethanol.

\section{Chemical and Physical Properties}

Indole-3-carbinol is an off-white powder with a melting point of $96^{\circ}$ to $99^{\circ} \mathrm{C}^{1}$. It is soluble in benzene, ethanol, and pentane ${ }^{2}$. Indole-3-carbinol is an unstable compound that undergoes rapid oligomerization in acid $\mathrm{pH}$ environments, like the stomach. At low $\mathrm{pH}$, a wide variety of condensation products are formed, ranging from linear and cyclic dimers, trimers, and tetramers to extended heterocyclic compounds such as indolocarbazoles ${ }^{3-6}$.

\section{Production, Use, and Human Exposure}

Indole-3-carbinol is a naturally formed breakdown product of glucosinolate glucobrassicin, a component found in cruciferous vegetables in the Brassica genus, which includes brussels sprouts, cauliflower, cabbage, kale, rape, turnips, and broccoli. Although indole-3-carbinol is not naturally occurring in these vegetables, it is readily formed from glucobrassicin via a thioglucosidase-mediated autolytic process during the simple mechanics of cutting, chewing, mashing, or cooking. Myrosinase, an enzyme that is usually compartmentalized within the plant cells, is released when the cells are physically damaged. Free myrosinase readily catalyzes the hydrolysis of glucobrassicin to indole-3-carbinol ${ }^{7}$. Indole-3-carbinol is also marketed and promoted as a dietary supplement that is available at health food stores, pharmacies, and via the internet. Indole-3-carbinol may be sold as the sole ingredient in products or in combination nutraceuticals that contain a variety of botanicals and/or vitamins. The health claims for indole3-carbinol-containing supplements include cancer prevention, antioxidant protection, hormone balance, immune system enhancement, and hepatic and intestinal detoxification.

The most widespread exposure of humans to indole-3-carbinol occurs through the consumption of glucobrassicin, the indole-3-carbinol precursor found in Brassica vegetables. Brassica vegetables are frequently consumed in the diets of both Western and Eastern cultures ${ }^{6}$. In the United States population, the daily intake of indole-3-carbinol from cruciferous vegetables has been estimated to be $2.6 \mathrm{mg}$ or less ${ }^{2}$. Estimated mean daily intake of glucobrassicin in the United Kingdom is approximately 12.5 and $7 \mathrm{mg}$ per person from fresh and cooked sources, respectively, and the average daily consumption of indole-3-carbinol is approximately $0.1 \mathrm{mg} / \mathrm{kg}$ body weight. Based on levels of brassica consumption in the Japanese diet, the 
average daily intake of indole precursors can range up to $112 \mathrm{mg}$ per day, which corresponds to a daily dose of approximately $1.6 \mathrm{mg} / \mathrm{kg}$ for a $70 \mathrm{~kg}$ person ${ }^{8 ;}{ }^{\text {. Carlson et }}$ al. ${ }^{10}$ reported concentrations of 3-indolylmethyl glucosinolates in broccoli (42.2 to $71.7 \mathrm{mmol} / 100 \mathrm{~g}$ fresh weight), brussels sprouts (327.8 to $469.4 \mathrm{mmol} / 100 \mathrm{~g}$ ), cauliflower (18.8 to $104.7 \mathrm{mmol} / 100 \mathrm{~g})$, collard greens (67.2 to $165.3 \mathrm{mmol} / 100 \mathrm{~g})$, kale (44.2 to $102.3 \mathrm{mmol} / 100 \mathrm{~g}$ ), mustard greens (4.2 to $12.2 \mathrm{mmol} / 100 \mathrm{~g}$ ), and kohlrabi (27.7 mmol/100 g).

Humans are also exposed to indole-3-carbinol as a dietary supplement. The daily dose of 200 to $400 \mathrm{mg}$ that has been used to investigate the therapeutic value of indole-3-carbinol in clinical trials is also the recommended dose indicated by package labeling on indole-3-carbinol products. These daily exposures are equivalent to 2.9 to $5.7 \mathrm{mg} / \mathrm{kg}$ for a $70 \mathrm{~kg}$ person.

\section{Regulatory Status}

While the U.S. Food and Drug Administration (FDA) does not regulate food that contains precursors of indole-3-carbinol, compounds that are marketed as dietary supplements are regulated under the Dietary Supplement Health and Education Act of 1994. Indole-3-carbinol is not listed by the FDA as a Generally Recognized as Safe substance.

\section{Absorption, Distribution, Metabolism, and Excretion}

\section{Experimental Animals}

The disposition of indole-3-carbinol has been studied in experimental animals. In rainbow trout, $25 \%$ of an oral dose of $40 \mathrm{mg} / \mathrm{kg}\left[{ }^{3} \mathrm{H}\right.$ ] indole-3-carbinol given via feed or gavage was recovered in the water 72 hours following exposure reflecting the excretion via urine and gills ${ }^{11}$.

Radioactivity in the liver between 48 and 72 hours following exposure accounted for 1\% to 1.5\% of the total administered dose. In goats, approximately $80 \%$ of the dose was recovered in urine 72 hours following a 2-hour infusion of $0.27 \mathrm{mmol} / \mathrm{kg}\left[{ }^{3} \mathrm{H}\right]$ indole-3-carbinol. Following exposure of F344 rats to 2,000 ppm [ $\left.{ }^{3} \mathrm{H}\right]$ indole-3-carbinol in the diet, steady-state excretion in urine and feces was achieved at 40 hours and 112 hours, respectively, following the beginning of exposure. Excretion in these two routes accounted for approximately $75 \%$ of the dose with about $77 \%$ of the excreted dose recovered in the feces ${ }^{12}$. Although not demonstrated in rats, biliary excretion has been shown to be a major route of excretion in rainbow trout, suggesting that the high fecal excretion in rats could be due to biliary excretion and not due to poor absorption. Following repeated gavage administration of $1 \mathrm{mmol} / \mathrm{kg}$ indole-3-carbinol in rats (a comparable daily dose as in the feeding study above) for 6 days followed by 1 day of dosing with $\left[{ }^{3} \mathrm{H}\right]$ indole3-carbinol, indole-3-carbinol-equivalents in the liver at 1.5, 3, and 6 hours, respectively, were $0.97 \%, 1.34 \%$, and $2.45 \%$ of the total administered dose. However, the concentration of indole3-carbinol equivalents in blood, kidney, tongue, or lung changed slightly over the same time period. Of the total radioactive equivalents in the liver, the extractable radioactivity decreased from $45 \%$ to $31 \%$ from 1.5 to 6 hours. In female CD-1 mice following gavage administration of $250 \mathrm{mg} / \mathrm{kg}$, indole-3-carbinol was rapidly absorbed, and the peak plasma concentration was reached within 15 minutes $^{13}$. The concentration in plasma fell below the limit of detection 1 hour following administration suggesting rapid excretion. As observed in rats, indole-3-carbinol was extensively distributed to tissues including the kidney, liver, heart, and brain, with the highest levels detected in the liver. 
Acidic incubation of indole-3-carbinol in vitro resulted in the formation of multiple condensation

products $^{14-16}$. The major products formed were the dimer, 3,3'-diindolylmethane (DIM), and two trimers, [2-(indol-3-yl-methyl)-indol-3-yl]indol-3-ylmethane (LTR) and 5,6,11,12,17,18-hexahydrodiindolo [2',3':4,5;2",3':7,8] cyclonona[1,2-b]indole (CTI). The formation of oligo-mers was strongly $\mathrm{pH}$ dependent. Incubation of indole-3-carbinol at $\mathrm{pHs}$ less than 3 resulted in approximately equal formation of the three oligomers, whereas incubation at pHs greater than 3 produced large amounts of DIM and LTR but not CTI; CTI was detected at pHs greater than $4.5^{14}$.

The metabolism of indole-3-carbinol has been investigated in in vitro preparations of rat and chicken embryo liver ${ }^{6}$. These studies demonstrated that the first step in the metabolic route was the formation of indole-3-carboxaldehyde, via both the mixed function oxidase and alcohol dehydrogenase systems. Further metabolism by these systems resulted in the formation of 5hydroxy-indole-carboxaldehyde and indole-3-carbox-ylic acid.

The oligomeric products of indole-3-carbinol are thought to be primarily responsible for eliciting the biological effects observed for indole-3-carbinol. In rats and fish, DIM, LTR, and 1-(3hydroxymethyl)-indolyl-3-indolyl-methane were detected in the liver following oral administration of indole-3-carbinol; the parent indole-3-carbinol was not detected ${ }^{11 ; 12 ; 14}$. The pattern of condensation products in the gastric contents strongly resembled the pattern obtained after in vitro acid condensation at pHs between 4.5 and 5 . In extracts from stomach tissue, small intestine, and liver, the patterns of oligomers were similar to that found in the stomach contents ${ }^{14}$. In addition, indolo[3,2b] carbazole (ICZ), a potent aryl hydrocarbon receptor (AhR) agonist was also identified ${ }^{14}$. When male Sprague Dawley rats were fed dietary indole-3-carbinol or a cabbage-supplemented diet (25\% cabbage) for 5 days, ICZ levels were increased in tissues and excreta ${ }^{5}$. In mice as in rats, acid condensation products were detected in plasma and in some tissues; the parent was also detected in plasma, suggesting that indole-3-carbinol was not completely converted to acid condensation products in the gut. In mice, ICZ was detected only in the liver ${ }^{13}$. The peak plasma concentrations of DIM and LTR were approximately one sixth and one tenth that of indole-3-carbinol and the levels persisted much longer than the parent. In addition to the acid condensation products, minor oxidative metabolites of indole-3-carbinol, indol-3-carboxylic acid and indole-3-carboxalde-hyde were also detected in plasma from mice. Tissues profiles of metabolites were similar to the plasma profile suggesting that these metabolites were in equilibrium with the plasma ${ }^{13}$. The proposed metabolic scheme for indole-3carbinol following oral administration in rodents is shown in Figure 2.

\section{Humans}

No published data are available regarding the absorption, distribution, metabolism, or excretion of indole-3-carbinol in humans.

\section{Toxicity}

\section{Experimental Animals}

The results of short-term studies suggest that the liver is a target organ for indole-3-carbinol. In male guinea pigs, indole-3-carbinol exposure induced steatosis in the liver, primarily in periportal hepatocytes ${ }^{17}$. In male CD-1 mice exposed to $500 \mathrm{or} 750 \mathrm{mg} / \mathrm{kg}$ per day in the feed for 
5 days, indole-3-carbinol significantly increased liver weight and microsomal protein content ${ }^{18}$. This study also showed that indole-3-carbinol alters cholesterol homeostasis. Doses of 500 and $750 \mathrm{mg} / \mathrm{kg}$ caused significant increases in hepatic acyl-CoA:cholesterol acyltransferase activity and lowered hepatic cholesterol levels. In male CD-1 mice exposed to $100 \mathrm{mg}$ indole-3$\mathrm{carbinol} / \mathrm{kg}$ for 10 days, hepatotoxicity was indicated by reduced glutathione levels and dosedependent increases in plasma alanine aminotransferase and ornithine transcarbamylase activities ${ }^{19}$. This dose also elicited a 16 -fold increase in ethoxyresorufin-O-deethylase activity. Increased liver weights and hepatic cytochrome P450 (CYP) activities were also observed in male and female CD rats exposed to $50 \mathrm{mg}$ indole-3-carbinol/kg body weight ${ }^{20}$. In Sprague Dawley rats exposed to $50 \mathrm{mg} / \mathrm{kg}$, indole-3-carbinol significantly induced total hepatic CYP levels in both sexes ${ }^{21}$. Hepatic CYP1A1 and CYP1A2 were significantly induced in both sexes. Indole-3-carbinol also induced expression of hepatic CYP3A2 and CYP1A1 in the colon of both sexes of rats.

The hepatic induction of CYP1A by indole-3-carbinol is mediated via the AhR, a transcription factor that plays a critical mechanistic role in the toxicity and carcinogenicity of 2,3,7,8tetrachlorodibenzo-p-dioxin (TCDD). Indole-3-carbinol and its major acid condensation products are AhR agonists ${ }^{22-24}$. The toxicological implication of indole-3-carbinol-induced AhR activation is not clearly understood.

Studies also suggest that the nervous system is a target organ for indole-3-carbinol. In male Sprague Dawley rats treated with a single subcutaneous dose of 225 , 300, or $500 \mathrm{mg} / \mathrm{kg}$, indole3 -carbinol induced sedation ataxia and loss of righting reflex and sleep ${ }^{25}$. Three out of four rats died 1 to 3 hours after exposure to $500 \mathrm{mg} / \mathrm{kg}$; animals were comatose before death. In male CD1 mice administered doses of 100 to $500 \mathrm{mg} / \mathrm{kg}$, indole-3-carbinol produced dose-dependent increases in neurological impairment, primarily associated with locomotion, as measured by subjective evaluation of appearance, posture, and motor activities ${ }^{19}$. At $500 \mathrm{mg} / \mathrm{kg}$, animals became comatose. In male guinea pigs, clinical signs of intoxication were apparent within 48 hours of administration (per os) of $0.3 \mathrm{mg}$ indole-3-carbinol $/ \mathrm{kg}$ body weight in $10 \%$ Cremophor ${ }^{\circledR}$ castor oil ${ }^{17}$. Signs included moderate depression, trembling, tachypnea, polypnea, irregular breathing, and increased vesicular lung sounds. In the lung, indole-3-carbinol induced interstitial pneumonia with septal hyperemia. 


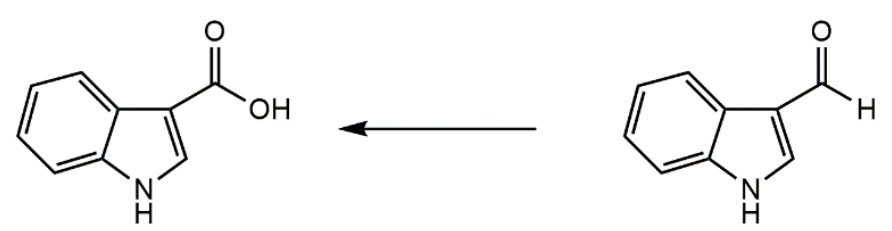

Indole-3-carboxylic acid

Indole-3-carboxaldehyde
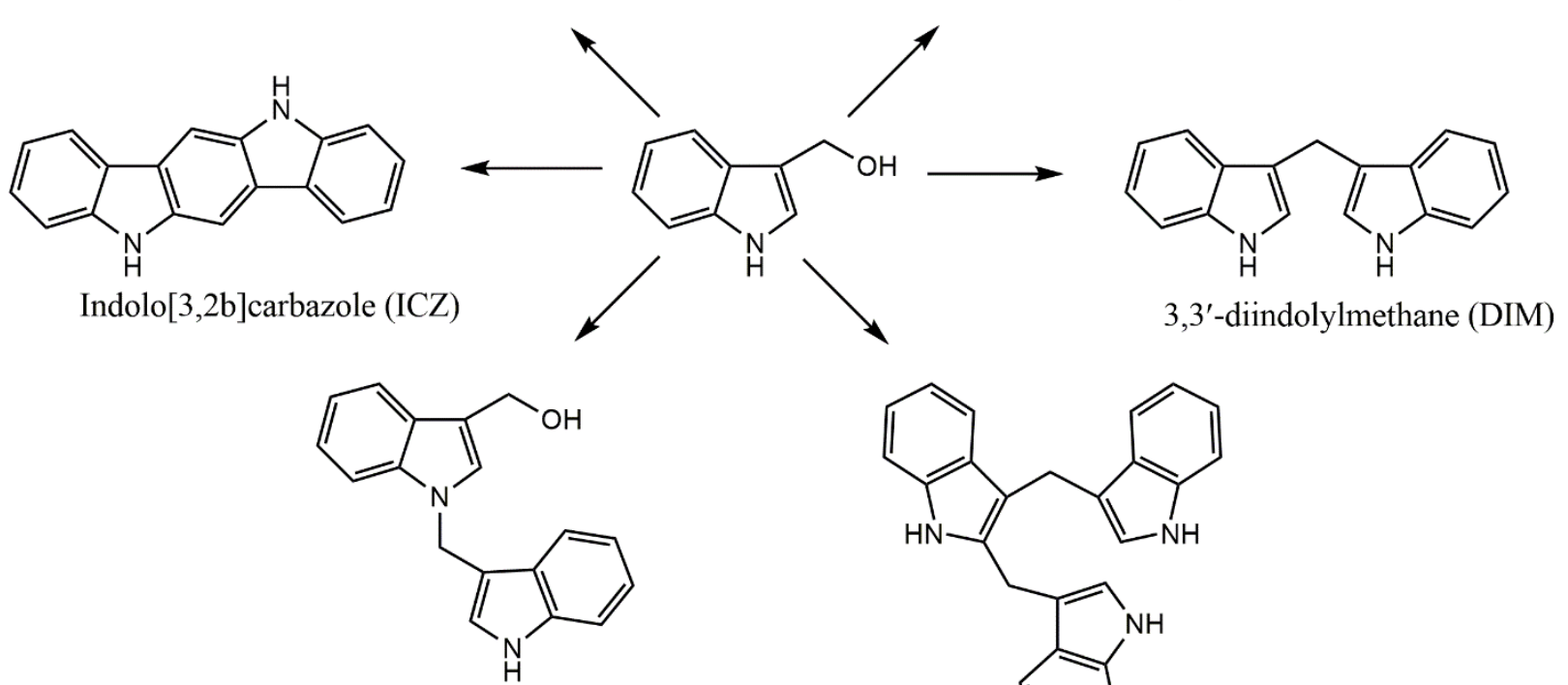

1-(3-hydroxymethyl)-indolyl-3-indolylmethane (HIIM)<smiles>c1ccc2c(Cc3[nH]c4ccccc4c3Cc3c[nH]c4ccccc34)c[nH]c2c1</smiles>

[2-(indol-3-yl-methyl)-indol-3-yl]indol-3-ylmethane (Linear trimer, LTR)

Figure 2. Proposed Metabolic Scheme for Indole-3-carbinol following Oral Administration in Rodents

Adapted from Stresser et al. ${ }^{12}$ and Anderton et al. ${ }^{13}$.

\section{Humans}

In general, most studies in humans have not reported overt toxicity of indole-3-carbinol. In a double-blind, placebo-controlled dose range-finding study, women at risk for breast cancer were administered up to $400 \mathrm{mg}$ indole-3-carbinol for 4 weeks $^{26}$. A slight increase in alanine aminotransferase, indicating hepatocellular membrane leakage, was observed in two participants (dose not specified). Doses of $300 \mathrm{mg}(4.2 \mathrm{mg} / \mathrm{kg}$ for a $70 \mathrm{~kg}$ person) increased the ratio of 2-hydroxyestrone to 16a-hydroxyestrone in the urine, which reflects a shift in estrogen metabolism towards less estrogenic metabolites. Similar results on urinary estrogen metabolites were observed in women with cervical dysplasia administered 200 or 400 mg indole-3carbinol/day ${ }^{27}$ and women with systemic lupus erythematosis administered $375 \mathrm{mg} /$ day for 3 months ${ }^{28}$. A single subject developed a skin rash that went away after cessation of treatment, and then reappeared after indole-3-carbinol was readministered ${ }^{28}$. 


\section{Reproductive and Developmental Toxicity}

\section{Experimental Animals}

Two investigative studies have reported on the results of in utero exposure to indole-3-carbinol on reproduction and development. Pregnant Harlan Sprague Dawley rats were administered indole-3-carbinol in corn oil via gavage (1 or $100 \mathrm{mg} / \mathrm{kg}$ body weight; three to five dams per dose group) on gestational day (GD) 15 and reproductive parameters were assessed in male offspring on postnatal day (PND) $62^{29}$. Indole-3-carbinol significantly decreased anogenital distance and crown-rump length on PND 1. When normalized to pup body weight on PND 1 however, there were no effects on these endpoints. Daily sperm production/g testicular parenchyma was significantly decreased at both doses of indole-3-carbinol; however, daily sperm production/testis was only reduced at $100 \mathrm{mg} / \mathrm{kg}$. Exposure did not adversely affect sperm number in the total epididymis or in the head plus body or tail of the epididymis, but did increase epididymal transit time of sperm by more than 1 day in rats exposed to $1 \mathrm{mg} / \mathrm{kg}$. Pregnant Holtzman rats (up to four dams/group) were administered either 200 or $300 \mathrm{mg}$ indole-3carbinol/kg on GDs 8 and $9^{25}$. Dams dosed with $300 \mathrm{mg} / \mathrm{kg}$ displayed lower body weight gain over GDs 9 to 11. Fetal weights on GD 20 were significantly depressed only in the $200 \mathrm{mg} / \mathrm{kg}$ group. There were no effects of indole-3-carbinol on embryonic death or fetal development.

\section{Humans}

No reports on the effect of indole-3-carbinol on reproduction or development in humans were found in the literature.

\section{Carcinogenicity}

\section{Experimental Animals}

No 2-year carcinogenicity studies of indole-3-carbinol in animals were found in the literature. However, several studies have investigated the effect of indole-3-carbinol on chemical-induced tumorigenesis. In initiation-promotion models of carcinogenesis, indole-3-carbinol is a tumor promoter that enhances colon tumors in rats and mice as well as pancreatic tumors in hamsters ${ }^{30}$; 31. Exposure to indole-3-carbinol promotes aflatoxin $\mathrm{B}_{1}$-initiated hepatocarcinogenesis in rainbow trout ${ }^{32}$ and thyroid gland tumors in Sprague Dawley rats ${ }^{33}$. In studies designed to assess inhibition, indole-3-carbinol did not induce tumors when administered without an initiator in Sprague Dawley rats, ACI/N rats, or ICR/Ha mice ${ }^{34-36}$. In female Donryu rats, a strain with a high incidence of endometrial cancer, exposure to 200, 500, or 1,000 ppm indole-3-carbinol in the diet for 660 days resulted in a dose-dependent decrease in the incidence of endometrial adenocarcinoma $^{37}$.

Dietary administration of indole-3-carbinol inhibited benzo[a]pyrene-induced neoplasia in the forestomach in ICR/Ha mice and 7,12-dimethylbenz[a]anthracene-induced mammary gland tumor formation in Sprague Dawley rats ${ }^{34}$. In female C3H/OuJ mice exposed to indole-3carbinol in the diet, mammary gland tumor incidence and multiplicity were significantly decreased, and tumor latency was prolonged ${ }^{38}$. In a three-generation study in Balb/cfC3H mice, exposure to indole-3-carbinol did not affect mammary gland tumor incidence, however the latency of mammary gland tumors was increased ${ }^{39}$. Inhibition of tumorigenesis has been 
observed for diethylnitrosamine-induced hepatocarcinogenesis in ACI/N and Sprague Dawley rats and in the infant mouse model ${ }^{35 ; 40 ; 41}$. Indole-3-carbinol also inhibits tumorigenesis in the lung in various animal models ${ }^{42-45}$.

\section{Humans}

Consumption of cruciferous vegetables has been associated with a decreased risk of cancer in humans $^{46-49}$. Based on epidemiologic evidence and results from animal studies, the National Research Council, Committee on Diet, Nutrition, and Cancer has recommended increased consumption of Brassica vegetables as a measure to decrease the incidence of human cancer. The anticarcinogenic activity of cruciferous vegetables has been attributed to indole-3-carbinol and its acid-condensation products ${ }^{3 ; 50-53}$.

Indole-3-carbinol has been promoted and investigated for treatment and prevention of various types of common cancers. Indole-3-carbinol has been shown to inhibit tumorigenesis in multiple tissues. As a result, it has been the focus of several early-phase clinical trials to assess efficacy in the treatment of breast cancer, cervical dysplasia, systemic lupus erythematosus, and recurrent respiratory papillomatosis in adults and children ${ }^{26-28 ; 52 ; 54-58}$. In general, doses in these clinical studies ranged between 200 and $400 \mathrm{mg}$ indole-3-carbinol per day.

\section{Genetic Toxicity}

The genotoxic potential of indole-3-carbinol has been evaluated in a limited number of bacterial mutagenicity assays and in vitro and in vivo mammalian cell tests. In the majority of tests, indole-3-carbinol gave negative results. However, indole-3-carbinol undergoes chemical reactions in the acidic environment of the stomach to produce compounds that are mutagenic or that can increase the mutagenic potential of other compounds.

Indole-3-carbinol was weakly mutagenic in Salmonella typhimurium strain TA100 [lowest effective dose (LED) of $312.5 \mu \mathrm{g} /$ plate] in the presence or absence of metabolic activation (S9 $\mathrm{mix})^{59}$. In contrast, no mutagenicity was detected with indole-3-carbinol in S. typhimurium strains TA98, TA1535, or TA1537 [highest ineffective dose (HID) of 1,250 $\mu \mathrm{g} / \mathrm{plate}$ ] or Escherichia coli strain WP2 uvrA/pKM101 (HID of 2,500 $\mu \mathrm{g} / \mathrm{plate}$ ), with or without S9 mix $^{59}$. Additionally, in a differential survival assay, E. coli deficient for nucleotide excision repair and recombinational repair were no more sensitive to indole-3-carbinol (5 $\mu \mathrm{g} / \mathrm{mL})$ than a strain proficient for these DNA repair pathways ${ }^{60}$.

Indole compounds present in Brassica vegetables (e.g., broccoli, brussels sprouts, and cabbage) may be precursors of mutagens formed during the process of digestion as, in the presence of nitrites, they can be converted to $\mathrm{N}$-nitroso compounds at low $\mathrm{pH}^{61-63}$. One study investigated whether indole-3-carbinol became mutagenic following reaction with sodium nitrite at $\mathrm{pH} 3$ at $37^{\circ} \mathrm{C}$. Under those conditions, indole-3-carbinol formed a nitrosated product that was reported to be directly mutagenic to E. coli WP2 uvrA/pKM101 (LED of $12.5 \mathrm{nmol}$ ) and to S. typhimurium strains TA98 and TA100 (LEDs of $12.5 \mathrm{nmol}$ and $50 \mathrm{nmol}$, respectively) ${ }^{64}$. Furthermore, the mutagenic effects of the nitrosated product were reduced to near-baseline levels in the presence of S9 mix, and the parent molecule, indole-3-carbinol, was not mutagenic in any of the three tester strains, with or without S9 mix $^{64}$. However, the authors did not report the concentration range that was used to study indole-3-carbinol and no actual data were provided, thus preventing independent assessment of the findings. 
No increase in mutant frequency was observed in mouse lymphoma L5178Y $t k^{+/}$cells treated with indole-3-carbinol in the presence of S9 (HID of $30 \mu \mathrm{g} / \mathrm{mL}$ ) or in the absence of S9 (HID of $325 \mu \mathrm{g} / \mathrm{mL})^{59}$. In vivo, no induction of chromosomal aberrations was observed in bone marrow cells of male Swiss albino mice following a single intraperitoneal injection of $500 \mathrm{mg} / \mathrm{kg}$ indole3 -carbinol ${ }^{65}$ or five daily exposures to $5 \mathrm{mg} / \mathrm{kg}$ indole-3-carbinol by gavage ${ }^{66}$. In a micronucleus assay, male and female Swiss-Webster mice were administered single doses of indole-3-carbinol $(375,750$, or $1,500 \mathrm{mg} / \mathrm{kg}$ ) by oral gavage and assessed at 24 or 48 hours postexposure for the frequency of micronucleated polychromatic erythrocytes (MN-PCE) in bone marrow ${ }^{59}$. Although no increases in the frequency of MN-PCEs were observed in male mice, a dose-dependent increase in the frequency of MN-PCEs was observed in female mice at 24 hours (LED of $750 \mathrm{mg} / \mathrm{kg})^{59}$.

Indole-3-carbinol has been studied for antimutagenic effects in bacterial mutagenicity assays and mammalian cell in vitro and in vivo assays. No clear evidence of antimutagenic activity was seen in the bacterial mutagenicity assays and effects were mixed for in vitro mammalian cell assays. Evidence of antimutagenicity in the in vivo studies is more consistent, with decreases reported for induction of cytogenetic damage (sister chromatid exchanges and micronuclei) and adduct formation in various tissues following treatment with known genotoxicants such as cyclophosphamide, benzo[a] pyrene, 2-amino-3-methylimidazo[4,5-f]quinoline, and tobacco smoke ${ }^{66-71}$.

In addition to N-nitrosation as described above, indole-3-carbinol can undergo acid condensation in the stomach to produce 3,3'-diindolylmethane. One study showed that 3,3'-diindolylmethane can increase the genotoxic effects of a known carcinogen, aflatoxin B1 (AFB1). Pre-incubating primary human hepatocytes for 48 hours with 3,3'-diindolylmethane at concentrations of 10 or $50 \mu \mathrm{M}$ increased the formation of AFB1-DNA adducts fourfold or sixfold, respectively ${ }^{72}$. Furthermore, incubation with 3,3'-diindolylmethane for 48 hours increased the expression of CYP1A1 and CYP1A2 and downregulated glutathione S-transferase mu 1 at the transcriptional level $^{72}$. Therefore, in primary human hepatocytes, 3,3'-diindolylmethane appears to increase the level of Phase I enzymes that produce the genotoxic form of AFB1 (AFB1-8,9-exo-epoxide) and to decrease one of the Phase II enzymes that can detoxify AFB1-8,9-exo-epoxide. No other studies on the genotoxic effects of 3,3'-diindolylmethane were identified.

In summary, results from the few evaluations of the genotoxicity of indole-3-carbinol that were identified in the literature were negative, except for one weakly positive bacterial mutagenicity test result and one study in which a significant increase was observed in MN-PCEs in female (but not male) mice following administration of indole-3-carbinol by oral gavage. In addition, several studies were identified that reported indole-3-carbinol-mediated inhibition of chemicalinduced genotoxicity in vivo. However, indole-3-carbinol may undergo reactions in the stomach to produce genotoxic compounds or compounds that can enhance the genotoxicity of other compounds through modulation of key metabolic processes.

\section{Study Rationale}

Indole-3-carbinol was nominated by the National Cancer Institute for toxicity and carcinogenicity testing because of its occurrence in natural products and its potential use as a breast cancer chemopreventive agent. There is very little known about the toxicity of long-term exposure. 


\section{Materials and Methods}

\section{Procurement and Characterization}

\section{Indole-3-carbinol}

Indole-3-carbinol was obtained from ChemPacific Corporation (Baltimore, MD) in one lot (CHP801001) that was used during the 3-month and 2-year studies. Identity and purity analyses were conducted by the analytical chemistry laboratory at Battelle Columbus Support Services (Columbus, $\mathrm{OH}$ ) and the study laboratories, Southern Research Institute (SRI) (Birmingham, AL) for the 3-month studies and Battelle Columbus Operations (Columbus, OH) for the 2-year studies (Appendix I). Karl Fischer titration and elemental analyses were performed by Galbraith Laboratories, Inc. (Knoxville, TN). Reports on analyses performed in support of the indole-3carbinol studies are on file at the National Institute of Environmental Health Sciences.

Lot CHP801001 of the test chemical, a yellow crystalline solid, was identified as indole-3carbinol by the analytical chemistry laboratory using infrared (IR) and proton and carbon-13 nuclear magnetic resonance (NMR) spectroscopy and by the study laboratories using IR and proton NMR (SRI only) spectroscopy. Karl Fischer titration was used to determine the water content; elemental analyses were used to determine the carbon, hydrogen, and nitrogen content; and the melting point was determined using a differential scanning calorimeter. The purity was determined by the analytical chemistry laboratory using high-performance liquid chromatography (HPLC) with ultraviolet detection. Karl Fischer titration indicated 1.3\% water. Elemental analyses for carbon, hydrogen, and nitrogen were consistent with the theoretical values, and the melting point was $99.3^{\circ} \mathrm{C}$, consistent with the manufacturer's Certificate of Analysis. HPLC analysis indicated one major peak with six impurities, each $0.1 \%$ or greater of the total peak area with a combined area of $2.8 \%$ of total peak area in the chromatogram. The overall purity of lot CHP801001 was determined to be approximately $97 \%$.

To ensure stability, the bulk chemical was stored at $-20^{\circ} \mathrm{C}$, protected from light in amber glass containers sealed with Teflon ${ }^{\circledR}$-lined lids. Periodic reanalyses of the bulk chemical were performed by the study laboratory twice during the 3-month studies and approximately every 6 months during the 2-year studies using HPLC; no degradation of the bulk chemical was detected.

\section{Corn Oil}

Corn oil was used as the vehicle for the formulations and was obtained from Red Diamond, Inc. (Birmingham, AL), in one lot that was used in the 3-month studies and from Spectrum Chemicals and Laboratory Products (Gardena, CA) in seven lots and Sigma-Aldrich (St. Louis, $\mathrm{MO}$ ) in three lots that were used in the 2-year studies. Analysis of the corn oil for peroxides was performed by potentiometric titration, and each lot was within the acceptable range of less than or equal to $3 \mathrm{mEq} / \mathrm{kg}$. 


\section{Preparation and Analysis of Dose Formulations}

The dose formulations were prepared by mixing the appropriate amount of milled indole-3carbinol with corn oil to achieve the required concentrations (Table I-2). Dose formulations were stored in amber glass vials sealed with Teflon ${ }^{\circledR}$-lined lids at $5^{\circ} \mathrm{C}$ for up to 41 days.

Homogeneity, gavageability, and resuspendability studies were performed on $60 \mathrm{mg} / \mathrm{mL}$ dose formulations, and stability studies were performed on $1 \mathrm{mg} / \mathrm{mL}$ formulations by the analytical chemistry laboratory using HPLC. Homogeneity was confirmed, and gavageability was confirmed for a 20-gauge gavage needle. Resuspendability was confirmed for dose formulations that had been stored for 14 days at $5^{\circ} \mathrm{C}$. Stability was confirmed for at least 8 days for formulations stored in amber glass vials sealed with Teflon ${ }^{\circledR}$-lined lids at $5^{\circ} \mathrm{C}$ and for 3 hours under simulated animal room conditions. The study laboratory at SRI performed stability studies with $1.6 \mathrm{mg} / \mathrm{mL}$ dose formulations using HPLC, and stability was confirmed for at least 41 days for dose formulations stored in amber glass vials sealed with Teflon ${ }^{\circledR}$-lined lids at $5^{\circ} \mathrm{C}$. Prior to the 2-year studies, the study laboratory at Battelle Columbus Operations performed homogeneity studies on $6.25,15,25$, and $60 \mathrm{mg} / \mathrm{mL}$ dose formulations and gavageability studies on $60 \mathrm{mg} / \mathrm{mL}$ dose formulations using HPLC; homogeneity and gavagability were confirmed.

Periodic analyses of the dose formulations of indole-3-carbinol were performed by the study laboratories using HPLC. During the 3-month studies, the dose formulations were analyzed three times; all 15 of the dose formulations for rats and all 15 for mice were within $10 \%$ of the target concentrations (Table I-3). Animal room samples of these dose formulations were also analyzed; all 15 for rats and all 15 for mice were within $10 \%$ of the target concentrations. During the 2-year studies, the dose formulations were analyzed approximately every 2 months (Table I-4). Of the dose formulations analyzed, all 72 for rats and all 36 for mice were within $10 \%$ of the target concentrations; all 24 of the animal room samples for rats and all 12 for mice were within $10 \%$ of the target concentrations.

\section{Animal Source}

Male and female F344/N rats and B6C3F1/N mice were obtained from the NTP colony maintained at Taconic Farms, Inc. (Germantown, NY) for the 3-month studies. For the 2-year studies, male and female Harlan Sprague Dawley rats and B6C3F1/N mice were obtained from Harlan Sprague Dawley, Inc. (Indianapolis, IN), and Taconic Farms, Inc. (Germantown, NY), respectively. In the interim between conducting the 3-month studies and the initiation of the 2-year studies, NTP discontinued use of the F344/N rat in toxicity and carcinogenicity studies.

NTP has previously conducted seven separate 2-year studies in Sprague Dawley rats based on prior observations by Kociba et al. ${ }^{73}$ that Sprague Dawley rats are sensitive to the effects of dioxin-like compounds ${ }^{74-80}$. Based on the role of indole-3-carbinol as an agonist ligand for the aryl hydrocarbon receptor, a dioxin-responsive mechanism, Harlan Sprague Dawley rats were selected for the 2-year study.

\section{Animal Welfare}

Animal care and use are in accordance with the Public Health Service Policy on Humane Care and Use of Animals. All animal studies were conducted in an animal facility accredited by the Association for the Assessment and Accreditation of Laboratory Animal Care International. 
Studies were approved by the Battelle Columbus Operations Animal Care and Use Committee and conducted in accordance with all relevant NIH and NTP animal care and use policies and applicable federal, state, and local regulations and guidelines.

\section{Three-month Studies}

The 3-month studies were conducted to evaluate the cumulative toxic effects of repeated exposure to indole-3-carbinol and to determine the appropriate doses to be used in the 2-year studies. The doses selected for the 3-month studies were based on study results supplied by the National Cancer Institute (NCI) that provided sufficient information in rats to determine appropriate doses to obtain a no-observed-adverse-effect level and a possible maximum tolerated dose. On receipt, the rats and mice were 4 to 5 weeks old. Rats were quarantined for 12 (females) or 13 (males) days and mice were quarantined for 14 (males) or 15 (females) days. Rats were 5 (females only) to 6 weeks old and mice were 6 to 7 weeks old on the first day of the studies. Before the studies began, five male and five female rats and mice were randomly selected for parasite evaluation and gross observation for evidence of disease. The health of the animals was monitored during the studies according to the protocols of the NTP Sentinel Animal Program (Appendix K).

Groups of 10 male and 10 female core study rats were administered $0,18.75,37.5,75$, 150, or $300 \mathrm{mg}$ indole-3-carbinol/kg body weight in corn oil by gavage, and groups of 10 male and 10 female mice were administered 0, 15.6, 31.25, 62.5, 125, or $250 \mathrm{mg} / \mathrm{kg}, 5$ days per week for 14 weeks. These initial doses were selected based on published literature, additional data provided from a 3-month rat study being conducted by the $\mathrm{NCI}^{20}$, and a pilot study conducted in Sprague Dawley rats (unpublished data). Crowell et al. ${ }^{20}$ demonstrated effects in rats at $100 \mathrm{mg} / \mathrm{kg}$; increased incidences of centrilobular hepatocyte hypertrophy, and increased liver, kidney, and spleen weights were observed in males, and increased liver and kidney weights were observed in females at this dose. In a pilot study, exposure to $200 \mathrm{mg} / \mathrm{kg}$ indole-3-carbinol for 6 weeks induced a 10\% decrease in body weight and a 20\% increase in liver weight. In mice, neurological effects and increased mortality have been observed at doses of $500 \mathrm{mg} / \mathrm{kg}$. Clinical pathology study groups of 10 male and 10 female rats were administered the same doses as the core study rats 5 days per week for 25 days. Vehicle control animals received the corn oil vehicle alone. Feed and water were available ad libitum. Rats and female mice were housed five per cage; male mice were housed individually. Clinical findings for core study rats and mice were recorded weekly. The animals were weighed initially; on day 2 (female mice), 3 (male animals), or 4 (female rats); weekly; and at the end of the studies. Details of the study design and animal maintenance are summarized in Table 1.

Liver (median, left, and right lobes) and lung (right lobe) samples were collected from 10 male and 10 female core study rats and mice at necropsy for determination of cytochrome P450 1A1 and 1A2 activities. Liver and lung samples were weighed, minced, and frozen until analysis. CYP1A1 in the liver and lung and CYP1A2 in the liver were measured based on the activities of 7-ethoxyresorufin-O-deethylase (EROD) or acetanilide-4-hydroxylase (A4H), respectively. Microsomal suspensions were prepared using the $\mathrm{CaCl}_{2}$ aggregation method described by Schenkman and Cinti ${ }^{81}$. The concentration of protein in each suspension was determined by the Lowry method $^{82}$. EROD activities were determined by spectrofluorometric methods, including those of Chang and Waxman ${ }^{83}$ for liver, and A4H activities were determined using HPLC ${ }^{84}$. 
Blood was collected from the retroorbital plexus of clinical pathology study rats under carbon dioxide anesthesia on days 4 and 25 and, using the same method, blood was collected from core study rats and mice at the end of the studies for hematology and clinical chemistry (rats) analyses. For hematology analyses, blood from each animal was collected into a tube containing EDTA. Erythrocyte, platelet, and leukocyte counts, hematocrit values, hemoglobin concentration, mean cell volume, mean cell hemoglobin, mean cell hemoglobin concentration, and reticulocyte counts were determined using an Advia ${ }^{\circledR} 120$ Hematology System (Bayer, Inc., Tarrytown, NY) and reagents from the instrument manufacturer. For clinical chemistry analyses, blood was collected into a tube containing no anticoagulant. Clinical chemistry analyses were performed using a Hitachi 911 Chemistry Analyzer (Roche Diagnostics Corporation, Indianapolis, IN). Reagents were supplied by the instrument manufacturer. The parameters measured are listed in Table 1.

At the end of the 3-month studies, samples were collected for sperm motility and vaginal cytology evaluations on core study rats administered to $0,75,150$, or $300 \mathrm{mg} / \mathrm{kg}$ and mice administered to $0,62.5,125$, or $250 \mathrm{mg} / \mathrm{kg}$. The parameters evaluated are listed in Table 1 . For 12 consecutive days prior to scheduled terminal euthanasia, the vaginal vaults of the females were moistened with saline, if necessary, and samples of vaginal fluid and cells were stained. Relative numbers of leukocytes, nucleated epithelial cells, and large squamous epithelial cells were determined and used to ascertain estrous cycle stage (i.e., diestrus, proestrus, estrus, and metestrus). Male animals were evaluated for sperm count and motility. The left testis and left epididymis were isolated and weighed. The tail of the epididymis (cauda epididymis) was then removed from the epididymal body (corpus epididymis) and weighed. Test yolk (rats) or modified Tyrode's buffer (mice) was applied to slides and a small incision was made at the distal border of the cauda epididymis. The sperm effluxing from the incision were dispersed in the buffer on the slides, and the numbers of motile and nonmotile spermatozoa were counted for five fields per slide by two observers. Following completion of sperm motility estimates, each left cauda epididymis was placed in buffered saline solution. Caudae were finely minced, and the tissue was incubated in the saline solution and then heat fixed at $65^{\circ} \mathrm{C}$. Sperm density was then determined microscopically with the aid of a hemacytometer. To quantify spermatogenesis, the testicular spermatid head count was determined by removing the tunica albuginea and homogenizing the left testis in phosphate-buffered saline containing $10 \%$ dimethyl sulfoxide. Homogenization-resistant spermatid nuclei were counted with a hemacytometer.

Necropsies were performed on all core study animals. The heart, right kidney, liver, lung, right testis, and thymus of core study rats and mice were weighed. Tissues for microscopic examination were fixed and preserved in $10 \%$ neutral buffered formalin (except eyes were first fixed in Davidson's solution), processed and trimmed, embedded in paraffin, sectioned to a thickness of 4 to $6 \mu \mathrm{m}$, and stained with hematoxylin and eosin. Complete histopathologic examinations were performed by the study laboratory pathologist on all vehicle control and $300 \mathrm{mg} / \mathrm{kg}$ core study rats and all vehicle control and $250 \mathrm{mg} / \mathrm{kg}$ mice. In rats, the liver, mesenteric lymph node, and small intestine were examined in all dosed groups; in mice, the liver and mesenteric lymph node (females only) were examined in all dosed groups. Table 1 lists the tissues and organs routinely examined.

After a review of the laboratory reports and selected histopathology slides by a quality assessment (QA) pathologist, the findings and reviewed slides were submitted to a NTP Pathology Working Group (PWG) coordinator for a second independent review. Any 
inconsistencies in the diagnoses made by the study laboratory and QA pathologists were resolved by the NTP pathology peer review process. Final diagnoses for reviewed lesions represent a consensus of the PWG or a consensus between the study laboratory pathologist, NTP pathologist, QA pathologist(s), and the PWG coordinator. Details of these review procedures have been described, in part, by Maronpot and Boorman ${ }^{85}$ and Boorman et al. ${ }^{86}$.

\section{Two-year Studies}

\section{Study Design}

Groups of 50 male and 50 female rats were administered 0, 75, 150, or 300 mg indole-3carbinol/kg body weight in corn oil gavage, and groups of 50 male and 50 female mice were administered 0, 62.5, 125, or $250 \mathrm{mg} / \mathrm{kg}, 5$ days per week for 104 (male rats) or 105 weeks. To further investigate the pathogenesis of treatment-related increased incidences of dilation of lymphatics of the duodenum, jejunum, and mesenteric lymph nodes observed in the 3-month studies $^{87}$, an additional five male rats per special intestine study group were administered indole3-carbinol for 1 or 4 weeks. To evaluate transcriptional changes in the liver, liver tissue from five vehicle control female rats and five $300 \mathrm{mg} / \mathrm{kg}$ female rats was collected and processed for microarray analyses at 3 months (Appendix L).

Rats were quarantined for 13 (males) or 14 (females) days and mice were quarantined for 18 (females) or 19 (males) days before the beginning of the studies. Five male and five female rats and mice were randomly selected for parasite evaluation and gross observation of disease. Rats and mice were approximately 6 to 7 weeks old at the beginning of the studies. The health of the animals was monitored during the studies according to the protocols of the NTP Sentinel Animal Program (Appendix K).

Male rats were housed up to three per cage, female rats and mice were housed up to five per cage, and male mice were housed individually. Feed and water were available ad libitum. Cages and racks were rotated every 2 weeks. Further details of animal maintenance are given in Table 1. Information on feed composition and contaminants is provided in Appendix J.

\section{Clinical Examinations and Pathology}

All animals were observed twice daily. Body weights of core study animals were recorded initially, weekly for the first 13 weeks, monthly thereafter, and upon study termination. Clinical findings were recorded at week 5, monthly, and upon study termination.

Complete necropsies and microscopic examinations were performed on all core study rats and mice. At necropsy, all organs and tissues were examined for grossly visible lesions, and all major tissues were fixed and preserved in 10\% neutral buffered formalin (except, initially, eyes were fixed in Davidson's solution and testes were fixed in modified Davidson's solution), processed and trimmed, embedded in paraffin, sectioned to a thickness of 4 to $6 \mu \mathrm{m}$, and stained with hematoxylin and eosin for microscopic examination. For all paired organs (e.g., adrenal gland, kidney, ovary), samples from each organ were examined. In the standard evaluation of the uterus, a transverse section through each uterine horn, approximately $0.5 \mathrm{~cm}$ cranial to the cervix, was collected for histopathological evaluation. In an extended review, all residual uterine, cervical and vaginal tissue stored in $10 \%$ neutral buffered formalin were opened (longitudinally) and examined for untrimmed potential gross lesions. All untrimmed potential lesions found were 
collected and in addition to the remaining segments of uterus were processed histologically and sectioned longitudinally for histopathological examination. Tissues examined microscopically are listed in Table 1.

Complete necropsies were performed on all special intestine study male rats. At necropsy, the small intestines (duodenum and jejunum) were collected, fixed, and preserved in 10\% neutral buffered formalin. Additionally, samples of the duodenum and jejunum were cryopreserved. Microscopic evaluations of the small intestine were performed on five special study male rats at 1 week and five special study male rats at 4 weeks. The cryosections of the small intestine (duodenum and jejunum) were stained with the Oil-red-O and Sudan Black histochemical stains that are specific for detecting fat (lipid) in tissue sections.

At 3 months, liver tissue from the left lateral lobe of five vehicle control female rats and five $300 \mathrm{mg} / \mathrm{kg}$ female rats was collected, frozen in liquid nitrogen, and transported to Battelle Biomedical Research Center (Columbus, $\mathrm{OH}$ ) to be processed for RNA isolation and purification, cDNA synthesis, and array hybridization (Appendix L).

Microscopic evaluations were completed by the study laboratory pathologist, and the pathology data were entered into the Toxicology Data Management System. The report, slides, paraffin blocks, residual wet tissues, and pathology data were sent to the NTP Archives for inventory, slide/block match, wet tissue audit, and storage. The slides, individual animal data records, and pathology tables were evaluated by an independent quality assessment laboratory. The individual animal records and tables were compared for accuracy, the slide and tissue counts were verified, and the histotechnique was evaluated. For the 2-year studies, a quality assessment pathologist evaluated slides from all tumors and all potential target organs, which included the liver of rats and mice, small intestine of rats, uterus of female rats, and nose and glandular stomach of mice.

The quality assessment report and the reviewed slides were submitted to the NTP PWG coordinator, who reviewed the selected tissues and addressed any inconsistencies in the diagnoses made by the laboratory and quality assessment pathologists. Representative histopathology slides containing examples of lesions related to chemical administration, examples of disagreements in diagnoses between the laboratory and quality assessment pathologists, or lesions of general interest were presented by the coordinator to the PWG for review. The PWG consisted of the quality assessment pathologist and other pathologists experienced in rodent toxicologic pathology. This group examined the tissues without any knowledge of dose groups. When the PWG consensus differed from the opinion of the laboratory pathologist, the diagnosis was changed. Final diagnoses for reviewed lesions represent a consensus between the laboratory pathologist, reviewing pathologist(s), and the PWG. Details of these review procedures have been described, in part, by Maronpot and Boorman ${ }^{85}$ and Boorman et al. ${ }^{86}$. For subsequent analyses of the pathology data, the decision of whether to evaluate the diagnosed lesions for each tissue type separately or combined was generally based on the guidelines of McConnell et al. ${ }^{88}$. 
Indole-3-carbinol, NTP TR 584

Table 1. Experimental Design and Materials and Methods in the Gavage Studies of Indole-3-carbinol

\begin{tabular}{|c|c|}
\hline Three-month Studies & Two-year Studies \\
\hline \multicolumn{2}{|l|}{ Study Laboratory } \\
\hline Southern Research Institute (Birmingham, AL) & Battelle Columbus Operations (Columbus, OH) \\
\hline \multicolumn{2}{|l|}{ Strain and Species } \\
\hline $\begin{array}{l}\text { F344/N rats } \\
\text { B6C3F1/N mice }\end{array}$ & $\begin{array}{l}\text { Harlan Sprague Dawley rats } \\
\text { B6C3F1/N mice }\end{array}$ \\
\hline \multicolumn{2}{|l|}{ Animal Source } \\
\hline Taconic Farms, Inc. (Germantown, NY) & $\begin{array}{l}\text { Rats: Harlan Sprague Dawley, Inc. (Indianapolis, IN) } \\
\text { Mice: Taconic Farms, Inc. (Germantown, NY) }\end{array}$ \\
\hline \multicolumn{2}{|l|}{ Time Held Before Studies } \\
\hline $\begin{array}{l}\text { Rats: } 12 \text { (females) or } 13 \text { (males) days } \\
\text { Mice: } 14 \text { (males) or } 15 \text { (females) days }\end{array}$ & $\begin{array}{l}\text { Rats: } 13 \text { (males) or } 14 \text { (females) days } \\
\text { Mice: } 18 \text { (females) or } 19 \text { (males) days }\end{array}$ \\
\hline \multicolumn{2}{|l|}{ Average Age When Studies Began } \\
\hline $\begin{array}{l}\text { Rats: } 5 \text { to } 6 \text { weeks } \\
\text { Mice: } 6 \text { to } 7 \text { weeks }\end{array}$ & 6 to 7 weeks \\
\hline \multicolumn{2}{|l|}{ Date of First Dose } \\
\hline $\begin{array}{l}\text { Core study rats: August } 30 \text { (females) or } 31 \text { (males), } \\
2004 \\
\text { Mice: September } 1 \text { (males) or } 2 \text { (females), } 2004\end{array}$ & $\begin{array}{l}\text { Rats: March } 14 \text { (males) or } 15 \text { (females), } 2007 \\
\text { Mice: April } 2 \text { (females) or } 3 \text { (males), } 2007\end{array}$ \\
\hline \multicolumn{2}{|l|}{ Duration of Dosing } \\
\hline 5 days per week for 14 weeks & 5 days per week for 104 (male rats) or 105 weeks \\
\hline \multicolumn{2}{|l|}{ Date of Last Dose } \\
\hline $\begin{array}{l}\text { Core study rats: November } 30 \text { (females) or December } \\
1 \text { (males), } 2004 \\
\text { Mice: December } 2 \text { (males) or } 3 \text { (females), } 2004\end{array}$ & $\begin{array}{l}\text { Rats: March } 10 \text { (males) or } 12 \text { (females), } 2009 \\
\text { Mice: March } 31 \text { (females) or April } 2 \text { (males), } 2009\end{array}$ \\
\hline \multicolumn{2}{|l|}{ Necropsy Dates } \\
\hline $\begin{array}{l}\text { Core study rats: December } 1 \text { (females) or } 2 \text { (males), } \\
2004 \\
\text { Mice: December } 3 \text { (males) or } 4 \text { (females), } 2004\end{array}$ & $\begin{array}{l}\text { Rats: March } 9 \text { to } 11 \text { (males) or } 12 \text { and } 13 \text { (females), } 2009 \\
\text { Mice: March } 30 \text { to April } 1 \text { (females) or April } 1 \text { to } 3 \\
\text { (males), } 2009\end{array}$ \\
\hline \multicolumn{2}{|l|}{ Average Age at Necropsy } \\
\hline 19 to 20 weeks & $\begin{array}{l}\text { Rats: } 110 \text { to } 111 \text { weeks } \\
\text { Mice: } 110 \text { (females) to } 112 \text { weeks }\end{array}$ \\
\hline \multicolumn{2}{|l|}{ Size of Study Groups } \\
\hline Core study: 10 male and 10 female rats and mice & Core study: 50 males and 50 females \\
\hline Clinical pathology study: 10 male and 10 female rats & $\begin{array}{l}\text { Special intestine study: } 10 \text { male rats } \\
\text { Microarray study: Five vehicle control female rats and } \\
\text { five } 300 \mathrm{mg} / \mathrm{kg} \text { female rats }\end{array}$ \\
\hline
\end{tabular}




\section{Method of Distribution}

Animals were distributed randomly into groups of approximately equal initial mean body weights.

\section{Animals per Cage}

Rats: 5

Mice: 1 (males) or 5 (females)

\section{Method of Animal Identification}

Tail tattoo

\section{Diet}

Irradiated NTP-2000 wafer feed (Zeigler Brothers, Inc., Gardners, PA), available ad libitum, changed at least once weekly

\section{Water}

Tap water (Birmingham municipal supply) via automatic watering system (Edstrom Industries, Waterford, WI), available ad libitum

\section{Cages}

Polycarbonate (Lab Products, Inc., Maywood, NJ), changed once (male mice) or twice weekly

\section{Bedding}

Irradiated hardwood bedding chips (P.J. Murphy Forest Irradiated Sani-Chips ${ }^{\circledR}$ (P.J. Murphy Forest Products, Products, Inc., Montville, NJ), changed once (male mice) or twice weekly

\section{Rack Filters}

Reemay ${ }^{\circledR}$ spun-bonded polyester (Andico, Birmingham, AL), changed every 2 weeks

\section{Racks}

Stainless steel (Lab Products, Inc., Maywood, NJ), changed every 2 weeks

\section{Animal Room Environment}

Temperature: $72^{\circ} \pm 3^{\circ} \mathrm{F}$

Relative humidity: $50 \% \pm 15 \%$

Room fluorescent light: 12 hours/day

Room air changes: at least 10/hour

\section{Doses}

Rats: $0,18.75,37.5,75,150$, or $300 \mathrm{mg} / \mathrm{kg}$ in corn oil by gavage (dosing volume $5 \mathrm{~mL} / \mathrm{kg}$ )

Mice: $0,15.6,31.25,62.5,125$, or $250 \mathrm{mg} / \mathrm{kg}$ in corn oil by gavage (dosing volume $10 \mathrm{~mL} / \mathrm{kg}$ )
Same as 3-month studies

Rats: 3 (males) or 5 (females)

Mice: 1 (males) or 5 (females)

Tail tattoo

Same as 3-month studies

Tap water (Columbus municipal supply) via automatic watering system (Edstrom Industries, Waterford, WI), available ad libitum

Same as 3-month studies except from Lab Products, Inc., Seaford, DE; rotated every 2 weeks Inc., Montville, NJ), changed once (male mice) or twice weekly

Spun-bonded polyester (Snow Filtration Co., Cincinnati, $\mathrm{OH})$, changed every 2 weeks

Stainless steel (Lab Products, Inc., Seaford, DE), changed and rotated every 2 weeks

Temperature: $72^{\circ} \pm 3^{\circ} \mathrm{F}$

Relative humidity: $50 \% \pm 15 \%$

Room fluorescent light: 12 hours/day

Room air changes: at least 10/hour

Rats: Core study rats and special intestine study male rats $0,75,150$, or $300 \mathrm{mg} / \mathrm{kg}$ and microarray study female rats 0 or $300 \mathrm{mg} / \mathrm{kg}$ in corn oil by gavage (dosing volume $5 \mathrm{~mL} / \mathrm{kg})$

Mice: $0,62.5,125$, or $250 \mathrm{mg} / \mathrm{kg}$ in corn oil by gavage (dosing volume $10 \mathrm{~mL} / \mathrm{kg}$ ) 


\section{Type and Frequency of Observation}

Observed twice daily; animals were weighed initially, day 2 (female mice), day 3 (male rats and mice), day 4 (female rats), weekly, and at the end of the studies; clinical findings for core study animals were recorded weekly.

\section{Method of Euthanasia}

Carbon dioxide asphyxiation

\section{Necropsy}

Necropsies were performed on all core study animals. Organs weighed were heart, right kidney, liver, lung, right testis, and thymus of core study rats and mice.

\section{Clinical Pathology}

Blood was collected from the retroorbital sinus of clinical pathology rats on days 4 and 25 and from core study rats and mice at the end of the studies for hematology and clinical chemistry (rats).

Hematology: hematocrit; hemoglobin concentration; erythrocyte, reticulocyte, nucleated erythrocyte, and platelet counts; mean cell volume; mean cell hemoglobin; mean cell hemoglobin concentration; and leukocyte count and differentials

Clinical chemistry: urea nitrogen, creatinine, glucose, total protein, albumin, alanine aminotransferase, alkaline phosphatase, creatine kinase, sorbitol dehydrogenase, and bile acids

\section{Histopathology}

Complete histopathology was performed on core study vehicle control rats and mice, $300 \mathrm{mg} / \mathrm{kg}$ rats, and $250 \mathrm{mg} / \mathrm{kg}$ mice. In addition to gross lesions and tissue masses, the following tissues were examined: adrenal gland, bone with marrow, brain, clitoral gland, esophagus, eyes, gallbladder (mice only), Harderian gland, heart, large intestine (cecum, colon, rectum), small intestine (duodenum, jejunum, ileum), kidney, liver, lungs, lymph nodes (mandibular and mesenteric), mammary gland, nose, ovary, pancreas, parathyroid gland, pituitary gland, preputial gland, prostate gland, salivary gland, seminal vesicle, skin, spleen, stomach (forestomach and glandular), testis (with epididymis), thymus, thyroid gland, trachea, urinary bladder, and uterus. In addition, the liver, mesenteric lymph node, and small intestine were examined in all dosed groups of rats, and the liver and mesenteric lymph node (females only) were examined in all dosed groups of mice.
Observed twice daily; core study animals were weighed initially, weekly for the first 13 weeks, monthly thereafter, and upon study termination; clinical findings for core study animals were recorded at week 5, monthly, and at the end of the studies.

Same as 3-month studies

Necropsies were performed on all core study animals and special intestine study male rats.

None

Complete histopathology was performed on all core study rats and mice. In addition to gross lesions and tissue masses, the following tissues were examined: adrenal gland, bone with marrow, brain, clitoral gland, esophagus, eyes, gallbladder (mice), Harderian gland, heart, large intestine (cecum, colon, rectum), small intestine (duodenum, jejunum, ileum), kidney, liver, lung, lymph nodes (mandibular and mesenteric), mammary gland, nose, ovary, pancreas, parathyroid gland, pituitary gland, preputial gland, prostate gland, salivary gland, seminal vesicle, skin, spleen, stomach (forestomach and glandular), testis (with epididymis), thymus, thyroid gland, trachea, urinary bladder, and uterus. The small intestines were examined in five special intestine study male rats at 1 week and five special intestine study male rats at 4 weeks. Extended gross and microscopic reviews were performed on the residual uterine wet tissue from all experimental groups of female rats. 


\section{Sperm Motility and Vaginal Cytology}

At the end of the studies, spermatid and sperm samples None were collected from $0,75,150$, and $300 \mathrm{mg} / \mathrm{kg}$ core study male rats and $0,62.5,125$, and $250 \mathrm{mg} / \mathrm{kg}$ male mice. The following parameters were evaluated: spermatid heads per testis and per gram testis, sperm motility, and sperm per cauda epididymis and per gram cauda epididymis. The left cauda, left epididymis, and left testis were weighed. Vaginal samples were collected for up to 12 consecutive days prior to the end of the studies from $0,75,150$, and $300 \mathrm{mg} / \mathrm{kg}$ core study female rats and $0,62.5,125$, and $250 \mathrm{mg} / \mathrm{kg}$ female mice.

\section{Cytochrome P450 Activities}

Liver (median, left, and right lobes) and lung (right

None lobe) samples were collected from 10 male and 10 female core study rats and mice at necropsy. CYP1A1 activity in the liver and lung and CYP1A2 activity in the liver were measured.

\section{Microarray Study}

None

Liver tissue of five vehicle control female rats and five $300 \mathrm{mg} / \mathrm{kg}$ female rats was collected and processed to evaluate transcriptional changes in the liver at a 3 months (Appendix L).

\section{Statistical Methods}

\section{Survival Analyses}

The probability of survival was estimated by the product-limit procedure of Kaplan and Meier ${ }^{89}$ and is presented in the form of graphs. Animals found dead of other than natural causes were censored; animals dying from natural causes were not censored. Statistical analyses for possible dose-related effects on survival used Cox's ${ }^{90}$ method for testing two groups for equality and Tarone's ${ }^{91}$ life table test to identify dose-related trends. All reported P values for the survival analyses are two sided.

\section{Calculation of Incidence}

The incidences of neoplasms or nonneoplastic lesions are presented in Table A-1, Table A-3, Table B-1, Table B-5. Table C-1, Table C-4, Table D-1, and Table D-4 as the numbers of animals bearing such lesions at a specific anatomic site and the numbers of animals with that site examined microscopically. For calculation of statistical significance, the incidences of most neoplasms (Table A-2, Table B-2, Table C-2, and Table D-2) and all nonneoplastic lesions are given as the numbers of animals affected at each site examined microscopically. However, when macroscopic examination was required to detect neoplasms in certain tissues (e.g., mesentery, pleura, peripheral nerve, skeletal muscle, tongue, tooth, and Zymbal's gland) before microscopic evaluation, the denominators consist of the number of animals that had a gross abnormality. When neoplasms had multiple potential sites of occurrence (e.g., leukemia or lymphoma), the 
denominators consist of the number of animals on which a necropsy was performed. Table A-2, Table B-2, Table C-2, and Table D-2 also give the survival-adjusted neoplasm rate for each group and each site-specific neoplasm. This survival-adjusted rate (based on the Poly-3 method described below) accounts for differential mortality by assigning a reduced risk of neoplasm, proportional to the third power of the fraction of time on study, only to site-specific, lesion-free animals that do not reach terminal euthanasia.

\section{Analysis of Neoplasm and Nonneoplastic Lesion Incidences}

The Poly-k test ${ }^{92-94}$ was used to assess neoplasm and nonneoplastic lesion prevalence. This test is a survival-adjusted quantal-response procedure that modifies the Cochran-Armitage linear trend test to take survival differences into account. More specifically, this method modifies the denominator in the quantal estimate of lesion incidence to approximate more closely the total number of animal years at risk. For analysis of a given site, each animal is assigned a risk weight. This value is one if the animal had a lesion at that site or if it survived until terminal euthanasia; if the animal died prior to terminal euthanasia and did not have a lesion at that site, its risk weight is the fraction of the entire study time that it survived, raised to the kth power.

This method yields a lesion prevalence rate that depends only upon the choice of a shape parameter for a Weibull hazard function describing cumulative lesion incidence over time ${ }^{92}$. Unless otherwise specified, a value of $k=3$ was used in the analysis of site-specific lesions. This value was recommended by Bailer and Portier ${ }^{92}$ following an evaluation of neoplasm onset time distributions for a variety of site-specific neoplasms in control F344/N rats and B6C3F1/N mice ${ }^{95}$. Bailer and Portier ${ }^{92}$ showed that the Poly-3 test gave valid results if the true value of $\mathrm{k}$ was anywhere in the range from 1 to 5 . A further advantage of the Poly-3 method is that it does not require lesion lethality assumptions. Variation introduced by the use of risk weights, which reflect differential mortality, was accommodated by adjusting the variance of the Poly-3 statistic as recommended by Bieler and Williams ${ }^{96}$.

Tests of significance included pairwise comparisons of each dosed group with controls and a test for an overall dose-related trend. Continuity-corrected Poly-3 tests were used in the analysis of lesion incidence, and reported $\mathrm{P}$ values are one sided. The significance of lower incidences or decreasing trends in lesions is represented as $1-\mathrm{P}$ with the letter $\mathrm{N}$ added (e.g., $\mathrm{P}=0.99$ is presented as $\mathrm{P}=0.01 \mathrm{~N}$ ).

\section{Analysis of Continuous Variables}

Two approaches were employed to assess the significance of pairwise comparisons between dosed and control groups in the analysis of continuous variables. Organ and body weight data, which historically have approximately normal distributions, were analyzed with the parametric multiple comparison procedures of Dunnett ${ }^{97}$ and Williams ${ }^{98 ;}$;9. Hematology, clinical chemistry, cytochrome P450, spermatid, and epididymal spermatozoal data, which have typically skewed distributions, were analyzed using the nonparametric multiple comparison methods of Shirley ${ }^{100}$ (as modified by Williams ${ }^{101}$ ) and Dunn ${ }^{102}$. Jonckheere's test ${ }^{103}$ was used to assess the significance of the dose-related trends and to determine whether a trend-sensitive test (Williams' or Shirley's test) was more appropriate for pairwise comparisons than a test that does not assume a monotonic dose-related trend (Dunnett's or Dunn's test). Prior to statistical analysis, extreme values identified by the outlier test of Dixon and Massey ${ }^{104}$ were examined by NTP personnel, 
and implausible values were eliminated from the analysis. Proportions of regular cycling females in each dosed group were compared to the control group using the Fisher exact test ${ }^{105}$. Tests for extended periods of estrus, diestrus, metestrus, and proestrus, as well as skipped estrus and skipped diestrus, were constructed based on a Markov chain model proposed by Girard and Sager ${ }^{106}$. For each dose group, a transition probability matrix was estimated for transitions among the proestrus, estrus, metestrus, and diestrus stages, with provision for extended stays within each stage as well as for skipping estrus or diestrus within a cycle. Equality of transition matrices among dose groups and between the control group and each dosed group was tested using chi-square statistics.

\section{Historical Control Data}

The concurrent control group represents the most valid comparison to the treated groups and is the only control group analyzed statistically in NTP bioassays. However, historical control data are often helpful in interpreting potential treatment-related effects, particularly for uncommon or rare neoplasm types. For meaningful comparisons, the conditions for studies in the historical control database must be generally similar. Significant factors affecting the background incidences of neoplasms at a variety of sites are diet, sex, strain/stock, and route of exposure. The NTP historical control database contains all 2-year studies for each species, sex, and strain/stock with histopathology findings in control animals completed within the most recent 5-year period $^{107-109}$. In general, the historical control database for a given study includes studies using the same route of administration, and the overall incidences of neoplasms in controls for all routes of administration are included for comparison, including the current study. In the historical control database for Sprague Dawley rats, there are only two studies, the current study of indole-3-carbinol and PCB 118. Because both studies were corn oil gavage studies, only same route of administration historical data are presented in this Technical Report for female Sprague Dawley rats.

\section{Quality Assurance Methods}

The 3-month and 2-year studies were conducted in compliance with Food and Drug Administration Good Laboratory Practice Regulations ${ }^{110}$. In addition, as records from the 3-month and 2-year studies were submitted to the NTP Archives, these studies were audited retrospectively by an independent quality assessment contractor. Separate audits covered completeness and accuracy of the pathology data, pathology specimens, final pathology tables, and a draft of this NTP Technical Report. Audit procedures and findings are presented in the reports and are on file at NIEHS. The audit findings were reviewed and assessed by NTP staff, and all comments were resolved or otherwise addressed during the preparation of this Technical Report.

\section{Genetic Toxicology}

The genetic toxicity of indole-3-carbinol was assessed by testing the ability of the chemical to induce mutations in various strains of Salmonella typhimurium and Escherichia coli, micronucleated erythrocytes in rat bone marrow, and increases in the frequency of micronucleated erythrocytes in mouse peripheral blood. Micronuclei (literally "small nuclei" or Howell-Jolly bodies) are biomarkers of induced structural or numerical chromosomal alterations and are formed when acentric fragments or whole chromosomes fail to incorporate into either of 
two daughter nuclei during cell division ${ }^{111 ; 112}$. The protocols for these studies and the results are given in Appendix E.

The genetic toxicity studies have evolved from an earlier effort by NTP to develop a comprehensive database permitting a critical anticipation of a chemical's carcinogenicity in experimental animals based on numerous considerations, including the molecular structure of the chemical and its observed effects in short-term in vitro and in vivo genetic toxicity tests (structure-activity relationships). The short-term tests were originally developed to clarify proposed mechanisms of chemical-induced DNA damage based on the relationship between electrophilicity and mutagenicity ${ }^{113}$ and the somatic mutation theory of cancer ${ }^{114 ; 115}$. However, it should be noted that not all cancers arise through genotoxic mechanisms.

DNA reactivity combined with Salmonella mutagenicity is highly correlated with induction of carcinogenicity in multiple species/sexes of rodents and at multiple tissue sites ${ }^{116}$. A positive response in the Salmonella test was shown to be the most predictive in vitro indicator for rodent carcinogenicity (89\% of the Salmonella mutagens are rodent carcinogens) ${ }^{117 ; 118}$. Additionally, no battery of tests that included the Salmonella test improved the predictivity of the Salmonella test alone. However, these other tests can provide useful information on the types of DNA and chromosomal damage induced by the chemical under investigation.

The predictivity for carcinogenicity of a positive response in acute in vivo bone marrow chromosome aberration or micronucleus tests appears to be less than that in the Salmonella test $^{119 ; 120}$. However, clearly positive results in long-term peripheral blood micronucleus tests have high predictivity for rodent carcinogenicity; a weak response in one sex only or negative results in both sexes in this assay do not correlate well with either negative or positive results in rodent carcinogenicity studies ${ }^{121}$. Because of the theoretical and observed associations between induced genetic damage and adverse effects in somatic and germ cells, the determination of in vivo genetic effects is important to the overall understanding of the risks associated with exposure to a particular chemical. 


\section{Results}

\section{Three-month Study in F344/N Rats}

All rats survived to the end of the study (Table 2). The mean body weight gain of males in the $300 \mathrm{mg} / \mathrm{kg}$ group was significantly less than that of the vehicle controls (Table 2; Figure 3).

There were no clinical findings related to administration of indole-3-carbinol.

Table 2. Survival and Body Weights of F344/N Rats in the Three-month Gavage Study of Indole-3-carbinol $^{\mathrm{a}}$

\begin{tabular}{cccccc}
\hline $\begin{array}{c}\text { Dose } \\
(\mathbf{m g} / \mathbf{k g})\end{array}$ & Survival $^{\mathbf{b}}$ & $\begin{array}{c}\text { Initial Body } \\
\text { Weight } \\
\mathbf{( g )}\end{array}$ & $\begin{array}{c}\text { Final Body } \\
\text { Weight } \\
\mathbf{( g )}\end{array}$ & $\begin{array}{c}\text { Change in Body } \\
\text { Weight } \\
\text { (g) }\end{array}$ & $\begin{array}{c}\text { Final Weight } \\
\text { Relative to } \\
\text { Controls } \\
\mathbf{( \% )}\end{array}$ \\
\hline Male & & & & & \\
0 & $10 / 10$ & $103 \pm 1$ & $309 \pm 5$ & $206 \pm 4$ & \\
18.75 & $10 / 10$ & $103 \pm 1$ & $320 \pm 9$ & $217 \pm 8$ & 103 \\
37.5 & $10 / 10$ & $107 \pm 1^{*}$ & $325 \pm 7$ & $218 \pm 7$ & 105 \\
75 & $10 / 10$ & $105 \pm 1$ & $321 \pm 8$ & $215 \pm 7$ & 104 \\
150 & $10 / 10$ & $104 \pm 1$ & $309 \pm 8$ & $204 \pm 8$ & 100 \\
300 & $10 / 10$ & $104 \pm 1$ & $285 \pm 6$ & $182 \pm 6 *$ & 92 \\
\hline Female & & & & & \\
0 & $10 / 10$ & $91 \pm 2$ & $189 \pm 2$ & $99 \pm 3$ & 101 \\
18.75 & $10 / 10$ & $90 \pm 1$ & $192 \pm 3$ & $102 \pm 3$ & 103 \\
37.5 & $10 / 10$ & $90 \pm 2$ & $195 \pm 2$ & $105 \pm 2$ & 98 \\
75 & $10 / 10$ & $90 \pm 1$ & $185 \pm 3$ & $95 \pm 3$ & 97 \\
150 & $10 / 10$ & $89 \pm 2$ & $183 \pm 3$ & $94 \pm 2$ & 97 \\
300 & $10 / 10$ & $91 \pm 2$ & $183 \pm 3$ & $92 \pm 3$ & \\
\hline
\end{tabular}

*Significantly different $(\mathrm{P} \leq 0.05)$ from the vehicle control group by Dunnett's test.

${ }^{a}$ Weights and weight changes are given as mean \pm standard error.

${ }^{b}$ Number of animals surviving at 14 weeks/number initially in group. 

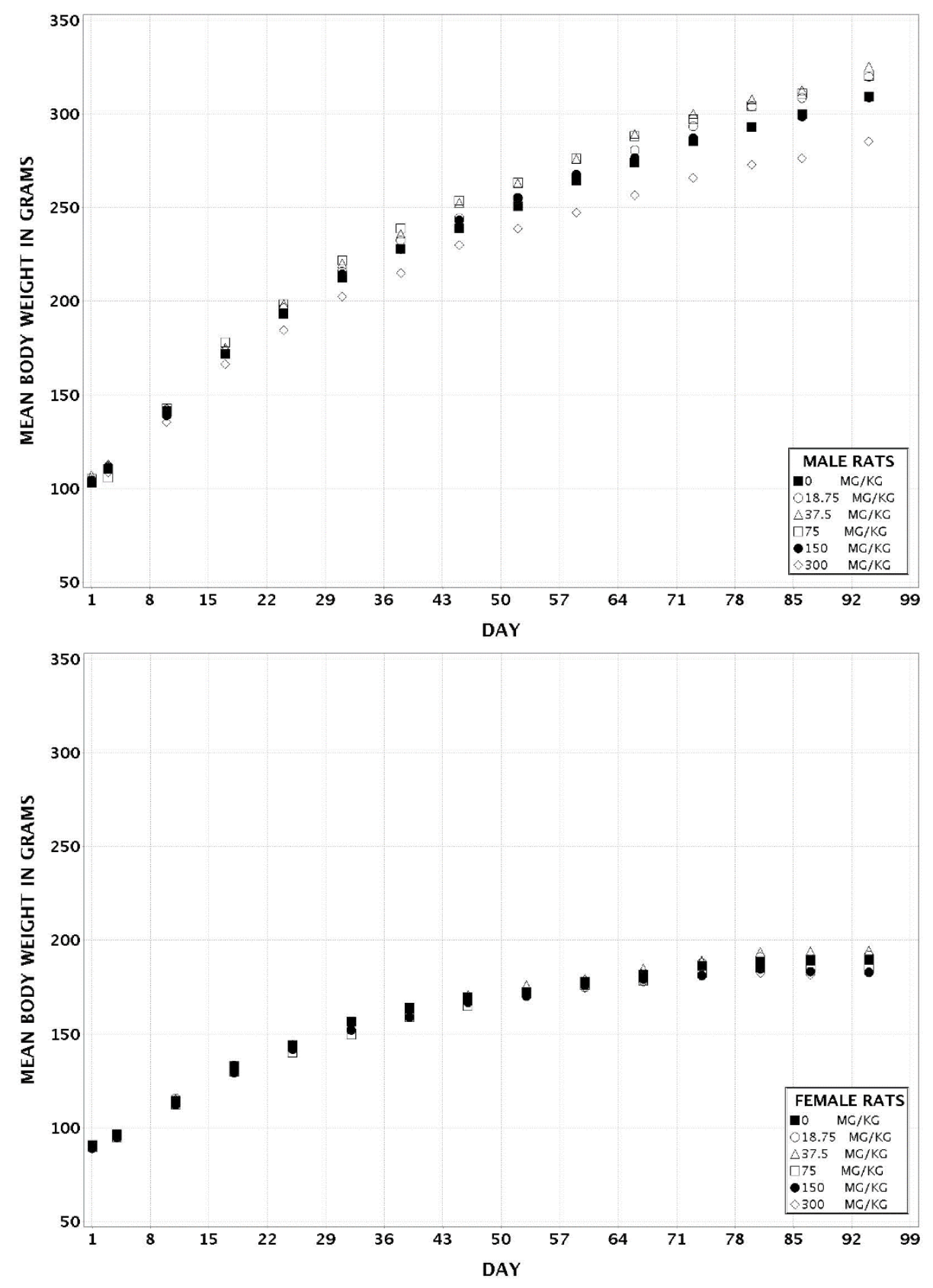

Figure 3. Growth Curves for F344/N Rats Administered Indole-3-carbinol by Gavage for Three Months

There were no changes in the hematology and clinical chemistry data of rats that were considered attributable to indole-3-carbinol administration (Table F-1).

The absolute and relative liver weights of all dosed groups of males and females were significantly increased compared to the vehicle controls (Table 3 and Table G-1). The relative kidney weights of $75 \mathrm{mg} / \mathrm{kg}$ or greater males and all dosed groups of females were significantly increased, as were the absolute kidney weights of $75 \mathrm{mg} / \mathrm{kg}$ males and 18.75, 37.5, and $300 \mathrm{mg} / \mathrm{kg}$ females. The absolute and relative thymus weights of $75 \mathrm{mg} / \mathrm{kg}$ or greater females 
were significantly decreased. Microscopically, there were no histopathologic lesions that correlated with these organ weight changes.

Table 3. Selected Organ Weights and Organ-Weight-to-Body-Weight Ratios for F344/N Rats in the Three-month Gavage Study of Indole-3-carbinol ${ }^{\mathrm{a}}$

\begin{tabular}{|c|c|c|c|c|c|c|}
\hline & $\begin{array}{l}\text { Vehicle } \\
\text { Control }\end{array}$ & $18.75 \mathrm{mg} / \mathrm{kg}$ & $37.5 \mathrm{mg} / \mathrm{kg}$ & 75 mg/kg & $150 \mathrm{mg} / \mathrm{kg}$ & $300 \mathrm{mg} / \mathrm{kg}$ \\
\hline n & 10 & 10 & 10 & 10 & 10 & 10 \\
\hline \multicolumn{7}{|l|}{ Male } \\
\hline $\begin{array}{l}\text { Necropsy } \\
\text { body wt }\end{array}$ & $309 \pm 5$ & $320 \pm 9$ & $325 \pm 7$ & $321 \pm 8$ & $309 \pm 8$ & $285 \pm 6$ \\
\hline \multicolumn{7}{|l|}{ R. Kidney } \\
\hline Absolute & $0.86 \pm 0.02$ & $0.94 \pm 0.02$ & $0.93 \pm 0.03$ & $0.97 \pm 0.03^{*}$ & $0.92 \pm 0.03$ & $0.90 \pm 0.02$ \\
\hline Relative & $2.785 \pm 0.038$ & $2.940 \pm 0.033$ & $2.844 \pm 0.054$ & $3.012 \pm 0.038^{* *}$ & $2.981 \pm 0.048^{* *}$ & $3.152 \pm 0.055^{* *}$ \\
\hline \multicolumn{7}{|l|}{ Liver } \\
\hline Absolute & $9.98 \pm 0.30$ & $11.19 \pm 0.40^{*}$ & $11.20 \pm 0.40^{*}$ & $11.85 \pm 0.36^{* *}$ & $12.07 \pm 0.38^{* *}$ & $12.57 \pm 0.33^{* *}$ \\
\hline Relative & $32.205 \pm 0.594$ & $34.924 \pm 0.481^{* *}$ & $34.382 \pm 0.716^{* *}$ & $36.941 \pm 0.435^{* *}$ & $39.050 \pm 0.472 * *$ & $44.047 \pm 0.729 * *$ \\
\hline
\end{tabular}

Female

Necropsy

body wt

$189 \pm 2$

$192 \pm 3$

$195 \pm 2$

$185 \pm 3$

$183 \pm 3$

$183 \pm 3$

R. Kidney

$\begin{array}{lcccccc}\text { Absolute } & 0.57 \pm 0.01 & 0.66 \pm 0.01^{* *} & 0.63 \pm 0.01^{*} & 0.61 \pm 0.01 & 0.62 \pm 0.01 & 0.63 \pm 0.02^{*} \\ \text { Relative } & 3.034 \pm 0.056 & 3.447 \pm 0.053^{* *} & 3.233 \pm 0.075^{* *} & 3.277 \pm 0.046^{* *} & 3.375 \pm 0.064^{* *} & 3.436 \pm 0.063^{* *}\end{array}$

Liver

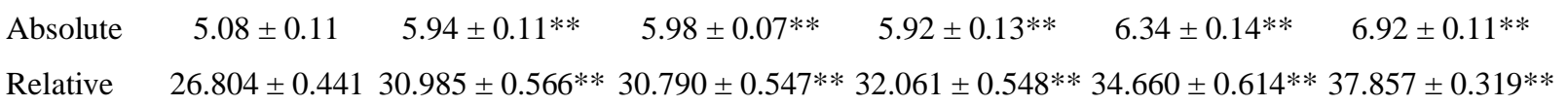

Thymus

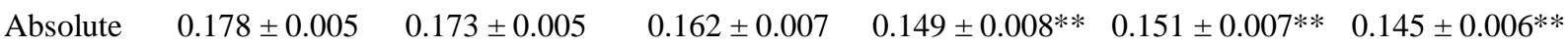

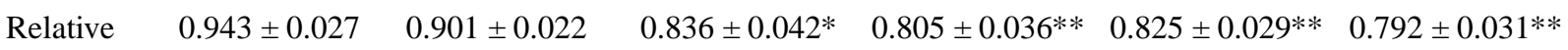

*Significantly different $(\mathrm{P} \leq 0.05)$ from the vehicle control group by Williams' or Dunnett's test.

$* * \mathrm{P} \leq 0.01$.

${ }^{a}$ Organ weights (absolute weights) and body weights are given in grams; organ-weight-to-body-weight ratios (relative weights) are given as mg organ weight/g body weight (mean \pm standard error).

Liver and lung samples were collected for determinations of P450 enzyme activities (Table 4). Microsomal suspensions were prepared from liver samples and were assayed for 7ethoxyresorufin- $O$-deethylase (EROD) activity (a marker for CYP1A1 activity) and acetanilide4-hydroxylase (A4H) activity (a marker for CYP1A2 activity). Microsomal samples from lung were analyzed for EROD activity only. In the liver, there were significant and dose-dependent increases in $\mathrm{A} 4 \mathrm{H}$ and EROD activities in all dosed groups of male and female rats. Maximal inductions of $\mathrm{A} 4 \mathrm{H}$ and EROD activities in males were greater than 5-fold and 100-fold, respectively, compared to the vehicle controls. In females, A4H and EROD activities were maximally increased 4.7-fold and 80-fold, respectively. Pulmonary EROD activity was 
Indole-3-carbinol, NTP TR 584

significantly increased in all dosed groups of females and in males administered $75 \mathrm{mg} / \mathrm{kg}$ or greater.

Table 4. Liver and Lung Cytochrome P450 Data for F344/N Rats in the Three-month Gavage Study of Indole-3-carbinol ${ }^{\mathrm{a}}$

\begin{tabular}{llllll}
\hline $\begin{array}{l}\text { Vehicle } \\
\text { Control }\end{array}$ & $18.75 \mathrm{mg} / \mathrm{kg}$ & $37.5 \mathrm{mg} / \mathrm{kg}$ & $75 \mathrm{mg} / \mathrm{kg}$ & $150 \mathrm{mg} / \mathrm{kg}$ & $300 \mathrm{mg} / \mathrm{kg}$ \\
\hline
\end{tabular}

Male

Liver Microsomes

n 2 ren

9

$10 \quad 10$

10

10

9

A4H (nmol/minute per mg microsomal protein)

$$
0.404 \pm 0.027 \quad 0.627 \pm 0.071^{*} \quad 0.999 \pm 0.067 * * \quad 1.187 \pm 0.086^{* *} \quad 1.546 \pm 0.071^{*} \quad 2.045 \pm 0.077^{* *}
$$

EROD (pmol/minute per mg microsomal protein)

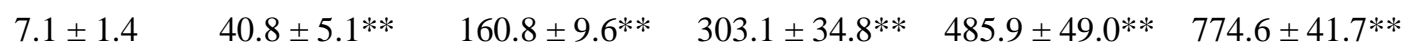

Lung Microsomes

n

8

$9 \quad 10$

10

10

10

EROD (pmol/minute per mg microsomal protein)

ND $\quad 0.033 \pm 0.023 \quad 0.008 \pm 0.008 \quad 0.160 \pm 0.042 * * 1.146 \pm 0.218^{* *} 1.227 \pm 0.250^{* *}$

Female

n

$10-10$

$10 \quad 10$

10

10

10

Liver Microsomes

A4H (nmol/minute per mg microsomal protein)

$$
0.343 \pm 0.0260 .652 \pm 0.069 * * \quad 0.826 \pm 0.017^{* *} 0.973 \pm 0.043^{* *} 1.408 \pm 0.105^{* *} 1.621 \pm 0.088^{* *}
$$

EROD (pmol/minute per mg microsomal protein)

$$
6.1 \pm 1.1 \quad 70.7 \pm 15.1^{* *} \quad 172.8 \pm 15.0^{* *} \quad 236.5 \pm 24.7 * * \quad 440.5 \pm 41.3^{* *} \quad 495.4 \pm 39.9^{* *}
$$

\section{Lung Microsomes}

EROD (pmol/minute per mg microsomal protein)

$$
0.006 \pm 0.0041 .050 \pm 0.205^{* *} 2.711 \pm 0.267 * * 2.671 \pm 0.540^{* *} \quad 2.747 \pm 0.364 * * 3.008 \pm 0.333^{* *}
$$

*Significantly different $(\mathrm{P} \leq 0.05)$ from the vehicle control group by Shirley’s test.

$* * \mathrm{P} \leq 0.01$.

$\mathrm{ND}=$ None detected.

a Data are presented as mean \pm standard error. A4H = acetanilide-4-hydroxylase; EROD = 7-ethoxyresorufin- $O$-deethylase;

There were no significant differences in reproductive tissue weights or spermatid or epididymal spermatozoal measurements of males administered 75,150 , or $300 \mathrm{mg} / \mathrm{kg}$ when compared to the vehicle control group; therefore, indole-3-carbinol did not exhibit the potential to be a reproductive toxicant in male rats (Table $\mathrm{H}-1$ ). Indole-3-carbinol did exhibit the potential to be a female reproductive toxicant based on the increased estrous cycle length (approximately 1 day), and an additional day of diestrus (Table H-2 and Table H-3; Figure H-1). This was manifested as the probability of extended diestrus being significantly higher in the $300 \mathrm{mg} / \mathrm{kg}$ group than in the vehicle control group when estrous cyclicity was analyzed using Markov transition matrix analysis. 
Dose-related increased incidences and severities of minimal to mild ectasia of the lymphatic vessels and lipidosis of the lamina propria occurred in the villi of the small intestines (duodenum and jejunum) of males and females (Table 5). Compared to the vehicle controls, these incidences were significantly increased in the duodenum of 150 and $300 \mathrm{mg} / \mathrm{kg}$ males and females and in the jejunum of $75 \mathrm{mg} / \mathrm{kg}$ or greater males and females. The severity of lymphatic ectasia was minimal to moderate in the jejunum and minimal to mild in the duodenum. Affected lymphatics were variably dilated, lined by a single layer of endothelial cells, and occasionally contained fibrillar amphophilic material. Within the stroma of affected villi, there were occasional macrophages that contained clear cytoplasmic vacuoles (lamina propria) consistent with accumulated lipid (fat). Lesions of moderate severity often disrupted the architecture of the villi. In general, lesions in the jejunum were more severe than those in the duodenum. The cytoplasmic vacuoles within macrophages in the lamina propria of the villi stained positively with special histochemical stains (Oil-red-O and Sudan Black) for lipid.

Table 5. Incidences of Selected Nonneoplastic Lesions in F344/N Rats in the Three-month Gavage Study of Indole-3-carbinol

$\begin{array}{lllll}\text { Vehicle } & 18.75 \mathrm{mg} / \mathrm{kg} \quad 37.5 \mathrm{mg} / \mathrm{kg} \quad 75 \mathrm{mg} / \mathrm{kg} \quad 150 \mathrm{mg} / \mathrm{kg} \quad 300 \mathrm{mg} / \mathrm{kg} \\ \text { Control }\end{array}$

\begin{tabular}{|c|c|c|c|c|c|c|}
\hline \multicolumn{7}{|l|}{ Male } \\
\hline Intestine Small, Duodenum ${ }^{\mathrm{a}}$ & 10 & 10 & 10 & 10 & 10 & 10 \\
\hline Lamina Propria, Lipidosis ${ }^{\mathrm{b}}$ & 0 & 0 & 0 & $1(1.0)^{\mathrm{c}}$ & $10 * *(1.2)$ & $10 * *(2.2)$ \\
\hline Lymphatic, Ectasia & 0 & 0 & 0 & $1(1.0)$ & $10 * *(1.2)$ & $10 * *(2.2)$ \\
\hline Intestine Small, Jejunum & 10 & 9 & 10 & 10 & 10 & 10 \\
\hline Lamina Propria, Lipidosis & 0 & $1(1.0)$ & $1(1.0)$ & $5^{* *}(1.0)$ & $10 * *(2.3)$ & $10 * *(3.5)$ \\
\hline Lymphatic, Ectasia & 0 & $1(1.0)$ & $1(1.0)$ & $5 * *(1.0)$ & $10 * *(2.3)$ & $10 * *(3.5)$ \\
\hline Lymph Node, Mesenteric & 7 & 10 & 9 & 10 & 10 & 10 \\
\hline Lipidosis & 0 & $2(1.0)$ & $1(1.0)$ & 0 & $5 *(1.4)$ & $10 * *(3.0)$ \\
\hline Lymphatic, Ectasia & 0 & $2(1.0)$ & $1(1.0)$ & 0 & $5 *(1.4)$ & $10 * *(3.0)$ \\
\hline \multicolumn{7}{|l|}{ Female } \\
\hline Intestine Small, Duodenum & 10 & 10 & 10 & 10 & 10 & 10 \\
\hline Lamina Propria, Lipidosis & 0 & 0 & 0 & 0 & $9 * *(1.0)$ & $10 * *(2.4)$ \\
\hline Lymphatic, Ectasia & 0 & 0 & 0 & 0 & $9 * *(1.0)$ & $10 * *(2.4)$ \\
\hline Intestine Small, Jejunum & 10 & 10 & 10 & 10 & 10 & 10 \\
\hline Lamina Propria, Lipidosis & 0 & 0 & $1(1.0)$ & $10 * *(1.0)$ & $10 * *(1.8)$ & $10 * *(3.0)$ \\
\hline Lymphatic, Ectasia & 0 & 0 & $1(1.0)$ & $10 * *(1.0)$ & $10 * *(1.8)$ & $10 * *(3.0)$ \\
\hline Lymph Node, Mesenteric & 10 & 10 & 10 & 10 & 10 & 10 \\
\hline Lipidosis & 0 & 0 & 0 & 0 & $3(1.3)$ & $9 * *(3.2)$ \\
\hline Lymphatic, Ectasia & 0 & 0 & 0 & 0 & $3(1.3)$ & $9 * *(3.2)$ \\
\hline
\end{tabular}


In the mesenteric lymph node, dose-related increased incidences and severities of lymphatic ectasia and lipidosis occurred in males and females. These incidences were significantly increased in 150 and $300 \mathrm{mg} / \mathrm{kg}$ males and $300 \mathrm{mg} / \mathrm{kg}$ females compared to the vehicle controls. These lesions occurred primarily in the subcapsular sinuses, occasionally in the cortex and paracortex, and infrequently in the medullary sinuses. Microscopically, the ectatic lymphatics were dilated and contained macrophages with foamy-appearing cytoplasm suggestive of accumulated lipid and occasional multiinucleated giant cells. Lesions of moderate or marked severity often disrupted the nodal architecture. The cytoplasmic vacuoles within macrophages stained positively with special histochemical stains (Oil-red-O and Sudan Black) for lipid.

Dose Selection Rationale: In the 3-month study, chemical-related increases in liver and kidney weights were observed in F344/N rats. Lymphatic ectasia occurred in the small intestine and mesenteric lymph node, but the lesions were not considered severe enough to compromise survival in the 2-year study. At $300 \mathrm{mg} / \mathrm{kg}$, there were $7 \%$ and $12 \%$ decreases in body weight gains of females and males, respectively, and an $8 \%$ decrease in terminal body weight in males. Decreased body weights were not considered sufficient to exclude $300 \mathrm{mg} / \mathrm{kg}$ as the highest dose in the 2-year study. There was concern, however, that higher doses would not be well tolerated. At $300 \mathrm{mg} / \mathrm{kg}$, the induction of hepatic EROD activity by indole-3-carbinol was greater than 100 -fold in treated males and 80-fold in treated females compared to the vehicle controls. These levels of induction were equivalent to or exceeded the levels of induction at which exposure to dioxin-like aryl hydrocarbon receptor ligands such as PCB 126 and TCDD induced moderate to marked hepatotoxicity in 2-year studies ${ }^{74 ;}$. The only liver toxicity observed in these studies at 14 weeks was hepatocyte hypertrophy, a lesion previously observed in some studies of indole-3carbinol. Based on the available data, the doses selected for the 2-year gavage study in Harlan Sprague Dawley rats were 75, 150, and $300 \mathrm{mg} / \mathrm{kg}$.

\section{Two-year Study in Sprague Dawley Rats}

\section{Survival}

Estimates of 2-year survival probabilities for male and female rats are shown in Table 6 and in the Kaplan-Meier survival curves (Figure 4). Survival of dosed groups was similar to that of the vehicle control groups.

\section{Body Weights and Clinical Findings}

Mean body weights of dosed groups of males and females were similar to those of the vehicle controls throughout the study (Figure 5; Table 7 and Table 8). No clinical findings related to the administration of indole-3-carbinol were observed in males or females. 
Indole-3-carbinol, NTP TR 584

Table 6. Survival of Sprague Dawley Rats in the Two-year Gavage Study of Indole-3-carbinol

\begin{tabular}{|c|c|c|}
\hline $\begin{array}{l}\text { Vehicle } \\
\text { Control }\end{array}$ & 75 mg/kg & $150 \mathrm{mg} / \mathrm{kg}$ \\
\hline
\end{tabular}

\section{Male}

Animals initially in study

$\begin{array}{cccc}50 & 50 & 50 & 50 \\ 0 & 0 & 0 & 1 \\ 14 & 23 & 15 & 20 \\ 16 & 14 & 18 & 17 \\ 20 & 13 & 17 & 12 \\ 40 & 26 & 34 & 25 \\ 644 & 617 & 640 & 615 \\ =0.224 & \mathrm{P}=0.084 & \mathrm{P}=0.557 & \mathrm{P}=0.100\end{array}$

Accidental death ${ }^{\mathrm{a}}$

Moribund

Natural deaths

Animals surviving to study termination

Percent probability of survival at end of study ${ }^{b}$

Mean survival (days) ${ }^{\mathrm{c}}$

$\mathrm{P}=0.224$

$\begin{array}{cccc}50 & 50 & 50 & 50 \\ 0 & 1 & 0 & 1 \\ 24 & 22 & 23 & 11 \\ 5 & 8 & 7 & 8 \\ 21 & 19^{\mathrm{e}} & 20^{\mathrm{e}} & 30 \\ 42 & 39 & 38 & 61 \\ 637 & 634 & 625 & 648 \\ =0.055 \mathrm{~N} & \mathrm{P}=1.000 & \mathrm{P}=0.837 & \mathrm{P}=0.079 \mathrm{~N}\end{array}$

Female

Animals initially in study

Accidental deaths ${ }^{\mathrm{a}}$

Moribund

Natural deaths

Animals surviving to study termination

Percent probability of survival at end of study

Mean survival (days)

$$
\mathrm{P}=0.055 \mathrm{~N}
$$$$
\mathrm{P}=1.000
$$

${ }^{a}$ Censored in the survival analyses.

baplan-Meier determinations.

${ }^{c}$ Mean of all deaths (uncensored, censored, and terminal euthanasia).

${ }^{\mathrm{d}}$ The result of the life table trend test ${ }^{91}$ is in the vehicle control column, and the results of the life table pairwise comparisons ${ }^{90}$ with the vehicle controls are in the dosed group columns. A negative trend or lower mortality in a dose group is indicated by $\mathbf{N}$. 'encludes one animal that died during the last week of the study. 
Indole-3-carbinol, NTP TR 584
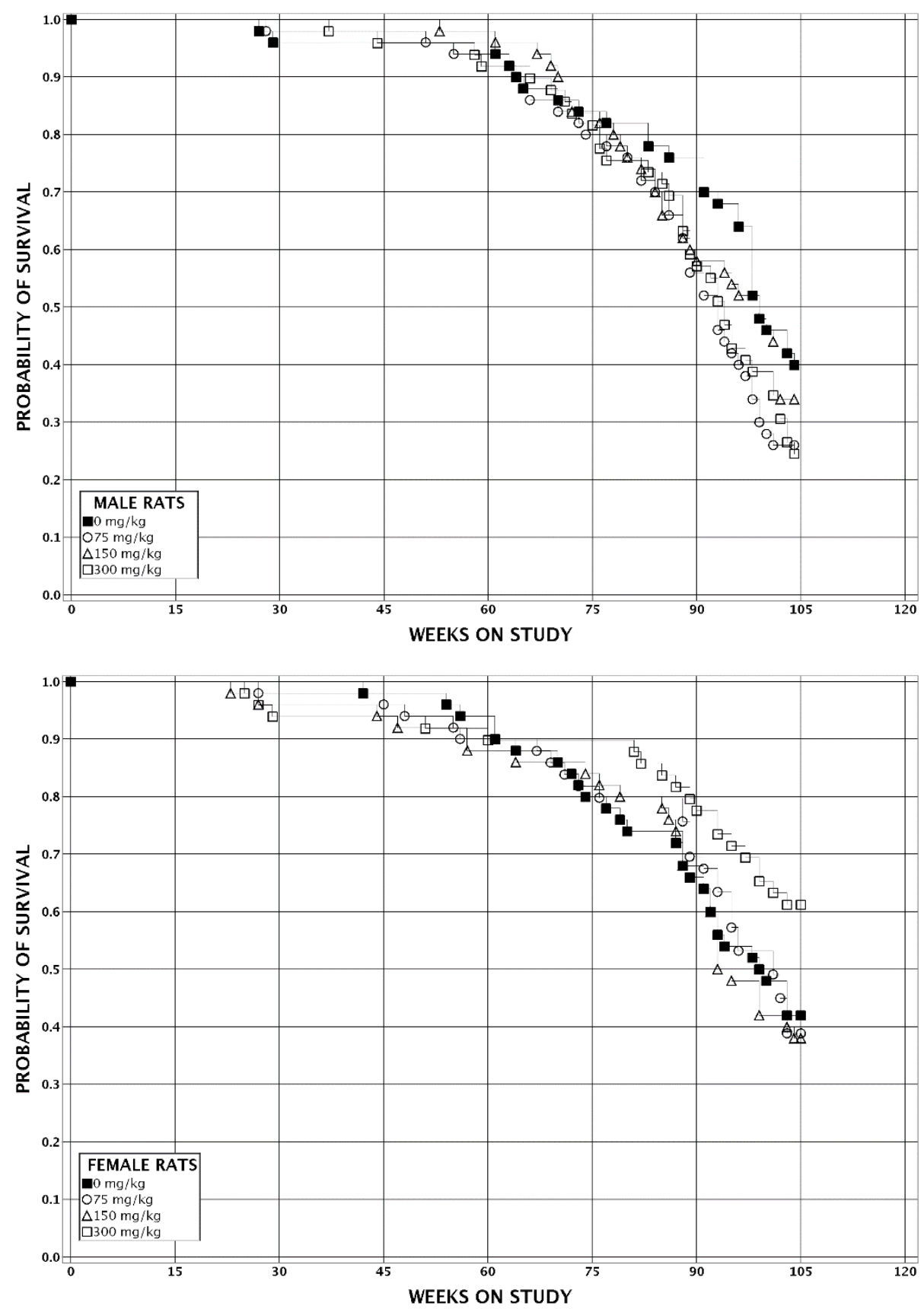

Figure 4. Kaplan-Meier Survival Curves for Sprague Dawley Rats Administered Indole-3-carbinol by Gavage for Two Years 
Indole-3-carbinol, NTP TR 584
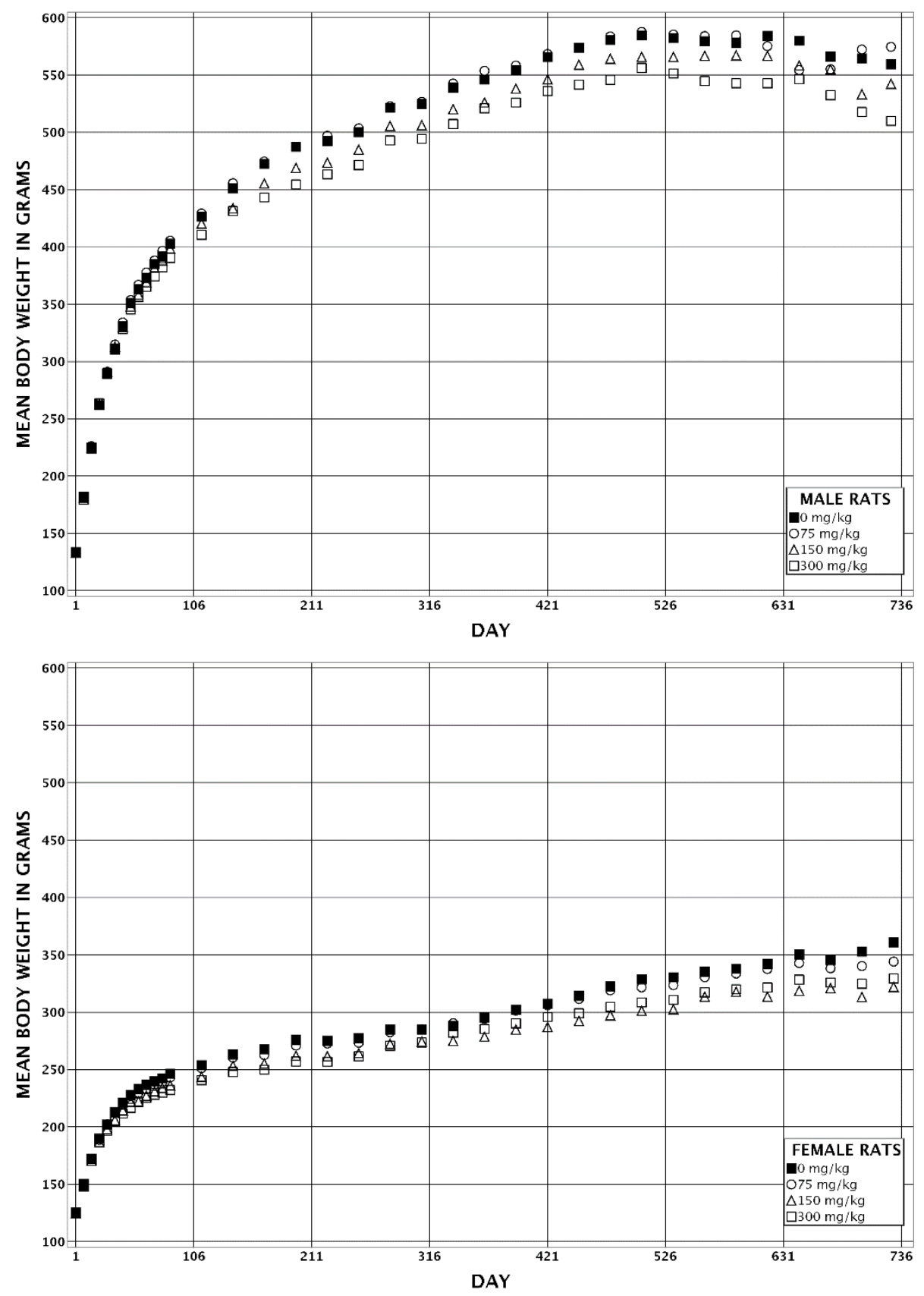

Figure 5. Growth Curves for Sprague Dawley Rats Administered Indole-3-carbinol by Gavage for Two Years 
Indole-3-carbinol, NTP TR 584

Table 7. Mean Body Weights and Survival of Male Sprague Dawley Rats in the Two-year Gavage Study of Indole-3-carbinol

\begin{tabular}{|c|c|c|c|c|c|c|c|c|c|c|c|}
\hline \multirow[b]{2}{*}{ Day } & \multicolumn{2}{|c|}{ Vehicle Control } & \multicolumn{3}{|c|}{75 mg/kg } & \multicolumn{3}{|c|}{150 mg/kg } & \multicolumn{3}{|c|}{$300 \mathrm{mg} / \mathrm{kg}$} \\
\hline & $\begin{array}{l}\text { Av. } \\
\text { Wt. } \\
\text { (g) }\end{array}$ & $\begin{array}{c}\text { No. of } \\
\text { Survivors }\end{array}$ & $\begin{array}{l}\text { Av. } \\
\text { Wt. } \\
\text { (g) }\end{array}$ & $\begin{array}{l}\text { Wt. (\% of } \\
\text { Controls) }\end{array}$ & $\begin{array}{c}\text { No. of } \\
\text { Survivors }\end{array}$ & $\begin{array}{l}\text { Av. } \\
\text { Wt. } \\
\text { (g) }\end{array}$ & $\begin{array}{l}\text { Wt. (\% of } \\
\text { Controls) }\end{array}$ & $\begin{array}{c}\text { No. of } \\
\text { Survivors }\end{array}$ & $\begin{array}{l}\text { Av. } \\
\text { Wt. } \\
\text { (g) }\end{array}$ & $\begin{array}{l}\text { Wt. (\% of } \\
\text { Controls) }\end{array}$ & $\begin{array}{c}\text { No. of } \\
\text { Survivors }\end{array}$ \\
\hline 1 & 134 & 50 & 134 & 100 & 50 & 133 & 100 & 50 & 133 & 100 & 50 \\
\hline 8 & 182 & 50 & 182 & 100 & 50 & 181 & 99 & 50 & 180 & 99 & 50 \\
\hline 15 & 225 & 50 & 226 & 101 & 50 & 225 & 100 & 50 & 224 & 100 & 50 \\
\hline 22 & 262 & 50 & 264 & 101 & 50 & 263 & 100 & 50 & 264 & 101 & 50 \\
\hline 29 & 289 & 50 & 291 & 101 & 50 & 291 & 101 & 50 & 290 & 100 & 50 \\
\hline 36 & 311 & 50 & 315 & 101 & 50 & 313 & 101 & 50 & 311 & 100 & 50 \\
\hline 43 & 331 & 50 & 334 & 101 & 50 & 330 & 100 & 50 & 328 & 99 & 50 \\
\hline 50 & 351 & 50 & 354 & 101 & 50 & 348 & 99 & 50 & 345 & 98 & 50 \\
\hline 57 & 363 & 50 & 367 & 101 & 50 & 359 & 99 & 50 & 357 & 98 & 50 \\
\hline 64 & 373 & 50 & 378 & 101 & 50 & 369 & 99 & 50 & 365 & 98 & 50 \\
\hline 71 & 385 & 50 & 388 & 101 & 50 & 382 & 99 & 50 & 375 & 97 & 50 \\
\hline 78 & 392 & 50 & 397 & 101 & 50 & 388 & 99 & 50 & 382 & 98 & 50 \\
\hline 85 & 403 & 50 & 406 & 101 & 50 & 399 & 99 & 50 & 391 & 97 & 50 \\
\hline 113 & 427 & 50 & 429 & 101 & 50 & 420 & 99 & 50 & 411 & 96 & 50 \\
\hline 141 & 451 & 50 & 456 & 101 & 50 & 434 & 96 & 50 & 432 & 96 & 50 \\
\hline 169 & 473 & 50 & 475 & 100 & 50 & 455 & 96 & 50 & 443 & 94 & 50 \\
\hline 197 & 488 & 48 & 488 & 100 & 49 & 469 & 96 & 50 & 455 & 93 & 49 \\
\hline 225 & 493 & 48 & 497 & 101 & 49 & 474 & 96 & 50 & 464 & 94 & 49 \\
\hline 253 & 500 & 48 & 504 & 101 & 49 & 485 & 97 & 50 & 472 & 94 & 49 \\
\hline 281 & 521 & 48 & 523 & 100 & 49 & 506 & 97 & 50 & 493 & 95 & 48 \\
\hline 309 & 525 & 48 & 527 & 100 & 49 & 506 & 97 & 50 & 495 & 94 & 47 \\
\hline 337 & 539 & 48 & 543 & 101 & 49 & 520 & 97 & 50 & 508 & 94 & 47 \\
\hline 365 & 546 & 48 & 554 & 101 & 48 & 526 & 96 & 50 & 521 & 95 & 47 \\
\hline 393 & 554 & 48 & 558 & 101 & 47 & 538 & 97 & 49 & 526 & 95 & 47 \\
\hline 421 & 566 & 47 & 568 & 100 & 47 & 546 & 97 & 49 & 536 & 95 & 45 \\
\hline 449 & 574 & 45 & 574 & 100 & 45 & 559 & 97 & 48 & 542 & 94 & 45 \\
\hline 477 & 581 & 44 & 584 & 101 & 43 & 564 & 97 & 47 & 546 & 94 & 43 \\
\hline 505 & 585 & 42 & 588 & 101 & 41 & 566 & 97 & 42 & 556 & 95 & 41 \\
\hline 533 & 582 & 42 & 586 & 101 & 39 & 566 & 97 & 41 & 552 & 95 & 38 \\
\hline 561 & 580 & 41 & 584 & 101 & 38 & 567 & 98 & 38 & 545 & 94 & 37 \\
\hline 589 & 578 & 39 & 585 & 101 & 35 & 567 & 98 & 35 & 543 & 94 & 35 \\
\hline 617 & 584 & 38 & 575 & 98 & 29 & 567 & 97 & 31 & 543 & 93 & 30 \\
\hline 645 & 580 & 34 & 554 & 96 & 26 & 558 & 96 & 29 & 547 & 94 & 26 \\
\hline 673 & 566 & 32 & 555 & 98 & 20 & 555 & 98 & 26 & 533 & 94 & 20 \\
\hline 701 & 565 & 23 & 572 & 101 & 14 & 533 & 95 & 23 & 518 & 92 & 18 \\
\hline \multicolumn{12}{|c|}{ Mean for Weeks } \\
\hline $1-13$ & 308 & & 310 & 101 & & 306 & 99 & & 304 & 99 & \\
\hline $14-52$ & 491 & & 494 & 101 & & 474 & 97 & & 464 & 95 & \\
\hline 53-101 & 572 & & 572 & 100 & & 555 & 97 & & 539 & 94 & \\
\hline
\end{tabular}


Table 8. Mean Body Weights and Survival of Female Sprague Dawley Rats in the Two-year Gavage Study of Indole-3-carbinol

\begin{tabular}{|c|c|c|c|c|c|c|c|c|c|c|c|}
\hline \multirow[b]{2}{*}{ Day } & \multicolumn{2}{|c|}{ Vehicle Control } & \multicolumn{3}{|c|}{75 mg/kg } & \multicolumn{3}{|c|}{$150 \mathrm{mg} / \mathrm{kg}$} & \multicolumn{3}{|c|}{300 mg/kg } \\
\hline & $\begin{array}{l}\text { Av. } \\
\text { Wt. } \\
\text { (g) }\end{array}$ & $\begin{array}{c}\text { No. of } \\
\text { Survivors }\end{array}$ & $\begin{array}{l}\text { Av. } \\
\text { Wt. } \\
\text { (g) }\end{array}$ & $\begin{array}{l}\text { Wt. (\% of } \\
\text { Controls) }\end{array}$ & $\begin{array}{c}\text { No. of } \\
\text { Survivors }\end{array}$ & $\begin{array}{l}\text { Av. } \\
\text { Wt. } \\
\text { (g) }\end{array}$ & $\begin{array}{l}\text { Wt. (\% of } \\
\text { Controls) }\end{array}$ & $\begin{array}{c}\text { No. of } \\
\text { Survivors }\end{array}$ & $\begin{array}{l}\text { Av. } \\
\text { Wt. } \\
\text { (g) }\end{array}$ & $\begin{array}{l}\text { Wt. (\% of } \\
\text { Controls) }\end{array}$ & $\begin{array}{c}\text { No. of } \\
\text { Survivors }\end{array}$ \\
\hline 1 & 126 & 50 & 125 & 100 & 50 & 125 & 99 & 50 & 125 & 100 & 50 \\
\hline 8 & 150 & 50 & 150 & 100 & 50 & 149 & 99 & 50 & 148 & 99 & 50 \\
\hline 15 & 172 & 50 & 172 & 100 & 50 & 170 & 99 & 50 & 171 & 99 & 50 \\
\hline 22 & 190 & 50 & 189 & 99 & 50 & 187 & 98 & 50 & 187 & 99 & 50 \\
\hline 29 & 202 & 50 & 203 & 100 & 50 & 198 & 98 & 50 & 197 & 97 & 50 \\
\hline 36 & 213 & 50 & 212 & 100 & 50 & 206 & 97 & 50 & 205 & 96 & 50 \\
\hline 43 & 221 & 50 & 220 & 99 & 50 & 215 & 97 & 50 & 212 & 96 & 50 \\
\hline 50 & 228 & 50 & 227 & 100 & 50 & 222 & 98 & 50 & 217 & 95 & 50 \\
\hline 57 & 233 & 50 & 230 & 99 & 50 & 222 & 95 & 50 & 222 & 95 & 50 \\
\hline 64 & 237 & 50 & 233 & 98 & 50 & 227 & 96 & 50 & 225 & 95 & 50 \\
\hline 71 & 240 & 50 & 237 & 99 & 50 & 231 & 96 & 50 & 228 & 95 & 50 \\
\hline 78 & 243 & 50 & 239 & 99 & 50 & 234 & 97 & 50 & 230 & 95 & 50 \\
\hline 85 & 246 & 50 & 243 & 99 & 50 & 236 & 96 & 50 & 233 & 94 & 50 \\
\hline 113 & 254 & 50 & 251 & 99 & 50 & 244 & 96 & 50 & 241 & 95 & 50 \\
\hline 141 & 263 & 50 & 260 & 99 & 50 & 253 & 96 & 50 & 248 & 94 & 50 \\
\hline 169 & 268 & 50 & 263 & 98 & 50 & 255 & 95 & 49 & 250 & 93 & 50 \\
\hline 197 & 276 & 50 & 271 & 98 & 49 & 262 & 95 & 48 & 257 & 93 & 46 \\
\hline 225 & 275 & 50 & 273 & 99 & 49 & 262 & 95 & 48 & 257 & 93 & 46 \\
\hline 253 & 277 & 50 & 273 & 99 & 49 & 264 & 95 & 48 & 262 & 94 & 46 \\
\hline 281 & 285 & 50 & 283 & 99 & 49 & 272 & 96 & 48 & 271 & 95 & 46 \\
\hline 309 & 285 & 49 & 285 & 100 & 49 & 275 & 96 & 47 & 274 & 96 & 46 \\
\hline 337 & 288 & 49 & 290 & 101 & 47 & 275 & 96 & 46 & 282 & 98 & 46 \\
\hline 365 & 295 & 49 & 294 & 100 & 47 & 279 & 94 & 46 & 286 & 97 & 45 \\
\hline 393 & 302 & 47 & 301 & 100 & 45 & 285 & 94 & 45 & 290 & 96 & 45 \\
\hline 421 & 307 & 47 & 306 & 100 & 44 & 287 & 93 & 44 & 296 & 96 & 44 \\
\hline 449 & 315 & 44 & 312 & 99 & 44 & 292 & 93 & 43 & 299 & 95 & 44 \\
\hline 477 & 323 & 44 & 319 & 99 & 43 & 297 & 92 & 43 & 305 & 94 & 44 \\
\hline 505 & 329 & 42 & 322 & 98 & 41 & 301 & 92 & 43 & 309 & 94 & 44 \\
\hline 533 & 331 & 40 & 324 & 98 & 39 & 303 & 92 & 41 & 311 & 94 & 44 \\
\hline 561 & 335 & 37 & 330 & 99 & 39 & 314 & 94 & 40 & 317 & 95 & 44 \\
\hline 589 & 338 & 37 & 334 & 99 & 39 & 318 & 94 & 40 & 320 & 95 & 42 \\
\hline 617 & 342 & 33 & 338 & 99 & 37 & 314 & 92 & 34 & 322 & 94 & 40 \\
\hline 645 & 350 & 28 & 343 & 98 & 31 & 319 & 91 & 28 & 329 & 94 & 36 \\
\hline 673 & 346 & 27 & 338 & 98 & 26 & 321 & 93 & 24 & 326 & 94 & 35 \\
\hline 701 & 353 & 24 & 340 & 96 & 26 & 313 & 89 & 21 & 325 & 92 & 31 \\
\hline \multicolumn{12}{|c|}{ Mean for Weeks } \\
\hline $1-13$ & 208 & & 206 & 99 & & 202 & 97 & & 200 & 96 & \\
\hline 14-52 & 275 & & 272 & 99 & & 262 & 95 & & 260 & 95 & \\
\hline 53-101 & 328 & & 323 & 98 & & 303 & 92 & & 310 & 95 & \\
\hline
\end{tabular}




\section{Pathology and Statistical Analyses}

This section describes the statistically significant or biologically noteworthy changes in the incidences of neoplasms and/or nonneoplastic lesions of the uterus, skin, small intestine (duodenum and jejunum), mesenteric lymph node, liver, thyroid gland, and pituitary gland. Summaries of the incidences of neoplasms and nonneoplastic lesions, statistical analyses of primary neoplasms that occurred with an incidence of at least 5\% in at least one animal group, and historical incidences for the neoplasms mentioned in this section are presented in Appendix A for male rats and Appendix B for female rats.

Uterus: In the standard evaluation, the incidences of adenocarcinoma occurred with a positive trend and were increased in all dosed groups (Table 9, Table B-1, and Table B-2). Although the increased incidences of adenocarcinoma were not statistically significant, the incidences in the 150 and $300 \mathrm{mg} / \mathrm{kg}$ groups exceeded the historical control range for corn oil gavage studies (Table 9 and Table B-3). In the extended evaluation, additional neoplasms and nonneoplastic proliferative lesions were diagnosed in the uteri of the vehicle control and dosed groups. In addition, previously undiagnosed types of neoplasms and nonneoplastic proliferative lesions were diagnosed in the uteri of the vehicle control and dosed groups including atypical hyperplasias, low incidences of adenomas in each of the dosed groups, and one adenosquamous carcinoma and one benign basosquamous tumor each in the $150 \mathrm{mg} / \mathrm{kg}$ group (Table 9 and Table B-2).

In the combined standard and extended evaluations, the incidence of adenocarcinoma was significantly increased in the $150 \mathrm{mg} / \mathrm{kg}$ group (Table 9). The incidences of adenoma were not significantly increased in the dosed groups; adenomas have not been observed in vehicle controls in gavage studies using female Harlan Sprague Dawley rats.

In the standard evaluation, a significantly increased incidence of squamous metaplasia of the endometrium occurred in the $150 \mathrm{mg} / \mathrm{kg}$ group (Table 9 and Table B-5). In the combined standard and extended evaluations, the incidence of squamous metaplasia of the endometrium was significantly decreased in the $300 \mathrm{mg} / \mathrm{kg}$ group (Table 9).

Uterine adenocarcinomas were large irregular, invasive masses that effaced the endometrium and commonly extended into the myometrium. They were composed of cuboidal to columnar neoplastic cells that formed irregular tubules, glandular structures or ducts that were surrounded by collagenous stroma. The neoplastic cells had scant eosinophilic cytoplasm and large vesicular nuclei and there was mild to marked pleomorphism and atypia. The neoplasm often contained areas of inflammation, hemorrhage, and/or necrosis (Figure 9 and Figure 10).

Uterine adenomas were solitary, non-invasive, nodular masses that were generally composed of a single layer of cuboidal to columnar epithelial cells typically arranged in glandular structures in which there was minimal to mild stratification or disorganized piling up of the epithelium (Figure 11 and Figure 12). The glandular structures were sometimes dilated and the neoplastic cells formed papillary infoldings or papillary projections into the lumen.

Atypical endometrial hyperplasia was observed in the luminal or glandular epithelium of the uterus. Epithelial atypia in the luminal epithelium consisted of a spectrum of changes that included increased cellular anisocytosis and anisokaryosis and increased cytoplasmic eosinophilia, epithelial blebbing, large cytoplasmic vacuoles and the presence of small papillary 
projections lined by disorganized epithelial cells. Occasionally, small branching papillary projections were characterized by epithelial lined, short, slender, fibrovascular stalks that extended into the uterine lumen (Figure 13). Epithelial atypia in the glands affected single glands or clusters that were separated by minimal amounts of stroma. The glands were lined by pseudostratified to stratified, cuboidal to tall columnar epithelial cells that exhibited cytoplasmic eosinophilia or basophilia, loss of cellular polarity, karyomegaly, mitoses, and minimal to mild cellular pleomorphism (Figure 14).

Squamous metaplasia of the endometrium was of minimal to moderate severity and consisted of replacement of the normal simple columnar epithelium lining the endometrium (uterine lumen) and occasional endometrial glands by keratinized squamous epithelium. In more severe cases, the uterine lumen was dilated and filled with varying amounts of keratin.

Table 9. Incidences of Neoplasms and Nonneoplastic Lesions of the Uterus in Female Sprague Dawley Rats in the Two-year Gavage Study of Indole-3-carbinol

\begin{tabular}{|c|c|c|c|c|}
\hline & $\begin{array}{l}\text { Vehicle } \\
\text { Control }\end{array}$ & $75 \mathrm{mg} / \mathrm{kg}$ & $150 \mathrm{mg} / \mathrm{kg}$ & $300 \mathrm{mg} / \mathrm{kg}$ \\
\hline \multicolumn{5}{|l|}{ Standard Evaluation } \\
\hline Number Necropsied & 50 & 50 & 50 & 50 \\
\hline Endometrium, Metaplasia, Squamous ${ }^{\mathrm{a}}$ & $12(1.9)^{\mathrm{b}}$ & $18(2.2)$ & $20 *(2.1)$ & $11(1.8)$ \\
\hline Adenocarcinoma, Multiple & 0 & 0 & 0 & 1 \\
\hline \multicolumn{5}{|l|}{ Adenocarcinoma (includes multiple) $^{\mathrm{c}}$} \\
\hline Overall rate ${ }^{\mathrm{d}}$ & $0 / 50(0 \%)$ & $1 / 50(2 \%)$ & $4 / 50(8 \%)$ & $4 / 50(8 \%)$ \\
\hline Adjusted rate ${ }^{\mathrm{e}}$ & $0.0 \%$ & $2.7 \%$ & $10.9 \%$ & $9.8 \%$ \\
\hline Terminal rate ${ }^{\mathrm{f}}$ & $0 / 21(0 \%)$ & 0/19 (0\%) & $1 / 19(5 \%)$ & 2/30 (7\%) \\
\hline First incidence (days) & $-^{\mathrm{h}}$ & 645 & 608 & 645 \\
\hline Poly-3 test ${ }^{\mathrm{g}}$ & $\mathrm{P}=0.040$ & $\mathrm{P}=0.503$ & $\mathrm{P}=0.059$ & $\mathrm{P}=0.074$ \\
\hline \multicolumn{5}{|l|}{ Extended Evaluation } \\
\hline Number Necropsied & 50 & 50 & 50 & 50 \\
\hline Endometrium, Metaplasia, Squamous & 38 & 33 & 35 & $27 * *$ \\
\hline Endometrium, Hyperplasia, Atypical & 15 & 14 & 21 & 16 \\
\hline Adenoma & 0 & 2 & 1 & 1 \\
\hline Basosquamous Tumor, Benign & 0 & 0 & 1 & 0 \\
\hline Adenocarcinoma, Multiple & 1 & 0 & 3 & 2 \\
\hline \multicolumn{5}{|l|}{ Adenocarcinoma (includes multiple) } \\
\hline Overall rate & $5 / 50(10 \%)$ & $3 / 50(6 \%)$ & $10 / 50(20 \%)$ & $8 / 50(16 \%)$ \\
\hline Adjusted rate & $13.7 \%$ & $8.2 \%$ & $26.9 \%$ & $19.8 \%$ \\
\hline Terminal rate & $3 / 21(14 \%)$ & 2/19 (11\%) & $5 / 19(26 \%)$ & $7 / 30(23 \%)$ \\
\hline First incidence (days) & 719 & 719 & 615 & 674 \\
\hline Poly-3 test & $P=0.158$ & $\mathrm{P}=0.351 \mathrm{~N}$ & $\mathrm{P}=0.127$ & $P=0.340$ \\
\hline
\end{tabular}


Indole-3-carbinol, NTP TR 584

\begin{tabular}{|c|c|c|c|c|}
\hline & $\begin{array}{l}\text { Vehicle } \\
\text { Control }\end{array}$ & $75 \mathrm{mg} / \mathrm{kg}$ & $150 \mathrm{mg} / \mathrm{kg}$ & $300 \mathrm{mg} / \mathrm{kg}$ \\
\hline \multicolumn{5}{|l|}{ Adenoma or Adenocarcinoma } \\
\hline Overall rate & $5 / 50(10 \%)$ & $5 / 50(10 \%)$ & $11 / 50(22 \%)$ & $9 / 50(18 \%)$ \\
\hline Adjusted rate & $13.7 \%$ & $13.5 \%$ & $29.6 \%$ & $22.3 \%$ \\
\hline Terminal rate & $3 / 21(14 \%)$ & $3 / 19(16 \%)$ & 6/19 (32\%) & $8 / 30(27 \%)$ \\
\hline First incidence (days) & 719 & 645 & 615 & 674 \\
\hline Poly-3 test & $P=0.146$ & $\mathrm{P}=0.624 \mathrm{~N}$ & $P=0.080$ & $P=0.247$ \\
\hline Adenosquamous Carcinoma & 0 & 0 & 1 & 0 \\
\hline \multicolumn{5}{|c|}{ Standard and Extended Evaluations (Combined) } \\
\hline Number Necropsied & 50 & 50 & 50 & 50 \\
\hline Endometrium, Metaplasia, Squamous & 38 & 33 & 36 & $27^{* *}$ \\
\hline Endometrium, Hyperplasia, Atypical & 15 & 14 & 21 & 16 \\
\hline Adenoma & 0 & 2 & 1 & 1 \\
\hline Basosquamous Tumor, Benign & 0 & 0 & 1 & 0 \\
\hline Adenocarcinoma, Multiple & 1 & 0 & 3 & 2 \\
\hline Adenocarcinoma (includes multiple) & 5 & 4 & $13^{*}$ & 10 \\
\hline \multicolumn{5}{|l|}{ Adenoma or Adenocarcinoma } \\
\hline Overall rate & $5 / 50(10 \%)$ & $5 / 50(10 \%)$ & $14 / 50$ (28\%) & $11 / 50(22 \%)$ \\
\hline Adjusted rate & $13.7 \%$ & $13.5 \%$ & $36.6 \%$ & $27.0 \%$ \\
\hline Terminal rate & $3 / 21(14 \%)$ & $3 / 19(16 \%)$ & 6/19 (32\%) & $9 / 30(30 \%)$ \\
\hline First incidence (days) & 719 & 645 & 608 & 645 \\
\hline Poly-3 test & $\mathrm{P}=0.052$ & $\mathrm{P}=0.624 \mathrm{~N}$ & $\mathrm{P}=0.018$ & $P=0.119$ \\
\hline Adenosquamous Carcinoma & 0 & 0 & 1 & 0 \\
\hline \multicolumn{5}{|c|}{ Adenocarcinoma or Adenosquamous Carcinoma } \\
\hline Overall rate & $5 / 50(10 \%)$ & $4 / 50(8 \%)$ & $13 / 50(26 \%)$ & $10 / 50(20 \%)$ \\
\hline Adjusted rate & $13.7 \%$ & $10.8 \%$ & $34.0 \%$ & $24.6 \%$ \\
\hline Terminal rate & $3 / 21(14 \%)$ & $2 / 19(11 \%)$ & $5 / 19(26 \%)$ & $8 / 30(27 \%)$ \\
\hline First incidence (days) & 719 & 645 & 608 & 645 \\
\hline Poly-3 test & $P=0.071$ & $\mathrm{P}=0.491 \mathrm{~N}$ & $P=0.033$ & $\mathrm{P}=0.177$ \\
\hline
\end{tabular}

*Significantly different $(\mathrm{P} \leq 0.05)$ from the vehicle control group by the Poly-3 test.

$* * \mathrm{P} \leq 0.01$.

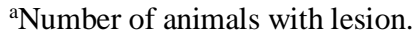

${ }^{\mathrm{b}}$ Average severity grade of lesions in affected animals: $1=$ minimal, $2=$ mild, $3=$ moderate, $4=$ marked.

'Historical incidence for 2-year gavage studies with corn oil vehicle control groups (mean \pm standard deviation): 2/102.

${ }^{\mathrm{d}}$ Number of animals with neoplasm per number of animals necropsied.

ePoly-3 estimated neoplasm incidence after adjustment for intercurrent mortality.

${ }_{\mathrm{f}}$ Observed incidence at terminal euthanasia.

gBeneath the vehicle control incidence is the $\mathrm{P}$ value associated with the trend test. Beneath the dosed group incidence are the $\mathrm{P}$ values corresponding to pairwise comparisons between the vehicle controls and that dosed group. The Poly-3 test accounts for differential mortality in animals that do not reach terminal euthanasia. A lower incidence in a dose group is indicated by $\mathbf{N}$.

hot applicable; no neoplasms in animal group. 
Skin: An increased incidence of fibroma occurred in $300 \mathrm{mg} / \mathrm{kg}$ females (Table 10, Table B-1, and Table B-2). Although the increased incidence of this neoplasm was not statistically significant, the incidence in $300 \mathrm{mg} / \mathrm{kg}$ females exceeded the historical control range for corn oil gavage studies (Table 10 and Table B-4). A single incidence each of fibrosarcoma occurred in vehicle control and $300 \mathrm{mg} / \mathrm{kg}$ females (Table 10 and Table B-1). The incidences of fibroma or fibrosarcoma (combined) occurred with a positive trend in females (Table 10 and Table B-2). Fibromas were circumscribed subcutaneous masses composed of well-differentiated fibrocytes interspersed between variable amounts of mature collagen stroma. Fibrosarcomas were large, invasive, masses that effaced the subcutaneous tissues and consisted of moderately welldifferentiated neoplastic fibroblasts arranged in interlacing bundles and whorls.

Table 10. Incidences of Neoplasms of the Skin in Female Sprague Dawley Rats in the Two-year Gavage Study of Indole-3-carbinol

\begin{tabular}{lcccc}
\hline & Vehicle Control & $\mathbf{7 5 ~} \mathbf{~} \mathbf{g} / \mathbf{k g}$ & $\mathbf{1 5 0} \mathbf{~} \mathbf{g} / \mathbf{k g}$ & $\mathbf{3 0 0} \mathbf{~} \mathbf{g} / \mathbf{k g}$ \\
\hline Number Necropsied & 50 & 50 & 50 & 50 \\
Fibroma $^{\mathrm{a}, \mathrm{b}}$ & 1 & 0 & 0 & 4 \\
Fibrosarcoma $^{\mathrm{c}}$ & 1 & 0 & 0 & 1 \\
Fibroma or Fibrosarcoma $^{\mathrm{d}}$ & & & & \\
Overall rate $^{\mathrm{e}}$ & $2 / 50(4 \%)$ & $0 / 50(0 \%)$ & $0 / 50(0 \%)$ & $5 / 50(10 \%)$ \\
Adjusted rate $^{\mathrm{f}}$ & $5.4 \%$ & $0.0 \%$ & $0.0 \%$ & $12.4 \%$ \\
Terminal rate $^{\mathrm{g}}$ & $1 / 21(5 \%)$ & $0 / 19(0 \%)$ & $0 / 19(0 \%)$ & $3 / 30(10 \%)$ \\
First incidence (days) & 645 & $-\mathcal{-}^{\mathrm{i}}$ & - & 692 \\
Poly-3 test $^{\mathrm{h}}$ & $\mathrm{P}=0.040$ & $\mathrm{P}=0.237 \mathrm{~N}$ & $\mathrm{P}=0.243 \mathrm{~N}$ & $\mathrm{P}=0.254$ \\
\hline
\end{tabular}

${ }^{\mathrm{a} N u m b e r}$ of animals with lesion.

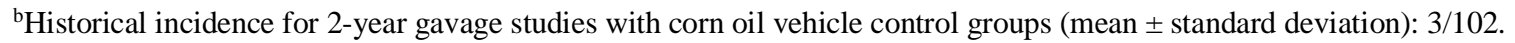

'Historical incidence for corn oil gavage studies: 1/102.

${ }^{\mathrm{d}}$ Historical incidence for corn oil gavage studies: 4/102.

${ }^{\text {e}}$ Number of animals with neoplasm per number of animals necropsied.

fPoly-3 estimated neoplasm incidence after adjustment for intercurrent mortality.

gObserved incidence at terminal euthanasia.

heneath the vehicle control incidence is the $\mathrm{P}$ value associated with the trend test. Beneath the dosed group incidence are the $\mathrm{P}$ values corresponding to pairwise comparisons between the vehicle controls and that dosed group. The Poly-3 test accounts for differential mortality in animals that do not reach terminal euthanasia. A lower incidence in a dose group is indicated by $\mathbf{N}$. ${ }^{i}$ Not applicable; no neoplasms in animal group.

Small Intestine: Significantly increased incidences of lymphatic ectasia of the duodenum and jejunum with generally increased severities occurred in 150 and $300 \mathrm{mg} / \mathrm{kg}$ males and females; two incidences of lymphatic ectasia occurred in the jejunum of $75 \mathrm{mg} / \mathrm{kg}$ males (Table 11, Table A-3, and Table B-5). Lymphatic ectasia did not occur in vehicle control animals.

Lymphatic ectasia was of minimal to marked severity and characterized by dilation of lymphatic vessels in villi of the duodenum and jejunum (Figure 15 and Figure 16). The dilated lymphatics varied in size and number, and were lined by flattened endothelial cells. The lumens of the dilated lymphatics were generally empty, but a few lymphatics contained minimal amounts of lacy, amphophilic material. In general, the jejunum was more frequently and severely affected than the duodenum. 
Mesenteric Lymph Node: Significantly increased incidences of lymphatic ectasia occurred in $300 \mathrm{mg} / \mathrm{kg}$ males and females (Table 11, Table A-3, and Table B-5). Single incidences of lymphatic ectasia occurred in a $150 \mathrm{mg} / \mathrm{kg}$ male and in a $150 \mathrm{mg} / \mathrm{kg}$ female. Lymphatic ectasia did not occur in vehicle control animals.

Lymphatic ectasia was of minimal to moderate severity and characterized by dilation of lymphatic vessels primarily in subcapsular and cortical regions of the nodes, but occasionally the paracortical and medullary lymphatics were affected. These dilated lymphatics varied in size and number, and were lined by flattened endothelial cells. In general, females were more frequently and severely affected than males (Figure 17 and Figure 18).

Liver: Incidences of cholangiofibrosis occurred in all dosed groups of males and in one $75 \mathrm{mg} / \mathrm{kg}$ female (Table 11, Table A-3, and Table B-5). The incidences of eosinophilic focus in 150 and $300 \mathrm{mg} / \mathrm{kg}$ females and of clear cell focus in $300 \mathrm{mg} / \mathrm{kg}$ females were significantly increased compared to the vehicle controls. The incidence of bile duct cyst was significantly increased in $300 \mathrm{mg} / \mathrm{kg}$ males.

Table 11. Incidences of Selected Nonneoplastic Lesions in Sprague Dawley Rats in the Two-year Gavage Study of Indole-3-carbinol

\begin{tabular}{|c|c|c|c|c|}
\hline & Vehicle Control & $75 \mathrm{mg} / \mathrm{kg}$ & $150 \mathrm{mg} / \mathrm{kg}$ & $300 \mathrm{mg} / \mathrm{kg}$ \\
\hline \multicolumn{5}{|l|}{ Male } \\
\hline Intestine Small, Duodenum ${ }^{a}$ & 43 & 48 & 47 & 48 \\
\hline Lymphatic, Ectasiab $^{\mathrm{b}}$ & 0 & 0 & $15^{* *}(1.5)^{\mathrm{c}}$ & $14^{* *}(1.4)$ \\
\hline Intestine Small, Jejunum & 40 & 39 & 40 & 42 \\
\hline Lymphatic, Ectasia & 0 & $2(1.0)$ & $27 * *(1.7)$ & $41 * *(2.0)$ \\
\hline Lymph Node, Mesenteric & 50 & 50 & 50 & 50 \\
\hline Lymphatic, Ectasia & 0 & 0 & $1(3.0)$ & $5^{*}(1.4)$ \\
\hline Liver & 50 & 50 & 50 & 50 \\
\hline Cholangiofibrosis & 0 & $1(2.0)$ & $3(2.7)$ & $1(2.0)$ \\
\hline Bile Duct, Cyst & 0 & 0 & $2(2.0)$ & $5^{*}(2.4)$ \\
\hline Thyroid Gland & 50 & 46 & 48 & 47 \\
\hline Follicular Cell, Hypertrophy & $21(1.8)$ & $34 * *(1.9)$ & $33 * *(2.1)$ & $36 * *(2.6)$ \\
\hline \multicolumn{5}{|l|}{ Female } \\
\hline Intestine Small, Duodenum & 48 & 47 & 48 & 47 \\
\hline Lymphatic, Ectasia & 0 & 0 & $16^{* *}(1.2)$ & $38 * *(1.5)$ \\
\hline Intestine Small, Jejunum & 47 & 46 & 48 & 48 \\
\hline Lymphatic, Ectasia & 0 & 0 & $30 * *(1.7)$ & $47^{* *}(2.5)$ \\
\hline Lymph Node, Mesenteric & 50 & 50 & 50 & 48 \\
\hline Lymphatic, Ectasia & 0 & 0 & $1(1.0)$ & $15^{* *}(1.7)$ \\
\hline
\end{tabular}


Indole-3-carbinol, NTP TR 584

\begin{tabular}{lcccc}
\hline & Vehicle Control & $\mathbf{7 5 ~} \mathbf{~} \mathbf{g} / \mathbf{k g}$ & $\mathbf{1 5 0} \mathbf{~} \mathbf{g} / \mathbf{k g}$ & $\mathbf{3 0 0} \mathbf{~} \mathbf{g} / \mathbf{k g}$ \\
\hline Liver & 50 & 50 & 50 & 48 \\
Cholangiofibrosis & 0 & $1(1.0)$ & 0 & 0 \\
Clear Cell Focus & 6 & 7 & 4 & $18^{* *}$ \\
Eosinophilic Focus & 0 & 4 & $5^{*}$ & $6^{*}$ \\
\hline
\end{tabular}

*Significantly different $(\mathrm{P} \leq 0.05)$ from the vehicle control group by the Poly-3 test.

$* * \mathrm{P} \leq 0.01$.

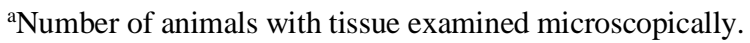

bNumber of animals with lesion.

${ }^{\mathrm{c}}$ Average severity grade of lesions in affected animals: $1=$ minimal, $2=$ mild, $3=$ moderate, $4=$ marked.

Cholangiofibrosis was of minimal to marked severity and consisted of sharply demarcated, nonencapsulated, focal to locally extensive lesions that effaced the hepatic parenchyma. Frequently, cholangiofibrosis occurred as nodular lesions that protruded from the capsular surface of the liver. At necropsy, increased incidences of liver masses and cysts were grossly observed in dosed groups of males and females compared to the vehicle controls. Parenchymal lesions consisted of variable amounts of dense collagenous connective tissue that surrounded multiple, variably sized, randomly distributed, dilated, atypical bile ducts that invariably contained eosinophilic to amphophilic, mucinous material and cellular debris (Figure 19 and Figure 20). The atypical ducts were lined by low cuboidal to columnar hyperbasophilic epithelial cells among which were scattered goblet-like or mucous cells. At times, the epithelium lining the bile ducts was absent, resulting in crescent-shaped structures. Moderate numbers of inflammatory cells were scattered through the collagenous tissue. Capsular lesions were encapsulated and primarily composed of dilated atypical ducts surrounded by lesser amounts of collagenous tissue than in lesions within the hepatic parenchyma (Figure 21 and Figure 22).

Eosinophilic foci were variably-sized discrete focal areas of enlarged hepatocytes in which the cytoplasm stained more lightly or darkly eosinophilic than that of the surrounding hepatocytes; in some cases the cytoplasm appeared granular (Figure 23). Clear cell foci were discrete focal areas of normal-sized to slightly enlarged hepatocytes that had clear cytoplasmic spaces around the cell nuclei (Figure 24).

Bile duct cysts were single to multiple, variably dilated bile ducts that disrupted the hepatic parenchyma. The dilated ducts were lined by flattened to cuboidal epithelial cells and occasionally contained fibrillar eosinophilic material.

Transcriptome analysis was performed on RNA extracted from microarray study female rat livers from the $300 \mathrm{mg} / \mathrm{kg}$ and vehicle control groups after 3 months of exposure. The observed effects on transcription were consistent with induction of xenobiotic metabolism related processes that are initiated through the activation of the aryl hydrocarbon receptor and oxidative stress sensing transcription factor nuclear factor, erythroid 2-like 2. A detailed breakdown of the transcriptomic results can be found in Appendix L.

Thyroid Gland: The incidences of follicular cell hypertrophy were significantly increased in all dosed groups of males compared to the vehicle controls, and the severities of the hypertrophy increased with increasing dose (Table 11 and Table A-3). 
Follicular cell hypertrophy was characterized by an increased height of follicular epithelial cells from normal cuboidal to tall columnar and a concomitant decrease in the diameter of the follicular lumens. In general, follicles contained decreased amounts of colloid and often contained aggregates of flocculent-appearing, amphophilic material. The following criteria were used when diagnosing follicular cell hypertrophy: minimal-30\% to $40 \%$ of follicles affected; mild $-40 \%$ to $60 \%$ of follicles affected; moderate- $60 \%$ to $80 \%$ of follicles affected; markedgreater than $80 \%$ of follicles affected.

Pituitary Gland: Incidences of adenoma occurred in all groups of males and females and the incidence of this neoplasm was significantly decreased in $300 \mathrm{mg} / \mathrm{kg}$ females (males: vehicle control, 5/50; 75 mg/kg, 8/49; 150 mg/kg, 9/50; 300 mg/kg, 8/47; females: vehicle control, 18/50, 75 mg/kg, 18/50, 150 mg/kg, 19/50, 300 mg/kg, 8/49) (Table A-1, Table A-2, Table B-1, and Table B-2).

\section{Three-month Study in Mice}

All mice survived to the end of the study (Table 12). The final mean body weights and mean body weight gains of dosed groups of males and females were similar to those of the vehicle controls (Table 12; Figure 6). There were no clinical findings related to administration of indole3-carbinol.

There were no changes in the hematology data of mice that were considered attributable to indole-3-carbinol administration (Table F-2).

Table 12. Survival and Body Weights of Mice in the Three-month Gavage Study of Indole-3-carbinol $^{\mathrm{a}}$

\begin{tabular}{cccccc}
\hline $\begin{array}{c}\text { Dose } \\
(\mathbf{m g} / \mathbf{k g})\end{array}$ & Survival $^{\mathbf{b}}$ & $\begin{array}{c}\text { Initial Body } \\
\text { Weight } \\
\text { (g) }\end{array}$ & $\begin{array}{c}\text { Final Body } \\
\text { Weight } \\
\mathbf{( g )}\end{array}$ & $\begin{array}{c}\text { Change in Body } \\
\text { Weight } \\
\text { (g) }\end{array}$ & $\begin{array}{c}\text { Final Weight } \\
\text { Relative to } \\
\text { Controls (\%) }\end{array}$ \\
\hline Male & & & & & \\
0 & $10 / 10$ & $23.6 \pm 0.2$ & $40.4 \pm 0.6$ & $16.8 \pm 0.6$ & \\
15.6 & $10 / 10$ & $23.6 \pm 0.2$ & $40.5 \pm 1.1$ & $17.0 \pm 1.1$ & 100 \\
31.25 & $10 / 10$ & $23.6 \pm 0.2$ & $41.5 \pm 0.9$ & $17.9 \pm 0.8$ & 103 \\
62.5 & $10 / 10$ & $23.6 \pm 0.1$ & $40.0 \pm 1.1$ & $16.4 \pm 1.2$ & 99 \\
125 & $10 / 10$ & $23.6 \pm 0.2$ & $42.2 \pm 1.6$ & $18.6 \pm 1.6$ & 104 \\
250 & $10 / 10$ & $23.7 \pm 0.3$ & $41.0 \pm 0.9$ & $17.2 \pm 0.8$ & 101 \\
\hline Female & & & & & \\
0 & $10 / 10$ & $19.5 \pm 0.2$ & $34.2 \pm 1.0$ & $14.6 \pm 1.0$ & 98 \\
15.6 & $10 / 10$ & $19.5 \pm 0.3$ & $33.5 \pm 1.0$ & $14.0 \pm 0.9$ & 99 \\
31.25 & $10 / 10$ & $19.5 \pm 0.3$ & $33.8 \pm 1.6$ & $14.3 \pm 1.7$ & 99 \\
62.5 & $10 / 10$ & $19.4 \pm 0.3$ & $33.7 \pm 0.6$ & $14.3 \pm 0.5$ & 97 \\
125 & $10 / 10$ & $19.4 \pm 0.3$ & $33.1 \pm 0.7$ & $13.7 \pm 0.6$ & 92 \\
250 & $10 / 10$ & $19.4 \pm 0.4$ & $31.4 \pm 1.3$ & $12.1 \pm 1.3$ & \\
\hline
\end{tabular}

${ }^{a}$ Weights and weight changes are given as mean \pm standard error. Differences from the vehicle control group are not significant by Dunnett's test.

bNumber of animals surviving at 14 weeks/number initially in group. 
Indole-3-carbinol, NTP TR 584
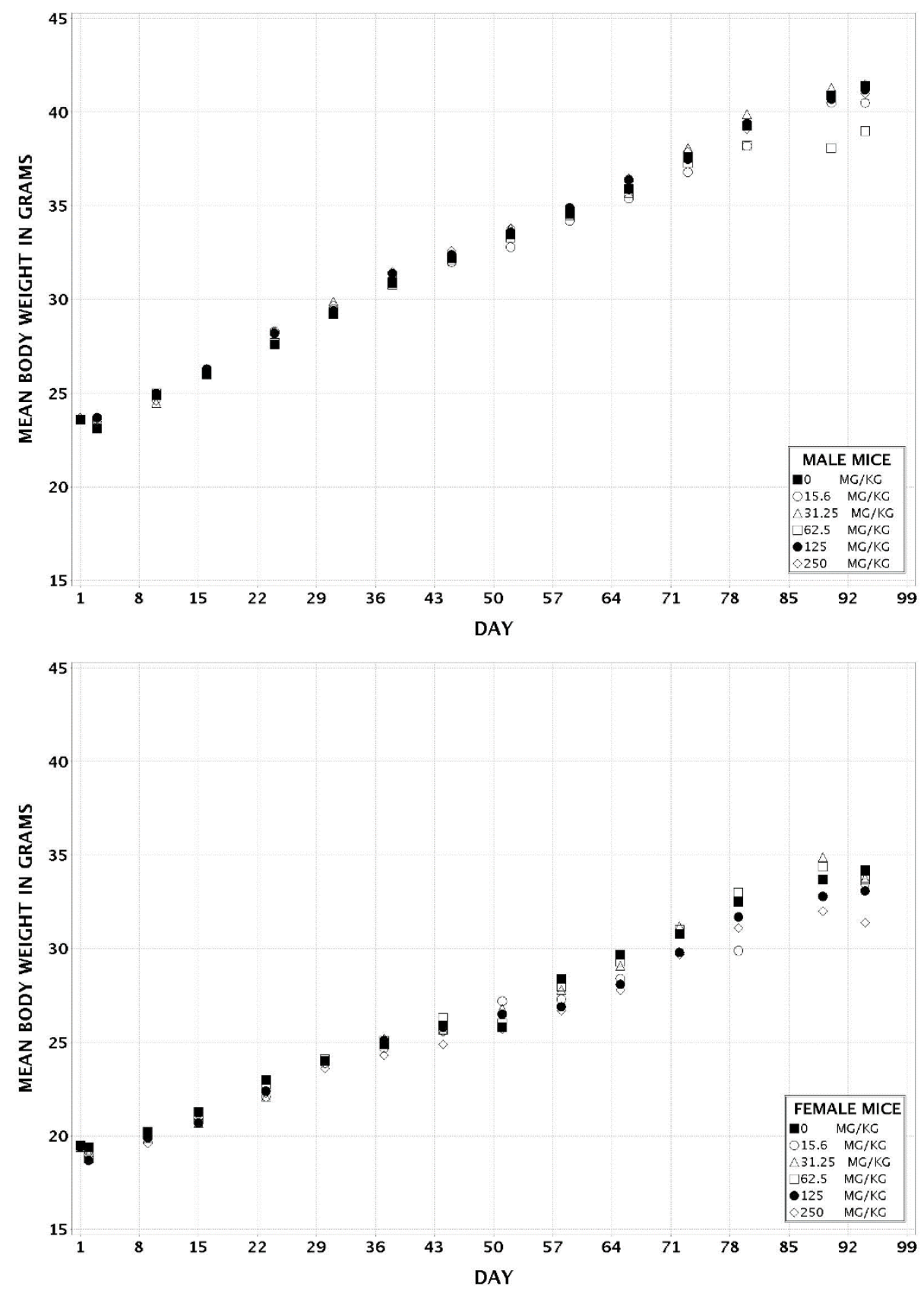

Figure 6. Growth Curves for Mice Administered Indole-3-carbinol by Gavage for Three Months

The absolute and relative liver weights of 125 and $250 \mathrm{mg} / \mathrm{kg}$ males and all dosed groups of females were significantly increased compared to the vehicle controls (Table 13 and Table G-2).

Liver and lung samples were collected for determinations of P450 enzyme activities (Table 14). Microsomal suspensions were prepared from liver samples and were assayed for 7ethoxyresorufin-O-deethylase (EROD) activity (a marker for CYP1A1 activity) and acetanilide4-hydroxylase (A4H) activity (a marker for CYP1A2 activity). Microsomal samples from lung were analyzed for EROD activity only. In the liver, there were significant and dose-dependent increases in $\mathrm{A} 4 \mathrm{H}$ activities in all dosed groups of male mice. Hepatic EROD activities were 
significantly increased in males administered $31.25 \mathrm{mg} / \mathrm{kg}$ or greater. Hepatic A4H and EROD activities were significantly increased in 125 and $250 \mathrm{mg} / \mathrm{kg}$ females. Maximal inductions of A4H and EROD activities in males were nearly 4-fold and 3-fold, respectively, compared to the vehicle controls. In females, A4H and EROD activities were maximally increased more than 2-fold and 3-fold, respectively. There were no treatment-related effects on pulmonary EROD activities in males or females.

Table 13. Liver Weights and Liver-to Body-Weight Ratios for Mice in the Three-month Gavage Study of Indole-3-carbinol ${ }^{\mathrm{a}}$

\begin{tabular}{|c|c|c|c|c|c|c|}
\hline & $\begin{array}{l}\text { Vehicle } \\
\text { Control }\end{array}$ & 15.6 mg/kg & 31.25 mg/kg & 62.5 mg/kg & 125 mg/kg & 250 mg/kg \\
\hline $\mathbf{n}$ & 10 & 10 & 10 & 10 & 10 & 10 \\
\hline \multicolumn{7}{|l|}{ Male } \\
\hline $\begin{array}{l}\text { Necropsy } \\
\text { body wt }\end{array}$ & $40.4 \pm 0.6$ & $40.5 \pm 1.1$ & $41.5 \pm 0.9$ & $40.0 \pm 1.1$ & $42.2 \pm 1.6$ & $41.0 \pm 0.9$ \\
\hline \multicolumn{7}{|l|}{ Liver } \\
\hline Absolute & $1.50 \pm 0.06$ & $1.46 \pm 0.07$ & $1.54 \pm 0.05$ & $1.54 \pm 0.05$ & $1.77 \pm 0.13^{*}$ & $1.82 \pm 0.06^{* *}$ \\
\hline Relative & $37.329 \pm 1.896$ & $35.868 \pm 0.799$ & $37.166 \pm 0.702$ & $38.520 \pm 0.632$ & $41.960 \pm 2.146^{*}$ & $44.333 \pm 0.817^{* *}$ \\
\hline \multicolumn{7}{|l|}{ Female } \\
\hline $\begin{array}{l}\text { Necropsy } \\
\text { body wt }\end{array}$ & $34.2 \pm 1.0$ & $33.5 \pm 1.0$ & $33.8 \pm 1.6$ & $33.7 \pm 0.6$ & $33.1 \pm 0.7$ & $31.4 \pm 1.3$ \\
\hline \multicolumn{7}{|l|}{ Liver } \\
\hline Absolute & $1.04 \pm 0.01$ & $1.21 \pm 0.05^{*}$ & $1.11 \pm 0.04^{*}$ & $1.16 \pm 0.03^{*}$ & $1.22 \pm 0.03^{* *}$ & $1.21 \pm 0.04^{* *}$ \\
\hline Relative & $30.559 \pm 0.696$ & $36.190 \pm 1.253^{* *}$ & $32.906 \pm 0.651^{* *}$ & $34.449 \pm 0.693^{* *}$ & $36.878 \pm 0.867 * *$ & $38.687 \pm 0.778^{* *}$ \\
\hline
\end{tabular}


Indole-3-carbinol, NTP TR 584

Table 14. Liver and Lung Cytochrome P450 Data for Mice in the Three-month Gavage Study of Indole-3-carbinol $^{\mathrm{a}}$

\begin{tabular}{|c|c|c|c|c|c|c|}
\hline & $\begin{array}{l}\text { Vehicle } \\
\text { Control }\end{array}$ & $15.6 \mathrm{mg} / \mathrm{kg}$ & $31.25 \mathrm{mg} / \mathrm{kg}$ & $62.5 \mathrm{mg} / \mathrm{kg}$ & $125 \mathrm{mg} / \mathrm{kg}$ & $250 \mathrm{mg} / \mathrm{kg}$ \\
\hline $\mathbf{n}$ & 10 & 10 & 10 & 10 & 10 & 10 \\
\hline \multicolumn{7}{|c|}{ Male } \\
\hline \multicolumn{7}{|c|}{ Liver Microsomes } \\
\hline \multicolumn{7}{|c|}{ A4H (nmol/minute per mg microsomal protein) } \\
\hline & $0.335 \pm 0.039$ & $0.449 \pm 0.011^{* *}$ & $0.515 \pm 0.024^{* *}$ & $0.984 \pm 0.037 * *$ & $1.124 \pm 0.087^{* *}$ & $1.293 \pm 0.109^{* *}$ \\
\hline \multicolumn{7}{|c|}{ EROD (pmol/minute per mg microsomal protein) } \\
\hline & $16.7 \pm 2.3$ & $20.2 \pm 1.1$ & $24.5 \pm 3.1^{*}$ & $36.2 \pm 3.5^{* *}$ & $45.4 \pm 8.4^{* *}$ & $43.0 \pm 10.4^{* *}$ \\
\hline \multicolumn{7}{|c|}{ Lung Microsomes } \\
\hline \multicolumn{7}{|c|}{ EROD (pmol/minute per mg microsomal protein) } \\
\hline & $0.112 \pm 0.062$ & $0.048 \pm 0.032$ & $0.011 \pm 0.011$ & $0.000 \pm 0.000$ & $0.000 \pm 0.000$ & $0.010 \pm 0.010$ \\
\hline
\end{tabular}

Female

Liver Microsomes

A4H (nmol/minute per mg microsomal protein)

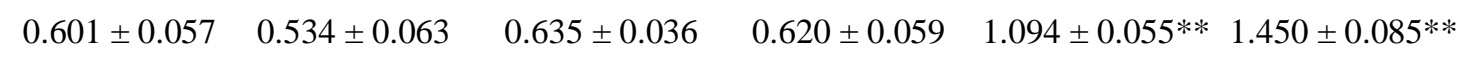

EROD (pmol/minute per mg microsomal protein)

$$
14.3 \pm 1.0 \quad 11.4 \pm 2.2 \quad 18.8 \pm 1.3 \quad 12.2 \pm 2.5 \quad 28.1 \pm 2.5^{* *} \quad 46.1 \pm 3.8^{* *}
$$

\section{Lung Microsomes}

EROD (pmol/minute per mg microsomal protein)

$$
\begin{array}{llllll}
0.005 \pm 0.005 & 0.011 \pm 0.011 & 0.059 \pm 0.059 & 0.000 \pm 0.000 & 0.075 \pm 0.056 & 0.139 \pm 0.071
\end{array}
$$

*Significantly different $(\mathrm{P} \leq 0.05)$ from the vehicle control group by Shirley’s test.

$* * \mathrm{P} \leq 0.01$.

aata are presented as mean \pm standard error. A4H = acetanilide-4-hydroxylase; EROD = 7-ethoxyresorufin-O-deethylase.

Sperm motility was significantly decreased in all dosed groups of males (Table H-4). The Markov transition matrix analyses of estrous cyclicity indicated that females in the $250 \mathrm{mg} / \mathrm{kg}$ group had a significantly higher probability of extended diestrus than the vehicle control females (Table H-5 and Table H-6; Figure H-2). Based on these results, indole-3-carbinol did exhibit the potential to be a reproductive toxicant in male and female mice.

No chemical-related histopathologic lesions were observed that could be attributed to the administration of indole-3-carbinol.

Dose Selection Rationale: There were no chemical-related effects on mortality, body weights, or lesion incidences in the 3-month study in mice. Chemical-related increases in liver weights were observed, and were consistent with increased hepatic cytochrome P450 activities. In $250 \mathrm{mg} / \mathrm{kg}$ females, there was an $8 \%$ decrease in final body weight and a slightly lower overall body weight gain. These effects were not considered sufficient to exclude $250 \mathrm{mg} / \mathrm{kg}$ as the highest dose in the 2-year study. However, studies in the literature at doses of $500 \mathrm{mg} / \mathrm{kg}$ have demonstrated treatment-related effects on survival and neurotoxicity; as a result, higher doses were not 
Indole-3-carbinol, NTP TR 584

considered for the 2-year study. The doses selected for the 2-year gavage study in B6C3F1/N mice were 62.5, 125, and $250 \mathrm{mg} / \mathrm{kg}$.

\section{Two-year Study in Mice}

\section{Survival}

Estimates of 2-year survival probabilities for male and female mice shown in Table 15 and in the Kaplan-Meier survival curves (Figure 7). Survival of $250 \mathrm{mg} / \mathrm{kg}$ females was significantly greater than that of the vehicle controls; survival of dosed groups of males was similar to that of the vehicle control group.

\section{Body Weights and Clinical Findings}

Mean body weights of dosed groups of male mice were similar to those of the vehicle controls throughout the study; however, those of $250 \mathrm{mg} / \mathrm{kg}$ female mice were at least $10 \%$ less than those of the vehicle controls between weeks 32 and 92 (Table 16 and Table 17; Figure 8). No clinical findings related to the administration of indole-3-carbinol were observed in males or females.

Table 15. Survival of Mice in the Two-year Gavage Study of Indole-3-carbinol

\begin{tabular}{|c|c|c|c|c|}
\hline & Vehicle Control & $62.5 \mathrm{mg} / \mathrm{kg}$ & $125 \mathrm{mg} / \mathrm{kg}$ & $250 \mathrm{mg} / \mathrm{kg}$ \\
\hline \multicolumn{5}{|l|}{ Male } \\
\hline Animals initially in study & 50 & 50 & 50 & 50 \\
\hline Accidental deaths $s^{\mathrm{a}}$ & 0 & 0 & 1 & 1 \\
\hline Moribund & 17 & 12 & 11 & 9 \\
\hline Natural deaths & 6 & 7 & 6 & 8 \\
\hline Animals surviving to study termination & $27^{\mathrm{e}}$ & 31 & 32 & 32 \\
\hline Percent probability of survival at end of study ${ }^{b}$ & 52 & 62 & 65 & 65 \\
\hline Mean survival (days) ${ }^{c}$ & 663 & 700 & 686 & 688 \\
\hline Survival analysis $^{\mathrm{d}}$ & $\mathrm{P}=0.202 \mathrm{~N}$ & $\mathrm{P}=0.256 \mathrm{~N}$ & $\mathrm{P}=0.182 \mathrm{~N}$ & $\mathrm{P}=0.192 \mathrm{~N}$ \\
\hline \multicolumn{5}{|l|}{ Female } \\
\hline Animals initially in study & 50 & 50 & 50 & 50 \\
\hline Accidental death $^{\mathrm{a}}$ & 1 & 0 & 0 & 0 \\
\hline Moribund & 10 & 6 & 15 & 3 \\
\hline Natural deaths & 6 & 4 & 9 & 2 \\
\hline Animals surviving to study termination & 33 & 40 & $26^{\mathrm{e}}$ & 45 \\
\hline Percent probability of survival at end of study & 67 & 80 & 52 & 90 \\
\hline Mean survival (days) & 702 & 704 & 672 & 713 \\
\hline Survival analysis & $\mathrm{P}=0.099 \mathrm{~N}$ & $\mathrm{P}=0.263 \mathrm{~N}$ & $P=0.082$ & $\mathrm{P}=0.016 \mathrm{~N}$ \\
\hline
\end{tabular}

${ }^{\mathrm{a}}$ Censored in the survival analyses.

${ }^{\mathrm{b}}$ Kaplan-Meier determinations.

cMean of all deaths (uncensored, censored, and terminal euthanasia).

${ }^{\mathrm{d}}$ The result of the life table trend test ${ }^{91}$ is in the vehicle control column, and the results of the life table pairwise comparisons ${ }^{90}$ with the vehicle controls are in the dosed group columns. A negative trend or lower mortality in a dose group is indicated by $\mathbf{N}$. eIncludes one animal that died during the last week of the study. 
Indole-3-carbinol, NTP TR 584
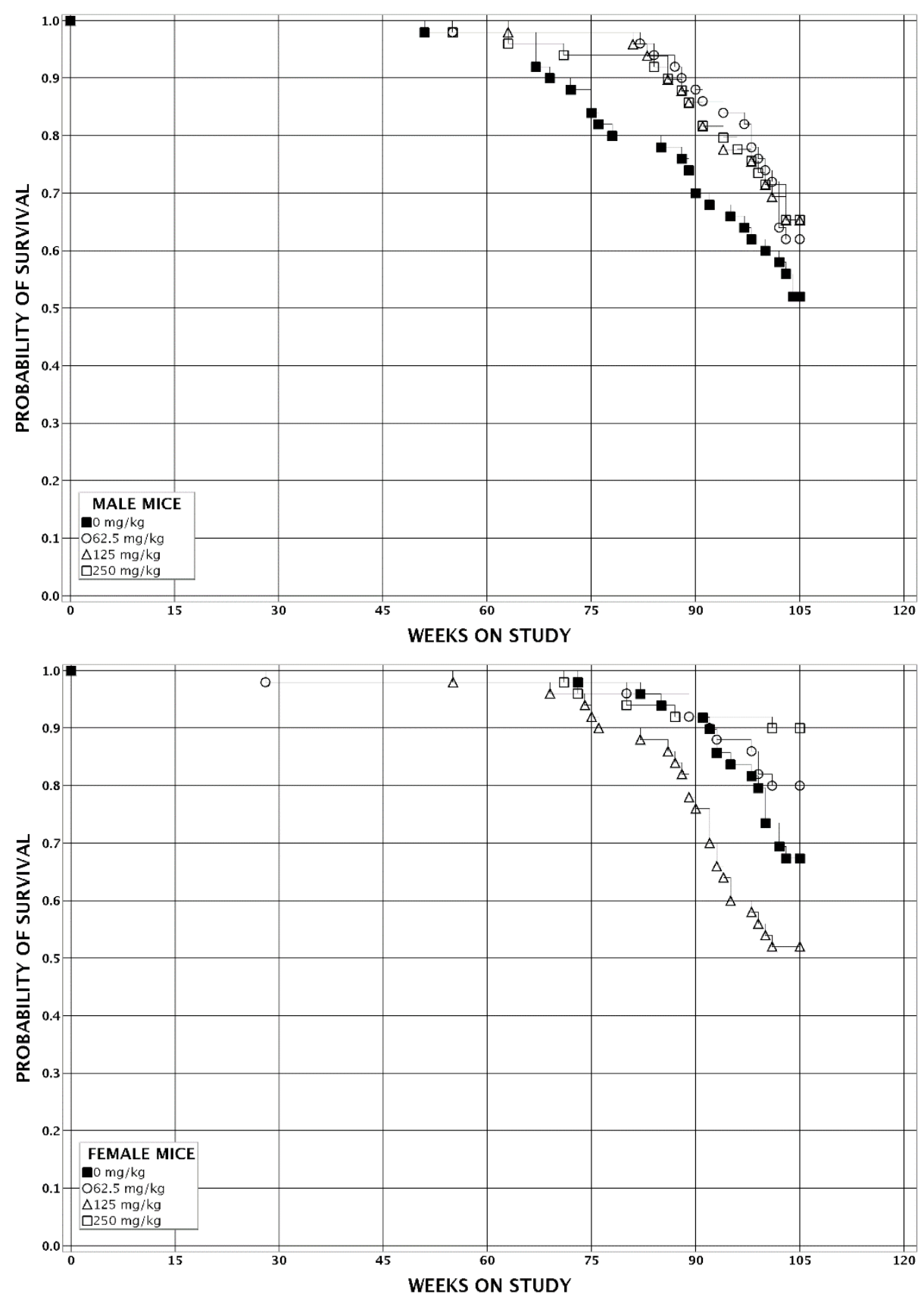

Figure 7. Kaplan-Meier Survival Curves for Mice Administered Indole-3-carbinol by Gavage for Two Years 
Indole-3-carbinol, NTP TR 584

Table 16. Mean Body Weights and Survival of Male Mice in the Two-year Gavage Study of Indole-3-carbinol

\begin{tabular}{|c|c|c|c|c|c|c|c|c|c|c|c|}
\hline \multirow[b]{2}{*}{ Day } & \multicolumn{3}{|c|}{ Vehicle Control } & \multicolumn{2}{|c|}{$62.5 \mathrm{mg} / \mathrm{kg}$} & \multicolumn{3}{|c|}{$125 \mathrm{mg} / \mathrm{kg}$} & \multicolumn{3}{|c|}{$250 \mathrm{mg} / \mathrm{kg}$} \\
\hline & $\begin{array}{l}\text { Av. } \\
\text { Wt. } \\
\text { (g) }\end{array}$ & $\begin{array}{l}\text { No. of } \\
\text { Survivors }\end{array}$ & $\begin{array}{l}\text { Av. } \\
\text { Wt. } \\
\text { (g) }\end{array}$ & $\begin{array}{l}\text { Wt. (\% of } \\
\text { Controls) }\end{array}$ & $\begin{array}{c}\text { No. of } \\
\text { Survivors }\end{array}$ & $\begin{array}{l}\text { Av. } \\
\text { Wt. } \\
\text { (g) }\end{array}$ & $\begin{array}{l}\text { Wt. (\% of } \\
\text { Controls) }\end{array}$ & $\begin{array}{c}\text { No. of } \\
\text { Survivors }\end{array}$ & $\begin{array}{l}\text { Av. } \\
\text { Wt. } \\
\text { (g) }\end{array}$ & $\begin{array}{l}\text { Wt. (\% of } \\
\text { Controls) }\end{array}$ & $\begin{array}{c}\text { No. of } \\
\text { Survivors }\end{array}$ \\
\hline 1 & 22.8 & 50 & 22.9 & 101 & 50 & 22.8 & 100 & 50 & 22.8 & 100 & 50 \\
\hline 8 & 23.9 & 50 & 24.1 & 101 & 50 & 24.1 & 101 & 50 & 24.1 & 101 & 50 \\
\hline 15 & 24.7 & 50 & 25.0 & 101 & 50 & 24.8 & 101 & 50 & 24.9 & 101 & 50 \\
\hline 22 & 26.4 & 50 & 26.7 & 101 & 50 & 26.6 & 101 & 50 & 26.5 & 101 & 50 \\
\hline 29 & 27.0 & 50 & 27.7 & 103 & 50 & 27.4 & 101 & 50 & 27.3 & 101 & 50 \\
\hline 36 & 28.8 & 50 & 29.5 & 103 & 50 & 29.3 & 102 & 50 & 28.9 & 100 & 50 \\
\hline 43 & 30.0 & 50 & 30.7 & 102 & 50 & 30.2 & 101 & 50 & 29.7 & 99 & 50 \\
\hline 50 & 31.7 & 50 & 32.2 & 101 & 50 & 31.6 & 100 & 50 & 31.1 & 98 & 50 \\
\hline 57 & 32.9 & 50 & 33.8 & 103 & 50 & 33.1 & 101 & 50 & 32.7 & 99 & 50 \\
\hline 64 & 33.7 & 50 & 34.7 & 103 & 50 & 33.5 & 100 & 50 & 33.3 & 99 & 50 \\
\hline 71 & 34.5 & 50 & 35.6 & 103 & 50 & 34.5 & 100 & 50 & 34.1 & 99 & 50 \\
\hline 78 & 36.4 & 50 & 37.7 & 104 & 50 & 36.4 & 100 & 50 & 36.1 & 99 & 50 \\
\hline 85 & 36.4 & 50 & 37.7 & 103 & 50 & 36.5 & 100 & 50 & 36.2 & 99 & 50 \\
\hline 113 & 39.8 & 50 & 42.1 & 106 & 50 & 40.8 & 103 & 50 & 40.5 & 102 & 50 \\
\hline 141 & 43.6 & 50 & 45.5 & 104 & 50 & 44.1 & 101 & 49 & 43.2 & 99 & 50 \\
\hline 169 & 46.1 & 50 & 48.0 & 104 & 50 & 47.1 & 102 & 49 & 45.8 & 99 & 50 \\
\hline 197 & 47.8 & 50 & 48.9 & 102 & 50 & 48.9 & 102 & 49 & 46.8 & 98 & 50 \\
\hline 225 & 50.8 & 50 & 51.9 & 102 & 50 & 51.9 & 102 & 49 & 49.7 & 98 & 50 \\
\hline 253 & 51.4 & 50 & 52.1 & 101 & 50 & 52.4 & 102 & 49 & 50.3 & 98 & 50 \\
\hline 281 & 51.9 & 50 & 52.6 & 101 & 50 & 52.9 & 102 & 49 & 51.5 & 99 & 50 \\
\hline 309 & 53.8 & 50 & 54.3 & 101 & 50 & 54.6 & 102 & 49 & 52.8 & 98 & 50 \\
\hline 337 & 54.0 & 50 & 54.5 & 101 & 50 & 54.6 & 101 & 49 & 52.7 & 98 & 50 \\
\hline 365 & 54.5 & 49 & 55.2 & 101 & 50 & 55.3 & 101 & 49 & 53.8 & 99 & 50 \\
\hline 393 & 54.5 & 49 & 55.9 & 103 & 49 & 55.4 & 102 & 49 & 54.6 & 100 & 49 \\
\hline 421 & 55.2 & 49 & 56.4 & 102 & 49 & 55.7 & 101 & 49 & 55.0 & 100 & 49 \\
\hline 449 & 55.6 & 49 & 56.6 & 102 & 49 & 55.9 & 101 & 48 & 55.6 & 100 & 48 \\
\hline 477 & 55.5 & 46 & 57.3 & 103 & 49 & 56.1 & 101 & 48 & 55.7 & 100 & 48 \\
\hline 505 & 55.6 & 44 & 57.1 & 103 & 49 & 55.5 & 100 & 48 & 56.0 & 101 & 47 \\
\hline 533 & 55.4 & 41 & 57.5 & 104 & 49 & 55.2 & 100 & 48 & 56.4 & 102 & 46 \\
\hline 561 & 54.7 & 40 & 56.5 & 103 & 49 & 53.8 & 98 & 48 & 55.6 & 102 & 46 \\
\hline 589 & 54.5 & 39 & 56.8 & 104 & 47 & 53.7 & 99 & 46 & 55.6 & 102 & 45 \\
\hline 617 & 54.6 & 38 & 55.9 & 102 & 45 & 53.1 & 97 & 42 & 55.0 & 101 & 42 \\
\hline 645 & 55.0 & 34 & 54.8 & 100 & 43 & 52.9 & 96 & 40 & 53.8 & 98 & 40 \\
\hline 673 & 54.4 & 32 & 53.8 & 99 & 41 & 53.0 & 98 & 38 & 52.5 & 97 & 38 \\
\hline 701 & 53.3 & 30 & 53.9 & 101 & 36 & 52.9 & 99 & 35 & 50.9 & 96 & 35 \\
\hline \multicolumn{12}{|c|}{ Mean for Weeks } \\
\hline $1-13$ & 29.9 & & 30.6 & 102 & & 30.1 & 101 & & 29.8 & 100 & \\
\hline $14-52$ & 48.8 & & 50.0 & 102 & & 49.7 & 102 & & 48.1 & 99 & \\
\hline 53-101 & 54.8 & & 56.0 & 102 & & 54.5 & 99 & & 54.7 & 100 & \\
\hline
\end{tabular}


Indole-3-carbinol, NTP TR 584

Table 17. Mean Body Weights and Survival of Female Mice in the Two-year Gavage Study of Indole-3-carbinol

\begin{tabular}{|c|c|c|c|c|c|c|c|c|c|c|c|}
\hline \multirow[b]{2}{*}{ Day } & \multicolumn{2}{|c|}{ Vehicle Control } & \multicolumn{3}{|c|}{$62.5 \mathrm{mg} / \mathrm{kg}$} & \multicolumn{3}{|c|}{$125 \mathrm{mg} / \mathrm{kg}$} & \multicolumn{3}{|c|}{$250 \mathrm{mg} / \mathrm{kg}$} \\
\hline & $\begin{array}{l}\text { Av. } \\
\text { Wt. } \\
\text { (g) }\end{array}$ & $\begin{array}{c}\text { No. of } \\
\text { Survivors }\end{array}$ & $\begin{array}{l}\text { Av. } \\
\text { Wt. } \\
\text { (g) }\end{array}$ & $\begin{array}{l}\text { Wt. (\% of } \\
\text { Controls) }\end{array}$ & $\begin{array}{c}\text { No. of } \\
\text { Survivors }\end{array}$ & $\begin{array}{l}\text { Av. } \\
\text { Wt. } \\
\text { (g) }\end{array}$ & $\begin{array}{l}\text { Wt. (\% of } \\
\text { Controls) }\end{array}$ & $\begin{array}{c}\text { No. of } \\
\text { Survivors }\end{array}$ & $\begin{array}{l}\text { Av. } \\
\text { Wt. } \\
\text { (g) }\end{array}$ & $\begin{array}{l}\text { Wt. (\% of } \\
\text { Controls) }\end{array}$ & $\begin{array}{c}\text { No. of } \\
\text { Survivors }\end{array}$ \\
\hline 1 & 18.6 & 50 & 18.6 & 100 & 50 & 18.5 & 99 & 50 & 18.8 & 101 & 50 \\
\hline 8 & 19.3 & 50 & 19.1 & 99 & 50 & 19.2 & 100 & 50 & 19.3 & 100 & 50 \\
\hline 15 & 20.3 & 50 & 20.0 & 99 & 50 & 20.1 & 99 & 50 & 20.3 & 100 & 50 \\
\hline 22 & 21.6 & 50 & 21.0 & 97 & 50 & 21.2 & 98 & 50 & 21.4 & 99 & 50 \\
\hline 29 & 22.5 & 50 & 21.9 & 98 & 50 & 22.2 & 99 & 50 & 22.0 & 98 & 50 \\
\hline 36 & 23.6 & 50 & 22.8 & 97 & 50 & 23.4 & 99 & 50 & 23.2 & 98 & 50 \\
\hline 43 & 24.8 & 50 & 23.9 & 97 & 50 & 24.8 & 100 & 50 & 24.6 & 99 & 50 \\
\hline 50 & 25.5 & 50 & 24.7 & 97 & 50 & 25.6 & 101 & 50 & 25.1 & 99 & 50 \\
\hline 57 & 26.5 & 50 & 25.9 & 98 & 50 & 26.8 & 101 & 50 & 25.9 & 98 & 50 \\
\hline 64 & 28.3 & 50 & 27.5 & 97 & 50 & 28.5 & 101 & 50 & 27.4 & 97 & 50 \\
\hline 71 & 29.0 & 50 & 28.8 & 99 & 50 & 29.7 & 103 & 50 & 28.6 & 99 & 50 \\
\hline 78 & 29.8 & 50 & 29.0 & 98 & 50 & 30.8 & 103 & 50 & 29.7 & 100 & 50 \\
\hline 85 & 30.0 & 50 & 30.1 & 100 & 50 & 31.2 & 104 & 50 & 30.3 & 101 & 50 \\
\hline 113 & 35.0 & 50 & 33.8 & 96 & 50 & 36.3 & 104 & 50 & 34.4 & 98 & 50 \\
\hline 141 & 37.7 & 50 & 36.2 & 96 & 50 & 39.0 & 103 & 50 & 36.9 & 98 & 50 \\
\hline 169 & 40.3 & 50 & 38.6 & 96 & 50 & 41.6 & 103 & 50 & 39.2 & 97 & 50 \\
\hline 197 & 44.4 & 50 & 41.7 & 94 & 49 & 44.2 & 99 & 50 & 40.9 & 92 & 50 \\
\hline 225 & 48.1 & 50 & 45.2 & 94 & 49 & 47.7 & 99 & 50 & 42.5 & 88 & 50 \\
\hline 253 & 50.9 & 50 & 47.8 & 94 & 49 & 50.0 & 98 & 50 & 43.7 & 86 & 50 \\
\hline 281 & 51.8 & 50 & 48.1 & 93 & 49 & 51.4 & 99 & 50 & 44.4 & 86 & 50 \\
\hline 309 & 56.9 & 50 & 53.0 & 93 & 49 & 56.3 & 99 & 50 & 47.5 & 84 & 50 \\
\hline 337 & 59.1 & 50 & 54.9 & 93 & 49 & 57.6 & 97 & 50 & 48.1 & 81 & 50 \\
\hline 365 & 61.9 & 50 & 57.5 & 93 & 49 & 59.6 & 96 & 50 & 49.0 & 79 & 50 \\
\hline 393 & 63.4 & 50 & 59.0 & 93 & 49 & 61.0 & 96 & 49 & 50.4 & 80 & 50 \\
\hline 421 & 63.0 & 50 & 59.7 & 95 & 49 & 61.5 & 98 & 49 & 50.8 & 81 & 50 \\
\hline 449 & 65.2 & 50 & 62.3 & 96 & 49 & 63.4 & 97 & 49 & 53.0 & 81 & 50 \\
\hline 477 & 65.2 & 50 & 62.6 & 96 & 49 & 63.2 & 97 & 49 & 53.8 & 83 & 50 \\
\hline 505 & 65.3 & 50 & 63.5 & 97 & 49 & 64.0 & 98 & 48 & 54.9 & 84 & 48 \\
\hline 533 & 65.8 & 49 & 63.8 & 97 & 49 & 63.6 & 97 & 45 & 55.7 & 85 & 48 \\
\hline 561 & 66.6 & 49 & 63.7 & 96 & 48 & 63.8 & 96 & 45 & 55.7 & 84 & 47 \\
\hline 589 & 66.3 & 46 & 64.1 & 97 & 48 & 62.5 & 94 & 44 & 55.7 & 84 & 47 \\
\hline 617 & 65.0 & 46 & 62.9 & 97 & 48 & 62.0 & 95 & 41 & 56.3 & 87 & 46 \\
\hline 645 & 62.6 & 44 & 61.8 & 99 & 45 & 61.0 & 97 & 34 & 55.8 & 89 & 46 \\
\hline 673 & 61.1 & 41 & 60.9 & 100 & 44 & 59.2 & 97 & 30 & 55.5 & 91 & 46 \\
\hline 701 & 59.2 & 36 & 60.9 & 103 & 40 & 58.4 & 99 & 27 & 55.5 & 94 & 46 \\
\hline \multicolumn{12}{|c|}{ Mean for Weeks } \\
\hline $1-13$ & 24.6 & & 24.1 & 98 & & 24.8 & 101 & & 24.4 & 99 & \\
\hline $14-52$ & 47.1 & & 44.4 & 94 & & 47.1 & 100 & & 42.0 & 89 & \\
\hline 53-101 & 63.9 & & 61.7 & 97 & & 61.8 & 97 & & 54.0 & 85 & \\
\hline
\end{tabular}


Indole-3-carbinol, NTP TR 584
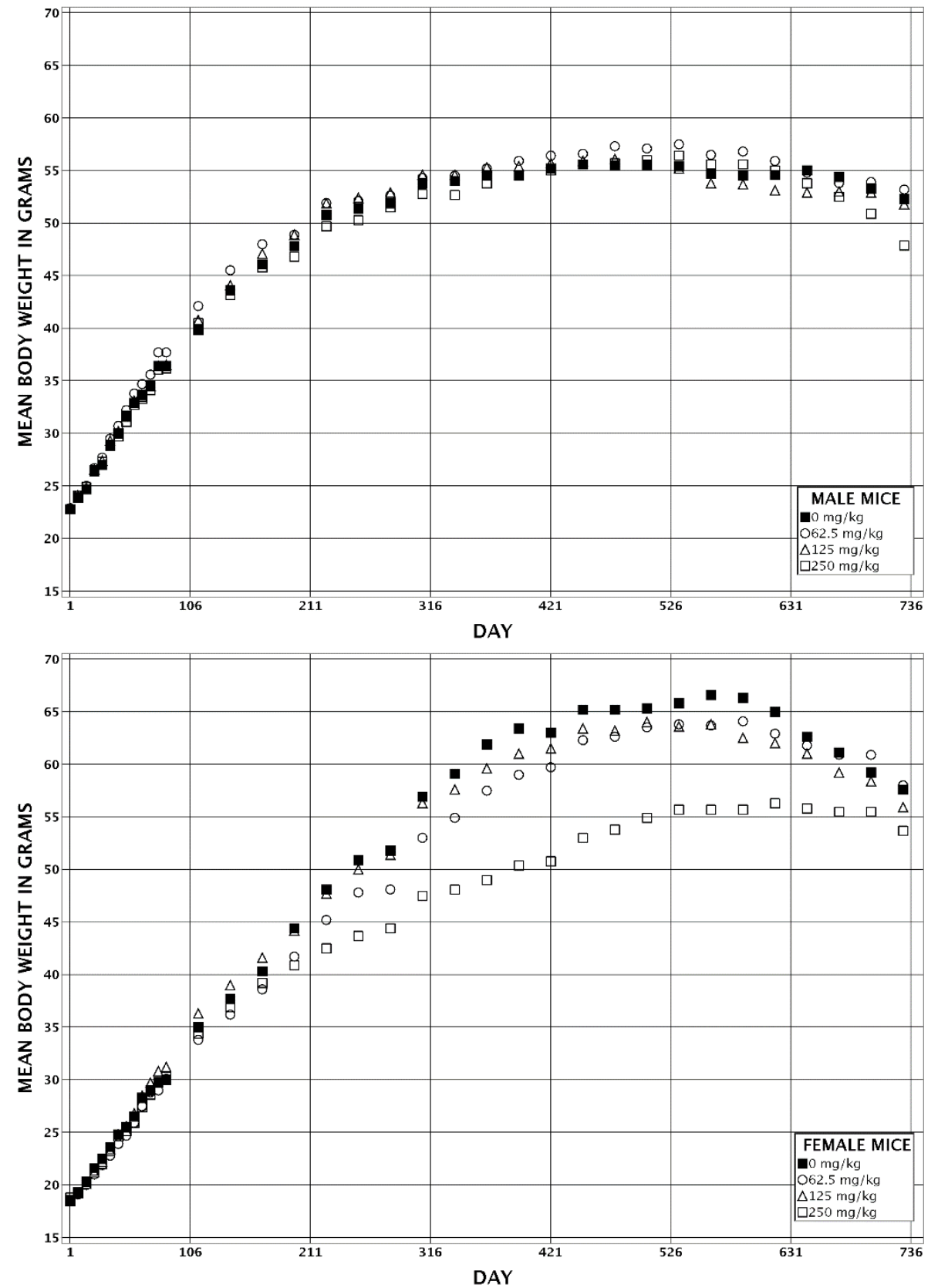

Figure 8. Growth Curves for Mice Administered Indole-3-carbinol by Gavage for Two Years 


\section{Pathology and Statistical Analyses}

This section describes the statistically significant or biologically noteworthy changes in the incidences of neoplasms and/or nonneoplastic lesions of the liver, glandular stomach, and nose. Summaries of the incidences of neoplasms and nonneoplastic lesions, statistical analyses of primary neoplasms that occurred with an incidence of at least $5 \%$ in at least one animal group, and historical incidences for the neoplasms mentioned in this section are presented in Appendix C for male mice and Appendix D for female mice.

Liver: Incidences of hepatocellular adenoma occurred with a positive trend in males and the incidence of this neoplasm was significantly increased in the $250 \mathrm{mg} / \mathrm{kg}$ group (Table 18, Table C-1, and Table C-2). In addition, the incidences of multiple hepatocellular adenoma were significantly increased in 62.5 and $250 \mathrm{mg} / \mathrm{kg}$ males.

Significantly increased incidences of hepatocellular carcinoma and multiple hepatocellular carcinoma occurred in $125 \mathrm{mg} / \mathrm{kg}$ males (Table 18, Table C-1, and Table C-2).

Incidences of hepatoblastoma occurred with a positive trend in males; the incidence of this neoplasm was significantly increased in the $250 \mathrm{mg} / \mathrm{kg}$ group and exceeded the historical control ranges for corn oil gavage studies and for all routes combined (Table 18, Table C-1, Table C-2, and Table C-3). In males, the incidences of multiple hepatoblastoma increased with increasing dose and the incidence of multiple hepatoblastoma was significantly increased in the $250 \mathrm{mg} / \mathrm{kg}$ group. One $125 \mathrm{mg} / \mathrm{kg}$ female and one $250 \mathrm{mg} / \mathrm{kg}$ female had a single hepatoblastoma (Table 18 and Table D-1).

Table 18. Incidences of Neoplasms and Nonneoplastic Lesions of the Liver in Mice in the Two-year Gavage Study of Indole-3-carbinol

\begin{tabular}{|c|c|c|c|c|}
\hline & $\begin{array}{l}\text { Vehicle } \\
\text { Control }\end{array}$ & $62.5 \mathrm{mg} / \mathrm{kg}$ & $125 \mathrm{mg} / \mathrm{kg}$ & $250 \mathrm{mg} / \mathrm{kg}$ \\
\hline \multicolumn{5}{|l|}{ Male } \\
\hline Number Examined Microscopically & 50 & 50 & 49 & 50 \\
\hline Clear Cell Focus ${ }^{\mathrm{a}}$ & 7 & $17^{*}$ & $22^{* *}$ & $20 * *$ \\
\hline Hepatocellular Adenoma, Multiple & 15 & $25^{*}$ & 16 & $33 * *$ \\
\hline \multicolumn{5}{|c|}{ Hepatocellular Adenoma (includes multiple) ${ }^{\mathrm{b}}$} \\
\hline Overall rate ${ }^{\mathrm{c}}$ & $26 / 50(52 \%)$ & $32 / 50$ (64\%) & $31 / 49$ (63\%) & $41 / 50(82 \%)$ \\
\hline Adjusted rate $^{\mathrm{d}}$ & $62.1 \%$ & $66.5 \%$ & $67.9 \%$ & $85.4 \%$ \\
\hline Terminal rate $\mathrm{e}^{\mathrm{e}}$ & 19/26 (73\%) & $21 / 31(68 \%)$ & 23/32 (72\%) & 28/32 (88\%) \\
\hline First incidence (days) & 463 & 381 & 437 & 385 \\
\hline Poly-3 test ${ }^{\mathrm{f}}$ & $P=0.005$ & $\mathrm{P}=0.411$ & $P=0.360$ & $P=0.006$ \\
\hline Hepatocellular Carcinoma, Multiple & 5 & 3 & $18^{* *}$ & 8 \\
\hline \multicolumn{5}{|c|}{ Hepatocellular Carcinoma (includes multiple) ${ }^{\mathrm{g}}$} \\
\hline Overall rate & $12 / 50(24 \%)$ & $11 / 50(22 \%)$ & 29/49 (59\%) & $14 / 50$ (28\%) \\
\hline Adjusted rate & $27.7 \%$ & $24.2 \%$ & $63.4 \%$ & $30.9 \%$ \\
\hline Terminal rate & 3/26 (12\%) & 8/31 (26\%) & 18/32 (56\%) & $8 / 32(25 \%)$ \\
\hline First incidence (days) & 465 & 682 & 567 & 385 \\
\hline
\end{tabular}


Indole-3-carbinol, NTP TR 584

\begin{tabular}{|c|c|c|c|c|}
\hline & $\begin{array}{l}\text { Vehicle } \\
\text { Control }\end{array}$ & $62.5 \mathrm{mg} / \mathrm{kg}$ & $125 \mathrm{mg} / \mathrm{kg}$ & $250 \mathrm{mg} / \mathrm{kg}$ \\
\hline Poly-3 test & $\mathrm{P}=0.217$ & $P=0.449 \mathrm{~N}$ & $\mathrm{P}<0.001$ & $\mathrm{P}=0.461$ \\
\hline \multicolumn{5}{|c|}{ Hepatocellular Adenoma or Carcinoma } \\
\hline Overall rate & 35/50 (70\%) & $36 / 50(72 \%)$ & $43 / 49(88 \%)$ & $44 / 50(88 \%)$ \\
\hline Adjusted rate & $77.6 \%$ & $74.6 \%$ & $90.6 \%$ & $91.1 \%$ \\
\hline Terminal rate & $21 / 26(81 \%)$ & 24/31 (77\%) & 29/32 (91\%) & $30 / 32(94 \%)$ \\
\hline First incidence (days) & 463 & 381 & 437 & 385 \\
\hline Poly-3 test & $\mathrm{P}=0.012$ & $\mathrm{P}=0.459 \mathrm{~N}$ & $P=0.062$ & $P=0.049$ \\
\hline Hepatoblastoma, Multiple & 0 & 1 & 3 & $7^{*}$ \\
\hline \multicolumn{5}{|l|}{ Hepatoblastoma (Includes Multiple) ${ }^{\mathrm{i}}$} \\
\hline Overall rate & $3 / 50(6 \%)$ & $4 / 50(8 \%)$ & $4 / 49(8 \%)$ & $14 / 50(28 \%)$ \\
\hline Adjusted rate & $7.6 \%$ & $8.9 \%$ & $9.3 \%$ & $31.6 \%$ \\
\hline Terminal rate & $3 / 26(12 \%)$ & 4/31 (13\%) & $3 / 32(9 \%)$ & $10 / 32(31 \%)$ \\
\hline First incidence (days) & $730(\mathrm{~T})$ & $730(\mathrm{~T})$ & 702 & 617 \\
\hline Poly-3 test & $\mathrm{P}<0.001$ & $P=0.567$ & $P=0.543$ & $\mathrm{P}=0.005$ \\
\hline \multicolumn{5}{|c|}{ Hepatocellular Carcinoma or Hepatoblastoma ${ }^{\mathrm{j}}$} \\
\hline Overall rate & $15 / 50(30 \%)$ & $13 / 50(26 \%)$ & $30 / 49(61 \%)$ & $25 / 50(50 \%)$ \\
\hline Adjusted rate & $34.6 \%$ & $28.7 \%$ & $65.6 \%$ & $54.5 \%$ \\
\hline Terminal rate & 6/26 (23\%) & 10/31 (32\%) & 19/32 (59\%) & $17 / 32(53 \%)$ \\
\hline First incidence (days) & 465 & 682 & 567 & 385 \\
\hline Poly-3 test & $\mathrm{P}=0.005$ & $\mathrm{P}=0.353 \mathrm{~N}$ & $\mathrm{P}=0.002$ & $\mathrm{P}=0.042$ \\
\hline \multicolumn{5}{|c|}{ Hepatocellular Adenoma, Hepatocellular Carcinoma, or Hepatoblastomak } \\
\hline Overall rate & $36 / 50(72 \%)$ & $36 / 50(72 \%)$ & $44 / 49(90 \%)$ & $45 / 50(90 \%)$ \\
\hline Adjusted rate & $79.8 \%$ & $74.6 \%$ & $92.7 \%$ & $92.8 \%$ \\
\hline Terminal rate & $22 / 26(85 \%)$ & 24/31 (77\%) & $30 / 32(94 \%)$ & $30 / 32(94 \%)$ \\
\hline First incidence (days) & 463 & 381 & 437 & 385 \\
\hline Poly-3 test & $\mathrm{P}=0.007$ & $\mathrm{P}=0.354 \mathrm{~N}$ & $\mathrm{P}=0.050$ & $\mathrm{P}=0.044$ \\
\hline \multicolumn{5}{|l|}{ Hepatocholangiocarcinoma ${ }^{1}$} \\
\hline Overall rate & $0 / 50(0 \%)$ & $3 / 50(6 \%)$ & $1 / 49(2 \%)$ & $0 / 50(0 \%)$ \\
\hline Adjusted rate & $0.0 \%$ & $6.6 \%$ & $2.3 \%$ & $0.0 \%$ \\
\hline Terminal rate & $0 / 26(0 \%)$ & $0 / 31(0 \%)$ & $1 / 32(3 \%)$ & $0 / 32(0 \%)$ \\
\hline First incidence (days) & $-{ }^{\mathrm{m}}$ & 630 & $730(\mathrm{~T})$ & - \\
\hline Poly-3 test & $\mathrm{P}=0.319 \mathrm{~N}$ & $P=0.146$ & $\mathrm{P}=0.515$ & $--^{\mathrm{n}}$ \\
\hline \multicolumn{5}{|l|}{ Female } \\
\hline Number Examined Microscopically & 50 & 50 & 50 & 50 \\
\hline Eosinophilic Focus & 16 & $26^{*}$ & $26^{*}$ & 21 \\
\hline Hepatocellular Adenoma, Multiple & 0 & 3 & 2 & 4 \\
\hline \multicolumn{5}{|c|}{ Hepatocellular Adenoma, (includes multiple) ${ }^{\circ}$} \\
\hline Overall rate & 7/50 (14\%) & $14 / 50(28 \%)$ & $8 / 50(16 \%)$ & $11 / 50(22 \%)$ \\
\hline Adjusted rate & $15.2 \%$ & $30.0 \%$ & $19.5 \%$ & $22.9 \%$ \\
\hline
\end{tabular}


Indole-3-carbinol, NTP TR 584

\begin{tabular}{lcccc}
\hline & $\begin{array}{c}\text { Vehicle } \\
\text { Control }\end{array}$ & $\mathbf{6 2 . 5} \mathbf{~ m g / k g}$ & $\mathbf{1 2 5} \mathbf{~ m g} / \mathbf{k g}$ & $\mathbf{2 5 0} \mathbf{~ m g / k g}$ \\
\hline Terminal rate & $5 / 33(15 \%)$ & $13 / 40(33 \%)$ & $7 / 26(27 \%)$ & $10 / 45(22 \%)$ \\
First incidence (days) & 568 & 687 & 621 & 603 \\
Poly-3 test & $\mathrm{P}=0.390$ & $\mathrm{P}=0.071$ & $\mathrm{P}=0.405$ & $\mathrm{P}=0.247$ \\
Hepatocellular Carcinoma, Multiple & 0 & 2 & 1 & 0 \\
Hepatocellular Carcinoma (includes multiple) & $6 / 50(12 \%)$ & $8 / 50(16 \%)$ & $9 / 50(18 \%)$ & $4 / 50(8 \%)$ \\
Overall rate & $13.2 \%$ & $17.1 \%$ & $21.5 \%$ & $8.4 \%$ \\
Adjusted rate & $5 / 33(15 \%)$ & $7 / 40(18 \%)$ & $5 / 26(19 \%)$ & $4 / 45(9 \%)$ \\
Terminal rate & 709 & 650 & 606 & $729(\mathrm{~T})$ \\
First incidence (days) & $\mathrm{P}=0.246 \mathrm{~N}$ & $\mathrm{P}=0.409$ & $\mathrm{P}=0.228$ & $\mathrm{P}=0.341 \mathrm{~N}$ \\
Poly-3 test & 0 & 0 & 1 & 1 \\
Hepatoblastoma & & & & \\
\hline
\end{tabular}

*Significantly different $(\mathrm{P} \leq 0.05)$ from the vehicle control group by the Poly-3 test.

$* * \mathrm{P} \leq 0.01$.

(T)Terminal euthanasia.

${ }^{a}$ Number of animals with lesion.

${ }^{b}$ Historical incidence for 2-year gavage studies with corn oil vehicle control groups (mean \pm standard deviation): $145 / 250$

(58.0\% $\pm 5.1 \%$ ), range 52\%-64\%; all routes: 594/949 (62.6\% $\pm 9.1 \%)$, range $48 \%-78 \%$.

'Number of animals with neoplasm per number of animals with liver examined microscopically.

${ }^{\mathrm{d} P o l y-3}$ estimated neoplasm incidence after adjustment for intercurrent mortality.

eobserved incidence at terminal euthanasia.

${ }^{\mathrm{f}}$ Beneath the vehicle control incidence is the $\mathrm{P}$ value associated with the trend test. Beneath the dosed group incidence are the $\mathrm{P}$ values corresponding to pairwise comparisons between the vehicle controls and that dosed group. The Poly-3 test accounts for differential mortality in animals that do not reach terminal euthanasia. A negative trend or a lower incidence in a dose group is indicated by $\mathbf{N}$.

gHistorical incidence for corn oil gavage studies: 87/250 (34.8\% $\pm 10.9 \%)$, range 22\%-44\%; all routes: 348/949

(36.7\% $\pm 11.4 \%$ ), range $22 \%-56 \%$.

hHistorical incidence for corn oil gavage studies: 189/250 (75.6\% $\pm 3.3 \%)$, range 70\%-78\%; all routes: 742/949 (78.2\% $\pm 7.2 \%)$, range $64 \%-90 \%$.

${ }^{i}$ Historical incidence for corn oil gavage studies: 9/250 (3.6\% $\pm 2.6 \%$ ), range $0 \%-6 \%$; all routes: 40/949 (4.2\% $\pm 3.5 \%$ ), range $0 \%-12 \%$.

${ }^{j}$ Historical incidence for corn oil gavage studies: 93/250 (37.2\% $\left.\pm 10.0 \%\right)$, range 24\%-48\%; all routes: 371/949

$(39.1 \% \pm 11.6 \%)$, range $22 \%-58 \%$.

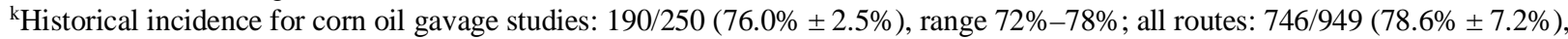
range 64\%-90\%.

${ }^{\mathrm{l} H i s t o r i c a l}$ incidence for corn oil gavage studies: 4/250 (1.6\% $\left.\pm 3.6 \%\right)$, range $0 \%-8 \%$; all routes: 10/949 (1.1\% $\left.\pm 2.2 \%\right)$, range $0 \%-8 \%$.

mot applicable; no neoplasms in animal group.

${ }^{n}$ Value of statistic cannot be computed.

${ }^{\circ}$ Historical incidence for corn oil gavage studies: 62/250 (24.8\% \pm 9.6\%), range 14\%-34\%; all routes: 378/948 (39.9\% \pm 18.7\%), range $14 \%-78 \%$.

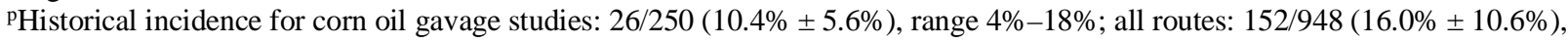
range $4 \%-46 \%$.

${ }^{\mathrm{q}}$ Historical incidence for corn oil gavage studies: $1 / 250(0.4 \% \pm 0.9 \%)$, range $0 \%-2 \%$; all routes: $4 / 948(0.4 \% \pm 0.8 \%)$, range $0 \%-2 \%$.

The combined incidences of hepatocellular adenoma, hepatocellular carcinoma, or hepatoblastoma occurred with a positive trend in males and were significantly increased in males administered 125 or $250 \mathrm{mg} / \mathrm{kg}$ (Table 18, Table C-1, and Table C-2). The combined incidences of hepatocellular carcinoma or hepatoblastoma were significantly increased in 125 and $250 \mathrm{mg} / \mathrm{kg}$ males. 
In males, a single hepatocholangioma occurred in the $250 \mathrm{mg} / \mathrm{kg}$ group (Table C-1). In addition, three and one hepatocholangiocarcinomas occurred in the 62.5 and $125 \mathrm{mg} / \mathrm{kg}$ groups, respectively (Table 18, Table $\mathrm{C}$ - 1 , and Table $\mathrm{C}$-2); these incidences were within the historical control ranges and were not dose-dependent (Table 18, Table C-1, and Table C-2).

The incidences of clear cell focus were significantly increased in all dosed groups of males and the incidences of eosinophilic focus were significantly increased in 62.5 and $125 \mathrm{mg} / \mathrm{kg}$ females (Table 18, Table C-4, and Table D-4).

Hepatocellular adenomas were relatively well-circumscribed, nodular masses that compressed the adjacent hepatic parenchyma (Figure 25 and Figure 26). They were composed of welldifferentiated hepatocytes that formed irregular hepatic plates that often compressed sinusoids and obliquely impinged on the adjacent parenchyma. In general, there was loss of normal lobular architecture with absence of central veins and portal tracts. The cytoplasm of the neoplastic cells varied tinctorially and often appeared eosinophilic, basophilic, clear, or mixed. When more than one hepatocellular adenoma was observed in an animal, the diagnosis of multiple hepatocellular adenoma was recorded.

Hepatocellular carcinomas were generally locally invasive, compressive, irregularly-shaped masses that effaced the normal hepatic parenchyma. Neoplastic hepatocytes were often pleomorphic and in some areas formed trabeculae three or more neoplastic hepatocytes thick (Figure 27 and Figure 28). Cellular atypia and mitotic figures were frequently observed. When more than one hepatocellular carcinoma was observed in an animal, the diagnosis of multiple hepatocellular carcinoma was recorded.

Hepatoblastomas were well-demarcated, irregular-shaped, hypercellular, deeply basophilic masses that occasionally occurred within hepatocellular carcinomas or adjacent to hepatocellular adenomas or hepatocellular carcinomas (Figure 29 and Figure 30). They were composed of sheets of small, elongate to spindle-shaped cells with hyperchromatic nuclei and scant, deeply basophilic cytoplasm that were separated by scant connective tissue stroma. The cells were sometimes arranged radially around small blood vessels and formed poorly defined rosettes or pseudoglandular structures. When more than one hepatoblastoma was observed in an animal, the diagnosis of multiple hepatoblastoma was recorded; when hepatoblastoma was observed within a hepatocellular carcinoma, only the hepatoblastoma was diagnosed. Hepatocholangiocarcinomas were morphologically similar to hepatocellular carcinomas, but also contained elements of malignant biliary epithelium. The one hepatocholangioma was morphologically similar to a hepatocellular adenoma, but also contained elements of benign neoplastic biliary epithelium.

Clear cell foci consisted of foci of hepatocytes that were of normal size to slightly enlarged containing cytoplasmic clear space and centrally located nuclei. Eosinophilic foci were characterized by focal regions of enlarged hepatocytes with granular, pink cytoplasm.

Glandular Stomach: The incidences of epithelium hyperplasia, chronic inflammation, and pigmentation were significantly increased in 125 and $250 \mathrm{mg} / \mathrm{kg}$ males and all dosed groups of females compared to the vehicle controls, and the severities generally increased with increasing dose (Table 19, Table C-4, and Table D-4).

Hyperplasia of the glandular stomach epithelium consisted of multifocal proliferation of the chief cells at the base of the gastric glands in the fundic region of the stomach. This change was subtle 
and of minimal severity. Compared to the glandular stomach of the vehicle controls (Figure 31), there was minimal increase in the number and disorganized crowding of the chief cells within the fundic glands; proliferating cells appeared to be slightly more basophilic than those in unaffected fundic glands (Figure 32). Chronic inflammation consisted of small aggregates of macrophages that were randomly distributed among the glands at the base of the mucosa and in the submucosa. The cytoplasm of macrophages contained a lightly staining, globular, golden yellow material presumed to be the test article and diagnosed as pigment. Multifocally, coarse aggregates of golden brown pigment similar to that within the macrophages were randomly distributed within minimally to mildly dilated gastric glands and the lamina propria. Mixed infiltrates of low numbers of lymphocytes and/or neutrophils were sparsely scattered within the lamina propria and submucosa.

Table 19. Incidences of Nonneoplastic Lesions of the Glandular Stomach in Mice in the Two-year Gavage Study of Indole-3-carbinol

\begin{tabular}{|c|c|c|c|c|}
\hline & Vehicle Control & $62.5 \mathrm{mg} / \mathrm{kg}$ & $125 \mathrm{mg} / \mathrm{kg}$ & $250 \mathrm{mg} / \mathrm{kg}$ \\
\hline \multicolumn{5}{|l|}{ Male } \\
\hline Number Examined Microscopically & 50 & 47 & 47 & 49 \\
\hline Epithelium, Hyperplasia $^{\mathrm{a}}$ & 0 & $1(1.0)^{\mathrm{b}}$ & $22 * *(1.2)$ & $40 * *(1.5)$ \\
\hline Inflammation, Chronic & $1(1.0)$ & $1(1.0)$ & $18 * *(1.0)$ & $45^{* *}(1.0)$ \\
\hline Pigmentation & 0 & $1(1.0)$ & $38 * *(1.0)$ & $48 * *(1.1)$ \\
\hline \multicolumn{5}{|l|}{ Female } \\
\hline Number Examined Microscopically & 48 & 50 & 49 & 50 \\
\hline Epithelium, Hyperplasia & $1(2.0)$ & $7 *(1.3)$ & $10 * *(1.2)$ & $35 * *(1.4)$ \\
\hline Inflammation, Chronic & 0 & $15^{* *}(1.0)$ & $29 * *(1.1)$ & $47 * *(1.3)$ \\
\hline Pigmentation & 0 & $15^{* *}(1.0)$ & $31 * *(1.2)$ & $49 * *(1.9)$ \\
\hline
\end{tabular}

*Significantly different $(\mathrm{P} \leq 0.05)$ from the vehicle control group by the Poly-3 test.

$* * \mathrm{P} \leq 0.01$.

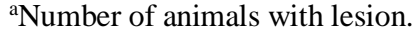

${ }^{\mathrm{b}}$ Average severity grade of lesions in affected animals: $1=$ minimal, $2=$ mild, $3=$ moderate, $4=$ marked.

Nose: Significantly increased incidences of nerve atrophy occurred in $250 \mathrm{mg} / \mathrm{kg}$ males and females compared to the vehicle controls (Table 20, Table C-4, and Table D-4). A single incidence of nerve atrophy without statistical significance occurred in a $125 \mathrm{mg} / \mathrm{kg}$ female. This lesion was most frequent in females.

Incidences of respiratory metaplasia of the olfactory epithelium were significantly increased in $250 \mathrm{mg} / \mathrm{kg}$ males and in 125 and $250 \mathrm{mg} / \mathrm{kg}$ females compared to the vehicle controls (Table 20, Table C-4, and Table D-4). The incidences of atrophy of the olfactory epithelium were significantly increased in 125 and $250 \mathrm{mg} / \mathrm{kg}$ males and $250 \mathrm{mg} / \mathrm{kg}$ females. Slightly increased incidences of mild to moderate olfactory epithelium degeneration occurred in $125 \mathrm{mg} / \mathrm{kg}$ males and in 125 and $250 \mathrm{mg} / \mathrm{kg}$ females. A significantly increased incidence of mild olfactory epithelium necrosis occurred in the $250 \mathrm{mg} / \mathrm{kg}$ males.

The incidences of respiratory epithelium hyaline droplet accumulation were significantly increased in 62.5 and $125 \mathrm{mg} / \mathrm{kg}$ males (Table 20 and Table C-4). The incidence of respiratory 
epithelium hyaline droplet accumulation was significantly decreased in $62.5 \mathrm{mg} / \mathrm{kg}$ females compared to the vehicle controls; however, the incidence and severity increased in $250 \mathrm{mg} / \mathrm{kg}$ females (Table 20 and Table D-4).

Significantly increased incidences of respiratory epithelium hyperplasia occurred in $250 \mathrm{mg} / \mathrm{kg}$ males and females (Table 20, Table C-4, and Table D-4). The severity was increased in $250 \mathrm{mg} / \mathrm{kg}$ females.

The incidence of inflammation was significantly increased in $250 \mathrm{mg} / \mathrm{kg}$ females (Table 20 and Table D-4). The incidence of inflammation was also increased in $125 \mathrm{mg} / \mathrm{kg}$ females, but not significantly.

Table 20. Incidences of Nonneoplastic Lesions of the Nose in Mice in the Two-year Gavage Study of Indole-3-carbinol

\begin{tabular}{|c|c|c|c|c|}
\hline & $\begin{array}{l}\text { Vehicle } \\
\text { Control }\end{array}$ & $62.5 \mathrm{mg} / \mathrm{kg}$ & $125 \mathrm{mg} / \mathrm{kg}$ & $250 \mathrm{mg} / \mathrm{kg}$ \\
\hline \multicolumn{5}{|l|}{ Male } \\
\hline Number Examined Microscopically & 50 & 50 & 50 & 50 \\
\hline Nerve, Atrophy ${ }^{\mathrm{a}}$ & 0 & 0 & 0 & $8^{* *}(2.0)$ \\
\hline $\begin{array}{l}\text { Olfactory Epithelium, Respiratory } \\
\text { Metaplasia }\end{array}$ & $14(1.1)^{\mathrm{b}}$ & $14(1.3)$ & $20(1.5)$ & $27 *(1.4)$ \\
\hline Olfactory Epithelium, Atrophy & $3(2.0)$ & $5(1.6)$ & $11 *(1.4)$ & $17 * *(1.5)$ \\
\hline Olfactory Epithelium, Degeneration & $1(1.0)$ & $1(1.0)$ & $4(1.8)$ & $2(2.0)$ \\
\hline Olfactory Epithelium, Necrosis & 0 & 0 & 0 & $6 *(2.2)$ \\
\hline $\begin{array}{l}\text { Respiratory Epithelium, Accumulation, } \\
\text { Hyaline Droplet }\end{array}$ & $18(1.2)$ & $34 * *(1.1)$ & $30 *(1.1)$ & $26(1.2)$ \\
\hline Respiratory Epithelium, Hyperplasia & $35(1.0)$ & $40(1.2)$ & $41(1.2)$ & $45 *(1.3)$ \\
\hline \multicolumn{5}{|l|}{ Female } \\
\hline Number Examined Microscopically & 50 & 50 & 50 & 50 \\
\hline Nerve, Atrophy & 0 & 0 & $1(2.0)$ & $50 * *(3.0)$ \\
\hline $\begin{array}{l}\text { Olfactory Epithelium, Respiratory } \\
\text { Metaplasia }\end{array}$ & $7(1.0)$ & $8(1.0)$ & $16^{*}(1.0)$ & $49 * *(2.9)$ \\
\hline Olfactory Epithelium, Atrophy & $1(1.0)$ & $2(1.0)$ & $3(2.0)$ & $45^{* *}(2.0)$ \\
\hline Olfactory Epithelium, Degeneration & 0 & 0 & $2(2.0)$ & $3(3.0)$ \\
\hline $\begin{array}{l}\text { Respiratory Epithelium, Accumulation, } \\
\text { Hyaline Droplet }\end{array}$ & $47(1.4)$ & $38 * *(1.1)$ & $42(1.1)$ & $50(2.4)$ \\
\hline Respiratory Epithelium, Hyperplasia & $32(1.0)$ & $31(1.0)$ & $38(1.0)$ & $50 * *(3.0)$ \\
\hline Inflammation & $4(1.5)$ & $1(1.0)$ & $8(1.1)$ & $39 * *(1.2)$ \\
\hline
\end{tabular}

*Significantly different $(\mathrm{P} \leq 0.05)$ from the vehicle control group by the Poly-3 test.

$* * \mathrm{P} \leq 0.01$.

${ }^{a}$ Number of animals with lesion.

${ }^{\mathrm{b}}$ Average severity grade of lesions in affected animals: $1=$ minimal, $2=$ mild, 3 = moderate, $4=$ marked. 
Atrophy of olfactory epithelium was of minimal to moderate severity primarily affecting segments of the ethmoid turbinates and nasal septum in the olfactory region of the Level III nasal histologic section and occasionally in olfactory epithelium lining the dorsal meatus of the Level II nasal histologic section. In affected segments, the height of the epithelium was noticeably shorter than that of unaffected segments of comparable segments in the vehicle controls (Figure 33 and Figure 34).

Nerve atrophy occurred predominantly in the lamina propria of segments of the turbinates (ethmoid) and nasal septum in the olfactory region of the Level III nasal histologic section and occasionally in olfactory epithelium lining the dorsal meatus of the Level II nasal histologic section. In affected segments, the nerve bundles were smaller in diameter and fewer in number than in comparable sections of the vehicle controls.histologic section. In affected segments, the nerve bundles were smaller in diameter and fewer in number than in comparable sections of the vehicle controls.

Olfactory epithelium respiratory metaplasia was observed frequently and affected the olfactory epithelium lining the nasal septum and ethmoturbinates in Level III, and occasionally the dorsal meatus of Level II, in predominantly dosed animals but occasionally in vehicle controls. The incidences and severities of this lesion increased with increasing dose. Olfactory epithelium respiratory metaplasia was of minimal to moderate severity and was characterized by replacement of olfactory epithelium by cuboidal to columnar, ciliated epithelial cells similar to those lining the maxilloturbinates (Figure 35 and Figure 36). Often, the metaplastic epithelium extended into the submocosal glands replacing duct and glandular epithelium.

Degeneration of the olfactory epithelium was characterized by segmental loss of and disorganization of the olfactory epithelia cells along with increased clear intercellular spaces with or without an overall decrease in height of the epithelium.

Olfactory epithelium necrosis, of minimal to moderate severity, was observed predominantly in ethmoid turbinates and occasionally the septum of Level III. Necrosis was characterized by disorganization of olfactory epithelial cells, which had scant eosinophilic cytoplasm and pyknotic nuclei. Degenerate neutrophils and fibrinous eosinophilic material were frequently associated with necrotic olfactory epithelial cells and occasionally effaced the epithelial cell layer.

Hyaline droplet accumulation in the respiratory epithelium was primarily observed in the ventral aspects of the nasal cavity, adjacent to the squamous-respiratory junction in the Level II histologic section and to the olfactory-respiratory junction in the Level III histologic section. Hyaline droplet accumulation consisted of intracytoplasmic, homogenous, eosinophilic, globular material in respiratory epithelial cells.

Hyperplasia of the respiratory epithelium was typically observed in the Level II histologic section and less frequently at the Level I section and was characterized by proliferation of ciliated columnar cells that occasionally formed villous structures that projected into nasal passages. Frequently, the hyperplastic cells extended into the submucosal glands that were often dilated. 
Inflammation was of minimal to mild severity and consisted of protein accumulations and/or infiltration of neutrophils and mononuclear inflammatory cells in the dorsal aspects of the nasal passages in the Level II and III histologic sections.

\section{Genetic Toxicology}

Indole-3-carbinol was tested in three independent bacterial mutagenicity studies, and results were varied. The first study, which employed Salmonella typhimurium strains TA97, TA98, TA100, TA1535, and TA1537 and used a concentration range of 3.3 to 3,333 $\mu$ g indole-3-carbinol/plate with and without $10 \%$ or $30 \%$ rat or hamster liver S9, yielded results that were judged to be equivocal in TA97 in the absence of S9 (Table E-1). A second study, using strains TA97, TA98, TA100, TA102, TA104, TA1535, and TA1537, and concentrations ranging from 33 to $10,000 \mu \mathrm{g} /$ plate, yielded weak positive responses in strain TA100 without and with 30\% hamster liver S9 (no mutagenicity was seen in this strain in the presence of other concentrations or species of S9) (Table E-1). Results of the third study were judged to be equivocal in $S$. typhimurium strain TA100 (concentration range 500 to 5,000 $\mu$ g/plate) in the presence of 10\% rat liver S9 and in Escherichia coli strain WP2 uvrA/pKM101 (concentration range of 500 to $7,500 \mu \mathrm{g} /$ plate) in the absence of S9 activation; no mutagenic activity was seen in this assay in S. typhimurium strain TA98, with or without S9, tested up to 5,000 $\mu$ g/plate (Table E-2).

In vivo, no increase in the frequency of micronucleated PCEs was seen in the bone marrow of male F344/N rats given three doses of indole-3-carbinol (500 to 2,000 mg/kg per day) via gavage; however, a significant decrease in the percent PCEs was seen in the bone marrow of treated rats, indicating that indole-3-carbinol was toxic to the bone marrow (Table E-3). In addition to the rat study, micronucleus frequencies in NCEs of male and female B6C3F1/N mice were assessed in peripheral blood following 3 months of daily gavage treatment with indole-3carbinol (15.6 to $250 \mathrm{mg} / \mathrm{kg}$ per day) in corn oil; no significant increases in micronucleated NCEs were seen in either sex, and no significant changes in percent PCEs occurred over the dose range tested (Table E-4).

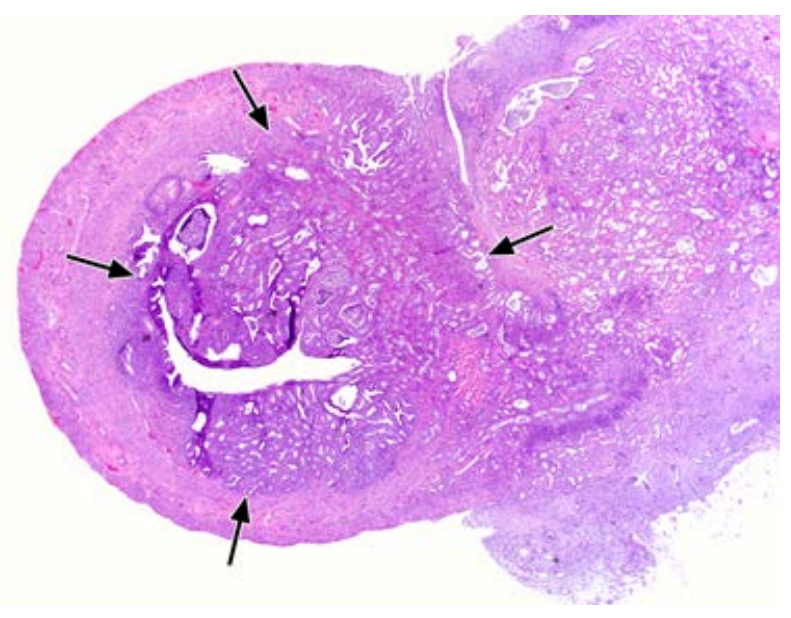

Figure 9. Adenocarcinoma of the Endometrium in the Uterus of a Female Harlan Sprague Dawley Rat Administered 300 mg/kg Indole-3-carbinol by Gavage for Two Years (H\&E)

Note the invasion through the myometrium and out to the serosal surface (arrows). 


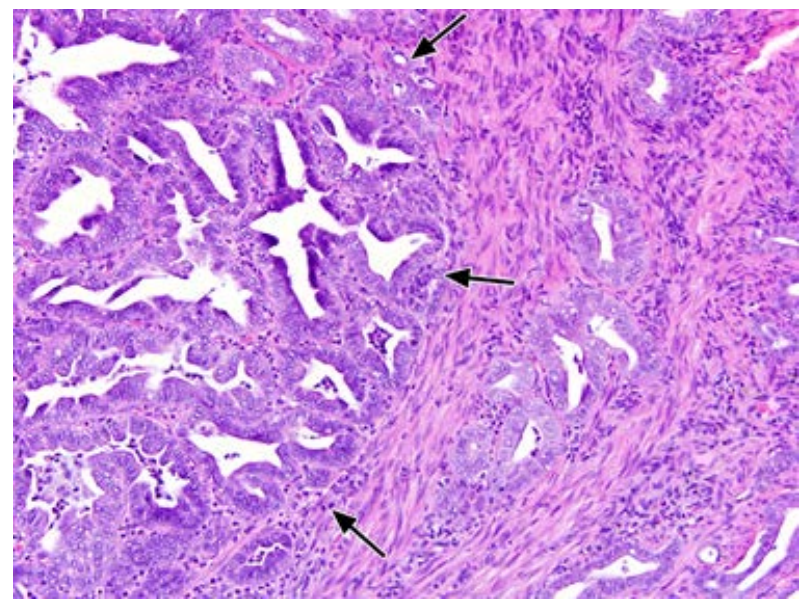

Figure 10. Higher Magnification of Figure 9 (H\&E)

Note the glandular neoplastic structures (arrows) invading into the myometrium.

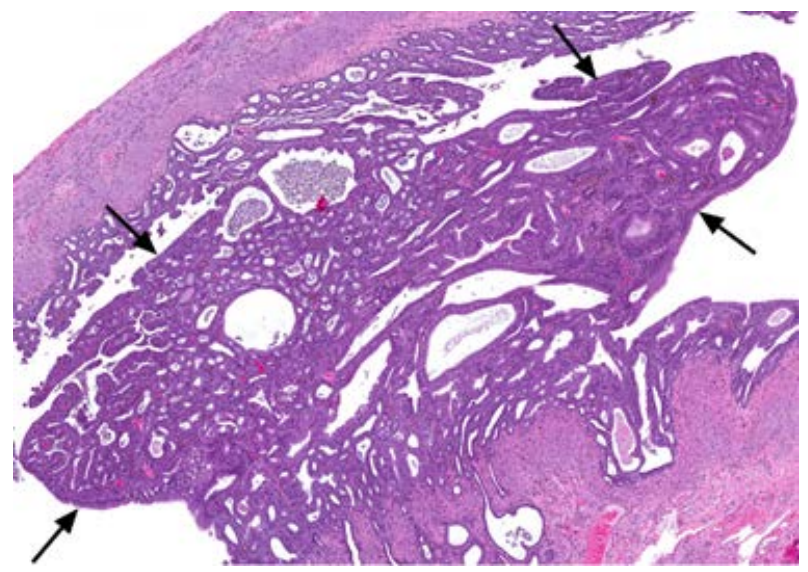

Figure 11. Adenoma (Arrows) of the Endometrium in the Uterus of a Female Harlan Sprague Dawley Rat Administered 300 mg/kg Indole-3-carbinol by Gavage for Two Years (H\&E)

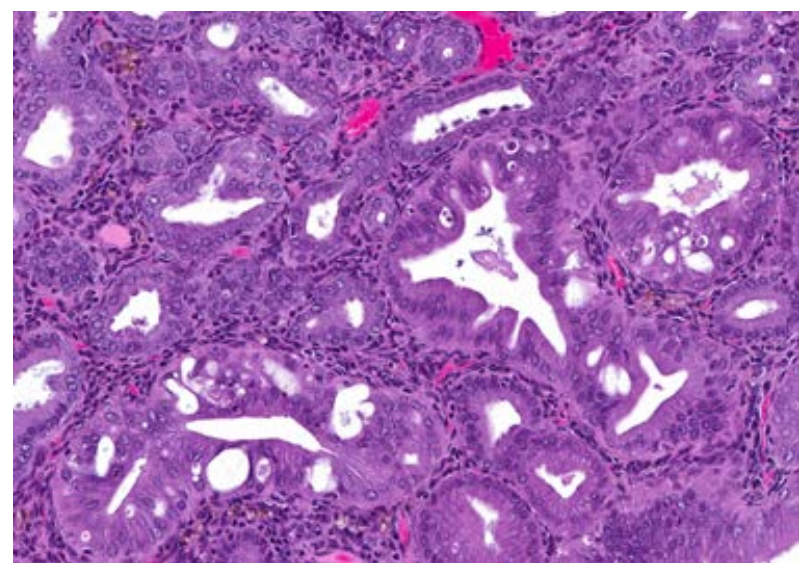

Figure 12. Higher Magnification of Figure 11 (H\&E)

The adenoma is composed of irregular glandular structures lined by cuboidal to columnar epithelium that is disorganized in some areas. 


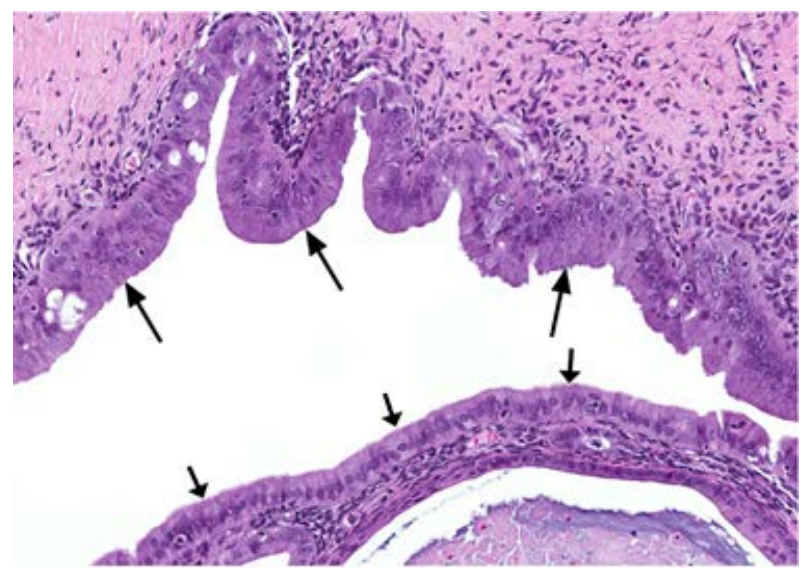

Figure 13. Atypical Hyperplasia of the Endometrium in the Uterus of a Female Harlan Sprague Dawley Rat Administered 300 mg/kg Indole-3-carbinol by Gavage for Two Years (H\&E)

Compared to the normal appearing epithelium lining the lumen of the uterus (thin arrows), a segment of the uterine lumen is lined by disorganized atypical epithelial cells (thick arrows).

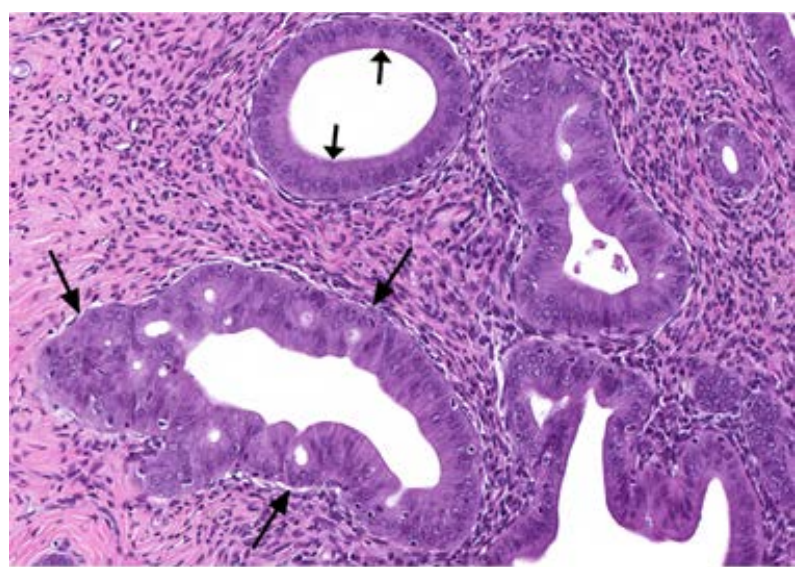

Figure 14. Atypical Hyperplasia of the Endometrium in the Uterus of a Female Harlan Sprague Dawley Rat Administered 300 mg/kg Indole-3-carbinol by Gavage for Two Years (H\&E)

Compared to the normal appearing glands (thin arrows), the affected glands are lined by disorganized atypical epithelial cells (thick arrows). 
Indole-3-carbinol, NTP TR 584

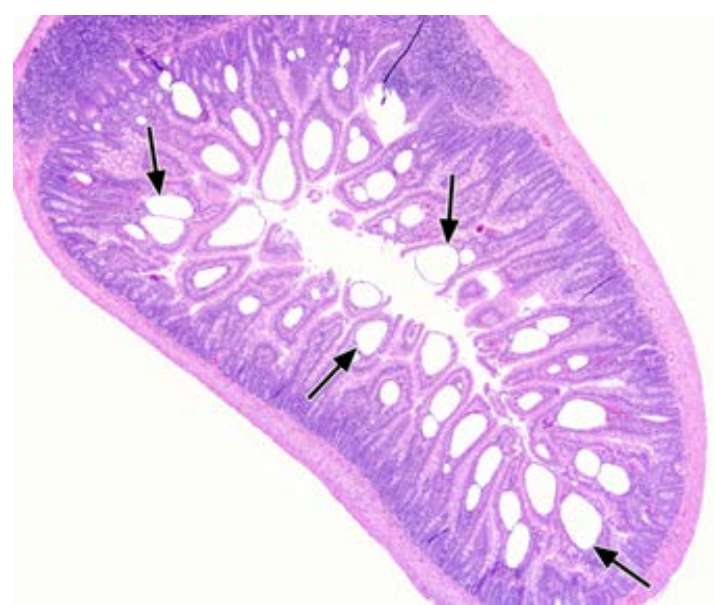

Figure 15. Lymphatic Ectasia in the Jejunum of a Female Harlan Sprague Dawley Rat Administered 300 mg/kg Indole-3-carbinol by Gavage for Two Years (H\&E)

Note multiple dilated lymphatics (arrows) in the lamina propria of the villi.

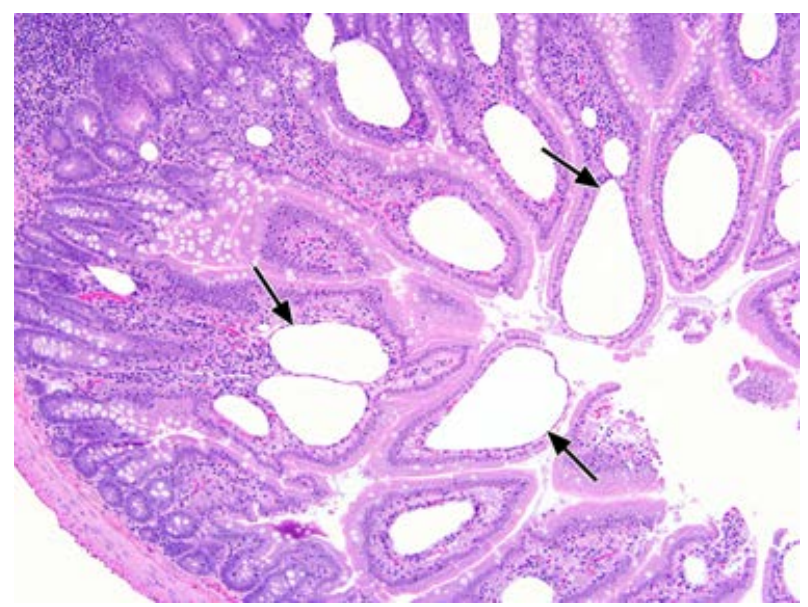

Figure 16. Higher Magnification of Figure 15 (H\&E)

A single layer of endothelial cells lines the dilated lymphatics (arrows). 
Indole-3-carbinol, NTP TR 584

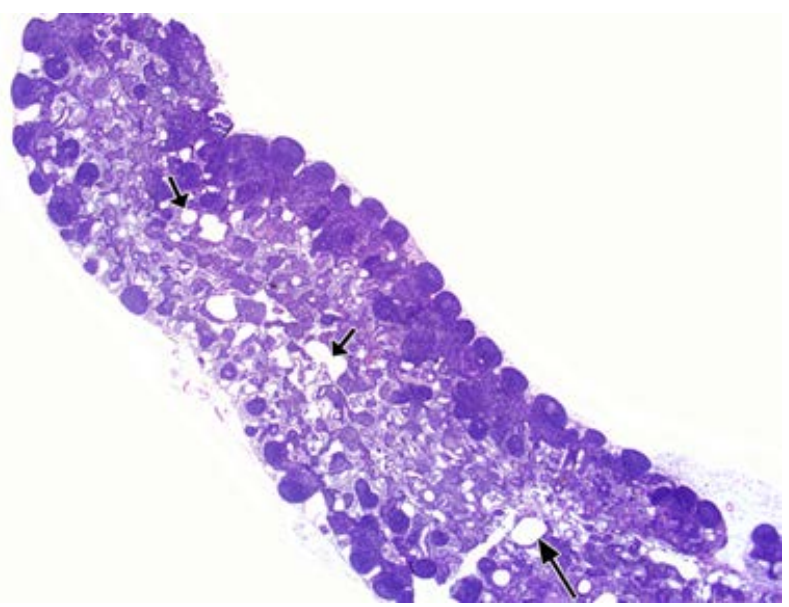

Figure 17. Lymphatic Ectasia in the Mesenteric Lymph Node of a Female Harlan Sprague Dawley Rat Administered $300 \mathrm{mg} / \mathrm{kg}$ Indole-3-carbinol by Gavage for Two Years (H\&E)

Note multiple dilated lymphatics in the medulla (arrows).

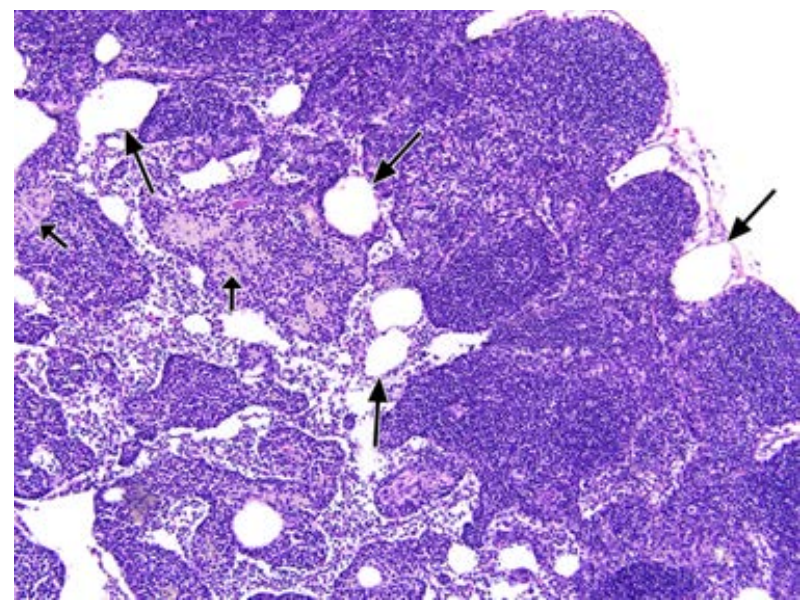

Figure 18. Higher Magnification of Figure 17 (H\&E)

Note the dilated lymphatics (long arrows) and aggregates of macrophages that have golden brown cytoplasmic pigment (short arrows). 
Indole-3-carbinol, NTP TR 584

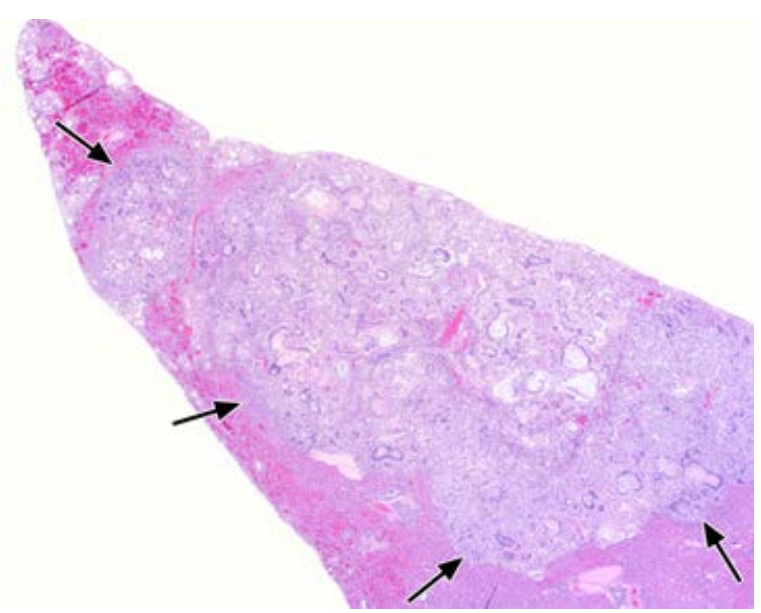

Figure 19. Cholangiofibrosis in the Liver of a Male Harlan Sprague Dawley Rat Administered $150 \mathrm{mg} / \mathrm{kg}$ Indole-3-carbinol by Gavage for Two Years (H\&E)

Note that the lesion has effaced an extensive area of the hepatic parenchyma (arrows).

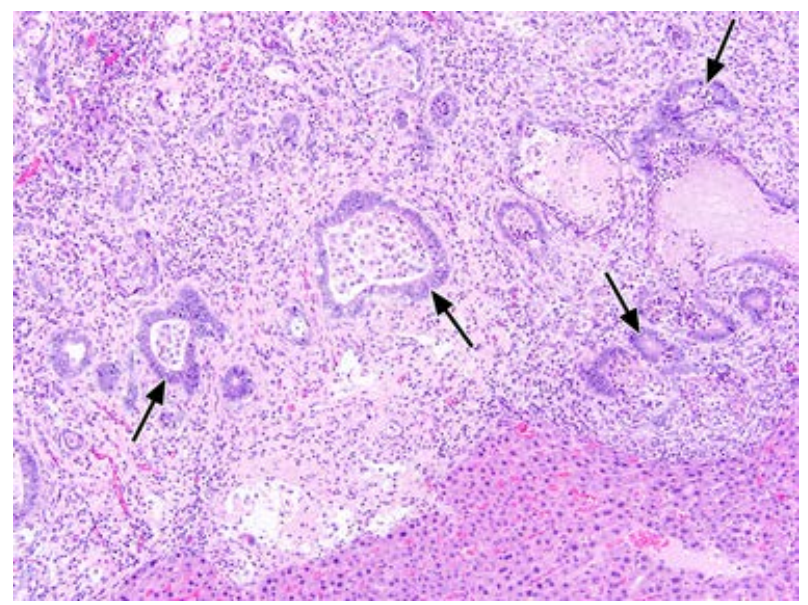

Figure 20. Higher Magnification of Figure 19 (H\&E)

Cholangiofibrosis consists of multiple, variably sized, irregular, atypical bile ducts surrounded by abundant fibrous tissue infiltrated by inflammatory cells (arrows). Ducts contain mucous material, exfoliated cells, and cellular debris. 
Indole-3-carbinol, NTP TR 584

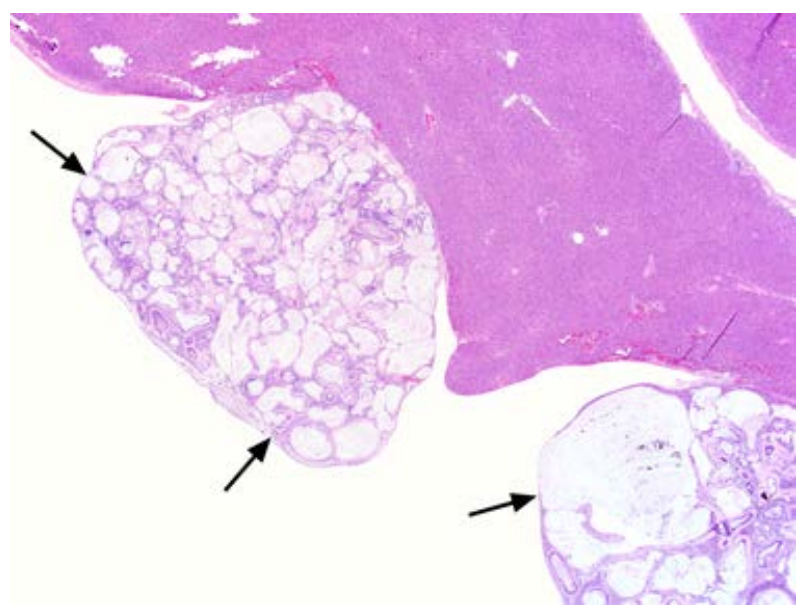

Figure 21. Cholangiofibrosis in the Liver of a Male Harlan Sprague Dawley Rat Administered $150 \mathrm{mg} / \mathrm{kg}$ Indole-3-carbinol by Gavage for Two Years (H\&E)

Note the nodular lesions (arrows) protruding from the capsular surface.

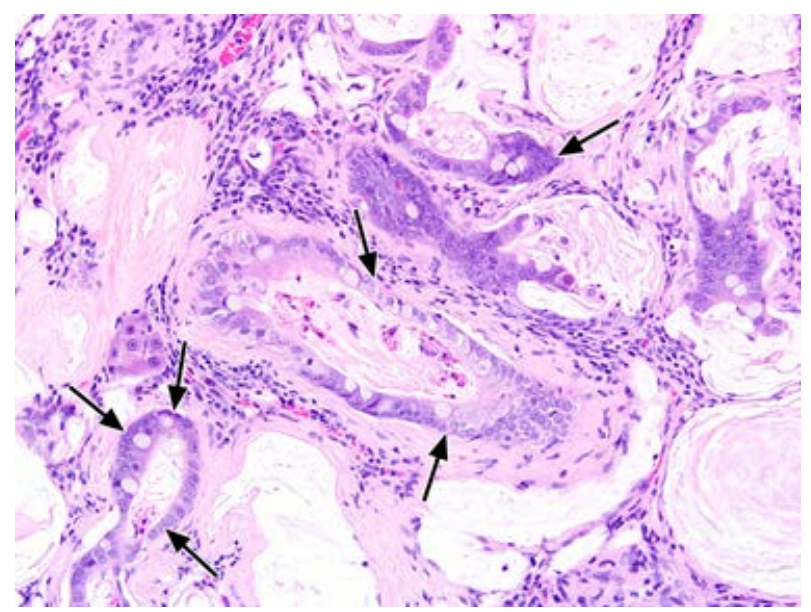

Figure 22. Higher Magnification of Figure 21 (H\&E)

Note the dilated bile ducts lined by atypical epithelial cells (arrows). The ducts contain mucous and cellular debris. 


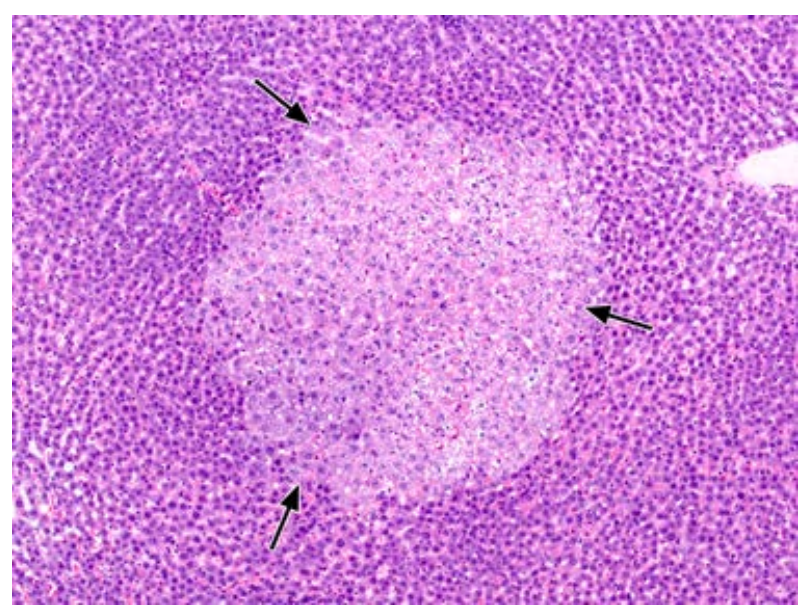

Figure 23. Eosinophilic Focus in the Liver of a Male Harlan Sprague Dawley Rat Administered $75 \mathrm{mg} / \mathrm{kg}$ Indole-3-carbinol by Gavage for Two Years (H\&E)

Note the discrete lesion (arrows) that is well-demarcated from the surrounding hepatic parenchyma.

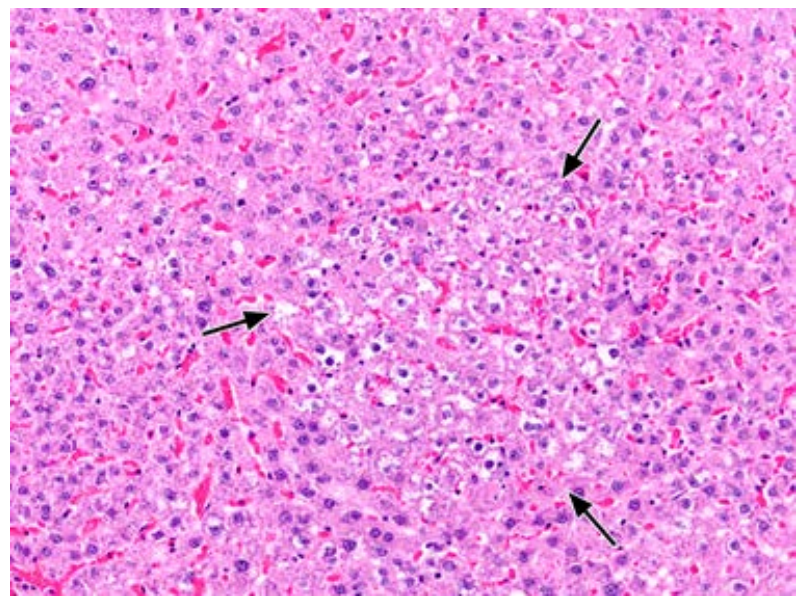

Figure 24. Clear Cell Focus (Arrows) in the Liver of a Male Harlan Sprague Dawley Rat Administered 75 mg/kg Indole-3-carbinol by Gavage for Two Years (H\&E) 
Indole-3-carbinol, NTP TR 584

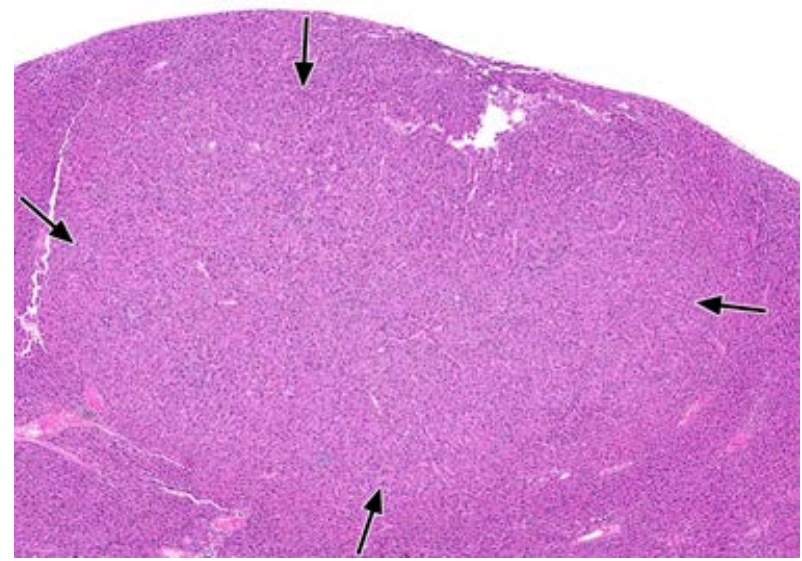

Figure 25. Hepatocellular Adenoma (Arrows) in the Liver of a Male B6C3F1/N Mouse Administered 250 mg/kg Indole-3-carbinol by Gavage for Two Years (H\&E)

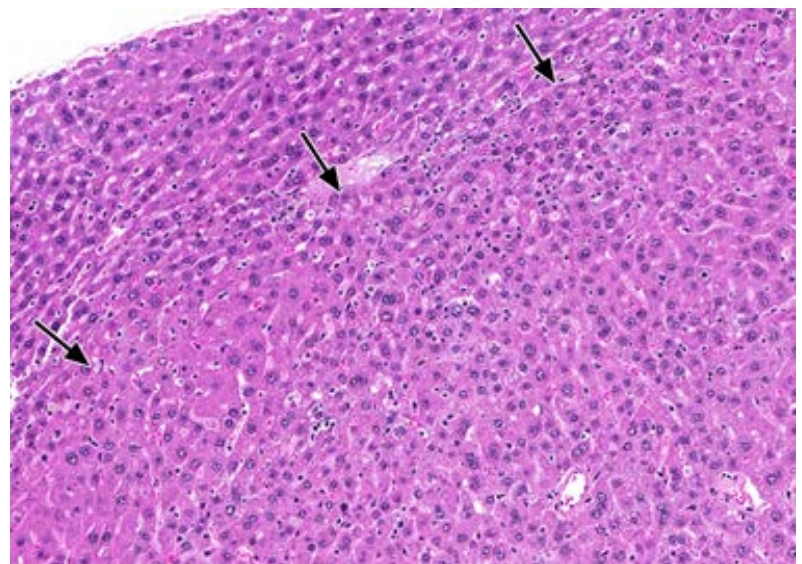

Figure 26. Higher Magnification of Figure 25 (H\&E)

Note the loss of normal hepatic architecture (left of arrows) within the adenoma (right of arrows) and distorted hepatic plates obliquely impinging upon the surrounding hepatic parenchyma. 


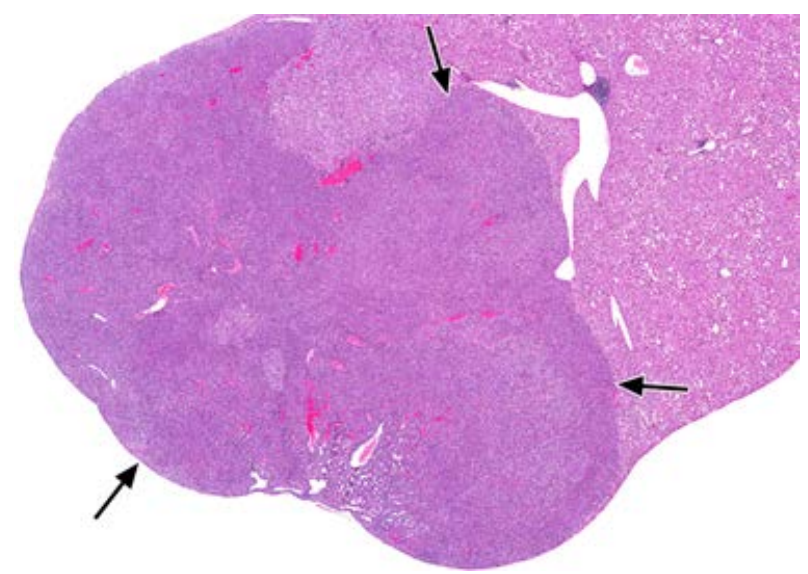

Figure 27. Large, Well-demarcated, Nodular Hepatocellular Carcinoma (Arrows) in a Male B6C3F1/N Mouse Administered 250 mg/kg Indole-3-carbinol by Gavage for Two Years (H\&E)

The neoplasm has completely effaced the hepatic parenchyma.

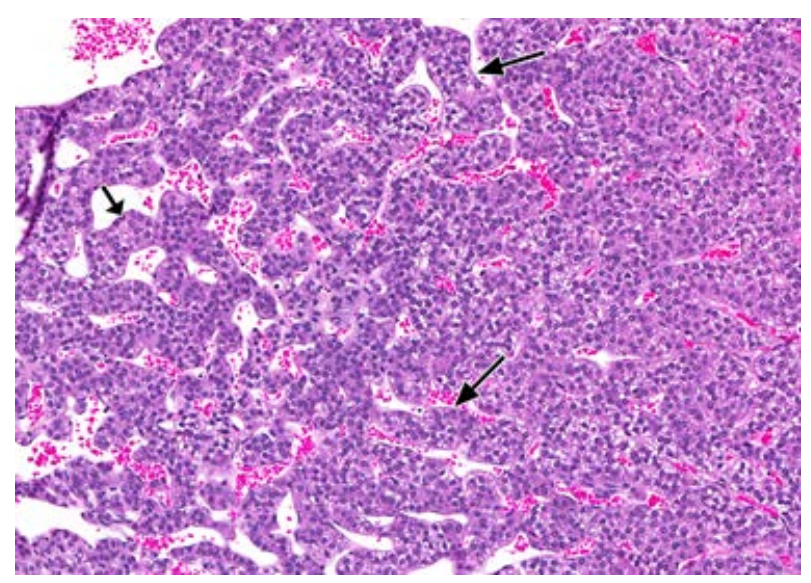

Figure 28. Higher Magnification of Figure 27 (H\&E)

Note the thick trabeculae of neoplastic hepatocytes (arrows) that are a characteristic feature of hepatocellular carcinoma. 


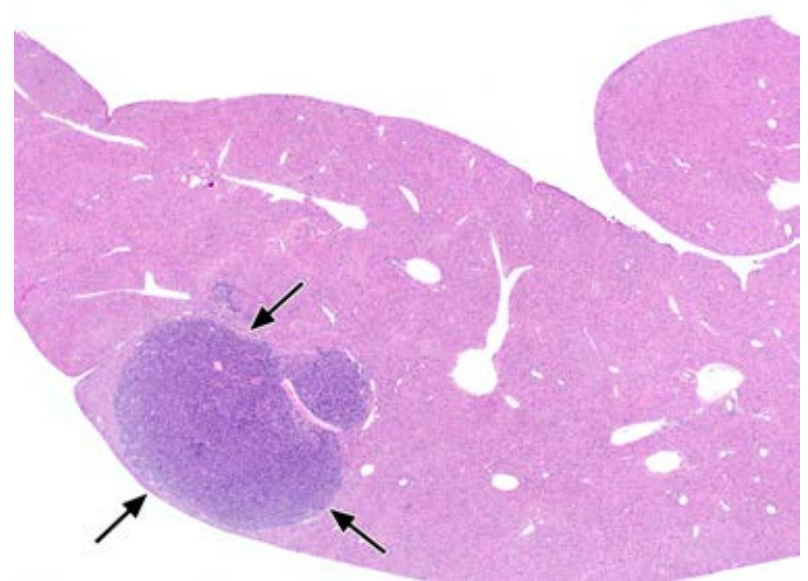

Figure 29. Hepatoblastoma (Arrows) in the Liver of a Male B6C3F1/N Mouse Administered $250 \mathrm{mg} / \mathrm{kg}$ Indole-3-carbinol by Gavage for Two Years (H\&E)

The neoplasm is well-demarcated from the surrounding hepatic parenchyma and stains deeply basophilic.

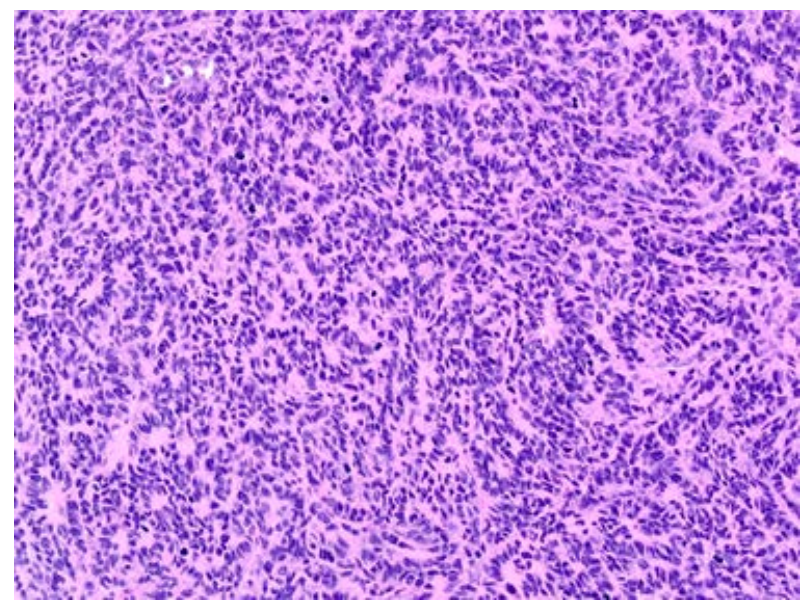

Figure 30. Higher Magnification of Figure 29 (H\&E)

Cells of the hepatoblastoma are small, elongate to spindle-shaped with hyperchromatic nuclei, and are separated by scant connective tissue stroma. 


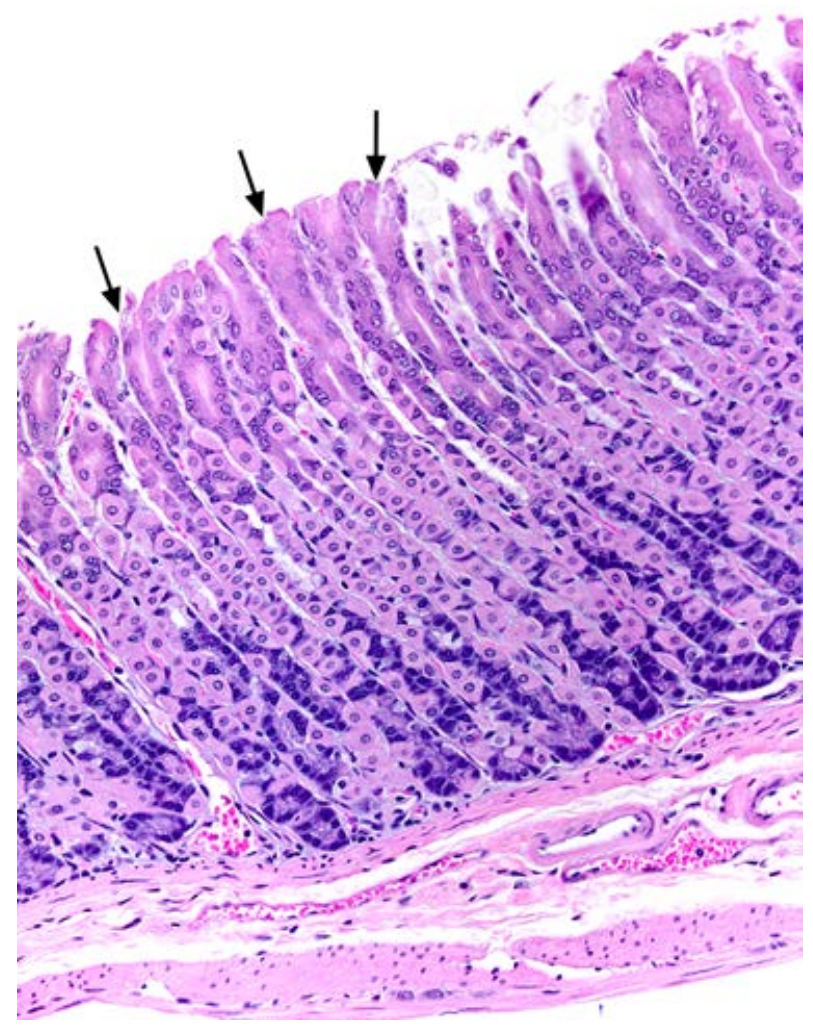

Figure 31. Normal Glandular Stomach Epithelium in a Vehicle Control Female B6C3F1/N Mouse in the Two-year Gavage Study of Indole-3-carbinol (H\&E)

Note elongate gastric glands (arrows) lined by large parietal cells with granular, eosinophilic cytoplasm, and deeply basophilic chief cells at the base of the glands. 


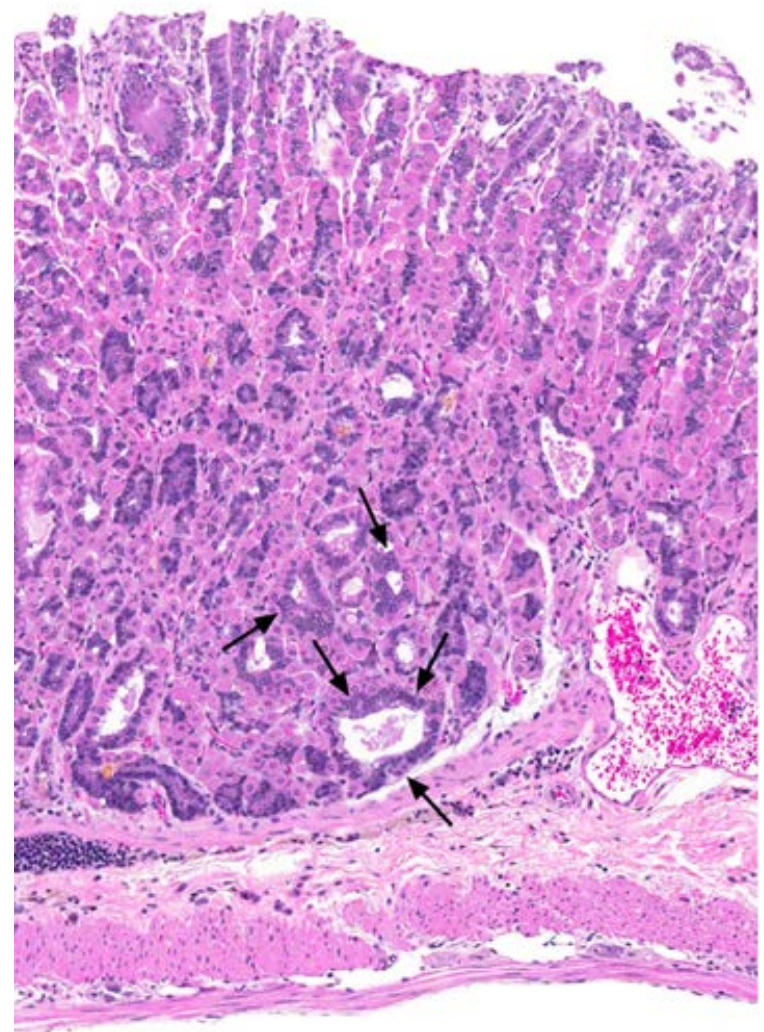

Figure 32. Hyperplasia of the Glandular Epithelium of the Stomach in a Male B6C3F1/N Mouse Administered 250 mg/kg Indole-3-carbinol by Gavage for Two Years (H\&E)

Note that there is disorganization (piling up) and a slight increase in the number of epithelial (chief) cells lining the gland (arrows).

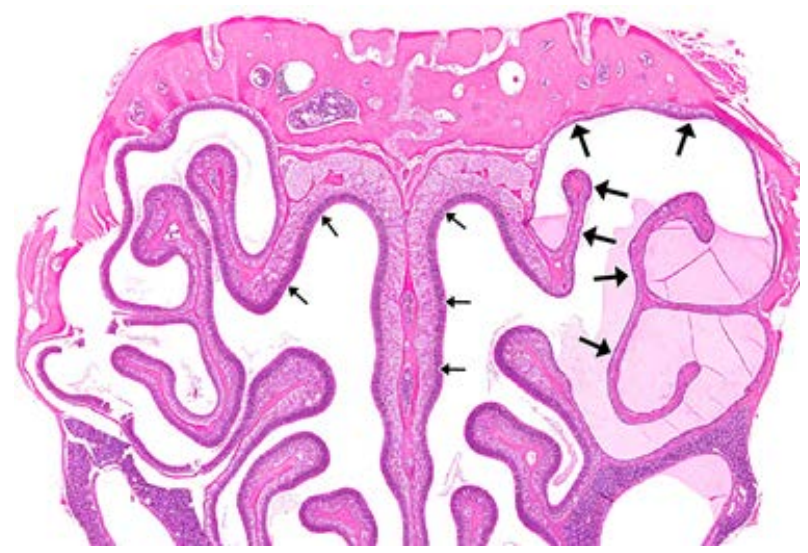

Figure 33. Low Magnification of the Ethmoid Turbinates (Level III Section) in the Nose of a Female B6C3F1/N Mouse Administered $250 \mathrm{mg} / \mathrm{kg}$ Indole-3-carbinol by Gavage for Two Years (H\&E)

Histologically normal olfactory epithelium is indicated on the left side and along the nasal septum of the nasal cavity (thin arrows). Note that the olfactory epithelium lining the dorsal meatus (thick arrows) is thinner (atrophy) than the normal epithelium. 


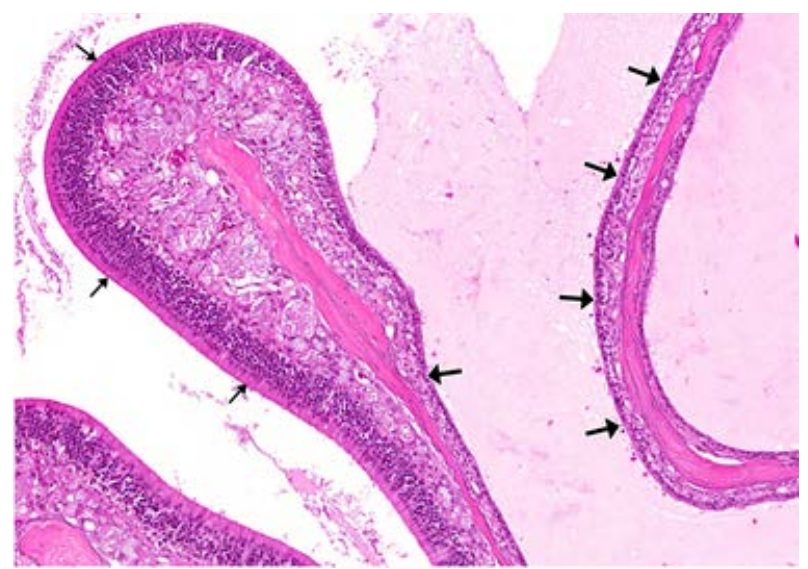

Figure 34. Higher Magnification of Figure 33 (H\&E)

Note that the height of segments of the olfactory epithelium is reduced (atrophy) to a single layer of cells (thick arrows) compared to the histologically normal olfactory epithelium (thin arrows).

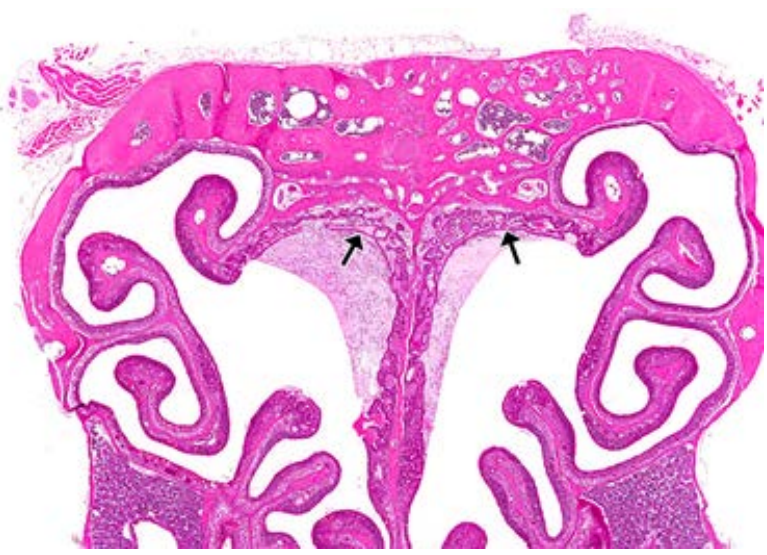

Figure 35. Low Magnification of the Ethmoid Turbinates (Level III Section) in the Nose of a Female B6C3F1/N Mouse Administered $250 \mathrm{mg} / \mathrm{kg}$ Indole-3-carbinol by Gavage for Two Years Showing Respiratory Epithelial Metaplasia in the Olfactory Epithelium (H\&E)

Note that the olfactory epithelium lining the upper one-third of the nasal septum and the adjacent meatuses is bilaterally replaced by a metaplastic respiratory epithelium (arrows). 


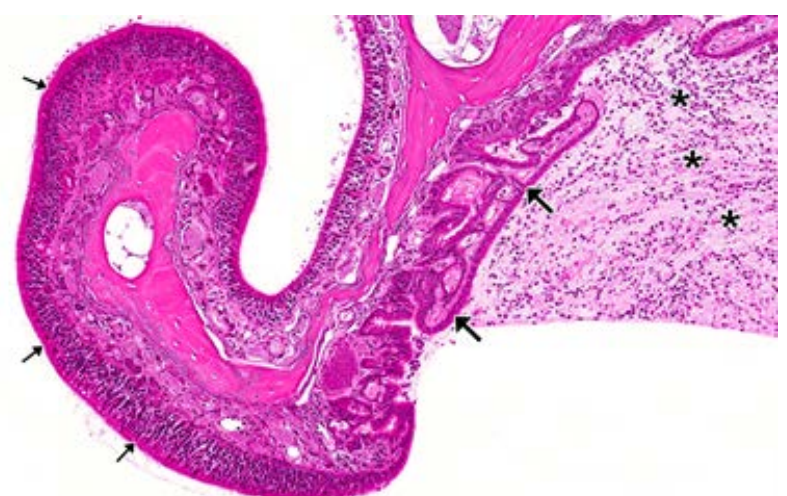

Figure 36. Higher Magnification of Figure 35 (H\&E)

Compared to histologically normal olfactory epithelium (thin arrows), in the affected segment, normal olfactory epithelium is replaced by ciliated, cuboidal to tall, columnar epithelial cells (thick arrows). The ciliated epithelium extends into and replaces the epithelium of the submucosal glands. Note inflammatory cells and cellular debris in the nasal passage (asterisks). 


\section{Discussion}

Indole-3-carbinol was nominated by the National Cancer Institute based on its growing use as a dietary supplement and its potential use as a therapeutic agent for the prevention of various types of cancer. While substantial evidence exists that indole-3-carbinol can reduce the risk of cancers induced by several known carcinogens when administered to animals, indole-3-carbinol can also function as an initiator and tumor promoter in certain models. The carcinogenic potential of indole-3-carbinol has not been studied in a 2-year bioassay.

The effects of gavage exposure to indole-3-carbinol for 3 months or 2 years were studied in male and female F344/N (3-month study) or Harlan Sprague Dawley (2-year study) rats and B6C3F1/N mice at doses up to $300 \mathrm{mg} / \mathrm{kg}$ for rats and $250 \mathrm{mg} / \mathrm{kg}$ for mice. In general, 3 months of exposure to indole-3-carbinol exhibited more effects in F344/ $\mathrm{N}$ rats than in mice. Decreased body weight gains were observed in male F344/N rats at $300 \mathrm{mg} / \mathrm{kg}$, but not in female F344/N rats or either sex of mice. In F344/ $\mathrm{N}$ rats, changes in organ weights were observed at all administered doses of indole-3-carbinol. Increased liver and kidney weights were observed in both sexes, and decreased thymus weights were observed only in female rats. No corresponding histopathology was noted in the liver, kidney, or thymus in either sex of F344/N rats. In male and female mice, the only treatment-related effects observed were increased liver weights in males at $125 \mathrm{mg} / \mathrm{kg}$ or greater and in females at $15.6 \mathrm{mg} / \mathrm{kg}$ or greater.

Increased liver weights have been reported in rats exposed to indole-3-carbinol in the presence and absence of hepatocyte hypertrophy ${ }^{20 ; 21}$. In the current 3-month studies, changes in liver weights in F344/N rats and mice were not accompanied by hepatocyte hypertrophy. In 28-day recovery studies, Crowell et al. ${ }^{20}$ demonstrated that the increased liver weights and hepatic hypertrophy induced by indole-3-carbinol were reversible effects.

In the liver, treatment-related increases in acetanilide-4-hydroxylase (A4H) and 7ethoxyresorufin-O-deethylase (EROD) activities were observed in both sexes of F344/N rats and mice and the magnitude of induction was greater in rats than in mice. The induction of hepatic

CYP1A1 and 1A2 has been widely demonstrated in repeat-dose studies of indole-3-carbinol ${ }^{20 ; 21 ;}$ 122-124. Other studies in the literature have demonstrated that exposure to indole-3-carbinol induces other hepatic enzymes in rodents, including glutathione-S-transferase, UDPglucuronosyl transferase, glutathione reductase, and quinone reductase ${ }^{19 ; 125 ; 126}$. In the current studies, increased pulmonary EROD activity was observed in F344/N rats, but not mice exposed to indole-3-carbinol.

Expression of CYP1A1 and 1A2 serves as a useful marker for activation of the aryl hydrocarbon receptor ${ }^{127-130}$. The induction of CYP1A1 is a very sensitive response to exposure to 2,3,7,8tetrachlorodibenzo-p-dioxin (TCDD) and other dioxin-like compounds ${ }^{131}$ which are potent inducers in many tissues including the liver, lung, kidney, nasal passages, and small intestine. In fact, in many cases the relative potency for induction of CYP1A1 has provided the basis for establishing Toxic Equivalency Factors for the activity of dioxin-like compounds ${ }^{132}$. While increased activity or expression of CYP1A1 and 1A2 are widely accepted as sensitive markers for exposure to AhR-agonists, the potential role of the induction of hepatic CYP1A1 and 1A2 enzymes in the mechanism of toxicity and carcinogenicity of dioxin-like compounds is not fully understood. However, it has been hypothesized that AhR-dependent induction of the CYP1 
family of cytochromes P450 may lead to induction of oxidative stress due to inefficient electron transfer during P450 metabolism ${ }^{133}$. CYP1A1 is also known to metabolize carcinogens like benzo[ $[a]$ pyrene and aflatoxin B1 to epoxide intermediates. Therefore, the induction of these enzymes may have implications in coexposures to indole-3-carbinol and other such chemicals in humans.

In both sexes of F344/N rats, indole-3-carbinol administration for 3 months increased the incidences of lymphatic ectasia in the duodenum and jejunum, lipidosis in the lamina propria of the small intestine, and lymphatic dilatation in the mesenteric lymph node. To further clarify and elucidate the pathogenesis of these lesions, special evaluations were conducted on tissues from 1-week and 4-week euthanasia of special intestine study male Sprague Dawley rats in the 2-year bioassay ${ }^{87}$. Because different rat strains were evaluated in the 3-month and 2-year studies, the concordance of the development of these lesions between F344/N rats and Harlan Sprague Dawley rats was also evaluated. In both strains of rats, treatment-related dilatation of lymphatics (lymphangiectasis) of the duodenum, jejunum, and mesenteric lymph node was observed at $150 \mathrm{mg} / \mathrm{kg}$ or greater. Electron microscopy and special staining with Oil-red-O and Sudan Black confirmed extracellular lipid accumulation within the villar lamina propria, lacteals, and macrophages ${ }^{87}$. While lymphatic ectasia in the small intestine or mesenteric lymph node was observed in both strains of rats, it was not observed in B6C3F1/N mice administered indole-3carbinol for 3 months or 2 years.

In the 2-year studies in Harlan Sprague Dawley rats, a positive trend in the incidences of uterine adenocarcinoma occurred in females; uterine masses observed grossly at necropsy were likely related to the increased incidences of uterine adenocarcinoma. The standard protocol for collection of uterine tissue outlined in the NTP Specifications for the Conduct of Toxicity and Carcinogenicity Studies requires that at necropsy a single transverse segment is collected from each uterine horn $0.5 \mathrm{~cm}$ from the cervix along with any grossly observed lesions; opening of the remaining segments of the horns is not required. The extended residual tissue review involved trimming, embedding and sectioning of the residual uterine tissue, cervix and vagina longitudinally. The reason for the residual tissue evaluation was to have a more comprehensive evaluation of the uteri in light of the occurrence of adenocarcinoma in the standard (initial) evaluation. During the extended review of the uteri, additional uterine masses were discovered mostly in uterine horns that were grossly dilated. The masses were small and would not have been discovered using the standard necropsy examination protocol unless the uterine horns were opened. During the extended microscopic evaluation, additional incidences of uterine adenocarcinomas and new incidences of adenomas were identified; adenomas were not diagnosed in the standard evaluation. In the combined standard and extended evaluations, the incidence of adenocarcinoma in the uterus was significantly increased in the $150 \mathrm{mg} / \mathrm{kg}$ group. The incidences of uterine adenoma were not significantly increased in the dosed groups.

Uterine adenocarcinomas are an uncommon background neoplasm in the Harlan Sprague Dawley rat. In the standard evaluation in the current 2-year study, no uterine adenocarcinomas were observed in vehicle control female rats. Based on the significant increase in the incidence of uterine adenocarcinomas in the $150 \mathrm{mg} / \mathrm{kg}$ group and the increased incidence in the $300 \mathrm{mg} / \mathrm{kg}$ group, these results were considered some evidence of carcinogenicity. In addition, the incidence of squamous metaplasia of the endometrium was significantly increased in $150 \mathrm{mg} / \mathrm{kg}$ female rats. 
In the skin of female Sprague Dawley rats, there was a positive trend in the incidences of fibroma or fibrosarcoma (combined). Fibromas or fibrosarcomas were only observed in the vehicle control and $300 \mathrm{mg} / \mathrm{kg}$ groups. Because the incidence observed in the $300 \mathrm{mg} / \mathrm{kg}$ group was not significantly increased compared to the vehicle controls, this was an uncertain finding that may have been related to indole-3-carbinol administration.

In the thyroid gland of male Sprague Dawley rats, there were general, dose-dependent increases in the incidences and severities of follicular cell hypertrophy. While the thyroid gland has not been identified as a target organ for indole-3-carbinol exposure, indole-3-carbinol at $0.25 \%$ in the diet (total daily intake not provided) has previously been shown to promote thyroid gland follicular cell adenomas and adenocarcinomas in an initiation-promotion model ${ }^{33}$.

In the 2-year mouse studies, body weights were decreased by greater than $10 \%$ in $250 \mathrm{mg} / \mathrm{kg}$ female mice from weeks 32 to 92, but no treatment-related decreases in survival were noted in males or females. In male mice, treatment-related neoplasms occurred only in the liver, while nonneoplastic lesions occurred in the glandular stomach and the nose. In female mice, treatmentrelated nonneoplastic lesions occurred in the liver, glandular stomach, and nose.

In the liver, there were generally positive trends for the incidences of hepatocellular adenoma, hepatocellular carcinoma, and hepatoblastoma as well as their combined incidences in indole-3carbinol-treated male mice in the 2-year studies. Increased incidences of hepatocellular adenoma and hepatoblastoma occurred in $250 \mathrm{mg} / \mathrm{kg}$ males and included increased numbers of mice with multiple lesions. A significant increase in the incidence of multiple adenoma also occurred in $62.5 \mathrm{mg} / \mathrm{kg}$ males. An increased incidence of hepatocellular carcinoma occurred in $125 \mathrm{mg} / \mathrm{kg}$ males, but not in males administered $250 \mathrm{mg} / \mathrm{kg}$. However, in early death animals the time to first observed incidence was 182 days less in the $250 \mathrm{mg} / \mathrm{kg}$ group than in the $125 \mathrm{mg} / \mathrm{kg}$ group, and 80 days less than in the vehicle controls. Although hepatocellular adenomas and hepatocellular carcinomas are relatively common neoplasms in male mice, the significantly increased incidences in indole-3-carbinol-treated males considerably exceeded the concurrent vehicle control and historical control rates. The combined incidences of hepatocellular adenoma, hepatocellular carcinoma, and hepatoblastoma were significantly increased in 125 and $250 \mathrm{mg} / \mathrm{kg}$ males. Based on the observed increases, the incidences of hepatocellular adenoma, hepatocellular carcinoma, and hepatoblastoma were considered related to indole-3-carbinol treatment and supportive of clear evidence of carcinogenicity in male mice.

Hepatic gene expression studies in $300 \mathrm{mg} / \mathrm{kg}$ females from the microarray study suggested the activation of multiple xenobiotic transcription factors in rat liver with the most pronounced activation being associated with AhR and Nrf2. Consistent with these findings was the upregulation of genes associated with xenobiotic metabolism which suggests the potential for indole-3-carbinol to modify drug efficacy and safety. These findings are largely similar to results from other transcriptomic studies of indole-3-carbinol.

In the current 2-year study, treatment-related nonneoplastic lesions occurred in the glandular stomach and the nose of both male and female mice. Indole-3-carbinol-induced hyperplasia, pigmentation, and chronic inflammation in the glandular stomach occurred at lower doses in females $(62.5 \mathrm{mg} / \mathrm{kg})$ than in males $(125 \mathrm{mg} / \mathrm{kg})$. The spectra of nasal lesions in the olfactory and respiratory epithelia were similar in males and females. In both sexes, incidences of nerve atrophy, respiratory metaplasia and atrophy of the olfactory epithelium, and hyperplasia of the 
respiratory epithelium were significantly increased in mice administered $250 \mathrm{mg} / \mathrm{kg}$. In addition, occurrences of olfactory epithelium necrosis and respiratory epithelium hyaline droplet accumulation in males and nasal inflammation in females were considered treatment-related. Nasal lesions have not been reported in shorter-term studies of indole-3-carbinol.

As an AhR agonist, indole-3-carbinol might be expected to elicit the same responses as other AhR agonists, most notably 2,3,7,8-tetrachlorodibenzo-p-dioxin (TCDD) and other dioxin-like compounds. However, it is important to note the absence of dioxin-like carcinogenicity in the current studies. In general, TCDD and other individual and relevant mixtures of dioxin-like compounds induce a characteristic spectrum of neoplasms in the three main target organs: liver, lung, and oral mucosa ${ }^{74-80 ; 134}$. In the liver, TCDD ${ }^{75}, 3,3^{\prime}, 4,4^{\prime}, 5$-pentachloro-biphenyl $(\mathrm{PCB} 126)^{74}, 2,3,4,7,8$-penta-chlorodibenzofuran (PeCDF) ${ }^{76}$, 2,3',4,4',5-pentachlorobiphenyl

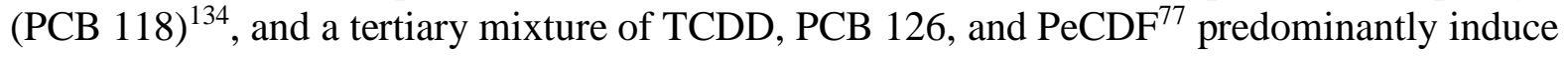
cholangiocarcinomas and hepatocellular adenomas. In addition to these neoplasms, a binary mixture of PCB 126 and PCB $153^{79}$ and a binary mixture of PCB 126 and PCB $118^{80}$ induce hepatocarcinomas. In general, exposure to these dioxin-like compounds induces cystic keratinizing epitheliomas in the lung ${ }^{74 ; 75 ; 77-80 ; 134}$. Gingival squamous cell carcinomas were induced by exposure to TCDD ${ }^{75}$, PCB $126^{74}, \mathrm{PeCDF}^{76}$, a binary mixture of PCB 126 and PCB $118^{80}$, and a binary mixture of PCB 126 and PCB $153^{79}$. None of these characteristic neoplasms induced by exposure to persistent dioxin-like compounds were observed in rats exposed to indole-3-carbinol, even at doses that elicited comparable fold-inductions of hepatic EROD activity, a marker of AhR activation. In addition to hepatic neoplasms, TCDD and other dioxin-like compounds induce dose-dependent increases in the incidences of a broad spectrum of nonneoplastic liver lesions, including hepatocyte hypertrophy, multinucleated hepatocytes, fatty change, bile duct hyperplasia and cyst, cholangiofibrosis, and oval cell hyperplasia ${ }^{74-77 ; 79 ; 80 ; 134 . ~}$ This broad spectrum of lesions was not observed in rats exposed to indole-3-carbinol for 2 years. Aside from increased liver weight, the only treatment-related lesions observed in the liver were increased incidences of clear cell and eosinophilic foci in female rats, and increased incidences of bile duct cysts in male rats.

Chronic exposure to dioxin-like compounds also induces nonneoplastic lesions in other tissues, including the lung, adrenal cortex, pancreas, kidney, heart, thyroid gland, and thymus ${ }^{74-77 ; 80 ; 134}$. The spectrum of toxicity and the specificity of target organs vary slightly between dioxin-like congeners, with exposure to some congeners affecting additional tissues. However, the general profiles of toxicity and carcinogenicity are similar. Therefore, the differences in the observed neoplastic and nonneoplastic responses between indole-3-carbinol and the dioxins demonstrate a clear difference between indole-3-carbinol and other persistent dioxin-like compounds. 
Indole-3-carbinol, NTP TR 584

\section{Conclusions}

Under the conditions of these 2-year gavage studies, there was no evidence of carcinogenic activity $^{a}$ of indole-3-carbinol in male Harlan Sprague Dawley rats administered 75, 150, or $300 \mathrm{mg} / \mathrm{kg}$. There was some evidence of carcinogenic activity of indole-3-carbinol in female Harlan Sprague Dawley rats based on increased incidences of malignant uterine neoplasms (primarily adenocarcinoma). The occurrences of fibroma and fibrosarcoma in the skin may have been related to indole-3-carbinol administration. There was clear evidence of carcinogenic activity of indole-3-carbinol in male B6C3F1/N mice based on increased incidences of liver neoplasms (hepatocellular adenoma, hepatocellular carcinoma, and hepatoblastoma). There was no evidence of carcinogenic activity of indole-3-carbinol in female B6C3F1/N mice administered $62.5,125$, or $250 \mathrm{mg} / \mathrm{kg}$.

Administration of indole-3-carbinol caused increased incidences of nonneoplastic lesions in the small intestine, mesenteric lymph node, and liver of male and female rats, the thyroid gland of male rats, the uterus of female rats, and the liver, glandular stomach, and nose of male and female mice.

aSee Explanation of Levels of Evidence of Carcinogenic Activity. A summary of the Peer Review Panel comments and the public discussion on this Technical Report appears in Appendix M. 


\section{References}

1. Aldrich Handbook of Fine Chemicals. Milwaukee, WI: Aldrich Chemical Company, Inc.; 1996. p. 849.

2. Broadbent TA, S Broadbent H. The chemistry and pharmacology of indole-3-carbinol (indole3-methanol) and 3-(methoxymethyl)indole. [Part II]. Curr Med Chem. 1998; 5:469-491.

3. Bradfield CA, Bjeldanes LF. Modification of carcinogen metabolism by indolylic autolysis products of Brassica oleraceae. Adv Exp Med Biol. 1991; 289:153-163.

http://dx.doi.org/10.1007/978-1-4899-2626-5_13

4. Wortelboer HM, De Kruif CA, Van Iersel AAJ, Falke HE, Noordhoek J, Blaauboer BJ. Acid reaction products of indole-3-carbinol and their effects on cytochrome P450 and phase II enzymes in rat and monkey hepatocytes. Biochem Pharmacol. 1992; 43(7):1439-1447. http://dx.doi.org/10.1016/0006-2952(92)90200-3

5. Kwon C-S, Grose KR, Riby J, Chen Y-H, Bjeldanes LF. In vivo production and enzymeinducing activity of indolo[3,2-b]carbazole. J Agric Food Chem. 1994; 42(11):2536-2540. http://dx.doi.org/10.1021/jf00047a030

6. Jongen WMF. Glucosinolates in Brassica: Occurrence and significance as cancer-modulating agents. Proc Nutr Soc. 1996; 55(1B):433-446. http://dx.doi.org/10.1079/PNS19960040

7. Holst B, Williamson G. A critical review of the bioavailability of glucosinolates and related compounds. Nat Prod Rep. 2004; 21(3):425-447. http://dx.doi.org/10.1039/b204039p

8. McDanell R, McLean AEM, Hanley AB, Heaney RK, Fenwick GR. Chemical and biological properties of indole glucosinolates (glucobrassicins): A review. Food Chem Toxicol. 1988; 26(1):59-70. http://dx.doi.org/10.1016/0278-6915(88)90042-7

9. Heaney RK, Fenwick GR. Natural toxins and protective factors in brassica species, including rapeseed. Nat Toxins. 1995; 3(4):233-237. http://dx.doi.org/10.1002/nt.2620030412

10. Carlson DG, Daxenbichler ME, VanEtten CH, Kwolek WF, Williams PH. Glucosinolates in crucifer vegetables: Broccoli, brussels sprouts, cauliflower, collards, kale, mustard greens, and kohlrabi. J Amer Soc Hort Sci. 1987; 112(1):173-178.

11. Dashwood RH, Uyetake L, Fong AT, Hendricks JD, Bailey GS. In vivo disposition of the natural anti-carcinogen indole-3-carbinol after PO administration to rainbow trout. Food Chem Toxicol. 1989; 27(6):385-392. http://dx.doi.org/10.1016/0278-6915(89)90144-0

12. Stresser DM, Williams DE, Griffin DA, Bailey GS. Mechanisms of tumor modulation by indole-3-carbinol. Disposition and excretion in male Fischer 344 rats. Drug Metab Dispos. 1995; 23(9):965.

13. Anderton MJ, Manson MM, Verschoyle RD, Gescher A, Lamb JH, Farmer PB, Steward WP, Williams ML. Pharmacokinetics and tissue disposition of indole-3-carbinol and its acid condensation products after oral administration to mice. Clin Cancer Res. 2004; 10(15):5233. http://dx.doi.org/10.1158/1078-0432.CCR-04-0163 
14. De Kruif CA, Marsman JW, Venekamp JC, Falke HE, Noordhoek J, Blaauboer BJ, Wortelboer HM. Structure elucidation of acid reaction products of indole-3-carbinol: Detection in vivo and enzyme induction in vitro. Chem Biol Interact. 1991; 80(3):303-315. http://dx.doi.org/10.1016/0009-2797(91)90090-T

15. Chang Y-C, Riby J, Chang GHF, Peng B, Firestone G, Bjeldanes LF. Cytostatic and antiestrogenic effects of 2-(indol-3-ylmethyl)-3,3'-diindolylmethane, a major in vivo product of dietary indole-3-carbinol. Biochem Pharmacol. 1999; 58(5):825-834. http://dx.doi.org/10.1016/S0006-2952(99)00165-3

16. Riby JE, Feng C, Chang Y-C, Schaldach CM, Firestone GL, Bjeldanes LF. The major cyclic trimeric product of indole-3-carbinol is a strong agonist of the estrogen receptor signaling pathway. Biochemistry. 2000; 39(5):910-918. http://dx.doi.org/10.1021/bi9919706

17. Gonzalez JM, Yusta B, Garcia C, Carpio M. Pulmonary and hepatic lesions in experimental 3-hydroxymethylindole intoxication. Vet Hum Toxiocol. 1986; 28:418-420.

18. LeBlanc GA, Stuart JD, Dunn SE, Baldwin WS. Effect of the plant compound indole-3carbinol on hepatic cholesterol homoeostasis. Food Chem Toxicol. 1994; 32(7):633-639. http://dx.doi.org/10.1016/0278-6915(94)90007-8

19. Shertzer HG, Sainsbury M. Intrinsic acute toxicity and hepatic enzyme inducing properties of the chemoprotectants indole-3-carbinol and 5,10-dihydroindeno[1,2-b]indole in mice. Food Chem Toxicol. 1991; 29(4):237-242. http://dx.doi.org/10.1016/0278-6915(91)90020-8

20. Crowell JA, Page JG, Levine BS, Tomlinson MJ, Hebert CD. Indole-3-carbinol, but not its major digestive product 3,3'-diindolylmethane, induces reversible hepatocyte hypertrophy and cytochromes P450. Toxicol Appl Pharmacol. 2006; 211(2):115-123.

http://dx.doi.org/10.1016/j.taap.2005.06.011

21. Leibelt DA, Hedstrom OR, Fischer KA, Pereira CB, Williams DE. Evaluation of chronic dietary exposure to indole-3-carbinol and absorption-enhanced 3,3'-diindolylmethane in Sprague-Dawley rats. Toxicol Sci. 2003; 74(1):10-21. http://dx.doi.org/10.1093/toxsci/kfg103

22. Johansson G, Gillner M, Hogberg B, Gustafsson J. The TCDD receptor in rat intestinal mucosa and its possible dietary ligands. Nutr Cancer. 2009; 3(3):134-144. http://dx.doi.org/10.1080/01635588109513715

23. Bjeldanes LF, Kim JY, Grose KR, Bartholomew JC, Bradfield CA. Aromatic hydrocarbon responsiveness-receptor agonists generated from indole-3-carbinol in vitro and in vivo: Comparisons with 2,3,7,8-tetrachlorodibenzo-p-dioxin. Proc Natl Acad Sci USA. 1991; 88(21):9543. http://dx.doi.org/10.1073/pnas.88.21.9543

24. Jellinck PH, Forkert PG, Riddick DS, Okey AB, Michnovicz JJ, Bradlow HL. Ah receptor binding properties of indole carbinols and induction of hepatic estradiol hydroxylation. Biochem Pharmacol. 1993; 45(5):1129-1136. http://dx.doi.org/10.1016/0006-2952(93)90258-X

25. Nishie K, Daxenbichler ME. Toxicology of glucosinolates, related compounds (nitriles, Rgoitrin, isothiocyanates) and vitamin U found in cruciferae. Food Cosmet Toxicol. 1980; 18(2):159-172. http://dx.doi.org/10.1016/0015-6264(80)90070-X 
26. Y.C. Wong G, Bradlow L, Sepkovic D, Mehl S, Mailman J, P. Osborne M. Dose-ranging study of Indole-3-Carbinol for breast cancer prevention. J Cell Biochem Suppl. 1997; 28-29:111-116.

27. Bell MC, Crowley-Nowick P, Bradlow HL, Sepkovic DW, Schmidt-Grimminger D, Howell P, Mayeaux EJ, Tucker A, Turbat-Herrera EA, Mathis JM. Placebo-controlled trial of indole-3carbinol in the treatment of CIN. Gynecol Oncol. 2000; 78(2):123-129. http://dx.doi.org/10.1006/gyno.2000.5847

28. McAlindon TE, Gulin J, Chen T, Klug T, Lahita R, Nuite M. Indole-3-carbinol in women with SLE: Effect on estrogen metabolism and disease activity. LUPUS. 2001; 10(11):779-783. http://dx.doi.org/10.1177/096120330101001104

29. Wilker C, Johnson L, Safe S. Effects of developmental exposure to indole-3-carbinol or 2,3,7,8-tetrachlorodibenzo-p-dioxin on reproductive potential of male rat offspring. Toxicol Appl Pharmacol. 1996; 141(1):68-75. http://dx.doi.org/10.1016/S0041-008X(96)80010-X

30. Pence BC, Buddingh F, Yang SP. Multiple dietary factors in the enhancement of dimethylhydrazine carcinogenesis: Main effect of indole-3-carbinol2. J Natl Cancer Inst. 1986; 77(1):269-276.

31. Dashwood RH. Indole-3-carbinol: Anticarcinogen or tumor promoter in brassica vegetables? Chem Biol Interact. 1998; 110(1):1-5. http://dx.doi.org/10.1016/S0009-2797(97)00115-4

32. Bailey GS, Hendricks JD, Shelton DW, Nixon JE, Pawlowski NE. Enhancement of carcinogenesis by the natural anticarcinogen indole-3-carbinol. J Natl Cancer Inst. 1987; 78(5):931-934.

33. Kim DJ, Han BS, Ahn B, Hasegawa R, Shirai T, Ito N, Tsuda H. Enhancement by indole-3carbinol of liver and thyroid gland neoplastic development in a rat medium-term multiorgan carcinogenesis model. Carcinogenesis. 1997; 18(2):377-381. http://dx.doi.org/10.1093/carcin/18.2.377

34. Wattenberg LW, Loub WD. Inhibition of polycyclic aromatic hydrocarbon-induced neoplasia by naturally occurring indoles. Cancer Res. 1978; 38(5):1410.

35. Tanaka T, Kojima T, Morishita Y, Mori H. Inhibitory effects of the natural products indole3-carbinol and sinigrin during initiation and promotion phases of 4-nitroquinoline 1-oxideinduced rat tongue carcinogenesis. Jpn J Cancer Res. 1992; 83(8):835-842.

http://dx.doi.org/10.1111/j.1349-7006.1992.tb01988.x

36. Grubbs C, Steele V, Casebolt T, M Juliana M, Eto I, M Whitaker L, H Dragnev K, J Kelloff G, L Lubet R. Chemoprevention of chemically-induced carcinogenesis by indole-3-carrbinol. Anticancer Res. 1995; 15:709-716.

37. Kojima T, Tanaka T, Mori H. Chemoprevention of spontaneous endometrial cancer in female Donryu rats by dietary indole-3-carbinol. Cancer Res. 1994; 54(6):1446.

38. Bradlow HL, Michnovicz JJ, Telang NT, Osborne MP. Effects of dietary indole-3-carbinol on estradiol metabolism and spontaneous mammary tumors in mice. Carcinogenesis. 1991; 12(9):1571-1574. http://dx.doi.org/10.1093/carcin/12.9.1571 
39. Malloy V, Bradlow L, Orentreich N. Interaction between a semisynthetic diet and indole-3carbinol on mammary tumor incidence in Balb/cfC3H mice. Anticancer Res. 1997; 17:43334337.

40. Kim DJ, Lee KK, Han BS, Ahn B, Bae JH, Jang JJ. Biphasic modifying effect of indole-3carbinol on diethylnitrosamine-induced preneoplastic glutathione S-transferase placental formpositive liver cell foci in Sprague-Dawley rats. Jpn J Cancer Res. 1994; 85(6):578-583. http://dx.doi.org/10.1111/j.1349-7006.1994.tb02399.x

41. Oganesian A, Hendricks JD, Williams DE. Long term dietary indole-3-carbinol inhibits diethylnitrosamine-initiated hepatocarcinogenesis in the infant mouse model. Cancer Lett. 1997; 118(1):87-94. http://dx.doi.org/10.1016/S0304-3835(97)00235-8

42. Morse MA, LaGreca SD, Amin SG, Chung F-L. Effects of indole-3-carbinol on lung tumorigenesis and DNA methylation induced by 4-(methylnitrosamino)-1-(3-pyridyl)-1butanone (NNK) and on the metabolism and disposition of NNK in A/J mice. Cancer Res. 1990; 50(9):2613.

43. Chung FL, Morse MA, Eklind KI, Xu Y. Inhibition of the tobacco-specific nitrosamineinduced lung tumorigenesis by compounds derived from cruciferous vegetables and green tea. Ann NY Acad Sci. 1993; 686(1):186-201. http://dx.doi.org/10.1111/j.1749-6632.1993.tb39174.x

44. Stoner GD, Adam-Rodwell G, Morse MA. Lung tumors in strain A mice: Application for studies in cancer chemoprevention. J Cell Biochem Suppl. 1993; 17F:95-103. http://dx.doi.org/10.1002/jcb.240531014

45. El-Bayoumy K, Upadhyaya P, Desai DH, Amin S, Hoffmann D, Wynder EL. Effects of 1, 4phenylenebis (methylene) selenocyanate, phenethyl isothiocyanate, indole-3-carbinol, and dlimonene individually and in combination on the tumorigenicity of the tobacco-specific nitrosamine 4-(methylnitrosamino)-1-(3-pyridyl)-1-butanone in A/J mouse lung. Anticancer Res. 1996; 16(5A):2709-2712.

46. Jain MG, Hislop GT, Howe GR, Ghadirian P. Plant foods, antioxidants, and prostate cancer risk: Findings from case-control studies in Canada. Nutr Cancer. 1999; 34(2):173-184. http://dx.doi.org/10.1207/S15327914NC3402_8

47. Kolonel LN, Hankin JH, Whittemore AS, Wu AH, Gallagher RP, Wilkens LR, John EM, Howe GR, Dreon DM, West DW et al. Vegetables, fruits, legumes and prostate cancer: A multiethnic case-control study. Cancer Epidemiol Biomarkers Prev. 2000; 9(8):795-804.

48. Voorrips LE, Goldbohm RA, van Poppel G, Sturmans F, Hermus RJJ, van den Brandt PA. Vegetable and fruit consumption and risks of colon and rectal cancer in a prospective cohort study: The Netherlands Cohort Study on Diet and Cancer. Am J Epidemiol. 2000; 152(11):10811092. http://dx.doi.org/10.1093/aje/152.11.1081

49. Terry P, Wolk A, Persson I, Magnusson C. Brassica vegetables and breast cancer risk. JAMA. 2001; 285(23):2975-2977. http://dx.doi.org/10.1001/jama.285.23.2975

50. Young TB, Wolf DA. Case-control study of proximal and distal colon cancer and diet in Wisconsin. Int J Cancer. 1988; 42(2):167-175. http://dx.doi.org/10.1002/ijc.2910420205 
51. Arnao M, Sanchez-Bravo J, Acosta M. Indole-3-carbinol as a scavenger of free radicals. Biochem Mol Biol Int. 1996; 39:1125-1134. http://dx.doi.org/10.1080/15216549600201302

52. Kelloff GJ, Boone CW, Crowell JA, Steele VE, Lubet RA, Doody LA, Malone WF, Hawk ET, Sigman CC. New agents for cancer chemoprevention. J Cell Biochem Suppl. 1996; 63(S26):1-28. https://doi.org/10.1002/jcb.240630703

53. Keck A-S, Finley JW. Cruciferous vegetables: Cancer protective mechanisms of glucosinolate hydrolysis products and selenium. Integr Cancer Ther. 2004; 3(1):5-12. http://dx.doi.org/10.1177/1534735403261831

54. Kelloff GJ, Crowell JA, Hawk ET, Steele VE, Lubet RA, Boone CW, Covey JM, Doody LA, Omenn GS, Greenwald P et al. Strategy and planning for chemopreventive drug development: Clinical development plans II. J Cell Biochem Suppl. 1996; 63(S26):54-71. http://dx.doi.org/10.1002/jcb.240630705

55. Rosen CA, Woodson GE, Thompson JW, Hengesteg AP, Bradlow HL. Preliminary results of the use of indole-3-carbinol for recurrent respiratory papillomatosis. Otolaryngol Head Neck Surg. 1998; 118(6):810-815. http://dx.doi.org/10.1016/S0194-5998(98)70274-8

56. Rosen CA, Bryson PC. Indole-3-Carbinol for recurrent respiratory papillomatosis: Long-term results. J Voice. 2004; 18(2):248-253. http://dx.doi.org/10.1016/j.jvoice.2003.05.005

57. Reed GA, Peterson KS, Smith HJ, Gray JC, Sullivan DK, Mayo MS, Crowell JA, Hurwitz A. A phase I study of indole-3-carbinol in women: Tolerability and effects. Cancer Epidemiol Biomarkers Prev. 2005; 14(8):1953-1960. http://dx.doi.org/10.1158/1055-9965.EPI-05-0121

58. Naik R, Nixon S, Lopes A, Godfrey K, Hatem MH, Monaghan JM. A randomized phase II trial of indole-3-carbinol in the treatment of vulvar intraepithelial neoplasia. Int J Gynecol Cancer. 2006; 16(2):786-790. http://dx.doi.org/10.1111/j.1525-1438.2006.00386.x

59. Doppalapudi RS, Riccio ES, Rausch LL, Shimon JA, Lee PS, Mortelmans KE, Kapetanovic IM, Crowell JA, Mirsalis JC. Evaluation of chemopreventive agents for genotoxic activity. Mutat Res. 2007; 629(2):148-160. http://dx.doi.org/10.1016/j.mrgentox.2007.02.004

60. Kassie F, Parzefall W, Musk S, Johnson I, Lamprecht G, Sontag G, Knasmuller S. Genotoxic effects of crude juices from Brassica vegetables and juices and extracts from phytopharmaceutical preparations and spices of cruciferous plants origin in bacterial and mammalian cells. Chem Biol Interact. 1996; 102(1):1-16. http://dx.doi.org/10.1016/00092797(96)03728-3

61. Ochiai M, Wakabayashi K, Sugimura T, Nagao M. Mutagenicities of indole and 30 derivatives after nitrite treatment. Mutat Res. 1986; 172(3):189-197.

http://dx.doi.org/10.1016/0165-1218(86)90056-X

62. Tiedink HGM, Davies JAR, van Broekhoven LW, van der Kamp HJ, Jongen WMF. Formation of mutagenic N-nitroso compounds in vegetable extracts upon nitrite treatment: A comparison with the glucosinolate content. Food Chem Toxicol. 1988; 26(11):947-954. http://dx.doi.org/10.1016/0278-6915(88)90093-2 
63. Tiedink HG, Davies JA, Jongen WM, van Broekhoven LW. Stability of mutagenic nitrosated products of indole compounds occurring in vegetables. IARC Sci Publ. 1991; 105:584-587.

64. Sasagawa C, Matsushima T. Mutagen formation on nitrite treatment of indole compounds derived from indole-glucosinolate. Mutat Res. 1991; 250(1):169-174. http://dx.doi.org/10.1016/0027-5107(91)90173-L

65. Agrawal RC, Kumar S. Prevention of chromosomal aberration in mouse bone marrow by indole-3-carbinol. Toxicol Lett. 1999; 106(2):137-141. http://dx.doi.org/10.1016/S03784274(99)00060-0

66. Shukla Y, Arora A, Taneja P. Antigenotoxic potential of certain dietary constituents. Teratog Carcinog Mutagen. 2003; 23(S1):323-335. http://dx.doi.org/10.1002/tcm.10059

67. Schut HAJ, Dashwood RH. Inhibition of DNA adduct formation of 2-amino-1-methyl-6phenylimidazo[4,5-b]pyridine (PhIP) by dietary indole-3-carbinol (I3C) in the mammary gland, colon, and liver of the female F-344 rat. Ann N Y Acad Sci. 1995; 768(1):210-214. http://dx.doi.org/10.1111/j.1749-6632.1995.tb12124.x

68. Xu M, Bailey AC, Hernaez JF, Taoka CR, Schut HAJ, Dashwood RH. Protection by green tea, black tea, and indole-3-carbinol against 2 amino-3-methylimidazo[4,5-f]quinolone induced DNA adducts and colonic aberrant crypts in the F344 rat. Carcinogenesis. 1996; 17(7):14291434. http://dx.doi.org/10.1093/carcin/17.7.1429

69. Xu M, Schut HA, Bjeldanes LF, Williams DE, Bailey GS, Dashwood RH. Inhibition of 2amino-3-methylimidazo[4,5-f] quinoline-DNA adducts by indole-3-carbinol: Dose-response studies in the rat colon. Carcinogenesis. 1997; 18(11):2149-2153. http://dx.doi.org/10.1093/carcin/18.11.2149

70. Schut HAJ, Cummings DA, Smale MHE, Josyula S, Friesen MD. DNA adducts of heterocyclic amines: Formation, removal and inhibition by dietary components. Mutat Res. 1997; 376(1):185-194. http://dx.doi.org/10.1016/S0027-5107(97)00042-0

71. Arif JM, Gairola CG, Kelloff GJ, Lubet RA, Gupta RC. Inhibition of cigarette smoke-related DNA adducts in rat tissues by indole-3-carbinol. Mutat Res. 2000; 452(1):11-18. http://dx.doi.org/10.1016/S0027-5107(00)00010-5

72. Gross-Steinmeyer K, Stapleton PL, Tracy JH, Bammler TK, Strom SC, Buhler DR, Eaton DL. Modulation of aflatoxin B1-mediated genotoxicity in primary cultures of human hepatocytes by diindolylmethane, curcumin, and xanthohumols. Toxicol Sci. 2009; 112(2):303-310. http://dx.doi.org/10.1093/toxsci/kfp206

73. Kociba RJ, Keyes DG, Beyer JE, Carreon RM, Wade CE, Dittenber DA, Kalnins RP, Frauson LE, Park CN, Barnard SD et al. Results of a two-year chronic toxicity and oncogenicity study of 2,3,7,8-tetrachlorodibenzo-p-dioxin in rats. Toxicol Appl Pharmacol. 1978; 46(2):279303. http://dx.doi.org/10.1016/0041-008X(78)90075-3

74. National Toxicology Program (NTP). Toxicology and carcinogenesis studies of 3,3',4,4',5pentachlorobiphenyl (PCB 126) (CASRN 57465-28-8) in female Harlan Sprague-Dawley rats (gavage studies). Research Triangle Park, NC: US Departments of Health and Human Services, 
Public Health Service, National Institutes of Health; 2006. Technical Report Series No. 520. NIH Publication No. 06-4454.

75. National Toxicology Program (NTP). Toxicology and carcinogenesis studies of 2,3,7,8tetrachlorodibenzo-p-dioxin (TCDD) (CASRN 1746-01-6) in female Harlan Sprague-Dawley rats (gavage studies). Research Triangle Park, NC: US Departments of Health and Human Services, Public Health Service, National Institutes of Health; 2006. Technical Report Series No. 521. NIH Publication No. 06-4455.

76. National Toxicology Program (NTP). Toxicology and carcinogenesis studies of 2,3,4,7,8pentachlorodibenzofuran (PeCDF) (CASRN 57117-31-4) in female Harlan Sprague-Dawley rats (gavage studies). Research Triangle Park, NC: US Departments of Health and Human Services, Public Health Service, National Institutes of Health; 2006. Technical Report Series No. 525. NIH Publication No. 06-4461.

77. National Toxicology Program (NTP). Toxicology and carcinogenesis studies of a mixture of TCDD, PeCDF, and PCB 126 (CASRNs 1746-01-6, 57117-31-4, and 57465-28-8) in female Harlan Sprague-Dawley rats (gavage studies). Research Triangle Park, NC: US Departments of Health and Human Services, Public Health Service, National Institutes of Health; 2006. Technical Report Series No. 526. NIH Publication No. 06-4462.

78. National Toxicology Program (NTP). Toxicology and carcinogenesis studies of 2,2',4,4',5,5'hexachlorobiphenyl (PCB 153) (CASRN 35065-27-1) in female Harlan Sprague-Dawley rats (gavage studies). Research Triangle Park, NC: US Departments of Health and Human Services, Public Health Service, National Institutes of Health; 2006. Technical Report Series No. 529. NIH Publication No. 06-4465.

79. National Toxicology Program (NTP). Toxicology and carcinogenesis studies of a binary mixture of PCB 126 and PCB 153 (CASRN 57465-28-8 and 35065-27-1) in female Harlan Sprague-Dawley rats (gavage studies). Research Triangle Park, NC: US Departments of Health and Human Services, Public Health Service, National Institutes of Health; 2006. Technical Report Series No. 530. NIH Publication No. 06-4466.

80. National Toxicology Program (NTP). Toxicology and carcinogenesis studies of a binary mixture of PCB 126 and PCB 118 (CASRN 57465-28-8 and 31508-00-6) in female Harlan Sprague-Dawley rats (gavage studies). Research Triangle Park, NC: US Departments of Health and Human Services, Public Health Service, National Institutes of Health; 2006. Technical Report Series No. 531. NIH Publication No. 07-4467.

81. Schenkman JB, Cinti DL. Preparation of microsomes with calcium. Methods Enzymol. 1978; 52:83-89. http://dx.doi.org/10.1016/S0076-6879(78)52008-9

82. Lowry OH, Rosebrough NJ, Farr AL, Randall RJ. Protein measurement with Folin phenol reagent. J Biol Chem. 1951; 193(1):265-275.

83. Chang TK, Waxman DJ. Enzymatic analysis of cDNA-expressed human CYP1A1, CYP1A2, and CYP1B1 with 7-ethoxyresorufin as substrate. Methods Mol Biol. 1998; 107:103-109. 
84. Hamm JT, Ross DG, Richardson VM, Diliberto JJ, Birnbaum LS. Methoxyresorufin: An inappropriate substrate for CYP1A2 in the mouse. Biochem Pharmacol. 1998; 56(12):16571660. http://dx.doi.org/10.1016/S0006-2952(98)00241-X

85. Maronpot RR, Boorman GA. Interpretation of rodent hepatocellular proliferative alterations and hepatocellular tumors in chemical safety assessment. Toxicol Pathol. 1982; 10(2):71-78. http://dx.doi.org/10.1177/019262338201000210

86. Boorman GA, Montgomery CA, Jr., Eustis SL, Wolfe MJ, McConnell EE, Hardisty JF. Quality assurance in pathology for rodent carcinogenicity studies In: Milman HA, Weisburger EK, editors. Handbook of Carcinogen Testing. Park Ridge, NJ: Noyes Publications; 1985. p. 345-357.

87. Boyle MC, Crabbs TA, Wyde ME, Painter JT, Hill GD, Malarkey DE, Lieuallen WG, Nyska A. Intestinal lymphangiectasis and lipidosis in rats following subchronic exposure to indole-3carbinol via oral gavage. Toxicol Pathol. 2012; 40(4):561-576.

http://dx.doi.org/10.1177/0192623311436178

88. McConnell EE, Solleveld HA, Swenberg JA, Boorman GA. Guidelines for combining neoplasms for evaluation of rodent carcinogenesis studies. J Natl Cancer Inst. 1986; 76(2):283-289.

89. Kaplan EL, Meier P. Nonparametric estimation from incomplete observations. J Am Stat Assoc. 1958;53:457-481. http://dx.doi.org/10.1080/01621459.1958.10501452

90. Cox DR. Regression models and life-tables. J R Stat Soc. 1972; B34:187-220.

91. Tarone RE. Tests for trend in life table analysis. Biometrika. 1975; 62(3):679-690. http://dx.doi.org/10.1093/biomet/62.3.679

92. Bailer AJ, Portier CJ. Effects of treatment-induced mortality and tumor-induced mortality on tests for carcinogenicity in small samples. Biometrics. 1988; 44:417-431. http://dx.doi.org/10.2307/2531856

93. Portier CJ, Bailer AJ. Testing for increased carcinogenicity using a survival-adjusted quantal response test. Fundam Appl Toxicol. 1989; 12(4):731-737. http:// dx.doi.org/10.1016/0272-0590(89)90004-3

94. Piegorsch WW, Bailer AJ. Statistics for environmental biology and toxicology. Section 6.3.2. London: Chapman and Hall; 1997.

95. Portier CJ, Hedges JC, Hoel DG. Age-specific models of mortality and tumor onset for historical control animals in the National Toxicology Program's carcinogenicity experiments. Cancer Res. 1986; 46(9):4372.

96. Bieler GS, Williams RL. Ratio estimates, the delta method, and quantal response tests for increased carcinogenicity. Biometrics. 1993; 49(3):793-801. http://dx.doi.org/10.2307/2532200

97. Dunnett CW. A multiple comparison procedure for comparing several treatments with a control. J Am Stat Assoc. 1955; 50(272):1096-1121.

http://dx.doi.org/10.1080/01621459.1955.10501294 
98. Williams DA. A test for differences between treatment means when several dose levels are compared with a zero dose control. Biometrics. 1971; 27:103-117.

http://dx.doi.org/10.2307/2528930

99. Williams DA. The comparison of several dose levels with a zero dose control. Biometrics. 1972; 28:519-531. http://dx.doi.org/10.2307/2556164

100. Shirley E. A non-parametric equivalent of Williams' test for contrasting increasing dose levels of a treatment. Biometrics. 1977; 33:386-389. http://dx.doi.org/10.2307/2529789

101. Williams DA. A note on Shirley's nonparametric test for comparing several dose levels with a zero-dose control. Biometrics. 1986; 42:183-186. http://dx.doi.org/10.2307/2531254

102. Dunn OJ. Multiple comparison using rank sums. Technometrics. 1964; 6:241-252. http://dx.doi.org/10.1080/00401706.1964.10490181

103. Jonckheere AR. A distribution-free k-sample test against ordered alternatives. Biometrika. 1954; 41(1-2):133-145. http://dx.doi.org/10.1093/biomet/41.1-2.133

104. Dixon WJ, Massey FJ, Jr. Introduction to statistical analysis. 2nd ed. New York: McGrawHIll Book Company, Inc.; 1957. p. 276-278, 412. http://dx.doi.org/10.2307/2332898

105. Gart JJ, Chu KC, Tarone RE. Statistical issues in interpretation of chronic bioassay tests for carcinogenicity. J Natl Cancer Inst. 1979; 62(4):957-974.

106. Girard DM, Sager DB. The use of Markov chains to detect subtle variation in reproductive cycling. Biometrics. 1987; 43:225-234. http://dx.doi.org/10.2307/2531963

107. Haseman JK, Rao GN. Effects of corn oil, time-related changes, and inter-laboratory variability on tumor occurrence in control Fischer 344 (F344/N) rats. Toxicol Pathol. 1992; 20(1):52-60. http://dx.doi.org/10.1177/019262339202000107

108. Haseman JK. Value of historical controls in the interpretation of rodent tumor data. Drug Inf J. 1992; 26(2):191-200. http://dx.doi.org/10.1177/009286159202600210

109. Haseman JK. Data analysis: Statistical analysis and use of historical control data. Regul Toxicol Pharmacol. 1995; 21(1):52-59. http://dx.doi.org/10.1006/rtph.1995.1009

110. Code of Federal Regulations (CFR). 21:Part 58.

111. Schmid W. The micronucleus test. Mutat Res. 1975; 31(1):9-15. http://dx.doi.org/10.1016/0165-1161(75)90058-8

112. Heddle JA, Hite M, Kirkhart B, Mavournin K, MacGregor JT, Newell GW, Salamone MF. The induction of micronuclei as a measure of genotoxicity: A report of the U.S. Environmental Protection Agency Gene-Tox Program. Mutat Res. 1983; 123(1):61-118. http://dx.doi.org/10.1016/0165-1110(83)90047-7

113. Miller JA, Miller EC. Ultimate chemical carcinogens as reactive mutagenic electrophiles In: Hiatt HH, Watson JD, Winsten JA, editors. Origins of Human Cancer. Cold Spring Harbor, NY: Cold Spring Harbor Laboratory; 1977. p. 605-627. 
114. Straus DS. Somatic mutation, cellular differentiation, and cancer causation. J Natl Cancer Inst. 1981; 67(2):233-241.

115. Crawford BD. Perspectives on the somatic mutation model of carcinogenesis In: Mehlman MA, Flamm WG, Lorentzen RJ, editors. Advances in Modern Environmental Toxicology: Mechanisms and Toxicity of Chemical Carcinogens and Mutagens. Princeton, NJ: Princeton Scientific Publishing Co. Inc.; 1985. p. 13-59.

116. Ashby J, Tennant RW. Definitive relationships among chemical structure, carcinogenicity and mutagenicity for 301 chemicals tested by the U.S. NTP. Mutat Res. 1991; 257(3):229-306. http://dx.doi.org/10.1016/0165-1110(91)90003-E

117. Tennant RW, Margolin BH, Shelby MD, Zeiger E, Haseman JK, Spalding J, Caspary W, Resnick M, Stasiewicz S, Anderson B et al. Prediction of chemical carcinogenicity in rodents from in vitro genetic toxicity assays. Science. 1987; 236(4804):933.

http://dx.doi.org/10.1126/science.3554512

118. Zeiger E, Haseman JK, Shelby MD, Margolin BH, Tennant RW, Holden HE. Evaluation of four in vitro genetic toxicity tests for predicting rodent carcinogenicity: Confirmation of earlier results with 41 additional chemicals. Environ Mol Mutagen. 1990; 16(S18):1-14. http://dx.doi.org/10.1002/em.2850160502

119. Shelby MD, Erexson GL, Hook GJ, Tice RR. Evaluation of a three-exposure mouse bone marrow micronucleus protocol: Results with 49 chemicals. Environ Mol Mutagen. 1993; 21(2):160-179. http://dx.doi.org/10.1002/em.2850210210

120. Shelby MD, Witt KL. Comparison of results from mouse bone marrow chromosome aberration and micronucleus tests. Environ Mol Mutagen. 1995; 25(4):302-313. http://dx.doi.org/10.1002/em.2850250407

121. Witt KL, Knapton A, Wehr CM, Hook GJ, Mirsalis J, Shelby MD, MacGregor JT. Micronucleated erythrocyte frequency in peripheral blood of B6C3F1 mice from short-term, prechronic, and chronic studies of the NTP carcinogenesis bioassay program. Environ Mol Mutagen. 2000; 36(3):163-194. http://dx.doi.org/10.1002/1098-2280(2000)36:3<163::AIDEM1>3.0.CO;2-P

122. Baldwin WS, Leblanc GA. The anti-carcinogenic plant compound indole-3-carbinol differentially modulates P450-mediated steroid hydroxylase activities in mice. Chem Biol Interact. 1992; 83(2):155-169. http://dx.doi.org/10.1016/0009-2797(92)90043-K

123. Horn TL, Reichert MA, Bliss RL, Malejka-Giganti D. Modulations of P450 mRNA in liver and mammary gland and P450 activities and metabolism of estrogen in liver by treatment of rats with indole-3-carbinol. Biochem Pharmacol. 2002; 64(3):393-404. http://dx.doi.org/10.1016/S0006-2952(02)01190-5

124. Szaefer H, Krajka-Kuazniak V, Bartoszek A, Baer-Dubowska W. Modulation of carcinogen metabolizing cytochromes P450 in rat liver and kidney by cabbage and sauerkraut juices: Comparison with the effects of indole-3-carbinol and phenethyl isothiocyanate. Phytother Res. 2012; 26(8):1148-1155. http://dx.doi.org/10.1002/ptr.3692 
125. Sparnins VL, Venegas PL, Wattenberg LW. Glutathione S-transferase activity: Enhancement by compounds inhibiting chemical carcinogenesis and by dietary constituents. J Natl Cancer Inst. 1982; 68:493-496.

126. Bradfield CA, Bjeldanes LF. Effect of dietary indole-3-carbinol on intestinal and hepatic monooxygenase, glutathione S-transferase and epoxide hydrolase activities in the rat. Food Chem Toxicol. 1984; 22(12):977-982. http://dx.doi.org/10.1016/0278-6915(84)90147-9

127. Whitlock Jr JP. Induction of cytochrome P4501A1. Annu Rev Pharmacol Toxicol. 1999; 39(1):103-125. http://dx.doi.org/10.1146/annurev.pharmtox.39.1.103

128. Denison MS, Nagy SR. Activation of the aryl hydrocarbon receptor by structurally diverse exogenous and endogenous chemicals. Annu Rev Pharmacol Toxicol. 2003; 43(1):309-334. http://dx.doi.org/10.1146/annurev.pharmtox.43.100901.135828

129. Mimura J, Fujii-Kuriyama Y. Functional role of AhR in the expression of toxic effects by TCDD. Biochim Biophys Acta. 2003; 1619(3):263-268. http://dx.doi.org/10.1016/S03044165(02)00485-3

130. Hankinson O. Role of coactivators in transcriptional activation by the aryl hydrocarbon receptor. Arch Biochem Biophys. 2005; 433(2):379-386.

http://dx.doi.org/10.1016/j.abb.2004.09.031

131. Whitlock JP. Mechanistic aspects of dioxin action. Chem Res Toxicol. 1993; 6(6):754-763. http://dx.doi.org/10.1021/tx00036a003

132. Van den Berg M, Birnbaum L, Bosveld AT, Brunstram B, Cook P, Feeley M, Giesy JP, Hanberg A, Hasegawa R, Kennedy SW et al. Toxic equivalency factors (TEFs) for PCBs, PCDDs, PCDFs for humans and wildlife. Environ Health Perspect. 1998; 106(12):775-792. http://dx.doi.org/10.1289/ehp.98106775

133. Park JY, Shigenaga MK, Ames BN. Induction of cytochrome P4501A1 by 2,3,7,8tetrachlorodibenzo-p-dioxin or indolo(3,2-b)carbazole is associated with oxidative DNA damage. Proc Natl Acad Sci USA. 1996; 93(6):2322-2327. http://dx.doi.org/10.1073/pnas.93.6.2322

134. National Toxicology Program (NTP). Toxicology and carcinogenesis studies of 2,3',4,4',5pentachlorobiphenyl (PCB 118) (CASRN 31508-00-6) in female Harlan Sprague-Dawley rats (gavage studies). Research Triangle Park, NC: US Departments of Health and Human Services, Public Health Service, National Institutes of Health; 2010. Technical Report Series No. 531. NIH Publication No. 07-4467.

135. Zeiger E, Anderson B, Haworth S, Lawlor T, Mortelmans K. Salmonella mutagenicity tests: V. Results from the testing of 311 chemicals. Environ Mol Mutagen. 1992; 19(S21):2-141. http://dx.doi.org/10.1002/em.2850190603

136. Macgregor JT, Wehr CM, Henika PR, Shelby MD. The in vivo erythrocyte micronucleus test: Measurement at steady state increases assay efficiency and permits integration with toxicity studies. Fundam Appl Toxicol. 1990; 14(3):513-522. http://dx.doi.org/10.1016/0272$\underline{\text { 0590(90)90255-I }}$ 
137. Hwu JR, Wein YS, Leu Y-J. Calcium metal in liquid ammonia for selective reduction of organic compounds. J Org Chem. 1996; 61(4):1493-1499. http://dx.doi.org/10.1021/jo951219c

138. Pouchert CJ, ed. The Aldrich Library of FT-IR Spectra, 2nd ed. Vol. 3, spectrum 3.3577C. Milwaukee, WI: Aldrich Chemical Company; 1997.

139. Irizarry RA, Bolstad BM, Collin F, Cope LM, Hobbs B, Speed TP. Summaries of Affymetrix GeneChip probe level data. Nucleic Acids Res. 2003; 31(4):e15. http://dx.doi.org/10.1093/nar/gng015

140. Omiecinski CJ, Vanden Heuvel JP, Perdew GH, Peters JM. Xenobiotic metabolism, disposition, and regulation by receptors: From biochemical phenomenon to predictors of major toxicities. Toxicol Sci. 2011; 120 Suppl 1:S49-75. http://dx.doi.org/10.1093/toxsci/kfq338

141. Dhakshinamoorthy S, Jaiswal AK. Small maf (MafG and MafK) proteins negatively regulate antioxidant response element-mediated expression and antioxidant induction of the NAD(P)H:Quinone oxidoreductase1 gene. J Biol Chem. 2000; 275(51):40134-40141. http://dx.doi.org/10.1074/jbc.M003531200

142. Kensler TW, Curphey TJ, Maxiutenko Y, Roebuck BD. Chemoprotection by organosulfur inducers of phase 2 enzymes: Dithiolethiones and dithiins. Drug Metabol Drug Interact. 2000; 17(1-4):3-22. http://dx.doi.org/10.1515/DMDI.2000.17.1-4.3

143. Kong AN, Yu R, Hebbar V, Chen C, Owuor E, Hu R, Ee R, Mandlekar S. Signal transduction events elicited by cancer prevention compounds. Mutat Res. 2001; 480-481:231241. http://dx.doi.org/10.1016/S0027-5107(01)00182-8

144. Kwak MK, Itoh K, Yamamoto M, Sutter TR, Kensler TW. Role of transcription factor Nrf2 in the induction of hepatic phase 2 and antioxidative enzymes in vivo by the cancer chemoprotective agent, 3H-1, 2-dimethiole-3-thione. Mol Med. 2001; 7(2):135-145. http://dx.doi.org/10.1007/BF03401947

145. Hernandez JP, Mota LC, Baldwin WS. Activation of CAR and PXR by dietary, environmental and occupational chemicals alters drug metabolism, intermediary metabolism, and cell proliferation. Curr Pharmacogenomics Person Med. 2009; 7(2):81-105. http://dx.doi.org/10.2174/187569209788654005

146. Ma Q. Role of nrf2 in oxidative stress and toxicity. Annu Rev Pharmacol Toxicol. 2013; 53:401-426. http://dx.doi.org/10.1146/annurev-pharmtox-011112-140320

147. Refetoff S. Inherited thyroxine-binding globulin abnormalities in man. Endocr Rev. 1989; 10(3):275-293. http://dx.doi.org/10.1210/edrv-10-3-275

148. Ameri K, Harris AL. Activating transcription factor 4. Int J Biochem Cell Biol. 2008; 40(1):14-21. http://dx.doi.org/10.1016/j.biocel.2007.01.020

149. Polyak K, Xia Y, Zweier JL, Kinzler KW, Vogelstein B. A model for p53-induced apoptosis. Nature. 1997; 389:300. http://dx.doi.org/10.1038/38525

150. Brew CT, Aronchik I, Hsu JC, Sheen JH, Dickson RB, Bjeldanes LF, Firestone GL. Indole3-carbinol activates the ATM signaling pathway independent of DNA damage to stabilize p53 
Indole-3-carbinol, NTP TR 584

and induce G1 arrest of human mammary epithelial cells. Int J Cancer. 2006; 118(4):857-868. http://dx.doi.org/10.1002/ijc.21445 


\section{Appendix A. Summary of Lesions in Male Sprague Dawley Rats in the Two-year Gavage Study of Indole-3-carbinol}

\section{Tables}

Table A-1. Summary of the Incidence of Neoplasms in Male Sprague Dawley Rats in the Two-year Gavage Study of Indole-3-carbinol.

Table A-2. Statistical Analysis of Primary Neoplasms in Male Sprague Dawley Rats in the Two-year Gavage Study of Indole-3-carbinol A-6

Table A-3. Summary of the Incidence of Nonneoplastic Lesions in Male Sprague Dawley Rats in the Two-year Gavage Study of Indole-3-carbinol 
Indole-3-carbinol, NTP TR 584

Table A-1. Summary of the Incidence of Neoplasms in Male Sprague Dawley Rats in the Two-year Gavage Study of Indole-3-carbinol ${ }^{\mathrm{a}}$

\begin{tabular}{|c|c|c|c|c|}
\hline & Vehicle Control & $75 \mathrm{mg} / \mathrm{kg}$ & $150 \mathrm{mg} / \mathrm{kg}$ & $300 \mathrm{mg} / \mathrm{kg}$ \\
\hline \multicolumn{5}{|l|}{ Disposition Summary } \\
\hline Animals Initially in Study & 50 & 50 & 50 & 50 \\
\hline \multicolumn{5}{|l|}{ Early deaths } \\
\hline Accidental death & - & - & - & 1 \\
\hline Moribund & 14 & 23 & 15 & 20 \\
\hline Natural deaths & 16 & 14 & 18 & 17 \\
\hline \multicolumn{5}{|l|}{ Survivors } \\
\hline Terminal euthanasia & 20 & 13 & 17 & 12 \\
\hline Animals examined microscopically & 50 & 50 & 50 & 50 \\
\hline \multicolumn{5}{|l|}{ Alimentary System } \\
\hline Esophagus & $(50)$ & $(50)$ & $(50)$ & $(50)$ \\
\hline Intestine large, cecum & $(50)$ & (50) & $(50)$ & $(50)$ \\
\hline Lipoma & - & $1(2 \%)$ & - & - \\
\hline Intestine large, colon & $(50)$ & $(50)$ & $(50)$ & $(50)$ \\
\hline Intestine large, rectum & $(50)$ & (50) & (50) & (50) \\
\hline Intestine small, duodenum & (43) & $(48)$ & $(47)$ & $(48)$ \\
\hline Carcinoma & - & $1(2 \%)$ & - & - \\
\hline Intestine small, ileum & (35) & $(43)$ & $(42)$ & (38) \\
\hline Intestine small, jejunum & $(40)$ & (39) & $(40)$ & $(42)$ \\
\hline Carcinoma & - & - & - & $1(2 \%)$ \\
\hline Liver & $(50)$ & $(50)$ & (50) & $(50)$ \\
\hline Carcinoma, metastatic, pancreas & - & - & - & $1(2 \%)$ \\
\hline Hepatocellular adenoma & $1(2 \%)$ & - & - & - \\
\hline Hepatocellular carcinoma & - & - & - & $1(2 \%)$ \\
\hline Mesentery & $(2)$ & (2) & (1) & $(0)$ \\
\hline Fat, sarcoma & - & - & $1(100 \%)$ & - \\
\hline Oral mucosa & $(50)$ & $(50)$ & $(50)$ & $(50)$ \\
\hline Squamous cell carcinoma & $1(2 \%)$ & - & $1(2 \%)$ & $1(2 \%)$ \\
\hline Pancreas & $(50)$ & (50) & $(50)$ & (49) \\
\hline Carcinoma & - & - & - & $1(2 \%)$ \\
\hline Mixed tumor malignant & - & - & $1(2 \%)$ & - \\
\hline Acinus, adenoma & $4(8 \%)$ & $1(2 \%)$ & $4(8 \%)$ & $4(8 \%)$ \\
\hline Acinus, adenoma, multiple & $1(2 \%)$ & $3(6 \%)$ & - & $1(2 \%)$ \\
\hline Salivary glands & $(50)$ & $(50)$ & (49) & $(50)$ \\
\hline
\end{tabular}


Indole-3-carbinol, NTP TR 584

\begin{tabular}{|c|c|c|c|c|}
\hline & Vehicle Control & 75 mg/kg & $150 \mathrm{mg} / \mathrm{kg}$ & $300 \mathrm{mg} / \mathrm{kg}$ \\
\hline Schwannoma malignant & $1(2 \%)$ & - & - & $1(2 \%)$ \\
\hline Stomach, forestomach & (50) & (50) & (50) & $(50)$ \\
\hline Squamous cell carcinoma & $1(2 \%)$ & - & $1(2 \%)$ & - \\
\hline Squamous cell papilloma & - & - & $1(2 \%)$ & - \\
\hline Stomach, glandular & (50) & $(50)$ & $(50)$ & (50) \\
\hline Tongue & (0) & $(0)$ & $(0)$ & (1) \\
\hline Squamous cell papilloma & - & - & - & $1(100 \%)$ \\
\hline Tooth & (1) & $(0)$ & (1) & (0) \\
\hline \multicolumn{5}{|l|}{ Cardiovascular System } \\
\hline Blood vessel & (50) & (50) & (50) & (50) \\
\hline $\begin{array}{l}\text { Aorta, fibrous histiocytoma, } \\
\text { metastatic, skin }\end{array}$ & - & - & - & $1(2 \%)$ \\
\hline Heart & $(50)$ & (50) & (50) & (50) \\
\hline Schwannoma malignant & $1(2 \%)$ & $1(2 \%)$ & - & - \\
\hline \multicolumn{5}{|l|}{ Endocrine System } \\
\hline Adrenal cortex & $(50)$ & (49) & $(50)$ & (50) \\
\hline Adenoma & - & $1(2 \%)$ & - & - \\
\hline Carcinoma & - & $1(2 \%)$ & - & $1(2 \%)$ \\
\hline Adrenal medulla & $(50)$ & (49) & (50) & $(50)$ \\
\hline Pheochromocytoma benign & $5(10 \%)$ & $5(10 \%)$ & $4(8 \%)$ & $3(6 \%)$ \\
\hline Pheochromocytoma complex & $1(2 \%)$ & - & - & - \\
\hline Pheochromocytoma malignant & $2(4 \%)$ & $1(2 \%)$ & $1(2 \%)$ & - \\
\hline Bilateral, pheochromocytoma benign & - & - & $1(2 \%)$ & $1(2 \%)$ \\
\hline Islets, pancreatic & $(50)$ & $(50)$ & $(50)$ & $(50)$ \\
\hline Adenoma & $2(4 \%)$ & $1(2 \%)$ & $1(2 \%)$ & - \\
\hline Parathyroid gland & (43) & (46) & $(50)$ & $(47)$ \\
\hline Carcinoma & - & $1(2 \%)$ & - & - \\
\hline Pituitary gland & $(50)$ & $(49)$ & $(50)$ & $(47)$ \\
\hline Pars distalis, adenoma & $5(10 \%)$ & $8(16 \%)$ & $9(18 \%)$ & $8(17 \%)$ \\
\hline Pars distalis, carcinoma & - & $1(2 \%)$ & - & - \\
\hline Thyroid gland & $(50)$ & $(46)$ & $(48)$ & $(47)$ \\
\hline Bilateral, c-cell, adenoma & $2(4 \%)$ & - & $1(2 \%)$ & - \\
\hline C-cell, adenoma & $11(22 \%)$ & $9(20 \%)$ & $8(17 \%)$ & $4(9 \%)$ \\
\hline C-cell, carcinoma & - & $1(2 \%)$ & $1(2 \%)$ & - \\
\hline Follicular cell, adenoma & - & $1(2 \%)$ & - & - \\
\hline
\end{tabular}


Indole-3-carbinol, NTP TR 584

\begin{tabular}{|c|c|c|c|c|}
\hline & Vehicle Control & $75 \mathrm{mg} / \mathrm{kg}$ & $150 \mathrm{mg} / \mathrm{kg}$ & $300 \mathrm{mg} / \mathrm{kg}$ \\
\hline \multicolumn{5}{|l|}{ General Body System } \\
\hline None & - & - & - & - \\
\hline \multicolumn{5}{|l|}{ Genital System } \\
\hline Epididymis & $(50)$ & $(50)$ & (50) & (50) \\
\hline Preputial gland & (50) & $(50)$ & (49) & (50) \\
\hline Prostate & (50) & $(50)$ & (50) & $(50)$ \\
\hline Seminal vesicle & (49) & $(50)$ & (50) & (50) \\
\hline Testes & (49) & (50) & (50) & (50) \\
\hline Interstitial cell, adenoma & $3(6 \%)$ & $2(4 \%)$ & $2(4 \%)$ & $2(4 \%)$ \\
\hline \multicolumn{5}{|l|}{ Hematopoietic System } \\
\hline Bone marrow & (50) & $(50)$ & (50) & (50) \\
\hline Lymph node & (4) & (8) & (8) & (6) \\
\hline Lymph node, mandibular & (50) & (50) & (49) & (50) \\
\hline Lymph node, mesenteric & $(50)$ & $(50)$ & (50) & (50) \\
\hline Hemangiosarcoma & $1(2 \%)$ & - & - & $1(2 \%)$ \\
\hline Spleen & (50) & (50) & (50) & (50) \\
\hline Thymus & (46) & (49) & (49) & $(47)$ \\
\hline Neural crest tumor & - & - & - & $1(2 \%)$ \\
\hline Thymoma benign & $1(2 \%)$ & $1(2 \%)$ & - & - \\
\hline \multicolumn{5}{|l|}{ Integumentary System } \\
\hline Mammary gland & (50) & (50) & (50) & $(50)$ \\
\hline Fibroadenoma & $1(2 \%)$ & $1(2 \%)$ & $1(2 \%)$ & - \\
\hline Skin & $(50)$ & $(50)$ & $(50)$ & $(50)$ \\
\hline Basal cell carcinoma & $1(2 \%)$ & $1(2 \%)$ & - & - \\
\hline Keratoacanthoma & $4(8 \%)$ & - & - & - \\
\hline Squamous cell carcinoma & $1(2 \%)$ & - & $1(2 \%)$ & - \\
\hline Squamous cell papilloma & $1(2 \%)$ & - & $1(2 \%)$ & $1(2 \%)$ \\
\hline Sebaceous gland, adenoma & - & $1(2 \%)$ & - & - \\
\hline Subcutaneous tissue, fibroma & $5(10 \%)$ & $2(4 \%)$ & $5(10 \%)$ & $2(4 \%)$ \\
\hline Subcutaneous tissue, fibrosarcoma & $1(2 \%)$ & $2(4 \%)$ & $1(2 \%)$ & $1(2 \%)$ \\
\hline $\begin{array}{l}\text { Subcutaneous tissue, fibrous } \\
\text { histiocytoma }\end{array}$ & $1(2 \%)$ & - & - & $1(2 \%)$ \\
\hline Subcutaneous tissue, sarcoma & - & $2(4 \%)$ & - & - \\
\hline $\begin{array}{l}\text { Subcutaneous tissue, schwannoma } \\
\text { malignant }\end{array}$ & - & - & $1(2 \%)$ & - \\
\hline
\end{tabular}


Indole-3-carbinol, NTP TR 584

\begin{tabular}{|c|c|c|c|c|}
\hline & Vehicle Control & 75 mg/kg & $150 \mathrm{mg} / \mathrm{kg}$ & $300 \mathrm{mg} / \mathrm{kg}$ \\
\hline \multicolumn{5}{|l|}{ Musculoskeletal System } \\
\hline Bone & $(50)$ & $(50)$ & (50) & $(50)$ \\
\hline Vertebra, osteoma & - & - & - & $1(2 \%)$ \\
\hline \multicolumn{5}{|l|}{ Nervous System } \\
\hline Brain & $(50)$ & $(50)$ & (50) & (50) \\
\hline Glioma malignant & - & $1(2 \%)$ & - & - \\
\hline Medulloblastoma & - & - & $1(2 \%)$ & - \\
\hline Meningioma malignant & - & $1(2 \%)$ & - & - \\
\hline Cerebellum, glioma malignant & - & - & - & $1(2 \%)$ \\
\hline Spinal cord & $(0)$ & $(2)$ & (1) & $(0)$ \\
\hline \multicolumn{5}{|l|}{ Respiratory System } \\
\hline Lung & (49) & (50) & (50) & (50) \\
\hline Alveolar/bronchiolar adenoma & - & - & $1(2 \%)$ & - \\
\hline Carcinoma, metastatic, adrenal cortex & - & - & - & $1(2 \%)$ \\
\hline Fibrous histiocytoma, metastatic, skin & - & - & - & $1(2 \%)$ \\
\hline Squamous cell carcinoma & - & $1(2 \%)$ & - & - \\
\hline Nose & (50) & (50) & (50) & (50) \\
\hline Trachea & (50) & (50) & (50) & $(50)$ \\
\hline \multicolumn{5}{|l|}{ Special Senses System } \\
\hline Ear & $(0)$ & $(0)$ & (1) & (0) \\
\hline Eye & (50) & (50) & (49) & (49) \\
\hline Harderian gland & (50) & (50) & (49) & (50) \\
\hline Lacrimal gland & (1) & $(2)$ & (4) & $(0)$ \\
\hline \multicolumn{5}{|l|}{ Urinary System } \\
\hline Kidney & $(50)$ & $(50)$ & (50) & (50) \\
\hline Renal tubule, adenoma & $1(2 \%)$ & - & - & $1(2 \%)$ \\
\hline Urinary bladder & $(50)$ & (50) & (50) & (50) \\
\hline \multicolumn{5}{|l|}{ Systemic Lesions } \\
\hline Multiple organs ${ }^{\mathrm{b}}$ & $(50)$ & (50) & (50) & $(50)$ \\
\hline Leukemia granulocytic & - & - & - & $1(2 \%)$ \\
\hline Lymphoma malignant & - & - & $1(2 \%)$ & $1(2 \%)$ \\
\hline Mesothelioma malignant & $1(2 \%)$ & $1(2 \%)$ & - & - \\
\hline
\end{tabular}


Indole-3-carbinol, NTP TR 584

\begin{tabular}{|c|c|c|c|c|}
\hline & Vehicle Control & $75 \mathrm{mg} / \mathrm{kg}$ & $150 \mathrm{mg} / \mathrm{kg}$ & $300 \mathrm{mg} / \mathrm{kg}$ \\
\hline \multicolumn{5}{|l|}{ Neoplasm Summary } \\
\hline Total animals with primary neoplasms ${ }^{\mathrm{c}}$ & 33 & 32 & 31 & 33 \\
\hline Total primary neoplasms & 60 & 53 & 50 & 42 \\
\hline Total animals with benign neoplasms & 27 & 23 & 26 & 25 \\
\hline Total benign neoplasms & 47 & 37 & 39 & 29 \\
\hline Total animals with malignant neoplasms & 12 & 15 & 10 & 12 \\
\hline Total malignant neoplasms & 13 & 16 & 11 & 12 \\
\hline Total animals with metastatic neoplasms & - & - & - & 3 \\
\hline Total metastatic neoplasms & - & - & - & 4 \\
\hline $\begin{array}{l}\text { Total animals with uncertain neoplasms } \\
\text { _benign or malignant }\end{array}$ & - & - & - & 1 \\
\hline Total uncertain neoplasms & - & - & - & 1 \\
\hline
\end{tabular}

${ }^{a}$ Number of animals examined microscopically at the site and the number of animals with neoplasm.

${ }^{b}$ Number of animals with any tissue examined microscopically.

'Primary neoplasms: all neoplasms except metastatic neoplasms.

Table A-2. Statistical Analysis of Primary Neoplasms in Male Sprague Dawley Rats in the Two-year Gavage Study of Indole-3-carbinol

\begin{tabular}{|c|c|c|c|c|}
\hline & Vehicle Control & $75 \mathrm{mg} / \mathrm{kg}$ & $150 \mathrm{mg} / \mathrm{kg}$ & $300 \mathrm{mg} / \mathrm{kg}$ \\
\hline \multicolumn{5}{|c|}{ Adrenal Medulla: Benign Pheochromocytoma } \\
\hline Overall rate $^{\mathrm{a}}$ & $5 / 50(10 \%)$ & $5 / 49(10 \%)$ & $5 / 50(10 \%)$ & $4 / 50$ (8\%) \\
\hline Adjusted rate ${ }^{\mathrm{b}}$ & $13.0 \%$ & $14.7 \%$ & $13.5 \%$ & $11.7 \%$ \\
\hline Terminal rate ${ }^{c}$ & $3 / 20(15 \%)$ & $2 / 13(15 \%)$ & $2 / 17(12 \%)$ & $3 / 12(25 \%)$ \\
\hline First incidence (days) & 645 & 571 & 593 & 621 \\
\hline Poly-3 test ${ }^{\mathrm{d}}$ & $\mathrm{P}=0.479 \mathrm{~N}$ & $P=0.549$ & $P=0.605$ & $\mathrm{P}=0.577 \mathrm{~N}$ \\
\hline \multicolumn{5}{|c|}{ Adrenal Medulla: Benign, Complex, or Malignant Pheochromocytoma } \\
\hline Overall rate & $8 / 50(16 \%)$ & $6 / 49(12 \%)$ & $6 / 50(12 \%)$ & $4 / 50(8 \%)$ \\
\hline Adjusted rate & $20.5 \%$ & $17.5 \%$ & $16.1 \%$ & $11.7 \%$ \\
\hline Terminal rate & 4/20 (20\%) & 2/13 (15\%) & $2 / 17(12 \%)$ & $3 / 12(25 \%)$ \\
\hline First incidence (days) & 634 & 571 & 593 & 621 \\
\hline Poly-3 test & $\mathrm{P}=0.190 \mathrm{~N}$ & $\mathrm{P}=0.491 \mathrm{~N}$ & $\mathrm{P}=0.422 \mathrm{~N}$ & $\mathrm{P}=0.243 \mathrm{~N}$ \\
\hline \multicolumn{5}{|l|}{ Pancreas: Adenoma } \\
\hline Overall rate & $5 / 50$ (10\%) & $4 / 50(8 \%)$ & $4 / 50(8 \%)$ & $5 / 49(10 \%)$ \\
\hline Adjusted rate & $13.0 \%$ & $11.8 \%$ & $10.9 \%$ & $14.8 \%$ \\
\hline Terminal rate & 3/20 (15\%) & 2/13 (15\%) & $2 / 17(12 \%)$ & $3 / 12(25 \%)$ \\
\hline First incidence (days) & 685 & 648 & 624 & 710 \\
\hline Poly-3 test & $P=0.474$ & $\mathrm{P}=0.579 \mathrm{~N}$ & $\mathrm{P}=0.527 \mathrm{~N}$ & $P=0.549$ \\
\hline
\end{tabular}


Indole-3-carbinol, NTP TR 584

\begin{tabular}{|c|c|c|c|c|}
\hline & Vehicle Control & 75 mg/kg & 150 mg/kg & $300 \mathrm{mg} / \mathrm{kg}$ \\
\hline \multicolumn{5}{|c|}{ Pancreas: Adenoma or Carcinoma } \\
\hline Overall rate & $5 / 50(10 \%)$ & $4 / 50(8 \%)$ & $4 / 50(8 \%)$ & $6 / 49(12 \%)$ \\
\hline Adjusted rate & $13.0 \%$ & $11.8 \%$ & $10.9 \%$ & $17.5 \%$ \\
\hline Terminal rate & $3 / 20(15 \%)$ & 2/13 (15\%) & 2/17 (12\%) & $3 / 12(25 \%)$ \\
\hline First incidence (days) & 685 & 648 & 624 & 589 \\
\hline Poly-3 test & $P=0.337$ & $P=0.579 N$ & $P=0.527 \mathrm{~N}$ & $P=0.418$ \\
\hline \multicolumn{5}{|c|}{ Pituitary Gland (Pars Distalis): Adenoma } \\
\hline Overall rate & $5 / 50(10 \%)$ & $8 / 49(16 \%)$ & $9 / 50(18 \%)$ & $8 / 47(17 \%)$ \\
\hline Adjusted rate & $12.9 \%$ & $23.3 \%$ & $23.4 \%$ & $23.3 \%$ \\
\hline Terminal rate & $2 / 20(10 \%)$ & 3/13 (23\%) & $1 / 17(6 \%)$ & $1 / 12(8 \%)$ \\
\hline First incidence (days) & 645 & 617 & 503 & 589 \\
\hline Poly-3 test & $P=0.191$ & $P=0.193$ & $P=0.180$ & $P=0.193$ \\
\hline \multicolumn{5}{|c|}{ Pituitary Gland (Pars Distalis): Adenoma or Carcinoma } \\
\hline Overall rate & $5 / 50(10 \%)$ & 9/49 (18\%) & $9 / 50(18 \%)$ & $8 / 47(17 \%)$ \\
\hline Adjusted rate & $12.9 \%$ & $25.9 \%$ & $23.4 \%$ & $23.3 \%$ \\
\hline Terminal rate & $2 / 20(10 \%)$ & $3 / 13(23 \%)$ & $1 / 17(6 \%)$ & $1 / 12(8 \%)$ \\
\hline First incidence (days) & 645 & 614 & 503 & 589 \\
\hline Poly-3 test & $P=0.218$ & $P=0.127$ & $P=0.180$ & $P=0.193$ \\
\hline \multicolumn{5}{|c|}{ Skin: Keratoacanthoma } \\
\hline Overall rate & $4 / 50(8 \%)$ & $0 / 50(0 \%)$ & $0 / 50(0 \%)$ & $0 / 50(0 \%)$ \\
\hline Adjusted rate & $10.4 \%$ & $0.0 \%$ & $0.0 \%$ & $0.0 \%$ \\
\hline Terminal rate & $2 / 20(10 \%)$ & $0 / 13(0 \%)$ & $0 / 17(0 \%)$ & $0 / 12(0 \%)$ \\
\hline First incidence (days) & 685 & $-{ }^{\mathrm{e}}$ & - & - \\
\hline Poly-3 test & $P=0.015 \mathrm{~N}$ & $P=0.079 \mathrm{~N}$ & $P=0.066 \mathrm{~N}$ & $\mathrm{P}=0.077 \mathrm{~N}$ \\
\hline \multicolumn{5}{|c|}{ Skin: Squamous Cell Papilloma or Keratoacanthoma } \\
\hline Overall rate & $5 / 50(10 \%)$ & $0 / 50(0 \%)$ & $1 / 50(2 \%)$ & $1 / 50(2 \%)$ \\
\hline Adjusted rate & $13.0 \%$ & $0.0 \%$ & $2.8 \%$ & $2.9 \%$ \\
\hline Terminal rate & $3 / 20(15 \%)$ & $0 / 13(0 \%)$ & $1 / 17(6 \%)$ & $0 / 12(0 \%)$ \\
\hline First incidence (days) & 685 & - & $727(\mathrm{~T})$ & 629 \\
\hline Poly-3 test & $P=0.075 N$ & $P=0.043 \mathrm{~N}$ & $P=0.112 \mathrm{~N}$ & $\mathrm{P}=0.129 \mathrm{~N}$ \\
\hline \multicolumn{5}{|c|}{ Skin: Squamous Cell Papilloma, Keratoacanthoma, or Squamous Cell Carcinoma } \\
\hline Overall rate & $5 / 50(10 \%)$ & $0 / 50(0 \%)$ & $2 / 50(4 \%)$ & $1 / 50(2 \%)$ \\
\hline Adjusted rate & $13.0 \%$ & $0.0 \%$ & $5.4 \%$ & $2.9 \%$ \\
\hline Terminal rate & $3 / 20(15 \%)$ & $0 / 13(0 \%)$ & $1 / 17(6 \%)$ & $0 / 12(0 \%)$ \\
\hline First incidence (days) & 685 & - & 624 & 629 \\
\hline Poly-3 test & $\mathrm{P}=0.104 \mathrm{~N}$ & $P=0.043 N$ & $P=0.233 N$ & $\mathrm{P}=0.129 \mathrm{~N}$ \\
\hline
\end{tabular}


Indole-3-carbinol, NTP TR 584

\begin{tabular}{|c|c|c|c|c|}
\hline & Vehicle Control & $75 \mathrm{mg} / \mathrm{kg}$ & $150 \mathrm{mg} / \mathrm{kg}$ & $300 \mathrm{mg} / \mathrm{kg}$ \\
\hline \multicolumn{5}{|c|}{ Skin: Squamous Cell Papilloma, Keratoacanthoma, Basal Cell Carcinoma, or Squamous Cell Carcinoma } \\
\hline Overall rate & $6 / 50(12 \%)$ & $1 / 50(2 \%)$ & $2 / 50(4 \%)$ & $1 / 50(2 \%)$ \\
\hline Adjusted rate & $15.5 \%$ & $3.0 \%$ & $5.4 \%$ & $2.9 \%$ \\
\hline Terminal rate & $3 / 20(15 \%)$ & $0 / 13(0 \%)$ & $1 / 17(6 \%)$ & $0 / 12(0 \%)$ \\
\hline First incidence (days) & 645 & 637 & 624 & 629 \\
\hline Poly-3 test & $P=0.048 \mathrm{~N}$ & $\mathrm{P}=0.079 \mathrm{~N}$ & $\mathrm{P}=0.147 \mathrm{~N}$ & $\mathrm{P}=0.077 \mathrm{~N}$ \\
\hline \multicolumn{5}{|c|}{ Skin (Subcutaneous Tissue): Fibroma } \\
\hline Overall rate & $5 / 50(10 \%)$ & $2 / 50(4 \%)$ & $5 / 50(10 \%)$ & $2 / 50(4 \%)$ \\
\hline Adjusted rate & $12.6 \%$ & $5.8 \%$ & $13.4 \%$ & $5.7 \%$ \\
\hline Terminal rate & $1 / 20(5 \%)$ & $0 / 13(0 \%)$ & $3 / 17(18 \%)$ & $0 / 12(0 \%)$ \\
\hline First incidence (days) & 575 & 597 & 500 & 401 \\
\hline Poly-3 test & $\mathrm{P}=0.286 \mathrm{~N}$ & $P=0.278 N$ & $P=0.596$ & $\mathrm{P}=0.267 \mathrm{~N}$ \\
\hline \multicolumn{5}{|c|}{ Skin (Subcutaneous Tissue): Fibrous Histiocytoma, Fibrosarcoma, or Sarcoma } \\
\hline Overall rate & $2 / 50(4 \%)$ & $4 / 50(8 \%)$ & $1 / 50(2 \%)$ & $2 / 50(4 \%)$ \\
\hline Adjusted rate & $5.0 \%$ & $11.2 \%$ & $2.7 \%$ & $5.7 \%$ \\
\hline Terminal rate & $0 / 20(0 \%)$ & $1 / 13(8 \%)$ & $0 / 17(0 \%)$ & $0 / 12(0 \%)$ \\
\hline First incidence (days) & 197 & 382 & 423 & 258 \\
\hline Poly-3 test & $\mathrm{P}=0.485 \mathrm{~N}$ & $P=0.286$ & $\mathrm{P}=0.526 \mathrm{~N}$ & $P=0.645$ \\
\hline \multicolumn{5}{|c|}{ Skin (Subcutaneous Tissue): Fibroma, Fibrous Histiocytoma, Fibrosarcoma, or Sarcoma } \\
\hline Overall rate & $7 / 50(14 \%)$ & $6 / 50(12 \%)$ & $6 / 50(12 \%)$ & $4 / 50(8 \%)$ \\
\hline Adjusted rate & $16.9 \%$ & $16.4 \%$ & $15.7 \%$ & $11.1 \%$ \\
\hline Terminal rate & $1 / 20(5 \%)$ & $1 / 13(8 \%)$ & $3 / 17(18 \%)$ & $0 / 12(0 \%)$ \\
\hline First incidence (days) & 197 & 382 & 423 & 258 \\
\hline Poly-3 test & $\mathrm{P}=0.275 \mathrm{~N}$ & $\mathrm{P}=0.597 \mathrm{~N}$ & $\mathrm{P}=0.562 \mathrm{~N}$ & $\mathrm{P}=0.341 \mathrm{~N}$ \\
\hline \multicolumn{5}{|l|}{ Testes: Adenoma } \\
\hline Overall rate & $3 / 49(6 \%)$ & $2 / 50(4 \%)$ & $2 / 50(4 \%)$ & $2 / 50(4 \%)$ \\
\hline Adjusted rate & $7.9 \%$ & $5.9 \%$ & $5.4 \%$ & $5.8 \%$ \\
\hline Terminal rate & $1 / 20(5 \%)$ & $1 / 13(8 \%)$ & $0 / 17(0 \%)$ & $1 / 12(8 \%)$ \\
\hline First incidence (days) & 635 & 671 & 570 & 532 \\
\hline Poly-3 test & $\mathrm{P}=0.446 \mathrm{~N}$ & $\mathrm{P}=0.553 \mathrm{~N}$ & $\mathrm{P}=0.506 \mathrm{~N}$ & $\mathrm{P}=0.543 \mathrm{~N}$ \\
\hline \multicolumn{5}{|c|}{ Thyroid Gland (C-Cell): Adenoma } \\
\hline Overall rate & $13 / 50(26 \%)$ & 9/46 (20\%) & 9/48 (19\%) & $4 / 47(9 \%)$ \\
\hline Adjusted rate & $32.5 \%$ & $27.5 \%$ & $24.2 \%$ & $12.0 \%$ \\
\hline Terminal rate & 7/20 (35\%) & 4/13 (31\%) & $3 / 17(18 \%)$ & $2 / 12(17 \%)$ \\
\hline First incidence (days) & 421 & 456 & 503 & 519 \\
\hline Poly-3 test & $\mathrm{P}=0.024 \mathrm{~N}$ & $\mathrm{P}=0.416 \mathrm{~N}$ & $\mathrm{P}=0.289 \mathrm{~N}$ & $\mathrm{P}=0.033 \mathrm{~N}$ \\
\hline
\end{tabular}


Indole-3-carbinol, NTP TR 584

\begin{tabular}{|c|c|c|c|c|}
\hline & Vehicle Control & 75 mg/kg & $150 \mathrm{mg} / \mathrm{kg}$ & $300 \mathrm{mg} / \mathrm{kg}$ \\
\hline \multicolumn{5}{|c|}{ Thyroid Gland (C-Cell): Adenoma or Carcinoma } \\
\hline Overall rate & $13 / 50(26 \%)$ & $10 / 46(22 \%)$ & 10/48 (21\%) & 4/47 (9\%) \\
\hline Adjusted rate & $32.5 \%$ & $30.2 \%$ & $26.6 \%$ & $12.0 \%$ \\
\hline Terminal rate & $7 / 20(35 \%)$ & $4 / 13(31 \%)$ & $3 / 17(18 \%)$ & $2 / 12(17 \%)$ \\
\hline First incidence (days) & 421 & 456 & 503 & 519 \\
\hline Poly-3 test & $\mathrm{P}=0.025 \mathrm{~N}$ & $P=0.516 \mathrm{~N}$ & $P=0.373 N$ & $P=0.033 N$ \\
\hline \multicolumn{5}{|c|}{ All Organs: Benign Neoplasms } \\
\hline Overall rate & 27/50 (54\%) & $23 / 50$ (46\%) & 26/50 (52\%) & $25 / 50$ (50\%) \\
\hline Adjusted rate & $63.7 \%$ & $58.1 \%$ & $62.3 \%$ & $62.2 \%$ \\
\hline Terminal rate & $12 / 20(60 \%)$ & $6 / 13(46 \%)$ & 10/17 (59\%) & $8 / 12(67 \%)$ \\
\hline First incidence (days) & 421 & 382 & 482 & 258 \\
\hline Poly-3 test & $P=0.544$ & $P=0.378 N$ & $\mathrm{P}=0.540 \mathrm{~N}$ & $\mathrm{P}=0.537 \mathrm{~N}$ \\
\hline \multicolumn{5}{|c|}{ All Organs: Malignant Neoplasms } \\
\hline Overall rate & $12 / 50(24 \%)$ & $15 / 50(30 \%)$ & $10 / 50(20 \%)$ & $12 / 50(24 \%)$ \\
\hline Adjusted rate & $29.3 \%$ & $37.1 \%$ & $25.2 \%$ & $30.8 \%$ \\
\hline Terminal rate & $5 / 20(25 \%)$ & $2 / 13(15 \%)$ & $1 / 17(6 \%)$ & $2 / 12(17 \%)$ \\
\hline First incidence (days) & 197 & 357 & 423 & 258 \\
\hline Poly-3 test & $P=0.480 \mathrm{~N}$ & $\mathrm{P}=0.301$ & $\mathrm{P}=0.438 \mathrm{~N}$ & $P=0.540$ \\
\hline \multicolumn{5}{|c|}{ All Organs: Benign or Malignant Neoplasms } \\
\hline Overall rate & $33 / 50$ (66\%) & $32 / 50(64 \%)$ & $31 / 50(62 \%)$ & $33 / 50(66 \%)$ \\
\hline Adjusted rate & $75.2 \%$ & $73.1 \%$ & $71.0 \%$ & $76.1 \%$ \\
\hline Terminal rate & $14 / 20$ (70\%) & 7/13 (54\%) & $11 / 17$ (65\%) & 9/12 (75\%) \\
\hline First incidence (days) & 197 & 357 & 423 & 258 \\
\hline Poly-3 test & $P=0.495$ & $\mathrm{P}=0.507 \mathrm{~N}$ & $\mathrm{P}=0.414 \mathrm{~N}$ & $P=0.564$ \\
\hline
\end{tabular}

(T) Terminal euthanasia.

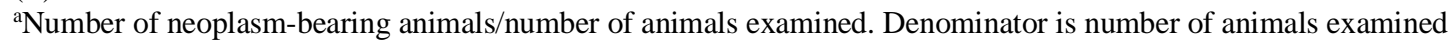

microscopically for adrenal medulla, pancreas, pituitary gland, testes, and thyroid gland; for other tissues, denominator is number of animals necropsied.

boly-3 estimated neoplasm incidence after adjustment for intercurrent mortality.

'Observed incidence at terminal euthanasia.

${ }^{d}$ Beneath the vehicle control incidence is the P value associated with the trend test. Beneath the dosed group incidence are the $\mathrm{P}$ values corresponding to pairwise comparisons between the vehicle controls and that dosed group. The Poly-3 test accounts for differential mortality in animals that do not reach terminal euthanasia. A negative trend or a lower incidence in a dose group is indicated by $\mathbf{N}$.

${ }^{\text {e}}$ Not applicable; no neoplasms in animal group. 
Indole-3-carbinol, NTP TR 584

Table A-3. Summary of the Incidence of Nonneoplastic Lesions in Male Sprague Dawley Rats in the Two-year Gavage Study of Indole-3-carbinol ${ }^{\mathrm{a}}$

\begin{tabular}{|c|c|c|c|c|}
\hline & Vehicle Control & $75 \mathrm{mg} / \mathrm{kg}$ & $150 \mathrm{mg} / \mathrm{kg}$ & $300 \mathrm{mg} / \mathrm{kg}$ \\
\hline \multicolumn{5}{|l|}{ Disposition Summary } \\
\hline Animals initially in study & 50 & 50 & 50 & 50 \\
\hline \multicolumn{5}{|l|}{ Early deaths } \\
\hline Accidental death & - & - & - & 1 \\
\hline Moribund & 14 & 23 & 15 & 20 \\
\hline Natural deaths & 16 & 14 & 18 & 17 \\
\hline \multicolumn{5}{|l|}{ Survivors } \\
\hline Terminal euthanasia & 20 & 13 & 17 & 12 \\
\hline Animals examined microscopically & 50 & 50 & 50 & 50 \\
\hline \multicolumn{5}{|l|}{ Alimentary System } \\
\hline Esophagus & $(50)$ & (50) & $(50)$ & $(50)$ \\
\hline Inflammation & - & $1(2 \%)$ & - & - \\
\hline Epithelium, hyperplasia & - & $1(2 \%)$ & - & - \\
\hline Intestine large, cecum & $(50)$ & $(50)$ & $(50)$ & $(50)$ \\
\hline Hyperplasia, lymphoid & - & $1(2 \%)$ & - & - \\
\hline Necrosis & $1(2 \%)$ & $1(2 \%)$ & - & - \\
\hline Intestine large, colon & $(50)$ & $(50)$ & $(50)$ & $(50)$ \\
\hline Erosion & $1(2 \%)$ & - & - & - \\
\hline Intestine large, rectum & $(50)$ & $(50)$ & $(50)$ & (50) \\
\hline Fibrosis & - & - & - & $1(2 \%)$ \\
\hline Intestine small, duodenum & $(43)$ & $(48)$ & $(47)$ & $(48)$ \\
\hline Lymphatic, ectasia & - & - & 15 (32\%) & $14(29 \%)$ \\
\hline Intestine small, ileum & (35) & (43) & $(42)$ & (38) \\
\hline Intestine small, jejunum & $(40)$ & (39) & $(40)$ & $(42)$ \\
\hline Lymphatic, ectasia & - & $2(5 \%)$ & 27 (68\%) & $41(98 \%)$ \\
\hline Liver & $(50)$ & $(50)$ & $(50)$ & $(50)$ \\
\hline Angiectasis & $1(2 \%)$ & - & - & - \\
\hline Basophilic focus & $3(6 \%)$ & - & $3(6 \%)$ & $1(2 \%)$ \\
\hline Cholangiofibrosis & - & $1(2 \%)$ & $3(6 \%)$ & $1(2 \%)$ \\
\hline Clear cell focus & $14(28 \%)$ & $13(26 \%)$ & $18(36 \%)$ & $9(18 \%)$ \\
\hline Degeneration, cystic & - & - & $1(2 \%)$ & - \\
\hline Eosinophilic focus & $10(20 \%)$ & $12(24 \%)$ & $10(20 \%)$ & $6(12 \%)$ \\
\hline Hepatodiaphragmatic nodule & $2(4 \%)$ & - & $1(2 \%)$ & $1(2 \%)$ \\
\hline Mixed cell focus & $4(8 \%)$ & $2(4 \%)$ & $1(2 \%)$ & $2(4 \%)$ \\
\hline Necrosis & $3(6 \%)$ & $3(6 \%)$ & $5(10 \%)$ & 7 (14\%) \\
\hline
\end{tabular}


Indole-3-carbinol, NTP TR 584

\begin{tabular}{|c|c|c|c|c|}
\hline & Vehicle Control & $75 \mathrm{mg} / \mathrm{kg}$ & $150 \mathrm{mg} / \mathrm{kg}$ & $300 \mathrm{mg} / \mathrm{kg}$ \\
\hline Artery, inflammation, chronic active & - & $1(2 \%)$ & - & - \\
\hline Bile duct, cyst & - & - & $1(2 \%)$ & $5(10 \%)$ \\
\hline Bile duct, cyst, multiple & - & - & $1(2 \%)$ & - \\
\hline Bile duct, fibrosis & $2(4 \%)$ & - & $1(2 \%)$ & $1(2 \%)$ \\
\hline Bile duct, hyperplasia & $7(14 \%)$ & $4(8 \%)$ & $3(6 \%)$ & $3(6 \%)$ \\
\hline Oval cell, hyperplasia & - & - & - & $1(2 \%)$ \\
\hline Periportal, inflammation, chronic active & $1(2 \%)$ & - & - & - \\
\hline Mesentery & $(2)$ & $(2)$ & (1) & $(0)$ \\
\hline Artery, inflammation, chronic active & $1(50 \%)$ & - & - & - \\
\hline Artery, mineralization & - & $1(50 \%)$ & - & - \\
\hline Fat, necrosis & $1(50 \%)$ & - & - & - \\
\hline Oral mucosa & $(50)$ & $(50)$ & $(50)$ & $(50)$ \\
\hline Foreign body & - & $1(2 \%)$ & - & - \\
\hline Hyperplasia, squamous & - & $1(2 \%)$ & $2(4 \%)$ & $1(2 \%)$ \\
\hline Sebaceous gland, ectopic tissue & - & - & - & $1(2 \%)$ \\
\hline Pancreas & $(50)$ & $(50)$ & $(50)$ & $(49)$ \\
\hline Angiectasis & - & - & - & $1(2 \%)$ \\
\hline Thrombosis & - & $1(2 \%)$ & - & - \\
\hline Acinus, atrophy & $1(2 \%)$ & $1(2 \%)$ & - & $2(4 \%)$ \\
\hline Acinus, hyperplasia & $16(32 \%)$ & $10(20 \%)$ & $14(28 \%)$ & $10(20 \%)$ \\
\hline Artery, inflammation, chronic active & $22(44 \%)$ & $32(64 \%)$ & 27 (54\%) & $21(43 \%)$ \\
\hline Salivary glands & (50) & (50) & (49) & (50) \\
\hline Metaplasia & - & $1(2 \%)$ & - & $2(4 \%)$ \\
\hline Stomach, forestomach & $(50)$ & $(50)$ & $(50)$ & $(50)$ \\
\hline Erosion & - & - & $1(2 \%)$ & - \\
\hline Hyperplasia & $19(38 \%)$ & $19(38 \%)$ & $19(38 \%)$ & $22(44 \%)$ \\
\hline Inflammation & $2(4 \%)$ & - & $4(8 \%)$ & $1(2 \%)$ \\
\hline Necrosis & $1(2 \%)$ & - & - & - \\
\hline Ulcer & $2(4 \%)$ & $1(2 \%)$ & $1(2 \%)$ & $1(2 \%)$ \\
\hline Stomach, glandular & $(50)$ & $(50)$ & $(50)$ & $(50)$ \\
\hline Epithelium, amyloid deposition & - & - & $1(2 \%)$ & - \\
\hline Epithelium, hyperplasia & $2(4 \%)$ & $5(10 \%)$ & $7(14 \%)$ & $3(6 \%)$ \\
\hline Epithelium, inflammation & - & $1(2 \%)$ & - & - \\
\hline Epithelium, inflammation, chronic active & $3(6 \%)$ & $4(8 \%)$ & $2(4 \%)$ & $1(2 \%)$ \\
\hline Epithelium, mineralization & $7(14 \%)$ & $12(24 \%)$ & $6(12 \%)$ & $10(20 \%)$ \\
\hline Epithelium, necrosis & $1(2 \%)$ & - & - & - \\
\hline Epithelium, ulcer & - & $1(2 \%)$ & - & - \\
\hline
\end{tabular}


Indole-3-carbinol, NTP TR 584

\begin{tabular}{|c|c|c|c|c|}
\hline & Vehicle Control & 75 mg/kg & $150 \mathrm{mg} / \mathrm{kg}$ & $300 \mathrm{mg} / \mathrm{kg}$ \\
\hline $\begin{array}{l}\text { Epithelium, muscularis, hyperplasia, } \\
\text { focal }\end{array}$ & - & $1(2 \%)$ & - & - \\
\hline Tongue & $(0)$ & $(0)$ & $(0)$ & (1) \\
\hline Tooth & (1) & $(0)$ & (1) & (0) \\
\hline Malformation & - & - & $1(100 \%)$ & - \\
\hline Necrosis & $1(100 \%)$ & - & - & - \\
\hline \multicolumn{5}{|l|}{ Cardiovascular System } \\
\hline Blood vessel & $(50)$ & $(50)$ & $(50)$ & $(50)$ \\
\hline Dilatation & $1(2 \%)$ & - & - & - \\
\hline Inflammation, chronic active & $30(60 \%)$ & 38 (76\%) & $30(60 \%)$ & 29 (58\%) \\
\hline Mineralization & - & $2(4 \%)$ & $1(2 \%)$ & $1(2 \%)$ \\
\hline Thrombosis & - & - & $1(2 \%)$ & - \\
\hline Heart & $(50)$ & $(50)$ & $(50)$ & $(50)$ \\
\hline Cardiomyopathy & 47 (94\%) & 48 (96\%) & 49 (98\%) & 48 (96\%) \\
\hline Mineralization & $1(2 \%)$ & - & - & $1(2 \%)$ \\
\hline Atrium, thrombosis & $1(2 \%)$ & $3(6 \%)$ & $3(6 \%)$ & $3(6 \%)$ \\
\hline Epicardium, inflammation & $1(2 \%)$ & $1(2 \%)$ & - & - \\
\hline Pericardium, inflammation & - & - & $1(2 \%)$ & - \\
\hline \multicolumn{5}{|l|}{ Endocrine System } \\
\hline Adrenal cortex & $(50)$ & (49) & $(50)$ & $(50)$ \\
\hline Atrophy & - & - & - & $1(2 \%)$ \\
\hline Degeneration, cystic & $4(8 \%)$ & $3(6 \%)$ & $5(10 \%)$ & $4(8 \%)$ \\
\hline Degeneration, fatty & $8(16 \%)$ & $13(27 \%)$ & $5(10 \%)$ & $16(32 \%)$ \\
\hline Hematopoietic cell proliferation & - & - & $1(2 \%)$ & - \\
\hline Hemorrhage & - & - & $1(2 \%)$ & - \\
\hline Hyperplasia & $14(28 \%)$ & $19(39 \%)$ & $19(38 \%)$ & $6(12 \%)$ \\
\hline Necrosis & $1(2 \%)$ & - & - & $2(4 \%)$ \\
\hline Thrombosis & - & $1(2 \%)$ & - & - \\
\hline Adrenal medulla & (50) & (49) & (50) & $(50)$ \\
\hline Angiectasis & $1(2 \%)$ & - & - & - \\
\hline Degeneration, fatty & - & $1(2 \%)$ & - & - \\
\hline Hyperplasia & $18(36 \%)$ & $22(45 \%)$ & $20(40 \%)$ & 19 (38\%) \\
\hline Islets, pancreatic & (50) & $(50)$ & $(50)$ & $(50)$ \\
\hline Hyperplasia & $3(6 \%)$ & $6(12 \%)$ & $1(2 \%)$ & $3(6 \%)$ \\
\hline Parathyroid gland & (43) & $(46)$ & $(50)$ & $(47)$ \\
\hline Hyperplasia & $3(7 \%)$ & $10(22 \%)$ & $13(26 \%)$ & 8 (17\%) \\
\hline Pituitary gland & $(50)$ & (49) & (50) & (47) \\
\hline
\end{tabular}


Indole-3-carbinol, NTP TR 584

\begin{tabular}{|c|c|c|c|c|}
\hline & Vehicle Control & $75 \mathrm{mg} / \mathrm{kg}$ & $150 \mathrm{mg} / \mathrm{kg}$ & $300 \mathrm{mg} / \mathrm{kg}$ \\
\hline Pars distalis, hyperplasia & 17 (34\%) & $18(37 \%)$ & $19(38 \%)$ & $12(26 \%)$ \\
\hline Thyroid gland & (50) & (46) & $(48)$ & $(47)$ \\
\hline Thrombosis & $1(2 \%)$ & - & - & - \\
\hline C-cell, hyperplasia & $6(12 \%)$ & $4(9 \%)$ & $4(8 \%)$ & 5 (11\%) \\
\hline C-cell, hypertrophy & - & - & - & $1(2 \%)$ \\
\hline Follicular cell, hyperplasia & - & $1(2 \%)$ & - & - \\
\hline Follicular cell, hypertrophy & $21(42 \%)$ & $34(74 \%)$ & 33 (69\%) & $36(77 \%)$ \\
\hline \multicolumn{5}{|l|}{ General Body System } \\
\hline None & - & - & - & - \\
\hline \multicolumn{5}{|l|}{ Genital System } \\
\hline Epididymis & (50) & $(50)$ & $(50)$ & $(50)$ \\
\hline Atrophy & $1(2 \%)$ & - & - & - \\
\hline Preputial gland & $(50)$ & $(50)$ & (49) & $(50)$ \\
\hline Cyst & $7(14 \%)$ & - & $3(6 \%)$ & $5(10 \%)$ \\
\hline Fibrosis & $1(2 \%)$ & - & - & - \\
\hline Inflammation & $2(4 \%)$ & - & - & $1(2 \%)$ \\
\hline Prostate & $(50)$ & (50) & $(50)$ & $(50)$ \\
\hline Atrophy & $1(2 \%)$ & $1(2 \%)$ & - & - \\
\hline Fibrosis & $1(2 \%)$ & $3(6 \%)$ & $2(4 \%)$ & $1(2 \%)$ \\
\hline Hyperplasia & $3(6 \%)$ & $4(8 \%)$ & $7(14 \%)$ & $3(6 \%)$ \\
\hline Inflammation, acute & $1(2 \%)$ & $6(12 \%)$ & - & $6(12 \%)$ \\
\hline Inflammation, chronic active & - & - & - & $1(2 \%)$ \\
\hline Necrosis & $1(2 \%)$ & - & - & - \\
\hline Seminal vesicle & (49) & $(50)$ & $(50)$ & $(50)$ \\
\hline Hypoplasia & - & - & $1(2 \%)$ & - \\
\hline Inflammation, acute & - & - & - & $2(4 \%)$ \\
\hline Testes & (49) & $(50)$ & $(50)$ & $(50)$ \\
\hline Atrophy & $30(61 \%)$ & 37 (74\%) & 35 (70\%) & $33(66 \%)$ \\
\hline Pigmentation, hemosiderin & - & - & $1(2 \%)$ & - \\
\hline Artery, inflammation, chronic active & $28(57 \%)$ & $34(68 \%)$ & $33(66 \%)$ & $33(66 \%)$ \\
\hline Interstitial cell, hyperplasia & $1(2 \%)$ & - & $1(2 \%)$ & $2(4 \%)$ \\
\hline \multicolumn{5}{|l|}{ Hematopoietic System } \\
\hline Bone marrow & (50) & (50) & (50) & (50) \\
\hline Hyperplasia & $1(2 \%)$ & $2(4 \%)$ & $4(8 \%)$ & $3(6 \%)$ \\
\hline Lymph node & (4) & (8) & (8) & (6) \\
\hline Angiectasis & - & - & - & $1(17 \%)$ \\
\hline Bronchial, hemorrhage & - & $1(13 \%)$ & $1(13 \%)$ & - \\
\hline
\end{tabular}


Indole-3-carbinol, NTP TR 584

\begin{tabular}{|c|c|c|c|c|}
\hline & Vehicle Control & $75 \mathrm{mg} / \mathrm{kg}$ & $150 \mathrm{mg} / \mathrm{kg}$ & $300 \mathrm{mg} / \mathrm{kg}$ \\
\hline Deep cervical, hemorrhage & - & $1(13 \%)$ & - & - \\
\hline Deep cervical, hyperplasia, plasma cell & - & $1(13 \%)$ & - & - \\
\hline Deep cervical, inflammation & - & $1(13 \%)$ & - & - \\
\hline Iliac, infiltration cellular, histiocyte & - & - & $1(13 \%)$ & - \\
\hline Lumbar, hemorrhage & - & - & $2(25 \%)$ & - \\
\hline Mediastinal, ectasia & - & $1(13 \%)$ & $1(13 \%)$ & - \\
\hline Mediastinal, hemorrhage & $1(25 \%)$ & $2(25 \%)$ & $3(38 \%)$ & $1(17 \%)$ \\
\hline Mediastinal, hyperplasia, lymphoid & - & $1(13 \%)$ & - & - \\
\hline Mediastinal, infiltration cellular, lipocyte & - & - & - & $1(17 \%)$ \\
\hline $\begin{array}{l}\text { Mediastinal, infiltration cellular, } \\
\text { histiocyte }\end{array}$ & - & $1(13 \%)$ & - & - \\
\hline Mediastinal, pigmentation, hemosiderin & - & - & - & $1(17 \%)$ \\
\hline Pancreatic, hemorrhage & - & $1(13 \%)$ & - & $1(17 \%)$ \\
\hline Renal, ectasia & $2(50 \%)$ & - & - & - \\
\hline Renal, hemorrhage & $1(25 \%)$ & $2(25 \%)$ & - & - \\
\hline Renal, pigmentation, hemosiderin & - & - & - & $1(17 \%)$ \\
\hline Lymph node, mandibular & $(50)$ & $(50)$ & $(49)$ & $(50)$ \\
\hline Ectasia & - & $1(2 \%)$ & $1(2 \%)$ & $1(2 \%)$ \\
\hline Hemorrhage & - & - & $1(2 \%)$ & - \\
\hline Hyperplasia, plasma cell & $1(2 \%)$ & - & - & - \\
\hline Lymph node, mesenteric & $(50)$ & $(50)$ & $(50)$ & $(50)$ \\
\hline Ectasia & - & $1(2 \%)$ & - & - \\
\hline Hemorrhage & - & $1(2 \%)$ & - & - \\
\hline Hyperplasia, lymphoid & $1(2 \%)$ & - & - & $1(2 \%)$ \\
\hline Lymphatic, ectasia & - & - & $1(2 \%)$ & $5(10 \%)$ \\
\hline Spleen & $(50)$ & $(50)$ & $(50)$ & $(50)$ \\
\hline Atrophy & - & - & - & $1(2 \%)$ \\
\hline Hematopoietic cell proliferation & $28(56 \%)$ & $33(66 \%)$ & $28(56 \%)$ & $29(58 \%)$ \\
\hline Inflammation & $1(2 \%)$ & - & - & - \\
\hline Necrosis & - & - & $1(2 \%)$ & - \\
\hline Thymus & $(46)$ & (49) & (49) & $(47)$ \\
\hline Atrophy & $41(89 \%)$ & $41(84 \%)$ & $42(86 \%)$ & $41(87 \%)$ \\
\hline Hemorrhage & - & - & $1(2 \%)$ & - \\
\hline Epithelial cell, hyperplasia & $1(2 \%)$ & - & - & - \\
\hline
\end{tabular}


Indole-3-carbinol, NTP TR 584

\begin{tabular}{|c|c|c|c|c|}
\hline & Vehicle Control & 75 mg/kg & $150 \mathrm{mg} / \mathrm{kg}$ & $300 \mathrm{mg} / \mathrm{kg}$ \\
\hline \multicolumn{5}{|l|}{ Integumentary System } \\
\hline Mammary gland & $(50)$ & $(50)$ & $(50)$ & $(50)$ \\
\hline Fibrosis & - & - & $1(2 \%)$ & - \\
\hline Inflammation & - & - & - & $1(2 \%)$ \\
\hline Skin & $(50)$ & $(50)$ & $(50)$ & $(50)$ \\
\hline Cyst epithelial inclusion & $5(10 \%)$ & $3(6 \%)$ & $2(4 \%)$ & $2(4 \%)$ \\
\hline Inflammation & $3(6 \%)$ & $2(4 \%)$ & - & $2(4 \%)$ \\
\hline Necrosis & - & $2(4 \%)$ & - & - \\
\hline Epidermis, ulcer & $1(2 \%)$ & - & - & - \\
\hline Sebaceous gland, hyperplasia & - & - & - & $1(2 \%)$ \\
\hline \multicolumn{5}{|l|}{ Musculoskeletal System } \\
\hline Bone & $(50)$ & $(50)$ & $(50)$ & $(50)$ \\
\hline Fibrous osteodystrophy & $12(24 \%)$ & $19(38 \%)$ & $13(26 \%)$ & $8(16 \%)$ \\
\hline Maxilla, fracture & - & - & $1(2 \%)$ & - \\
\hline \multicolumn{5}{|l|}{ Nervous System } \\
\hline Brain & $(50)$ & $(50)$ & $(50)$ & $(50)$ \\
\hline Cyst epithelial inclusion & - & - & - & $1(2 \%)$ \\
\hline Gliosis & - & $2(4 \%)$ & - & - \\
\hline Necrosis & - & - & - & $2(4 \%)$ \\
\hline Spinal cord & $(0)$ & (2) & (1) & $(0)$ \\
\hline Degeneration & - & $2(100 \%)$ & $1(100 \%)$ & - \\
\hline \multicolumn{5}{|l|}{ Respiratory System } \\
\hline Lung & (49) & $(50)$ & $(50)$ & $(50)$ \\
\hline Edema & - & - & $1(2 \%)$ & $1(2 \%)$ \\
\hline Fibrosis & - & - & - & $1(2 \%)$ \\
\hline Foreign body & - & $1(2 \%)$ & $1(2 \%)$ & - \\
\hline Hemorrhage & - & - & $1(2 \%)$ & $1(2 \%)$ \\
\hline Hemorrhage, multifocal & $3(6 \%)$ & $3(6 \%)$ & $7(14 \%)$ & $7(14 \%)$ \\
\hline Inflammation, granulomatous & - & - & $1(2 \%)$ & - \\
\hline Inflammation, chronic & 17 (35\%) & $21(42 \%)$ & $24(48 \%)$ & $23(46 \%)$ \\
\hline Mineralization & - & - & - & $1(2 \%)$ \\
\hline Necrosis & $1(2 \%)$ & - & - & - \\
\hline Alveolar epithelium, hyperplasia & $1(2 \%)$ & - & $1(2 \%)$ & $1(2 \%)$ \\
\hline Alveolus, infiltration cellular, histiocyte & 19 (39\%) & $22(44 \%)$ & $19(38 \%)$ & $13(26 \%)$ \\
\hline Alveolus, mineralization & $1(2 \%)$ & $2(4 \%)$ & $1(2 \%)$ & $1(2 \%)$ \\
\hline Nose & $(50)$ & $(50)$ & $(50)$ & $(50)$ \\
\hline Foreign body & $4(8 \%)$ & $12(24 \%)$ & $11(22 \%)$ & $10(20 \%)$ \\
\hline
\end{tabular}


Indole-3-carbinol, NTP TR 584

\begin{tabular}{|c|c|c|c|c|}
\hline & Vehicle Control & 75 mg/kg & $150 \mathrm{mg} / \mathrm{kg}$ & $300 \mathrm{mg} / \mathrm{kg}$ \\
\hline Fungus & $1(2 \%)$ & - & - & - \\
\hline Inflammation & $1(2 \%)$ & - & - & - \\
\hline Inflammation, chronic active & $11(22 \%)$ & $15(30 \%)$ & $16(32 \%)$ & $15 .(30 \%)$ \\
\hline Thrombosis & - & $1(2 \%)$ & - & - \\
\hline Respiratory epithelium, hyperplasia & $2(4 \%)$ & 2.(4\%) & $4(8 \%)$ & 7 (14\%) \\
\hline $\begin{array}{l}\text { Respiratory epithelium, metaplasia, } \\
\text { squamous }\end{array}$ & - & - & $2(4 \%)$ & - \\
\hline Trachea & (50) & $(50)$ & $(50)$ & $(50)$ \\
\hline Inflammation & $1(2 \%)$ & 1.(2\%) & $1(2 \%)$ & - \\
\hline \multicolumn{5}{|l|}{ Special Senses System } \\
\hline Ear & $(0)$ & $(0)$ & (1) & $(0)$ \\
\hline External ear, hyperplasia, squamous & - & - & $1(100 \%)$ & - \\
\hline Eye & $(50)$ & $(50)$ & (49) & (49) \\
\hline Cataract & - & - & - & $1(2 \%)$ \\
\hline Anterior chamber, inflammation, acute & $2(4 \%)$ & $1(2 \%)$ & $4(8 \%)$ & $3(6 \%)$ \\
\hline Cornea, inflammation, acute & $20(40 \%)$ & $25(50 \%)$ & 17 (35\%) & $21(43 \%)$ \\
\hline Retina, fibrosis & - & - & - & $1(2 \%)$ \\
\hline Harderian gland & (50) & $(50)$ & (49) & $(50)$ \\
\hline Angiectasis & - & - & - & $1(2 \%)$ \\
\hline Pigmentation, porphyrin & $1(2 \%)$ & - & - & - \\
\hline Lacrimal gland & (1) & (2) & (4) & $(0)$ \\
\hline Degeneration & $1(100 \%)$ & $2(100 \%)$ & $4(100 \%)$ & - \\
\hline \multicolumn{5}{|l|}{ Urinary System } \\
\hline Kidney & $(50)$ & $(50)$ & $(50)$ & $(50)$ \\
\hline Cyst & - & - & - & $1(2 \%)$ \\
\hline Metaplasia, osseous & - & $1(2 \%)$ & - & - \\
\hline Nephropathy & $50(100 \%)$ & 49 (98\%) & 49 (98\%) & $50(100 \%)$ \\
\hline Renal tubule, dilatation & - & - & $1(2 \%)$ & - \\
\hline Urinary bladder & (50) & (50) & $(50)$ & (50) \\
\hline Hemorrhage & - & - & - & $1(2 \%)$ \\
\hline Inflammation & - & - & - & $1(2 \%)$ \\
\hline
\end{tabular}

a Number of animals examined microscopically at the site and the number of animals with lesion. 


\section{Appendix B. Summary of Lesions in Female Sprague Dawley Rats in the Two-year Gavage Study of Indole-3-carbinol}

\section{Tables}

Table B-1. Summary of the Incidence of Neoplasms in Female Sprague Dawley Rats in the Two-year Gavage Study of Indole-3-carbinol

Table B-2. Statistical Analysis of Primary Neoplasms in Female Sprague Dawley Rats in the Two-year Gavage Study of Indole-3-carbinol

Table B-3. Historical Incidence of Carcinoma of the Uterus in Control Female Sprague Dawley Rats

Table B-4. Historical Incidence of Skin Neoplasms in Control Female Sprague Dawley Rats.

Table B-5. Summary of the Incidence of Nonneoplastic Lesions in Female Sprague Dawley Rats in the Two-year Gavage Study of Indole-3-carbinol. 
Table B-1. Summary of the Incidence of Neoplasms in Female Sprague Dawley Rats in the Two-year Gavage Study of Indole-3-carbinol ${ }^{\mathrm{a}}$

\begin{tabular}{|c|c|c|c|c|}
\hline & $\begin{array}{l}\text { Vehicle } \\
\text { Control }\end{array}$ & $75 \mathrm{mg} / \mathrm{kg}$ & $150 \mathrm{mg} / \mathrm{kg}$ & $300 \mathrm{mg} / \mathrm{kg}$ \\
\hline \multicolumn{5}{|l|}{ Disposition Summary } \\
\hline Animals initially in study & 50 & 50 & 50 & 50 \\
\hline \multicolumn{5}{|l|}{ Early deaths } \\
\hline Accidental deaths & - & 1 & - & 1 \\
\hline Moribund & 24 & 22 & 23 & 11 \\
\hline Natural deaths & 5 & 8 & 7 & 8 \\
\hline \multicolumn{5}{|l|}{ Survivors } \\
\hline Died last week of study & - & 1 & 1 & - \\
\hline Terminal euthanasia & 21 & 18 & 19 & 30 \\
\hline Animals examined microscopically & 50 & 50 & 50 & 50 \\
\hline \multicolumn{5}{|l|}{ Alimentary System } \\
\hline Esophagus & (50) & (50) & (50) & $(50)$ \\
\hline Intestine large, cecum & (50) & $(50)$ & (50) & $(48)$ \\
\hline Intestine large, colon & (50) & $(50)$ & $(50)$ & $(48)$ \\
\hline Intestine large, rectum & (50) & (50) & (50) & $(50)$ \\
\hline Intestine small, duodenum & (48) & $(47)$ & (48) & $(47)$ \\
\hline Intestine small, ileum & $(47)$ & $(47)$ & $(48)$ & $(47)$ \\
\hline Intestine small, jejunum & (47) & (46) & (48) & $(48)$ \\
\hline Liver & $(50)$ & $(50)$ & (50) & (48) \\
\hline Adenocarcinoma, metastatic, uterus & - & - & $2(4 \%)$ & $1(2 \%)$ \\
\hline Hepatocellular adenoma & $2(4 \%)$ & $1(2 \%)$ & $4(8 \%)$ & - \\
\hline Hepatocellular carcinoma & - & $1(2 \%)$ & - & - \\
\hline Mesentery & $(0)$ & (2) & $(2)$ & $(1)$ \\
\hline Fat, adenocarcinoma, metastatic, uterus & - & $1(50 \%)$ & $1(50 \%)$ & $1(100 \%)$ \\
\hline $\begin{array}{l}\text { Fat, squamous cell carcinoma, metastatic, } \\
\text { uterus }\end{array}$ & - & - & $1(50 \%)$ & - \\
\hline Oral mucosa & $(50)$ & $(50)$ & $(50)$ & $(50)$ \\
\hline Squamous cell carcinoma & - & - & - & $2(4 \%)$ \\
\hline Pancreas & $(50)$ & (49) & (49) & $(48)$ \\
\hline Adenocarcinoma, metastatic, uterus & - & $1(2 \%)$ & $1(2 \%)$ & $1(2 \%)$ \\
\hline Sarcoma stromal, metastatic, uterus & $1(2 \%)$ & - & - & - \\
\hline Acinus, adenoma & - & $1(2 \%)$ & - & $1(2 \%)$ \\
\hline Salivary glands & (50) & (49) & (48) & $(50)$ \\
\hline Stomach, forestomach & $(50)$ & $(50)$ & $(50)$ & (49) \\
\hline
\end{tabular}


Indole-3-carbinol, NTP TR 584

\begin{tabular}{|c|c|c|c|c|}
\hline & $\begin{array}{l}\text { Vehicle } \\
\text { Control }\end{array}$ & $75 \mathrm{mg} / \mathrm{kg}$ & $150 \mathrm{mg} / \mathrm{kg}$ & $300 \mathrm{mg} / \mathrm{kg}$ \\
\hline Adenocarcinoma, metastatic, uterus & - & - & - & $1(2 \%)$ \\
\hline Sarcoma stromal, metastatic, uterus & $1(2 \%)$ & - & - & - \\
\hline Squamous cell carcinoma & $1(2 \%)$ & - & - & $1(2 \%)$ \\
\hline Stomach, glandular & (50) & (49) & $(50)$ & (49) \\
\hline Adenocarcinoma, metastatic, uterus & - & - & - & $1(2 \%)$ \\
\hline Carcinoma & - & $1(2 \%)$ & - & - \\
\hline Tongue & (1) & (1) & $(0)$ & (1) \\
\hline Tooth & $(0)$ & (1) & $(0)$ & $(0)$ \\
\hline \multicolumn{5}{|l|}{ Cardiovascular System } \\
\hline Blood vessel & $(50)$ & $(50)$ & $(50)$ & $(50)$ \\
\hline $\begin{array}{l}\text { Aorta, squamous cell carcinoma, } \\
\text { metastatic, lung }\end{array}$ & - & - & $1(2 \%)$ & - \\
\hline Heart & (50) & (50) & $(50)$ & (49) \\
\hline Hemangioma & - & - & $1(2 \%)$ & - \\
\hline Schwannoma malignant & - & $2(4 \%)$ & - & - \\
\hline $\begin{array}{l}\text { Squamous cell carcinoma, metastatic, } \\
\text { lung }\end{array}$ & - & - & $1(2 \%)$ & - \\
\hline \multicolumn{5}{|l|}{ Endocrine System } \\
\hline Adrenal cortex & $(50)$ & (50) & (50) & $(48)$ \\
\hline Adenocarcinoma, metastatic, uterus & - & - & - & $1(2 \%)$ \\
\hline Adenoma & - & $1(2 \%)$ & $1(2 \%)$ & - \\
\hline Sarcoma stromal, metastatic, uterus & $1(2 \%)$ & - & - & - \\
\hline Adrenal medulla & $(50)$ & (50) & $(50)$ & $(48)$ \\
\hline Pheochromocytoma benign & - & $2(4 \%)$ & $2(4 \%)$ & - \\
\hline Islets, pancreatic & (50) & $(50)$ & $(50)$ & $(50)$ \\
\hline Adenoma & - & $1(2 \%)$ & - & - \\
\hline Carcinoma & - & - & $1(2 \%)$ & - \\
\hline Parathyroid gland & $(47)$ & (49) & $(46)$ & (49) \\
\hline Pituitary gland & $(50)$ & $(50)$ & $(50)$ & (49) \\
\hline $\begin{array}{l}\text { Schwannoma malignant, metastatic, } \\
\text { peripheral nerve }\end{array}$ & - & $1(2 \%)$ & - & - \\
\hline Pars distalis, adenoma & $18(36 \%)$ & $18(36 \%)$ & $19(38 \%)$ & $8(16 \%)$ \\
\hline Thyroid gland & (49) & (49) & $(48)$ & $(47)$ \\
\hline C-cell, adenoma & $11(22 \%)$ & $9(18 \%)$ & $5(10 \%)$ & $10(21 \%)$ \\
\hline C-cell, carcinoma & - & $1(2 \%)$ & - & $1(2 \%)$ \\
\hline Follicular cell, adenoma & - & - & $1(2 \%)$ & - \\
\hline
\end{tabular}


Indole-3-carbinol, NTP TR 584

\begin{tabular}{|c|c|c|c|c|}
\hline & $\begin{array}{l}\text { Vehicle } \\
\text { Control }\end{array}$ & $75 \mathrm{mg} / \mathrm{kg}$ & $150 \mathrm{mg} / \mathrm{kg}$ & $300 \mathrm{mg} / \mathrm{kg}$ \\
\hline \multicolumn{5}{|l|}{ General Body System } \\
\hline None & - & - & - & - \\
\hline \multicolumn{5}{|l|}{ Genital System } \\
\hline Clitoral gland & (50) & $(50)$ & $(50)$ & $(50)$ \\
\hline Ovary & (50) & $(50)$ & $(50)$ & (49) \\
\hline Adenocarcinoma, metastatic, uterus & - & - & $1(2 \%)$ & - \\
\hline Granulosa cell tumor benign & - & - & $1(2 \%)$ & - \\
\hline Granulosa-theca tumor benign & $1(2 \%)$ & - & - & - \\
\hline Tubulostromal carcinoma & $1(2 \%)$ & - & - & - \\
\hline Uterus & (50) & (50) & $(50)$ & $(50)$ \\
\hline Adenocarcinoma & - & $1(2 \%)$ & $4(8 \%)$ & $3(6 \%)$ \\
\hline Adenocarcinoma, multiple & - & - & - & $1(2 \%)$ \\
\hline Polyp stromal & $10(20 \%)$ & $10(20 \%)$ & $13(26 \%)$ & $9(18 \%)$ \\
\hline Sarcoma stromal & $1(2 \%)$ & - & $2(4 \%)$ & $1(2 \%)$ \\
\hline Sarcoma stromal, multiple & $1(2 \%)$ & - & - & - \\
\hline Squamous cell carcinoma & - & - & $1(2 \%)$ & - \\
\hline Vagina & $(2)$ & (1) & (0) & $(2)$ \\
\hline Polyp & $1(50 \%)$ & - & - & - \\
\hline Sarcoma stromal, metastatic, uterus & - & - & - & $1(50 \%)$ \\
\hline Schwannoma malignant & $1(50 \%)$ & $1(100 \%)$ & - & $1(50 \%)$ \\
\hline \multicolumn{5}{|l|}{ Hematopoietic System } \\
\hline Bone marrow & (50) & $(50)$ & $(50)$ & $(50)$ \\
\hline Lymph node & (7) & (6) & (4) & (3) \\
\hline $\begin{array}{l}\text { Pancreatic, sarcoma stromal, metastatic, } \\
\text { uterus }\end{array}$ & $1(14 \%)$ & - & - & - \\
\hline $\begin{array}{l}\text { Renal, adenocarcinoma, metastatic, } \\
\text { uterus }\end{array}$ & - & - & - & $1(33 \%)$ \\
\hline Lymph node, mandibular & $(50)$ & (49) & (49) & $(50)$ \\
\hline Lymph node, mesenteric & $(50)$ & $(50)$ & $(50)$ & $(48)$ \\
\hline Adenocarcinoma, metastatic, uterus & - & - & - & $1(2 \%)$ \\
\hline Sarcoma stromal, metastatic, uterus & $1(2 \%)$ & - & - & - \\
\hline Spleen & $(50)$ & $(50)$ & $(50)$ & $(48)$ \\
\hline Thymus & $(47)$ & $(50)$ & $(49)$ & $(50)$ \\
\hline Adenocarcinoma, metastatic, uterus & - & - & - & $1(2 \%)$ \\
\hline $\begin{array}{l}\text { Squamous cell carcinoma, metastatic, } \\
\text { lung }\end{array}$ & - & - & $1(2 \%)$ & - \\
\hline
\end{tabular}


Indole-3-carbinol, NTP TR 584

\begin{tabular}{|c|c|c|c|c|}
\hline & $\begin{array}{l}\text { Vehicle } \\
\text { Control }\end{array}$ & 75 mg/kg & $150 \mathrm{mg} / \mathrm{kg}$ & $300 \mathrm{mg} / \mathrm{kg}$ \\
\hline \multicolumn{5}{|l|}{ Integumentary System } \\
\hline Mammary gland & (50) & $(50)$ & $(50)$ & $(50)$ \\
\hline Adenoma & $3(6 \%)$ & $1(2 \%)$ & - & $2(4 \%)$ \\
\hline Carcinoma & $3(6 \%)$ & $2(4 \%)$ & $2(4 \%)$ & - \\
\hline Fibroadenoma & $18(36 \%)$ & $23(46 \%)$ & $20(40 \%)$ & 17 (34\%) \\
\hline Fibroadenoma, multiple & $11(22 \%)$ & $8(16 \%)$ & $4(8 \%)$ & $6(12 \%)$ \\
\hline Myoepithelioma & $1(2 \%)$ & - & - & - \\
\hline Skin & $(50)$ & (50) & $(50)$ & $(50)$ \\
\hline Basal cell carcinoma & - & - & $1(2 \%)$ & - \\
\hline Fibrosarcoma & - & - & - & $1(2 \%)$ \\
\hline Hemangiosarcoma & $1(2 \%)$ & - & - & - \\
\hline Schwannoma malignant & $1(2 \%)$ & - & - & - \\
\hline Subcutaneous tissue, fibroma & $1(2 \%)$ & - & - & $4(8 \%)$ \\
\hline Subcutaneous tissue, fibrosarcoma & $1(2 \%)$ & - & - & - \\
\hline Subcutaneous tissue, sarcoma & - & - & - & $1(2 \%)$ \\
\hline $\begin{array}{l}\text { Subcutaneous tissue, schwannoma } \\
\text { malignant }\end{array}$ & - & - & $1(2 \%)$ & - \\
\hline \multicolumn{5}{|l|}{ Musculoskeletal System } \\
\hline Bone & $(50)$ & $(50)$ & $(50)$ & $(50)$ \\
\hline Cranium, osteoma & - & - & - & $1(2 \%)$ \\
\hline Periosteum, sarcoma & - & - & - & $1(2 \%)$ \\
\hline Skeletal muscle & (1) & $(0)$ & (1) & (1) \\
\hline Adenocarcinoma, metastatic, uterus & - & - & - & $1(100 \%)$ \\
\hline Sarcoma & $1(100 \%)$ & - & - & - \\
\hline \multicolumn{5}{|l|}{ Nervous System } \\
\hline Brain & (50) & $(50)$ & $(50)$ & $(50)$ \\
\hline Glioma malignant & - & - & - & $1(2 \%)$ \\
\hline Granular cell tumor benign & $1(2 \%)$ & - & - & - \\
\hline Peripheral nerve & $(0)$ & $(2)$ & (0) & $(0)$ \\
\hline Trigeminal, schwannoma malignant & - & $1(50 \%)$ & - & - \\
\hline Spinal cord & $(0)$ & (1) & $(0)$ & $(0)$ \\
\hline \multicolumn{5}{|l|}{ Respiratory System } \\
\hline Lung & $(50)$ & $(50)$ & $(50)$ & (49) \\
\hline Adenocarcinoma, metastatic, uterus & - & $1(2 \%)$ & $1(2 \%)$ & $1(2 \%)$ \\
\hline Alveolar/bronchiolar adenoma & $1(2 \%)$ & - & $1(2 \%)$ & - \\
\hline
\end{tabular}


Indole-3-carbinol, NTP TR 584

\begin{tabular}{|c|c|c|c|c|}
\hline & $\begin{array}{l}\text { Vehicle } \\
\text { Control }\end{array}$ & $75 \mathrm{mg} / \mathrm{kg}$ & $150 \mathrm{mg} / \mathrm{kg}$ & $300 \mathrm{mg} / \mathrm{kg}$ \\
\hline Cystic keratinizing epithelioma & - & $1(2 \%)$ & - & - \\
\hline Squamous cell carcinoma, multiple & - & - & $1(2 \%)$ & - \\
\hline Mediastinum, sarcoma & $1(2 \%)$ & - & - & - \\
\hline Nose & (49) & $(50)$ & (49) & $(50)$ \\
\hline Trachea & $(50)$ & $(50)$ & $(50)$ & $(50)$ \\
\hline \multicolumn{5}{|l|}{ Special Senses System } \\
\hline Eye & $(50)$ & $(50)$ & $(50)$ & $(50)$ \\
\hline Harderian gland & $(50)$ & $(50)$ & $(50)$ & $(50)$ \\
\hline $\begin{array}{l}\text { Schwannoma malignant, metastatic, } \\
\text { peripheral nerve }\end{array}$ & - & $1(2 \%)$ & - & - \\
\hline \multicolumn{5}{|l|}{ Urinary System } \\
\hline Kidney & $(50)$ & $(50)$ & (50) & $(48)$ \\
\hline Adenocarcinoma, metastatic, uterus & - & - & - & $1(2 \%)$ \\
\hline $\begin{array}{l}\text { Capsule, adenocarcinoma, metastatic, } \\
\text { uterus }\end{array}$ & - & - & $1(2 \%)$ & - \\
\hline Renal tubule, adenoma & - & - & $1(2 \%)$ & - \\
\hline Renal tubule, adenoma, multiple & - & & $1(2 \%)$ & - \\
\hline Urinary bladder & (49) & $(50)$ & $(50)$ & $(50)$ \\
\hline Squamous cell carcinoma & - & - & $1(2 \%)$ & - \\
\hline \multicolumn{5}{|l|}{ Systemic Lesions } \\
\hline Multiple organs ${ }^{b}$ & $(50)$ & (50) & $(50)$ & $(50)$ \\
\hline Histiocytic sarcoma & $1(2 \%)$ & - & - & - \\
\hline Leukemia mononuclear & - & $2(4 \%)$ & - & - \\
\hline Lymphoma malignant & - & - & $1(2 \%)$ & $1(2 \%)$ \\
\hline \multicolumn{5}{|l|}{ Neoplasm Summary } \\
\hline Total animals with primary neoplasms ${ }^{\mathrm{c}}$ & 46 & 44 & 46 & 37 \\
\hline Total primary neoplasms & 93 & 88 & 89 & 73 \\
\hline Total animals with benign neoplasms & 40 & 40 & 41 & 35 \\
\hline Total benign neoplasms & 79 & 76 & 74 & 58 \\
\hline Total animals with malignant neoplasms & 14 & 11 & 15 & 15 \\
\hline Total malignant neoplasms & 14 & 12 & 15 & 15 \\
\hline Total animals with metastatic neoplasms & 1 & 2 & 4 & 3 \\
\hline Total metastatic neoplasms & 5 & 5 & 11 & 13 \\
\hline
\end{tabular}

${ }^{a}$ Number of animals examined microscopically at the site and the number of animals with neoplasm.

${ }^{b}$ Number of animals with any tissue examined microscopically.

'Primary neoplasms: all neoplasms except metastatic neoplasms. 
Indole-3-carbinol, NTP TR 584

Table B-2. Statistical Analysis of Primary Neoplasms in Female Sprague Dawley Rats in the Two-year Gavage Study of Indole-3-carbinol

\begin{tabular}{|c|c|c|c|c|}
\hline & $\begin{array}{l}\text { Vehicle } \\
\text { Control }\end{array}$ & $75 \mathrm{mg} / \mathrm{kg}$ & $150 \mathrm{mg} / \mathrm{kg}$ & $300 \mathrm{mg} / \mathrm{kg}$ \\
\hline \multicolumn{5}{|c|}{ Liver: Hepatocellular Adenoma } \\
\hline Overall rate $^{\mathrm{a}}$ & $2 / 50(4 \%)$ & $1 / 50(2 \%)$ & $4 / 50(8 \%)$ & $0 / 48(0 \%)$ \\
\hline Adjusted rate ${ }^{\mathrm{b}}$ & $5.5 \%$ & $2.7 \%$ & $11.0 \%$ & $0.0 \%$ \\
\hline Terminal rate ${ }^{c}$ & $2 / 21(10 \%)$ & $1 / 19(5 \%)$ & $2 / 19(11 \%)$ & $0 / 30(0 \%)$ \\
\hline First incidence (days) & $729(\mathrm{~T})$ & $729(\mathrm{~T})$ & 596 & $-^{\mathrm{e}}$ \\
\hline Poly-3 test ${ }^{\mathrm{d}}$ & $\mathrm{P}=0.227 \mathrm{~N}$ & $\mathrm{P}=0.498 \mathrm{~N}$ & $\mathrm{P}=0.335$ & $\mathrm{P}=0.216 \mathrm{~N}$ \\
\hline \multicolumn{5}{|c|}{ Liver: Hepatocellular Adenoma or Carcinoma } \\
\hline Overall rate & $2 / 50(4 \%)$ & $2 / 50(4 \%)$ & $4 / 50(8 \%)$ & $0 / 48(0 \%)$ \\
\hline Adjusted rate & $5.5 \%$ & $5.4 \%$ & $11.0 \%$ & $0.0 \%$ \\
\hline Terminal rate & $2 / 21(10 \%)$ & $1 / 19(5 \%)$ & $2 / 19(11 \%)$ & 0/30 (0\%) \\
\hline First incidence (days) & $729(\mathrm{~T})$ & 616 & 596 & - \\
\hline Poly-3 test & $\mathrm{P}=0.184 \mathrm{~N}$ & $\mathrm{P}=0.689 \mathrm{~N}$ & $\mathrm{P}=0.335$ & $\mathrm{P}=0.216 \mathrm{~N}$ \\
\hline \multicolumn{5}{|c|}{ Mammary Gland: Fibroadenoma } \\
\hline Overall rate & $29 / 50(58 \%)$ & $31 / 50(62 \%)$ & $24 / 50(48 \%)$ & $23 / 50(46 \%)$ \\
\hline Adjusted rate & $66.6 \%$ & $71.5 \%$ & $58.4 \%$ & $53.5 \%$ \\
\hline Terminal rate & $11 / 21(52 \%)$ & $11 / 19(58 \%)$ & $10 / 19(53 \%)$ & $14 / 30(47 \%)$ \\
\hline First incidence (days) & 288 & 420 & 329 & 356 \\
\hline Poly-3 test & $\mathrm{P}=0.058 \mathrm{~N}$ & $\mathrm{P}=0.389$ & $\mathrm{P}=0.283 \mathrm{~N}$ & $\mathrm{P}=0.147 \mathrm{~N}$ \\
\hline \multicolumn{5}{|c|}{ Mammary Gland: Adenoma } \\
\hline Overall rate & $3 / 50(6 \%)$ & $1 / 50(2 \%)$ & $0 / 50(0 \%)$ & $2 / 50(4 \%)$ \\
\hline Adjusted rate & $8.2 \%$ & $2.7 \%$ & $0.0 \%$ & $5.0 \%$ \\
\hline Terminal rate & $1 / 21(5 \%)$ & $0 / 19(0 \%)$ & 0/19 (0\%) & $2 / 30(7 \%)$ \\
\hline First incidence (days) & 681 & 645 & - & $729(\mathrm{~T})$ \\
\hline Poly-3 test & $\mathrm{P}=0.436 \mathrm{~N}$ & $\mathrm{P}=0.301 \mathrm{~N}$ & $\mathrm{P}=0.122 \mathrm{~N}$ & $P=0.459 N$ \\
\hline \multicolumn{5}{|c|}{ Mammary Gland: Fibroadenoma or Adenoma } \\
\hline Overall rate & $30 / 50(60 \%)$ & $31 / 50$ (62\%) & $24 / 50$ (48\%) & $25 / 50(50 \%)$ \\
\hline Adjusted rate & $68.6 \%$ & $71.5 \%$ & $58.4 \%$ & $58.2 \%$ \\
\hline Terminal rate & $11 / 21(52 \%)$ & $11 / 19$ (58\%) & $10 / 19(53 \%)$ & $16 / 30(53 \%)$ \\
\hline First incidence (days) & 288 & 420 & 329 & 356 \\
\hline Poly-3 test & $\mathrm{P}=0.106 \mathrm{~N}$ & $P=0.471$ & $\mathrm{P}=0.217 \mathrm{~N}$ & $\mathrm{P}=0.210 \mathrm{~N}$ \\
\hline \multicolumn{5}{|c|}{ Mammary Gland: Carcinoma } \\
\hline Overall rate & $3 / 50(6 \%)$ & $2 / 50(4 \%)$ & $2 / 50(4 \%)$ & $0 / 50(0 \%)$ \\
\hline Adjusted rate & $7.8 \%$ & $5.4 \%$ & $5.6 \%$ & $0.0 \%$ \\
\hline Terminal rate & $0 / 21(0 \%)$ & $1 / 19(5 \%)$ & 2/19 (11\%) & $0 / 30(0 \%)$ \\
\hline
\end{tabular}


Indole-3-carbinol, NTP TR 584

\begin{tabular}{|c|c|c|c|c|}
\hline & $\begin{array}{l}\text { Vehicle } \\
\text { Control }\end{array}$ & 75 mg/kg & $150 \mathrm{mg} / \mathrm{kg}$ & $300 \mathrm{mg} / \mathrm{kg}$ \\
\hline First incidence (days) & 446 & 705 & 729 (T) & - \\
\hline Poly-3 test & $\mathrm{P}=0.077 \mathrm{~N}$ & $P=0.522 \mathrm{~N}$ & $P=0.536 \mathrm{~N}$ & $\mathrm{P}=0.111 \mathrm{~N}$ \\
\hline \multicolumn{5}{|c|}{ Mammary Gland: Adenoma or Carcinoma } \\
\hline Overall rate & $6 / 50(12 \%)$ & $3 / 50(6 \%)$ & $2 / 50(4 \%)$ & $2 / 50(4 \%)$ \\
\hline Adjusted rate & $15.5 \%$ & $8.1 \%$ & $5.6 \%$ & $5.0 \%$ \\
\hline Terminal rate & $1 / 21(5 \%)$ & $1 / 19(5 \%)$ & 2/19 (11\%) & $2 / 30(7 \%)$ \\
\hline First incidence (days) & 446 & 645 & $729(\mathrm{~T})$ & $729(\mathrm{~T})$ \\
\hline Poly-3 test & $\mathrm{P}=0.084 \mathrm{~N}$ & $P=0.261 \mathrm{~N}$ & $P=0.158 N$ & $\mathrm{P}=0.119 \mathrm{~N}$ \\
\hline \multicolumn{5}{|c|}{ Mammary Gland: Fibroadenoma, Adenoma, or Carcinoma } \\
\hline Overall rate & $32 / 50(64 \%)$ & $31 / 50(62 \%)$ & $25 / 50(50 \%)$ & $25 / 50(50 \%)$ \\
\hline Adjusted rate & $70.8 \%$ & $71.5 \%$ & $60.8 \%$ & $58.2 \%$ \\
\hline Terminal rate & $11 / 21(52 \%)$ & 11/19 (58\%) & 11/19 (58\%) & $16 / 30(53 \%)$ \\
\hline First incidence (days) & 288 & 420 & 329 & 356 \\
\hline Poly-3 test & $P=0.076 \mathrm{~N}$ & $P=0.566$ & $P=0.216 \mathrm{~N}$ & $\mathrm{P}=0.146 \mathrm{~N}$ \\
\hline \multicolumn{5}{|c|}{ Pituitary Gland (Pars Distalis): Adenoma } \\
\hline Overall rate & $18 / 50$ (36\%) & 18/50 (36\%) & 19/50 (38\%) & $8 / 49(16 \%)$ \\
\hline Adjusted rate & $45.6 \%$ & $45.1 \%$ & $49.0 \%$ & $19.8 \%$ \\
\hline Terminal rate & 8/21 (38\%) & 10/19 (53\%) & 11/19 (58\%) & 3/30 (10\%) \\
\hline First incidence (days) & 552 & 420 & 516 & 571 \\
\hline Poly-3 test & $P=0.006 \mathrm{~N}$ & $P=0.574 N$ & $P=0.469$ & $\mathrm{P}=0.011 \mathrm{~N}$ \\
\hline \multicolumn{5}{|c|}{ Skin (Subcutaneous Tissue): Fibroma } \\
\hline Overall rate & $1 / 50(2 \%)$ & $0 / 50(0 \%)$ & $0 / 50(0 \%)$ & $4 / 50(8 \%)$ \\
\hline Adjusted rate & $2.7 \%$ & $0.0 \%$ & $0.0 \%$ & $9.9 \%$ \\
\hline Terminal rate & $1 / 21(5 \%)$ & $0 / 19(0 \%)$ & $0 / 19(0 \%)$ & 3/30 (10\%) \\
\hline First incidence (days) & $729(\mathrm{~T})$ & - & - & 701 \\
\hline Poly-3 test & $P=0.029$ & $\mathrm{P}=0.499 \mathrm{~N}$ & $\mathrm{P}=0.504 \mathrm{~N}$ & $P=0.209$ \\
\hline \multicolumn{5}{|c|}{ Skin (Subcutaneous Tissue): Fibroma or Fibrosarcoma } \\
\hline Overall rate & $2 / 50(4 \%)$ & $0 / 50(0 \%)$ & $0 / 50(0 \%)$ & $5 / 50(10 \%)$ \\
\hline Adjusted rate & $5.4 \%$ & $0.0 \%$ & $0.0 \%$ & $12.4 \%$ \\
\hline Terminal rate & $1 / 21(5 \%)$ & 0/19 (0\%) & 0/19 (0\%) & 3/30 (10\%) \\
\hline First incidence (days) & 645 & - & - & 692 \\
\hline Poly-3 test & $P=0.040$ & $\mathrm{P}=0.237 \mathrm{~N}$ & $\mathrm{P}=0.243 \mathrm{~N}$ & $\mathrm{P}=0.254$ \\
\hline \multicolumn{5}{|c|}{ Skin (Subcutaneous Tissue): Fibroma, Fibrosarcoma, or Sarcoma } \\
\hline Overall rate & $2 / 50(4 \%)$ & 0/50 (0\%) & $0 / 50(0 \%)$ & 6/50 (12\%) \\
\hline Adjusted rate & $5.4 \%$ & $0.0 \%$ & $0.0 \%$ & $14.9 \%$ \\
\hline
\end{tabular}


Indole-3-carbinol, NTP TR 584

\begin{tabular}{|c|c|c|c|c|}
\hline & $\begin{array}{l}\text { Vehicle } \\
\text { Control }\end{array}$ & $75 \mathrm{mg} / \mathrm{kg}$ & $150 \mathrm{mg} / \mathrm{kg}$ & $300 \mathrm{mg} / \mathrm{kg}$ \\
\hline Terminal rate & $1 / 21(5 \%)$ & 0/19 (0\%) & $0 / 19(0 \%)$ & 4/30 (13\%) \\
\hline First incidence (days) & 645 & - & - & 692 \\
\hline Poly-3 test & $P=0.013$ & $\mathrm{P}=0.237 \mathrm{~N}$ & $\mathrm{P}=0.243 \mathrm{~N}$ & $P=0.163$ \\
\hline \multicolumn{5}{|c|}{ Thyroid Gland (C-Cell): Adenoma } \\
\hline Overall rate & $11 / 49(22 \%)$ & 9/49 (18\%) & $5 / 48(10 \%)$ & $10 / 47(21 \%)$ \\
\hline Adjusted rate & $29.8 \%$ & $23.9 \%$ & $13.9 \%$ & $25.0 \%$ \\
\hline Terminal rate & 8/21 (38\%) & 6/19 (32\%) & 2/19 (11\%) & 7/30 (23\%) \\
\hline First incidence (days) & 558 & 313 & 631 & 605 \\
\hline Poly-3 test & $\mathrm{P}=0.385 \mathrm{~N}$ & $\mathrm{P}=0.375 \mathrm{~N}$ & $\mathrm{P}=0.082 \mathrm{~N}$ & $\mathrm{P}=0.413 \mathrm{~N}$ \\
\hline \multicolumn{5}{|c|}{ Thyroid Gland (C-Cell): Adenoma or Carcinoma } \\
\hline Overall rate & $11 / 49(22 \%)$ & $10 / 49(20 \%)$ & $5 / 48(10 \%)$ & $11 / 47(23 \%)$ \\
\hline Adjusted rate & $29.8 \%$ & $26.6 \%$ & $13.9 \%$ & $27.4 \%$ \\
\hline Terminal rate & $8 / 21(38 \%)$ & $7 / 19(37 \%)$ & $2 / 19(11 \%)$ & $7 / 30(23 \%)$ \\
\hline First incidence (days) & 558 & 313 & 631 & 605 \\
\hline Poly-3 test & $\mathrm{P}=0.457 \mathrm{~N}$ & $P=0.478 N$ & $P=0.082 N$ & $P=0.508 \mathrm{~N}$ \\
\hline \multicolumn{5}{|c|}{ Uterus: Stromal Polyp (Standard Evaluation) } \\
\hline Overall rate & $10 / 50(20 \%)$ & $10 / 50$ (20\%) & $13 / 50(26 \%)$ & 9/50 (18\%) \\
\hline Adjusted rate & $26.6 \%$ & $26.2 \%$ & $34.7 \%$ & $22.1 \%$ \\
\hline Terminal rate & 7/21 (33\%) & $5 / 19(26 \%)$ & $8 / 19(42 \%)$ & 6/30 (20\%) \\
\hline First incidence (days) & 422 & 420 & 611 & 622 \\
\hline Poly-3 test & $\mathrm{P}=0.379 \mathrm{~N}$ & $\mathrm{P}=0.589 \mathrm{~N}$ & $\mathrm{P}=0.300$ & $\mathrm{P}=0.420 \mathrm{~N}$ \\
\hline \multicolumn{5}{|c|}{ Uterus: Stromal Polyp or Stromal Sarcoma (Standard Evaluation) } \\
\hline Overall rate & $12 / 50(24 \%)$ & $10 / 50(20 \%)$ & $15 / 50(30 \%)$ & 9/50 (18\%) \\
\hline Adjusted rate & $31.3 \%$ & $26.2 \%$ & $38.6 \%$ & $22.1 \%$ \\
\hline Terminal rate & 7/21 (33\%) & $5 / 19(26 \%)$ & $8 / 19(42 \%)$ & $6 / 30(20 \%)$ \\
\hline First incidence (days) & 422 & 420 & 393 & 622 \\
\hline Poly-3 test & $\mathrm{P}=0.260 \mathrm{~N}$ & $\mathrm{P}=0.405 \mathrm{~N}$ & $\mathrm{P}=0.330$ & $\mathrm{P}=0.249 \mathrm{~N}$ \\
\hline \multicolumn{5}{|c|}{ Uterus: Adenocarcinoma (Standard Evaluation) } \\
\hline Overall rate & $0 / 50(0 \%)$ & $1 / 50(2 \%)$ & $4 / 50(8 \%)$ & $4 / 50(8 \%)$ \\
\hline Adjusted rate & $0.0 \%$ & $2.7 \%$ & $10.9 \%$ & $9.8 \%$ \\
\hline Terminal rate & $0 / 21(0 \%)$ & 0/19 (0\%) & $1 / 19(5 \%)$ & $2 / 30(7 \%)$ \\
\hline First incidence (days) & - & 645 & 608 & 645 \\
\hline Poly-3 test & $P=0.040$ & $P=0.503$ & $\mathrm{P}=0.059$ & $\mathrm{P}=0.074$ \\
\hline
\end{tabular}


Indole-3-carbinol, NTP TR 584

\begin{tabular}{|c|c|c|c|c|}
\hline & $\begin{array}{l}\text { Vehicle } \\
\text { Control }\end{array}$ & $75 \mathrm{mg} / \mathrm{kg}$ & $150 \mathrm{mg} / \mathrm{kg}$ & $300 \mathrm{mg} / \mathrm{kg}$ \\
\hline \multicolumn{5}{|c|}{ Uterus: Adenocarcinoma (Extended Evaluation) } \\
\hline Overall rate & $5 / 50(10 \%)$ & $3 / 50(6 \%)$ & $10 / 50(20 \%)$ & $8 / 50(16 \%)$ \\
\hline Adjusted rate & $13.7 \%$ & $8.2 \%$ & $26.9 \%$ & $19.8 \%$ \\
\hline Terminal rate & 3/21 (14\%) & $2 / 19(11 \%)$ & $5 / 19(26 \%)$ & $7 / 30(23 \%)$ \\
\hline First incidence (days) & 719 & 719 & 615 & 674 \\
\hline Poly-3 test & $\mathrm{P}=0.158$ & $\mathrm{P}=0.351 \mathrm{~N}$ & $P=0.127$ & $\mathrm{P}=0.340$ \\
\hline \multicolumn{5}{|c|}{ Uterus: Adenoma or Adenocarcinoma (Extended Evaluation) } \\
\hline Overall rate & $5 / 50(10 \%)$ & $5 / 50(10 \%)$ & 11/50 (22\%) & 9/50 (18\%) \\
\hline Adjusted rate & $13.7 \%$ & $13.5 \%$ & $29.6 \%$ & $22.3 \%$ \\
\hline Terminal rate & $3 / 21(14 \%)$ & $3 / 19(16 \%)$ & 6/19 (32\%) & $8 / 30(27 \%)$ \\
\hline First incidence (days) & 719 & 645 & 615 & 674 \\
\hline Poly-3 test & $\mathrm{P}=0.146$ & $\mathrm{P}=0.624 \mathrm{~N}$ & $P=0.080$ & $P=0.247$ \\
\hline \multicolumn{5}{|c|}{ Uterus: Adenoma or Adenocarcinoma (Standard and Extended Evaluation) } \\
\hline Overall rate & $5 / 50(10 \%)$ & $5 / 50(10 \%)$ & $14 / 50$ (28\%) & $11 / 50(22 \%)$ \\
\hline Adjusted rate & $13.7 \%$ & $13.5 \%$ & $36.6 \%$ & $27.0 \%$ \\
\hline Terminal rate & 3/21 (14\%) & $3 / 19(16 \%)$ & 6/19 (32\%) & 9/30 (30\%) \\
\hline First incidence (days) & 719 & 645 & 608 & 645 \\
\hline Poly-3 test & $\mathrm{P}=0.052$ & $\mathrm{P}=0.624 \mathrm{~N}$ & $\mathrm{P}=0.018$ & $P=0.119$ \\
\hline \multicolumn{5}{|c|}{ Uterus: Adenocarcinoma or Adenosquamous Carcinoma (Standard and Extended Evaluations) } \\
\hline Overall rate & $5 / 50(10 \%)$ & $4 / 50(8 \%)$ & $13 / 50(26 \%)$ & $10 / 50(20 \%)$ \\
\hline Adjusted rate & $13.7 \%$ & $10.8 \%$ & $34.0 \%$ & $24.6 \%$ \\
\hline Terminal rate & 3/21 (14\%) & 2/19 (11\%) & $5 / 19(26 \%)$ & 8/30 (27\%) \\
\hline First incidence (days) & 719 & 645 & 608 & 645 \\
\hline Poly-3 test & $\mathrm{P}=0.071$ & $\mathrm{P}=0.491 \mathrm{~N}$ & $P=0.033$ & $\mathrm{P}=0.177$ \\
\hline \multicolumn{5}{|c|}{ All Organs: Benign Neoplasms } \\
\hline Overall rate & $40 / 50(80 \%)$ & 40/50 (80\%) & $41 / 50(82 \%)$ & $35 / 50(70 \%)$ \\
\hline Adjusted rate & $89.5 \%$ & $88.9 \%$ & $91.7 \%$ & $79.1 \%$ \\
\hline Terminal rate & 19/21 (91\%) & 18/19 (95\%) & 18/19 (95\%) & $22 / 30(73 \%)$ \\
\hline First incidence (days) & 288 & 313 & 307 & 356 \\
\hline Poly-3 test & $\mathrm{P}=0.067 \mathrm{~N}$ & $P=0.609 N$ & $P=0.508$ & $\mathrm{P}=0.127 \mathrm{~N}$ \\
\hline \multicolumn{5}{|c|}{ All Organs: Malignant Neoplasms } \\
\hline Overall rate & $14 / 50(28 \%)$ & $11 / 50(22 \%)$ & 15/50 (30\%) & $15 / 50(30 \%)$ \\
\hline Adjusted rate & $33.4 \%$ & $27.7 \%$ & $36.7 \%$ & $35.8 \%$ \\
\hline Terminal rate & 3/21 (14\%) & 4/19 (21\%) & 6/19 (32\%) & 8/30 (27\%) \\
\hline First incidence (days) & 425 & 187 & 160 & 605 \\
\hline
\end{tabular}


Indole-3-carbinol, NTP TR 584

\begin{tabular}{|c|c|c|c|c|}
\hline & $\begin{array}{l}\text { Vehicle } \\
\text { Control }\end{array}$ & $75 \mathrm{mg} / \mathrm{kg}$ & $150 \mathrm{mg} / \mathrm{kg}$ & $300 \mathrm{mg} / \mathrm{kg}$ \\
\hline Poly-3 test & $P=0.356$ & $\mathrm{P}=0.374 \mathrm{~N}$ & $P=0.464$ & $P=0.498$ \\
\hline \multicolumn{5}{|c|}{ All Organs: Benign or Malignant Neoplasms } \\
\hline Overall rate & $46 / 50(92 \%)$ & $44 / 50(88 \%)$ & $46 / 50$ (92\%) & $37 / 50(74 \%)$ \\
\hline Adjusted rate & $95.3 \%$ & $92.6 \%$ & $96.6 \%$ & $83.6 \%$ \\
\hline Terminal rate & 19/21 (91\%) & 18/19 (95\%) & 18/19 (95\%) & $24 / 30(80 \%)$ \\
\hline First incidence (days) & 288 & 187 & 160 & 356 \\
\hline Poly-3 test & $\mathrm{P}=0.029 \mathrm{~N}$ & $\mathrm{P}=0.445 \mathrm{~N}$ & $P=0.577$ & $\mathrm{P}=0.057 \mathrm{~N}$ \\
\hline
\end{tabular}

(T) Terminal euthanasia.

${ }^{a}$ Number of neoplasm-bearing animals/number of animals examined. Denominator is number of animals examined microscopically for liver, pituitary gland, and thyroid gland; for other tissues, denominator is number of animals necropsied. bPoly-3 estimated neoplasm incidence after adjustment for intercurrent mortality.

'Observed incidence at terminal euthanasia.

${ }^{\mathrm{d} B e n e a t h}$ the vehicle control incidence is the $\mathrm{P}$ value associated with the trend test. Beneath the dosed group incidence are the $\mathrm{P}$ values corresponding to pairwise comparisons between the vehicle controls and that dosed group. The Poly- 3 test accounts for differential mortality in animals that do not reach terminal euthanasia. A negative trend or a lower incidence in a dose group is indicated by $\mathbf{N}$.

eNot applicable; no neoplasms in animal group.

Table B-3. Historical Incidence of Carcinoma of the Uterus in Control Female Sprague Dawley Rats $^{\mathrm{a}}$

\begin{tabular}{lc}
\hline \multicolumn{1}{c}{ Study (Study Start) } & Incidence in Controls \\
\hline Historical Incidence: Corn Oil Gavage Studies $^{\mathbf{b}}$ & \\
Indole-3-carbinol (March 2007) & $0 / 50$ \\
PCB 118 (March 2004) & $2 / 52$ \\
Total & $2 / 102(2.0 \%)$ \\
Mean \pm standard deviation & $1.9 \% \pm 2.7 \%$ \\
Range & $0 \%-4 \%$ \\
\hline
\end{tabular}

aData as of June 2013.

bSame data for overall historical incidence for all routes.

Table B-4. Historical Incidence of Skin Neoplasms in Control Female Sprague Dawley Rats ${ }^{\mathrm{a}}$

\begin{tabular}{|c|c|c|}
\hline Study (Study Start) & Fibroma & Fibrosarcoma \\
\hline
\end{tabular}

\begin{tabular}{lccc}
\hline \multicolumn{2}{l}{ Historical Incidence: Corn Oil Gavage Studies } & & \\
Indole-3-carbinol (March 2007) & $1 / 50$ & $1 / 50$ & $2 / 50$ \\
PCB 118 (March 2004) & $2 / 52$ & $0 / 52$ & $2 / 52$ \\
Total (\%) & $3 / 102(2.9 \%)$ & $1 / 102(1.0 \%)$ & $4 / 102(3.9 \%)$ \\
Mean \pm standard deviation & $2.9 \% \pm 1.3 \%$ & $1.0 \% \pm 1.4 \%$ & $3.9 \% \pm 0.1 \%$ \\
Range & $2 \%-4 \%$ & $0 \%-2 \%$ & $4 \%$ \\
\hline
\end{tabular}

aData as of June 2013.

bSame data for overall historical incidence for all routes. 
Table B-5. Summary of the Incidence of Nonneoplastic Lesions in Female Sprague Dawley Rats in the Two-year Gavage Study of Indole-3-carbinol ${ }^{a}$

\begin{tabular}{|c|c|c|c|c|}
\hline & Vehicle Control & $75 \mathrm{mg} / \mathrm{kg}$ & $150 \mathrm{mg} / \mathrm{kg}$ & $300 \mathrm{mg} / \mathrm{kg}$ \\
\hline \multicolumn{5}{|l|}{ Disposition Summary } \\
\hline Animals initially in study & 50 & 50 & 50 & 50 \\
\hline \multicolumn{5}{|l|}{ Early deaths } \\
\hline Accidental deaths & - & 1 & - & 1 \\
\hline Moribund & 24 & 22 & 23 & 11 \\
\hline Natural deaths & 5 & 8 & 7 & 8 \\
\hline \multicolumn{5}{|l|}{ Survivors } \\
\hline Died last week of study & - & 1 & 1 & - \\
\hline Terminal euthanasia & 21 & 18 & 19 & 30 \\
\hline Animals examined microscopically & 50 & 50 & 50 & 50 \\
\hline \multicolumn{5}{|l|}{ Alimentary System } \\
\hline Esophagus & $(50)$ & $(50)$ & $(50)$ & $(50)$ \\
\hline Inflammation & - & $2(4 \%)$ & - & - \\
\hline Perforation & - & - & - & $1(2 \%)$ \\
\hline Intestine large, cecum & $(50)$ & $(50)$ & $(50)$ & (48) \\
\hline Intestine large, colon & (50) & $(50)$ & $(50)$ & $(48)$ \\
\hline Intestine large, rectum & (50) & $(50)$ & $(50)$ & (50) \\
\hline Metaplasia & - & - & $1(2 \%)$ & - \\
\hline Intestine small, duodenum & $(48)$ & $(47)$ & $(48)$ & $(47)$ \\
\hline Lymphatic, ectasia & - & - & $16(33 \%)$ & 38 (81\%) \\
\hline Intestine small, ileum & (47) & $(47)$ & (48) & (47) \\
\hline Intestine small, jejunum & $(47)$ & (46) & (48) & (48) \\
\hline Lymphatic, ectasia & - & - & $30(63 \%)$ & 47 (98\%) \\
\hline Liver & (50) & $(50)$ & $(50)$ & $(48)$ \\
\hline Angiectasis & $1(2 \%)$ & - & $2(4 \%)$ & $3(6 \%)$ \\
\hline Basophilic focus & $14(28 \%)$ & $6(12 \%)$ & $11(22 \%)$ & $14(29 \%)$ \\
\hline Cholangiofibrosis & - & $1(2 \%)$ & - & - \\
\hline Clear cell focus & $6(12 \%)$ & $7(14 \%)$ & $4(8 \%)$ & $18(38 \%)$ \\
\hline Eosinophilic focus & - & $4(8 \%)$ & $5(10 \%)$ & $6(13 \%)$ \\
\hline Hepatodiaphragmatic nodule & - & - & $3(6 \%)$ & $2(4 \%)$ \\
\hline Inflammation, chronic & - & - & - & $1(2 \%)$ \\
\hline Mixed cell focus & $5(10 \%)$ & $1(2 \%)$ & $5(10 \%)$ & $4(8 \%)$ \\
\hline Necrosis & $1(2 \%)$ & $2(4 \%)$ & - & - \\
\hline Pigmentation & $1(2 \%)$ & - & - & - \\
\hline
\end{tabular}


Indole-3-carbinol, NTP TR 584

\begin{tabular}{|c|c|c|c|c|}
\hline & Vehicle Control & $75 \mathrm{mg} / \mathrm{kg}$ & $150 \mathrm{mg} / \mathrm{kg}$ & $300 \mathrm{mg} / \mathrm{kg}$ \\
\hline Thrombosis & - & $1(2 \%)$ & - & - \\
\hline Bile duct, cyst & $2(4 \%)$ & $1(2 \%)$ & $4(8 \%)$ & $4(8 \%)$ \\
\hline Bile duct, cyst, multiple & $2(4 \%)$ & $1(2 \%)$ & $2(4 \%)$ & $2(4 \%)$ \\
\hline Bile duct, hyperplasia & $2(4 \%)$ & - & - & - \\
\hline Centrilobular, degeneration & - & $1(2 \%)$ & - & - \\
\hline Mesentery & $(0)$ & $(2)$ & (2) & $(1)$ \\
\hline Artery, inflammation, chronic active & - & $1(50 \%)$ & - & - \\
\hline Oral mucosa & (50) & (50) & $(50)$ & $(50)$ \\
\hline Hyperplasia, squamous & - & - & $1(2 \%)$ & $2(4 \%)$ \\
\hline Pancreas & (50) & (49) & (49) & $(48)$ \\
\hline Acinus, hyperplasia & $1(2 \%)$ & $3(6 \%)$ & - & $1(2 \%)$ \\
\hline Artery, inflammation, chronic active & $1(2 \%)$ & $4(8 \%)$ & $3(6 \%)$ & $6(13 \%)$ \\
\hline Salivary glands & (50) & (49) & (48) & (50) \\
\hline Inflammation & $1(2 \%)$ & - & - & - \\
\hline Stomach, forestomach & $(50)$ & $(50)$ & $(50)$ & (49) \\
\hline Hyperplasia & $2(4 \%)$ & $5(10 \%)$ & $7(14 \%)$ & $3(6 \%)$ \\
\hline Inflammation & $1(2 \%)$ & - & - & $1(2 \%)$ \\
\hline Pigmentation, melanin & - & $1(2 \%)$ & - & - \\
\hline Ulcer & $1(2 \%)$ & - & - & - \\
\hline Stomach, glandular & (50) & (49) & $(50)$ & (49) \\
\hline Erosion & - & - & $1(2 \%)$ & - \\
\hline Ulcer & $1(2 \%)$ & - & - & - \\
\hline Epithelium, hyperplasia & $1(2 \%)$ & - & $1(2 \%)$ & - \\
\hline Epithelium, mineralization & - & - & $1(2 \%)$ & - \\
\hline Tongue & (1) & $(1)$ & $(0)$ & (1) \\
\hline Cyst & - & - & - & $1(100 \%)$ \\
\hline Erosion & $1(100 \%)$ & - & - & - \\
\hline Inflammation & - & $1(100 \%)$ & - & - \\
\hline Tooth & $(0)$ & (1) & $(0)$ & $(0)$ \\
\hline \multicolumn{5}{|l|}{ Cardiovascular System } \\
\hline Blood vessel & $(50)$ & $(50)$ & $(50)$ & $(50)$ \\
\hline Degeneration, focal & - & - & $1(2 \%)$ & - \\
\hline Inflammation, chronic active & $1(2 \%)$ & $3(6 \%)$ & $4(8 \%)$ & 7 (14\%) \\
\hline Thrombosis & - & - & $1(2 \%)$ & - \\
\hline Heart & (50) & (50) & (50) & (49) \\
\hline Cardiomyopathy & $24(48 \%)$ & $24(48 \%)$ & $24(48 \%)$ & 24 (49\%) \\
\hline
\end{tabular}


Indole-3-carbinol, NTP TR 584

\begin{tabular}{|c|c|c|c|c|}
\hline & Vehicle Control & $75 \mathrm{mg} / \mathrm{kg}$ & $150 \mathrm{mg} / \mathrm{kg}$ & $300 \mathrm{mg} / \mathrm{kg}$ \\
\hline Atrium, thrombosis & - & $1(2 \%)$ & - & - \\
\hline Epicardium, inflammation & $1(2 \%)$ & $3(6 \%)$ & - & $1(2 \%)$ \\
\hline Pericardium, inflammation & - & - & $2(4 \%)$ & - \\
\hline \multicolumn{5}{|l|}{ Endocrine System } \\
\hline Adrenal cortex & (50) & $(50)$ & $(50)$ & (48) \\
\hline Degeneration, cystic & $16(32 \%)$ & $12(24 \%)$ & $11(22 \%)$ & $15(31 \%)$ \\
\hline Degeneration, fatty & $5(10 \%)$ & $3(6 \%)$ & - & $1(2 \%)$ \\
\hline Hyperplasia & $18(36 \%)$ & $11(22 \%)$ & $18(36 \%)$ & 15 (31\%) \\
\hline Hypertrophy & $1(2 \%)$ & - & - & - \\
\hline Inflammation & - & $1(2 \%)$ & - & $1(2 \%)$ \\
\hline Necrosis & - & - & $1(2 \%)$ & $1(2 \%)$ \\
\hline Thrombosis & - & $1(2 \%)$ & $1(2 \%)$ & - \\
\hline Bilateral, degeneration, cystic & $1(2 \%)$ & - & - & - \\
\hline Adrenal medulla & (50) & $(50)$ & $(50)$ & $(48)$ \\
\hline Hyperplasia & 15 (30\%) & $13(26 \%)$ & $11(22 \%)$ & $12(25 \%)$ \\
\hline Islets, pancreatic & (50) & $(50)$ & $(50)$ & (50) \\
\hline Parathyroid gland & (47) & (49) & (46) & (49) \\
\hline Hyperplasia & - & $2(4 \%)$ & - & - \\
\hline Pituitary gland & (50) & $(50)$ & $(50)$ & (49) \\
\hline Pars distalis, hyperplasia & 19 (38\%) & $14(28 \%)$ & $18(36 \%)$ & $24(49 \%)$ \\
\hline Thyroid gland & (49) & (49) & (48) & (47) \\
\hline C-cell, hyperplasia & $7(14 \%)$ & $5(10 \%)$ & $6(13 \%)$ & $5(11 \%)$ \\
\hline Follicular cell, hyperplasia & $1(2 \%)$ & - & $1(2 \%)$ & $1(2 \%)$ \\
\hline Follicular cell, hypertrophy & 27 (55\%) & $23(47 \%)$ & $24(50 \%)$ & $30(64 \%)$ \\
\hline
\end{tabular}

\section{General Body System}

None

\section{Genital System}

Clitoral gland

(50)

Cyst

5 (10\%)

$2(4 \%)$

$3(6 \%)$

4 (8\%)

Inflammation

$1(2 \%)$

$-$

2 (4\%)

Ovary

(50)

(50)

Angiectasis

Atrophy

40 (80\%)

$-$

1 (2\%)

Cyst

17 (34\%)

23 (46\%)

37 (74\%)

30 (61\%)

Inflammation, acute

$13(26 \%)$

$16(32 \%)$

16 (33\%)

Inflammation, chronic

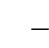

$-$

$1(2 \%)$

1 (2\%)

$-$

$-$

$1(2 \%)$ 
Indole-3-carbinol, NTP TR 584

\begin{tabular}{|c|c|c|c|c|}
\hline & Vehicle Control & $75 \mathrm{mg} / \mathrm{kg}$ & $150 \mathrm{mg} / \mathrm{kg}$ & $300 \mathrm{mg} / \mathrm{kg}$ \\
\hline Inflammation, chronic active & $1(2 \%)$ & $1(2 \%)$ & - & $1(2 \%)$ \\
\hline Necrosis, fibrinoid & - & - & $1(2 \%)$ & - \\
\hline Uterus & (50) & $(50)$ & (50) & $(50)$ \\
\hline Cyst & - & - & $2(4 \%)$ & - \\
\hline Dilatation & $9(18 \%)$ & $9(18 \%)$ & $7(14 \%)$ & $5(10 \%)$ \\
\hline Inflammation, histiocytic, focal & $1(2 \%)$ & - & - & - \\
\hline Inflammation, acute & $5(10 \%)$ & $12(24 \%)$ & $6(12 \%)$ & $9(18 \%)$ \\
\hline Thrombosis & - & - & $1(2 \%)$ & $1(2 \%)$ \\
\hline Endometrium, hyperplasia, cystic & 29 (58\%) & $28(56 \%)$ & $29(58 \%)$ & $21(42 \%)$ \\
\hline Endometrium, metaplasia, squamous & $12(24 \%)$ & $18(36 \%)$ & $20(40 \%)$ & $11(22 \%)$ \\
\hline Vagina & $(2)$ & (1) & $(0)$ & $(2)$ \\
\hline \multicolumn{5}{|l|}{ Hematopoietic System } \\
\hline Bone marrow & (50) & $(50)$ & (50) & (50) \\
\hline Atrophy & - & $1(2 \%)$ & $1(2 \%)$ & - \\
\hline Hyperplasia & $21(42 \%)$ & $20(40 \%)$ & $23(46 \%)$ & 17 (34\%) \\
\hline Lymph node & (7) & (6) & (4) & (3) \\
\hline Axillary, hyperplasia, lymphoid & - & - & - & $1(33 \%)$ \\
\hline Iliac, hyperplasia, lymphoid & - & $1(17 \%)$ & - & - \\
\hline Iliac, infiltration cellular, histiocyte & - & - & - & $1(33 \%)$ \\
\hline Inguinal, hemorrhage & - & $1(17 \%)$ & - & - \\
\hline Inguinal, hyperplasia, lymphoid & $1(14 \%)$ & - & $1(25 \%)$ & - \\
\hline Lumbar, hemorrhage & $2(29 \%)$ & $1(17 \%)$ & - & - \\
\hline Lumbar, hyperplasia, lymphoid & $1(14 \%)$ & $1(17 \%)$ & - & - \\
\hline Lumbar, infiltration cellular, histiocyte & - & $1(17 \%)$ & $1(25 \%)$ & - \\
\hline Mediastinal, ectasia & - & - & $1(25 \%)$ & - \\
\hline Mediastinal, hemorrhage & $2(29 \%)$ & - & $1(25 \%)$ & - \\
\hline Mediastinal, infiltration cellular, histiocyte & $1(14 \%)$ & - & - & - \\
\hline Lymph node, mandibular & (50) & (49) & (49) & $(50)$ \\
\hline Lymph node, mesenteric & $(50)$ & $(50)$ & $(50)$ & $(48)$ \\
\hline Hemorrhage & - & - & - & $1(2 \%)$ \\
\hline Pigmentation, hemosiderin & $1(2 \%)$ & - & - & - \\
\hline Lymphatic, ectasia & - & - & $1(2 \%)$ & $15(31 \%)$ \\
\hline Spleen & $(50)$ & $(50)$ & $(50)$ & $(48)$ \\
\hline Hematopoietic cell proliferation & $36(72 \%)$ & $32(64 \%)$ & $40(80 \%)$ & $33(69 \%)$ \\
\hline Thymus & $(47)$ & $(50)$ & (49) & (50) \\
\hline Atrophy & 38 (81\%) & 31 (62\%) & 34 (69\%) & $38(76 \%)$ \\
\hline
\end{tabular}


Indole-3-carbinol, NTP TR 584

\begin{tabular}{|c|c|c|c|c|}
\hline & Vehicle Control & 75 mg/kg & $150 \mathrm{mg} / \mathrm{kg}$ & $300 \mathrm{mg} / \mathrm{kg}$ \\
\hline Hemorrhage & - & - & - & $1(2 \%)$ \\
\hline Inflammation & - & $1(2 \%)$ & $1(2 \%)$ & - \\
\hline Epithelial cell, hyperplasia & $1(2 \%)$ & $4(8 \%)$ & $3(6 \%)$ & - \\
\hline \multicolumn{5}{|l|}{ Integumentary System } \\
\hline Mammary gland & (50) & (50) & (50) & (50) \\
\hline Galactocele & $1(2 \%)$ & - & - & - \\
\hline Hyperplasia & $8(16 \%)$ & $3(6 \%)$ & 7 (14\%) & 7 (14\%) \\
\hline Inflammation & $1(2 \%)$ & $1(2 \%)$ & - & - \\
\hline Inflammation, chronic & $1(2 \%)$ & - & - & - \\
\hline Duct, dilatation & $1(2 \%)$ & - & - & - \\
\hline Skin & $(50)$ & $(50)$ & (50) & (50) \\
\hline Cyst epithelial inclusion & - & $1(2 \%)$ & - & $1(2 \%)$ \\
\hline Fibrosis & - & - & - & $1(2 \%)$ \\
\hline \multicolumn{5}{|l|}{ Musculoskeletal System } \\
\hline Bone & (50) & (50) & (50) & (50) \\
\hline Fibrous osteodystrophy & - & $1(2 \%)$ & $1(2 \%)$ & - \\
\hline Skeletal muscle & (1) & $(0)$ & (1) & (1) \\
\hline Degeneration & - & - & $1(100 \%)$ & - \\
\hline \multicolumn{5}{|l|}{ Nervous System } \\
\hline Brain & (50) & $(50)$ & (50) & $(50)$ \\
\hline Gliosis & - & $1(2 \%)$ & - & - \\
\hline Hemorrhage & - & - & - & $1(2 \%)$ \\
\hline Inflammation & - & $1(2 \%)$ & - & - \\
\hline Necrosis & $1(2 \%)$ & - & - & $1(2 \%)$ \\
\hline Meninges, pigmentation, lipofuscin & - & $1(2 \%)$ & - & - \\
\hline Peripheral nerve & $(0)$ & (2) & $(0)$ & $(0)$ \\
\hline Spinal cord & $(0)$ & (1) & $(0)$ & $(0)$ \\
\hline Hemorrhage & - & $1(100 \%)$ & - & - \\
\hline Necrosis & - & $1(100 \%)$ & - & - \\
\hline \multicolumn{5}{|l|}{ Respiratory System } \\
\hline Lung & $(50)$ & $(50)$ & $(50)$ & $(49)$ \\
\hline Atelectasis & - & $1(2 \%)$ & - & - \\
\hline Foreign body & - & $2(4 \%)$ & - & - \\
\hline Inflammation, granulomatous & $1(2 \%)$ & - & - & - \\
\hline Inflammation, chronic & $34(68 \%)$ & $30(60 \%)$ & $38(76 \%)$ & 35 (71\%) \\
\hline Alveolar epithelium, hyperplasia & - & - & - & $1(2 \%)$ \\
\hline
\end{tabular}


Indole-3-carbinol, NTP TR 584

\begin{tabular}{|c|c|c|c|c|}
\hline & Vehicle Control & $75 \mathrm{mg} / \mathrm{kg}$ & $150 \mathrm{mg} / \mathrm{kg}$ & $300 \mathrm{mg} / \mathrm{kg}$ \\
\hline Alveolar epithelium, metaplasia, squamous & - & - & - & $1(2 \%)$ \\
\hline Alveolus, infiltration cellular, histiocyte & $13(26 \%)$ & $16(32 \%)$ & $11(22 \%)$ & $9(18 \%)$ \\
\hline Bronchiole, hyperplasia & - & $1(2 \%)$ & $1(2 \%)$ & - \\
\hline Mediastinum, inflammation & - & $1(2 \%)$ & - & - \\
\hline Pleura, inflammation, chronic active & - & $1(2 \%)$ & - & - \\
\hline Nose & (49) & $(50)$ & (49) & $(50)$ \\
\hline Angiectasis & - & - & $1(2 \%)$ & - \\
\hline Foreign body & $3(6 \%)$ & $2(4 \%)$ & $3(6 \%)$ & $3(6 \%)$ \\
\hline Inflammation, chronic active & $5(10 \%)$ & $4(8 \%)$ & $5(10 \%)$ & $6(12 \%)$ \\
\hline Glands, olfactory epithelium, hyperplasia & - & $1(2 \%)$ & - & - \\
\hline Olfactory epithelium, atrophy & - & - & $2(4 \%)$ & $5(10 \%)$ \\
\hline Olfactory epithelium, degeneration & $1(2 \%)$ & - & $1(2 \%)$ & - \\
\hline Respiratory epithelium, hyperplasia & - & - & $3(6 \%)$ & $2(4 \%)$ \\
\hline Respiratory epithelium, metaplasia, squamous & - & - & $1(2 \%)$ & $1(2 \%)$ \\
\hline Trachea & (50) & $(50)$ & $(50)$ & $(50)$ \\
\hline Epithelium, necrosis & - & $1(2 \%)$ & - & - \\
\hline \multicolumn{5}{|l|}{ Special Senses System } \\
\hline Eye & $(50)$ & $(50)$ & $(50)$ & $(50)$ \\
\hline Cataract & $1(2 \%)$ & - & - & - \\
\hline Inflammation & - & $1(2 \%)$ & $1(2 \%)$ & - \\
\hline Harderian gland & $(50)$ & $(50)$ & $(50)$ & $(50)$ \\
\hline Hyperplasia, focal & - & - & $1(2 \%)$ & - \\
\hline Inflammation & - & $1(2 \%)$ & - & - \\
\hline \multicolumn{5}{|l|}{ Urinary System } \\
\hline Kidney & $(50)$ & $(50)$ & $(50)$ & $(48)$ \\
\hline Nephropathy & 45 (90\%) & $41(82 \%)$ & $42(84 \%)$ & $43(90 \%)$ \\
\hline Pelvis, inflammation & - & - & - & $1(2 \%)$ \\
\hline Urinary bladder & (49) & $(50)$ & $(50)$ & $(50)$ \\
\hline Hyperplasia & - & - & $1(2 \%)$ & - \\
\hline
\end{tabular}




\section{Appendix C. Summary of Lesions in Male Mice in the Two-year Gavage Study of Indole-3-carbinol}

\section{Tables}

Table C-1. Summary of the Incidence of Neoplasms in Male Mice in the Two-year Gavage Study of Indole-3-carbinol

Table C-2. Statistical Analysis of Primary Neoplasms in Male Mice in the Two-year

Gavage Study of Indole-3-carbinol

Table C-3. Historical Incidence of Liver Neoplasms in Control Male B6C3F1/N Mice a ........ C-11

Table C-4. Summary of the Incidence of Nonneoplastic Lesions in Male Mice in the Two-year Gavage Study of Indole-3-carbinol 
Indole-3-carbinol, NTP TR 584

Table C-1. Summary of the Incidence of Neoplasms in Male Mice in the Two-year Gavage Study of Indole-3-carbinol $^{\mathrm{a}}$

$\begin{aligned} & \text { Vehicle } \\ & \text { Control }\end{aligned} \quad 62.5 \mathrm{mg} / \mathrm{kg} \quad 125 \mathrm{mg} / \mathrm{kg} \quad 250 \mathrm{mg} / \mathrm{kg}$

\section{Disposition Summary}

Animals initially in study

50

50

50

50

Early deaths

Accidental deaths

Moribund

- $\quad-$

1

Natural deaths

17

6

12

1

9

Survivors

Died last week of study

Terminal euthanasia

Animals examined microscopically

\section{Alimentary System}

Esophagus

Gallbladder

$1(2 \%)$

Carcinoma, metastatic, pancreas

$-$

Squamous cell carcinoma, metastatic, stomach,

$1(2 \%)$ forestomach

Intestine large, cecum

Intestine large, colon

Intestine large, rectum

Intestine small, duodenum

Adenoma

$2(4 \%)$

Carcinoma

Intestine small, ileum

Intestine small, jejunum

Adenoma

$1(2 \%)$

Liver

$$
\begin{aligned}
& \text { Alveolar/bronchiolar carcinoma, metastatic, lung } \\
& \text { Carcinoma, metastatic, intestine small, duodenum } \\
& \text { Fibrous histiocytoma } \\
& \text { Hemangioma } \\
& \text { Hemangiosarcoma } \\
& \text { Hemangiosarcoma, multiple } \\
& \text { Hepatoblastoma } \\
& \text { Hepatoblastoma, multiple } \\
& \text { Hepatocellular adenoma } \\
& \text { Hepatocellular adenoma, multiple }
\end{aligned}
$$

- $\quad 1(2 \%)$

$-$

$1(2 \%)$

$-$

$1(2 \%)$

(50) (49)

- -

$-\quad 1(2 \%)$

$-$

$-$

$-$

$1(2 \%)$

$3(6 \%)$

$2(4 \%)$

$-$

$1(2 \%)$

$1(2 \%)$

$2(4 \%)$

$1(2 \%)$

$2(4 \%)$

$3(6 \%)$

$3(6 \%)$

$1(2 \%)$

$-$

$1(2 \%)$

$1(2 \%)$

$7(14 \%)$

$11(22 \%)$

7 (14\%)

$3(6 \%)$

$7(14 \%)$

15 (30\%)

25 (50\%)

15 (31\%)

8 (16\%)

16 (33\%)

33 (66\%) 
Indole-3-carbinol, NTP TR 584

\begin{tabular}{|c|c|c|c|c|}
\hline & $\begin{array}{l}\text { Vehicle } \\
\text { Control }\end{array}$ & $62.5 \mathrm{mg} / \mathrm{kg}$ & $125 \mathrm{mg} / \mathrm{kg}$ & $250 \mathrm{mg} / \mathrm{kg}$ \\
\hline Hepatocellular carcinoma & $7(14 \%)$ & $8(16 \%)$ & $11(22 \%)$ & $6(12 \%)$ \\
\hline Hepatocellular carcinoma, multiple & $5(10 \%)$ & $3(6 \%)$ & $18(37 \%)$ & $8(16 \%)$ \\
\hline Hepatocholangiocarcinoma & - & $3(6 \%)$ & $1(2 \%)$ & - \\
\hline Hepatocholangioma & - & - & - & $1(2 \%)$ \\
\hline $\begin{array}{l}\text { Squamous cell carcinoma, metastatic, stomach, } \\
\text { forestomach }\end{array}$ & $1(2 \%)$ & - & - & - \\
\hline Mesentery & (7) & (3) & $(2)$ & $(2)$ \\
\hline Fibrosarcoma & - & $1(33 \%)$ & - & - \\
\hline Hepatoblastoma, metastatic, liver & - & $1(33 \%)$ & - & - \\
\hline Hepatocellular carcinoma, metastatic, liver & $1(14 \%)$ & - & - & - \\
\hline $\begin{array}{l}\text { Leiomyosarcoma, metastatic, stomach, } \\
\text { forestomach }\end{array}$ & - & $1(33 \%)$ & - & - \\
\hline $\begin{array}{l}\text { Squamous cell carcinoma, metastatic, stomach, } \\
\text { forestomach }\end{array}$ & $1(14 \%)$ & - & - & - \\
\hline Oral mucosa & $(0)$ & (1) & $(0)$ & $(2)$ \\
\hline Pancreas & $(50)$ & $(50)$ & $(50)$ & $(50)$ \\
\hline Carcinoma & - & - & $1(2 \%)$ & - \\
\hline Hemangioma & - & $1(2 \%)$ & - & - \\
\hline Hepatocellular carcinoma, metastatic, liver & $1(2 \%)$ & - & - & - \\
\hline Leiomyosarcoma, metastatic, stomach, glandular & - & $1(2 \%)$ & - & - \\
\hline $\begin{array}{l}\text { Squamous cell carcinoma, metastatic, stomach, } \\
\text { forestomach }\end{array}$ & $1(2 \%)$ & - & - & - \\
\hline Salivary glands & $(50)$ & $(50)$ & $(50)$ & $(50)$ \\
\hline Adenoma & $1(2 \%)$ & - & - & - \\
\hline Hemangiosarcoma & $1(2 \%)$ & - & - & - \\
\hline Stomach, forestomach & $(50)$ & $(50)$ & $(50)$ & $(50)$ \\
\hline Squamous cell carcinoma & $1(2 \%)$ & - & - & - \\
\hline Squamous cell papilloma & $1(2 \%)$ & $1(2 \%)$ & $1(2 \%)$ & - \\
\hline Squamous cell papilloma, multiple & - & $1(2 \%)$ & - & - \\
\hline Stomach, glandular & $(50)$ & $(47)$ & $(47)$ & $(49)$ \\
\hline Carcinoma & - & - & $1(2 \%)$ & - \\
\hline Carcinoma, metastatic, intestine small, duodenum & - & - & - & $1(2 \%)$ \\
\hline Carcinoma, metastatic, pancreas & - & - & $1(2 \%)$ & - \\
\hline Leiomyosarcoma & & $1(2 \%)$ & - & - \\
\hline Tooth & (30) & (37) & $(32)$ & $(32)$ \\
\hline \multicolumn{5}{|l|}{ Cardiovascular System } \\
\hline Blood vessel & (49) & $(50)$ & (49) & (49) \\
\hline Heart & $(50)$ & $(50)$ & $(50)$ & (50) \\
\hline
\end{tabular}


Indole-3-carbinol, NTP TR 584

\begin{tabular}{|c|c|c|c|c|}
\hline & $\begin{array}{l}\text { Vehicle } \\
\text { Control }\end{array}$ & $62.5 \mathrm{mg} / \mathrm{kg}$ & $125 \mathrm{mg} / \mathrm{kg}$ & $250 \mathrm{mg} / \mathrm{kg}$ \\
\hline Alveolar/bronchiolar carcinoma, metastatic, lung & - & $1(2 \%)$ & - & - \\
\hline Hemangiosarcoma & - & $1(2 \%)$ & - & - \\
\hline \multicolumn{5}{|l|}{ Endocrine system } \\
\hline Adrenal cortex & $(50)$ & $(50)$ & $(50)$ & $(50)$ \\
\hline Adenoma & - & - & $2(4 \%)$ & - \\
\hline Alveolar/bronchiolar carcinoma, metastatic, lung & - & $1(2 \%)$ & - & - \\
\hline Adrenal medulla & $(50)$ & $(50)$ & $(50)$ & $(50)$ \\
\hline Pheochromocytoma benign & - & $1(2 \%)$ & - & - \\
\hline Islets, pancreatic & $(50)$ & $(50)$ & $(50)$ & (50) \\
\hline Adenoma & $1(2 \%)$ & - & - & $1(2 \%)$ \\
\hline Parathyroid gland & $(48)$ & $(44)$ & $(48)$ & $(44)$ \\
\hline Pituitary gland & $(50)$ & $(50)$ & $(50)$ & $(50)$ \\
\hline Pars intermedia, adenoma & - & $1(2 \%)$ & $1(2 \%)$ & - \\
\hline Thyroid gland & $(50)$ & $(50)$ & $(50)$ & (50) \\
\hline C-cell, adenoma & - & $1(2 \%)$ & - & $1(2 \%)$ \\
\hline Follicular cell, adenoma & - & - & $1(2 \%)$ & $1(2 \%)$ \\
\hline \multicolumn{5}{|l|}{ General Body System } \\
\hline None & - & - & - & - \\
\hline \multicolumn{5}{|l|}{ Genital System } \\
\hline Coagulating gland & $(1)$ & $(0)$ & $(0)$ & $(0)$ \\
\hline Epididymis & $(50)$ & $(50)$ & $(50)$ & $(50)$ \\
\hline Hepatocellular carcinoma, metastatic, liver & $1(2 \%)$ & - & - & - \\
\hline Leiomyosarcoma, metastatic, stomach, glandular & - & $1(2 \%)$ & - & - \\
\hline Preputial gland & $(50)$ & $(50)$ & $(50)$ & (50) \\
\hline Prostate & $(50)$ & $(50)$ & $(50)$ & (50) \\
\hline $\begin{array}{l}\text { Squamous cell carcinoma, metastatic, stomach, } \\
\text { forestomach }\end{array}$ & $1(2 \%)$ & - & - & - \\
\hline Seminal vesicle & $(50)$ & $(50)$ & $(50)$ & (50) \\
\hline Testes & $(50)$ & (50) & (50) & $(50)$ \\
\hline Bilateral, interstitial cell, adenoma & - & - & - & $1(2 \%)$ \\
\hline Interstitial cell, adenoma & - & $1(2 \%)$ & - & $1(2 \%)$ \\
\hline \multicolumn{5}{|l|}{ Hematopoietic System } \\
\hline Bone marrow & $(50)$ & $(50)$ & $(50)$ & $(50)$ \\
\hline Hemangiosarcoma & $1(2 \%)$ & $1(2 \%)$ & $1(2 \%)$ & - \\
\hline Lymph node & $(2)$ & $(2)$ & $(0)$ & (4) \\
\hline Lymph node, mandibular & $(50)$ & $(50)$ & (50) & (50) \\
\hline Lymph node, mesenteric & (48) & (48) & (49) & $(50)$ \\
\hline
\end{tabular}


Indole-3-carbinol, NTP TR 584

\begin{tabular}{|c|c|c|c|c|}
\hline & $\begin{array}{l}\text { Vehicle } \\
\text { Control }\end{array}$ & $62.5 \mathrm{mg} / \mathrm{kg}$ & $125 \mathrm{mg} / \mathrm{kg}$ & $250 \mathrm{mg} / \mathrm{kg}$ \\
\hline Hemangiosarcoma & - & $1(2 \%)$ & - & - \\
\hline Spleen & $(50)$ & $(50)$ & $(50)$ & $(50)$ \\
\hline Hemangiosarcoma & $1(2 \%)$ & $1(2 \%)$ & $1(2 \%)$ & $1(2 \%)$ \\
\hline Thymus & $(49)$ & $(47)$ & $(46)$ & $(45)$ \\
\hline Alveolar/bronchiolar carcinoma, metastatic, lung & - & - & - & $1(2 \%)$ \\
\hline Hepatocellular carcinoma, metastatic, liver & - & - & $1(2 \%)$ & - \\
\hline $\begin{array}{l}\text { Squamous cell carcinoma, metastatic, stomach, } \\
\text { forestomach }\end{array}$ & $1(2 \%)$ & - & - & - \\
\hline Thymoma benign & $1(2 \%)$ & - & - & - \\
\hline \multicolumn{5}{|l|}{ Integumentary System } \\
\hline Mammary gland & $(2)$ & $(0)$ & $(0)$ & $(0)$ \\
\hline Skin & $(50)$ & $(50)$ & $(50)$ & $(50)$ \\
\hline Epidermis, keratoacanthoma & - & - & $1(2 \%)$ & - \\
\hline Subcutaneous tissue, fibrosarcoma & $1(2 \%)$ & $1(2 \%)$ & - & - \\
\hline Subcutaneous tissue, hemangiosarcoma & - & $2(4 \%)$ & $1(2 \%)$ & - \\
\hline Subcutaneous tissue, lipoma & - & - & - & $1(2 \%)$ \\
\hline Subcutaneous tissue, neural crest tumor & - & - & $1(2 \%)$ & - \\
\hline Subcutaneous tissue, schwannoma malignant & - & - & $1(2 \%)$ & - \\
\hline \multicolumn{5}{|l|}{ Musculoskeletal System } \\
\hline Bone & $(50)$ & $(50)$ & $(50)$ & $(50)$ \\
\hline Skeletal muscle & (0) & $(0)$ & $(2)$ & $(0)$ \\
\hline Hemangiosarcoma & - & - & $2(100 \%)$ & - \\
\hline \multicolumn{5}{|l|}{ Nervous System } \\
\hline Brain & $(50)$ & $(50)$ & $(50)$ & $(50)$ \\
\hline Granular cell tumor benign & $1(2 \%)$ & - & - & - \\
\hline \multicolumn{5}{|l|}{ Respiratory System } \\
\hline Lung & $(50)$ & $(50)$ & $(50)$ & $(50)$ \\
\hline Alveolar/bronchiolar adenoma & $4(8 \%)$ & $6(12 \%)$ & $6(12 \%)$ & $8(16 \%)$ \\
\hline Alveolar/bronchiolar adenoma, multiple & - & $1(2 \%)$ & $2(4 \%)$ & - \\
\hline Alveolar/bronchiolar carcinoma & $3(6 \%)$ & $7(14 \%)$ & $5(10 \%)$ & $4(8 \%)$ \\
\hline Alveolar/bronchiolar carcinoma, multiple & $1(2 \%)$ & - & - & $1(2 \%)$ \\
\hline Carcinoma, metastatic, harderian gland & - & - & - & $1(2 \%)$ \\
\hline Hepatoblastoma, metastatic, liver & - & $1(2 \%)$ & $1(2 \%)$ & $2(4 \%)$ \\
\hline Hepatocellular carcinoma, metastatic, liver & $6(12 \%)$ & $3(6 \%)$ & $10(20 \%)$ & $4(8 \%)$ \\
\hline Hepatocholangiocarcinoma, metastatic, liver & - & $1(2 \%)$ & - & - \\
\hline Nose & $(50)$ & $(50)$ & $(50)$ & $(50)$ \\
\hline Trachea & $(50)$ & $(50)$ & $(50)$ & $(50)$ \\
\hline
\end{tabular}


Indole-3-carbinol, NTP TR 584

\begin{tabular}{|c|c|c|c|c|}
\hline & $\begin{array}{l}\text { Vehicle } \\
\text { Control }\end{array}$ & $62.5 \mathrm{mg} / \mathrm{kg}$ & $125 \mathrm{mg} / \mathrm{kg}$ & $250 \mathrm{mg} / \mathrm{kg}$ \\
\hline \multicolumn{5}{|l|}{ Special Senses System } \\
\hline Eye & $(50)$ & $(50)$ & $(50)$ & $(50)$ \\
\hline Harderian gland & $(50)$ & $(50)$ & $(50)$ & $(50)$ \\
\hline Adenoma & $6(12 \%)$ & $6(12 \%)$ & $7(14 \%)$ & $3(6 \%)$ \\
\hline Carcinoma & $1(2 \%)$ & $1(2 \%)$ & - & $1(2 \%)$ \\
\hline Bilateral, adenoma & - & $1(2 \%)$ & - & - \\
\hline \multicolumn{5}{|l|}{ Urinary System } \\
\hline Kidney & $(50)$ & $(50)$ & $(50)$ & $(50)$ \\
\hline Alveolar/bronchiolar carcinoma, metastatic, lung & - & $1(2 \%)$ & - & $1(2 \%)$ \\
\hline Hepatocellular carcinoma, metastatic, liver & $1(2 \%)$ & - & - & - \\
\hline Urethra & $(0)$ & (1) & $(0)$ & $(0)$ \\
\hline Urinary bladder & $(50)$ & $(50)$ & $(50)$ & (50) \\
\hline \multicolumn{5}{|l|}{ Systemic Lesions } \\
\hline Multiple organs $^{\mathrm{b}}$ & $(50)$ & $(50)$ & $(50)$ & $(50)$ \\
\hline Histiocytic sarcoma & $2(4 \%)$ & $1(2 \%)$ & - & $1(2 \%)$ \\
\hline Lymphoma malignant & $3(6 \%)$ & $1(2 \%)$ & $1(2 \%)$ & $5(10 \%)$ \\
\hline \multicolumn{5}{|l|}{ Neoplasm Summary } \\
\hline Total animals with primary neoplasms ${ }^{c}$ & 48 & 45 & 48 & 48 \\
\hline Total primary neoplasms & 78 & 95 & 103 & 107 \\
\hline Total animals with benign neoplasms & 33 & 40 & 39 & 42 \\
\hline Total benign neoplasms & 44 & 53 & 53 & 61 \\
\hline Total animals with malignant neoplasms & 27 & 26 & 36 & 35 \\
\hline Total malignant neoplasms & 34 & 42 & 49 & 46 \\
\hline Total animals with metastatic neoplasms & 7 & 7 & 10 & 9 \\
\hline Total metastatic neoplasms & 16 & 13 & 14 & 11 \\
\hline $\begin{array}{l}\text { Total animals with uncertain neoplasms- } \\
\text { benign or malignant }\end{array}$ & - & - & 1 & - \\
\hline Total uncertain neoplasms & - & - & 1 & - \\
\hline
\end{tabular}

${ }^{a}$ Number of animals examined microscopically at the site and the number of animals with neoplasm.

${ }^{b}$ Number of animals with any tissue examined microscopically.

'Primary neoplasms: all neoplasms except metastatic neoplasms. 
Indole-3-carbinol, NTP TR 584

Table C-2. Statistical Analysis of Primary Neoplasms in Male Mice in the Two-year Gavage Study of Indole-3-carbinol

\section{Vehicle Control $\quad 62.5 \mathrm{mg} / \mathrm{kg} \quad 125 \mathrm{mg} / \mathrm{kg} \quad 250 \mathrm{mg} / \mathrm{kg}$}

\begin{tabular}{|c|c|c|c|c|}
\hline \multicolumn{5}{|c|}{ Harderian Gland: Adenoma } \\
\hline Overall rate ${ }^{\mathrm{a}}$ & 6/50 (12\%) & 7/50 (14\%) & 7/50 (14\%) & $3 / 50(6 \%)$ \\
\hline Adjusted rate $^{\mathrm{b}}$ & $14.5 \%$ & $15.4 \%$ & $15.6 \%$ & $6.7 \%$ \\
\hline Terminal rate $\mathrm{c}^{\mathrm{C}}$ & $2 / 26(8 \%)$ & $5 / 31(16 \%)$ & 4/32 (13\%) & $1 / 32(3 \%)$ \\
\hline First incidence (days) & 463 & 616 & 437 & 437 \\
\hline Poly-3 test ${ }^{\mathrm{d}}$ & $\mathrm{P}=0.144 \mathrm{~N}$ & $\mathrm{P}=0.571$ & $\mathrm{P}=0.564$ & $\mathrm{P}=0.205 \mathrm{~N}$ \\
\hline \multicolumn{5}{|c|}{ Harderian Gland: Adenoma or Carcinoma } \\
\hline Overall rate & $7 / 50(14 \%)$ & $8 / 50(16 \%)$ & $7 / 50(14 \%)$ & $4 / 50(8 \%)$ \\
\hline Adjusted rate & $16.7 \%$ & $17.6 \%$ & $15.6 \%$ & $9.0 \%$ \\
\hline Terminal rate & $2 / 26(8 \%)$ & 5/31 (16\%) & 4/32 (13\%) & $2 / 32(6 \%)$ \\
\hline First incidence (days) & 463 & 616 & 437 & 437 \\
\hline Poly-3 test & $\mathrm{P}=0.152 \mathrm{~N}$ & $P=0.568$ & $\mathrm{P}=0.560 \mathrm{~N}$ & $\mathrm{P}=0.226 \mathrm{~N}$ \\
\hline \multicolumn{5}{|c|}{ Liver: Hemangiosarcoma } \\
\hline Overall rate & $4 / 50(8 \%)$ & $4 / 50(8 \%)$ & $1 / 49(2 \%)$ & $3 / 50(6 \%)$ \\
\hline Adjusted rate & $10.0 \%$ & $8.8 \%$ & $2.3 \%$ & $6.8 \%$ \\
\hline Terminal rate & $2 / 26(8 \%)$ & 1/31 (3\%) & 0/32 (0\%) & 0/32 (0\%) \\
\hline First incidence (days) & 626 & 630 & 596 & 495 \\
\hline Poly-3 test & $\mathrm{P}=0.321 \mathrm{~N}$ & $\mathrm{P}=0.571 \mathrm{~N}$ & $\mathrm{P}=0.155 \mathrm{~N}$ & $P=0.445 N$ \\
\hline \multicolumn{5}{|c|}{ Liver: Hepatocellular Adenoma } \\
\hline Overall rate & $26 / 50$ (52\%) & $32 / 50$ (64\%) & $31 / 49$ (63\%) & $41 / 50(82 \%)$ \\
\hline Adjusted rate & $62.1 \%$ & $66.5 \%$ & $67.9 \%$ & $85.4 \%$ \\
\hline Terminal rate & 19/26 (73\%) & 21/31 (68\%) & 23/32 (72\%) & 28/32 (88\%) \\
\hline First incidence (days) & 463 & 381 & 437 & 385 \\
\hline Poly-3 test & $P=0.005$ & $\mathrm{P}=0.411$ & $\mathrm{P}=0.360$ & $P=0.006$ \\
\hline \multicolumn{5}{|c|}{ Liver: Hepatocellular Carcinoma } \\
\hline Overall rate & $12 / 50(24 \%)$ & $11 / 50(22 \%)$ & 29/49 (59\%) & $14 / 50(28 \%)$ \\
\hline Adjusted rate & $27.7 \%$ & $24.2 \%$ & $63.4 \%$ & $30.9 \%$ \\
\hline Terminal rate & $3 / 26(12 \%)$ & 8/31 (26\%) & $18 / 32(56 \%)$ & 8/32 (25\%) \\
\hline First incidence (days) & 465 & 682 & 567 & 385 \\
\hline Poly-3 test & $\mathrm{P}=0.217$ & $\mathrm{P}=0.449 \mathrm{~N}$ & $\mathrm{P}<0.001$ & $\mathrm{P}=0.461$ \\
\hline \multicolumn{5}{|c|}{ Liver: Hepatocellular Adenoma or Carcinoma } \\
\hline Overall rate & $35 / 50(70 \%)$ & $36 / 50$ (72\%) & 43/49 (88\%) & $44 / 50$ (88\%) \\
\hline Adjusted rate & $77.6 \%$ & $74.6 \%$ & $90.6 \%$ & $91.1 \%$ \\
\hline
\end{tabular}


Indole-3-carbinol, NTP TR 584

\begin{tabular}{|c|c|c|c|c|}
\hline & Vehicle Control & $62.5 \mathrm{mg} / \mathrm{kg}$ & $125 \mathrm{mg} / \mathrm{kg}$ & $250 \mathrm{mg} / \mathrm{kg}$ \\
\hline Terminal rate & 21/26 (81\%) & 24/31 (77\%) & 29/32 (91\%) & 30/32 (94\%) \\
\hline First incidence (days) & 463 & 381 & 437 & 385 \\
\hline Poly-3 test & $P=0.012$ & $\mathrm{P}=0.459 \mathrm{~N}$ & $P=0.062$ & $P=0.049$ \\
\hline \multicolumn{5}{|c|}{ Liver: Hepatoblastoma } \\
\hline Overall rate & $3 / 50(6 \%)$ & $4 / 50(8 \%)$ & $4 / 49(8 \%)$ & $14 / 50(28 \%)$ \\
\hline Adjusted rate & $7.6 \%$ & $8.9 \%$ & $9.3 \%$ & $31.6 \%$ \\
\hline Terminal rate & 3/26 (12\%) & 4/31 (13\%) & 3/32 (9\%) & 10/32 (31\%) \\
\hline First incidence (days) & $730(\mathrm{~T})$ & $730(\mathrm{~T})$ & 702 & 617 \\
\hline Poly-3 test & $\mathrm{P}<0.001$ & $P=0.567$ & $P=0.543$ & $\mathrm{P}=0.005$ \\
\hline \multicolumn{5}{|c|}{ Liver: Hepatocellular Carcinoma or Hepatoblastoma } \\
\hline Overall rate & $15 / 50(30 \%)$ & $13 / 50(26 \%)$ & $30 / 49(61 \%)$ & $25 / 50(50 \%)$ \\
\hline Adjusted rate & $34.6 \%$ & $28.7 \%$ & $65.6 \%$ & $54.5 \%$ \\
\hline Terminal rate & $6 / 26(23 \%)$ & 10/31 (32\%) & 19/32 (59\%) & $17 / 32(53 \%)$ \\
\hline First incidence (days) & 465 & 682 & 567 & 385 \\
\hline Poly-3 test & $\mathrm{P}=0.005$ & $\mathrm{P}=0.353 \mathrm{~N}$ & $\mathrm{P}=0.002$ & $\mathrm{P}=0.042$ \\
\hline \multicolumn{5}{|c|}{ Liver: Hepatocellular Adenoma, Hepatocellular Carcinoma, or Hepatoblastoma } \\
\hline Overall rate & $36 / 50$ (72\%) & $36 / 50$ (72\%) & $44 / 49(90 \%)$ & $45 / 50(90 \%)$ \\
\hline Adjusted rate & $79.8 \%$ & $74.6 \%$ & $92.7 \%$ & $92.8 \%$ \\
\hline Terminal rate & 22/26 (85\%) & 24/31 (77\%) & 30/32 (94\%) & 30/32 (94\%) \\
\hline First incidence (days) & 463 & 381 & 437 & 385 \\
\hline Poly-3 test & $\mathrm{P}=0.007$ & $\mathrm{P}=0.354 \mathrm{~N}$ & $\mathrm{P}=0.050$ & $\mathrm{P}=0.044$ \\
\hline \multicolumn{5}{|c|}{ Liver: Hepatocholangiocarcinoma } \\
\hline Overall rate & $0 / 50(0 \%)$ & $3 / 50(6 \%)$ & $1 / 49(2 \%)$ & $0 / 50(0 \%)$ \\
\hline Adjusted rate & $0.0 \%$ & $6.6 \%$ & $2.3 \%$ & $0.0 \%$ \\
\hline Terminal rate & $0 / 26(0 \%)$ & 0/31 (0\%) & $1 / 32(3 \%)$ & 0/32 (0\%) \\
\hline First incidence (days) & $-^{\mathrm{e}}$ & 630 & $730(\mathrm{~T})$ & - \\
\hline Poly-3 test & $\mathrm{P}=0.319 \mathrm{~N}$ & $P=0.146$ & $P=0.515$ & $-{ }^{f}$ \\
\hline \multicolumn{5}{|c|}{ Lung: Alveolar/Bronchiolar Adenoma } \\
\hline Overall rate & $4 / 50(8 \%)$ & 7/50 (14\%) & $8 / 50(16 \%)$ & $8 / 50(16 \%)$ \\
\hline Adjusted rate & $10.1 \%$ & $15.4 \%$ & $17.8 \%$ & $17.9 \%$ \\
\hline Terminal rate & 4/26 (15\%) & $5 / 31(16 \%)$ & 4/32 (13\%) & $5 / 32(16 \%)$ \\
\hline First incidence (days) & $730(\mathrm{~T})$ & 631 & 437 & 495 \\
\hline Poly-3 test & $\mathrm{P}=0.230$ & $\mathrm{P}=0.342$ & $\mathrm{P}=0.241$ & $\mathrm{P}=0.238$ \\
\hline \multicolumn{5}{|c|}{ Lung: Alveolar/Bronchiolar Carcinoma } \\
\hline Overall rate & $4 / 50(8 \%)$ & 7/50 (14\%) & 5/50 (10\%) & 5/50 (10\%) \\
\hline
\end{tabular}


Indole-3-carbinol, NTP TR 584

\begin{tabular}{|c|c|c|c|c|}
\hline & Vehicle Control & $62.5 \mathrm{mg} / \mathrm{kg}$ & $125 \mathrm{mg} / \mathrm{kg}$ & $250 \mathrm{mg} / \mathrm{kg}$ \\
\hline Adjusted rate & $10.0 \%$ & $15.4 \%$ & $11.4 \%$ & $11.4 \%$ \\
\hline Terminal rate & $3 / 26(12 \%)$ & 4/31 (13\%) & $3 / 32(9 \%)$ & $4 / 32(13 \%)$ \\
\hline First incidence (days) & 680 & 682 & 655 & 586 \\
\hline Poly-3 test & $\mathrm{P}=0.523 \mathrm{~N}$ & $P=0.337$ & $P=0.561$ & $P=0.560$ \\
\hline \multicolumn{5}{|c|}{ Lung: Alveolar/Bronchiolar Adenoma or Carcinoma } \\
\hline Overall rate & $8 / 50(16 \%)$ & $12 / 50(24 \%)$ & $13 / 50(26 \%)$ & $11 / 50(22 \%)$ \\
\hline Adjusted rate & $20.0 \%$ & $26.2 \%$ & $28.6 \%$ & $24.6 \%$ \\
\hline Terminal rate & $7 / 26(27 \%)$ & 7/31 (23\%) & 7/32 (22\%) & $8 / 32(25 \%)$ \\
\hline First incidence (days) & 680 & 631 & 437 & 495 \\
\hline Poly-3 test & $P=0.420$ & $\mathrm{P}=0.338$ & $\mathrm{P}=0.252$ & $\mathrm{P}=0.405$ \\
\hline \multicolumn{5}{|c|}{ Small Intestine (Site Unspecified): Adenoma } \\
\hline Overall rate & $3 / 50(6 \%)$ & $0 / 50(0 \%)$ & $1 / 50(2 \%)$ & $1 / 50(2 \%)$ \\
\hline Adjusted rate & $7.5 \%$ & $0.0 \%$ & $2.3 \%$ & $2.3 \%$ \\
\hline Terminal rate & $2 / 26(8 \%)$ & $0 / 31(0 \%)$ & $1 / 32(3 \%)$ & $1 / 32(3 \%)$ \\
\hline First incidence (days) & 728 & - & $730(\mathrm{~T})$ & $730(\mathrm{~T})$ \\
\hline Poly-3 test & $\mathrm{P}=0.308 \mathrm{~N}$ & $\mathrm{P}=0.098 \mathrm{~N}$ & $\mathrm{P}=0.273 \mathrm{~N}$ & $\mathrm{P}=0.274 \mathrm{~N}$ \\
\hline \multicolumn{5}{|c|}{ Small Intestine (Site Unspecified): Adenoma or Carcinoma } \\
\hline Overall rate & $3 / 50(6 \%)$ & $0 / 50(0 \%)$ & $1 / 50(2 \%)$ & $3 / 50(6 \%)$ \\
\hline Adjusted rate & $7.5 \%$ & $0.0 \%$ & $2.3 \%$ & $6.9 \%$ \\
\hline Terminal rate & $2 / 26(8 \%)$ & 0/31 (0\%) & $1 / 32(3 \%)$ & 3/32 (9\%) \\
\hline First incidence (days) & 728 & - & $730(\mathrm{~T})$ & $730(\mathrm{~T})$ \\
\hline Poly-3 test & $P=0.415$ & $\mathrm{P}=0.098 \mathrm{~N}$ & $\mathrm{P}=0.273 \mathrm{~N}$ & $\mathrm{P}=0.621 \mathrm{~N}$ \\
\hline \multicolumn{5}{|c|}{ All Organs: Hemangiosarcoma } \\
\hline Overall rate & $4 / 50(8 \%)$ & $5 / 50(10 \%)$ & $5 / 50(10 \%)$ & $3 / 50(6 \%)$ \\
\hline Adjusted rate & $10.0 \%$ & $10.9 \%$ & $11.1 \%$ & $6.8 \%$ \\
\hline Terminal rate & $2 / 26(8 \%)$ & $1 / 31(3 \%)$ & 1/32 (3\%) & 0/32 (0\%) \\
\hline First incidence (days) & 626 & 630 & 596 & 495 \\
\hline Poly-3 test & $\mathrm{P}=0.337 \mathrm{~N}$ & $P=0.581$ & $P=0.570$ & $\mathrm{P}=0.445 \mathrm{~N}$ \\
\hline \multicolumn{5}{|c|}{ All Organs: Hemangioma or Hemangiosarcoma } \\
\hline Overall rate & $4 / 50(8 \%)$ & $6 / 50(12 \%)$ & $5 / 50(10 \%)$ & $4 / 50(8 \%)$ \\
\hline Adjusted rate & $10.0 \%$ & $13.1 \%$ & $11.1 \%$ & $9.0 \%$ \\
\hline Terminal rate & 2/26 (8\%) & 2/31 (7\%) & 1/32 (3\%) & 1/32 (3\%) \\
\hline First incidence (days) & 626 & 630 & 596 & 495 \\
\hline Poly-3 test & $\mathrm{P}=0.430 \mathrm{~N}$ & $P=0.454$ & $P=0.570$ & $\mathrm{P}=0.588 \mathrm{~N}$ \\
\hline
\end{tabular}


Indole-3-carbinol, NTP TR 584

\section{Vehicle Control $\quad 62.5 \mathrm{mg} / \mathrm{kg} \quad 125 \mathrm{mg} / \mathrm{kg} \quad 250 \mathrm{mg} / \mathrm{kg}$}

\section{All Organs: Malignant Lymphoma}

Overall rate

Adjusted rate

Terminal rate

First incidence (days)

Poly-3 test

\section{All Organs: Benign Neoplasms}

Overall rate

Adjusted rate

Terminal rate

First incidence (days)

Poly-3 test

All Organs: Malignant Neoplasms

Overall rate

Adjusted rate

Terminal rate

First incidence (days)

Poly-3 test
$3 / 50(6 \%)$

$1 / 50(2 \%)$

$2.2 \%$

$1 / 26(4 \%)$

711

$\mathrm{P}=0.164$

$\mathrm{P}=0.262 \mathrm{~N}$

$1 / 50(2 \%)$

$5 / 50(10 \%)$

$11.3 \%$

3/32 (9\%)

631

$\mathrm{P}=0.413$

\section{All Organs: Benign or Malignant Neoplasms}

Overall rate

Adjusted rate

Terminal rate

First incidence (days)

Poly-3 test

$\begin{array}{cccc}33 / 50(66 \%) & 40 / 50(80 \%) & 39 / 50(78 \%) & 42 / 50(84 \%) \\ 77.2 \% & 81.8 \% & 82.8 \% & 86.1 \% \\ 23 / 26(89 \%) & 26 / 31(84 \%) & 28 / 32(88 \%) & 28 / 32(88 \%) \\ 463 & 381 & 437 & 385 \\ \mathrm{P}=0.166 & \mathrm{P}=0.381 & \mathrm{P}=0.331 & \mathrm{P}=0.182\end{array}$

$\begin{array}{cccc}27 / 50(54 \%) & 26 / 50(52 \%) & 36 / 50(72 \%) & 35 / 50(70 \%) \\ 58.2 \% & 54.5 \% & 75.4 \% & 73.8 \% \\ 10 / 26(39 \%) & 13 / 31(42 \%) & 21 / 32(66 \%) & 23 / 32(72 \%) \\ 465 & 381 & 567 & 385 \\ P=0.022 & P=0.437 N & P=0.055 & P=0.078\end{array}$

(T) Terminal euthanasia.

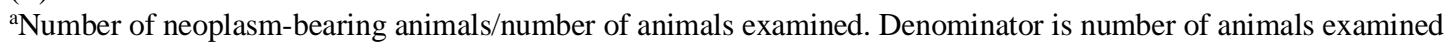
microscopically for liver and lung; for other tissues, denominator is number of animals necropsied.

${ }^{b}$ Poly-3 estimated neoplasm incidence after adjustment for intercurrent mortality.

cObserved incidence at terminal euthanasia.

${ }^{\mathrm{d} B e n e a t h}$ the vehicle control incidence is the $\mathrm{P}$ value associated with the trend test. Beneath the dosed group incidence are the $\mathrm{P}$ values corresponding to pairwise comparisons between the vehicle controls and that dosed group. The Poly- 3 test accounts for differential mortality in animals that do not reach terminal euthanasia. A negative trend or a lower incidence in a dose group is indicated by $\mathbf{N}$.

'Not applicable; no neoplasms in animal group.

fValue of statistic cannot be computed. 
Indole-3-carbinol, NTP TR 584

Table C-3. Historical Incidence of Liver Neoplasms in Control Male B6C3F1/N Mice ${ }^{\mathrm{a}}$

\begin{tabular}{|c|c|c|c|c|}
\hline Study (Study Start) & $\begin{array}{c}\text { Hepatocellular } \\
\text { Adenoma }\end{array}$ & $\begin{array}{c}\text { Hepatocellular } \\
\text { Carcinoma }\end{array}$ & $\begin{array}{l}\text { Hepatocellular } \\
\text { Adenoma or } \\
\text { Hepatocellular } \\
\text { Carcinoma }\end{array}$ & Hepatoblastoma \\
\hline \multicolumn{5}{|c|}{ Historical Incidence: Corn Oil Gavage Studies } \\
\hline Ginkgo biloba extract (March 2005) & $31 / 50$ & $22 / 50$ & $39 / 50$ & $3 / 50$ \\
\hline Indole-3-carbinol (April 2007) & $26 / 50$ & $12 / 50$ & $35 / 50$ & $3 / 50$ \\
\hline Kava kava extract (August 2004) & $27 / 50$ & $20 / 50$ & $38 / 50$ & $0 / 50$ \\
\hline$N, N$-Dimethyl-p-toluidine (October 2004) & $29 / 50$ & $22 / 50$ & $38 / 50$ & $1 / 50$ \\
\hline Tetrabromobisphenol A (August 2007) & $32 / 50$ & $11 / 50$ & $39 / 50$ & $2 / 50$ \\
\hline Total (\%) & $145 / 250(58.0 \%)$ & $87 / 250(34.8 \%)$ & $189 / 250(75.6 \%)$ & $9 / 250(3.6 \%)$ \\
\hline Mean \pm standard deviation & $58.0 \% \pm 5.1 \%$ & $34.8 \% \pm 10.9 \%$ & $75.6 \% \pm 3.3 \%$ & $3.6 \% \pm 2.6 \%$ \\
\hline Range & $52 \%-64 \%$ & $22 \%-44 \%$ & $70 \%-78 \%$ & $0 \%-6 \%$ \\
\hline \multicolumn{5}{|l|}{ Overall Historical Incidence: All Routes } \\
\hline Total (\%) & $594 / 949(62.6 \%)$ & $348 / 949$ (36.7\%) & $742 / 949(78.2 \%)$ & $40 / 949(4.2 \%)$ \\
\hline Mean \pm standard deviation & $62.6 \% \pm 9.1 \%$ & $36.7 \% \pm 11.4 \%$ & $78.2 \% \pm 7.2 \%$ & $4.2 \% \pm 3.5 \%$ \\
\hline \multirow[t]{2}{*}{ Range } & $48 \%-78 \%$ & $22 \%-56 \%$ & $64 \%-90 \%$ & $0 \%-12 \%$ \\
\hline & & $\begin{array}{l}\text { Hepatocellular } \\
\text { Carcinoma or } \\
\text { Hepatoblastoma }\end{array}$ & $\begin{array}{l}\text { Hepatocellular } \\
\text { Adenoma, } \\
\text { Hepatocellular } \\
\text { Carcinoma, or } \\
\text { Hepatoblastoma }\end{array}$ & $\begin{array}{c}\text { Hepatocholangio } \\
\text {-carcinoma }\end{array}$ \\
\hline \multicolumn{5}{|c|}{ Historical Incidence: Corn Oil Gavage Studies } \\
\hline Ginkgo biloba extract (March 2005) & & $24 / 50$ & $39 / 50$ & $0 / 50$ \\
\hline Indole-3-carbinol (April 2007) & & $15 / 50$ & $36 / 50$ & $0 / 50$ \\
\hline Kava kava extract (August 2004) & & $20 / 50$ & $38 / 50$ & $4 / 50$ \\
\hline$N, N$-Dimethyl-p-toluidine (October 2004) & & $22 / 50$ & $38 / 50$ & $0 / 50$ \\
\hline Tetrabromobisphenol A (August 2007) & & $12 / 50$ & $39 / 50$ & $0 / 50$ \\
\hline Total (\%) & & $93 / 250(37.2 \%)$ & $190 / 250(76.0 \%)$ & $4 / 250(1.6 \%)$ \\
\hline Mean \pm standard deviation & & $37.2 \% \pm 10.0 \%$ & $76.0 \% \pm 2.5 \%$ & $1.6 \% \pm 3.6 \%$ \\
\hline Range & & $24 \%-48 \%$ & $72 \%-78 \%$ & $0 \%-8 \%$ \\
\hline \multicolumn{5}{|l|}{ Overall Historical Incidence: All Routes } \\
\hline Total (\%) & & $371 / 949$ (39.1\%) & 746/949 (78.6\%) & $10 / 949(1.1 \%)$ \\
\hline Mean \pm standard deviation & & $39.1 \% \pm 11.6 \%$ & $78.6 \% \pm 7.2 \%$ & $1.1 \% \pm 2.2 \%$ \\
\hline Range & & $22 \%-58 \%$ & $64 \%-90 \%$ & $0 \%-8 \%$ \\
\hline
\end{tabular}

aData as of June 2013. 
Indole-3-carbinol, NTP TR 584

Table C-4. Summary of the Incidence of Nonneoplastic Lesions in Male Mice in the Two-year Gavage Study of Indole-3-carbinol ${ }^{\mathrm{a}}$

$\begin{aligned} & \text { Vehicle } \\ & \text { Control }\end{aligned} \quad 62.5 \mathrm{mg} / \mathrm{kg} \quad 125 \mathrm{mg} / \mathrm{kg} \quad 250 \mathrm{mg} / \mathrm{kg}$

\section{Disposition Summary}

Animals initially in study

50

50

50

50

Early deaths

Accidental deaths

Moribund

$\begin{array}{cccc}- & - & 1 & 1 \\ 17 & 12 & 11 & 9 \\ 6 & 7 & 6 & 8\end{array}$

Natural deaths

Survivors

Died last week of study

Terminal euthanasia

1

26

50

Animals examined microscopically

\section{Alimentary System}

Esophagus

Mineralization

Gallbladder

Atrophy

Inflammation, suppurative

Intestine large, cecum

Ulcer

Intestine large, colon

Inflammation, chronic

Intestine large, rectum

Inflammation, chronic

Intestine small, duodenum

Ulcer

Epithelium, atrophy

Intestine small, ileum

Intestine small, jejunum

Atrophy

Inflammation, suppurative

Lymphangiectasis

Peyer's patch, hyperplasia

Liver

Basophilic focus

Clear cell focus
(50)

$-$

(50)

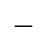

$-$

(50)

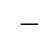

(50)

$1(2 \%)$

(50)

(50)

$1(2 \%)$

(50)

(49)

$-$

$-$

(50)

(50)

$1(2 \%)$

$1(2 \%)$

$1(2 \%)$

(50)

(50)

$1(2 \%)$

(50)

(50)

(50)

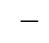

$-$

(50)

$1(2 \%)$

$1(2 \%)$

$1(2 \%)$

- $\quad 1(2 \%)$

(50)

(50)

(50)

$1(2 \%)$

(50)

\section{$-$}

-

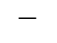

1 (2\%)

$-$

1 (2\%)

(50)

(50)

(49)
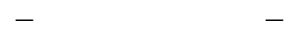

$-$

$-$

$-$

2 (4\%)

$1(2 \%)$

1 (2\%)

7 (14\%)
4 (8\%)

(50)

(49)

5 (10\%)

17 (34\%) 
Indole-3-carbinol, NTP TR 584

\begin{tabular}{|c|c|c|c|c|}
\hline & $\begin{array}{l}\text { Vehicle } \\
\text { Control }\end{array}$ & $62.5 \mathrm{mg} / \mathrm{kg}$ & $125 \mathrm{mg} / \mathrm{kg}$ & $250 \mathrm{mg} / \mathrm{kg}$ \\
\hline Eosinophilic focus & $29(58 \%)$ & 35 (70\%) & $35(71 \%)$ & $33(66 \%)$ \\
\hline Fatty change & $29(58 \%)$ & 27 (54\%) & $28(57 \%)$ & $37(74 \%)$ \\
\hline Hepatodiaphragmatic nodule & - & $1(2 \%)$ & - & $1(2 \%)$ \\
\hline Inflammation, chronic & - & - & - & $1(2 \%)$ \\
\hline Mixed cell focus & $1(2 \%)$ & $6(12 \%)$ & $6(12 \%)$ & $5(10 \%)$ \\
\hline Necrosis & $3(6 \%)$ & $2(4 \%)$ & $2(4 \%)$ & $2(4 \%)$ \\
\hline Necrosis, multifocal & $1(2 \%)$ & - & - & - \\
\hline Artery, thrombosis & - & - & - & $1(2 \%)$ \\
\hline Bile duct, hyperplasia & - & - & - & $1(2 \%)$ \\
\hline Oval cell, hyperplasia & - & - & - & $1(2 \%)$ \\
\hline Periportal, fibrosis & - & - & - & $1(2 \%)$ \\
\hline Mesentery & (7) & (3) & (2) & $(2)$ \\
\hline Fat, necrosis & $4(57 \%)$ & - & $2(100 \%)$ & $2(100 \%)$ \\
\hline Oral mucosa & $(0)$ & (1) & $(0)$ & (2) \\
\hline Pancreas & (50) & (50) & $(50)$ & $(50)$ \\
\hline Inflammation, chronic & - & - & - & $2(4 \%)$ \\
\hline Acinus, atrophy & $1(2 \%)$ & - & $1(2 \%)$ & - \\
\hline Duct, cyst & - & $1(2 \%)$ & - & - \\
\hline Salivary glands & (50) & (50) & (50) & (50) \\
\hline Infiltration cellular, mononuclear cell & $23(46 \%)$ & $30(60 \%)$ & 27 (54\%) & $22(44 \%)$ \\
\hline Duct, sublingual gland, hyperplasia & - & - & $1(2 \%)$ & - \\
\hline Stomach, forestomach & $(50)$ & (50) & $(50)$ & (50) \\
\hline Inflammation, chronic & - & $1(2 \%)$ & $4(8 \%)$ & $1(2 \%)$ \\
\hline Ulcer & $6(12 \%)$ & $5(10 \%)$ & 7 (14\%) & $1(2 \%)$ \\
\hline Epithelium, hyperplasia & $2(4 \%)$ & $2(4 \%)$ & $4(8 \%)$ & $2(4 \%)$ \\
\hline Stomach, glandular & $(50)$ & $(47)$ & $(47)$ & (49) \\
\hline Erosion & $1(2 \%)$ & - & - & - \\
\hline Inflammation, chronic & $1(2 \%)$ & $1(2 \%)$ & $18(38 \%)$ & 45 (92\%) \\
\hline Mineralization & $5(10 \%)$ & $2(4 \%)$ & - & - \\
\hline Pigmentation & - & $1(2 \%)$ & 38 (81\%) & $48(98 \%)$ \\
\hline Epithelium, atrophy & - & - & - & $1(2 \%)$ \\
\hline Epithelium, hyperplasia & - & $1(2 \%)$ & $22(47 \%)$ & $40(82 \%)$ \\
\hline Tooth & (30) & (37) & (32) & (32) \\
\hline Dysplasia & 30 (100\%) & 36 (97\%) & 32 (100\%) & 32 (100\%) \\
\hline
\end{tabular}


Indole-3-carbinol, NTP TR 584

\begin{tabular}{|c|c|c|c|c|}
\hline & $\begin{array}{l}\text { Vehicle } \\
\text { Control }\end{array}$ & $62.5 \mathrm{mg} / \mathrm{kg}$ & $125 \mathrm{mg} / \mathrm{kg}$ & $250 \mathrm{mg} / \mathrm{kg}$ \\
\hline \multicolumn{5}{|l|}{ Cardiovascular System } \\
\hline Blood vessel & (49) & $(50)$ & (49) & (49) \\
\hline $\begin{array}{l}\text { Adventitia, pulmonary vein, infiltration } \\
\text { cellular, polymorphonuclear }\end{array}$ & $1(2 \%)$ & - & - & - \\
\hline Aorta, mineralization & - & $1(2 \%)$ & - & - \\
\hline Heart & (50) & $(50)$ & $(50)$ & $(50)$ \\
\hline Cardiomyopathy & $11(22 \%)$ & 7 (14\%) & $18(36 \%)$ & $8(16 \%)$ \\
\hline Inflammation, chronic & $1(2 \%)$ & - & - & - \\
\hline Artery, inflammation, chronic & $1(2 \%)$ & - & - & - \\
\hline Artery, mineralization & - & $1(2 \%)$ & - & - \\
\hline Myocardium, mineralization & - & $3(6 \%)$ & - & $1(2 \%)$ \\
\hline Valve, inflammation & - & - & - & $1(2 \%)$ \\
\hline \multicolumn{5}{|l|}{ Endocrine System } \\
\hline Adrenal cortex & (50) & $(50)$ & $(50)$ & $(50)$ \\
\hline Hyperplasia & $1(2 \%)$ & - & - & $1(2 \%)$ \\
\hline Hypertrophy & - & $4(8 \%)$ & $2(4 \%)$ & - \\
\hline Vacuolization cytoplasmic & - & $2(4 \%)$ & - & - \\
\hline Adrenal medulla & (50) & (50) & $(50)$ & (50) \\
\hline Hyperplasia & $2(4 \%)$ & - & $1(2 \%)$ & $2(4 \%)$ \\
\hline Islets, pancreatic & $(50)$ & $(50)$ & $(50)$ & (50) \\
\hline Hyperplasia & $1(2 \%)$ & - & $1(2 \%)$ & - \\
\hline Parathyroid gland & (48) & (44) & (48) & (44) \\
\hline Pituitary gland & $(50)$ & (50) & (50) & $(50)$ \\
\hline Cyst & $2(4 \%)$ & $1(2 \%)$ & $1(2 \%)$ & $1(2 \%)$ \\
\hline Pars intermedia, hyperplasia & - & $1(2 \%)$ & - & - \\
\hline Thyroid gland & (50) & (50) & $(50)$ & (50) \\
\hline Inflammation, chronic & - & - & - & $1(2 \%)$ \\
\hline Follicle, cyst & $1(2 \%)$ & - & - & - \\
\hline Follicle, degeneration & - & - & - & $1(2 \%)$ \\
\hline \multicolumn{5}{|l|}{ General Body System } \\
\hline None & - & - & - & - \\
\hline \multicolumn{5}{|l|}{ Genital System } \\
\hline Coagulating gland & (1) & $(0)$ & (0) & $(0)$ \\
\hline Epididymis & $(50)$ & $(50)$ & (50) & (50) \\
\hline Angiectasis & - & - & - & $1(2 \%)$ \\
\hline
\end{tabular}


Indole-3-carbinol, NTP TR 584

\begin{tabular}{|c|c|c|c|c|}
\hline & $\begin{array}{l}\text { Vehicle } \\
\text { Control }\end{array}$ & $62.5 \mathrm{mg} / \mathrm{kg}$ & $125 \mathrm{mg} / \mathrm{kg}$ & $250 \mathrm{mg} / \mathrm{kg}$ \\
\hline Granuloma sperm & - & - & - & $1(2 \%)$ \\
\hline Infiltration cellular, mononuclear cell & $1(2 \%)$ & $6(12 \%)$ & $3(6 \%)$ & $5(10 \%)$ \\
\hline Inflammation, chronic & - & $2(4 \%)$ & - & - \\
\hline Preputial gland & (50) & $(50)$ & $(50)$ & $(50)$ \\
\hline Cyst & $14(28 \%)$ & $10(20 \%)$ & $6(12 \%)$ & $6(12 \%)$ \\
\hline Inflammation, suppurative & $2(4 \%)$ & - & - & - \\
\hline Inflammation, granulomatous & - & $2(4 \%)$ & - & $2(4 \%)$ \\
\hline Prostate & (50) & $(50)$ & $(50)$ & $(50)$ \\
\hline Hyperplasia & $2(4 \%)$ & - & $1(2 \%)$ & $1(2 \%)$ \\
\hline Inflammation, suppurative & - & - & $1(2 \%)$ & - \\
\hline Inflammation, chronic & $1(2 \%)$ & - & - & $1(2 \%)$ \\
\hline Seminal vesicle & (50) & (50) & (50) & (50) \\
\hline Atrophy & $1(2 \%)$ & - & - & - \\
\hline Testes & (50) & (50) & $(50)$ & $(50)$ \\
\hline Inflammation, granulomatous & $1(2 \%)$ & - & - & - \\
\hline Inflammation, chronic & $1(2 \%)$ & - & - & - \\
\hline Mineralization & $1(2 \%)$ & - & - & - \\
\hline Germinal epithelium, atrophy & $2(4 \%)$ & - & $3(6 \%)$ & $3(6 \%)$ \\
\hline \multicolumn{5}{|l|}{ Hematopoietic System } \\
\hline Bone marrow & (50) & (50) & $(50)$ & $(50)$ \\
\hline Lymph node & $(2)$ & (2) & (0) & (4) \\
\hline Mediastinal, hyperplasia & - & - & - & $1(25 \%)$ \\
\hline Pancreatic, inflammation & - & - & - & $1(25 \%)$ \\
\hline Pancreatic, necrosis & - & $1(50 \%)$ & - & - \\
\hline Renal, inflammation & - & - & - & $1(25 \%)$ \\
\hline Lymph node, mandibular & (50) & (50) & $(50)$ & $(50)$ \\
\hline Hyperplasia, lymphoid & - & $1(2 \%)$ & - & $1(2 \%)$ \\
\hline Lymph node, mesenteric & (48) & (48) & (49) & $(50)$ \\
\hline Hematopoietic cell proliferation & $1(2 \%)$ & - & - & - \\
\hline Hyperplasia, lymphoid & - & - & - & $2(4 \%)$ \\
\hline Inflammation & - & - & - & $1(2 \%)$ \\
\hline Spleen & $(50)$ & $(50)$ & $(50)$ & $(50)$ \\
\hline Accessory spleen & - & - & $1(2 \%)$ & - \\
\hline Atrophy & - & - & - & $1(2 \%)$ \\
\hline Hematopoietic cell proliferation & $16(32 \%)$ & $16(32 \%)$ & $24(48 \%)$ & $20(40 \%)$ \\
\hline
\end{tabular}


Indole-3-carbinol, NTP TR 584

\begin{tabular}{|c|c|c|c|c|}
\hline & $\begin{array}{l}\text { Vehicle } \\
\text { Control }\end{array}$ & $62.5 \mathrm{mg} / \mathrm{kg}$ & $125 \mathrm{mg} / \mathrm{kg}$ & $250 \mathrm{mg} / \mathrm{kg}$ \\
\hline Necrosis & $1(2 \%)$ & - & - & - \\
\hline Lymphoid follicle, atrophy & $2(4 \%)$ & - & - & - \\
\hline Thymus & (49) & $(47)$ & $(46)$ & $(45)$ \\
\hline Atrophy & $44(90 \%)$ & $44(94 \%)$ & $45(98 \%)$ & $39(87 \%)$ \\
\hline Ectopic parathyroid gland & - & $1(2 \%)$ & - & $1(2 \%)$ \\
\hline \multicolumn{5}{|l|}{ Integumentary System } \\
\hline Mammary gland & (2) & $(0)$ & $(0)$ & (0) \\
\hline Skin & $(50)$ & $(50)$ & $(50)$ & $(50)$ \\
\hline Ulcer & $3(6 \%)$ & $2(4 \%)$ & $3(6 \%)$ & $1(2 \%)$ \\
\hline Subcutaneous tissue, inflammation, acute & - & - & $1(2 \%)$ & - \\
\hline $\begin{array}{l}\text { Subcutaneous tissue, inflammation, } \\
\text { chronic }\end{array}$ & - & - & $2(4 \%)$ & - \\
\hline \multicolumn{5}{|l|}{ Musculoskeletal System } \\
\hline Bone & $(50)$ & (50) & (50) & $(50)$ \\
\hline Hyperostosis & - & - & $1(2 \%)$ & $1(2 \%)$ \\
\hline Skeletal muscle & $(0)$ & $(0)$ & $(2)$ & $(0)$ \\
\hline \multicolumn{5}{|l|}{ Nervous System } \\
\hline Brain & $(50)$ & (50) & $(50)$ & $(50)$ \\
\hline Meninges, inflammation, chronic & - & $1(2 \%)$ & - & - \\
\hline \multicolumn{5}{|l|}{ Respiratory System } \\
\hline Lung & $(50)$ & (50) & $(50)$ & $(50)$ \\
\hline Infiltration cellular, histiocyte & - & $2(4 \%)$ & - & $1(2 \%)$ \\
\hline Inflammation, chronic & - & $1(2 \%)$ & $1(2 \%)$ & $1(2 \%)$ \\
\hline Pigmentation, hemosiderin & - & $1(2 \%)$ & - & - \\
\hline Thrombosis & - & - & $1(2 \%)$ & - \\
\hline Alveolar epithelium, hyperplasia & $3(6 \%)$ & $4(8 \%)$ & $5(10 \%)$ & - \\
\hline $\begin{array}{l}\text { Mediastinum, inflammation, } \\
\text { granulomatous }\end{array}$ & - & - & $1(2 \%)$ & - \\
\hline Nose & $(50)$ & $(50)$ & $(50)$ & $(50)$ \\
\hline Foreign body & $3(6 \%)$ & $1(2 \%)$ & - & $3(6 \%)$ \\
\hline Inflammation & 15 (30\%) & $11(22 \%)$ & 12 (24\%) & $16(32 \%)$ \\
\hline Polyp, inflammatory & $1(2 \%)$ & $2(4 \%)$ & $3(6 \%)$ & $1(2 \%)$ \\
\hline Respiratory metaplasia & - & - & $1(2 \%)$ & - \\
\hline Nerve, atrophy & - & - & - & $8(16 \%)$ \\
\hline Nerve, olfactory epithelium, atrophy & - & $1(2 \%)$ & - & - \\
\hline
\end{tabular}


Indole-3-carbinol, NTP TR 584

\begin{tabular}{|c|c|c|c|c|}
\hline & $\begin{array}{l}\text { Vehicle } \\
\text { Control }\end{array}$ & $62.5 \mathrm{mg} / \mathrm{kg}$ & $125 \mathrm{mg} / \mathrm{kg}$ & $250 \mathrm{mg} / \mathrm{kg}$ \\
\hline $\begin{array}{l}\text { Olfactory epithelium, accumulation, } \\
\text { hyaline droplet }\end{array}$ & $4(8 \%)$ & $4(8 \%)$ & $5(10 \%)$ & $5(10 \%)$ \\
\hline Olfactory epithelium, atrophy & $3(6 \%)$ & $5(10 \%)$ & $11(22 \%)$ & $17(34 \%)$ \\
\hline Olfactory epithelium, degeneration & $1(2 \%)$ & $1(2 \%)$ & $4(8 \%)$ & $2(4 \%)$ \\
\hline $\begin{array}{l}\text { Olfactory epithelium, metaplasia, } \\
\text { squamous }\end{array}$ & - & - & - & $2(4 \%)$ \\
\hline Olfactory epithelium, necrosis & - & - & - & $6(12 \%)$ \\
\hline $\begin{array}{l}\text { Olfactory epithelium, respiratory } \\
\text { metaplasia }\end{array}$ & $14(28 \%)$ & $14(28 \%)$ & $20(40 \%)$ & 27 (54\%) \\
\hline $\begin{array}{l}\text { Respiratory epithelium, accumulation, } \\
\text { hyaline droplet }\end{array}$ & $18(36 \%)$ & $34(68 \%)$ & $30(60 \%)$ & $26(52 \%)$ \\
\hline Respiratory epithelium, hyperplasia & 35 (70\%) & $40(80 \%)$ & $41(82 \%)$ & 45 (90\%) \\
\hline $\begin{array}{l}\text { Respiratory epithelium, metaplasia, } \\
\text { squamous }\end{array}$ & - & - & - & $1(2 \%)$ \\
\hline Respiratory epithelium, necrosis & - & - & - & $2(4 \%)$ \\
\hline Trachea & (50) & (50) & $(50)$ & $(50)$ \\
\hline \multicolumn{5}{|l|}{ Special Senses System } \\
\hline Eye & (50) & (50) & (50) & $(50)$ \\
\hline Phthisis bulbi & $1(2 \%)$ & $1(2 \%)$ & - & - \\
\hline $\begin{array}{l}\text { Anterior chamber, inflammation, } \\
\text { suppurative }\end{array}$ & - & $1(2 \%)$ & - & $1(2 \%)$ \\
\hline Cornea, inflammation, suppurative & - & $1(2 \%)$ & - & - \\
\hline Cornea, inflammation, chronic & $2(4 \%)$ & $3(6 \%)$ & $2(4 \%)$ & $1(2 \%)$ \\
\hline $\begin{array}{l}\text { Optic nerve, infiltration cellular, } \\
\text { mononuclear cell }\end{array}$ & - & - & - & $1(2 \%)$ \\
\hline Harderian gland & (50) & (50) & $(50)$ & $(50)$ \\
\hline Hyperplasia & $1(2 \%)$ & $4(8 \%)$ & $5(10 \%)$ & $3(6 \%)$ \\
\hline Inflammation, suppurative & $1(2 \%)$ & $1(2 \%)$ & - & - \\
\hline Inflammation, chronic & - & $1(2 \%)$ & - & - \\
\hline \multicolumn{5}{|l|}{ Urinary System } \\
\hline Kidney & $(50)$ & (50) & $(50)$ & $(50)$ \\
\hline Infarct & $3(6 \%)$ & $2(4 \%)$ & - & - \\
\hline Inflammation, acute & - & $2(4 \%)$ & - & - \\
\hline Inflammation, chronic & $1(2 \%)$ & - & - & $1(2 \%)$ \\
\hline Metaplasia, osseous & $1(2 \%)$ & $1(2 \%)$ & $1(2 \%)$ & $1(2 \%)$ \\
\hline Mineralization & $4(8 \%)$ & $8(16 \%)$ & $1(2 \%)$ & $2(4 \%)$ \\
\hline Nephropathy & 42 (84\%) & $50(100 \%)$ & 45 (90\%) & 43 (86\%) \\
\hline Thrombosis & - & $1(2 \%)$ & - & - \\
\hline
\end{tabular}


Indole-3-carbinol, NTP TR 584

\begin{tabular}{|c|c|c|c|c|}
\hline & $\begin{array}{l}\text { Vehicle } \\
\text { Control }\end{array}$ & $62.5 \mathrm{mg} / \mathrm{kg}$ & $125 \mathrm{mg} / \mathrm{kg}$ & $250 \mathrm{mg} / \mathrm{kg}$ \\
\hline Papilla, necrosis & $1(2 \%)$ & - & - & $1(2 \%)$ \\
\hline Pelvis, inflammation, suppurative & $2(4 \%)$ & $2(4 \%)$ & $1(2 \%)$ & - \\
\hline Renal tubule, cyst & $1(2 \%)$ & $3(6 \%)$ & $2(4 \%)$ & $2(4 \%)$ \\
\hline Renal tubule, hyperplasia & - & - & $1(2 \%)$ & - \\
\hline Renal tubule, pigmentation & $1(2 \%)$ & $1(2 \%)$ & $1(2 \%)$ & $5(10 \%)$ \\
\hline Renal tubule, vacuolization cytoplasmic & $1(2 \%)$ & - & - & - \\
\hline Urethra & $(0)$ & (1) & (0) & $(0)$ \\
\hline Inflammation, acute & - & $1(100 \%)$ & - & - \\
\hline Urinary bladder & (50) & (50) & $(50)$ & $(50)$ \\
\hline Hemorrhage & - & $1(2 \%)$ & - & - \\
\hline Inflammation, chronic & $1(2 \%)$ & $2(4 \%)$ & - & - \\
\hline
\end{tabular}

${ }^{a}$ Number of animals examined microscopically at the site and the number of animals with lesion. 


\section{Appendix D. Summary of Lesions in Female Mice in the Two-year Gavage Study of Indole-3-carbinol}

\section{Tables}

Table D-1. Summary of the Incidence of Neoplasms in Female Mice in the Two-year Gavage Study of Indole-3-carbinol

Table D-2. Statistical Analysis of Primary Neoplasms in Female Mice in the Two-year

Gavage Study of Indole-3-carbinol ..............................................................

Table D-3. Historical Incidence of Liver Neoplasms in Control Female B6C3F1/N Mice........D-9

Table D-4. Summary of the Incidence of Nonneoplastic Lesions in Female Mice in the

Two-year Gavage Study of Indole-3-carbinol.............................................. D-10 
Indole-3-carbinol, NTP TR 584

Table D-1. Summary of the Incidence of Neoplasms in Female Mice in the Two-year Gavage Study of Indole-3-carbinol ${ }^{\mathrm{a}}$

\begin{tabular}{|c|c|c|c|c|}
\hline & $\begin{array}{l}\text { Vehicle } \\
\text { Control }\end{array}$ & $62.5 \mathrm{mg} / \mathrm{kg}$ & $125 \mathrm{mg} / \mathrm{kg}$ & $250 \mathrm{mg} / \mathrm{kg}$ \\
\hline \multicolumn{5}{|l|}{ Disposition Summary } \\
\hline Animals initially in study & 50 & 50 & 50 & 50 \\
\hline \multicolumn{5}{|l|}{ Early deaths } \\
\hline Accidental death & 1 & - & - & - \\
\hline Moribund & 10 & 6 & 15 & 3 \\
\hline Natural deaths & 6 & 4 & 9 & 2 \\
\hline \multicolumn{5}{|l|}{ Survivors } \\
\hline Died last week of study & - & - & 1 & - \\
\hline Terminal euthanasia & 33 & 40 & 25 & 45 \\
\hline Animals examined microscopically & 50 & 50 & 50 & 50 \\
\hline \multicolumn{5}{|l|}{ Alimentary System } \\
\hline Esophagus & $(50)$ & (50) & $(50)$ & $(50)$ \\
\hline Squamous cell carcinoma & - & - & $1(2 \%)$ & - \\
\hline Gallbladder & (49) & $(50)$ & $(50)$ & $(50)$ \\
\hline $\begin{array}{l}\text { Leiomyosarcoma, metastatic, intestine } \\
\text { small, jejunum }\end{array}$ & $1(2 \%)$ & - & - & - \\
\hline Intestine large, cecum & $(50)$ & $(50)$ & $(50)$ & $(50)$ \\
\hline Leiomyosarcoma & $1(2 \%)$ & - & - & - \\
\hline Intestine large, colon & $(50)$ & $(50)$ & $(50)$ & $(50)$ \\
\hline Intestine large, rectum & $(50)$ & $(50)$ & $(50)$ & $(50)$ \\
\hline Intestine small, duodenum & $(50)$ & $(50)$ & $(50)$ & $(50)$ \\
\hline Intestine small, ileum & $(50)$ & $(50)$ & $(50)$ & $(50)$ \\
\hline Intestine small, jejunum & $(50)$ & $(50)$ & $(50)$ & $(50)$ \\
\hline Leiomyosarcoma & $1(2 \%)$ & - & - & - \\
\hline Liver & $(50)$ & $(50)$ & $(50)$ & $(50)$ \\
\hline Hemangiosarcoma, multiple & - & - & $1(2 \%)$ & - \\
\hline Hepatoblastoma & - & - & $1(2 \%)$ & $1(2 \%)$ \\
\hline Hepatocellular adenoma & $7(14 \%)$ & $11(22 \%)$ & $6(12 \%)$ & $7(14 \%)$ \\
\hline Hepatocellular adenoma, multiple & - & $3(6 \%)$ & $2(4 \%)$ & $4(8 \%)$ \\
\hline Hepatocellular carcinoma & $6(12 \%)$ & $6(12 \%)$ & $8(16 \%)$ & $4(8 \%)$ \\
\hline Hepatocellular carcinoma, multiple & - & $2(4 \%)$ & $1(2 \%)$ & - \\
\hline Mesentery & (3) & (7) & (7) & (6) \\
\hline Carcinoma, metastatic, kidney & - & $1(14 \%)$ & - & - \\
\hline Hemangioma & - & - & $1(14 \%)$ & - \\
\hline Pancreas & $(50)$ & $(50)$ & $(50)$ & $(50)$ \\
\hline
\end{tabular}


Indole-3-carbinol, NTP TR 584

\begin{tabular}{|c|c|c|c|c|}
\hline & $\begin{array}{l}\text { Vehicle } \\
\text { Control }\end{array}$ & $62.5 \mathrm{mg} / \mathrm{kg}$ & $125 \mathrm{mg} / \mathrm{kg}$ & $250 \mathrm{mg} / \mathrm{kg}$ \\
\hline Salivary glands & (50) & (49) & (50) & $(50)$ \\
\hline Stomach, forestomach & $(50)$ & $(50)$ & $(50)$ & $(50)$ \\
\hline Squamous cell papilloma & $1(2 \%)$ & - & - & - \\
\hline Stomach, glandular & $(48)$ & (50) & (49) & $(50)$ \\
\hline Tongue & $(0)$ & $(0)$ & (0) & (1) \\
\hline Squamous cell papilloma & - & - & - & $1(100 \%)$ \\
\hline Tooth & (6) & (9) & (9) & $(11)$ \\
\hline \multicolumn{5}{|l|}{ Cardiovascular System } \\
\hline Blood vessel & (48) & $(50)$ & $(50)$ & $(50)$ \\
\hline Hemangioma & $1(2 \%)$ & - & - & - \\
\hline Heart & (50) & (50) & $(50)$ & (50) \\
\hline \multicolumn{5}{|l|}{ Endocrine System } \\
\hline Adrenal cortex & (49) & $(50)$ & $(50)$ & $(50)$ \\
\hline Adenoma & $1(2 \%)$ & - & - & - \\
\hline Subcapsular, adenoma & $1(2 \%)$ & - & - & - \\
\hline Adrenal medulla & $(49)$ & $(50)$ & $(50)$ & $(50)$ \\
\hline Islets, pancreatic & $(50)$ & (50) & $(50)$ & $(50)$ \\
\hline Adenoma & - & $1(2 \%)$ & - & - \\
\hline Carcinoma & - & - & $1(2 \%)$ & - \\
\hline Parathyroid gland & (43) & (48) & $(49)$ & $(46)$ \\
\hline Pituitary gland & (49) & (50) & $(50)$ & (49) \\
\hline Pars distalis, adenoma & $3(6 \%)$ & $4(8 \%)$ & - & $1(2 \%)$ \\
\hline Pars intermedia, adenoma & $1(2 \%)$ & - & - & - \\
\hline Thyroid gland & $(50)$ & (50) & (49) & $(50)$ \\
\hline C-cell, carcinoma & $1(2 \%)$ & - & - & - \\
\hline Follicular cell, adenoma & $1(2 \%)$ & - & - & $1(2 \%)$ \\
\hline \multicolumn{5}{|l|}{ General Body System } \\
\hline Tissue NOS & $(0)$ & $(0)$ & $(1)$ & $(0)$ \\
\hline \multicolumn{5}{|l|}{ Genital System } \\
\hline Clitoral gland & (49) & (50) & $(50)$ & $(50)$ \\
\hline Ovary & $(50)$ & (50) & $(50)$ & $(50)$ \\
\hline Cystadenoma & $1(2 \%)$ & $4(8 \%)$ & $1(2 \%)$ & $4(8 \%)$ \\
\hline Hemangioma & - & - & - & $1(2 \%)$ \\
\hline Bilateral, tubulostromal adenoma & $1(2 \%)$ & - & - & - \\
\hline Uterus & $(50)$ & $(50)$ & $(50)$ & $(50)$ \\
\hline Carcinoma & - & $1(2 \%)$ & - & - \\
\hline Polyp stromal & - & - & - & $4(8 \%)$ \\
\hline
\end{tabular}


Indole-3-carbinol, NTP TR 584

\begin{tabular}{|c|c|c|c|c|}
\hline & $\begin{array}{l}\text { Vehicle } \\
\text { Control }\end{array}$ & $62.5 \mathrm{mg} / \mathrm{kg}$ & $125 \mathrm{mg} / \mathrm{kg}$ & $250 \mathrm{mg} / \mathrm{kg}$ \\
\hline \multicolumn{5}{|l|}{ Hematopoietic System } \\
\hline Bone marrow & (50) & $(50)$ & $(50)$ & $(50)$ \\
\hline Lymph node & (9) & (5) & (6) & (1) \\
\hline Lymph node, mandibular & $(50)$ & (49) & (50) & (50) \\
\hline Lymph node, mesenteric & $(49)$ & (49) & $(49)$ & (49) \\
\hline Hemangiosarcoma & - & - & $1(2 \%)$ & - \\
\hline Spleen & $(50)$ & $(50)$ & $(50)$ & $(50)$ \\
\hline Thymus & (49) & $(50)$ & $(50)$ & $(50)$ \\
\hline $\begin{array}{l}\text { Alveolar/bronchiolar carcinoma, metastatic, } \\
\text { lung }\end{array}$ & - & - & $1(2 \%)$ & - \\
\hline \multicolumn{5}{|l|}{ Integumentary System } \\
\hline Mammary gland & $(50)$ & $(50)$ & $(50)$ & $(50)$ \\
\hline Carcinoma & $1(2 \%)$ & - & - & - \\
\hline Skin & $(50)$ & $(50)$ & $(50)$ & $(50)$ \\
\hline Squamous cell carcinoma & - & - & $1(2 \%)$ & - \\
\hline Subcutaneous tissue, fibrosarcoma & - & - & $2(4 \%)$ & - \\
\hline Subcutaneous tissue, hemangioma & - & - & - & $1(2 \%)$ \\
\hline $\begin{array}{l}\text { Subcutaneous tissue, hemangiosarcoma, } \\
\text { multiple }\end{array}$ & - & - & $1(2 \%)$ & - \\
\hline Subcutaneous tissue, sarcoma & $1(2 \%)$ & - & - & - \\
\hline \multicolumn{5}{|l|}{ Musculoskeletal System } \\
\hline Bone & $(50)$ & $(50)$ & $(50)$ & $(50)$ \\
\hline Skeletal muscle & $(0)$ & $(1)$ & $(2)$ & $(0)$ \\
\hline Fibrosarcoma & - & - & $1(50 \%)$ & - \\
\hline Rhabdomyosarcoma & - & - & $1(50 \%)$ & - \\
\hline Schwannoma malignant & - & $1(100 \%)$ & - & - \\
\hline \multicolumn{5}{|l|}{ Nervous System } \\
\hline Brain & $(50)$ & $(50)$ & $(50)$ & $(50)$ \\
\hline Meningioma benign & - & $1(2 \%)$ & - & - \\
\hline \multicolumn{5}{|l|}{ Respiratory System } \\
\hline Lung & $(50)$ & $(50)$ & $(50)$ & $(50)$ \\
\hline Alveolar/bronchiolar adenoma & $1(2 \%)$ & $1(2 \%)$ & $3(6 \%)$ & $2(4 \%)$ \\
\hline Alveolar/bronchiolar carcinoma & $2(4 \%)$ & $2(4 \%)$ & $3(6 \%)$ & - \\
\hline Alveolar/bronchiolar carcinoma, multiple & - & $1(2 \%)$ & - & - \\
\hline Carcinoma, metastatic, harderian gland & - & $1(2 \%)$ & - & - \\
\hline Carcinoma, metastatic, thyroid gland & $1(2 \%)$ & - & - & - \\
\hline Hepatocellular carcinoma, metastatic, liver & $2(4 \%)$ & $2(4 \%)$ & $2(4 \%)$ & - \\
\hline
\end{tabular}


Indole-3-carbinol, NTP TR 584

\begin{tabular}{|c|c|c|c|c|}
\hline & $\begin{array}{l}\text { Vehicle } \\
\text { Control }\end{array}$ & $62.5 \mathrm{mg} / \mathrm{kg}$ & $125 \mathrm{mg} / \mathrm{kg}$ & $250 \mathrm{mg} / \mathrm{kg}$ \\
\hline Squamous cell carcinoma, metastatic, skin & - & - & $1(2 \%)$ & - \\
\hline Nose & $(50)$ & $(50)$ & $(50)$ & $(50)$ \\
\hline Trachea & $(50)$ & $(50)$ & $(50)$ & $(50)$ \\
\hline \multicolumn{5}{|l|}{ Special Senses System } \\
\hline Ear & $(2)$ & $(0)$ & (0) & $(0)$ \\
\hline Eye & $(50)$ & $(50)$ & $(50)$ & $(50)$ \\
\hline Harderian gland & $(50)$ & $(50)$ & $(50)$ & $(50)$ \\
\hline Adenoma & $3(6 \%)$ & $7(14 \%)$ & $7(14 \%)$ & - \\
\hline Carcinoma & - & $1(2 \%)$ & $1(2 \%)$ & $1(2 \%)$ \\
\hline \multicolumn{5}{|l|}{ Urinary System } \\
\hline Kidney & $(50)$ & $(50)$ & $(50)$ & (50) \\
\hline Bilateral, renal tubule, carcinoma & - & $1(2 \%)$ & - & - \\
\hline Renal tubule, carcinoma, multiple & - & - & - & $1(2 \%)$ \\
\hline Urinary bladder & $(50)$ & $(50)$ & $(50)$ & $(50)$ \\
\hline \multicolumn{5}{|l|}{ Systemic Lesions } \\
\hline Multiple organs ${ }^{\mathrm{b}}$ & $(50)$ & $(50)$ & $(50)$ & $(50)$ \\
\hline Histiocytic sarcoma & $1(2 \%)$ & - & - & $1(2 \%)$ \\
\hline Lymphoma malignant & $6(12 \%)$ & $9(18 \%)$ & $6(12 \%)$ & $3(6 \%)$ \\
\hline \multicolumn{5}{|l|}{ Neoplasm Summary } \\
\hline Total animals with primary neoplasms ${ }^{c}$ & 30 & 32 & 36 & 26 \\
\hline Total primary neoplasms & 42 & 56 & 50 & 37 \\
\hline Total animals with benign neoplasms & 17 & 21 & 17 & 20 \\
\hline Total benign neoplasms & 22 & 32 & 20 & 26 \\
\hline Total animals with malignant neoplasms & 19 & 19 & 26 & 9 \\
\hline Total malignant neoplasms & 20 & 24 & 30 & 11 \\
\hline Total animals with metastatic neoplasms & 4 & 4 & 4 & - \\
\hline Total metastatic neoplasms & 4 & 4 & 4 & - \\
\hline
\end{tabular}

${ }^{a}$ Number of animals examined microscopically at the site and the number of animals with neoplasm.

${ }^{b}$ Number of animals with any tissue examined microscopically.

'Primary neoplasms: all neoplasms except metastatic neoplasms. 
Indole-3-carbinol, NTP TR 584

Table D-2. Statistical Analysis of Primary Neoplasms in Female Mice in the Two-year Gavage Study of Indole-3-carbinol

\begin{tabular}{|c|c|c|c|c|}
\hline & Vehicle Control & $62.5 \mathrm{mg} / \mathrm{kg}$ & $125 \mathrm{mg} / \mathrm{kg}$ & $250 \mathrm{mg} / \mathrm{kg}$ \\
\hline \multicolumn{5}{|c|}{ Harderian Gland: Adenoma } \\
\hline Overall rate $\mathrm{e}^{\mathrm{a}}$ & $3 / 50(6 \%)$ & $7 / 50(14 \%)$ & 7/50 (14\%) & $0 / 50(0 \%)$ \\
\hline Adjusted rate ${ }^{b}$ & $6.6 \%$ & $15.0 \%$ & $16.6 \%$ & $0.0 \%$ \\
\hline Terminal rate $\mathrm{c}^{\mathrm{C}}$ & 3/33 (9\%) & $5 / 40(13 \%)$ & $5 / 26(19 \%)$ & $0 / 45(0 \%)$ \\
\hline First incidence (days) & $729(\mathrm{~T})$ & 681 & 381 & $-^{\mathrm{e}}$ \\
\hline Poly-3 test ${ }^{\mathrm{d}}$ & $\mathrm{P}=0.072 \mathrm{~N}$ & $\mathrm{P}=0.170$ & $\mathrm{P}=0.128$ & $\mathrm{P}=0.111 \mathrm{~N}$ \\
\hline \multicolumn{5}{|c|}{ Harderian Gland: Adenoma or Carcinoma } \\
\hline Overall rate & $3 / 50(6 \%)$ & $8 / 50(16 \%)$ & $8 / 50(16 \%)$ & $1 / 50(2 \%)$ \\
\hline Adjusted rate & $6.6 \%$ & $17.1 \%$ & $19.0 \%$ & $2.1 \%$ \\
\hline Terminal rate & 3/33 (9\%) & $6 / 40(15 \%)$ & 6/26 (23\%) & $0 / 45(0 \%)$ \\
\hline First incidence (days) & $729(\mathrm{~T})$ & 681 & 381 & 603 \\
\hline Poly-3 test & $\mathrm{P}=0.134 \mathrm{~N}$ & $\mathrm{P}=0.108$ & $\mathrm{P}=0.076$ & $\mathrm{P}=0.286 \mathrm{~N}$ \\
\hline \multicolumn{5}{|c|}{ Liver: Hepatocellular Adenoma } \\
\hline Overall rate & $7 / 50$ (14\%) & $14 / 50(28 \%)$ & $8 / 50(16 \%)$ & $11 / 50(22 \%)$ \\
\hline Adjusted rate & $15.2 \%$ & $30.0 \%$ & $19.5 \%$ & $22.9 \%$ \\
\hline Terminal rate & 5/33 (15\%) & $13 / 40(33 \%)$ & $7 / 26(27 \%)$ & $10 / 45(22 \%)$ \\
\hline First incidence (days) & 568 & 687 & 621 & 603 \\
\hline Poly-3 test & $\mathrm{P}=0.390$ & $\mathrm{P}=0.071$ & $\mathrm{P}=0.405$ & $P=0.247$ \\
\hline \multicolumn{5}{|c|}{ Liver: Hepatocellular Carcinoma } \\
\hline Overall rate & 6/50 (12\%) & $8 / 50(16 \%)$ & 9/50 (18\%) & $4 / 50(8 \%)$ \\
\hline Adjusted rate & $13.2 \%$ & $17.1 \%$ & $21.5 \%$ & $8.4 \%$ \\
\hline Terminal rate & 5/33 (15\%) & $7 / 40(18 \%)$ & $5 / 26(19 \%)$ & $4 / 45(9 \%)$ \\
\hline First incidence (days) & 709 & 650 & 606 & $729(\mathrm{~T})$ \\
\hline Poly-3 test & $\mathrm{P}=0.246 \mathrm{~N}$ & $P=0.409$ & $\mathrm{P}=0.228$ & $\mathrm{P}=0.341 \mathrm{~N}$ \\
\hline \multicolumn{5}{|c|}{ Liver: Hepatocellular Adenoma or Carcinoma } \\
\hline Overall rate & $12 / 50(24 \%)$ & $19 / 50(38 \%)$ & $16 / 50(32 \%)$ & $14 / 50(28 \%)$ \\
\hline Adjusted rate & $26.1 \%$ & $40.5 \%$ & $37.9 \%$ & $29.2 \%$ \\
\hline Terminal rate & 10/33 (30\%) & $17 / 40(43 \%)$ & $11 / 26(42 \%)$ & $13 / 45(29 \%)$ \\
\hline First incidence (days) & 568 & 650 & 606 & 603 \\
\hline Poly-3 test & $P=0.503 N$ & $P=0.103$ & $\mathrm{P}=0.165$ & $P=0.460$ \\
\hline \multicolumn{5}{|c|}{ Liver: Hepatocellular Carcinoma or Hepatoblastoma } \\
\hline Overall rate & $6 / 50(12 \%)$ & $8 / 50(16 \%)$ & $10 / 50(20 \%)$ & $4 / 50(8 \%)$ \\
\hline Adjusted rate & $13.2 \%$ & $17.1 \%$ & $23.9 \%$ & $8.4 \%$ \\
\hline Terminal rate & 5/33 (15\%) & $7 / 40(18 \%)$ & 6/26 (23\%) & 4/45 (9\%) \\
\hline
\end{tabular}


Indole-3-carbinol, NTP TR 584

\begin{tabular}{|c|c|c|c|c|}
\hline & Vehicle Control & $62.5 \mathrm{mg} / \mathrm{kg}$ & $125 \mathrm{mg} / \mathrm{kg}$ & $250 \mathrm{mg} / \mathrm{kg}$ \\
\hline First incidence (days) & 709 & 650 & 606 & $729(\mathrm{~T})$ \\
\hline Poly-3 test & $\mathrm{P}=0.257 \mathrm{~N}$ & $P=0.409$ & $P=0.154$ & $\mathrm{P}=0.341 \mathrm{~N}$ \\
\hline \multicolumn{5}{|c|}{ Liver: Hepatocellular Adenoma, Hepatocellular Carcinoma, or Hepatoblastoma } \\
\hline Overall rate & $12 / 50(24 \%)$ & 19/50 (38\%) & $17 / 50(34 \%)$ & $14 / 50(28 \%)$ \\
\hline Adjusted rate & $26.1 \%$ & $40.5 \%$ & $40.3 \%$ & $29.2 \%$ \\
\hline Terminal rate & 10/33 (30\%) & $17 / 40(43 \%)$ & $12 / 26(46 \%)$ & $13 / 45(29 \%)$ \\
\hline First incidence (days) & 568 & 650 & 606 & 603 \\
\hline Poly-3 test & $\mathrm{P}=0.511 \mathrm{~N}$ & $P=0.103$ & $P=0.113$ & $P=0.460$ \\
\hline \multicolumn{5}{|c|}{ Lung: Alveolar/Bronchiolar Adenoma } \\
\hline Overall rate & $1 / 50(2 \%)$ & $1 / 50(2 \%)$ & $3 / 50(6 \%)$ & $2 / 50(4 \%)$ \\
\hline Adjusted rate & $2.2 \%$ & $2.2 \%$ & $7.3 \%$ & $4.2 \%$ \\
\hline Terminal rate & 0/33 (0\%) & $1 / 40(3 \%)$ & $2 / 26(8 \%)$ & $2 / 45(4 \%)$ \\
\hline First incidence (days) & 634 & $729(\mathrm{~T})$ & 639 & $729(\mathrm{~T})$ \\
\hline Poly-3 test & $P=0.343$ & $\mathrm{P}=0.757 \mathrm{~N}$ & $\mathrm{P}=0.267$ & $\mathrm{P}=0.514$ \\
\hline \multicolumn{5}{|c|}{ Lung: Alveolar/Bronchiolar Carcinoma } \\
\hline Overall rate & $2 / 50(4 \%)$ & $3 / 50(6 \%)$ & $3 / 50(6 \%)$ & $0 / 50(0 \%)$ \\
\hline Adjusted rate & $4.4 \%$ & $6.5 \%$ & $7.2 \%$ & $0.0 \%$ \\
\hline Terminal rate & $2 / 33(6 \%)$ & $3 / 40(8 \%)$ & $0 / 26(0 \%)$ & $0 / 45(0 \%)$ \\
\hline First incidence (days) & $729(\mathrm{~T})$ & $729(\mathrm{~T})$ & 526 & - \\
\hline Poly-3 test & $P=0.151 \mathrm{~N}$ & $\mathrm{P}=0.511$ & $\mathrm{P}=0.463$ & $\mathrm{P}=0.227 \mathrm{~N}$ \\
\hline \multicolumn{5}{|c|}{ Lung: Alveolar/Bronchiolar Adenoma or Carcinoma } \\
\hline Overall rate & $3 / 50(6 \%)$ & $4 / 50(8 \%)$ & $6 / 50(12 \%)$ & $2 / 50(4 \%)$ \\
\hline Adjusted rate & $6.6 \%$ & $8.6 \%$ & $14.2 \%$ & $4.2 \%$ \\
\hline Terminal rate & 2/33 (6\%) & $4 / 40(10 \%)$ & $2 / 26(8 \%)$ & $2 / 45(4 \%)$ \\
\hline First incidence (days) & 634 & $729(\mathrm{~T})$ & 526 & $729(\mathrm{~T})$ \\
\hline Poly-3 test & $\mathrm{P}=0.385 \mathrm{~N}$ & $P=0.509$ & $P=0.203$ & $\mathrm{P}=0.482 \mathrm{~N}$ \\
\hline \multicolumn{5}{|l|}{ Ovary: Cystadenoma } \\
\hline Overall rate & $1 / 50(2 \%)$ & $4 / 50(8 \%)$ & $1 / 50(2 \%)$ & $4 / 50(8 \%)$ \\
\hline Adjusted rate & $2.2 \%$ & $8.6 \%$ & $2.4 \%$ & $8.4 \%$ \\
\hline Terminal rate & $0 / 33(0 \%)$ & $4 / 40(10 \%)$ & $0 / 26(0 \%)$ & $4 / 45(9 \%)$ \\
\hline First incidence (days) & 695 & $729(\mathrm{~T})$ & 638 & $729(\mathrm{~T})$ \\
\hline Poly-3 test & $\mathrm{P}=0.232$ & $P=0.185$ & $P=0.737$ & $P=0.193$ \\
\hline \multicolumn{5}{|c|}{ Pituitary Gland (Pars Distalis): Adenoma } \\
\hline Overall rate & $3 / 49(6 \%)$ & $4 / 50(8 \%)$ & $0 / 50(0 \%)$ & $1 / 49(2 \%)$ \\
\hline Adjusted rate & $6.8 \%$ & $8.6 \%$ & $0.0 \%$ & $2.2 \%$ \\
\hline Terminal rate & 3/32 (9\%) & $4 / 40(10 \%)$ & $0 / 26(0 \%)$ & 1/44 (2\%) \\
\hline
\end{tabular}


Indole-3-carbinol, NTP TR 584

\begin{tabular}{|c|c|c|c|c|}
\hline & Vehicle Control & $62.5 \mathrm{mg} / \mathrm{kg}$ & $125 \mathrm{mg} / \mathrm{kg}$ & $250 \mathrm{mg} / \mathrm{kg}$ \\
\hline First incidence (days) & $729(\mathrm{~T})$ & $729(\mathrm{~T})$ & - & $729(\mathrm{~T})$ \\
\hline Poly-3 test & $\mathrm{P}=0.108 \mathrm{~N}$ & $\mathrm{P}=0.525$ & $\mathrm{P}=0.135 \mathrm{~N}$ & $\mathrm{P}=0.288 \mathrm{~N}$ \\
\hline \multicolumn{5}{|l|}{ Uterus: Stromal Polyp } \\
\hline Overall rate & $0 / 50(0 \%)$ & $0 / 50(0 \%)$ & $0 / 50(0 \%)$ & $4 / 50(8 \%)$ \\
\hline Adjusted rate & $0.0 \%$ & $0.0 \%$ & $0.0 \%$ & $8.4 \%$ \\
\hline Terminal rate & $0 / 33(0 \%)$ & $0 / 40(0 \%)$ & $0 / 26(0 \%)$ & $4 / 45(9 \%)$ \\
\hline First incidence (days) & - & - & - & $729(\mathrm{~T})$ \\
\hline Poly-3 test & $P=0.003$ & $-^{\mathrm{f}}$ & - & $P=0.067$ \\
\hline \multicolumn{5}{|c|}{ All Organs: Hemangioma or Hemangiosarcoma } \\
\hline Overall rate & $1 / 50(2 \%)$ & $0 / 50(0 \%)$ & $3 / 50(6 \%)$ & $2 / 50(4 \%)$ \\
\hline Adjusted rate & $2.2 \%$ & $0.0 \%$ & $7.2 \%$ & $4.2 \%$ \\
\hline Terminal rate & $0 / 33(0 \%)$ & $0 / 40(0 \%)$ & $0 / 26(0 \%)$ & $2 / 45(4 \%)$ \\
\hline First incidence (days) & 709 & - & 621 & $729(\mathrm{~T})$ \\
\hline Poly-3 test & $\mathrm{P}=0.258$ & $\mathrm{P}=0.496 \mathrm{~N}$ & $\mathrm{P}=0.274$ & $\mathrm{P}=0.516$ \\
\hline \multicolumn{5}{|c|}{ All Organs: Malignant Lymphoma } \\
\hline Overall rate & $6 / 50(12 \%)$ & 9/50 (18\%) & $6 / 50(12 \%)$ & $3 / 50(6 \%)$ \\
\hline Adjusted rate & $12.9 \%$ & $18.9 \%$ & $14.4 \%$ & $6.3 \%$ \\
\hline Terminal rate & 0/33 (0\%) & $6 / 40(15 \%)$ & 3/26 (12\%) & $3 / 45(7 \%)$ \\
\hline First incidence (days) & 634 & 558 & 597 & $729(\mathrm{~T})$ \\
\hline Poly-3 test & $\mathrm{P}=0.116 \mathrm{~N}$ & $P=0.301$ & $\mathrm{P}=0.540$ & $\mathrm{P}=0.233 \mathrm{~N}$ \\
\hline \multicolumn{5}{|c|}{ All Organs: Benign Neoplasms } \\
\hline Overall rate & $17 / 50$ (34\%) & $21 / 50(42 \%)$ & $17 / 50(34 \%)$ & $20 / 50(40 \%)$ \\
\hline Adjusted rate & $36.5 \%$ & $44.8 \%$ & $39.1 \%$ & $41.7 \%$ \\
\hline Terminal rate & 12/33 (36\%) & $18 / 40$ (45\%) & $11 / 26(42 \%)$ & $19 / 45(42 \%)$ \\
\hline First incidence (days) & 568 & 681 & 381 & 603 \\
\hline Poly-3 test & $P=0.427$ & $P=0.274$ & $P=0.485$ & $P=0.382$ \\
\hline \multicolumn{5}{|c|}{ All Organs: Malignant Neoplasms } \\
\hline Overall rate & 19/50 (38\%) & 19/50 (38\%) & $26 / 50(52 \%)$ & 9/50 (18\%) \\
\hline Adjusted rate & $39.4 \%$ & $39.6 \%$ & $56.5 \%$ & $18.3 \%$ \\
\hline Terminal rate & 8/33 (24\%) & $14 / 40$ (35\%) & 10/26 (39\%) & $6 / 45(13 \%)$ \\
\hline First incidence (days) & 569 & 558 & 526 & 491 \\
\hline Poly-3 test & $\mathrm{P}=0.015 \mathrm{~N}$ & $\mathrm{P}=0.576$ & $\mathrm{P}=0.070$ & $\mathrm{P}=0.017 \mathrm{~N}$ \\
\hline \multicolumn{5}{|c|}{ All Organs: Benign or Malignant Neoplasms } \\
\hline Overall rate & $30 / 50$ (60\%) & $32 / 50$ (64\%) & $36 / 50$ (72\%) & $26 / 50$ (52\%) \\
\hline Adjusted rate & $61.5 \%$ & $66.2 \%$ & $75.2 \%$ & $52.8 \%$ \\
\hline Terminal rate & 18/33 (55\%) & $25 / 40$ (63\%) & $17 / 26(65 \%)$ & $23 / 45$ (51\%) \\
\hline
\end{tabular}


Indole-3-carbinol, NTP TR 584

\begin{tabular}{|c|c|c|c|c|}
\hline & Vehicle Control & $62.5 \mathrm{mg} / \mathrm{kg}$ & $125 \mathrm{mg} / \mathrm{kg}$ & $250 \mathrm{mg} / \mathrm{kg}$ \\
\hline First incidence (days) & 568 & 558 & 381 & 491 \\
\hline Poly-3 test & $\mathrm{P}=0.182 \mathrm{~N}$ & $\mathrm{P}=0.393$ & $P=0.105$ & $\mathrm{P}=0.252 \mathrm{~N}$ \\
\hline
\end{tabular}

(T) Terminal euthanasia.

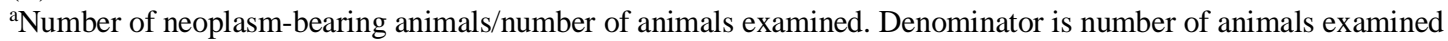

microscopically for liver, lung, ovary, and pituitary gland; for other tissues, denominator is number of animals necropsied.

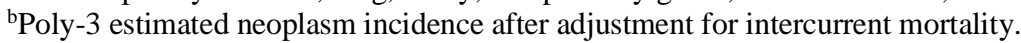

'Observed incidence at terminal euthanasia.

${ }^{\mathrm{d} B e n e a t h}$ the vehicle control incidence is the $\mathrm{P}$ value associated with the trend test. Beneath the dosed group incidence are the $\mathrm{P}$ values corresponding to pairwise comparisons between the vehicle controls and that dosed group. The Poly-3 test accounts for differential mortality in animals that do not reach terminal euthanasia. A negative trend or a lower incidence in a dose group is indicated by $\mathbf{N}$.

eNot applicable; no neoplasms in animal group.

fValue of statistic cannot be computed.

Table D-3. Historical Incidence of Liver Neoplasms in Control Female B6C3F1/N Mice ${ }^{\mathrm{a}}$

\begin{tabular}{lccc}
\hline \multicolumn{1}{c}{ Study (Study Start) } & $\begin{array}{c}\text { Hepatocellular } \\
\text { Adenoma }\end{array}$ & $\begin{array}{c}\text { Hepatocellular } \\
\text { Carcinoma }\end{array}$ & Hepatoblastoma \\
\hline Historical Incidence: Corn Oil Gavage Studies & & & \\
Ginkgo biloba extract (March 2005) & $17 / 50$ & $9 / 50$ & $1 / 50$ \\
Indole-3-carbinol (April 2007) & $7 / 50$ & $6 / 50$ & $0 / 50$ \\
Kava kava extract (August 2004) & $8 / 50$ & $3 / 50$ & $0 / 50$ \\
$N, N$-Dimethyl-p-toluidine (October 2004) & $17 / 50$ & $6 / 50$ & $0 / 50$ \\
Tetrabromobisphenol A (August 2007) & $13 / 50$ & $2 / 50$ & $0 / 50$ \\
Total (\%) & $62 / 250(24.8 \%)$ & $26 / 250(10.4 \%)$ & $1 / 250(0.4 \%)$ \\
Mean \pm standard deviation & $24.8 \% \pm 9.6 \%$ & $10.4 \% \pm 5.6 \%$ & $0.4 \% \pm 0.9 \%$ \\
Range & $14 \%-34 \%$ & $4 \%-18 \%$ & $0 \%-2 \%$ \\
Overall Historical Incidence: All Routes & & & $4 / 948(0.4 \%)$ \\
Total (\%) & $378 / 948(39.9 \%)$ & $152 / 948(16.0 \%)$ & $0.4 \% \pm 0.8 \%$ \\
Mean \pm standard deviation & $39.9 \% \pm 18.7 \%$ & $16.0 \% \pm 10.6 \%$ & $0 \%-2 \%$ \\
Range & $14 \%-78 \%$ & $4 \%-46 \%$ & \\
\hline
\end{tabular}

aData as of June 2013. 
Indole-3-carbinol, NTP TR 584

Table D-4. Summary of the Incidence of Nonneoplastic Lesions in Female Mice in the Two-year Gavage Study of Indole-3-carbinol ${ }^{\mathrm{a}}$

$\begin{aligned} & \text { Vehicle } \\ & \text { Control }\end{aligned} \quad 62.5 \mathrm{mg} / \mathrm{kg} \quad 125 \mathrm{mg} / \mathrm{kg} \quad 250 \mathrm{mg} / \mathrm{kg}$

\section{Disposition Summary}

Animals initially in study

50

50

50

50

Early deaths

Accidental death

Moribund

10

Natural deaths

6

6

-
15

$-$

Survivors

Died last week of study

Terminal euthanasia

$-$

33

40

45

Animals examined microscopically

50

50

25

50

\section{Alimentary System}

Esophagus

(50)

(50)

Gallbladder

(50)

$$
\text { Cyst }
$$

Inflammation, chronic

Intestine large, cecum

$1(2 \%)$

Intestine large, colon

$1(2 \%)$

Intestine large, rectum

Intestine small, duodenum

Intestine small, ileum

Ulcer

$1(2 \%)$

Epithelium, hyperplasia

$1(2 \%)$

Intestine small, jejunum

Ulcer

$1(2 \%)$

Liver

(50)

(50)

Angiectasis

Basophilic focus

$1(2 \%)$

$4(8 \%)$

$6(12 \%)$

7 (14\%)

$5(10 \%)$

Clear cell focus

Eosinophilic focus

Fatty change

Hematopoietic cell proliferation

Hemorrhage

$3(6 \%)$

$2(4 \%)$

$1(2 \%)$

$1(2 \%)$

$16(32 \%)$

26 (52\%)

$26(52 \%)$

$21(42 \%)$

36 (72\%)

39 (78\%)

$35(70 \%)$

$40(80 \%)$

$2(4 \%)$

$-\quad 2(4 \%)$

Hepatodiaphragmatic nodule

- $\quad-$

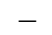

$-$

- $\quad 1(2 \%)$

Hyperplasia, lymphoid

- $\quad-\quad-$


Indole-3-carbinol, NTP TR 584

\begin{tabular}{|c|c|c|c|c|}
\hline & $\begin{array}{l}\text { Vehicle } \\
\text { Control }\end{array}$ & $62.5 \mathrm{mg} / \mathrm{kg}$ & $125 \mathrm{mg} / \mathrm{kg}$ & $250 \mathrm{mg} / \mathrm{kg}$ \\
\hline Inflammation, chronic & - & $1(2 \%)$ & - & - \\
\hline Mixed cell focus & $2(4 \%)$ & $4(8 \%)$ & $5(10 \%)$ & $1(2 \%)$ \\
\hline Necrosis & $1(2 \%)$ & $1(2 \%)$ & $1(2 \%)$ & - \\
\hline Bile duct, cyst & - & $1(2 \%)$ & - & - \\
\hline Centrilobular, necrosis & - & - & $1(2 \%)$ & - \\
\hline Mesentery & (3) & (7) & (7) & (6) \\
\hline Fat, necrosis & $3(100 \%)$ & $6(86 \%)$ & $7(100 \%)$ & $5(83 \%)$ \\
\hline Pancreas & (50) & (50) & (50) & (50) \\
\hline Angiectasis & - & $1(2 \%)$ & - & - \\
\hline Inflammation, chronic & $1(2 \%)$ & $1(2 \%)$ & - & - \\
\hline Acinus, atrophy & - & - & - & $1(2 \%)$ \\
\hline Acinus, hyperplasia & $1(2 \%)$ & $2(4 \%)$ & $1(2 \%)$ & - \\
\hline Duct, cyst & $1(2 \%)$ & - & - & - \\
\hline Salivary glands & (50) & (49) & (50) & (50) \\
\hline Hyperplasia & $1(2 \%)$ & - & - & - \\
\hline Duct, cyst & - & - & - & $1(2 \%)$ \\
\hline Stomach, forestomach & (50) & (50) & (50) & (50) \\
\hline Inflammation, chronic & $1(2 \%)$ & - & - & - \\
\hline Ulcer & $9(18 \%)$ & $4(8 \%)$ & $8(16 \%)$ & $3(6 \%)$ \\
\hline Epithelium, hyperplasia & - & - & $1(2 \%)$ & - \\
\hline Stomach, glandular & (48) & (50) & (49) & (50) \\
\hline Erosion & - & - & - & $1(2 \%)$ \\
\hline Hyperplasia, lymphoid & $1(2 \%)$ & - & - & - \\
\hline Inflammation, chronic & - & $15(30 \%)$ & $29(59 \%)$ & 47 (94\%) \\
\hline Mineralization & $2(4 \%)$ & - & $3(6 \%)$ & - \\
\hline Pigmentation & - & $15(30 \%)$ & $31(63 \%)$ & 49 (98\%) \\
\hline Epithelium, hyperplasia & $1(2 \%)$ & 7 (14\%) & $10(20 \%)$ & 35 (70\%) \\
\hline Tongue & $(0)$ & $(0)$ & (0) & (1) \\
\hline Tooth & (6) & (9) & (9) & (11) \\
\hline Dysplasia & $6(100 \%)$ & $9(100 \%)$ & $9(100 \%)$ & $10(91 \%)$ \\
\hline \multicolumn{5}{|l|}{ Cardiovascular System } \\
\hline Blood vessel & $(48)$ & (50) & $(50)$ & (50) \\
\hline Mineralization & - & - & $1(2 \%)$ & - \\
\hline Aorta, inflammation, chronic & - & - & - & $1(2 \%)$ \\
\hline Heart & (50) & (50) & (50) & (50) \\
\hline
\end{tabular}


Indole-3-carbinol, NTP TR 584

\begin{tabular}{|c|c|c|c|c|}
\hline & $\begin{array}{l}\text { Vehicle } \\
\text { Control }\end{array}$ & $62.5 \mathrm{mg} / \mathrm{kg}$ & $125 \mathrm{mg} / \mathrm{kg}$ & $250 \mathrm{mg} / \mathrm{kg}$ \\
\hline Cardiomyopathy & $4(8 \%)$ & $3(6 \%)$ & $1(2 \%)$ & $2(4 \%)$ \\
\hline Inflammation, chronic & - & $1(2 \%)$ & - & - \\
\hline Artery, inflammation, chronic & - & - & $1(2 \%)$ & $1(2 \%)$ \\
\hline Myocardium, mineralization & $2(4 \%)$ & - & - & $1(2 \%)$ \\
\hline \multicolumn{5}{|l|}{ Endocrine System } \\
\hline Adrenal cortex & (49) & $(50)$ & $(50)$ & $(50)$ \\
\hline Hematopoietic cell proliferation & $1(2 \%)$ & - & - & - \\
\hline Hyperplasia & $1(2 \%)$ & $1(2 \%)$ & - & - \\
\hline Inflammation, chronic & $1(2 \%)$ & - & - & - \\
\hline Necrosis & - & $1(2 \%)$ & - & $1(2 \%)$ \\
\hline Vacuolization cytoplasmic & $1(2 \%)$ & $1(2 \%)$ & $1(2 \%)$ & - \\
\hline Adrenal medulla & (49) & $(50)$ & $(50)$ & $(50)$ \\
\hline Hyperplasia & $1(2 \%)$ & $1(2 \%)$ & $3(6 \%)$ & - \\
\hline Islets, pancreatic & $(50)$ & $(50)$ & (50) & $(50)$ \\
\hline Hyperplasia & $5(10 \%)$ & $1(2 \%)$ & $4(8 \%)$ & $1(2 \%)$ \\
\hline Parathyroid gland & (43) & $(48)$ & (49) & $(46)$ \\
\hline Pituitary gland & (49) & $(50)$ & $(50)$ & (49) \\
\hline Cyst & - & $2(4 \%)$ & - & - \\
\hline Pars distalis, hyperplasia & $5(10 \%)$ & $18(36 \%)$ & $6(12 \%)$ & $4(8 \%)$ \\
\hline Pars distalis, vacuolization cytoplasmic & - & - & - & $1(2 \%)$ \\
\hline Pars intermedia, hyperplasia & $1(2 \%)$ & - & - & - \\
\hline Thyroid gland & $(50)$ & $(50)$ & (49) & $(50)$ \\
\hline Inflammation, chronic & - & - & $1(2 \%)$ & - \\
\hline Follicle, hyperplasia & & - & - & $1(2 \%)$ \\
\hline \multicolumn{5}{|l|}{ General Body System } \\
\hline Tissue NOS & $(0)$ & $(0)$ & (1) & (0) \\
\hline Inflammation, chronic & - & - & $1(100 \%)$ & - \\
\hline \multicolumn{5}{|l|}{ Genital System } \\
\hline Clitoral gland & (49) & $(50)$ & $(50)$ & (50) \\
\hline Ovary & $(50)$ & (50) & $(50)$ & (50) \\
\hline Angiectasis & - & - & $1(2 \%)$ & $1(2 \%)$ \\
\hline Cyst & $16(32 \%)$ & 15 (30\%) & $9(18 \%)$ & $8(16 \%)$ \\
\hline Inflammation, chronic & $1(2 \%)$ & - & $1(2 \%)$ & - \\
\hline Mineralization & - & - & $1(2 \%)$ & - \\
\hline Thrombosis & $2(4 \%)$ & - & $2(4 \%)$ & $2(4 \%)$ \\
\hline
\end{tabular}


Indole-3-carbinol, NTP TR 584

\begin{tabular}{|c|c|c|c|c|}
\hline & $\begin{array}{l}\text { Vehicle } \\
\text { Control }\end{array}$ & $62.5 \mathrm{mg} / \mathrm{kg}$ & $125 \mathrm{mg} / \mathrm{kg}$ & $250 \mathrm{mg} / \mathrm{kg}$ \\
\hline Uterus & $(50)$ & $(50)$ & $(50)$ & $(50)$ \\
\hline Inflammation, suppurative & - & $1(2 \%)$ & - & - \\
\hline Inflammation, chronic & - & $4(8 \%)$ & - & - \\
\hline Metaplasia, squamous & - & - & $1(2 \%)$ & - \\
\hline Necrosis & $1(2 \%)$ & - & - & - \\
\hline Endometrium, hyperplasia, cystic & $36(72 \%)$ & $32(64 \%)$ & $30(60 \%)$ & $34(68 \%)$ \\
\hline \multicolumn{5}{|l|}{ Hematopoietic System } \\
\hline Bone marrow & (50) & $(50)$ & $(50)$ & $(50)$ \\
\hline Lymph node & (9) & (5) & (6) & (1) \\
\hline Lumbar, ectasia & $1(11 \%)$ & - & - & - \\
\hline Lumbar, hemorrhage & - & - & $1(17 \%)$ & - \\
\hline Renal, ectasia & $2(22 \%)$ & $1(20 \%)$ & $2(33 \%)$ & - \\
\hline Lymph node, mandibular & $(50)$ & (49) & $(50)$ & (50) \\
\hline Lymph node, mesenteric & (49) & (49) & (49) & (49) \\
\hline Spleen & (50) & $(50)$ & $(50)$ & $(50)$ \\
\hline Angiectasis & $1(2 \%)$ & - & - & - \\
\hline Hematopoietic cell proliferation & $30(60 \%)$ & $30(60 \%)$ & 27 (54\%) & $23(46 \%)$ \\
\hline Hyperplasia, granulocytic & - & - & $1(2 \%)$ & - \\
\hline Hyperplasia, lymphoid & - & $1(2 \%)$ & - & - \\
\hline Hyperplasia, mast cell & - & - & - & $1(2 \%)$ \\
\hline Pigmentation, hemosiderin & $1(2 \%)$ & - & - & - \\
\hline Lymphoid follicle, atrophy & - & $1(2 \%)$ & - & - \\
\hline Lymphoid follicle, hyperplasia & $2(4 \%)$ & - & - & - \\
\hline Thymus & $(49)$ & $(50)$ & $(50)$ & $(50)$ \\
\hline Atrophy & $42(86 \%)$ & $40(80 \%)$ & $40(80 \%)$ & $41(82 \%)$ \\
\hline Hyperplasia & - & $1(2 \%)$ & - & - \\
\hline \multicolumn{5}{|l|}{ Integumentary System } \\
\hline Mammary gland & (50) & $(50)$ & $(50)$ & $(50)$ \\
\hline Inflammation, chronic & $1(2 \%)$ & - & - & - \\
\hline Skin & $(50)$ & (50) & $(50)$ & $(50)$ \\
\hline Ulcer & $1(2 \%)$ & - & $1(2 \%)$ & - \\
\hline Subcutaneous tissue, fibrosis & $1(2 \%)$ & - & - & - \\
\hline Subcutaneous tissue, hemorrhage & - & - & - & $1(2 \%)$ \\
\hline Subcutaneous tissue, inflammation, chronic & - & - & $1(2 \%)$ & - \\
\hline
\end{tabular}


Indole-3-carbinol, NTP TR 584

\begin{tabular}{|c|c|c|c|c|}
\hline & $\begin{array}{l}\text { Vehicle } \\
\text { Control }\end{array}$ & $62.5 \mathrm{mg} / \mathrm{kg}$ & $125 \mathrm{mg} / \mathrm{kg}$ & $250 \mathrm{mg} / \mathrm{kg}$ \\
\hline \multicolumn{5}{|l|}{ Musculoskeletal System } \\
\hline Bone & $(50)$ & $(50)$ & $(50)$ & $(50)$ \\
\hline Fibro-osseous lesion & $9(18 \%)$ & $13(26 \%)$ & $11(22 \%)$ & 17 (34\%) \\
\hline Fracture & $1(2 \%)$ & - & - & - \\
\hline Skeletal muscle & $(0)$ & (1) & (2) & $(0)$ \\
\hline \multicolumn{5}{|l|}{ Nervous System } \\
\hline Brain & $(50)$ & $(50)$ & $(50)$ & (50) \\
\hline Hemorrhage & $1(2 \%)$ & - & - & - \\
\hline Inflammation, granulomatous & - & - & - & $1(2 \%)$ \\
\hline Hypothalamus, compression & $2(4 \%)$ & - & - & - \\
\hline Meninges, inflammation, chronic & - & $1(2 \%)$ & - & - \\
\hline \multicolumn{5}{|l|}{ Respiratory System } \\
\hline Lung & (50) & (50) & $(50)$ & (50) \\
\hline Hemorrhage & - & - & - & $1(2 \%)$ \\
\hline Hyperplasia, lymphoid & - & $1(2 \%)$ & - & - \\
\hline Inflammation, chronic & - & $1(2 \%)$ & - & - \\
\hline Metaplasia, osseous & - & - & $1(2 \%)$ & - \\
\hline Thrombosis & $1(2 \%)$ & - & - & - \\
\hline Alveolar epithelium, hyperplasia & $1(2 \%)$ & $2(4 \%)$ & $1(2 \%)$ & $2(4 \%)$ \\
\hline Alveolus, infiltration cellular, histiocyte & $1(2 \%)$ & $1(2 \%)$ & $1(2 \%)$ & $1(2 \%)$ \\
\hline Mediastinum, inflammation, chronic & $1(2 \%)$ & - & - & - \\
\hline Nose & (50) & (50) & (50) & (50) \\
\hline Foreign body & - & - & $1(2 \%)$ & $2(4 \%)$ \\
\hline Inflammation & $4(8 \%)$ & $1(2 \%)$ & $8(16 \%)$ & $39(78 \%)$ \\
\hline Nerve, atrophy & - & - & $1(2 \%)$ & $50(100 \%)$ \\
\hline Nerve, olfactory epithelium, atrophy & $1(2 \%)$ & $1(2 \%)$ & - & - \\
\hline $\begin{array}{l}\text { Olfactory epithelium, accumulation, } \\
\text { hyaline droplet }\end{array}$ & $18(36 \%)$ & $27(54 \%)$ & $21(42 \%)$ & $44(88 \%)$ \\
\hline Olfactory epithelium, atrophy & $1(2 \%)$ & $2(4 \%)$ & $3(6 \%)$ & 45 (90\%) \\
\hline Olfactory epithelium, degeneration & - & - & $2(4 \%)$ & $3(6 \%)$ \\
\hline Olfactory epithelium, necrosis & - & - & $2(4 \%)$ & - \\
\hline $\begin{array}{l}\text { Olfactory epithelium, respiratory } \\
\text { metaplasia }\end{array}$ & $7(14 \%)$ & $8(16 \%)$ & $16(32 \%)$ & 49 (98\%) \\
\hline $\begin{array}{l}\text { Respiratory epithelium, accumulation, } \\
\text { hyaline droplet }\end{array}$ & 47 (94\%) & $38(76 \%)$ & $42(84 \%)$ & $50(100 \%)$ \\
\hline Respiratory epithelium, hyperplasia & $32(64 \%)$ & 31 (62\%) & 38 (76\%) & $50(100 \%)$ \\
\hline
\end{tabular}


Indole-3-carbinol, NTP TR 584

\begin{tabular}{|c|c|c|c|c|}
\hline & $\begin{array}{l}\text { Vehicle } \\
\text { Control }\end{array}$ & $62.5 \mathrm{mg} / \mathrm{kg}$ & $125 \mathrm{mg} / \mathrm{kg}$ & $250 \mathrm{mg} / \mathrm{kg}$ \\
\hline $\begin{array}{l}\text { Respiratory epithelium, metaplasia, } \\
\text { squamous }\end{array}$ & $1(2 \%)$ & - & - & - \\
\hline Respiratory epithelium, necrosis & $2(4 \%)$ & - & $1(2 \%)$ & - \\
\hline Trachea & $(50)$ & (50) & $(50)$ & $(50)$ \\
\hline \multicolumn{5}{|l|}{ Special Senses System } \\
\hline Ear & (2) & $(0)$ & $(0)$ & $(0)$ \\
\hline Eye & $(50)$ & (50) & $(50)$ & $(50)$ \\
\hline Cornea, inflammation, chronic & $1(2 \%)$ & - & $1(2 \%)$ & $1(2 \%)$ \\
\hline Lens, cataract & - & $1(2 \%)$ & $1(2 \%)$ & $1(2 \%)$ \\
\hline Harderian gland & $(50)$ & $(50)$ & $(50)$ & $(50)$ \\
\hline Dilatation & - & - & - & $6(12 \%)$ \\
\hline Hyperplasia & $2(4 \%)$ & $3(6 \%)$ & $5(10 \%)$ & $9(18 \%)$ \\
\hline Infiltration cellular, mononuclear cell & $24(48 \%)$ & $18(36 \%)$ & $8(16 \%)$ & $22(44 \%)$ \\
\hline \multicolumn{5}{|l|}{ Urinary System } \\
\hline Kidney & $(50)$ & (50) & $(50)$ & $(50)$ \\
\hline Infarct & $3(6 \%)$ & - & $1(2 \%)$ & - \\
\hline Metaplasia, osseous & - & - & - & $1(2 \%)$ \\
\hline Mineralization & $4(8 \%)$ & $3(6 \%)$ & $4(8 \%)$ & - \\
\hline Nephropathy & $24(48 \%)$ & $25(50 \%)$ & $19(38 \%)$ & $20(40 \%)$ \\
\hline Artery, inflammation, chronic & - & - & - & $1(2 \%)$ \\
\hline Renal tubule, accumulation, hyaline droplet & - & - & - & $1(2 \%)$ \\
\hline Renal tubule, cyst & - & - & $1(2 \%)$ & $1(2 \%)$ \\
\hline Renal tubule, pigmentation & $1(2 \%)$ & - & - & - \\
\hline Urinary bladder & $(50)$ & $(50)$ & $(50)$ & $(50)$ \\
\hline Infiltration cellular, mononuclear cell & $3(6 \%)$ & - & - & - \\
\hline Transitional epithelium, hyperplasia & - & - & - & $1(2 \%)$ \\
\hline
\end{tabular}




\section{Appendix E. Genetic Toxicology}

\section{Table of Contents}

E.1. Bacterial Mutagenicity Test Protocol ..................................................................... E-2

E.2. Rat Bone Marrow Micronucleus Test Protocol.......................................................... E-2

E.3. Mouse Peripheral Blood Micronucleus Test Protocol ................................................... E-3

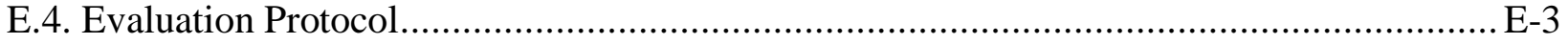

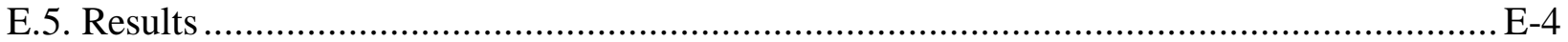

\section{Tables}

Table E-1. Mutagenicity of Indole-3-carbinol in Salmonella typhimurium ............................ E-5

Table E-2. Mutagenicity of Indole-3-carbinol in Bacterial Tester Strains.

Table E-3. Induction of Micronuclei in Bone Marrow Polychromatic Erythrocytes of Male F344/N Rats Following Treatment with Indole-3-carbinol by Gavage for Three Days

Table E-4. Frequency of Micronuclei in Peripheral Blood Erythrocytes of Mice Following Treatment with Indole-3-carbinol by Gavage for Three Months 


\section{E.1. Bacterial Mutagenicity Test Protocol}

Testing procedures used in the first two studies, conducted at BioReliance Corporation (Rockville, MD) followed protocols reported by Zeiger et al. ${ }^{135}$; in the study conducted by SITEK Research Laboratories (Rockville, MD) using the same chemical lot (CHP801001) that was used in the 3-month and 2-year bioassays, a slightly modified procedure was used, and that is described in detail below. Indole-3-carbinol was tested as a coded sample. In the studies conducted at BioReliance Corporation, indole-3-carbinol was incubated with the Salmonella typhimurium tester strains TA97, TA98, TA100, TA102, TA104, TA1535, and TA1537 either in buffer or $10 \%$ or $30 \%$ S9 mix (metabolic activation enzymes and cofactors from Aroclor 1254induced male Sprague Dawley rat or Syrian hamster liver) for 20 minutes at $37^{\circ} \mathrm{C}$. Top agar supplemented with L-histidine and d-biotin was added, and the contents of the tubes were mixed and poured onto the surfaces of minimal glucose agar plates. Histidine-independent mutant colonies arising on these plates were counted following incubation for 2 days at $37^{\circ} \mathrm{C}$.

The modified protocol used at SITEK Research Laboratories used only 10\% rat liver S9 for exogenous metabolic activation and employed tryptophan-dependent Escherichia coli strain WP2 uvrA/pKM101 as a bacterial tester strain in addition to S. typhimurium strains TA98 and TA100. Incubation of bacterial strains with indole-3-carbinol and subsequent plating were carried out as described above for the traditional protocol with the addition of tryptophandeficient medium for the $E$. coli tester strain.

In both studies, each trial consisted of triplicate plates of concurrent positive and negative controls and at least five doses of indole-3-carbinol. At both laboratories, the highest concentration tested was limited by toxicity. All trials were repeated except TA1537 without S9.

In this assay, a positive response is defined as a reproducible, dose-related increase in histidineor tryptophan-independent (revertant) colonies in any one strain/activation combination. An equivocal response is defined as an increase in revertants that is not dose related, is not reproducible, or is not of sufficient magnitude to support a determination of mutagenicity. A negative response is obtained when no increase in revertant colonies is observed following chemical treatment. There is no minimum percentage or fold increase required for a chemical to be judged positive or weakly positive, although positive calls are typically reserved for increases in mutant colonies that are at least twofold over background.

\section{E.2. Rat Bone Marrow Micronucleus Test Protocol}

Indole-3-carbinol (500, 1,000, or 2,000 mg/kg per day) was administered to male F344/ $\mathrm{N}$ rats, five per treatment group, once daily for 3 days by gavage, a protocol similar to that presented by Shelby et al. ${ }^{119}$. The positive control was $25 \mathrm{mg} / \mathrm{kg}$ cyclophosphamide and the vehicle control was corn oil. The rats were euthanized 24 hours after the third dosing, and blood smears were prepared from bone marrow cells obtained from the femurs. Air-dried smears were fixed and stained with acridine orange; 2,000 polychromatic erythrocytes (PCEs; reticulocytes) were scored for frequency of micronucleated cells in three to five rats per dose group. In addition, the percentage of PCEs among 200 erythrocytes in the bone marrow was determined for each animal as a measure of bone marrow toxicity. 
The results were tabulated as the mean of the pooled results from all animals within a treatment group plus or minus the standard error of the mean. The frequency of micronucleated cells among PCEs was analyzed by a statistical software package that tested for increasing trend over dose groups with a one-tailed Cochran-Armitage trend test, followed by pairwise comparisons between each dosed group and the vehicle control group. In the presence of excess binomial variation, as detected by a binomial dispersion test, the binomial variance of the CochranArmitage test was adjusted upward in proportion to the excess variation. In the micronucleus test, an individual trial is considered positive if the trend test $\mathrm{P}$ value is less than or equal to 0.025 or if the $\mathrm{P}$ value for any single dosed group is less than or equal to 0.025 divided by the number of dosed groups. A final call of positive for micronucleus induction is preferably based on reproducibly positive trials (as noted above). Ultimately, the final call is determined by the scientific staff after considering the results of statistical analyses, the reproducibility of any effects observed, and the magnitudes of those effects.

\section{E.3. Mouse Peripheral Blood Micronucleus Test Protocol}

A detailed discussion of this assay is presented by MacGregor et al. ${ }^{136}$. At the end of the 3-month toxicity study, peripheral blood samples were obtained from male and female mice. Smears were immediately prepared and fixed in absolute methanol. The methanol-fixed slides were shipped to the genetic toxicity testing laboratory (ILS, Inc., Research Triangle Park, NC) where they were stained with acridine orange and coded. Slides were scanned to determine the frequency of micronucleated cells in 2,000 normochromatic erythrocytes (NCEs) in each of five mice per dose group. In addition, the percentage of PCEs in a population of 1,000 erythrocytes was determined as a measure of bone marrow toxicity.

The peripheral blood results were tabulated as described for PCEs in the rat bone marrow micronucleus test. Results of the 3-month studies were accepted without repeat tests, because additional test data could not be obtained.

\section{E.4. Evaluation Protocol}

These are the basic guidelines for arriving at an overall assay result for assays performed by the National Toxicology Program. Statistical as well as biological factors are considered. For an individual assay, the statistical procedures for data analysis have been described in the preceding protocols. There have been instances, however, in which multiple samples of a chemical were tested in the same assay, and different results were obtained among these samples and/or among laboratories. Results from more than one aliquot or from more than one laboratory are not simply combined into an overall result. Rather, all the data are critically evaluated, particularly with regard to pertinent protocol variations, in determining the weight of evidence for an overall conclusion of chemical activity in an assay. In addition to multiple aliquots, the in vitro assays have another variable that must be considered in arriving at an overall test result. In vitro assays are conducted with and without exogenous metabolic activation. Results obtained in the absence of activation are not combined with results obtained in the presence of activation; each testing condition is evaluated separately. The summary table in the Abstract of this Technical Report presents a result that represents a scientific judgment of the overall evidence for activity of the chemical in an assay. 
Indole-3-carbinol, NTP TR 584

\section{E.5. Results}

Indole-3-carbinol was tested in three independent bacterial mutagenicity studies, and results were varied. The first study, which employed S. typhimurium strains TA97, TA98, TA100, TA1535, and TA1537 and used a concentration range of 3.3 to 3,333 $\mu$ g indole-3-carbinol/plate with and without $10 \%$ or $30 \%$ rat or hamster liver S9, yielded results that were judged to be equivocal in TA97 in the absence of S9 (Table E-1). A second study, using strains TA97, TA98, TA100, TA102, TA104, TA1535, and TA1537, and concentrations ranging from 33 to 10,000 $\mu \mathrm{g} / \mathrm{plate}$, yielded weak positive responses in strain TA100 without and with 30\% hamster liver S9 (no mutagenicity was seen in this strain in the presence of other concentrations or species of S9) (Table E-1). Results of the third study were judged to be equivocal in S. typhimurium strain TA100 (concentration range 500 to 5,000 $\mu$ g/plate) in the presence of $10 \%$ rat liver S9 and in $E$. coli strain WP2 uvrA/pKM101 (concentration range of 500 to 7,500 $\mu \mathrm{g} / \mathrm{plate}$ ) in the absence of S9 activation; no mutagenic activity was seen in this assay in S. typhimurium strain TA98, with or without S9, tested up to 5,000 $\mu$ g/plate (Table E-2).

In vivo, no increase in the frequency of micronucleated PCEs was seen in the bone marrow of male F344/N rats given three doses of indole-3-carbinol (500 to 2,000 $\mathrm{mg} / \mathrm{kg}$ per day) via gavage; however, a significant decrease in the percent PCEs was seen in the bone marrow of treated rats, indicating that indole-3-carbinol was toxic to the bone marrow (Table E-3). In addition to the rat study, micronucleus frequencies in NCEs of male and female B6C3F1/N mice were assessed in peripheral blood following 3 months of daily gavage treatment with indole-3carbinol (15.6 to $250 \mathrm{mg} / \mathrm{kg}$ per day) in corn oil; no significant increases in micronucleated NCEs were seen in either sex, and no significant changes in percent PCEs occurred over the dose range tested (Table E-4). 
Indole-3-carbinol, NTP TR 584

Table E-1. Mutagenicity of Indole-3-carbinol in Salmonella typhimurium ${ }^{\text {a }}$

\begin{tabular}{|c|c|c|c|c|c|c|c|}
\hline Strain & $\begin{array}{c}\text { Dose } \\
(\mu \mathrm{g} / \text { plate })\end{array}$ & Without S9 & Without S9 & $\begin{array}{c}\text { With } 10 \% \\
\text { Hamster S9 }\end{array}$ & $\begin{array}{c}\text { With } 30 \% \\
\text { Hamster S9 }\end{array}$ & $\begin{array}{c}\text { With } 10 \% \\
\text { Rat S9 }\end{array}$ & $\begin{array}{c}\text { With } 10 \% \\
\text { Rat S9 }\end{array}$ \\
\hline \multicolumn{8}{|l|}{ Study 1} \\
\hline \multirow[t]{9}{*}{ TA100 } & 0 & $98 \pm 4$ & $122 \pm 10$ & $122 \pm 6$ & $87 \pm 3$ & $117 \pm 14$ & $158 \pm 9$ \\
\hline & 3.3 & & $118 \pm 7$ & & & & \\
\hline & 33 & $92 \pm 6$ & $109 \pm 6$ & $121 \pm 9$ & $85 \pm 5$ & $138 \pm 8$ & \\
\hline & 100 & $111 \pm 10$ & $127 \pm 2$ & $122 \pm 5$ & $90 \pm 1$ & $141 \pm 3$ & $163 \pm 11$ \\
\hline & 333 & $107 \pm 9$ & $151 \pm 9$ & $147 \pm 4$ & $80 \pm 7$ & $177 \pm 4$ & $172 \pm 3$ \\
\hline & 1,000 & $98 \pm 12$ & $163 \pm 12$ & $122 \pm 17$ & $80 \pm 8$ & $206 \pm 17$ & $162 \pm 20$ \\
\hline & 1,500 & & & & & & $139 \pm 19$ \\
\hline & 2,000 & $34 \pm 4^{\mathrm{b}}$ & & $86 \pm 13^{b}$ & & $148 \pm 5$ & $74 \pm 7$ \\
\hline & 3,333 & & & & $63 \pm 8^{b}$ & & \\
\hline Trial summary & & Negative & Negative & Negative & Negative & Equivocal & Negative \\
\hline \multirow[t]{2}{*}{ Positive control } & & $218 \pm 12$ & $327 \pm 9$ & $813 \pm 96$ & $231 \pm 46$ & $854 \pm 30$ & $635 \pm 34$ \\
\hline & $\begin{array}{c}\text { Dose } \\
(\mu \mathrm{g} / \text { plate })\end{array}$ & $\begin{array}{c}\text { With } 30 \% \text { Rat } \\
\text { S9 } \\
\end{array}$ & & & & & \\
\hline \multirow[t]{6}{*}{ TA100 (continued) } & 0 & $84 \pm 3$ & & & & & \\
\hline & 33 & $81 \pm 7$ & & & & & \\
\hline & 100 & $76 \pm 9$ & & & & & \\
\hline & 333 & $76 \pm 4$ & & & & & \\
\hline & 1,000 & $80 \pm 6^{b}$ & & & & & \\
\hline & 3,333 & $38 \pm 4^{\mathrm{b}}$ & & & & & \\
\hline Trial summary & & Negative & & & & & \\
\hline \multirow[t]{2}{*}{ Positive control } & & $208 \pm 10$ & & & & & \\
\hline & $\begin{array}{c}\text { Dose } \\
(\mu \mathrm{g} / \text { plate })\end{array}$ & Without S9 & Without S9 & Without S9 & $\begin{array}{c}\text { With } 10 \% \\
\text { Hamster S9 }\end{array}$ & $\begin{array}{c}\text { With } 10 \% \\
\text { Hamster S9 }\end{array}$ & $\begin{array}{c}\text { With } 30 \% \\
\text { Hamster S9 }\end{array}$ \\
\hline \multirow[t]{9}{*}{ TA97 } & 0 & $197 \pm 10$ & $94 \pm 11$ & $134 \pm 9$ & $164 \pm 6$ & $123 \pm 4$ & $130 \pm 20$ \\
\hline & 3.3 & & & $109 \pm 14$ & & & \\
\hline & 33 & $187 \pm 13$ & $92 \pm 1$ & $118 \pm 4$ & $177 \pm 12$ & & $109 \pm 4$ \\
\hline & 100 & $203 \pm 10$ & $99 \pm 5$ & $108 \pm 5$ & $98 \pm 13$ & $119 \pm 7$ & $109 \pm 5$ \\
\hline & 333 & $249 \pm 1$ & $106 \pm 2$ & $155 \pm 1$ & $147 \pm 7$ & $103 \pm 6$ & $99 \pm 15$ \\
\hline & 667 & & $119 \pm 10$ & & & & \\
\hline & 1,000 & $203 \pm 6$ & $101 \pm 11$ & $159 \pm 4$ & $185 \pm 14$ & $109 \pm 14$ & $109 \pm 8$ \\
\hline & 2,000 & $0^{\mathrm{d}}$ & & & $202 \pm 14$ & $58 \pm 10$ & $123 \pm 10$ \\
\hline & 2,500 & & & & & $0^{\mathrm{b}}$ & \\
\hline Trial summary & & Equivocal & Equivocal & Negative & Equivocal & Negative & Negative \\
\hline Positive control & & $740 \pm 78$ & $214 \pm 24$ & $328 \pm 6$ & $958 \pm 82$ & $1,265 \pm 30$ & $536 \pm 40$ \\
\hline
\end{tabular}


Indole-3-carbinol, NTP TR 584

\begin{tabular}{|c|c|c|c|c|c|c|c|}
\hline & $\begin{array}{c}\text { Dose } \\
(\mu \mathrm{g} / \text { plate })\end{array}$ & $\begin{array}{c}\text { With } 10 \% \text { Rat } \\
\text { S9 }\end{array}$ & $\begin{array}{c}\text { With } 10 \% \\
\text { Rat S9 }\end{array}$ & $\begin{array}{c}\text { With } 30 \% \\
\text { Rat S9 }\end{array}$ & & & \\
\hline \multirow[t]{7}{*}{ TA97 (continued) } & 0 & $158 \pm 10$ & $125 \pm 9$ & $160 \pm 11$ & & & \\
\hline & 33 & $116 \pm 10$ & & $156 \pm 8$ & & & \\
\hline & 100 & $166 \pm 7$ & $116 \pm 4$ & $160 \pm 8$ & & & \\
\hline & 333 & $170 \pm 21$ & $111 \pm 7$ & $145 \pm 8$ & & & \\
\hline & 1,000 & $193 \pm 1$ & $101 \pm 11$ & $96 \pm 7$ & & & \\
\hline & 1,500 & & $94 \pm 7$ & & & & \\
\hline & 2,000 & $122 \pm 15$ & $68 \pm 5$ & $115 \pm 6$ & & & \\
\hline Trial summary & & Equivocal & Negative & Negative & & & \\
\hline \multirow[t]{2}{*}{ Positive control } & & $1,443 \pm 199$ & $1,135 \pm 37$ & $324 \pm 35$ & & & \\
\hline & $\begin{array}{c}\text { Dose } \\
(\mu \mathrm{g} / \text { plate }) \\
\end{array}$ & Without S9 & Without S9 & $\begin{array}{c}\text { With } 10 \% \\
\text { Hamster S9 }\end{array}$ & $\begin{array}{c}\text { With } 30 \% \\
\text { Hamster S9 }\end{array}$ & $\begin{array}{c}\text { With } 10 \% \\
\text { Rat S9 } \\
\end{array}$ & $\begin{array}{c}\text { With } 30 \% \\
\text { Rat S9 } \\
\end{array}$ \\
\hline \multirow[t]{8}{*}{ TA98 } & 0 & $26 \pm 1$ & $15 \pm 1$ & $24 \pm 3$ & $19 \pm 2$ & $21 \pm 3$ & $24 \pm 2$ \\
\hline & 3.3 & & $18 \pm 0$ & & & & \\
\hline & 33 & $21 \pm 3$ & $20 \pm 3$ & $28 \pm 2$ & $22 \pm 1$ & $23 \pm 3$ & $22 \pm 2$ \\
\hline & 100 & $21 \pm 2$ & $17 \pm 3$ & $25 \pm 4$ & $17 \pm 3$ & $25 \pm 2$ & $24 \pm 4$ \\
\hline & 333 & $24 \pm 3$ & $19 \pm 0$ & $24 \pm 4$ & $21 \pm 2$ & $30 \pm 3$ & $21 \pm 2$ \\
\hline & 1,000 & $14 \pm 2$ & $18 \pm 2$ & $24 \pm 3$ & $21 \pm 4$ & $26 \pm 1$ & $25 \pm 3$ \\
\hline & 2,000 & $13 \pm 2$ & & $16 \pm 2^{b}$ & & $12 \pm 1^{\mathrm{b}}$ & \\
\hline & 3,333 & & & & $14 \pm 2^{b}$ & & $14 \pm 1^{\mathrm{b}}$ \\
\hline Trial summary & & Negative & Negative & Negative & Negative & Negative & Negative \\
\hline Positive control & & $68 \pm 5$ & $90 \pm 5$ & $751 \pm 22$ & $55 \pm 2$ & $457 \pm 66$ & $82 \pm 13$ \\
\hline \multirow[t]{8}{*}{ TA1535 } & 0 & $15 \pm 1$ & $15 \pm 3$ & $16 \pm 2$ & $18 \pm 1$ & $13 \pm 0$ & $19 \pm 1$ \\
\hline & 3.3 & & $19 \pm 1$ & & & & \\
\hline & 33 & $19 \pm 3$ & $17 \pm 2$ & $11 \pm 2$ & $19 \pm 3$ & $18 \pm 0$ & $20 \pm 3$ \\
\hline & 100 & $23 \pm 4$ & $20 \pm 4$ & $14 \pm 3$ & $17 \pm 2$ & $12 \pm 2$ & $12 \pm 3$ \\
\hline & 333 & $18 \pm 2$ & $19 \pm 2$ & $13 \pm 3$ & $10 \pm 3$ & $12 \pm 1$ & $12 \pm 2$ \\
\hline & 1,000 & $19 \pm 3$ & $18 \pm 0$ & $12 \pm 4$ & $13 \pm 1$ & $14 \pm 4$ & $10 \pm 2$ \\
\hline & 2,000 & Toxic & & $10 \pm 1$ & & $9 \pm 1$ & \\
\hline & 3,333 & & & & $4 \pm 2^{\mathrm{e}}$ & & $0^{\mathrm{e}}$ \\
\hline Trial summary & & Negative & Negative & Negative & Negative & Negative & Negative \\
\hline \multirow[t]{2}{*}{ Positive control } & & $531 \pm 92$ & $364 \pm 34$ & $80 \pm 13$ & $210 \pm 11$ & $355 \pm 60$ & $79 \pm 5$ \\
\hline & $\begin{array}{c}\text { Dose } \\
(\mu \mathrm{g} / \text { plate })\end{array}$ & Without S9 & & & & & \\
\hline \multirow[t]{6}{*}{ TA1537 } & 0 & $5 \pm 0$ & & & & & \\
\hline & 33 & $8 \pm 3$ & & & & & \\
\hline & 100 & $5 \pm 2$ & & & & & \\
\hline & 333 & $4 \pm 1$ & & & & & \\
\hline & 1,000 & $7 \pm 1$ & & & & & \\
\hline & 1,500 & $3 \pm 2^{b}$ & & & & & \\
\hline Trial summary & & Negative & & & & & \\
\hline Positive control & & $13 \pm 1$ & & & & & \\
\hline
\end{tabular}


Indole-3-carbinol, NTP TR 584

\begin{tabular}{|c|c|c|c|c|c|c|c|}
\hline & $\begin{array}{c}\text { Dose } \\
(\mu \mathrm{g} / \text { plate })\end{array}$ & Without S9 & Without S9 & $\begin{array}{c}\text { With 10\% } \\
\text { Hamster S9 }\end{array}$ & $\begin{array}{c}\text { With 30\% } \\
\text { Hamster S9 }\end{array}$ & $\begin{array}{c}\text { With 30\% } \\
\text { Hamster S9 }\end{array}$ & $\begin{array}{c}\text { With } 10 \% \\
\text { Rat S9 }\end{array}$ \\
\hline \multicolumn{8}{|l|}{ Study 2} \\
\hline \multirow[t]{7}{*}{ TA102 } & 0 & $277 \pm 27$ & $366 \pm 5$ & $347 \pm 22$ & $358 \pm 9$ & $281 \pm 11$ & $348 \pm 11$ \\
\hline & 33 & $270 \pm 28$ & $378 \pm 6$ & $429 \pm 22$ & $357 \pm 10$ & & $436 \pm 24$ \\
\hline & 100 & $262 \pm 8$ & $362 \pm 6$ & $421 \pm 14$ & $397 \pm 31$ & $291 \pm 21$ & $405 \pm 33$ \\
\hline & 333 & $280 \pm 25$ & $345 \pm 14$ & $394 \pm 11$ & $389 \pm 23$ & $295 \pm 13$ & $416 \pm 49$ \\
\hline & 1,000 & $185 \pm 10$ & $255 \pm 50$ & $333 \pm 7$ & $434 \pm 25$ & $220 \pm 12$ & $320 \pm 18$ \\
\hline & 1,500 & & & & & $217 \pm 11$ & \\
\hline & 3,333 & $30 \pm 3^{d}$ & $84 \pm 5^{d}$ & $82 \pm 30^{d}$ & $167 \pm 32^{\mathrm{e}}$ & $96 \pm 9^{e}$ & $40 \pm 7^{d}$ \\
\hline Trial summary & & Negative & Negative & Negative & Equivocal & Negative & Negative \\
\hline \multirow[t]{2}{*}{ Positive control } & & $875 \pm 17$ & $1,435 \pm 32$ & $1,849 \pm 123$ & $1,383 \pm 22$ & $1,503 \pm 46$ & $1,714 \pm 98$ \\
\hline & $\begin{array}{c}\text { Dose } \\
(\mu \mathrm{g} / \mathrm{plate})\end{array}$ & $\begin{array}{c}\text { With } 30 \% \text { Rat } \\
\text { S9 }\end{array}$ & $\begin{array}{c}\text { With } 30 \% \\
\text { Rat S9 }\end{array}$ & & & & \\
\hline \multirow[t]{7}{*}{ TA102 (continued) } & 0 & $340 \pm 21$ & $233 \pm 14$ & & & & \\
\hline & 33 & $370 \pm 22$ & & & & & \\
\hline & 100 & $408 \pm 11$ & $203 \pm 6$ & & & & \\
\hline & 333 & $388 \pm 8$ & $224 \pm 4$ & & & & \\
\hline & 1,000 & $422 \pm 19$ & $148 \pm 12$ & & & & \\
\hline & 1,500 & & $115 \pm 6$ & & & & \\
\hline & 3,333 & $163 \pm 30^{e}$ & $102 \pm 8^{e}$ & & & & \\
\hline Trial summary & & Equivocal & Negative & & & & \\
\hline \multirow[t]{2}{*}{ Positive control } & & $1,107 \pm 66$ & $1,529 \pm 46$ & & & & \\
\hline & $\begin{array}{c}\text { Dose } \\
(\mu \mathrm{g} / \mathrm{plate})\end{array}$ & Without S9 & Without S9 & $\begin{array}{c}\text { With } 10 \% \\
\text { Hamster S9 }\end{array}$ & $\begin{array}{c}\text { With } 30 \% \\
\text { Hamster S9 }\end{array}$ & $\begin{array}{c}\text { With } 10 \% \\
\text { Rat S9 }\end{array}$ & $\begin{array}{c}\text { With } 30 \% \\
\text { Rat S9 }\end{array}$ \\
\hline \multirow[t]{6}{*}{ TA104 } & 0 & $222 \pm 11$ & $207 \pm 2$ & $249 \pm 18$ & $225 \pm 8$ & $206 \pm 13$ & $221 \pm 7$ \\
\hline & 33 & $205 \pm 9$ & $122 \pm 18$ & $187 \pm 7$ & $180 \pm 21$ & $184 \pm 7$ & $219 \pm 13$ \\
\hline & 100 & $213 \pm 6$ & $163 \pm 11$ & $216 \pm 19$ & $181 \pm 10$ & $204 \pm 17$ & $244 \pm 17$ \\
\hline & 333 & $180 \pm 19$ & $147 \pm 14$ & $175 \pm 19$ & $183 \pm 19$ & $204 \pm 15$ & $207 \pm 3$ \\
\hline & 1,000 & $185 \pm 14$ & $89 \pm 3$ & $208 \pm 23$ & $213 \pm 2$ & $181 \pm 9$ & $237 \pm 22$ \\
\hline & 3,333 & $0^{\mathrm{d}}$ & $47 \pm 11^{\mathrm{d}}$ & $205 \pm 5^{\mathrm{d}}$ & $38 \pm 1^{d}$ & $207 \pm 26^{d}$ & $46 \pm 3^{d}$ \\
\hline Trial summary & & Negative & Negative & Negative & Negative & Negative & Negative \\
\hline \multirow[t]{2}{*}{ Positive control } & & $705 \pm 14$ & $624 \pm 6$ & $1,080 \pm 34$ & $1,112 \pm 36$ & $1,173 \pm 17$ & $744 \pm 61$ \\
\hline & $\begin{array}{c}\text { Dose } \\
(\mu \mathrm{g} / \text { plate })\end{array}$ & Without S9 & Without S9 & Without S9 & $\begin{array}{c}\text { With 10\% } \\
\text { Hamster S9 }\end{array}$ & $\begin{array}{c}\text { With 10\% } \\
\text { Hamster S9 }\end{array}$ & $\begin{array}{c}\text { With 30\% } \\
\text { Hamster S9 }\end{array}$ \\
\hline \multirow[t]{8}{*}{ TA100 } & 0 & $90 \pm 0$ & $104 \pm 8$ & $149 \pm 7$ & $174 \pm 7$ & $139 \pm 9$ & $97 \pm 10$ \\
\hline & 33 & $76 \pm 10$ & $120 \pm 5$ & $141 \pm 11$ & $180 \pm 16$ & $146 \pm 7$ & $89 \pm 4$ \\
\hline & 100 & $114 \pm 5$ & $122 \pm 7$ & $161 \pm 7$ & $185 \pm 4$ & $138 \pm 10$ & $105 \pm 6$ \\
\hline & 333 & $123 \pm 7$ & $144 \pm 4$ & $196 \pm 23$ & $213 \pm 4$ & $170 \pm 5$ & $144 \pm 10$ \\
\hline & 1,000 & $133 \pm 19$ & $199 \pm 7$ & $166 \pm 3$ & $229 \pm 20$ & $183 \pm 6$ & $156 \pm 5$ \\
\hline & 1,200 & & $183 \pm 9$ & & & & \\
\hline & 1,500 & $46 \pm 44^{\mathrm{b}}$ & & & & $130 \pm 9^{e}$ & $149 \pm 18$ \\
\hline & 3,333 & & & $0^{\mathrm{d}}$ & $0^{\mathrm{d}}$ & & \\
\hline Trial summary & & Weakly Positive & $\begin{array}{l}\text { Weakly } \\
\text { Positive }\end{array}$ & Negative & Negative & Negative & $\begin{array}{l}\text { Weakly } \\
\text { Positive }\end{array}$ \\
\hline Positive control & & $225 \pm 20$ & $432 \pm 47$ & $594 \pm 33$ & $1,428 \pm 58$ & $845 \pm 79$ & $296 \pm 25$ \\
\hline
\end{tabular}


Indole-3-carbinol, NTP TR 584

\begin{tabular}{|c|c|c|c|c|c|c|c|}
\hline & $\begin{array}{c}\text { Dose } \\
(\mu \mathrm{g} / \text { plate) }\end{array}$ & $\begin{array}{c}\text { With 30\% } \\
\text { Hamster S9 }\end{array}$ & $\begin{array}{c}\text { With } 10 \% \\
\text { Rat S9 }\end{array}$ & $\begin{array}{c}\text { With } 10 \% \\
\text { Rat S9 }\end{array}$ & $\begin{array}{c}\text { With 30\% } \\
\text { Rat S9 }\end{array}$ & & \\
\hline \multirow[t]{8}{*}{ TA100 (continued) } & 0 & $102 \pm 2$ & $163 \pm 2$ & $121 \pm 16$ & $101 \pm 6$ & & \\
\hline & 33 & $104 \pm 2$ & $200 \pm 6$ & $141 \pm 16$ & $105 \pm 3$ & & \\
\hline & 100 & $106 \pm 2$ & $181 \pm 6$ & $120 \pm 7$ & $94 \pm 11$ & & \\
\hline & 333 & $132 \pm 11$ & $217 \pm 14$ & $178 \pm 15$ & $107 \pm 11$ & & \\
\hline & 1,000 & $157 \pm 16$ & $239 \pm 26$ & $158 \pm 16$ & $124 \pm 10$ & & \\
\hline & 1,200 & $191 \pm 8$ & & & & & \\
\hline & 1,500 & & & $132 \pm 9^{e}$ & $79 \pm 4^{e}$ & & \\
\hline & 3,333 & & $0^{\mathrm{d}}$ & & & & \\
\hline Trial summary & & Weakly Positive & Equivocal & Negative & Negative & & \\
\hline \multirow[t]{2}{*}{ Positive control } & & $956 \pm 35$ & $1,259 \pm 205$ & $595 \pm 37$ & $357 \pm 27$ & & \\
\hline & $\begin{array}{c}\text { Dose } \\
(\mu \mathrm{g} / \mathrm{plate}) \\
\end{array}$ & Without S9 & Without S9 & $\begin{array}{c}\text { With 10\% } \\
\text { Hamster S9 } \\
\end{array}$ & $\begin{array}{c}\text { With 30\% } \\
\text { Hamster S9 } \\
\end{array}$ & $\begin{array}{c}\text { With } 30 \% \\
\text { Hamster S9 }\end{array}$ & $\begin{array}{c}\text { With } 10 \% \\
\text { Rat S9 } \\
\end{array}$ \\
\hline \multirow[t]{8}{*}{ TA97 } & 0 & $114 \pm 2$ & $156 \pm 15$ & $186 \pm 29$ & $151 \pm 11$ & $132 \pm 6$ & $201 \pm 12$ \\
\hline & 33 & $110 \pm 9$ & $148 \pm 9$ & $203 \pm 13$ & $149 \pm 6$ & & $184 \pm 19$ \\
\hline & 100 & $107 \pm 8$ & $167 \pm 9$ & $211 \pm 5$ & $157 \pm 8$ & $159 \pm 8$ & $168 \pm 2$ \\
\hline & 333 & $126 \pm 4$ & $151 \pm 6$ & $229 \pm 32$ & $148 \pm 12$ & $160 \pm 9$ & $160 \pm 4$ \\
\hline & 1,000 & $133 \pm 4$ & $168 \pm 10$ & $208 \pm 17$ & $162 \pm 9$ & $185 \pm 6$ & $211 \pm 10$ \\
\hline & 1,500 & $108 \pm 12^{\mathrm{e}}$ & & & $210 \pm 6^{e}$ & $194 \pm 12$ & \\
\hline & 2,000 & & & & & $124 \pm 13$ & \\
\hline & 3,333 & & $0^{\mathrm{d}}$ & $0^{\mathrm{d}}$ & & & $0^{\mathrm{d}}$ \\
\hline Trial summary & & Negative & Negative & Negative & Equivocal & Equivocal & Negative \\
\hline \multirow[t]{2}{*}{ Positive control } & & $394 \pm 2$ & $476 \pm 32$ & $1,320 \pm 31$ & $1,680 \pm 75$ & $1,458 \pm 59$ & $1,671 \pm 307$ \\
\hline & $\begin{array}{c}\text { Dose } \\
(\mu \mathrm{g} / \text { plate }) \\
\end{array}$ & $\begin{array}{c}\text { With } 30 \% \text { Rat } \\
\text { S9 }\end{array}$ & $\begin{array}{c}\text { With } 30 \% \\
\text { Rat S9 } \\
\end{array}$ & & & & \\
\hline \multirow[t]{7}{*}{ TA97 (continued) } & 0 & $151 \pm 3$ & $145 \pm 8$ & & & & \\
\hline & 33 & $143 \pm 2$ & & & & & \\
\hline & 100 & $142 \pm 8$ & $132 \pm 2$ & & & & \\
\hline & 333 & $146 \pm 6$ & $164 \pm 11$ & & & & \\
\hline & 1,000 & $177 \pm 5$ & $167 \pm 8$ & & & & \\
\hline & 1,500 & $193 \pm 12^{\mathrm{e}}$ & $175 \pm 20$ & & & & \\
\hline & 2,000 & & $95 \pm 11$ & & & & \\
\hline Trial summary & & Equivocal & Equivocal & & & & \\
\hline \multirow[t]{2}{*}{ Positive control } & & $553 \pm 16$ & $463 \pm 15$ & & & & \\
\hline & $\begin{array}{c}\text { Dose } \\
(\mu \mathrm{g} / \text { plate })\end{array}$ & Without S9 & Without S9 & $\begin{array}{c}\text { With 10\% } \\
\text { Hamster S9 }\end{array}$ & $\begin{array}{c}\text { With 30\% } \\
\text { Hamster S9 }\end{array}$ & $\begin{array}{c}\text { With } 10 \% \\
\text { Rat S9 }\end{array}$ & $\begin{array}{c}\text { With } 30 \% \\
\text { Rat S9 }\end{array}$ \\
\hline \multirow[t]{7}{*}{ TA98 } & 0 & $15 \pm 2$ & $18 \pm 3$ & $18 \pm 5$ & $17 \pm 1$ & $21 \pm 3$ & $19 \pm 2$ \\
\hline & 33 & $13 \pm 3$ & $15 \pm 1$ & $21 \pm 4$ & $11 \pm 1$ & $18 \pm 2$ & $11 \pm 1$ \\
\hline & 100 & $13 \pm 1$ & $16 \pm 2$ & $21 \pm 5$ & $17 \pm 4$ & $21 \pm 2$ & $13 \pm 1$ \\
\hline & 333 & $13 \pm 1$ & $14 \pm 3$ & $21 \pm 6$ & $12 \pm 1$ & $27 \pm 4$ & $19 \pm 1$ \\
\hline & 1,000 & $10 \pm 2$ & $9 \pm 2$ & $18 \pm 3$ & $13 \pm 2$ & $20 \pm 1$ & $17 \pm 5$ \\
\hline & 1,500 & $0^{\mathrm{b}}$ & & & $6 \pm 1$ & & $9 \pm 2^{\mathrm{b}}$ \\
\hline & 3,333 & & $0^{\mathrm{d}}$ & $0^{\mathrm{d}}$ & & $0^{\mathrm{d}}$ & \\
\hline Trial summary & & Negative & Negative & Negative & Negative & Negative & Negative \\
\hline Positive control & & $115 \pm 2$ & $100 \pm 7$ & $1,536 \pm 136$ & $246 \pm 8$ & $629 \pm 141$ & $129 \pm 18$ \\
\hline
\end{tabular}


Indole-3-carbinol, NTP TR 584

\begin{tabular}{|c|c|c|c|c|c|c|c|}
\hline & $\begin{array}{c}\text { Dose } \\
(\mu \mathrm{g} / \text { plate })\end{array}$ & Without S9 & Without S9 & Without S9 & $\begin{array}{c}\text { With 10\% } \\
\text { Hamster S9 }\end{array}$ & $\begin{array}{c}\text { With } 30 \% \\
\text { Hamster S9 }\end{array}$ & $\begin{array}{c}\text { With } 30 \% \\
\text { Hamster S9 }\end{array}$ \\
\hline \multirow[t]{9}{*}{ TA1535 } & 0 & $14 \pm 2$ & $14 \pm 1$ & $18 \pm 2$ & $14 \pm 1$ & $10 \pm 1$ & $13 \pm 1$ \\
\hline & 33 & $13 \pm 2$ & & $18 \pm 3$ & $13 \pm 1$ & $13 \pm 0$ & \\
\hline & 100 & $14 \pm 1$ & & $19 \pm 3$ & $14 \pm 1$ & $10 \pm 1$ & \\
\hline & 333 & $13 \pm 1$ & $15 \pm 2$ & $20 \pm 1$ & $13 \pm 1$ & $13 \pm 1$ & $12 \pm 2$ \\
\hline & 1,000 & $15 \pm 1$ & $10 \pm 1$ & $18 \pm 2$ & $13 \pm 1$ & $13 \pm 1$ & $12 \pm 1$ \\
\hline & 1,200 & $12 \pm 1$ & & & & $14 \pm 3$ & \\
\hline & 1,500 & & $8 \pm 0$ & & & & $9 \pm 1$ \\
\hline & 3,333 & & $0^{\mathrm{d}}$ & $0^{\mathrm{d}}$ & $0^{\mathrm{d}}$ & & $2^{\mathrm{d}}$ \\
\hline & 10,000 & & $0^{\mathrm{d}}$ & & & & $0^{\mathrm{d}}$ \\
\hline Trial summary & & Negative & Negative & Negative & Negative & Negative & Negative \\
\hline \multirow[t]{2}{*}{ Positive control } & & $130 \pm 5$ & $166 \pm 4$ & $256 \pm 39$ & $287 \pm 24$ & $66 \pm 3$ & $155 \pm 23$ \\
\hline & $\begin{array}{c}\text { Dose } \\
(\mu \mathrm{g} / \text { plate })\end{array}$ & $\begin{array}{c}\text { With } 10 \% \text { Rat } \\
\text { S9 }\end{array}$ & $\begin{array}{c}\text { With 30\% } \\
\text { Rat S9 }\end{array}$ & & & & \\
\hline \multirow{7}{*}{$\begin{array}{l}\text { TA1535 } \\
\text { (continued) }\end{array}$} & 0 & $15 \pm 0$ & $13 \pm 3$ & & & & \\
\hline & 33 & $14 \pm 2$ & $12 \pm 1$ & & & & \\
\hline & 100 & $17 \pm 3$ & $10^{\mathrm{f}}$ & & & & \\
\hline & 333 & $15 \pm 1$ & $18 \pm 2$ & & & & \\
\hline & 1,000 & $12 \pm 2$ & $12 \pm 1$ & & & & \\
\hline & 1,500 & & $8 \pm 2^{e}$ & & & & \\
\hline & 3,333 & $0^{\mathrm{d}}$ & & & & & \\
\hline Trial summary & & Negative & Negative & & & & \\
\hline \multirow[t]{2}{*}{ Positive control } & & $422 \pm 19$ & $95 \pm 6$ & & & & \\
\hline & $\begin{array}{c}\text { Dose } \\
(\mu \mathrm{g} / \text { plate })\end{array}$ & $\begin{array}{c}\text { With } 30 \% \\
\text { Hamster S9 }\end{array}$ & $\begin{array}{c}\text { With 30\% } \\
\text { Rat S9 }\end{array}$ & & & & \\
\hline \multirow[t]{6}{*}{ TA1537 } & 0 & $9 \pm 2$ & $9 \pm 2$ & & & & \\
\hline & 100 & $8 \pm 1$ & $7 \pm 1$ & & & & \\
\hline & 333 & $8 \pm 1$ & $10 \pm 1$ & & & & \\
\hline & 1,000 & $9 \pm 2$ & $8 \pm 1$ & & & & \\
\hline & 1,500 & $5 \pm 1$ & $5 \pm 1$ & & & & \\
\hline & 2,000 & $4 \pm 1^{\mathrm{e}}$ & $5 \pm 1^{\mathrm{e}}$ & & & & \\
\hline Trial summary & & Negative & Negative & & & & \\
\hline Positive control & & $530 \pm 58$ & $143 \pm 22$ & & & & \\
\hline $\begin{array}{l}\text { aStudies were pe } \\
\text { plates. The detail } \\
\text { bSlight toxicity. } \\
\text { 'The positive cor } \\
\text { and TA1537), 4- } \\
\text { control for metab } \\
\text { for TA102. } \\
\text { dSlight toxicity a } \\
\text { eprecipitate on pl } \\
\text { fContamination. }\end{array}$ & $\begin{array}{l}\text { ned at BioRe } \\
\text { rotocol is pre } \\
\text { s in the abser } \\
\text {-O-phenylen } \\
\text { activation w } \\
\text { recipitate on }\end{array}$ & $\begin{array}{l}\text { eliance Corporatio } \\
\text { esented by Zeiger } \\
\text { nce of metabolic a } \\
\text { lediamine (TA98), } \\
\text { vith all strains was } \\
\text { plate. }\end{array}$ & $\begin{array}{l}\text { n. Data are pre } \\
\text { et al. }{ }^{135} .0 \mu \mathrm{g} / \\
\text { mitomycin-C } \\
\text { 2-aminoanthr }\end{array}$ & $\begin{array}{l}\text { ented as rever } \\
\text { late was the sc } \\
\text { odium azide ( } \\
\text { TA102), and n } \\
\text { cene, except } 2\end{array}$ & $\begin{array}{l}\text { nts/plate (mea } \\
\text { vent control. } \\
\text { A100 and TA1 } \\
\text { ethyl methanes } \\
\text { minoanthracer }\end{array}$ & $\begin{array}{l} \pm \text { standard errc } \\
\text { 35), 9-aminoa } \\
\text { lfonate (TA10 } \\
\text { or sterigmatoc }\end{array}$ & $\begin{array}{l}\text { from three } \\
\text { idine (TA97 } \\
\text { The positive } \\
\text { stin was used }\end{array}$ \\
\hline
\end{tabular}


Indole-3-carbinol, NTP TR 584

Table E-2. Mutagenicity of Indole-3-carbinol in Bacterial Tester Strains ${ }^{\text {a }}$

\begin{tabular}{|c|c|c|c|c|c|c|c|}
\hline Strain & $\begin{array}{c}\text { Dose } \\
\text { ( } \mu \text { g/plate) }\end{array}$ & Without S9 & Without S9 & Without S9 & $\begin{array}{c}\text { With } 10 \% \\
\text { Rat S9 }\end{array}$ & $\begin{array}{c}\text { With } 10 \% \\
\text { Rat S9 }\end{array}$ & $\begin{array}{c}\text { With } 10 \% \\
\text { Rat S9 }\end{array}$ \\
\hline \multirow[t]{7}{*}{ TA100 } & 0 & $44 \pm 3$ & $103 \pm 7$ & $81 \pm 4$ & $80 \pm 2$ & $61 \pm 3$ & $94 \pm 2$ \\
\hline & 100 & & $109 \pm 4$ & $90 \pm 0$ & & & \\
\hline & 500 & $63 \pm 2$ & $124 \pm 6$ & $129 \pm 1$ & $109 \pm 4$ & $84 \pm 2$ & $126 \pm 5$ \\
\hline & 1,500 & $47 \pm 1$ & $60 \pm 8$ & $75 \pm 3$ & $142 \pm 13$ & $84 \pm 3$ & $174 \pm 4$ \\
\hline & 2,500 & $32 \pm 1$ & $20 \pm 1^{\mathrm{b}}$ & $26 \pm 2$ & $74 \pm 1$ & $84 \pm 6$ & $123 \pm 5$ \\
\hline & 3,500 & $9 \pm 1^{b}$ & Toxic & $12 \pm 3^{b}$ & $45 \pm 5$ & $44 \pm 8^{c}$ & $23 \pm 3^{b}$ \\
\hline & 5,000 & Toxic & & & $6 \pm 3^{b}$ & $26 \pm 8^{c}$ & $5^{c}$ \\
\hline Trial summary & & Negative & Negative & Negative & Equivocal & Negative & Equivocal \\
\hline Positive control $^{\mathrm{d}}$ & & $420 \pm 24$ & $844 \pm 95$ & $737 \pm 40$ & $1,108 \pm 18$ & $831 \pm 93$ & $1,086 \pm 2$ \\
\hline \multirow[t]{7}{*}{ TA98 } & 0 & $24 \pm 1$ & $21 \pm 1$ & $16 \pm 2$ & $30 \pm 2$ & $34 \pm 2$ & $25 \pm 4$ \\
\hline & 100 & $21 \pm 4$ & & $24 \pm 1$ & & & \\
\hline & 500 & $17 \pm 2$ & $24 \pm 1$ & $20 \pm 1$ & $30 \pm 1$ & $37 \pm 1$ & $27 \pm 4$ \\
\hline & 1,500 & $11 \pm 2$ & $23 \pm 1$ & $9 \pm 1$ & $30 \pm 2$ & $27 \pm 2$ & $19 \pm 3$ \\
\hline & 2,500 & $4 \pm 1^{\mathrm{b}}$ & $15 \pm 1$ & $6 \pm 1^{b}$ & $27 \pm 2$ & $17 \pm 4$ & $9 \pm 2$ \\
\hline & 3,500 & Toxic & Toxic & Toxic & $11 \pm 1^{\mathrm{b}}$ & $11 \pm 0$ & $3 \pm 1^{b}$ \\
\hline & 5,000 & & Toxic & & $7 \pm 1^{b}$ & $3 \pm 1^{\mathrm{c}}$ & Toxic \\
\hline Trial summary & & Negative & Negative & Negative & Negative & Negative & Negative \\
\hline Positive control & & $441 \pm 43$ & $654 \pm 17$ & $411 \pm 41$ & $1,533 \pm 29$ & $787 \pm 4$ & $787 \pm 91$ \\
\hline \multicolumn{8}{|c|}{ Escherichia coli WP2 uvrA/pKM101 (Analogous to TA102) } \\
\hline & 0 & $136 \pm 5$ & $164 \pm 20$ & $188 \pm 4$ & $185 \pm 4$ & $188 \pm 6$ & $225 \pm 15$ \\
\hline & 500 & $193 \pm 5$ & $204 \pm 7$ & $241 \pm 5$ & & & \\
\hline & 1,500 & $218 \pm 8$ & $212 \pm 5$ & $279 \pm 11$ & $238 \pm 9$ & $261 \pm 3$ & $248 \pm 10$ \\
\hline & 2,500 & $194 \pm 10$ & $199 \pm 9$ & $234 \pm 11$ & $217 \pm 1$ & $235 \pm 9$ & $355 \pm 2$ \\
\hline & 3,500 & $139 \pm 9$ & $111 \pm 2$ & $173 \pm 9$ & $194 \pm 6$ & $178 \pm 9$ & $342 \pm 2$ \\
\hline & 5,000 & $87 \pm 8$ & $59 \pm 4^{b}$ & $128 \pm 9^{c}$ & $184 \pm 6$ & $115 \pm 5$ & $98 \pm 3^{b}$ \\
\hline & 7,500 & Toxic & Toxic & & $104 \pm 9$ & $63 \pm 5^{b}$ & $242 \pm 1^{\mathrm{c}}$ \\
\hline & 10,000 & & & & $65 \pm 12^{b}$ & Toxic & \\
\hline Trial summary & & Equivocal & Negative & Equivocal & Negative & Negative & Equivocal \\
\hline Positive control & & $1,992 \pm 81$ & $2,139 \pm 53$ & $1,810 \pm 66$ & $1,144 \pm 12$ & $1,080 \pm 17$ & $1,298 \pm 51$ \\
\hline
\end{tabular}

aStudy was performed at SITEK Research Laboratories using the same lot of indole-3-carbinol (CHP801001) used in the 3-month and 2-year bioassays and a modification of the protocol presented by Zeiger et al. ${ }^{135}$. Data are presented as revertants/plate (mean \pm standard error) from three plates. $0 \mu \mathrm{g} /$ plate was the solvent control.

bSlight toxicity.

'Precipitate on plate.

${ }^{\mathrm{d}}$ The positive controls in the absence of metabolic activation were sodium azide (TA100), 4-nitro-o-phenylenediamine (TA98), and methyl methanesulfonate (E. coli). The positive control for metabolic activation with all strains was 2-aminoanthracene. 
Indole-3-carbinol, NTP TR 584

Table E-3. Induction of Micronuclei in Bone Marrow Polychromatic Erythrocytes of Male F344/N Rats Following Treatment with Indole-3-carbinol by Gavage for Three Days ${ }^{\text {a }}$

\begin{tabular}{lccccc}
\hline & $\begin{array}{c}\text { Dose } \\
(\mathbf{m g} / \mathbf{k g})\end{array}$ & $\begin{array}{c}\text { Number of Rats } \\
\text { with Erythrocytes } \\
\text { Scored }\end{array}$ & $\begin{array}{c}\text { Micronucleated } \\
\text { PCEs/1,000 PCEs }\end{array}$ & P Value $^{\mathbf{c}}$ & PCEs (\%) $^{\mathbf{b}}$ (\%) \\
\hline Corn oil $^{\mathrm{d}}$ & 0 & 5 & $1.00 \pm 0.22$ & & $51.100 \pm 3.98$ \\
Indole-3-carbinol & 500 & 5 & $1.22 \pm 0.33$ & 0.3227 & $27.560 \pm 8.24$ \\
& 1,000 & 3 & $1.33 \pm 0.17$ & 0.2713 & $41.500 \pm 2.50$ \\
& 2,000 & 4 & $0.83 \pm 0.38$ & 0.6306 & $27.375 \pm 8.30$ \\
& & & $\mathrm{P}=0.611^{\mathrm{e}}$ & & \\
Cyclophosphamide $^{\mathrm{f}}$ & 25 & 4 & $26.17 \pm 5.17$ & 0.0000 & $7.075 \pm 2.21$ \\
\hline
\end{tabular}

a Study was performed at ILS, Inc., using a protocol similar to that presented by Shelby et al. ${ }^{119}$; PCE = polychromatic erythrocyte.

${ }^{\mathrm{b}}$ Mean \pm standard error.

'Pairwise comparison with the vehicle control group; dosed group values are significant at $\mathrm{P} \leq 0.008$; positive control values are significant at $\mathrm{P} \leq 0.05$.

${ }^{\mathrm{d}}$ Vehicle control.

'Significance of micronucleated PCEs/1,000 PCEs tested by the one-tailed trend test; significant at P $\leq 0.025$.

fPositive control. 
Indole-3-carbinol, NTP TR 584

Table E-4. Frequency of Micronuclei in Peripheral Blood Erythrocytes of Mice Following Treatment with Indole-3-carbinol by Gavage for Three Months ${ }^{a}$

\begin{tabular}{|c|c|c|c|c|c|}
\hline & $\begin{array}{c}\text { Dose } \\
\text { (mg/kg) }\end{array}$ & $\begin{array}{c}\text { Number of Mice } \\
\text { with Erythrocytes } \\
\text { Scored }\end{array}$ & $\begin{array}{l}\text { Micronucleated } \\
\text { NCEs/1,000 NCEs }\end{array}$ & P Value ${ }^{c}$ & $\operatorname{PCEs}^{\mathrm{b}}(\%)$ \\
\hline \multicolumn{6}{|l|}{ Male } \\
\hline Corn oil $^{\mathrm{d}}$ & 0 & 5 & $2.10 \pm 0.19$ & & $3.62 \pm 0.31$ \\
\hline \multirow[t]{6}{*}{ Indole-3-carbinol } & 15.6 & 5 & $1.50 \pm 0.27$ & 0.8416 & $3.68 \pm 0.16$ \\
\hline & 31.25 & 5 & $1.80 \pm 0.25$ & 0.6847 & $3.74 \pm 0.29$ \\
\hline & 62.5 & 5 & $2.00 \pm 0.35$ & 0.5621 & $3.84 \pm 0.29$ \\
\hline & 125 & 5 & $1.90 \pm 0.29$ & 0.6242 & $3.84 \pm 0.28$ \\
\hline & 250 & 5 & $1.60 \pm 0.33$ & 0.7947 & $3.52 \pm 0.34$ \\
\hline & \multicolumn{5}{|c|}{$P=0.663^{e}$} \\
\hline \multicolumn{6}{|l|}{ Female } \\
\hline Corn oil & 0 & 5 & $1.10 \pm 0.37$ & & $3.18 \pm 0.23$ \\
\hline \multirow[t]{6}{*}{ Indole-3-carbinol } & 15.6 & 5 & $1.40 \pm 0.19$ & 0.2741 & $2.60 \pm 0.49$ \\
\hline & 31.25 & 5 & $1.60 \pm 0.29$ & 0.1678 & $3.78 \pm 0.52$ \\
\hline & 62.5 & 5 & $1.60 \pm 0.33$ & 0.1678 & $3.24 \pm 0.41$ \\
\hline & 125 & 5 & $1.80 \pm 0.34$ & 0.0967 & $3.40 \pm 0.29$ \\
\hline & 250 & 5 & $1.90 \pm 0.33$ & 0.0719 & $3.12 \pm 0.19$ \\
\hline & \multicolumn{5}{|c|}{$\mathrm{P}=0.087$} \\
\hline \multicolumn{6}{|c|}{$\begin{array}{l}\text { aStudy was performed at ILS, Inc. The detailed protocol is presented by MacGregor et al. }{ }^{136} \text {; NCE = normochrc } \\
\text { PCE = polychromatic erythrocyte. } \\
\text { bMean } \pm \text { standard error. } \\
\text { 'Pairwise comparison with the vehicle control group; dosed group values are significant at } \mathrm{P} \leq 0.005 \text {. } \\
\text { dVehicle control. } \\
\text { eSignificance of micronucleated NCEs } / 1,000 \text { NCEs tested by the one-tailed trend test; significant at } \mathrm{P} \leq 0.025 \text {. }\end{array}$} \\
\hline
\end{tabular}


Indole-3-carbinol, NTP TR 584

\section{Appendix F. Clinical Pathology Results}

\section{Tables}

Table F-1. Hematology and Clinical Chemistry Data for F344/N Rats in the Three-month Gavage Study of Indole-3-carbinol ........................................................ F-2

Table F-2. Hematology Data for Mice in the Three-month Gavage Study of

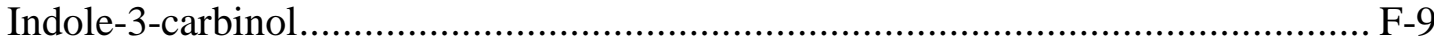


Indole-3-carbinol, NTP TR 584

Table F-1. Hematology and Clinical Chemistry Data for F344/N Rats in the Three-month Gavage Study of Indole-3-carbinol ${ }^{\mathrm{a}}$

\begin{tabular}{|c|c|c|c|c|c|c|}
\hline & Vehicle Control & $18.75 \mathrm{mg} / \mathrm{kg}$ & $37.5 \mathrm{mg} / \mathrm{kg}$ & 75 mg/kg & $150 \mathrm{mg} / \mathrm{kg}$ & $300 \mathrm{mg} / \mathrm{kg}$ \\
\hline \multicolumn{7}{|l|}{ Male } \\
\hline \multicolumn{7}{|l|}{ Hematology } \\
\hline \multicolumn{7}{|l|}{$\mathbf{n}$} \\
\hline Day 4 & 10 & 10 & 10 & 10 & 10 & 10 \\
\hline Day 25 & 9 & 10 & 9 & 10 & 9 & 9 \\
\hline Week 14 & 10 & 10 & 10 & 10 & 9 & 10 \\
\hline \multicolumn{7}{|c|}{ Hematocrit (auto) (\%) } \\
\hline Day 4 & $45.0 \pm 0.8$ & $45.4 \pm 0.6$ & $44.5 \pm 0.4$ & $45.1 \pm 0.5$ & $44.3 \pm 0.4$ & $47.3 \pm 1.5$ \\
\hline Day 25 & $45.9 \pm 0.4$ & $46.5 \pm 0.5$ & $44.6 \pm 0.6$ & $46.0 \pm 0.7$ & $45.4 \pm 0.5$ & $45.0 \pm 0.2$ \\
\hline Week 14 & $48.5 \pm 0.3$ & $47.8 \pm 0.4$ & $48.8 \pm 0.3$ & $48.7 \pm 0.4$ & $48.4 \pm 0.5$ & $48.5 \pm 0.6$ \\
\hline \multicolumn{7}{|c|}{ Hematocrit (spun) (\%) } \\
\hline Day 4 & $44.3 \pm 0.6$ & $44.7 \pm 0.6$ & $43.9 \pm 0.3$ & $44.3 \pm 0.4$ & $44.0 \pm 0.5$ & $46.3 \pm 1.4$ \\
\hline Day 25 & $46.2 \pm 0.3$ & $46.2 \pm 0.5$ & $45.0 \pm 0.5$ & $46.1 \pm 0.5$ & $45.6 \pm 0.6$ & $44.9 \pm 0.3$ \\
\hline Week 14 & $47.5 \pm 0.3$ & $46.8 \pm 0.4$ & $47.7 \pm 0.3$ & $47.8 \pm 0.5$ & $47.2 \pm 0.4$ & $47.3 \pm 0.4$ \\
\hline \multicolumn{7}{|c|}{ Hemoglobin (g/dL) } \\
\hline Day 4 & $14.9 \pm 0.3$ & $14.9 \pm 0.2$ & $14.7 \pm 0.1$ & $14.9 \pm 0.2$ & $14.7 \pm 0.1$ & $15.5 \pm 0.5$ \\
\hline Day 25 & $15.7 \pm 0.2$ & $15.8 \pm 0.2$ & $15.2 \pm 0.3$ & $15.8 \pm 0.2$ & $15.4 \pm 0.2$ & $15.4 \pm 0.1$ \\
\hline Week 14 & $15.9 \pm 0.1$ & $15.7 \pm 0.1$ & $16.1 \pm 0.1$ & $16.1 \pm 0.1$ & $15.9 \pm 0.2$ & $15.8 \pm 0.2$ \\
\hline \multicolumn{7}{|c|}{ Erythrocytes $\left(10^{6} / \mu \mathrm{L}\right)$} \\
\hline Day 4 & $7.39 \pm 0.12$ & $7.43 \pm 0.11$ & $7.30 \pm 0.07$ & $7.43 \pm 0.07$ & $7.36 \pm 0.05$ & $7.86 \pm 0.25$ \\
\hline Day 25 & $8.30 \pm 0.09$ & $8.38 \pm 0.10$ & $8.09 \pm 0.09$ & $8.31 \pm 0.13$ & $8.16 \pm 0.09$ & $8.25 \pm 0.06$ \\
\hline Week 14 & $8.49 \pm 0.05$ & $8.38 \pm 0.08$ & $8.67 \pm 0.06$ & $8.64 \pm 0.07$ & $8.63 \pm 0.08$ & $8.71 \pm 0.12$ \\
\hline \multicolumn{7}{|c|}{ Reticulocytes $\left(10^{5} / \mu \mathrm{L}\right)$} \\
\hline Day 4 & $5.49 \pm 0.37$ & $5.83 \pm 0.35$ & $5.66 \pm 0.39$ & $5.59 \pm 0.33$ & $5.13 \pm 0.19$ & $4.49 \pm 0.22 *$ \\
\hline Day 25 & $2.87 \pm 0.14$ & $3.05 \pm 0.08$ & $2.93 \pm 0.12$ & $2.82 \pm 0.14$ & $2.78 \pm 0.19$ & $2.47 \pm 0.17$ \\
\hline Week 14 & $2.14 \pm 0.04$ & $2.03 \pm 0.05$ & $1.96 \pm 0.04$ & $1.98 \pm 0.05$ & $2.04 \pm 0.09$ & $1.93 \pm 0.10$ \\
\hline \multicolumn{7}{|c|}{ Reticulocytes (\%) } \\
\hline Day 4 & $7.48 \pm 0.57$ & $7.90 \pm 0.55$ & $7.77 \pm 0.54$ & $7.52 \pm 0.46$ & $6.97 \pm 0.28$ & $5.81 \pm 0.41^{*}$ \\
\hline Day 25 & $3.48 \pm 0.18$ & $3.64 \pm 0.12$ & $3.63 \pm 0.16$ & $3.43 \pm 0.20$ & $3.44 \pm 0.26$ & $2.99 \pm 0.22$ \\
\hline Week 14 & $2.52 \pm 0.05$ & $2.43 \pm 0.06$ & $2.27 \pm 0.06$ & $2.29 \pm 0.07$ & $2.36 \pm 0.10$ & $2.24 \pm 0.12$ \\
\hline \multicolumn{7}{|c|}{ Nucleated erythrocytes/100 leukocytes } \\
\hline Day 4 & $0.5 \pm 0.2$ & $0.9 \pm 0.3$ & $0.2 \pm 0.1$ & $0.7 \pm 0.2$ & $0.7 \pm 0.2$ & $0.6 \pm 0.3$ \\
\hline Day 25 & $0.1 \pm 0.1$ & $0.1 \pm 0.1$ & $0.2 \pm 0.1$ & $0.2 \pm 0.1$ & $0.2 \pm 0.2$ & $0.3 \pm 0.2$ \\
\hline Week 14 & $0.2 \pm 0.2$ & $0.1 \pm 0.1$ & $0.0 \pm 0.0$ & $0.0 \pm 0.0$ & $0.0 \pm 0.0$ & $0.0 \pm 0.0$ \\
\hline \multicolumn{7}{|c|}{ Mean cell volume (fL) } \\
\hline Day 4 & $60.8 \pm 0.3$ & $61.2 \pm 0.5$ & $61.0 \pm 0.2$ & $60.7 \pm 0.3$ & $60.2 \pm 0.3$ & $60.2 \pm 0.3$ \\
\hline Day 25 & $55.4 \pm 0.3$ & $55.5 \pm 0.3$ & $55.2 \pm 0.2$ & $55.3 \pm 0.3$ & $55.6 \pm 0.3$ & $54.6 \pm 0.4$ \\
\hline
\end{tabular}


Indole-3-carbinol, NTP TR 584

\begin{tabular}{|c|c|c|c|c|c|c|}
\hline & Vehicle Control & $18.75 \mathrm{mg} / \mathrm{kg}$ & $37.5 \mathrm{mg} / \mathrm{kg}$ & 75 mg/kg & $150 \mathrm{mg} / \mathrm{kg}$ & $300 \mathrm{mg} / \mathrm{kg}$ \\
\hline Week 14 & $57.1 \pm 0.2$ & $57.0 \pm 0.2$ & $56.4 \pm 0.3$ & $56.4 \pm 0.2^{*}$ & $56.1 \pm 0.2^{* *}$ & $55.6 \pm 0.2^{* *}$ \\
\hline \multicolumn{7}{|c|}{ Mean cell hemoglobin (pg) } \\
\hline Day 4 & $20.1 \pm 0.1$ & $20.1 \pm 0.2$ & $20.1 \pm 0.1$ & $20.0 \pm 0.2$ & $20.0 \pm 0.1$ & $19.8 \pm 0.1$ \\
\hline Day 25 & $18.9 \pm 0.1$ & $18.9 \pm 0.1$ & $18.7 \pm 0.2$ & $19.0 \pm 0.1$ & $18.9 \pm 0.1$ & $18.6 \pm 0.1$ \\
\hline Week 14 & $18.7 \pm 0.1$ & $18.7 \pm 0.1$ & $18.6 \pm 0.1$ & $18.6 \pm 0.1$ & $18.4 \pm 0.1^{* *}$ & $18.1 \pm 0.1^{* *}$ \\
\hline \multicolumn{7}{|c|}{ Mean cell hemoglobin concentration (g/dL) } \\
\hline Day 4 & $33.1 \pm 0.2$ & $32.9 \pm 0.2$ & $33.0 \pm 0.2$ & $32.9 \pm 0.2$ & $33.1 \pm 0.1$ & $32.8 \pm 0.2$ \\
\hline Day 25 & $34.2 \pm 0.2$ & $34.1 \pm 0.2$ & $34.0 \pm 0.3$ & $34.3 \pm 0.2$ & $34.0 \pm 0.1$ & $34.1 \pm 0.2$ \\
\hline Week 14 & $32.7 \pm 0.1$ & $32.8 \pm 0.1$ & $33.0 \pm 0.2$ & $33.0 \pm 0.1$ & $32.8 \pm 0.1$ & $32.6 \pm 0.1$ \\
\hline \multicolumn{7}{|c|}{ Platelets $\left(10^{3} / \mu \mathrm{L}\right)$} \\
\hline Day 4 & $972.3 \pm 40.4$ & $1,004.3 \pm 20.3$ & $934.5 \pm 23.8$ & $1,090.1 \pm 47.6$ & $1,015.7 \pm 34.4$ & $1,117.9 \pm 48.0$ \\
\hline Day 25 & $752.3 \pm 20.6$ & $795.3 \pm 24.7$ & $815.1 \pm 47.0$ & $775.5 \pm 29.3$ & $803.2 \pm 30.0$ & $805.4 \pm 26.6$ \\
\hline Week 14 & $588.3 \pm 18.8$ & $600.7 \pm 11.3$ & $604.1 \pm 8.3$ & $602.8 \pm 8.1$ & $618.3 \pm 12.9$ & $658.5 \pm 15.7^{* *}$ \\
\hline \multicolumn{7}{|c|}{ Leukocytes $\left(10^{3} / \mu \mathrm{L}\right)$} \\
\hline Day 4 & $8.87 \pm 0.27$ & $9.03 \pm 0.22$ & $8.42 \pm 0.45$ & $9.65 \pm 0.36$ & $8.91 \pm 0.19$ & $8.00 \pm 0.75$ \\
\hline Day 25 & $9.63 \pm 0.46$ & $9.36 \pm 0.83$ & $9.79 \pm 0.61$ & $9.67 \pm 0.31$ & $9.11 \pm 0.84$ & $9.97 \pm 0.91$ \\
\hline Week 14 & $7.78 \pm 0.56$ & $7.76 \pm 0.47$ & $7.94 \pm 0.50$ & $8.06 \pm 0.46$ & $8.20 \pm 0.78$ & $7.76 \pm 0.70$ \\
\hline \multicolumn{7}{|c|}{ Segmented neutrophils $\left(10^{3} / \mu \mathrm{L}\right)$} \\
\hline Day 4 & $1.08 \pm 0.04$ & $1.15 \pm 0.07$ & $1.10 \pm 0.06$ & $1.22 \pm 0.07$ & $1.08 \pm 0.06$ & $1.18 \pm 0.07$ \\
\hline Day 25 & $0.82 \pm 0.04$ & $0.78 \pm 0.07$ & $0.89 \pm 0.06$ & $0.85 \pm 0.04$ & $0.81 \pm 0.06$ & $0.99 \pm 0.06$ \\
\hline Week 14 & $1.13 \pm 0.04$ & $1.15 \pm 0.06$ & $1.11 \pm 0.04$ & $1.01 \pm 0.04$ & $1.12 \pm 0.05$ & $1.16 \pm 0.05$ \\
\hline \multicolumn{7}{|c|}{ Lymphocytes $\left(10^{3} / \mu \mathrm{L}\right)$} \\
\hline Day 4 & $7.38 \pm 0.24$ & $7.51 \pm 0.19$ & $6.96 \pm 0.40$ & $7.96 \pm 0.31$ & $7.42 \pm 0.16$ & $6.39 \pm 0.67$ \\
\hline Day 25 & $8.38 \pm 0.44$ & $8.15 \pm 0.73$ & $8.42 \pm 0.53$ & $8.46 \pm 0.30$ & $7.91 \pm 0.74$ & $8.50 \pm 0.82$ \\
\hline Week 14 & $6.32 \pm 0.55$ & $6.30 \pm 0.42$ & $6.53 \pm 0.49$ & $6.74 \pm 0.45$ & $6.74 \pm 0.72$ & $6.33 \pm 0.65$ \\
\hline \multicolumn{7}{|c|}{ Monocytes $\left(10^{3} / \mu \mathrm{L}\right)$} \\
\hline Day 4 & $0.20 \pm 0.01$ & $0.19 \pm 0.01$ & $0.18 \pm 0.01$ & $0.24 \pm 0.02$ & $0.21 \pm 0.01$ & $0.22 \pm 0.03$ \\
\hline Day 25 & $0.15 \pm 0.01$ & $0.15 \pm 0.01$ & $0.14 \pm 0.02$ & $0.12 \pm 0.02$ & $0.14 \pm 0.02$ & $0.19 \pm 0.03$ \\
\hline Week 14 & $0.12 \pm 0.01$ & $0.11 \pm 0.01$ & $0.11 \pm 0.01$ & $0.10 \pm 0.01$ & $0.11 \pm 0.01$ & $0.11 \pm 0.01$ \\
\hline \multicolumn{7}{|c|}{ Basophils $\left(10^{3} / \mu \mathrm{L}\right)$} \\
\hline Day 4 & $0.033 \pm 0.005$ & $0.037 \pm 0.004$ & $0.028 \pm 0.005$ & $0.032 \pm 0.003$ & $0.039 \pm 0.007$ & $0.036 \pm 0.008$ \\
\hline Day 25 & $0.069 \pm 0.010$ & $0.066 \pm 0.013$ & $0.069 \pm 0.013$ & $0.063 \pm 0.010$ & $0.052 \pm 0.012$ & $0.066 \pm 0.013$ \\
\hline Week 14 & $0.059 \pm 0.007$ & $0.049 \pm 0.008$ & $0.045 \pm 0.009$ & $0.066 \pm 0.005$ & $0.066 \pm 0.010$ & $0.043 \pm 0.008$ \\
\hline \multicolumn{7}{|c|}{ Eosinophils $\left(10^{3} / \mu \mathrm{L}\right)$} \\
\hline Day 4 & $0.04 \pm 0.00$ & $0.03 \pm 0.00$ & $0.04 \pm 0.00$ & $0.04 \pm 0.00$ & $0.03 \pm 0.00$ & $0.02 \pm 0.00^{*}$ \\
\hline Day 25 & $0.06 \pm 0.01$ & $0.05 \pm 0.01$ & $0.04 \pm 0.01$ & $0.04 \pm 0.01$ & $0.04 \pm 0.01$ & $0.05 \pm 0.01$ \\
\hline Week 14 & $0.08 \pm 0.01$ & $0.07 \pm 0.00^{*}$ & $0.07 \pm 0.00^{*}$ & $0.06 \pm 0.00^{* *}$ & $0.07 \pm 0.01^{*}$ & $0.04 \pm 0.01^{* *}$ \\
\hline \multicolumn{7}{|c|}{ Large unstained cells $\left(10^{3} / \mu \mathrm{L}\right)$} \\
\hline Day 4 & $0.142 \pm 0.011$ & $0.109 \pm 0.010$ & $0.115 \pm 0.012$ & $0.150 \pm 0.008$ & $0.137 \pm 0.012$ & $0.145 \pm 0.023$ \\
\hline
\end{tabular}


Indole-3-carbinol, NTP TR 584

\begin{tabular}{|c|c|c|c|c|c|c|}
\hline & Vehicle Control & $18.75 \mathrm{mg} / \mathrm{kg}$ & 37.5 mg/kg & 75 mg/kg & $150 \mathrm{mg} / \mathrm{kg}$ & 300 mg/kg \\
\hline Day 25 & $0.150 \pm 0.037$ & $0.167 \pm 0.034$ & $0.224 \pm 0.052$ & $0.137 \pm 0.018$ & $0.162 \pm 0.051$ & $0.178 \pm 0.026$ \\
\hline Week 14 & $0.081 \pm 0.009$ & $0.080 \pm 0.008$ & $0.074 \pm 0.008$ & $0.091 \pm 0.005$ & $0.091 \pm 0.012$ & $0.081 \pm 0.011$ \\
\hline
\end{tabular}

\section{Clinical Chemistry}

n

\begin{tabular}{|c|c|c|c|c|c|c|}
\hline Day 4 & 10 & 10 & 10 & 10 & 10 & 10 \\
\hline Day 25 & 10 & 10 & 10 & 10 & 10 & 9 \\
\hline Week 14 & 10 & 10 & 10 & 10 & 10 & 10 \\
\hline
\end{tabular}

Urea nitrogen (mg/dL)

Day 4

Day 25

Week 14

Creatinine (mg/dL)

Day 4

Day 25

Week 14

Glucose (mg/dL)

Day 4

Day 25

Week 14

Total protein (g/dL)

Day 4

Day 25

Week 14

Albumin (g/dL)

Day 4

Day 25

Week 14

Alanine aminotransferase (IU/L)

Day 4

Day 25

Week 14

$77 \pm 3$

$64 \pm 2$

$61 \pm 3$

Alkaline phosphatase (IU/L)

Day 4

$633 \pm 14$

Day 25

Week 14

$445 \pm 8$

$223 \pm 3$

Creatine kinase (IU/L)

Day 4

Day 25

$628 \pm 61$

$520 \pm 70$

$242 \pm 33$

Week 14
$13.1 \pm 0.6$

$13.6 \pm 0.6$

$15.2 \pm 0.5$

$13.4 \pm 0.4$

$12.8 \pm 0.4$

$13.4 \pm 0.5$

$14.1 \pm 0.7^{*}$

$13.4 \pm 0.5$

$14.4 \pm 0.5$

$13.3 \pm 0.5^{* *}$

$13.4 \pm 0.3^{* *}$

$15.1 \pm 1.1$

$11.9 \pm 0.5$

$13.3 \pm 0.5$

$0.10 \pm 0.00$

$0.11 \pm 0.01$

$0.12 \pm 0.01$

$0.11 \pm 0.01$

$0.12 \pm 0.01$

$0.23 \pm 0.02$

$0.20 \pm 0.01$

$0.22 \pm 0.01$

$0.22 \pm 0.01$

$0.20 \pm 0.00$

$0.28 \pm 0.01$

$0.32 \pm 0.01$

$0.30 \pm 0.00$

$0.29 \pm 0.01$

$0.31 \pm 0.02$

$123 \pm 2$

$123 \pm 1$

$121 \pm 2$

$125 \pm 2$

$116 \pm 3$

$126 \pm 4$

$123 \pm 2$

$138 \pm 3$

$122 \pm 2$

$135 \pm 3$

$126 \pm 2$

$128 \pm 3$

$134 \pm 3$

$136 \pm 3$

$130 \pm 3$

$5.7 \pm 0.1 \quad 5.8 \pm 0.0$

$5.7 \pm 0.1$

$5.6 \pm 0.1$

$5.6 \pm 0.1$

$6.7 \pm 0.1$

$6.6 \pm 0.1$

$6.7 \pm 0.1$

$6.7 \pm 0.1$

$6.4 \pm 0.1$

$6.7 \pm 0.1$

$6.9 \pm 0.0$

$6.8 \pm 0.1$

$6.8 \pm 0.1$

$6.4 \pm 0.1$

$4.3 \pm 0.0$

$4.3 \pm 0.0$

$4.7 \pm 0.1$

$4.6 \pm 0.1$

$4.7 \pm 0.0$

$4.9 \pm 0.0$

$4.3 \pm 0.0$

$4.2 \pm 0.0$

$4.2 \pm 0.1$

$4.7 \pm 0.1$

$4.7 \pm 0.0$

$4.6 \pm 0.0$

$4.9 \pm 0.0$

$4.9 \pm 0.0$

$4.6 \pm 0.1$
$79 \pm 3$

$80 \pm 2$

$61 \pm 2$

$53 \pm 2$

$55 \pm 2$

$631 \pm 18$

$617 \pm 12$

$224 \pm 6$

$77 \pm 2$

$65 \pm 2$

$59 \pm 4$

$459 \pm 11$

$232 \pm 6$

$591 \pm 53$

$464 \pm 67$

$587 \pm 46$

$368 \pm 35$

$256 \pm 27$

$243 \pm 34$
$437 \pm 9$
$656 \pm 18$

$416 \pm 13$

$206 \pm 7$

$622 \pm 68$

$376 \pm 41$

$230 \pm 17$
$79 \pm 3$

$58 \pm 1$

$49 \pm 1^{* *}$

$691 \pm 17 *$

$380 \pm 30 * *$

$198 \pm 4^{* *}$

$178 \pm 4^{* *}$
$545 \pm 42$

$340 \pm 35$

$267 \pm 38$
$540 \pm 45$

$430 \pm 37$

$284 \pm 34$ 
Indole-3-carbinol, NTP TR 584

\begin{tabular}{|c|c|c|c|c|c|c|}
\hline & Vehicle Control & $18.75 \mathrm{mg} / \mathrm{kg}$ & $37.5 \mathrm{mg} / \mathrm{kg}$ & $75 \mathrm{mg} / \mathrm{kg}$ & $150 \mathrm{mg} / \mathrm{kg}$ & $300 \mathrm{mg} / \mathrm{kg}$ \\
\hline \multicolumn{7}{|c|}{ Sorbitol dehydrogenase (IU/L) } \\
\hline Day 4 & $6 \pm 1^{b}$ & $9 \pm 1^{\mathrm{b}}$ & $8 \pm 2^{\mathrm{c}}$ & $8 \pm 1 * b$ & $9 \pm 1^{*}$ & $13 \pm 2^{* *}$ \\
\hline Day 25 & $9 \pm 2^{\mathrm{b}}$ & $10 \pm 2$ & $10 \pm 1^{b}$ & $10 \pm 2^{\mathrm{d}}$ & $11 \pm 1^{\mathrm{b}}$ & $12 \pm 2$ \\
\hline Week 14 & $18 \pm 1$ & $17 \pm 1$ & $15 \pm 1$ & $17 \pm 1$ & $13 \pm 2$ & $17 \pm 2$ \\
\hline \multicolumn{7}{|c|}{ Bile acids $(\mu \mathrm{mol} / \mathrm{L})$} \\
\hline Day 4 & $18.8 \pm 1.8$ & $20.1 \pm 2.0$ & $21.7 \pm 1.8$ & $24.7 \pm 1.1^{* *}$ & $28.2 \pm 2.0^{* *}$ & $29.6 \pm 1.4^{* *}$ \\
\hline Day 25 & $17.8 \pm 0.9$ & $16.5 \pm 1.8$ & $14.9 \pm 0.9$ & $22.5 \pm 1.8$ & $23.4 \pm 1.7$ & $21.3 \pm 1.3$ \\
\hline Week 14 & $19.5 \pm 2.4$ & $20.0 \pm 2.6$ & $18.6 \pm 2.6$ & $18.0 \pm 1.5$ & $19.1 \pm 1.0$ & $21.3 \pm 1.3$ \\
\hline
\end{tabular}

\section{Female}

Hematology

n

\begin{tabular}{lcccccc} 
Day 4 & 9 & 10 & 9 & 10 & 10 & 9 \\
Day 25 & 8 & 10 & 10 & 8 & 10 & 9 \\
Week 14 & 10 & 10 & 10 & 10 & 10 & 10 \\
\hline
\end{tabular}

Hematocrit (auto) (\%)

Day 4

$48.7 \pm 0.7$

$50.4 \pm 0.8$

$49.8 \pm 1.0$

$48.8 \pm 1.2$

$50.1 \pm 1.0$

Day 25

$47.3 \pm 0.7$

$46.7 \pm 0.8$

$47.0 \pm 0.5$

$46.8 \pm 0.6$

$47.8 \pm 0.6$

$47.3 \pm 0.3$

Week 14

$47.8 \pm 0.4$

$46.8 \pm 0.3$

$46.2 \pm 0.3^{*}$

$47.0 \pm 0.5$

$46.0 \pm 0.3^{*}$

$46.7 \pm 0.4$

Hematocrit (spun) (\%)

Day 4

Day 25

Week 14

Hemoglobin (g/dL)

Day 4

Day 25

Week 14

Erythrocytes $\left(10^{6} / \mu \mathrm{L}\right)$

Day 4

Day 25

Week 14

$48.5 \pm 0.8$

$46.6 \pm 0.5$

$47.3 \pm 0.4$

$47.9 \pm 0.6$

$50.0 \pm 0.6$

$45.9 \pm 0.7$

$46.5 \pm 0.5$

$46.7 \pm 0.3$

$46.5 \pm 0.3$

$49.4 \pm 0.9$

$48.6 \pm 1.0$

$50.3 \pm 0.9$

$46.4 \pm 0.5$

$47.3 \pm 0.5$

$47.2 \pm 0.4$

$46.8 \pm 0.4$

$45.8 \pm 0.2^{*}$

$46.6 \pm 0.4$

$15.8 \pm 0.3$

$15.8 \pm 0.2$

$16.2 \pm 0.3$

$16.4 \pm 0.4$

$15.9 \pm 0.4$

$16.4 \pm 0.3$

$15.5 \pm 0.2$

$15.5 \pm 0.2$

$15.7 \pm 0.1$

$15.5 \pm 0.2$

$15.4 \pm 0.1$

$15.2 \pm 0.1^{* *}$

$15.4 \pm 0.1^{*}$

$15.8 \pm 0.1$

$15.6 \pm 0.1$

$15.6 \pm 0.1$

$8.02 \pm 0.10$

$7.99 \pm 0.13$

$8.20 \pm 0.12$

$8.17 \pm 0.16$

$15.1 \pm 0.1^{* *}$

$15.2 \pm 0.1 * *$

Reticulocytes $\left(10^{5} / \mu \mathrm{L}\right)$

Day 4

Day 25

$8.56 \pm 0.08$

$8.37 \pm 0.15$

$8.49 \pm 0.08$

$8.47 \pm 0.12$

$8.11 \pm 0.05$

$8.02 \pm 0.07$

$8.24 \pm 0.07$

$7.96 \pm 0.19$

$8.35 \pm 0.15$

$8.22 \pm 0.06$

$4.10 \pm 0.36$

$4.29 \pm 0.38$

$4.48 \pm 0.43$

$3.82 \pm 0.34$

$2.07 \pm 0.14$

$1.89 \pm 0.09$

$1.96 \pm 0.07$

$1.91 \pm 0.08$

$1.88 \pm 0.09$

$1.80 \pm 0.08$

$8.63 \pm 0.08$

$8.63 \pm 0.07$

Week 14

$1.76 \pm 0.04$

$5.13 \pm 0.48$

$5.38 \pm 0.49$

$5.49 \pm 0.53$

$4.75 \pm 0.52$

$4.97 \pm 0.48$

$3.74 \pm 0.34 *$

Day 25

$2.05 \pm 0.14$

$2.49 \pm 0.19$

$2.21 \pm 0.10$

$2.30 \pm 0.12$

$2.21 \pm 0.12$

$2.07 \pm 0.09$

Week 14

$2.36 \pm 0.12$

$2.36 \pm 0.12$

$2.20 \pm 0.09$

$2.23 \pm 0.05$

$2.27 \pm 0.07$ 
Indole-3-carbinol, NTP TR 584

\begin{tabular}{|c|c|c|c|c|c|c|}
\hline & Vehicle Control & $18.75 \mathrm{mg} / \mathrm{kg}$ & $37.5 \mathrm{mg} / \mathrm{kg}$ & $75 \mathrm{mg} / \mathrm{kg}$ & $150 \mathrm{mg} / \mathrm{kg}$ & $300 \mathrm{mg} / \mathrm{kg}$ \\
\hline \multicolumn{7}{|c|}{ Nucleated erythrocytes/100 leukocytes } \\
\hline Day 4 & $0.7 \pm 0.3$ & $0.3 \pm 0.2$ & $0.8 \pm 0.4$ & $1.0 \pm 0.3$ & $0.9 \pm 0.3$ & $0.7 \pm 0.4$ \\
\hline Day 25 & $0.1 \pm 0.1$ & $0.2 \pm 0.1$ & $0.2 \pm 0.1$ & $0.3 \pm 0.3$ & $0.2 \pm 0.1$ & $0.1 \pm 0.1$ \\
\hline Week 14 & $0.2 \pm 0.2$ & $0.4 \pm 0.2$ & $0.2 \pm 0.1$ & $0.2 \pm 0.1$ & $0.1 \pm 0.1$ & $0.2 \pm 0.1$ \\
\hline \multicolumn{7}{|c|}{ Mean cell volume (fL) } \\
\hline Day 4 & $60.9 \pm 0.3$ & $61.0 \pm 0.2$ & $61.5 \pm 0.3$ & $61.0 \pm 0.2$ & $61.2 \pm 0.4$ & $60.0 \pm 0.3$ \\
\hline Day 25 & $55.3 \pm 0.4$ & $55.8 \pm 0.3$ & $55.4 \pm 0.3$ & $55.3 \pm 0.3$ & $55.5 \pm 0.5$ & $54.8 \pm 0.4$ \\
\hline Week 14 & $58.1 \pm 0.1$ & $57.7 \pm 0.1^{* *}$ & $57.6 \pm 0.2^{* *}$ & $57.0 \pm 0.1^{* *}$ & $56.4 \pm 0.2^{* *}$ & $55.5 \pm 0.1^{* *}$ \\
\hline \multicolumn{7}{|c|}{ Mean cell hemoglobin (pg) } \\
\hline Day 4 & $19.8 \pm 0.1$ & $19.8 \pm 0.2$ & $19.8 \pm 0.1$ & $20.0 \pm 0.1$ & $20.0 \pm 0.1$ & $19.7 \pm 0.2$ \\
\hline Day 25 & $18.1 \pm 0.1$ & $18.5 \pm 0.1$ & $18.5 \pm 0.1$ & $18.3 \pm 0.1$ & $18.3 \pm 0.1$ & $18.0 \pm 0.1$ \\
\hline Week 14 & $19.0 \pm 0.1$ & $19.0 \pm 0.0$ & $18.9 \pm 0.1^{*}$ & $18.7 \pm 0.0^{* *}$ & $18.5 \pm 0.1^{* *}$ & $18.0 \pm 0.1^{* *}$ \\
\hline \multicolumn{7}{|c|}{ Mean cell hemoglobin concentration (g/dL) } \\
\hline Day 4 & $32.4 \pm 0.3$ & $32.4 \pm 0.2$ & $32.2 \pm 0.3$ & $32.8 \pm 0.2$ & $32.6 \pm 0.2$ & $32.8 \pm 0.2$ \\
\hline Day 25 & $32.8 \pm 0.2$ & $33.1 \pm 0.2$ & $33.4 \pm 0.2$ & $33.1 \pm 0.2$ & $33.0 \pm 0.2$ & $32.9 \pm 0.1$ \\
\hline Week 14 & $32.8 \pm 0.1$ & $32.8 \pm 0.1$ & $32.9 \pm 0.2$ & $32.7 \pm 0.1$ & $32.8 \pm 0.2$ & $32.5 \pm 0.1$ \\
\hline \multicolumn{7}{|c|}{ Platelets $\left(10^{3} / \mu \mathrm{L}\right)$} \\
\hline Day 4 & $955.8 \pm 39.5$ & $983.1 \pm 46.2$ & $979.3 \pm 58.0$ & $962.5 \pm 23.3$ & $926.6 \pm 46.6$ & $1,077.2 \pm 50.4$ \\
\hline Day 25 & $755.6 \pm 29.7$ & $729.4 \pm 20.9$ & $729.0 \pm 20.5$ & $721.0 \pm 27.4$ & $787.4 \pm 34.2$ & $711.4 \pm 33.7$ \\
\hline Week 14 & $573.2 \pm 19.6$ & $588.1 \pm 12.9$ & $554.1 \pm 18.9$ & $621.4 \pm 13.6$ & $590.1 \pm 15.0$ & $610.7 \pm 21.6$ \\
\hline \multicolumn{7}{|c|}{ Leukocytes $\left(10^{3} / \mu \mathrm{L}\right)$} \\
\hline Day 4 & $10.37 \pm 0.34$ & $9.82 \pm 0.21$ & $9.00 \pm 0.25^{*}$ & $10.32 \pm 0.42$ & $10.08 \pm 0.35$ & $10.75 \pm 0.35$ \\
\hline Day 25 & $9.64 \pm 0.55$ & $9.54 \pm 0.36$ & $9.22 \pm 0.68$ & $9.56 \pm 0.81$ & $9.93 \pm 0.64$ & $10.37 \pm 0.51$ \\
\hline Week 14 & $6.40 \pm 0.36$ & $6.50 \pm 0.30$ & $6.40 \pm 0.44$ & $6.49 \pm 0.38$ & $6.58 \pm 0.36$ & $7.20 \pm 0.38$ \\
\hline \multicolumn{7}{|c|}{ Segmented neutrophils $\left(10^{3} / \mu \mathrm{L}\right)$} \\
\hline Day 4 & $1.03 \pm 0.04$ & $1.05 \pm 0.05$ & $1.04 \pm 0.06$ & $1.08 \pm 0.06$ & $1.15 \pm 0.08$ & $1.30 \pm 0.07 * *$ \\
\hline Day 25 & $0.84 \pm 0.06$ & $0.98 \pm 0.08$ & $0.92 \pm 0.10$ & $0.96 \pm 0.10$ & $0.83 \pm 0.07$ & $1.08 \pm 0.06$ \\
\hline Week 14 & $1.08 \pm 0.07$ & $1.19 \pm 0.07$ & $1.23 \pm 0.08$ & $1.28 \pm 0.07$ & $1.03 \pm 0.05$ & $1.14 \pm 0.04$ \\
\hline \multicolumn{7}{|c|}{ Lymphocytes $\left(10^{3} / \mu \mathrm{L}\right)$} \\
\hline Day 4 & $8.89 \pm 0.28$ & $8.38 \pm 0.22$ & $7.62 \pm 0.21^{*}$ & $8.80 \pm 0.37$ & $8.53 \pm 0.30$ & $8.96 \pm 0.31$ \\
\hline Day 25 & $8.40 \pm 0.49$ & $8.13 \pm 0.35$ & $7.90 \pm 0.56$ & $8.19 \pm 0.70$ & $8.63 \pm 0.53$ & $8.76 \pm 0.52$ \\
\hline Week 14 & $5.05 \pm 0.30$ & $5.02 \pm 0.26$ & $4.88 \pm 0.40$ & $4.94 \pm 0.34$ & $5.27 \pm 0.33$ & $5.78 \pm 0.31$ \\
\hline \multicolumn{7}{|c|}{ Monocytes $\left(10^{3} / \mu \mathrm{L}\right)$} \\
\hline Day 4 & $0.23 \pm 0.02$ & $0.20 \pm 0.01$ & $0.19 \pm 0.01$ & $0.23 \pm 0.02$ & $0.22 \pm 0.02$ & $0.27 \pm 0.02$ \\
\hline Day 25 & $0.16 \pm 0.02$ & $0.15 \pm 0.01$ & $0.13 \pm 0.02$ & $0.15 \pm 0.02$ & $0.17 \pm 0.02$ & $0.19 \pm 0.02$ \\
\hline Week 14 & $0.09 \pm 0.01$ & $0.10 \pm 0.01$ & $0.11 \pm 0.01$ & $0.11 \pm 0.01$ & $0.10 \pm 0.01$ & $0.09 \pm 0.01$ \\
\hline \multicolumn{7}{|c|}{ Basophils $\left(10^{3} / \mu \mathrm{L}\right)$} \\
\hline Day 4 & $0.037 \pm 0.004$ & $0.031 \pm 0.004$ & $0.026 \pm 0.002$ & $0.034 \pm 0.004$ & $0.031 \pm 0.004$ & $0.037 \pm 0.003$ \\
\hline Day 25 & $0.050 \pm 0.008$ & $0.064 \pm 0.011$ & $0.061 \pm 0.011$ & $0.074 \pm 0.010$ & $0.070 \pm 0.013$ & $0.082 \pm 0.010$ \\
\hline
\end{tabular}


Indole-3-carbinol, NTP TR 584

\begin{tabular}{|c|c|c|c|c|c|c|}
\hline & Vehicle Control & $18.75 \mathrm{mg} / \mathrm{kg}$ & $37.5 \mathrm{mg} / \mathrm{kg}$ & $75 \mathrm{mg} / \mathrm{kg}$ & $150 \mathrm{mg} / \mathrm{kg}$ & $300 \mathrm{mg} / \mathrm{kg}$ \\
\hline Week 14 & $0.045 \pm 0.008$ & $0.061 \pm 0.011$ & $0.047 \pm 0.008$ & $0.050 \pm 0.006$ & $0.050 \pm 0.008$ & $0.053 \pm 0.008$ \\
\hline \multicolumn{7}{|c|}{ Eosinophils $\left(10^{3} / \mu \mathrm{L}\right)$} \\
\hline Day 4 & $0.04 \pm 0.01$ & $0.03 \pm 0.00$ & $0.04 \pm 0.00$ & $0.04 \pm 0.00$ & $0.03 \pm 0.00$ & $0.04 \pm 0.01$ \\
\hline Day 25 & $0.06 \pm 0.01$ & $0.06 \pm 0.01$ & $0.06 \pm 0.01$ & $0.05 \pm 0.01$ & $0.04 \pm 0.00^{* *}$ & $0.05 \pm 0.01^{*}$ \\
\hline Week 14 & $0.08 \pm 0.01$ & $0.06 \pm 0.01$ & $0.05 \pm 0.01$ & $0.05 \pm 0.00^{*}$ & $0.06 \pm 0.01^{*}$ & $0.04 \pm 0.00^{* *}$ \\
\hline \multicolumn{7}{|c|}{ Large unstained cells $\left(10^{3} / \mu \mathrm{L}\right)$} \\
\hline Day 4 & $0.143 \pm 0.018$ & $0.116 \pm 0.005$ & $0.097 \pm 0.008^{*}$ & $0.130 \pm 0.012$ & $0.120 \pm 0.011$ & $0.174 \pm 0.019$ \\
\hline Day 25 & $0.136 \pm 0.014$ & $0.155 \pm 0.032$ & $0.151 \pm 0.024$ & $0.141 \pm 0.019$ & $0.188 \pm 0.061$ & $0.217 \pm 0.044$ \\
\hline Week 14 & $0.054 \pm 0.008$ & $0.077 \pm 0.020$ & $0.079 \pm 0.016$ & $0.067 \pm 0.007$ & $0.077 \pm 0.011$ & $0.087 \pm 0.023$ \\
\hline
\end{tabular}

\section{Clinical Chemistry}

n

\begin{tabular}{|c|c|c|c|c|c|c|}
\hline Day 4 & 10 & 10 & 10 & 10 & 10 & 9 \\
\hline Day 25 & 10 & 10 & 10 & 10 & 10 & 9 \\
\hline Week 14 & 10 & 10 & 10 & 10 & 10 & 10 \\
\hline
\end{tabular}

Urea nitrogen (mg/dL)

Day 4

Day 25

Week 14

Creatinine (mg/dL)

Day 4

Day 25

Week 14

Glucose (mg/dL)

Day 4

Day 25

Week 14

Total protein (g/dL)

Day 4

Day 25

Week 14

Albumin (g/dL)

\section{Day 4}

Day 25

Week 14

Alanine aminotransferase (IU/L)

$$
\text { Day } 4
$$

Day 25

Week 14

$65 \pm 2$

\begin{abstract}
$11.7 \pm 0.6$
\end{abstract}
$12.6 \pm 0.6$

$11.8 \pm 0.6$

$13.2 \pm 0.6$

$13.6 \pm 0.5$

$12.6 \pm 0.6$

$$
12.6 \pm 0.4
$$

$14.8 \pm 0.3$

$16.4 \pm 0.5$

$15.2 \pm 0.5$

$15.0 \pm 0.5$

$14.1 \pm 0.4^{*}$

$13.9 \pm 0.3$

$12.8 \pm 0.4$

$13.1 \pm 0.4$

$12.2 \pm 0.7$

$12.3 \pm 0.5$

$0.17 \pm 0.02$

$0.14 \pm 0.02$

$0.17 \pm 0.02$

$0.18 \pm 0.01$

$0.19 \pm 0.02$

$0.26 \pm 0.02$

$0.24 \pm 0.02$

$0.28 \pm 0.01$

$0.28 \pm 0.01$

$0.27 \pm 0.02$

$0.30 \pm 0.00$

$135 \pm 7$

$133 \pm 3$

$128 \pm 4$

$131 \pm 2$

$137 \pm 6$

$136 \pm 4$

$0.17 \pm 0.02$

$0.19 \pm 0.01$

$0.22 \pm 0.01$

$0.22 \pm 0.01$

$0.27 \pm 0.02$

$0.29 \pm 0.01$

$130 \pm 3$

$124 \pm 2$

$124 \pm 4$

$116 \pm 3^{b}$

$117 \pm 4$

$128 \pm 2$

$127 \pm 2$

$5.9 \pm 0.1$

$6.0 \pm 0.1$

$6.0 \pm 0.1$

$6.0 \pm 0.1$

$6.1 \pm 0.1$

$6.4 \pm 0.1$

$6.5 \pm 0.1$

$6.4 \pm 0.1$

$6.4 \pm 0.0$

$6.6 \pm 0.1$

$6.0 \pm 0.1$

$6.6 \pm 0.1$

$6.9 \pm 0.1$

$6.6 \pm 0.1$

$6.8 \pm 0.1$

$6.7 \pm 0.1$

$6.4 \pm 0.1$

$6.3 \pm 0.1$

$4.4 \pm 0.1$

$4.5 \pm 0.0$

$4.5 \pm 0.0$

$4.5 \pm 0.0$

$4.5 \pm 0.1$

$4.4 \pm 0.1$

$4.7 \pm 0.1$

$4.8 \pm 0.1$

$4.7 \pm 0.0$

$4.7 \pm 0.0$

$4.8 \pm 0.1$

$4.7 \pm 0.1$

$4.9 \pm 0.1$

$5.1 \pm 0.1$

$4.9 \pm 0.0$

$5.0 \pm 0.1$

$5.0 \pm 0.1$

$4.7 \pm 0.0$

$66 \pm 2$

$67 \pm 3$

$65 \pm 3$

$69 \pm 2$

$80 \pm 2 * *$

$48 \pm 2$

$52 \pm 1$

$49 \pm 2$

$47 \pm 2$

$58 \pm 2$

$42 \pm 2 * *$

$45 \pm 2$ **

$36 \pm 1^{* *}$

$35 \pm 1^{* *}$

$47 \pm 1^{* *}$ 
Indole-3-carbinol, NTP TR 584

\begin{tabular}{|c|c|c|c|c|c|c|}
\hline & Vehicle Control & $18.75 \mathrm{mg} / \mathrm{kg}$ & $37.5 \mathrm{mg} / \mathrm{kg}$ & $75 \mathrm{mg} / \mathrm{kg}$ & $150 \mathrm{mg} / \mathrm{kg}$ & $300 \mathrm{mg} / \mathrm{kg}$ \\
\hline \multicolumn{7}{|c|}{ Alkaline phosphatase (IU/L) } \\
\hline Day 4 & $553 \pm 11$ & $583 \pm 15$ & $585 \pm 19$ & $571 \pm 13$ & $576 \pm 12$ & $610 \pm 18$ \\
\hline Day 25 & $381 \pm 8$ & $382 \pm 9$ & $371 \pm 11$ & $348 \pm 8^{*}$ & $327 \pm 13^{* *}$ & $328 \pm 11^{* *}$ \\
\hline Week 14 & $205 \pm 4$ & $180 \pm 5^{* *}$ & $189 \pm 9 *$ & $190 \pm 6^{*}$ & $164 \pm 5^{* *}$ & $155 \pm 6^{* *}$ \\
\hline \multicolumn{7}{|c|}{ Creatine kinase (IU/L) } \\
\hline Day 4 & $447 \pm 52$ & $445 \pm 46$ & $463 \pm 35$ & $462 \pm 39$ & $395 \pm 26^{\mathrm{b}}$ & $415 \pm 37$ \\
\hline Day 25 & $453 \pm 77$ & $468 \pm 51$ & $438 \pm 58$ & $534 \pm 91$ & $424 \pm 40$ & $426 \pm 25$ \\
\hline Week 14 & $187 \pm 17$ & $229 \pm 30$ & $238 \pm 20$ & $189 \pm 22$ & $152 \pm 19$ & $187 \pm 21$ \\
\hline \multicolumn{7}{|c|}{ Sorbitol dehydrogenase (IU/L) } \\
\hline Day 4 & $7 \pm 1^{b}$ & $7 \pm 1^{b}$ & $3 \pm 1^{\mathrm{b}}$ & $5 \pm 1^{b}$ & $7 \pm 1$ & $8 \pm 2$ \\
\hline Day 25 & $10 \pm 3^{\mathrm{e}}$ & $10 \pm 2$ & $12 \pm 2^{\mathrm{d}}$ & $8 \pm 2^{d}$ & $11 \pm 2$ & $10 \pm 1$ \\
\hline Week 14 & $15 \pm 1$ & $14 \pm 1$ & $16 \pm 1$ & $15 \pm 2$ & $12 \pm 1$ & $14 \pm 1$ \\
\hline \multicolumn{7}{|c|}{ Bile acids $(\mu \mathrm{mol} / \mathrm{L})$} \\
\hline Day 4 & $17.2 \pm 3.3$ & $16.7 \pm 1.6$ & $13.9 \pm 0.7$ & $18.2 \pm 1.1$ & $19.8 \pm 2.1$ & $20.2 \pm 1.5^{*}$ \\
\hline Day 25 & $17.7 \pm 2.3$ & $16.0 \pm 0.9$ & $19.9 \pm 2.6$ & $20.2 \pm 1.7$ & $24.5 \pm 2.7^{*}$ & $23.0 \pm 1.9 *$ \\
\hline Week 14 & $15.8 \pm 1.9$ & $18.4 \pm 3.1$ & $16.8 \pm 1.5$ & $14.4 \pm 1.2$ & $17.2 \pm 1.6$ & $20.0 \pm 2.1$ \\
\hline
\end{tabular}

*Significantly different $(\mathrm{P} \leq 0.05)$ from the vehicle control group by Dunn’s or Shirley's test.

$* * \mathrm{P} \leq 0.01$.

${ }^{\text {a }}$ Data are presented as mean \pm standard error. Statistical tests were performed on unrounded data.

${ }^{b} \mathrm{n}=9$.

${ }^{c} n=7$.

${ }^{\mathrm{d}} \mathrm{n}=8$.

$\mathrm{e}_{\mathrm{n}}=6$. 
Indole-3-carbinol, NTP TR 584

Table F-2. Hematology Data for Mice in the Three-month Gavage Study of Indole-3-carbinol ${ }^{\mathrm{a}}$

\begin{tabular}{|c|c|c|c|c|c|c|}
\hline & $\begin{array}{l}\text { Vehicle } \\
\text { Control }\end{array}$ & $15.6 \mathrm{mg} / \mathrm{kg}$ & $31.25 \mathrm{mg} / \mathrm{kg}$ & $62.5 \mathrm{mg} / \mathrm{kg}$ & $125 \mathrm{mg} / \mathrm{kg}$ & $250 \mathrm{mg} / \mathrm{kg}$ \\
\hline $\mathbf{n}$ & 10 & 10 & 10 & 10 & 10 & 10 \\
\hline \multicolumn{7}{|l|}{ Male } \\
\hline Hematocrit (auto) (\%) & $51.0 \pm 0.6$ & $50.9 \pm 0.3$ & $51.2 \pm 0.3$ & $50.5 \pm 0.7$ & $52.6 \pm 0.6$ & $53.0 \pm 0.5 *$ \\
\hline Hematocrit (spun) (\%) & $50.0 \pm 0.5$ & $50.0 \pm 0.3$ & $49.8 \pm 0.3$ & $49.6 \pm 0.6$ & $51.8 \pm 0.6^{*}$ & $52.3 \pm 0.5^{* *}$ \\
\hline Hemoglobin (g/dL) & $17.1 \pm 0.2$ & $17.0 \pm 0.1$ & $17.2 \pm 0.1$ & $16.9 \pm 0.2$ & $17.5 \pm 0.2^{*}$ & $17.8 \pm 0.2^{* *}$ \\
\hline Erythrocytes $\left(10^{6} / \mu \mathrm{L}\right)$ & $10.19 \pm 0.14$ & $10.27 \pm 0.05$ & $10.25 \pm 0.07$ & $10.14 \pm 0.13$ & $10.58 \pm 0.14^{*}$ & $10.73 \pm 0.11^{* *}$ \\
\hline Reticulocytes $\left(10^{5} / \mu \mathrm{L}\right)$ & $3.23 \pm 0.10$ & $3.13 \pm 0.05$ & $3.01 \pm 0.04$ & $3.15 \pm 0.09$ & $3.11 \pm 0.08$ & $3.09 \pm 0.06$ \\
\hline Reticulocytes (\%) & $3.19 \pm 0.10$ & $3.04 \pm 0.05$ & $2.96 \pm 0.05$ & $3.11 \pm 0.11$ & $2.93 \pm 0.08^{*}$ & $2.88 \pm 0.06^{*}$ \\
\hline $\begin{array}{l}\text { Nucleated erythrocytes/ } \\
100 \text { leukocytes }\end{array}$ & $0.00 \pm 0.00$ & $0.00 \pm 0.00$ & $0.00 \pm 0.00$ & $0.00 \pm 0.00$ & $0.00 \pm 0.00$ & $0.00 \pm 0.00$ \\
\hline Mean cell volume (fL) & $50.1 \pm 0.3$ & $49.6 \pm 0.2$ & $49.9 \pm 0.3$ & $49.8 \pm 0.2$ & $49.7 \pm 0.2$ & $49.4 \pm 0.3 *$ \\
\hline Mean cell hemoglobin (pg) & $16.8 \pm 0.1$ & $16.6 \pm 0.1$ & $16.8 \pm 0.1$ & $16.7 \pm 0.1$ & $16.6 \pm 0.1$ & $16.6 \pm 0.1$ \\
\hline $\begin{array}{r}\text { Mean cell hemoglobin } \\
\text { concentration (g/dL) }\end{array}$ & $33.5 \pm 0.1$ & $33.5 \pm 0.1$ & $33.6 \pm 0.1$ & $33.5 \pm 0.1$ & $33.4 \pm 0.2$ & $33.5 \pm 0.1$ \\
\hline Platelets $\left(10^{3} / \mu \mathrm{L}\right)$ & $853.0 \pm 22.2$ & $906.4 \pm 40.5$ & $866.3 \pm 36.4$ & $900.1 \pm 34.4$ & $838.8 \pm 35.2$ & $900.2 \pm 28.9$ \\
\hline Leukocytes $\left(10^{3} / \mu \mathrm{L}\right)$ & $4.26 \pm 0.33$ & $4.55 \pm 0.22$ & $4.64 \pm 0.23$ & $4.48 \pm 0.35$ & $4.74 \pm 0.28$ & $4.89 \pm 0.39$ \\
\hline $\begin{array}{l}\text { Segmented neutrophils } \\
\qquad\left(10^{3} / \mu \mathrm{L}\right)\end{array}$ & $0.55 \pm 0.04$ & $0.53 \pm 0.04$ & $0.52 \pm 0.02$ & $0.55 \pm 0.05$ & $0.58 \pm 0.04$ & $0.58 \pm 0.03$ \\
\hline Lymphocytes $\left(10^{3} / \mu \mathrm{L}\right)$ & $3.36 \pm 0.28$ & $3.66 \pm 0.18$ & $3.82 \pm 0.23$ & $3.58 \pm 0.30$ & $3.80 \pm 0.24$ & $4.00 \pm 0.35$ \\
\hline Monocytes $\left(10^{3} / \mu \mathrm{L}\right)$ & $0.05 \pm 0.01$ & $0.05 \pm 0.00$ & $0.05 \pm 0.01$ & $0.06 \pm 0.01$ & $0.07 \pm 0.01$ & $0.05 \pm 0.01$ \\
\hline Basophils $\left(10^{3} / \mu \mathrm{L}\right)$ & $0.007 \pm 0.002$ & $0.006 \pm 0.002$ & $0.011 \pm 0.002$ & $0.008 \pm 0.002$ & $0.009 \pm 0.003$ & $0.007 \pm 0.002$ \\
\hline Eosinophils $\left(10^{3} / \mu \mathrm{L}\right)$ & $0.27 \pm 0.03$ & $0.28 \pm 0.03$ & $0.21 \pm 0.02$ & $0.27 \pm 0.02$ & $0.26 \pm 0.04$ & $0.23 \pm 0.04$ \\
\hline $\begin{array}{l}\text { Large unstained cells } \\
\qquad\left(10^{3} / \mu \mathrm{L}\right)\end{array}$ & $0.017 \pm 0.004$ & $0.015 \pm 0.002$ & $0.022 \pm 0.002$ & $0.017 \pm 0.003$ & $0.020 \pm 0.004$ & $0.022 \pm 0.004$ \\
\hline \multicolumn{7}{|l|}{ Female } \\
\hline Hematocrit (auto) (\%) & $51.5 \pm 0.4$ & $50.3 \pm 1.4$ & $53.0 \pm 0.4$ & $51.5 \pm 0.5$ & $51.1 \pm 0.6$ & $52.1 \pm 0.7$ \\
\hline Hematocrit (spun) (\%) & $50.3 \pm 0.5$ & $49.5 \pm 1.2$ & $51.9 \pm 0.3$ & $50.8 \pm 0.4$ & $50.5 \pm 0.6$ & $51.0 \pm 0.6$ \\
\hline Hemoglobin (g/dL) & $17.1 \pm 0.2$ & $16.6 \pm 0.4$ & $17.5 \pm 0.1$ & $17.2 \pm 0.1$ & $17.0 \pm 0.2$ & $17.3 \pm 0.2$ \\
\hline Erythrocytes $\left(10^{6} / \mu \mathrm{L}\right)$ & $10.30 \pm 0.11$ & $9.99 \pm 0.25$ & $10.57 \pm 0.10$ & $10.31 \pm 0.08$ & $10.25 \pm 0.13$ & $10.50 \pm 0.12$ \\
\hline Reticulocytes $\left(10^{5} / \mu \mathrm{L}\right)$ & $2.98 \pm 0.16$ & $2.64 \pm 0.22$ & $3.48 \pm 0.27$ & $2.86 \pm 0.18$ & $2.71 \pm 0.10$ & $2.64 \pm 0.21$ \\
\hline Reticulocytes (\%) & $2.89 \pm 0.16$ & $2.66 \pm 0.22$ & $3.31 \pm 0.26$ & $2.77 \pm 0.17$ & $2.64 \pm 0.13$ & $2.54 \pm 0.20$ \\
\hline $\begin{array}{l}\text { Nucleated erythrocytes/ } \\
100 \text { leukocytes }\end{array}$ & $0.00 \pm 0.00$ & $0.00 \pm 0.00$ & $0.00 \pm 0.00$ & $0.00 \pm 0.00$ & $0.00 \pm 0.00$ & $0.00 \pm 0.00$ \\
\hline Mean cell volume (fL) & $50.1 \pm 0.2$ & $50.4 \pm 0.4$ & $50.2 \pm 0.2$ & $50.0 \pm 0.2$ & $49.9 \pm 0.2$ & $49.6 \pm 0.2$ \\
\hline Mean cell hemoglobin (pg) & $16.6 \pm 0.1$ & $16.7 \pm 0.1$ & $16.6 \pm 0.1$ & $16.7 \pm 0.1$ & $16.6 \pm 0.1$ & $16.4 \pm 0.1$ \\
\hline $\begin{array}{l}\text { Mean cell hemoglobin } \\
\text { concentration (g/dL) }\end{array}$ & $33.1 \pm 0.1$ & $33.0 \pm 0.2$ & $33.1 \pm 0.1$ & $33.5 \pm 0.2$ & $33.3 \pm 0.1$ & $33.2 \pm 0.1$ \\
\hline Platelets $\left(10^{3} / \mu \mathrm{L}\right)$ & $643.3 \pm 36.7$ & $750.3 \pm 49.4$ & $734.3 \pm 52.7$ & $744.1 \pm 20.8$ & $647.2 \pm 36.2$ & $658.5 \pm 21.1$ \\
\hline
\end{tabular}


Indole-3-carbinol, NTP TR 584

\begin{tabular}{|c|c|c|c|c|c|c|}
\hline & $\begin{array}{l}\text { Vehicle } \\
\text { Control }\end{array}$ & $15.6 \mathrm{mg} / \mathrm{kg}$ & $31.25 \mathrm{mg} / \mathrm{kg}$ & $62.5 \mathrm{mg} / \mathrm{kg}$ & $125 \mathrm{mg} / \mathrm{kg}$ & $250 \mathrm{mg} / \mathrm{kg}$ \\
\hline Leukocytes $\left(10^{3} / \mu \mathrm{L}\right)$ & $2.63 \pm 0.22$ & $3.78 \pm 0.60$ & $2.84 \pm 0.17$ & $3.27 \pm 0.15$ & $2.97 \pm 0.18$ & $2.83 \pm 0.18$ \\
\hline $\begin{array}{l}\text { Segmented neutrophils } \\
\qquad\left(10^{3} / \mu \mathrm{L}\right)\end{array}$ & $0.28 \pm 0.05$ & $0.77 \pm 0.44$ & $0.34 \pm 0.05$ & $0.43 \pm 0.04$ & $0.31 \pm 0.03$ & $0.29 \pm 0.04$ \\
\hline Lymphocytes $\left(10^{3} / \mu \mathrm{L}\right)$ & $2.11 \pm 0.18$ & $2.77 \pm 0.22$ & $2.29 \pm 0.16$ & $2.65 \pm 0.13$ & $2.44 \pm 0.16$ & $2.30 \pm 0.13$ \\
\hline Monocytes $\left(10^{3} / \mu \mathrm{L}\right)$ & $0.02 \pm 0.00$ & $0.03 \pm 0.01$ & $0.03 \pm 0.00$ & $0.03 \pm 0.01$ & $0.03 \pm 0.01$ & $0.03 \pm 0.01$ \\
\hline Basophils $\left(10^{3} / \mu \mathrm{L}\right)$ & $0.006 \pm 0.002$ & $0.006 \pm 0.002$ & $0.007 \pm 0.002$ & $0.007 \pm 0.002$ & $0.006 \pm 0.002$ & $0.006 \pm 0.002$ \\
\hline Eosinophils $\left(10^{3} / \mu \mathrm{L}\right)$ & $0.21 \pm 0.02$ & $0.19 \pm 0.03$ & $0.17 \pm 0.03$ & $0.14 \pm 0.04$ & $0.17 \pm 0.03$ & $0.19 \pm 0.03$ \\
\hline $\begin{array}{l}\text { Large unstained cells } \\
\qquad\left(10^{3} / \mu \mathrm{L}\right)\end{array}$ & $0.009 \pm 0.002$ & $0.017 \pm 0.003$ & $0.010 \pm 0.001$ & $0.014 \pm 0.002$ & $0.012 \pm 0.001$ & $0.010 \pm 0.001$ \\
\hline
\end{tabular}

*Significantly different $(\mathrm{P} \leq 0.05)$ from the vehicle control group by Dunn’s or Shirley’s test.

$* * \mathrm{P} \leq 0.01$.

a Data are presented as mean \pm standard error. Statistical tests were performed on unrounded data. 
Indole-3-carbinol, NTP TR 584

\section{Appendix G. Organ Weights and Organ-Weight-to-Body- Weight Ratios}

\section{Tables}

Table G-1. Organ Weights and Organ-Weight-to-Body-Weight Ratios for F344/N Rats in the Three-month Gavage Study of Indole-3-carbinol ........................................

Table G-2. Organ Weights and Organ-Weight-to-Body-Weight Ratios for Mice in the Three-month Gavage Study of Indole-3-carbinol 
Indole-3-carbinol, NTP TR 584

Table G-1. Organ Weights and Organ-Weight-to-Body-Weight Ratios for F344/N Rats in the Three-month Gavage Study of Indole-3-carbinol ${ }^{\mathrm{a}}$

\begin{tabular}{|c|c|c|c|c|c|c|}
\hline & Vehicle Control & $18.75 \mathrm{mg} / \mathrm{kg}$ & $37.5 \mathrm{mg} / \mathrm{kg}$ & 75 mg/kg & $150 \mathrm{mg} / \mathrm{kg}$ & $300 \mathrm{mg} / \mathrm{kg}$ \\
\hline $\mathbf{n}$ & 10 & 10 & 10 & 10 & 10 & 10 \\
\hline \multicolumn{7}{|l|}{ Male } \\
\hline $\begin{array}{l}\text { Necropsy } \\
\text { body wt }\end{array}$ & $309 \pm 5$ & $320 \pm 9$ & $325 \pm 7$ & $321 \pm 8$ & $309 \pm 8$ & $285 \pm 6$ \\
\hline \multicolumn{7}{|l|}{ Heart } \\
\hline Absolute & $0.77 \pm 0.02$ & $0.82 \pm 0.02$ & $0.83 \pm 0.02$ & $0.82 \pm 0.01$ & $0.78 \pm 0.02$ & $0.75 \pm 0.02$ \\
\hline Relative & $2.483 \pm 0.034$ & $2.577 \pm 0.035$ & $2.561 \pm 0.030$ & $2.562 \pm 0.023$ & $2.528 \pm 0.032$ & $2.618 \pm 0.038 *$ \\
\hline \multicolumn{7}{|l|}{ R. Kidney } \\
\hline Absolute & $0.86 \pm 0.02$ & $0.94 \pm 0.02$ & $0.93 \pm 0.03$ & $0.97 \pm 0.03^{*}$ & $0.92 \pm 0.03$ & $0.90 \pm 0.02$ \\
\hline Relative & $2.785 \pm 0.038$ & $2.940 \pm 0.033$ & $2.844 \pm 0.054$ & $3.012 \pm 0.038^{* *}$ & $2.981 \pm 0.048^{* *}$ & $3.152 \pm 0.055^{* *}$ \\
\hline \multicolumn{7}{|l|}{ Liver } \\
\hline Absolute & $9.98 \pm 0.30$ & $11.19 \pm 0.40^{*}$ & $11.20 \pm 0.40^{*}$ & $11.85 \pm 0.36^{* *}$ & $12.07 \pm 0.38^{* *}$ & $12.57 \pm 0.33^{* *}$ \\
\hline Relative & $32.205 \pm 0.594$ & $34.924 \pm 0.481^{* *}$ & $34.382 \pm 0.716^{* *}$ & $36.941 \pm 0.435^{* *}$ & $39.050 \pm 0.472^{* *}$ & $44.047 \pm 0.729 * *$ \\
\hline \multicolumn{7}{|l|}{ Lung } \\
\hline Absolute & $1.31 \pm 0.06$ & $1.43 \pm 0.03$ & $1.43 \pm 0.06$ & $1.36 \pm 0.04$ & $1.45 \pm 0.08$ & $1.28 \pm 0.04$ \\
\hline Relative & $4.229 \pm 0.164$ & $4.474 \pm 0.113$ & $4.399 \pm 0.196$ & $4.240 \pm 0.075$ & $4.676 \pm 0.226$ & $4.486 \pm 0.131$ \\
\hline \multicolumn{7}{|l|}{ R. Testis } \\
\hline Absolute & $1.299 \pm 0.020$ & $1.311 \pm 0.020$ & $1.449 \pm 0.075^{*}$ & $1.334 \pm 0.025$ & $1.355 \pm 0.028$ & $1.292 \pm 0.029$ \\
\hline Relative & $4.201 \pm 0.055$ & $4.113 \pm 0.059$ & $4.477 \pm 0.265$ & $4.178 \pm 0.099$ & $4.396 \pm 0.064$ & $4.531 \pm 0.080$ \\
\hline \multicolumn{7}{|l|}{ Thymus } \\
\hline Absolute & $0.185 \pm 0.008$ & $0.187 \pm 0.008$ & $0.207 \pm 0.007$ & $0.191 \pm 0.006$ & $0.182 \pm 0.006$ & $0.170 \pm 0.007$ \\
\hline Relative & $0.597 \pm 0.021$ & $0.590 \pm 0.030$ & $0.635 \pm 0.019$ & $0.597 \pm 0.019$ & $0.591 \pm 0.019$ & $0.596 \pm 0.027$ \\
\hline \multicolumn{7}{|l|}{ Female } \\
\hline $\begin{array}{l}\text { Necropsy } \\
\text { body wt }\end{array}$ & $189 \pm 2$ & $192 \pm 3$ & $195 \pm 2$ & $185 \pm 3$ & $183 \pm 3$ & $183 \pm 3$ \\
\hline \multicolumn{7}{|l|}{ Heart } \\
\hline Absolute & $0.53 \pm 0.01$ & $0.56 \pm 0.01$ & $0.54 \pm 0.00$ & $0.53 \pm 0.01$ & $0.54 \pm 0.01$ & $0.53 \pm 0.01$ \\
\hline Relative & $2.784 \pm 0.036$ & $2.911 \pm 0.036$ & $2.782 \pm 0.030$ & $2.844 \pm 0.050$ & $2.926 \pm 0.045^{*}$ & $2.913 \pm 0.026$ \\
\hline \multicolumn{7}{|l|}{ R. Kidney } \\
\hline Absolute & $0.57 \pm 0.01$ & $0.66 \pm 0.01^{* *}$ & $0.63 \pm 0.01^{*}$ & $0.61 \pm 0.01$ & $0.62 \pm 0.01$ & $0.63 \pm 0.02^{*}$ \\
\hline Relative & $3.034 \pm 0.056$ & $3.447 \pm 0.053^{* *}$ & $3.233 \pm 0.075^{* *}$ & $3.277 \pm 0.046^{* *}$ & $3.375 \pm 0.064^{* *}$ & $3.436 \pm 0.063^{* *}$ \\
\hline \multicolumn{7}{|l|}{ Liver } \\
\hline Absolute & $5.08 \pm 0.11$ & $5.94 \pm 0.11^{* *}$ & $5.98 \pm 0.07^{* *}$ & $5.92 \pm 0.13^{* *}$ & $6.34 \pm 0.14^{* *}$ & $6.92 \pm 0.11^{* *}$ \\
\hline Relative & $26.804 \pm 0.441$ & $30.985 \pm 0.566^{* *}$ & $30.790 \pm 0.547^{* *}$ & $32.061 \pm 0.548^{* *}$ & $34.660 \pm 0.614^{* *}$ & $37.857 \pm 0.319 * *$ \\
\hline \multicolumn{7}{|c|}{ ( } \\
\hline Absolute & $0.92 \pm 0.03$ & $0.99 \pm 0.03$ & $0.93 \pm 0.02$ & $0.92 \pm 0.03$ & $0.94 \pm 0.03$ & $0.98 \pm 0.02$ \\
\hline Relative & $4.874 \pm 0.151$ & $5.144 \pm 0.149$ & $4.781 \pm 0.139$ & $4.982 \pm 0.182$ & $5.121 \pm 0.170$ & $5.386 \pm 0.176$ \\
\hline \multicolumn{7}{|l|}{ Thymus } \\
\hline Absolute & $0.178 \pm 0.005$ & $0.173 \pm 0.005$ & $0.162 \pm 0.007$ & $0.149 \pm 0.008^{* *}$ & $0.151 \pm 0.007^{* *}$ & $0.145 \pm 0.006^{* *}$ \\
\hline Relative & $0.943 \pm 0.027$ & $0.901 \pm 0.022$ & $0.836 \pm 0.042 *$ & $0.805 \pm 0.036^{* *}$ & $0.825 \pm 0.029 * *$ & $0.792 \pm 0.031^{* *}$ \\
\hline
\end{tabular}

*Significantly different $(\mathrm{P} \leq 0.05)$ from the vehicle control group by Williams' or Dunnett's test.

$* * \mathrm{P} \leq 0.01$.

${ }^{a}$ Organ weights (absolute weights) and body weights are given in grams; organ-weight-to-body-weight ratios (relative weights) are given as mg organ weight/g body weight (mean \pm standard error). 
Indole-3-carbinol, NTP TR 584

Table G-2. Organ Weights and Organ-Weight-to-Body-Weight Ratios for Mice in the Three-month Gavage Study of Indole-3-carbinol ${ }^{\mathrm{a}}$

\begin{tabular}{|c|c|c|c|c|c|c|}
\hline & Vehicle Control & $15.6 \mathrm{mg} / \mathrm{kg}$ & $31.25 \mathrm{mg} / \mathrm{kg}$ & $62.5 \mathrm{mg} / \mathrm{kg}$ & $125 \mathrm{mg} / \mathrm{kg}$ & $250 \mathrm{mg} / \mathrm{kg}$ \\
\hline $\mathbf{n}$ & 10 & 10 & 10 & 10 & 10 & 10 \\
\hline \multicolumn{7}{|l|}{ Male } \\
\hline $\begin{array}{l}\text { Necropsy } \\
\text { body wt }\end{array}$ & $40.4 \pm 0.6$ & $40.5 \pm 1.1$ & $41.5 \pm 0.9$ & $40.0 \pm 1.1$ & $42.2 \pm 1.6$ & $41.0 \pm 0.9$ \\
\hline \multicolumn{7}{|l|}{ Heart } \\
\hline Absolute & $0.14 \pm 0.00$ & $0.14 \pm 0.00$ & $0.14 \pm 0.00$ & $0.14 \pm 0.00$ & $0.15 \pm 0.00$ & $0.14 \pm 0.00$ \\
\hline Relative & $3.496 \pm 0.073$ & $3.418 \pm 0.066$ & $3.453 \pm 0.100$ & $3.413 \pm 0.085$ & $3.476 \pm 0.135$ & $3.330 \pm 0.088$ \\
\hline \multicolumn{7}{|l|}{ R. Kidney } \\
\hline Absolute & $0.28 \pm 0.01$ & $0.28 \pm 0.01$ & $0.29 \pm 0.01$ & $0.29 \pm 0.01$ & $0.29 \pm 0.01$ & $0.28 \pm 0.01$ \\
\hline Relative & $7.009 \pm 0.152$ & $6.908 \pm 0.212$ & $7.063 \pm 0.204$ & $7.137 \pm 0.195$ & $6.928 \pm 0.321$ & $6.891 \pm 0.166$ \\
\hline \multicolumn{7}{|l|}{ Liver } \\
\hline Absolute & $1.50 \pm 0.06$ & $1.46 \pm 0.07$ & $1.54 \pm 0.05$ & $1.54 \pm 0.05$ & $1.77 \pm 0.13^{*}$ & $1.82 \pm 0.06^{* *}$ \\
\hline Relative & $37.329 \pm 1.896$ & $35.868 \pm 0.799$ & $37.166 \pm 0.702$ & $38.520 \pm 0.632$ & $41.960 \pm 2.146 *$ & $44.333 \pm 0.817 * *$ \\
\hline \multicolumn{7}{|l|}{ Lung } \\
\hline Absolute & $0.24 \pm 0.02$ & $0.22 \pm 0.01$ & $0.22 \pm 0.01$ & $0.22 \pm 0.01$ & $0.24 \pm 0.01$ & $0.23 \pm 0.02$ \\
\hline Relative & $5.916 \pm 0.421$ & $5.532 \pm 0.321$ & $5.411 \pm 0.341$ & $5.406 \pm 0.307$ & $5.751 \pm 0.384$ & $5.665 \pm 0.348$ \\
\hline \multicolumn{7}{|l|}{ R. Testis } \\
\hline Absolute & $0.120 \pm 0.002$ & $0.121 \pm 0.004$ & $0.117 \pm 0.003^{b}$ & $0.116 \pm 0.003$ & $0.118 \pm 0.002$ & $0.109 \pm 0.006$ \\
\hline Relative & $2.985 \pm 0.068$ & $3.008 \pm 0.122$ & $2.828 \pm 0.077^{\mathrm{b}}$ & $2.920 \pm 0.121$ & $2.830 \pm 0.135$ & $2.656 \pm 0.143$ \\
\hline \multicolumn{7}{|l|}{ Thymus } \\
\hline Absolute & $0.032 \pm 0.002$ & $0.029 \pm 0.002$ & $0.031 \pm 0.001$ & $0.031 \pm 0.003$ & $0.031 \pm 0.004$ & $0.035 \pm 0.002$ \\
\hline Relative & $0.779 \pm 0.047$ & $0.728 \pm 0.049$ & $0.738 \pm 0.038$ & $0.784 \pm 0.066$ & $0.743 \pm 0.094$ & $0.846 \pm 0.048$ \\
\hline \multicolumn{7}{|l|}{ Female } \\
\hline $\begin{array}{l}\text { Necropsy } \\
\text { body wt }\end{array}$ & $34.2 \pm 1.0$ & $33.5 \pm 1.0$ & $33.8 \pm 1.6$ & $33.7 \pm 0.6$ & $33.1 \pm 0.7$ & $31.4 \pm 1.3$ \\
\hline \multicolumn{7}{|l|}{ Heart } \\
\hline Absolute & $0.12 \pm 0.00$ & $0.13 \pm 0.00$ & $0.12 \pm 0.00$ & $0.12 \pm 0.00$ & $0.12 \pm 0.00$ & $0.12 \pm 0.00$ \\
\hline Relative & $3.505 \pm 0.117$ & $3.860 \pm 0.158$ & $3.606 \pm 0.153$ & $3.577 \pm 0.104$ & $3.578 \pm 0.061$ & $3.699 \pm 0.130$ \\
\hline \multicolumn{7}{|l|}{ R. Kidney } \\
\hline Absolute & $0.17 \pm 0.00$ & $0.18 \pm 0.00$ & $0.17 \pm 0.00$ & $0.17 \pm 0.01$ & $0.17 \pm 0.00$ & $0.17 \pm 0.01$ \\
\hline Relative & $4.995 \pm 0.117$ & $5.303 \pm 0.119$ & $5.069 \pm 0.187$ & $5.094 \pm 0.172$ & $5.128 \pm 0.135$ & $5.351 \pm 0.178$ \\
\hline \multicolumn{7}{|l|}{ Liver } \\
\hline Absolute & $1.04 \pm 0.01$ & $1.21 \pm 0.05^{*}$ & $1.11 \pm 0.04^{*}$ & $1.16 \pm 0.03^{*}$ & $1.22 \pm 0.03^{* *}$ & $1.21 \pm 0.04^{* *}$ \\
\hline Relative & $30.559 \pm 0.696$ & $36.190 \pm 1.253^{* *}$ & $32.906 \pm 0.651^{* *}$ & $34.449 \pm 0.693 * *$ & $36.878 \pm 0.867 * *$ & $38.687 \pm 0.778 * *$ \\
\hline \multicolumn{7}{|l|}{ Lung } \\
\hline Absolute & $0.23 \pm 0.01$ & $0.22 \pm 0.01$ & $0.20 \pm 0.01^{*}$ & $0.21 \pm 0.01$ & $0.23 \pm 0.01$ & $0.23 \pm 0.01$ \\
\hline Relative & $6.764 \pm 0.244$ & $6.673 \pm 0.348$ & $5.863 \pm 0.199$ & $6.243 \pm 0.217$ & $7.055 \pm 0.265$ & $7.408 \pm 0.352$ \\
\hline \multicolumn{7}{|l|}{ Thymus } \\
\hline Absolute & $0.041 \pm 0.001$ & $0.036 \pm 0.001$ & $0.038 \pm 0.002$ & $0.041 \pm 0.002$ & $0.042 \pm 0.002$ & $0.031 \pm 0.002^{* *}$ \\
\hline Relative & $1.204 \pm 0.039$ & $1.087 \pm 0.045$ & $1.158 \pm 0.104$ & $1.218 \pm 0.063$ & $1.285 \pm 0.068$ & $1.019 \pm 0.087$ \\
\hline
\end{tabular}

*Significantly different $(\mathrm{P} \leq 0.05)$ from the vehicle control group by Williams' or Dunnett’s test. $* * \mathrm{P} \leq 0.01$

${ }^{a}$ Organ weights (absolute weights) and body weights are given in grams; organ-weight-to-body-weight ratios (relative weights) are given as mg organ weight/g body weight (mean \pm standard error).

${ }^{b} \mathrm{n}=9$. 


\section{Appendix H. Reproductive Tissue Evaluations and Estrous Cycle Characterization}

\section{Tables}

Table H-1. Summary of Reproductive Tissue Evaluations for Male F344/N Rats in the Three-month Gavage Study of Indole-3-carbinol

Table H-2. Estrous Cycle Characterization for Female F344/N Rats in the Three-month Gavage Study of Indole-3-carbinol

Table H-3. Results of Vaginal Cytology Study Using the Transition Matrix Approach in Female F344/N Rats Administered Indole-3-carbinol by Gavage for Three Months

Table H-4. Summary of Reproductive Tissue Evaluations for Male Mice in the Threemonth Gavage Study of Indole-3-carbinol

Table H-5. Estrous Cycle Characterization for Female Mice in the Three-month Gavage Study of Indole-3-carbinol

Table H-6. Results of Vaginal Cytology Study Using the Transition Matrix Approach in Female Mice Administered Indole-3-carbinol by Gavage for Three Months

\section{Figures}

Figure H-1. Vaginal Cytology Plots for Female F344/N Rats in the Three-month Gavage Study of Indole-3-carbinol.

Figure H-2. Vaginal Cytology Plots for Female Mice in the Three-month Gavage Study of Indole-3-carbinol. 
Indole-3-carbinol, NTP TR 584

Table H-1. Summary of Reproductive Tissue Evaluations for Male F344/N Rats in the Three-month Gavage Study of Indole-3-carbinol ${ }^{\mathrm{a}}$

\begin{tabular}{lcccc}
\hline & Vehicle Control & $\mathbf{7 5 ~} \mathbf{~} \mathbf{g} / \mathbf{k g}$ & $\mathbf{1 5 0} \mathbf{~} \mathbf{g} / \mathbf{k g}$ & $\mathbf{3 0 0} \mathbf{~} \mathbf{g} / \mathbf{k g}$ \\
\hline $\mathbf{n}$ & 10 & 10 & 10 & 10 \\
\hline Weights (g) & & & & \\
$\quad$ Necropsy body wt & $309 \pm 5$ & $321 \pm 8$ & $309 \pm 8$ & $285 \pm 6^{*}$ \\
$\quad$ L. Cauda epididymis & $0.1657 \pm 0.0055$ & $0.1687 \pm 0.0083$ & $0.1588 \pm 0.0067$ & $0.1591 \pm 0.0099$ \\
L. Epididymis & $0.4403 \pm 0.0125$ & $0.4414 \pm 0.0145$ & $0.4249 \pm 0.0101$ & $0.4074 \pm 0.0148$ \\
$\quad$ L. Testis & $1.3959 \pm 0.0246$ & $1.4735 \pm 0.0264$ & $1.4580 \pm 0.0235$ & $1.4157 \pm 0.0308$ \\
Spermatid measurements & & & & \\
$\quad$ Spermatid heads (10\%/testis) & $160.4 \pm 6.7$ & $168.4 \pm 7.8$ & $149.3 \pm 6.4$ & $170.9 \pm 12.6$ \\
$\quad$ Spermatid heads (106/g testis) & $153.4 \pm 6.0$ & $160.6 \pm 9.4$ & $143.4 \pm 5.4$ & $164.1 \pm 9.5$ \\
Epididymal spermatozoal measurements & & & & \\
$\quad$ Sperm motility (\%) & $84.60 \pm 1.62$ & $83.66 \pm 1.85$ & $82.23 \pm 1.66$ & $82.52 \pm 1.78$ \\
$\quad$ Sperm (10\%/cauda epididymis) & $94.03 \pm 2.43$ & $83.90 \pm 2.90$ & $89.39 \pm 4.30$ & $92.04 \pm 4.57$ \\
$\quad$ Sperm (10\%/g cauda epididymis) & $571.6 \pm 19.0$ & $505.7 \pm 26.3$ & $571.8 \pm 35.7$ & $590.9 \pm 31.4$ \\
\hline
\end{tabular}

*Significantly different $(\mathrm{P} \leq 0.05)$ from the vehicle control group by Dunnett’s test.

aData are presented as mean \pm standard error. Differences from the vehicle control group are not significant by Dunnett's test (tissue weights) or Dunn’s test (spermatid and epididymal spermatozoal measurements).

Table H-2. Estrous Cycle Characterization for Female F344/N Rats in the Three-month Gavage Study of Indole-3-carbinol ${ }^{\mathrm{a}}$

\begin{tabular}{lcccc}
\hline & Vehicle Control & $\mathbf{7 5 ~} \mathbf{~ g / / k g}$ & $\mathbf{1 5 0 ~} \mathbf{~ g} / \mathbf{k g}$ & $\mathbf{3 0 0} \mathbf{~} \mathbf{g} / \mathbf{k g}$ \\
\hline Number weighed at necropsy & 10 & 10 & 10 & 10 \\
$\quad$ Necropsy body wt (g) & $189 \pm 2$ & $185 \pm 3$ & $183 \pm 3$ & $183 \pm 3$ \\
$\begin{array}{l}\text { Proportion of regular cycling } \\
\text { females }\end{array}$ & $7 / 7$ & $8 / 8$ & $9 / 10$ & $8 / 9$ \\
Estrous cycle length (days) $^{\mathrm{b}}$ & $5.00 \pm 0.11^{\mathrm{c}}$ & $5.13 \pm 0.13^{\mathrm{d}}$ & $5.25 \pm 0.13$ & $5.89 \pm 0.31^{* \mathrm{e}}$ \\
Estrous stages $^{\mathrm{f}}$ (\% of cycle) & & & & \\
Diestrus & 45.8 & 50.0 & 52.5 & 60.0 \\
Proestrus & 15.0 & 13.3 & 16.7 & 9.2 \\
Estrus & 20.8 & 19.2 & 19.2 & 20.0 \\
Metestrus & 6.7 & 6.7 & 11.7 & 10.8 \\
Uncertain diagnoses & 11.7 & 10.8 & 0.0 & 0.0 \\
\hline
\end{tabular}

*Significantly different $(\mathrm{P} \leq 0.05)$ from the vehicle control group by Dunn's test.

${ }^{a}$ Necropsy body weights and estrous cycle length data are presented as mean \pm standard error. Differences from the vehicle control group are not significant by Dunnett's test (body weight). Tests for equality of transition probability matrices among all groups and between the vehicle control group and each dosed group indicated a significantly higher probability of extended diestrus in the $300 \mathrm{mg} / \mathrm{kg}$ group compared to the vehicle control group.

${ }^{b}$ Number of females with a regular cycle/number of females cycling.

'Estrous cycle was longer than 12 days or unclear in three of 10 animals.

${ }^{\mathrm{d}}$ Estrous cycle was longer than 12 days or unclear in two of 10 animals.

${ }^{\text {e}}$ Estrous cycle was longer than 12 days or unclear in one of 10 animals.

fEvidence shows that 150 and $300 \mathrm{mg} / \mathrm{kg}$ females differ significantly (Wilkes' Criterion, $\mathrm{P} \leq 0.05$ ) from the vehicle control females in the relative length of time spent in the estrous stages. 
Table H-3. Results of Vaginal Cytology Study Using the Transition Matrix Approach in Female F344/N Rats Administered Indole-3-carbinol by Gavage for Three Months

\begin{tabular}{|c|c|c|c|}
\hline Stage & Comparison $^{\mathbf{a}}$ & P Value & Trend $^{b}$ \\
\hline Overall Tests & Overall & $<0.001$ & \\
\hline Overall Tests & Low vs. Controls & 0.983 & - \\
\hline Overall Tests & Mid vs. Controls & 0.89 & $\mathrm{~N}$ \\
\hline Overall Tests & High vs. Controls & $<0.001$ & - \\
\hline Extended Estrus & Overall & 0.519 & \\
\hline Extended Estrus & Low vs. Controls & 0.572 & - \\
\hline Extended Estrus & Mid vs. Controls & 0.543 & $\mathrm{~N}$ \\
\hline Extended Estrus & High vs. Controls & 0.239 & - \\
\hline Extended Diestrus & Overall & 0.001 & \\
\hline Extended Diestrus & Low vs. Controls & 0.967 & $\mathrm{~N}$ \\
\hline Extended Diestrus & Mid vs. Controls & 0.861 & $\mathrm{~N}$ \\
\hline Extended Diestrus & High vs. Controls & $<0.001$ & - \\
\hline Extended Metestrus & Overall & 1 & \\
\hline Extended Metestrus & Low vs. Controls & 1 & - \\
\hline Extended Metestrus & Mid vs. Controls & 1 & - \\
\hline Extended Metestrus & High vs. Controls & 1 & - \\
\hline Extended Proestrus & Overall & 0.985 & \\
\hline Extended Proestrus & Low vs. Controls & 1 & - \\
\hline Extended Proestrus & Mid vs. Controls & 0.604 & - \\
\hline Extended Proestrus & High vs. Controls & 1 & - \\
\hline Skipped Estrus & Overall & 1 & \\
\hline Skipped Estrus & Low vs. Controls & 1 & - \\
\hline Skipped Estrus & Mid vs. Controls & 0.924 & - \\
\hline Skipped Estrus & High vs. Controls & 1 & - \\
\hline Skipped Diestrus & Overall & 1 & \\
\hline Skipped Diestrus & Low vs. Controls & 1 & - \\
\hline Skipped Diestrus & Mid vs. Controls & 1 & - \\
\hline Skipped Diestrus & High vs. Controls & 1 & - \\
\hline \multicolumn{4}{|c|}{ Summary of Significant Groups } \\
\hline Overall Tests & High vs. Controls & $<0.001$ & - \\
\hline Extended Diestrus & High vs. Controls & $<0.001$ & - \\
\hline
\end{tabular}


Indole-3-carbinol, NTP TR 584

Table H-4. Summary of Reproductive Tissue Evaluations for Male Mice in the Three-month Gavage Study of Indole-3-carbinol ${ }^{\mathrm{a}}$

\begin{tabular}{|c|c|c|c|c|}
\hline & Vehicle Control & $62.5 \mathrm{mg} / \mathrm{kg}$ & $125 \mathrm{mg} / \mathrm{kg}$ & $250 \mathrm{mg} / \mathrm{kg}$ \\
\hline $\mathbf{n}$ & 10 & 10 & 10 & 9 \\
\hline \multicolumn{5}{|l|}{ Weights (g) } \\
\hline Necropsy body wt & $40.4 \pm 0.6$ & $40.0 \pm 1.1$ & $42.2 \pm 1.6$ & $40.8 \pm 1.0$ \\
\hline L. Cauda epididymis & $0.0245 \pm 0.0010$ & $0.0255 \pm 0.0013$ & $0.0231 \pm 0.0013$ & $0.0232 \pm 0.0010$ \\
\hline L. Epididymis & $0.0583 \pm 0.0019$ & $0.0589 \pm 0.0014$ & $0.0555 \pm 0.0030$ & $0.0530 \pm 0.0020$ \\
\hline L. Testis & $0.1274 \pm 0.0023$ & $0.1244 \pm 0.0026$ & $0.1254 \pm 0.0014$ & $0.1202 \pm 0.0028$ \\
\hline \multicolumn{5}{|l|}{ Spermatid measurements } \\
\hline Spermatid heads ( $10^{6} /$ testis) & $20.99 \pm 1.28$ & $22.56 \pm 1.11$ & $22.63 \pm 1.60$ & $24.81 \pm 0.47$ \\
\hline Spermatid heads ( $10^{6} / g$ testis) & $378.9 \pm 52.3$ & $417.9 \pm 28.0$ & $478.1 \pm 47.0$ & $507.8 \pm 76.5$ \\
\hline \multicolumn{5}{|c|}{ Epididymal spermatozoal measurements } \\
\hline Sperm motility (\%) & $83.71 \pm 0.50$ & $79.68 \pm 1.09 * *$ & $79.12 \pm 0.96 * *$ & $74.26 \pm 0.80 * *$ \\
\hline Sperm (10\%/cauda epididymis) & $18.04 \pm 0.58$ & $18.97 \pm 1.04$ & $18.52 \pm 0.90$ & $18.73 \pm 2.35$ \\
\hline Sperm (106/g cauda epididymis) & $742.9 \pm 28.9$ & $747.1 \pm 30.7$ & $820.0 \pm 54.9$ & $800.5 \pm 94.9$ \\
\hline
\end{tabular}

**Significantly different $(\mathrm{P} \leq 0.01)$ from the vehicle control group by Shirley’s test.

a Data are presented as mean \pm standard error. Differences from the vehicle control group are not significant by Dunnett’s test (body and tissue weights) or Dunn's test (spermatid measurements, sperm per cauda epididymis, and sperm per gram cauda epididymis).

Table H-5. Estrous Cycle Characterization for Female Mice in the Three-month Gavage Study of Indole-3-carbinol ${ }^{\mathrm{a}}$

\begin{tabular}{lcccc}
\hline & Vehicle Control & $\mathbf{6 2 . 5} \mathbf{~} \mathbf{g} / \mathbf{k g}$ & $\mathbf{1 2 5} \mathbf{~} \mathbf{g} / \mathbf{k g}$ & $\mathbf{2 5 0} \mathbf{~} \mathbf{g} / \mathbf{k g}$ \\
\hline Number weighed at necropsy & 10 & 10 & 10 & 10 \\
$\quad$ Necropsy body wt (g) & $34.2 \pm 1.0$ & $33.7 \pm 0.6$ & $33.1 \pm 0.7$ & $31.4 \pm 1.3$ \\
Proportion of regular cycling females ${ }^{\mathrm{b}}$ & $8 / 10$ & $8 / 10$ & $7 / 10$ & $5 / 9$ \\
Estrous cycle length (days) & $4.06 \pm 0.13$ & $4.65 \pm 0.51$ & $4.29 \pm 0.17$ & $4.17 \pm 0.33^{c}$ \\
Estrous stages (\% of cycle) & & & & \\
Diestrus & 31.7 & 40.8 & 30.0 & 40.0 \\
Proestrus & 0.0 & 1.7 & 1.7 & 3.3 \\
Estrus & 46.7 & 38.3 & 46.7 & 37.5 \\
Metestrus & 21.7 & 19.2 & 21.7 & 19.2 \\
\hline
\end{tabular}

${ }^{a}$ Necropsy body weights and estrous cycle length data are presented as mean \pm standard error. Differences from the vehicle control group are not significant by Dunnett's test (body weight) or Dunn's test (estrous cycle length). By multivariate analysis of variance, dosed females do not differ significantly from the vehicle control females in the relative length of time spent in the estrous stages. Tests for equality of transition probability matrices among all groups and between the vehicle control group and each dosed group indicated a significantly higher probability of extended diestrus in the $250 \mathrm{mg} / \mathrm{kg}$ group compared to the vehicle control group.

${ }^{b}$ Number of females with a regular cycle/number of females cycling.

${ }^{\mathrm{c}}$ One of 10 animals was excluded from mean due to cycle length longer than 9 days. 
Indole-3-carbinol, NTP TR 584

Table H-6. Results of Vaginal Cytology Study Using the Transition Matrix Approach in Female Mice Administered Indole-3-carbinol by Gavage for Three Months

\begin{tabular}{|c|c|c|c|}
\hline Stage & Comparison $^{\mathrm{a}}$ & P Value & Trend $^{b}$ \\
\hline Overall Tests & Overall & 0.024 & \\
\hline Overall Tests & Low vs. Controls & 0.437 & - \\
\hline Overall Tests & Mid vs. Controls & 0.426 & - \\
\hline Overall Tests & High vs. Controls & 0.004 & - \\
\hline Extended Estrus & Overall & 0.793 & \\
\hline Extended Estrus & Low vs. Controls & 0.77 & $\mathrm{~N}$ \\
\hline Extended Estrus & Mid vs. Controls & 0.351 & - \\
\hline Extended Estrus & High vs. Controls & 0.775 & - \\
\hline Extended Diestrus & Overall & 0.052 & \\
\hline Extended Diestrus & Low vs. Controls & 0.065 & - \\
\hline Extended Diestrus & Mid vs. Controls & 0.946 & $\mathrm{~N}$ \\
\hline Extended Diestrus & High vs. Controls & 0.031 & - \\
\hline Extended Metestrus & Overall & 1 & \\
\hline Extended Metestrus & Low vs. Controls & 1 & - \\
\hline Extended Metestrus & Mid vs. Controls & 1 & - \\
\hline Extended Metestrus & High vs. Controls & 1 & - \\
\hline Extended Proestrus & Overall & 1 & \\
\hline Extended Proestrus & Low vs. Controls & 1 & - \\
\hline Extended Proestrus & Mid vs. Controls & 1 & - \\
\hline Extended Proestrus & High vs. Controls & 1 & - \\
\hline Skipped Estrus & Overall & 1 & \\
\hline Skipped Estrus & Low vs. Controls & 1 & - \\
\hline Skipped Estrus & Mid vs. Controls & 1 & - \\
\hline Skipped Estrus & High vs. Controls & 1 & - \\
\hline Skipped Diestrus & Overall & 1 & \\
\hline Skipped Diestrus & Low vs. Controls & 1 & - \\
\hline Skipped Diestrus & Mid vs. Controls & 1 & - \\
\hline Skipped Diestrus & High vs. Controls & 1 & - \\
\hline \multicolumn{4}{|c|}{ Summary of Significant Groups } \\
\hline Overall Tests & High vs. Controls & 0.004 & - \\
\hline Extended Diestrus & High vs. Controls & 0.031 & - \\
\hline
\end{tabular}

${ }^{a}$ Controls = Vehicle Control, Low $=62.5 \mathrm{mg} / \mathrm{kg}$, Mid $=125 \mathrm{mg} / \mathrm{kg}$, High $=250 \mathrm{mg} / \mathrm{kg}$.

${ }^{b} \mathrm{~N}$ means that the treated group had a lower probability of transitioning to the relevant abnormal state (extended estrus, extended diestrus, extended metestrus, extended proestrus, skipped estrus, or skipped diestrus) than did the vehicle control group. 


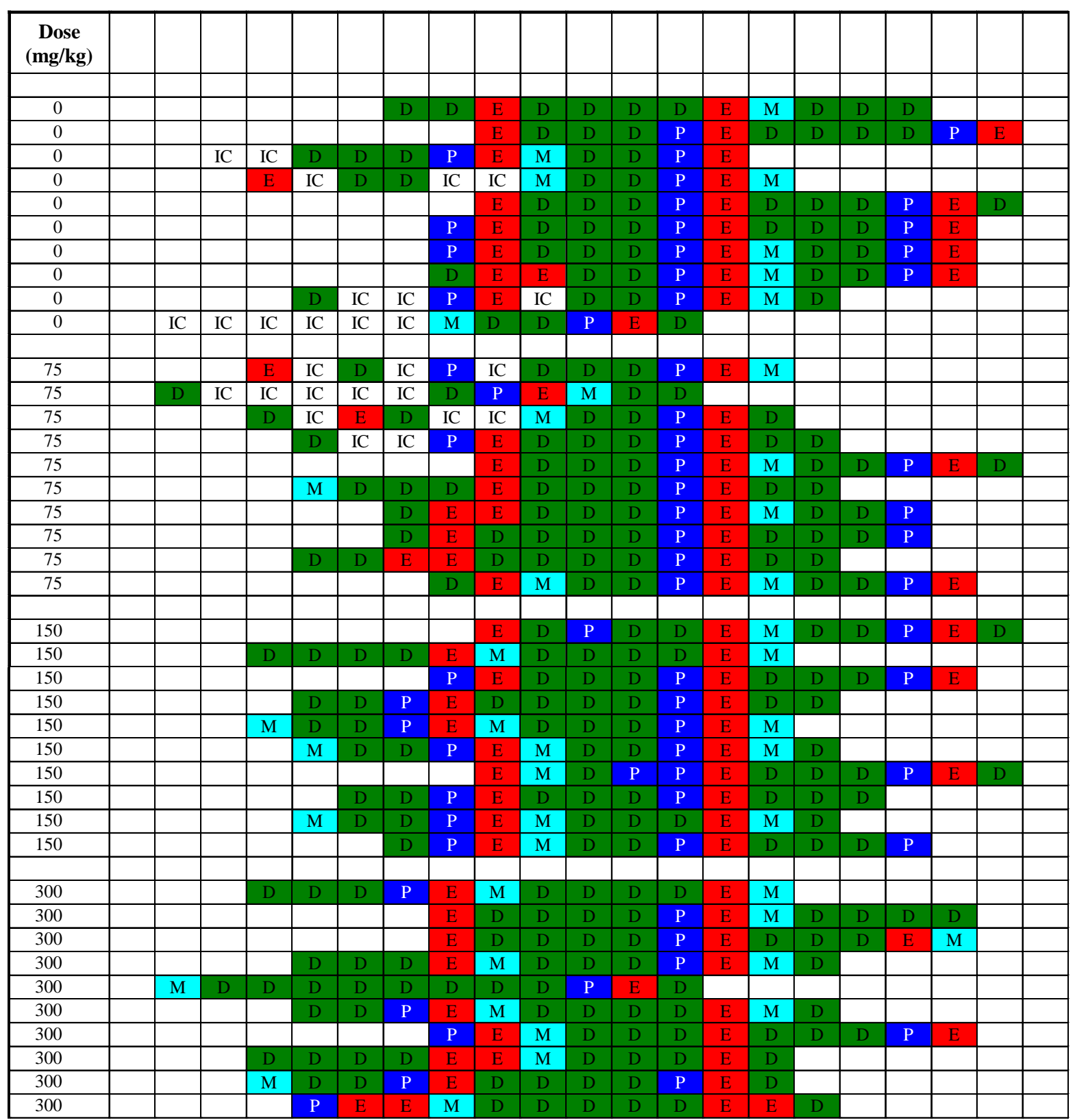

Figure H-1. Vaginal Cytology Plots for Female F344/N Rats in the Three-month Gavage Study of Indole-3-carbinol

IC = Insufficient number of cells to determine stage; $\mathrm{D}=$ diestrus, $\mathrm{P}=$ proestrus, $\mathrm{E}=$ estrus, $\mathrm{M}=$ metestrus 


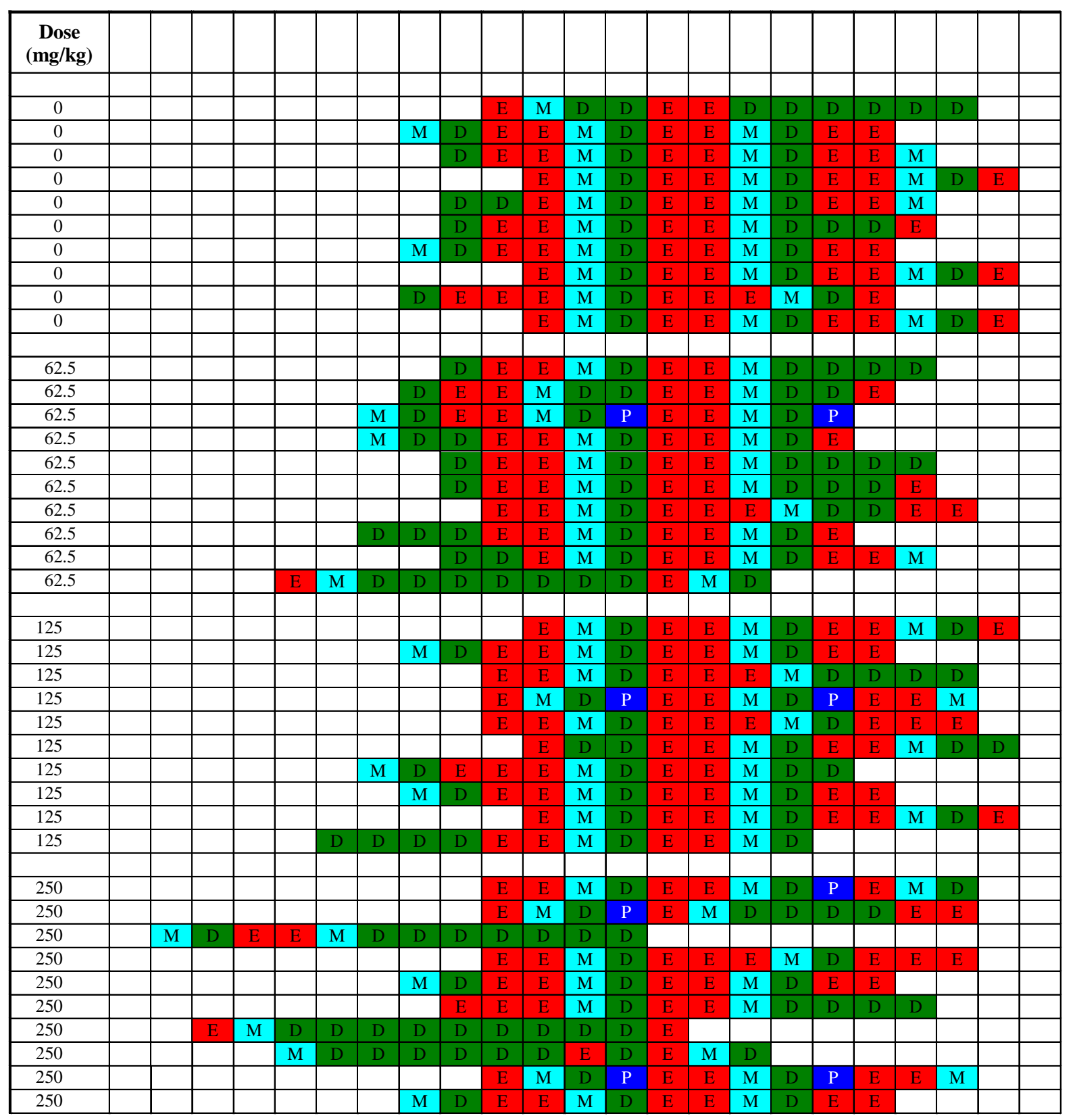

Figure H-2. Vaginal Cytology Plots for Female Mice in the Three-month Gavage Study of Indole-3-carbinol

$\mathrm{D}=$ diestrus, $\mathrm{P}=$ proestrus, $\mathrm{E}=$ estrus, $\mathrm{M}=$ metestrus 


\section{Appendix I. Chemical Characterization and Dose Formulation Studies}

\section{Table of Contents}

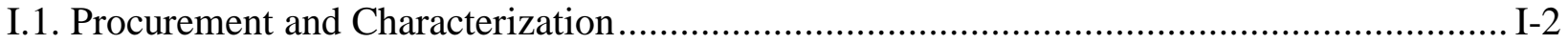

I.2. Preparation and Analysis of Dose Formulations ................................................. I-3

\section{Tables}

Table I-1. High-Performance Liquid Chromatography Systems Used in the Gavage

Studies of Indole-3-carbinol

Table I-2. Preparation and Storage of Dose Formulations in the Gavage Studies of Indole-3--carbinol

Table I-3. Results of Analyses of Dose Formulations Administered to F344/N Rats and Mice in the Three-month Gavage Studies of Indole-3-carbinol.

Table I-4. Results of Analyses of Dose Formulations Administered to Sprague Dawley

Rats and Mice in the Two-year Gavage Studies of Indole-3-carbinol....

\section{Figures}

Figure I-1. Infrared Absorption Spectrum of Indole-3-carbinol.

Figure I-2. Proton Nuclear Magnetic Resonance Spectrum of Indole-3-carbinol 
Indole-3-carbinol, NTP TR 584

\section{I.1. Procurement and Characterization}

\section{I.1.1. Indole-3-carbinol}

Indole-3-carbinol was obtained from ChemPacific Corporation (Baltimore, MD) in one lot (CHP801001) that was used during the 3-month and 2-year studies. Identity and purity analyses were conducted by the analytical chemistry laboratory at Battelle Columbus Support Services (Columbus, $\mathrm{OH}$ ) and the study laboratories, Southern Research Institute (SRI) (Birmingham, $\mathrm{AL}$ ) for the 3-month studies and Battelle Columbus Operations (Columbus, $\mathrm{OH}$ ) for the 2-year studies. Karl Fischer titration and elemental analyses were performed by Galbraith Laboratories, Inc. (Knoxville, TN). Reports on analyses performed in support of the indole-3-carbinol studies are on file at the National Institute of Environmental Health Sciences.

Lot CHP801001 of the test chemical, a yellow crystalline solid, was identified as indole-3carbinol by the analytical chemistry laboratory using infrared (IR) and proton and carbon-13 nuclear magnetic resonance (NMR) spectroscopy and by the study laboratories using IR and proton NMR (SRI only) spectroscopy. All spectra were consistent with the literature spectra ${ }^{137}$; 138 and the structure of indole-3-carbinol. Representative IR and proton NMR spectra are presented in Figure I-1 and Figure I-2, respectively.

For lot CHP801001, Karl Fischer titration was used to determine the water content; elemental analyses were used to determine the carbon, hydrogen, and nitrogen content; and the melting point was determined using a differential scanning calorimeter. The purity was determined by the analytical chemistry laboratory using high-performance liquid chromatography (HPLC) with ultraviolet detection by systems A and B (Table I-1).

Karl Fischer titration indicated 1.3\% water. Elemental analyses for carbon, hydrogen, and nitrogen were consistent with the theoretical values, and the melting point was $99.3^{\circ} \mathrm{C}$, consistent with the manufacturer's Certificate of Analysis. HPLC analysis indicated one major peak with six impurities, each $0.1 \%$ or greater of the total peak area with a combined area of $2.8 \%$ of total peak area in the chromatogram. The overall purity of lot CHP801001 was determined to be approximately $97 \%$.

Stability studies of the bulk chemical were performed by the analytical chemistry laboratory using HPLC by system B. These studies indicated that indole-3-carbinol was stable as a bulk chemical for at least 2 weeks when stored in sealed amber glass vials protected from light at temperatures up to $25^{\circ} \mathrm{C}$. However, at $60^{\circ} \mathrm{C}$, physical changes were observed in the test chemical. To ensure stability, the bulk chemical was stored at $-20^{\circ} \mathrm{C}$, protected from light in amber glass containers sealed with Teflon ${ }^{\circledR}$-lined lids. Periodic reanalyses of the bulk chemical were performed by the study laboratory twice during the 3-month studies and approximately every 6 months during the 2-year studies using HPLC by a system similar to system B; no degradation of the bulk chemical was detected.

\section{I.1.2. Corn Oil}

Corn oil was used as the vehicle for the formulations and was obtained from Red Diamond, Inc. (Birmingham, AL), in one lot that was used in the 3-month studies and from Spectrum Chemicals and Laboratory Products (Gardena, CA) in seven lots and from Sigma-Aldrich (St. Louis, MO) in three lots that were used in the 2-year studies. Analysis of the corn oil for 
peroxides was performed by potentiometric titration, and each lot was within the acceptable range of less than or equal to $3 \mathrm{mEq} / \mathrm{kg}$.

\section{I.2. Preparation and Analysis of Dose Formulations}

The dose formulations were prepared by mixing the appropriate amount of milled indole-3carbinol with corn oil to achieve the required concentrations (Table I-2). The dose formulations were prepared seven times during the 3-month studies and approximately every 4 weeks during the 2-year studies. Dose formulations were stored in amber glass vials sealed with Teflon ${ }^{\circledR}$-lined lids at $5^{\circ} \mathrm{C}$ for up to 41 days.

Homogeneity, gavageability, and resuspendability studies were performed on $60 \mathrm{mg} / \mathrm{mL}$ dose formulations, and stability studies were performed on $1 \mathrm{mg} / \mathrm{mL}$ formulations by the analytical chemistry laboratory using HPLC by system B (Table I-1). To achieve acceptable chromatography, all samples containing corn oil were clarified by centrifuging 1-mL aliquots of each dose formulation for approximately 5 minutes at 14,000 rpm, then diluting (1:1) with the mobile phase prior to HPLC analysis. Homogeneity was confirmed, and gavageability was confirmed for a 20-gauge gavage needle. Resuspendability was confirmed for dose formulations that had been stored for 14 days at $5^{\circ} \mathrm{C}$. Stability was confirmed for at least 8 days for $1 \mathrm{mg} / \mathrm{mL}$ formulations stored in amber glass vials sealed with Teflon ${ }^{\circledR}$-lined lids at $5^{\circ} \mathrm{C}$ and for 3 hours under simulated animal room conditions. The study laboratory at SRI performed stability studies with $1.6 \mathrm{mg} / \mathrm{mL}$ dose formulations using HPLC by a system similar to system B, and stability was confirmed for at least 41 days for dose formulations stored in amber glass vials sealed with Teflon ${ }^{\circledR}$-lined lids at $5{ }^{\circ} \mathrm{C}$. Prior to the 2-year studies, the study laboratory at Battelle Columbus Operations performed homogeneity studies on $6.25,15,25$, and $60 \mathrm{mg} / \mathrm{mL}$ dose formulations and gavageability studies on $60 \mathrm{mg} / \mathrm{mL}$ dose formulations using HPLC by a system similar to system B; homogeneity and gavagability were confirmed.

Periodic analyses of the dose formulations of indole-3-carbinol were performed by the study laboratories using HPLC by systems similar to system B. During the 3-month studies, the dose formulations were analyzed three times; all 15 dose formulations for rats and all 15 for mice were within $10 \%$ of the target concentrations (Table I-3). Animal room samples of these dose formulations were also analyzed; all 15 for rats and all 15 for mice were within $10 \%$ of the target concentrations. During the 2-year studies, the dose formulations were analyzed approximately every 2 months (Table I-4). Of the dose formulations analyzed, all 72 for rats and all 36 for mice were within $10 \%$ of the target concentrations; all 24 of the animal room samples for rats and all 12 for mice were within $10 \%$ of the target concentrations. 
Indole-3-carbinol, NTP TR 584

Table I-1. High-Performance Liquid Chromatography Systems Used in the Gavage Studies of Indole-3-carbinol $^{\mathrm{a}}$

\begin{tabular}{|c|c|c|}
\hline Detection System & Column & Solvent System \\
\hline \multicolumn{3}{|l|}{ System A } \\
\hline $\begin{array}{l}\text { Photodiode array } \\
\text { Ultraviolet ( } 220 \text { to } 360 \mathrm{~nm} \text { ) light }\end{array}$ & $\begin{array}{l}\text { Prodigy }^{\mathrm{TM}} \text { C18 ODS(3), } 250 \mathrm{~mm} \times \\
4.6 \mathrm{~mm}, 5 \mu \mathrm{m} \text { (Phenomenex, } \\
\text { Torrance, CA) }\end{array}$ & $\begin{array}{l}\text { 80:20 Methanol:1\% aqueous acetic } \\
\text { acid solution, isocratic; flow rate } \\
0.8 \mathrm{~mL} / \text { minute }\end{array}$ \\
\hline \multicolumn{3}{|l|}{ System B } \\
\hline Ultraviolet $(280 \mathrm{~nm})$ light & $\begin{array}{l}\text { Prodigy }{ }^{\mathrm{TM}} \mathrm{C} 18,250 \mathrm{~mm} \times 4.6 \mathrm{~mm} \text {, } \\
5 \mu \mathrm{m} \text { (Phenomenex) }\end{array}$ & $\begin{array}{l}80: 20 \text { Methanol: } 1.5 \% \text { aqueous acetic } \\
\text { acid solution, isocratic; flow rate } \\
0.8 \mathrm{~mL} / \text { minute }\end{array}$ \\
\hline
\end{tabular}

aThe high-performance liquid chromatographs were manufactured by Waters, Inc. (Milford, MA) (System A), or Spectra Physics (San Jose, CA) (System B).

Table I-2. Preparation and Storage of Dose Formulations in the Gavage Studies of Indole-3--carbinol

\begin{tabular}{lll}
\hline & Three-month Studies & Two-year Studies \\
\hline Preparation & &
\end{tabular}

Prior to the preparation of dose formulations, portions of frozen indole-3-carbinol were milled for approximately 15 seconds in an IKA Works M20 Universal mill, then passed through a 40-mesh sieve, and placed in high density polyethylene wide-mouth containers, sealed with Teflon ${ }^{\circledR}$-lined lids, rolled and shaken for approximately 15 minutes.

To prepare the dose formulations, containers of milled indole-3-carbinol were allowed to warm to room temperature. For each dose formulation, the appropriate amount of milled indole-3-carbinol was weighed into a weigh boat or beaker and transferred to a mortar; corn oil was added in increments and the mixture was ground with a pestle into a paste-like mixture, transferred to an Erlenmeyer flask with corn oil rinses, diluted to final volume and sonicated for approximately 10 minutes, and cooled by an ice water bath. A stir bar was added and the mixture was stirred for at least 2 hours to ensure homogeneity. Formulations were prepared seven times during the 3-month studies.

\section{Chemical Lot Number}

CHP801001

\section{CHP801001}

\section{Maximum Storage Time}

41 days

41 days

\section{Storage Conditions}

The dose formulations were stored in amber glass vials sealed with Teflon ${ }^{\circledR}$-lined lids at $5^{\circ} \mathrm{C}$.

\section{Study Laboratory}

Southern Research Institute (Birmingham, AL)
Same as 3-month studies except that dose formulations were prepared approximately every 4 weeks.
The dose formulations were stored in amber glass vials sealed with Teflon ${ }^{\circledR}$-lined lids at $5^{\circ} \mathrm{C}$. 
Indole-3-carbinol, NTP TR 584

Table I-3. Results of Analyses of Dose Formulations Administered to F344/N Rats and Mice in the Three-month Gavage Studies of Indole-3-carbinol

\begin{tabular}{|c|c|c|c|c|}
\hline Date Prepared & Date Analyzed & $\begin{array}{c}\text { Target } \\
\text { Concentration } \\
(\mathbf{m g} / \mathbf{m L})\end{array}$ & $\begin{array}{c}\text { Determined } \\
\text { Concentration } \\
(\mathrm{mg} / \mathrm{mL})\end{array}$ & $\begin{array}{c}\text { Difference } \\
\text { from Target } \\
\text { (\%) }\end{array}$ \\
\hline \multicolumn{5}{|l|}{ Rats } \\
\hline \multirow[t]{10}{*}{ August 23, 2004} & August 24-25, 2004 & 3.75 & $3.52 \pm 0.061$ & -6 \\
\hline & & 7.50 & $7.25 \pm 0.33$ & -3 \\
\hline & & 15 & $13.6 \pm 0.14$ & -9 \\
\hline & & 30 & $29.1 \pm 0.10$ & -3 \\
\hline & & 60 & $63.0 \pm 0.41$ & +5 \\
\hline & September 13-14, 2004 & 3.75 & $3.60 \pm 0.090$ & -4 \\
\hline & & 7.50 & $7.33 \pm 0.019$ & -2 \\
\hline & & 15 & $14.9 \pm 0.0085$ & -1 \\
\hline & & 30 & $30.0 \pm 0.37$ & 0 \\
\hline & & 60 & $60.8 \pm 0.38$ & +1 \\
\hline \multirow[t]{10}{*}{ October 5, 2004} & October 6-7, 2004 & 3.75 & $3.68 \pm 0.089$ & -2 \\
\hline & & 7.50 & $7.30 \pm 0.20$ & -3 \\
\hline & & 15 & $15.0 \pm 0.024$ & 0 \\
\hline & & 30 & $29.8 \pm 0.19$ & -1 \\
\hline & & 60 & $59.2 \pm 0.25$ & -1 \\
\hline & October 26-27, 2004 & 3.75 & $3.60 \pm 0.060$ & -4 \\
\hline & & 7.50 & $7.22 \pm 0.39$ & -4 \\
\hline & & 15 & $15.0 \pm 0.62$ & 0 \\
\hline & & 30 & $29.4 \pm 0.25$ & -2 \\
\hline & & 60 & $56.0 \pm 0.27$ & -7 \\
\hline \multirow[t]{10}{*}{ November 15, 2004} & November 16-17, 2004 & 3.75 & $3.54 \pm 0.074$ & -6 \\
\hline & & 7.50 & $7.03 \pm 0.068$ & -6 \\
\hline & & 15 & $14.7 \pm 0.42$ & -2 \\
\hline & & 30 & $29.6 \pm 0.34$ & -1 \\
\hline & & 60 & $56.8 \pm 0.83$ & -5 \\
\hline & December 8-9, 2004 & 3.75 & $3.44 \pm 0.025$ & -8 \\
\hline & & 7.50 & $6.91 \pm 0.038$ & -8 \\
\hline & & 15 & $14.4 \pm 0.30$ & -4 \\
\hline & & 30 & $29.9 \pm 0.047$ & 0 \\
\hline & & 60 & $58.7 \pm 3.2$ & -2 \\
\hline
\end{tabular}


Indole-3-carbinol, NTP TR 584

\begin{tabular}{|c|c|c|c|c|}
\hline Date Prepared & Date Analyzed & $\begin{array}{c}\text { Target } \\
\text { Concentration } \\
(\mathrm{mg} / \mathrm{mL})\end{array}$ & $\begin{array}{c}\text { Determined } \\
\text { Concentration }^{\mathrm{a}} \\
(\mathrm{mg} / \mathrm{mL})\end{array}$ & $\begin{array}{c}\text { Difference } \\
\text { from Target } \\
(\%)\end{array}$ \\
\hline \multicolumn{5}{|l|}{ Mice } \\
\hline \multirow[t]{10}{*}{ August 23, 2004} & August 24-25, 2004 & 1.56 & $1.52 \pm 0.0080$ & -3 \\
\hline & & 3.13 & $3.03 \pm 0.082$ & -3 \\
\hline & & 6.25 & $6.21 \pm 0.081$ & -1 \\
\hline & & 12.5 & $12.1 \pm 0.021$ & -3 \\
\hline & & 25 & $23.7 \pm 0.46$ & -5 \\
\hline & September 13-14, 2004 & 1.56 & $1.55 \pm 0.00026$ & -1 \\
\hline & & 3.13 & $2.83 \pm 0.039$ & -10 \\
\hline & & 6.25 & $5.86 \pm 0.12$ & -6 \\
\hline & & 12.5 & $11.5 \pm 0.032$ & -8 \\
\hline & & 25 & $24.2 \pm 0.13$ & -3 \\
\hline \multirow[t]{10}{*}{ October 5, 2004} & October 6-7, 2004 & 1.56 & $1.55 \pm 0.0013$ & -1 \\
\hline & & 3.13 & $2.86 \pm 0.033$ & -9 \\
\hline & & 6.25 & $5.89 \pm 0.022$ & -6 \\
\hline & & 12.5 & $11.9 \pm 0.0074$ & -5 \\
\hline & & 25 & $25.1 \pm 0.12$ & 0 \\
\hline & October 26-27, 2004 & 1.56 & $1.52 \pm 0.0059$ & -3 \\
\hline & & 3.13 & $2.84 \pm 0.019$ & -9 \\
\hline & & 6.25 & $5.92 \pm 0.092$ & -5 \\
\hline & & 12.5 & $11.8 \pm 0.63$ & -6 \\
\hline & & 25 & $25.2 \pm 0.090$ & +1 \\
\hline \multirow[t]{10}{*}{ November 15, 2004} & November 16-17, 2004 & 1.56 & $1.57 \pm 0.031$ & +1 \\
\hline & & 3.13 & $2.97 \pm 0.0038$ & -5 \\
\hline & & 6.25 & $6.26 \pm 0.017$ & 0 \\
\hline & & 12.5 & $12.4 \pm 0.080$ & -1 \\
\hline & & 25 & $25.1 \pm 0.98$ & 0 \\
\hline & December 8-9, 2004 & 1.56 & $1.58 \pm 0.012$ & +1 \\
\hline & & 3.13 & $3.00 \pm 0.051$ & -4 \\
\hline & & 6.25 & $6.25 \pm 0.080$ & 0 \\
\hline & & 12.5 & $12.1 \pm 0.083$ & -3 \\
\hline & & 25 & $25.2 \pm 0.20$ & +1 \\
\hline
\end{tabular}

${ }^{\mathrm{a}}$ Results of duplicate analyses. For rats, dosing volume $=5 \mathrm{~mL} / \mathrm{kg} ; 3.75 \mathrm{mg} / \mathrm{mL}=18.75 \mathrm{mg} / \mathrm{kg}, 7.50 \mathrm{mg} / \mathrm{mL}=37.5 \mathrm{mg} / \mathrm{kg}$, $15 \mathrm{mg} / \mathrm{mL}=75 \mathrm{mg} / \mathrm{kg}, 30 \mathrm{mg} / \mathrm{mL}=150 \mathrm{mg} / \mathrm{kg}, 60 \mathrm{mg} / \mathrm{mL}=300 \mathrm{mg} / \mathrm{kg}$. For mice, dosing volume $=10 \mathrm{~mL} / \mathrm{kg}$; $1.56 \mathrm{mg} / \mathrm{mL}=15.6 \mathrm{mg} / \mathrm{kg}, 3.13 \mathrm{mg} / \mathrm{mL}=31.25 \mathrm{mg} / \mathrm{kg}, 6.25 \mathrm{mg} / \mathrm{mL}=62.5 \mathrm{mg} / \mathrm{kg}, 12.5 \mathrm{mg} / \mathrm{mL}=125 \mathrm{mg} / \mathrm{kg}$, $25 \mathrm{mg} / \mathrm{mL}=250 \mathrm{mg} / \mathrm{kg}$.

bAnimal room samples. 
Indole-3-carbinol, NTP TR 584

Table I-4. Results of Analyses of Dose Formulations Administered to Sprague Dawley Rats and Mice in the Two-year Gavage Studies of Indole-3-carbinol

\begin{tabular}{|c|c|c|c|c|}
\hline Date Prepared & Date Analyzed & $\begin{array}{c}\text { Target } \\
\text { Concentration } \\
(\mathrm{mg} / \mathrm{mL})\end{array}$ & $\begin{array}{c}\text { Determined } \\
\text { Concentration }^{\mathrm{a}} \\
(\mathrm{mg} / \mathrm{mL})\end{array}$ & $\begin{array}{c}\text { Difference } \\
\text { from Target } \\
(\%)\end{array}$ \\
\hline \multicolumn{5}{|l|}{ Rats } \\
\hline \multirow[t]{12}{*}{ March 5, 2007} & March 8, 2007 & 15 & $15.1 \pm 0.2$ & +1 \\
\hline & & 15 & $15.0 \pm 0.0$ & 0 \\
\hline & & 30 & $30.2 \pm 0.2$ & +1 \\
\hline & & 30 & $30.6 \pm 0.2$ & +2 \\
\hline & & 60 & $61.9 \pm 0.3$ & +3 \\
\hline & & 60 & $61.6 \pm 0.1$ & +3 \\
\hline & April 12, 2007b & 15 & $15.5 \pm 0.1$ & +3 \\
\hline & & 15 & $15.4 \pm 0.2$ & +3 \\
\hline & & 30 & $30.6 \pm 0.1$ & +2 \\
\hline & & 30 & $30.5 \pm 0.1$ & +2 \\
\hline & & 60 & $62.0 \pm 0.1$ & +3 \\
\hline & & 60 & $60.7 \pm 0.2$ & +1 \\
\hline \multirow[t]{6}{*}{ May 2, 2007} & May 2, 2007 & 15 & $15.5 \pm 0.1$ & +3 \\
\hline & & 15 & $15.4 \pm 0.1$ & +3 \\
\hline & & 30 & $31.3 \pm 0.1$ & +4 \\
\hline & & 30 & $31.4 \pm 0.3$ & +5 \\
\hline & & 60 & $64.3 \pm 0.2$ & +7 \\
\hline & & 60 & $63.1 \pm 0.1$ & +5 \\
\hline \multirow[t]{6}{*}{ July 23, 2007} & July 24, 2007 & 15 & $15.8 \pm 0.1$ & +5 \\
\hline & & 15 & $15.8 \pm 0.1$ & +5 \\
\hline & & 30 & $31.3 \pm 0.2$ & +4 \\
\hline & & 30 & $31.8 \pm 0.1$ & +6 \\
\hline & & 60 & $63.4 \pm 0.4$ & +6 \\
\hline & & 60 & $63.6 \pm 0.1$ & +6 \\
\hline \multirow[t]{8}{*}{ September 17, 2007} & September 19, 2007 & 15 & $15.5 \pm 0.1$ & +3 \\
\hline & & 15 & $15.5 \pm 0.2$ & +3 \\
\hline & & 30 & $31.3 \pm 0.1$ & +4 \\
\hline & & 30 & $30.9 \pm 0.2$ & +3 \\
\hline & & 60 & $63.5 \pm 0.2$ & +6 \\
\hline & & 60 & $63.4 \pm 0.4$ & +6 \\
\hline & October 25, 2007b & 15 & $15.4 \pm 0.1$ & +3 \\
\hline & & 15 & $15.6 \pm 0.1$ & +4 \\
\hline
\end{tabular}


Indole-3-carbinol, NTP TR 584

\begin{tabular}{|c|c|c|c|c|}
\hline Date Prepared & Date Analyzed & $\begin{array}{c}\text { Target } \\
\text { Concentration } \\
(\mathbf{m g} / \mathbf{m L})\end{array}$ & $\begin{array}{c}\text { Determined } \\
\text { Concentration } \\
(\mathbf{m g} / \mathbf{m L})\end{array}$ & $\begin{array}{c}\text { Difference } \\
\text { from Target } \\
(\%)\end{array}$ \\
\hline \multirow{10}{*}{ November 12, 2007} & \multirow{10}{*}{ November 14, 2007} & 30 & $29.9 \pm 0.3$ & 0 \\
\hline & & 30 & $31.2 \pm 0.4$ & +4 \\
\hline & & 60 & $63.5 \pm 0.1$ & +6 \\
\hline & & 60 & $62.2 \pm 0.7$ & +4 \\
\hline & & 15 & $15.4^{\mathrm{c}}$ & +3 \\
\hline & & 15 & $15.6 \pm 0.2$ & +4 \\
\hline & & 30 & $30.9 \pm 0.2$ & +3 \\
\hline & & 30 & $30.9 \pm 0.2$ & +3 \\
\hline & & 60 & $62.9 \pm 0.5$ & +5 \\
\hline & & 60 & $62.3 \pm 0.4$ & +4 \\
\hline \multirow[t]{6}{*}{ January 8, 2008} & \multirow[t]{6}{*}{ January 9, 2008} & 15 & $15.4 \pm 0.1$ & +3 \\
\hline & & 15 & $15.5 \pm 0.0$ & +3 \\
\hline & & 30 & $31.1 \pm 0.1$ & +4 \\
\hline & & 30 & $31.1 \pm 0.2$ & +4 \\
\hline & & 60 & $62.9 \pm 0.1$ & +5 \\
\hline & & 60 & $63.2 \pm 0.4$ & +5 \\
\hline \multirow[t]{12}{*}{ March 31, 2008} & \multirow[t]{6}{*}{ April 2, 2008} & 15 & $15.5 \pm 0.1$ & +3 \\
\hline & & 15 & $15.5 \pm 0.3$ & +3 \\
\hline & & 30 & $31.7 \pm 0.4$ & +6 \\
\hline & & 30 & $31.2 \pm 0.2$ & +4 \\
\hline & & 60 & $63.0 \pm 0.2$ & +5 \\
\hline & & 60 & $63.2 \pm 0.5$ & +5 \\
\hline & \multirow[t]{6}{*}{ May $13,2008^{b}$} & 15 & $14.5 \pm 0.6$ & -3 \\
\hline & & 15 & $15.2 \pm 0.1$ & +1 \\
\hline & & 30 & $30.1 \pm 0.4$ & 0 \\
\hline & & 30 & $30.8 \pm 0.6$ & +3 \\
\hline & & 60 & $62.8 \pm 1.3$ & +5 \\
\hline & & 60 & $62.1 \pm 0.3$ & +4 \\
\hline \multirow[t]{6}{*}{ June 23, 2008} & \multirow[t]{6}{*}{ June 24, 2008} & 15 & $15.3 \pm 0.1$ & +2 \\
\hline & & 15 & $15.6 \pm 0.0$ & +4 \\
\hline & & 30 & $31.1 \pm 0.2$ & +4 \\
\hline & & 30 & $30.7 \pm 0.2$ & +2 \\
\hline & & 60 & $60.5 \pm 0.1$ & +1 \\
\hline & & 60 & $62.1 \pm 0.0$ & +4 \\
\hline
\end{tabular}


Indole-3-carbinol, NTP TR 584

\begin{tabular}{|c|c|c|c|c|}
\hline Date Prepared & Date Analyzed & $\begin{array}{c}\text { Target } \\
\text { Concentration } \\
(\mathrm{mg} / \mathrm{mL})\end{array}$ & $\begin{array}{c}\text { Determined } \\
\text { Concentration }^{\mathrm{a}} \\
(\mathrm{mg} / \mathrm{mL})\end{array}$ & $\begin{array}{c}\text { Difference } \\
\text { from Target } \\
(\%)\end{array}$ \\
\hline \multirow[t]{6}{*}{ August 15, 2008} & August 20, 2008 & 15 & $15.2 \pm 0.1$ & +1 \\
\hline & & 15 & $15.5 \pm 0.1$ & +3 \\
\hline & & 30 & $30.8 \pm 0.3$ & +3 \\
\hline & & 30 & $30.7 \pm 0.0$ & +2 \\
\hline & & 60 & $62.6 \pm 0.3$ & +4 \\
\hline & & 60 & $61.3 \pm 0.6$ & +2 \\
\hline \multirow[t]{12}{*}{ October 13, 2008} & October 14, 2008 & 15 & $15.3 \pm 0.1$ & +2 \\
\hline & & 15 & $15.7 \pm 0.1$ & +5 \\
\hline & & 30 & $31.2 \pm 0.3$ & +4 \\
\hline & & 30 & $31.1 \pm 0.1$ & +4 \\
\hline & & 60 & $61.4 \pm 0.1$ & +2 \\
\hline & & 60 & $63.0 \pm 0.3$ & +5 \\
\hline & November 26, 2008b & 15 & $15.2 \pm 0.1$ & +1 \\
\hline & & 15 & $15.7 \pm 0.2$ & +5 \\
\hline & & 30 & $31.8 \pm 0.7$ & +6 \\
\hline & & 30 & $31.0 \pm 0.3$ & +3 \\
\hline & & 60 & $63.7 \pm 1.2$ & +6 \\
\hline & & 60 & $62.4 \pm 0.7$ & +4 \\
\hline \multirow[t]{6}{*}{ December 8, 2008} & December 9, 2008 & 15 & $15.1 \pm 0.6$ & +1 \\
\hline & & 15 & $15.1 \pm 0.5$ & +1 \\
\hline & & 30 & $30.1 \pm 2.1$ & 0 \\
\hline & & 30 & $30.6 \pm 0.6$ & +2 \\
\hline & & 60 & $60.7 \pm 1.5$ & +1 \\
\hline & & 60 & $61.6 \pm 1.3$ & +3 \\
\hline \multirow[t]{6}{*}{ February 2, 2009} & February 5, 2009 & 15 & $15.2 \pm 0.1$ & +1 \\
\hline & & 15 & $15.4 \pm 0.1$ & +3 \\
\hline & & 30 & $30.9 \pm 0.2$ & +3 \\
\hline & & 30 & $30.6 \pm 0.3$ & +2 \\
\hline & & 60 & $62.5 \pm 0.4$ & +4 \\
\hline & & 60 & $61.6 \pm 0.3$ & +3 \\
\hline
\end{tabular}


Indole-3-carbinol, NTP TR 584

\begin{tabular}{|c|c|c|c|c|}
\hline Date Prepared & Date Analyzed & $\begin{array}{c}\text { Target } \\
\text { Concentration } \\
(\mathrm{mg} / \mathrm{mL})\end{array}$ & $\begin{array}{c}\text { Determined } \\
\text { Concentration }^{\mathrm{a}} \\
(\mathrm{mg} / \mathrm{mL})\end{array}$ & $\begin{array}{c}\text { Difference } \\
\text { from Target } \\
(\%)\end{array}$ \\
\hline \multicolumn{5}{|l|}{ Mice } \\
\hline \multirow[t]{6}{*}{ March 5, 2007} & March 8, 2007 & 6.25 & $6.24 \pm 0.51$ & 0 \\
\hline & & 12.5 & $12.7 \pm 0.1$ & +2 \\
\hline & & 25 & $25.1 \pm 0.2$ & 0 \\
\hline & April 12, 2007b & 6.25 & $6.64 \pm 0.09$ & +6 \\
\hline & & 12.5 & $13.0 \pm 0.0$ & +4 \\
\hline & & 25 & $25.7 \pm 2.9$ & +3 \\
\hline \multirow[t]{3}{*}{ May 2, 2007} & May 2, 2007 & 6.25 & $6.50 \pm 0.06$ & +4 \\
\hline & & 12.5 & $12.9 \pm 0.1$ & +3 \\
\hline & & 25 & $25.7 \pm 0.3$ & +3 \\
\hline \multirow[t]{3}{*}{ July 23, 2007} & July 24, 2007 & 6.25 & $6.27 \pm 0.01$ & 0 \\
\hline & & 12.5 & $13.0 \pm 0.1$ & +4 \\
\hline & & 25 & $26.2 \pm 0.2$ & +5 \\
\hline \multirow[t]{6}{*}{ September 17, 2007} & September 19, 2007 & 6.25 & $6.40 \pm 0.02$ & +2 \\
\hline & & 12.5 & $12.7 \pm 0.1$ & +2 \\
\hline & & 25 & $25.8 \pm 0.1$ & +3 \\
\hline & October $25,2007^{\mathrm{b}}$ & 6.25 & $6.43 \pm 0.01$ & +3 \\
\hline & & 12.5 & $12.3 \pm 0.1$ & -2 \\
\hline & & 25 & $25.2 \pm 1.3$ & +1 \\
\hline \multirow[t]{3}{*}{ November 12, 2007} & November 14, 2007 & 6.25 & $6.30 \pm 0.03$ & +1 \\
\hline & & 12.5 & $12.6 \pm 0.0$ & +1 \\
\hline & & 25 & $25.8 \pm 0.2$ & +3 \\
\hline \multirow[t]{3}{*}{ January 8, 2008} & January 9, 2008 & 6.25 & $6.46 \pm 0.01$ & +3 \\
\hline & & 12.5 & $12.6 \pm 0.2$ & +1 \\
\hline & & 25 & $25.7 \pm 0.1$ & +3 \\
\hline \multirow[t]{6}{*}{ March 31, 2008} & April 2, 2008 & 6.25 & $6.36 \pm 0.10$ & +2 \\
\hline & & 12.5 & $12.6 \pm 0.1$ & +1 \\
\hline & & 25 & $26.1 \pm 0.2$ & +4 \\
\hline & May 13, 2008b & 6.25 & $6.55 \pm 0.04$ & +5 \\
\hline & & 12.5 & $13.0 \pm 0.1$ & +4 \\
\hline & & 25 & $25.7 \pm 0.0$ & +3 \\
\hline \multirow[t]{3}{*}{ June 23, 2008} & June 24, 2008 & 6.25 & $6.50 \pm 0.03$ & +4 \\
\hline & & 12.5 & $12.8 \pm 0.0$ & +2 \\
\hline & & 25 & $26.1 \pm 0.1$ & +4 \\
\hline
\end{tabular}


Indole-3-carbinol, NTP TR 584

\begin{tabular}{llccc}
\hline Date Prepared & Date Analyzed & $\begin{array}{c}\text { Target } \\
\text { Concentration } \\
(\mathbf{m g} / \mathbf{m L})\end{array}$ & $\begin{array}{c}\text { Determined } \\
\text { Concentration } \\
(\mathbf{m g} / \mathbf{m L})\end{array}$ & $\begin{array}{c}\text { Difference } \\
\text { from Target } \\
\mathbf{( \% )}\end{array}$ \\
\hline August 15, 2008 & August 20, 2008 & 6.25 & $6.57 \pm 0.01$ & +5 \\
October 13, 2008 & October 14, 2008 & 25 & $12.7 \pm 0.0$ & +2 \\
& & 6.25 & $26.2 \pm 0.1$ & +5 \\
& & 12.5 & $6.61 \pm 0.05$ & +6 \\
& November 26, 2008 & 25 & $13.1 \pm 0.1$ & +5 \\
& & 6.25 & $26.2 \pm 0.2$ & +5 \\
December 8, 2008 & 12.5 & $6.45 \pm 0.07$ & +3 \\
& December 9, 2008 & 25 & $12.5 \pm 0.2$ & 0 \\
& & 6.25 & $26.3 \pm 0.2$ & +5 \\
February 2, 2009 & February 5, 2009 & 12.5 & $6.20 \pm 0.15$ & -1 \\
& & 25 & $12.1 \pm 0.2$ & -3 \\
& & 6.25 & $26.1 \pm 0.5$ & +4 \\
& & 12.5 & $6.27 \pm 0.03$ & 0 \\
\hline
\end{tabular}

${ }^{\mathrm{a}}$ Results of duplicate analyses. For rats, dosing volume $=5 \mathrm{~mL} / \mathrm{kg} ; 15 \mathrm{mg} / \mathrm{mL}=75 \mathrm{mg} / \mathrm{kg}, 30 \mathrm{mg} / \mathrm{mL}=150 \mathrm{mg} / \mathrm{kg}$, $60 \mathrm{mg} / \mathrm{mL}=300 \mathrm{mg} / \mathrm{kg}$. For mice, dosing volume $=10 \mathrm{~mL} / \mathrm{kg} ; 6.25 \mathrm{mg} / \mathrm{mL}=62.5 \mathrm{mg} / \mathrm{kg}, 12.5 \mathrm{mg} / \mathrm{mL}=125 \mathrm{mg} / \mathrm{kg}$, $25 \mathrm{mg} / \mathrm{mL}=250 \mathrm{mg} / \mathrm{kg}$.

${ }^{\mathrm{b}}$ Animal room samples.

'One value only.

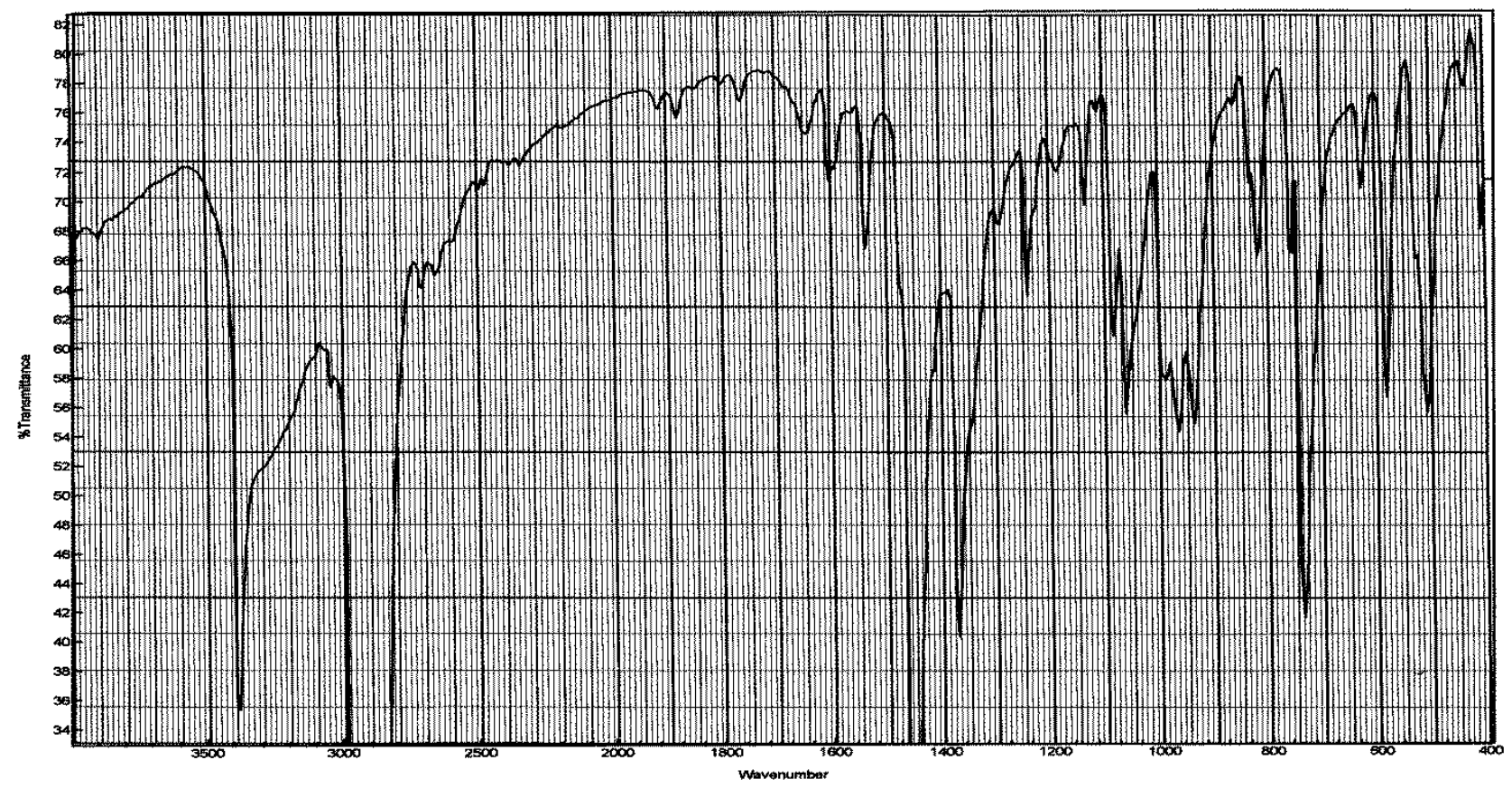

Figure I-1. Infrared Absorption Spectrum of Indole-3-carbinol 
Indole-3-carbinol, NTP TR 584

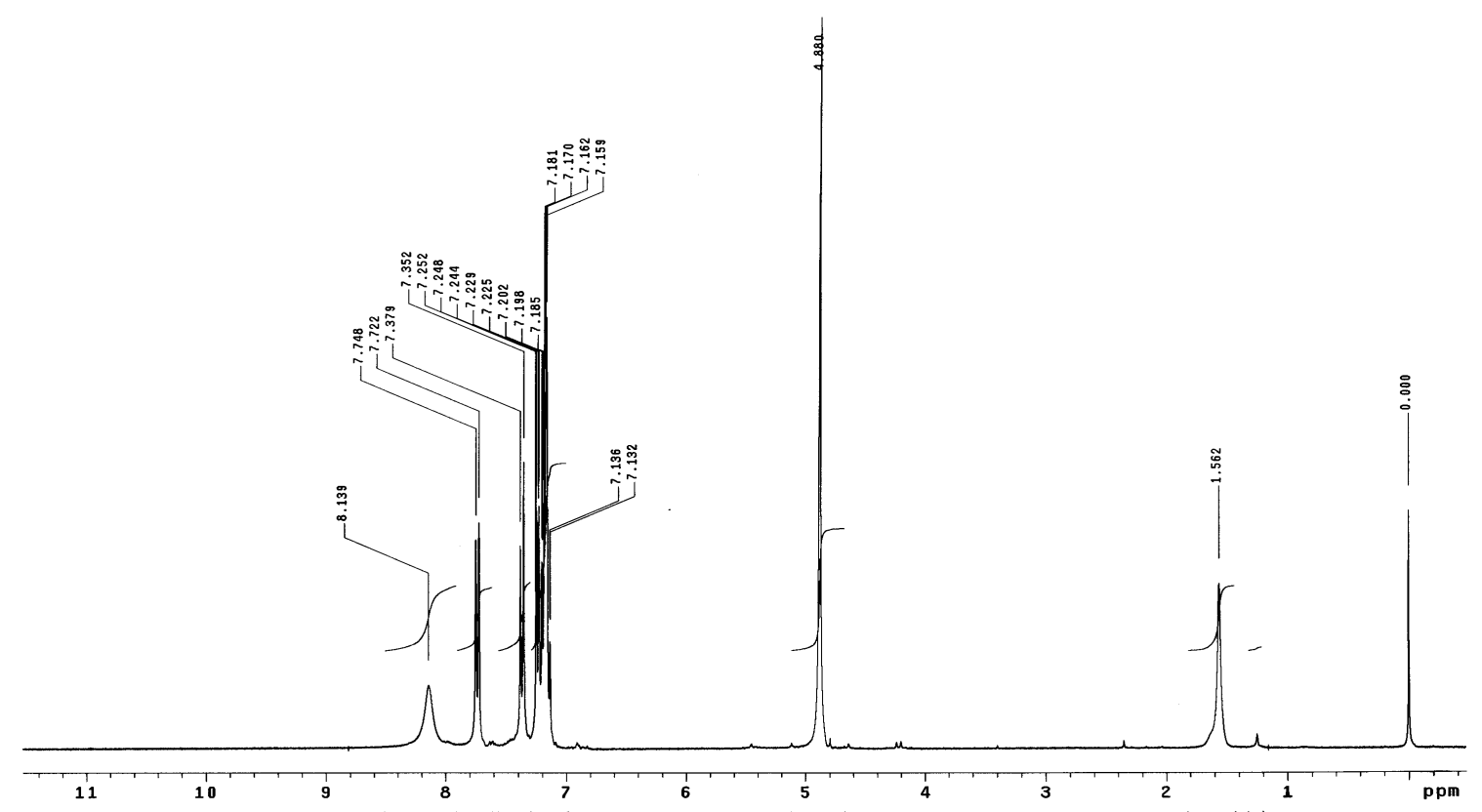

Figure I-2. Proton Nuclear Magnetic Resonance Spectrum of Indole-3-carbinol 


\section{Appendix J. Ingredients, Nutrient Composition, and Contaminant Levels in NTP-2000 Rat and Mouse Ration}

\section{Tables}

Table J-1. Ingredients of NTP-2000 Rat and Mouse Ration $\mathrm{J}-2$

Table J-2. Vitamins and Minerals in NTP-2000 Rat and Mouse Ration ..................................J-3

Table J-3. Nutrient Composition of NTP-2000 Rat and Mouse Ration..................................... J-4

Table J-4. Contaminant Levels in NTP-2000 Rat and Mouse Ration ................................... J-6 
Indole-3-carbinol, NTP TR 584

Table J-1. Ingredients of NTP-2000 Rat and Mouse Ration

Ingredients

Ground hard winter wheat

Ground \#2 yellow shelled corn

Wheat middlings

Oat hulls

Alfalfa meal (dehydrated, $17 \%$ protein)

Purified cellulose

Soybean meal (49\% protein)

Fish meal (60\% protein)

Corn oil (without preservatives)

Soy oil (without preservatives)

Dried brewer's yeast

Calcium carbonate (USP)

Vitamin premix ${ }^{\mathrm{a}}$

Mineral premix ${ }^{\mathrm{b}}$

Calcium phosphate, dibasic (USP)

Sodium chloride

Choline chloride (70\% choline)

Methionine

${ }^{a}$ Wheat middlings as carrier

${ }^{\mathrm{b}}$ Calcium carbonate as carrier.
Percent by Weight

22.26

22.18

15.0

8.5

7.5

5.5

5.0

4.0

3.0

3.0

1.0

0.9

0.5

0.5

0.4

0.3

0.26

0.2 
Indole-3-carbinol, NTP TR 584

Table J-2. Vitamins and Minerals in NTP-2000 Rat and Mouse Ration ${ }^{\mathrm{a}}$

\begin{tabular}{lcl}
\hline & Amount & \multicolumn{1}{c}{ Source } \\
\hline Vitamins & & \\
$\mathrm{A}$ & $4,000 \mathrm{IU}$ & Stabilized vitamin A palmitate or acetate \\
$\mathrm{D}$ & $1,000 \mathrm{IU}$ & D-activated animal sterol \\
$\mathrm{K}$ & $1.0 \mathrm{mg}$ & Menadione sodium bisulfite complex \\
$\alpha$-Tocopheryl acetate & $100 \mathrm{IU}$ & \\
Niacin & $23 \mathrm{mg}$ & - \\
Folic acid & $1.1 \mathrm{mg}$ & \\
$d$-Pantothenic acid & $10 \mathrm{mg}$ & $d$-Calcium pantothenate \\
Riboflavin & $3.3 \mathrm{mg}$ & \\
Thiamine & $4 \mathrm{mg}$ & Thiamine mononitrate \\
B 12 & $52 \mathrm{\mu g}$ & \\
Pyridoxine & $6.3 \mathrm{mg}$ & Pyridoxine hydrochloride \\
Biotin & $0.2 \mathrm{mg}$ & $d$-Biotin \\
\hline Minerals & & \\
Magnesium & $514 \mathrm{mg}$ & Magnesium oxide \\
Iron & $35 \mathrm{mg}$ & Iron sulfate \\
Zinc & $12 \mathrm{mg}$ & Zinc oxide \\
Manganese & $10 \mathrm{mg}$ & Manganese oxide \\
Copper & $2.0 \mathrm{mg}$ & Copper sulfate \\
Iodine & $0.2 \mathrm{mg}$ & Calcium iodate \\
Chromium & $0.2 \mathrm{mg}$ & Chromium acetate \\
\hline
\end{tabular}

aper kg of finished product. 
Indole-3-carbinol, NTP TR 584

Table J-3. Nutrient Composition of NTP-2000 Rat and Mouse Ration

\begin{tabular}{|c|c|c|c|}
\hline Nutrient & Mean \pm Standard Deviation & Range & Number of Samples \\
\hline Protein (\% by weight) & $14.7 \pm 0.71$ & $13.7-15.9$ & 24 \\
\hline Crude Fat (\% by weight) & $8.2 \pm 0.28$ & 7.7-8.8 & 24 \\
\hline Crude Fiber (\% by weight) & $9.1 \pm 0.54$ & $8.1-10.3$ & 24 \\
\hline Ash (\% by weight) & $5.1 \pm 0.25$ & $4.4-5.4$ & 24 \\
\hline \multicolumn{4}{|c|}{ Amino Acids (\% of total diet) } \\
\hline Arginine & $0.786 \pm 0.070$ & $0.670-0.970$ & 23 \\
\hline Cystine & $0.220 \pm 0.024$ & $0.150-0.250$ & 23 \\
\hline Glycine & $0.700 \pm 0.040$ & $0.620-0.800$ & 23 \\
\hline Histidine & $0.351 \pm 0.076$ & $0.270-0.680$ & 23 \\
\hline Isoleucine & $0.546 \pm 0.043$ & $0.430-0.660$ & 23 \\
\hline Leucine & $1.095 \pm 0.066$ & $0.960-1.240$ & 23 \\
\hline Lysine & $0.705 \pm 0.116$ & $0.310-0.860$ & 23 \\
\hline Methionine & $0.409 \pm 0.045$ & $0.260-0.490$ & 23 \\
\hline Phenylalanine & $0.628 \pm 0.039$ & $0.540-0.720$ & 23 \\
\hline Threonine & $0.506 \pm 0.042$ & $0.430-0.610$ & 23 \\
\hline Tryptophan & $0.150 \pm 0.028$ & $0.110-0.200$ & 23 \\
\hline Tyrosine & $0.405 \pm 0.063$ & $0.280-0.540$ & 23 \\
\hline Valine & $0.664 \pm 0.043$ & $0.550-0.730$ & 23 \\
\hline \multicolumn{4}{|c|}{ Essential Fatty Acids (\% of total diet) } \\
\hline Linoleic & $3.95 \pm 0.254$ & $3.49-4.55$ & 23 \\
\hline Linolenic & $0.30 \pm 0.031$ & $0.21-0.35$ & 23 \\
\hline \multicolumn{4}{|l|}{ Vitamins } \\
\hline Vitamin A (IU/kg) & $3,601 \pm 77$ & $2,350-5,720$ & 24 \\
\hline Vitamin D (IU/kg) & $1,000^{\mathrm{a}}$ & - & - \\
\hline$\alpha$-Tocopherol (ppm) & $80.3 \pm 21.56$ & $27.0-124.0$ & 23 \\
\hline Thiamine (ppm) ${ }^{\mathrm{b}}$ & $6.9 \pm 1.13$ & $5.1-9.0$ & 24 \\
\hline Riboflavin (ppm) & $7.7 \pm 2.87$ & $4.20-17.50$ & 23 \\
\hline Niacin (ppm) & $79.2 \pm 8.97$ & $66.4-98.2$ & 23 \\
\hline Pantothenic acid (ppm) & $27.0 \pm 12.35$ & $17.4-81.0$ & 23 \\
\hline Pyridoxine (ppm) ${ }^{\mathrm{b}}$ & $9.54 \pm 1.94$ & $6.44-13.7$ & 23 \\
\hline Folic acid (ppm) & $1.61 \pm 0.47$ & $1.15-3.27$ & 23 \\
\hline Biotin (ppm) & $0.32 \pm 0.10$ & $0.20-0.704$ & 23 \\
\hline Vitamin $B_{12}(\mathrm{ppb})$ & $53.4 \pm 38$ & $18.3-174.0$ & 23 \\
\hline Choline (ppm) ${ }^{\mathrm{b}}$ & $2,773 \pm 590$ & $1,160-3,790$ & 23 \\
\hline
\end{tabular}


Indole-3-carbinol, NTP TR 584

\begin{tabular}{lccc}
\hline \multicolumn{1}{c}{ Nutrient } & Mean \pm Standard Deviation & Range & Number of Samples \\
\hline Minerals & & & \\
Calcium (\%) & $0.920 \pm 0.048$ & $0.808-1.020$ & 24 \\
Phosphorus (\%) & $0.555 \pm 0.066$ & $0.471-0.822$ & 24 \\
Potassium (\%) & $0.667 \pm 0.030$ & $0.626-0.733$ & 23 \\
Chloride (\%) & $0.385 \pm 0.038$ & $0.300-0.474$ & 23 \\
Sodium (\%) & $0.189 \pm 0.016$ & $0.160-0.222$ & 23 \\
Magnesium (\%) & $0.216 \pm 0.061$ & $0.185-0.490$ & 23 \\
Sulfur (\%) & $0.170 \pm 0.029$ & $0.116-0.209$ & 14 \\
Iron (ppm) & $187 \pm 38.6$ & $135-311$ & 23 \\
Manganese (ppm) & $51.0 \pm 10.19$ & $21.0-73.1$ & 23 \\
Zinc (ppm) & $53.6 \pm 8.34$ & $43.3-78.5$ & 23 \\
Copper (ppm) & $7.10 \pm 2.540$ & $3.21-16.3$ & 23 \\
Iodine (ppm) & $0.503 \pm 0.201$ & $0.158-0.972$ & 23 \\
Chromium (ppm) & $0.696 \pm 0.269$ & $0.330-1.380$ & 23 \\
Cobalt (ppm) & $0.248 \pm 0.163$ & $0.094-0.864$ & 21 \\
\hline
\end{tabular}

aFrom formulation.

${ }^{\mathrm{b}}$ As hydrochloride (thiamine and pyridoxine) or chloride (choline). 
Indole-3-carbinol, NTP TR 584

Table J-4. Contaminant Levels in NTP-2000 Rat and Mouse Ration ${ }^{\mathrm{a}}$

\begin{tabular}{|c|c|c|c|}
\hline & $\begin{array}{c}\text { Mean } \pm \text { Standard } \\
\text { Deviation }^{b}\end{array}$ & Range & Number of Samples \\
\hline \multicolumn{4}{|l|}{ Contaminants } \\
\hline Arsenic (ppm) & $0.24 \pm 0.050$ & $0.16-0.40$ & 24 \\
\hline Cadmium (ppm) & $0.06 \pm 0.010$ & $0.05-0.10$ & 24 \\
\hline Lead (ppm) & $0.10 \pm 0.020$ & $0.07-0.16$ & 24 \\
\hline Mercury (ppm) & $<0.02$ & - & 24 \\
\hline Selenium (ppm) & $0.30 \pm 0.255$ & $0.14-1.02$ & 24 \\
\hline Aflatoxins (ppb) & $<5.00$ & - & 24 \\
\hline Nitrate nitrogen $(\mathrm{ppm})^{\mathrm{c}}$ & $17.8 \pm 8.15$ & $10.0-42.3$ & 24 \\
\hline Nitrite nitrogen $(\mathrm{ppm})^{\mathrm{c}}$ & $<0.61$ & - & 24 \\
\hline BHA (ppm) $)^{\mathrm{d}}$ & $<1.0$ & - & 24 \\
\hline BHT (ppm) $)^{\mathrm{d}}$ & $<1.0$ & - & 24 \\
\hline Aerobic plate count (CFU/g) & $10 \pm 0.0$ & $10.0-10.0$ & 24 \\
\hline Coliform (MPN/g) & $3.0 \pm 0.0$ & $3.0-3.0$ & 24 \\
\hline Escherichia coli (MPN/g) & $<10$ & - & 24 \\
\hline Salmonella (MPN/g) & Negative & - & 24 \\
\hline Total nitrosoamines (ppb) ${ }^{\mathrm{e}}$ & $8.8 \pm 6.32$ & $2.0-28.0$ & 24 \\
\hline$N$-Nitrosodimethylamine (ppb) ${ }^{\mathrm{e}}$ & $2.0 \pm 2.27$ & $0.9-10.3$ & 24 \\
\hline$N$-Nitrosopyrrolidine (ppb) ${ }^{\mathrm{e}}$ & $6.8 \pm 5.03$ & $1.0-17.7$ & 24 \\
\hline \multicolumn{4}{|l|}{ Pesticides (ppm) } \\
\hline$\alpha-\mathrm{BHC}$ & $<0.01$ & - & 23 \\
\hline$\beta$-BHC & $<0.02$ & - & 23 \\
\hline$\gamma$-BHC & $<0.01$ & - & 23 \\
\hline$\delta$-BHC & $<0.01$ & - & 23 \\
\hline Heptachlor & $<0.01$ & - & 23 \\
\hline Aldrin & $<0.01$ & - & 23 \\
\hline Heptachlor epoxide & $<0.01$ & - & 23 \\
\hline $\mathrm{DDE}$ & $<0.01$ & - & 23 \\
\hline DDD & $<0.01$ & - & 23 \\
\hline DDT & $<0.01$ & - & 23 \\
\hline HCB & $<0.01$ & - & 23 \\
\hline Mirex & $<0.01$ & - & 23 \\
\hline Methoxychlor & $<0.05$ & - & 23 \\
\hline Dieldrin & $<0.01$ & - & 23 \\
\hline Endrin & $<0.01$ & - & 23 \\
\hline
\end{tabular}


Indole-3-carbinol, NTP TR 584

\begin{tabular}{lccc}
\hline & $\begin{array}{c}\text { Mean } \pm \text { Standard } \\
\text { Deviation }\end{array}$ & Range & Number of Samples \\
\hline Telodrin & $<0.01$ & - & 23 \\
Chlordane & $<0.05$ & - & 23 \\
Toxaphene & $<0.10$ & - & 23 \\
Estimated PCBs & $<0.20$ & - & 23 \\
Ronnel & $<0.01$ & - & 23 \\
Ethion & $<0.02$ & - & 23 \\
Trithion & $<0.05$ & - & 23 \\
Diazinon & $<0.10$ & - & 23 \\
Methyl chlorpyrifos & $0.059 \pm 0.053$ & - & 24 \\
Methyl parathion & $<0.02$ & - & 23 \\
Ethyl parathion & $<0.02$ & $-0.020-0.180$ & 24 \\
Malathion & $0.055 \pm 0.044$ & -0.194 & 23 \\
Endosulfan I & $<0.01$ & - & 23 \\
Endosulfan II & $<0.01$ & - & 23 \\
Endosulfan sulfate & $<0.03$ & - & 23 \\
\hline
\end{tabular}

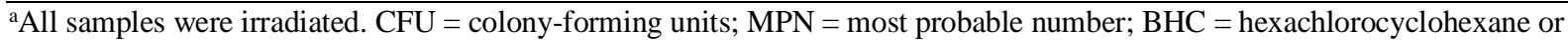
benzene hexachloride.

${ }^{\mathrm{b}}$ For values less than the limit of detection, the detection limit is given as the mean.

'Sources of contamination: alfalfa, grains, and fish meal.

${ }^{\mathrm{d}}$ Sources of contamination: soy oil and fish meal.

eAll values were corrected for percent recovery. 
Indole-3-carbinol, NTP TR 584

\section{Appendix K. Sentinel Animal Program \\ Table of Contents}

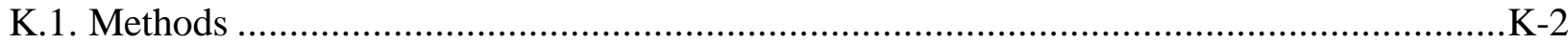

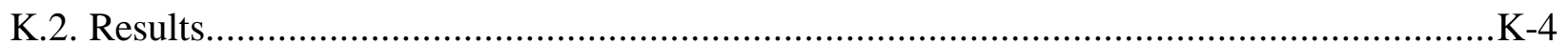

\section{Tables}

Table K-1. Laboratory Methods and Agents Tested for in the Sentinel Animal Program .........K-2 


\section{K.1. Methods}

Rodents used in the National Toxicology Program are produced in optimally clean facilities to eliminate potential pathogens that may affect study results. The Sentinel Animal Program is part of the periodic monitoring of animal health that occurs during the toxicological evaluation of compounds. Under this program, the disease state of the rodents is monitored via sera or feces from extra (sentinel) or dosed animals in the study rooms. The sentinel animals and the study animals are subject to identical environmental conditions. Furthermore, the sentinel animals come from the same production source and weanling groups as the animals used for the studies of test compounds.

Blood samples were collected from each animal and allowed to clot and the serum was separated. Additionally, fecal samples were collected and tested for Helicobacter species. All samples were processed appropriately and evaluated for the presence of pathogens. Samples were sent to BioReliance Corporation (Rockville, MD) or the Research Animal Diagnostic Laboratory (RADIL) at the University of Missouri (Columbia, MO). The laboratory methods and agents for which testing was performed are tabulated below; the times at which samples were collected during the studies are also listed.

Table K-1. Laboratory Methods and Agents Tested for in the Sentinel Animal Program

\begin{tabular}{ll}
\hline \multicolumn{1}{c}{ Method and Test } & \multicolumn{1}{c}{ Time of Collection } \\
\hline Rats & \\
Three-month Study & \\
ELISA & Study termination \\
PVM (pneumonia virus of mice) & Study termination \\
RCV/SDA (rat coronavirus/sialodacryoadenitis virus) & Study termination \\
Sendai & \\
Immunofluorescence Assay & Study termination \\
Parvovirus & \\
Two-year Study & \\
ELISA & End of quarantine, 4 weeks, 6 months \\
PVM & End of quarantine, 4 weeks, 6 months \\
RCV/SDA & End of quarantine, 4 weeks, 6 months \\
Sendai & \\
Immunofluorescence Assay & End of quarantine, 4 weeks, 6 months \\
Parvovirus & 4 weeks, 6 months \\
RCV/SDA & \\
Multiplex Fluorescent Immunoassay & 12 and 18 months, study termination \\
H-1 (Toolan's H-1 virus) & 12 and 18 months, study termination \\
KRV (Kilham rat Virus) & 12 and 18 months, study termination \\
Mycoplasma pulmonis &
\end{tabular}


Indole-3-carbinol, NTP TR 584

\begin{tabular}{ll}
\hline \multicolumn{1}{c}{ Method and Test } & \multicolumn{1}{c}{ Time of Collection } \\
\hline Parvo NS-1 & 12 and 18 months, study termination \\
PVM & 12 and 18 months, study termination \\
RCV/SDA & 12 and 18 months, study termination \\
RMV (rat minute virus) & 12 and 18 months, study termination \\
RPV (rat parvovirus) & 12 and 18 months, study termination \\
RTV (rat theilovirus) & 12 and 18 months, study termination \\
Sendai & 12 and 18 months, study termination \\
TMEV GDVII (Theiler's murine encephalomyelitis virus- -12 and 18 months, study termination \\
mouse poliovirus, strain GDVII)
\end{tabular}

\section{Mice}

Three-month Study

ELISA

Ectromelia virus

Study termination

EDIM (epizootic diarrhea of infant mice)

Study termination

LCM (lymphocytic choriomeningitis virus)

Study termination

Mouse adeno virus-1 (Mad-1)

Study termination

MHV (mouse hepatitis virus)

Study termination

MMV VP2 (mouse minute virus)

Study termination

MPV VP2 (mouse parvovirus)

Study termination

PVM

Study termination

Reovirus

Study termination

Sendai

Study termination

TMEV GDVII

Study termination

Immunofluorescence Assay

LCM

Study termination

Two-year Study

ELISA

Ectromelia virus

End of quarantine, 4 weeks, 6 months

EDIM

LCM

End of quarantine, 4 weeks, 6 months

End of quarantine, 4 weeks, 6 months

Mad-1

End of quarantine, 4 weeks, 6 months

MHV

End of quarantine, 4 weeks, 6 months

MMV VP2

End of quarantine, 4 weeks, 6 months

MPV VP2

End of quarantine, 4 weeks, 6 months

PVM

End of quarantine, 4 weeks, 6 months

Reovirus

End of quarantine, 4 weeks, 6 months 
Indole-3-carbinol, NTP TR 584

\begin{tabular}{ll}
\hline \multicolumn{1}{c}{ Method and Test } & \multicolumn{1}{c}{ Time of Collection } \\
\hline Sendai & End of quarantine, 4 weeks, 6 months \\
TMEV GDVII & End of quarantine, 4 weeks, 6 months \\
Immunofluorescence Assay & \\
MHV & 4 weeks \\
MPV & End of quarantine \\
LCM & 4 weeks \\
Multiplex Fluorescent Immunoassay & \\
Ectromelia virus & 12 and 18 months, study termination \\
EDIM & 12 and 18 months, study termination \\
LCM & 12 and 18 months, study termination \\
MHV & 12 and 18 months, study termination \\
MMV & 12 and 18 months, study termination \\
MNV (mouse norovirus) & 12 and 18 months, study termination \\
MPV & 12 and 18 months, study termination \\
M. pulmonis & 12 and 18 months, study termination \\
Parvo NS-1 & 12 and 18 months, study termination \\
PVM & 12 and 18 months, study termination \\
Reovirus 3 & 12 and 18 months, study termination \\
Sendai & 12 and 18 months, study termination \\
TMEV GDVII & 12 and 18 months, study termination \\
Polymerase Chain Reaction & \\
Helicobacter species & 18 months \\
\hline
\end{tabular}

\section{K.2. Results}

All test results were negative. 


\section{Appendix L. Microarray Analysis}

\section{Table of Contents}

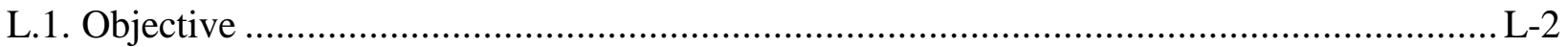

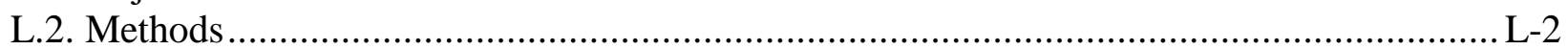

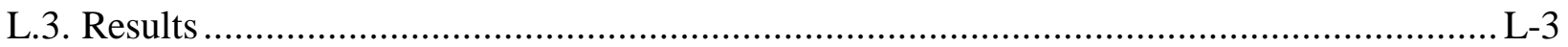

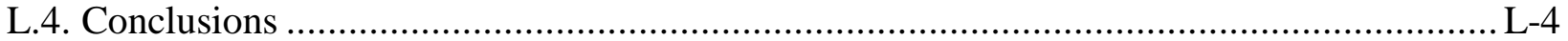

\section{Tables}

Table L-1. Top Five Up- and Down-regulated Genes in the Liver of Female Sprague Dawley Rats Administered Daily Doses of $300 \mathrm{mg} / \mathrm{kg}$ Indole-3-carbinol by Gavage for Three Months. L-6

Table L-2. Enriched Ingenuity Pathway Analysis ${ }^{\mathrm{TM}}$ Disease or Biological Functions that Exhibit Activation/inhibition in the Liver of Female Sprague Dawley Rats Administered Daily Doses of $300 \mathrm{mg} / \mathrm{kg}$ Indole-3-carbinol by Gavage for Three Months.

Table L-3. Enriched Ingenuity Pathway Analysis ${ }^{\mathrm{TM}}$ Toxicity Functions that Exhibit Activation/inhibition in the Liver of Female Sprague Dawley Rats Administered Daily Doses of $300 \mathrm{mg} / \mathrm{kg}$ Indole-3-carbinol by Gavage for Three Months

Table L-4. Ingenuity Pathway Analysis ${ }^{\mathrm{TM}}$ Canonical Pathways Exhibiting Significant $(\mathrm{P}<0.05)$ Enrichment in the Liver of Female Sprague Dawley Rats Administered Daily Doses of $300 \mathrm{mg} / \mathrm{kg}$ Indole-3-carbinol by Gavage for Three Months

Table L-5. Enriched Ingenuity Pathway Analysis ${ }^{\mathrm{TM}}$ Transcription Factors that Exhibit Activation/inhibition in the Liver of Female Sprague Dawley Rats Administered Daily Doses of $300 \mathrm{mg} / \mathrm{kg}$ Indole-3-carbinol by Gavage for Three Months.

Table L-6. Enriched Ingenuity Pathway Analysis ${ }^{\mathrm{TM}}$ Chemical Signaling Patterns that Exhibit Activation/inhibition in the Liver of Female Sprague Dawley Rats Administered Daily Doses of $300 \mathrm{mg} / \mathrm{kg}$ Indole-3-carbinol by Gavage for Three Months 


\section{L.1. Objective}

The objective of the microarray study was to evaluate the transcriptional changes in liver from rats exposed to 0 or $300 \mathrm{mg} / \mathrm{kg}$ indole-3-carbinol. At 3 months, livers were analyzed from female Harlan Sprague Dawley rats in the 2-year gavage study of indole-3-carbinol.

\section{L.2. Methods}

\section{L.2.1. RNA Isolation, cDNA Synthesis, and Array Hybridization}

Liver tissues were excised from five female rats gavaged with corn oil (vehicle control group) and five female rats gavaged with $300 \mathrm{mg} / \mathrm{kg}$ of indole-3-carbinol in corn oil (treated group) Monday through Friday for 3 months and on the termination day approximately 3 hours prior to tissue harvest.

Tissues were immediately frozen in liquid nitrogen at collection and transported to the Battelle Biomedical Research Center (Columbus, $\mathrm{OH}$ ). The liver tissues were removed and added to lysis buffer. Each sample was then homogenized for 45 seconds using OmniTip ${ }^{\mathrm{TM}}$ plastic disposable probes (Omni International, Marietta, GA). Following homogenization, samples were centrifuged and the RNA was extracted from the supernatant using the Qiagen RNeasy Midi Kit (Qiagen, Valencia, CA). RNA concentration and purity were determined by ultraviolet analysis using a NanoDrop 1000 spectrophotometer (NanoDrop Technologies, Wilmington, DE). All samples were evaluated by gel electrophoresis using the FlashGel ${ }^{\circledR}$ RNA cassette system (Lonza, Rockland, ME).

Total RNA (5 $\mu \mathrm{g})$ was used to synthesize double-stranded cDNA for each sample using Affymetrix GeneChip ${ }^{\circledR}$ 3'-Amplification One-Cycle cDNA Synthesis Reagents (Affymetrix, Inc., Santa Clara, CA) according to the manufacturer's protocol. All incubation steps were performed using a GeneAmp ${ }^{\circledR} 2400$ thermal cycler (PerkinElmer, Waltham, MA). The cDNA served as a template to synthesize biotin-labeled antisense cRNA using an IVT Labeling Kit (Affymetrix, Inc.). Labeled cRNA was fragmented and hybridized to the Affymetrix Rat Genome 230 2.0 Array as described in the Affymetrix GeneChip ${ }^{\circledR}$ protocol. Chip hybridization, washing, and staining were performed according to the Affymetrix-recommended protocol EukGE-Ws2v5. After washing, the chips were scanned using an Affymetrix GeneChip Scanner 3000 and the acquired data was then further processed by the Affymetrix GeneChip ${ }^{\circledR}$ Operating Software (GCOS) version 1.2.

\section{L.2.2. Microarray Analysis}

The 10 .CEL files representing the 10 liver samples from the study were normalized using the robust multiarray algorithm (RMA) ${ }^{139}$ using GeneSpring v12.6 (Agilent Technologies, Santa Clara, CA). Normalized expression values were baseline-transformed to the median of all samples. A Principal Component Analysis demonstrated clear separation of the samples by treatment group. All chips had a Gapdh $3^{\prime} / 5^{\prime}$ hybridization rate ranging from 1.1 to 1.6. Probesets were filtered to 26,253 (from 31,099) based on signal intensity values, specifically a probeset was retained if its expression values were between 20.0 and 100 percent in 1 out of 10 samples. Identification of probe sets that exhibited a statistically significant difference in intensity was determined by t-test (unpaired) with a P value of $<0.05$ and the Benjamini-Hochburg post-hoc 
test. Probesets that were significantly altered were further filtered using a minimum 1.5-fold difference.

\section{L.2.3. Ingenuity Pathway Analysis ${ }^{\mathrm{TM}}$}

The 343 probesets exhibiting significant $(\mathrm{P}<0.05)$ differential expression and $>1.5$-fold change (up- or down-regulation) were analyzed using the Ingenuity Pathway Analysis ${ }^{\mathrm{TM}}$ (IPA) (Ingenuity Systems $^{\circledR}$, Inc., Redwood City, CA; <www.ingenuity.com $>$ ). The probeset list when translated into genes in IPA corresponded to a total of 243 altered genes (referred to as differentially expressed genes (DEGs). The IPA Core Analysis was performed on February 12, 2014. Enrichment and activation analysis was carried out for IPA Disease or Biological Functions, Toxicity Functions, and Upstream Analysis.

Enrichment $\mathrm{P}$ values were calculated using a right-tailed Fisher's Exact Test combined with a Benjamini-Hochberg method of multiple testing correction. The $\mathrm{P}$ value $(\mathrm{P}<0.05)$ was determined by how many DEGs overlapped genes annotated into gene groups (e.g., canonical pathways, biological processes). Activation/inhibition analysis was performed using the annotation in the Ingenuity ${ }^{\circledR}$ Knowledge Base. In order to determine if there was plausible activation/inhibition of Disease or Biological Functions, Toxicity Functions, Transcription Factors, and Chemical Signaling Signatures, a Z-score was calculated. The Z-score was determined by concordance of observed patterns of regulation (up or down) with known effects on biological functions or effects of transcription factors/chemicals (activation or inhibition of target genes) as annotated in the Ingenuity ${ }^{\circledR}$ Knowledge Base. Based on the Z-score, a potential transcription factor was deemed to be activated (Z-score $>2$ ), inhibited (Z-score $<-2$ ), or not affected.

\section{L.3. Results}

\section{L.3.1. Differential Gene Expression}

Whole-rat genome Affymetrix 2302.0 microarrays were used to assess the effect of $300 \mathrm{mg} / \mathrm{kg}$ per day of indole-3-carbinol for 3 months on female Sprague Dawley rat liver. Significant differential expression between treated and vehicle control animals occurred in 973 probesets (mapped to 751 Entrez Gene IDs). Of 973 altered probesets, 553 were up-regulated and 420 were down-regulated. When the 973 probesets were subjected to a $>1.5$ cutoff, then 343 probesets (corresponding to 243 genes) were altered. The genes represented by the 343 probesets were used for analysis in IPA. The top five up- and down-regulated genes are shown in Table L-1.

The IPA Disease or Biological Functions and liver Toxicity Functions that were activated by indole-3-carbinol treatment primarily relate to phase I and phase 2 xenobiotic/endogenous chemical metabolism and redox pathways. The enriched/activated functions are listed in Table L-2 and Table L-3.

Consistent with the Disease or Biological Functions enrichment results, indole-3-carbinol treatment caused notable differential expression of genes involved in phase I and phase II xenobiotic/endogenous chemical metabolism along with the signaling pathways that mediate the changes in expression of these genes (e.g., Nrf2-mediated Oxidative Stress Response, Aryl Hydrocarbon Receptor Signaling, and Pxr/Rxr Activation). The enriched pathways are listed in Table L-4. 
Transcription factor enrichment/activation in IPA suggests a strong activation of Nrf2 (Nfe2l2) signaling along with notable activation of a variety of hepatic chemical response transcription factors Car (Nrli3), Pxr (Nrli2) and AhR/Arnt ${ }^{140}$. Results of the transcription factor upstream analysis are shown in Table L-5.

Chemical response pattern activation analysis in IPA is consistent with other results above. Chemicals known to activate Nrf2 (e.g., 1,2-dithiol-3-thione, tert-butyl-hydroquinone, oltipraz, and butylated hydroxyanisole) ${ }^{141-144}$, AhR/Arnt (e.g., tetrachlorodibenzodioxin, benzo(a)pyrene, 2,3,4,7,8-pentachlorodibenzofuran, and 3-methylcholanthrene) ${ }^{128}$ and Car/Pxr signaling (e.g., phenobarbital) ${ }^{145}$ exhibit similar patterns of gene expression compared to indole-3-carbinol. Results of the chemical response pattern activation analysis are shown in Table L-6.

\section{L.4. Conclusions}

The results strongly suggest that treatment with indole-3-carbinol strongly activates AhR and Nrf2 (Nfe2l2) signaling, but also appears to activate Car (Nrli3)/Pxr (Nrli2) signaling. These finding are most clearly demonstrated in the transcription factor enrichment analysis where Nfe2l2 has the strongest activation signal followed by Car/Pxr and then AhR/Arnt. Nfe2l2 coordinates the cellular defense against the cytotoxic effects of oxidative stress ${ }^{146}$. The finding that Nfe2l2 is activated is supported by the activation of glutathione conjugation and enrichment of the oxidative stress signaling and glutathione-mediated detoxification. Additionally, the gene expression pattern elicited by indole-3-carbinol treatment parallels strongly the gene expression patterns of 1,2-dithiol-3-thione, a soft electrophile known to activate Nfe2l2 signaling through effects on the redox status of cellular sulfhydryl groups. Activation of AhR/Arnt along with Car/Pxr is supported by the activation/enrichment of a number of biological functions/pathways associated with xenobiotic and endogenous chemicals and enrichment, a result consistent with hepatocyte hypertrophy/liver enlargement. Patterns of gene expression produced by 2,3,7,8tetrachlorodibenzo-p-dioxin (TCDD), benzo(a)pyrene, and 3-methylcholanthrene (prototype AhR activators) that have been curated in IPA strongly parallel patterns of gene expression produced by indole-3-carbinol. The patterns of AhR gene expression are supported by the high level of induction of Cyp1a1 and Cyp1b1. The finding that AhR signaling is activated by indole3-carbinol treatment is consistent with previous findings that acid condensates of indole-3carbinol formed in the gut are high affinity ligands for the $\mathrm{AhR}^{23}$. From the standpoint of chemical signatures, Car/Pxr activation by indole-3-carbinol is supported by the positive correlation patterns of gene expression from phenobarbital curated in IPA and the strong induction of Cyp2b1/2b2 (a documented target of Car/Pxr) by indole-3-carbinol.

Other notable findings:

1. Down-regulation of Serpina7 (gene that encodes the major thyroid hormone transport protein, TBG, in serum) may have effects on systemic thyroid hormone signaling ${ }^{147}$.

2. Activation of Atf4 is likely related to endoplasmic reticulum stress that may be due to increased protein synthesis associated with xenobiotic metabolizing enzyme induction in combination with soft electrophile stress produced by indole-3-carbinol that can cause proteins to unfold ${ }^{148}$. 
Indole-3-carbinol, NTP TR 584

3. Activation of Tp63 along with enrichment of p53 signaling and Gadd45 signaling is potentially a secondary effect of the soft electrophile-induced oxidative stress and subsequent change in redox status ${ }^{149}$ that can be elicited by indole-3-carbinol ${ }^{150}$. 
Indole-3-carbinol, NTP TR 584

Table L-1. Top Five Up- and Down-regulated Genes in the Liver of Female Sprague Dawley Rats Administered Daily Doses of $300 \mathrm{mg} / \mathrm{kg}$ Indole-3-carbinol by Gavage for Three Months

\begin{tabular}{lccc}
\hline Probeset ID & Gene Symbol & Entrez Gene & $\begin{array}{c}\text { Fold Change } \\
\text { Indole-3-carbinol } \\
\text { Treated versus } \\
\text { Vehicle Control }\end{array}$ \\
\hline $\begin{array}{l}\text { Up-regulated } \\
\text { 1370269_at }\end{array}$ & Cyp1a1 & 24296 & 334.6 \\
1368990_at & Cyp1b1 & 25426 & 75.1 \\
1371076_at & Cyp2b1///Cyp2b2///LOC100362704 & $100362704 / / / 24300 / / / 361523$ & 32.4 \\
1374056_at & Rbm24 & 690139 & 29.6 \\
1373188_at & Scn4b & 315611 & 17.1 \\
Down-regulated & & & -4.3 \\
1369657_at & Cpa1 & 24269 & -4.4 \\
1370387_at & Cyp3a9 & 171352 & -4.9 \\
1371143_at & Serpina7 & 81806 & -5.5 \\
1387053_at & Fmo1 & 25256 & -16.0 \\
1367896_at & Car3 & 54232 & \\
\hline
\end{tabular}


Indole-3-carbinol, NTP TR 584

Table L-2. Enriched Ingenuity Pathway Analysis ${ }^{\mathrm{TM}}$ Disease or Biological Functions that Exhibit Activation/inhibition in the Liver of Female Sprague Dawley Rats Administered Daily Doses of $300 \mathrm{mg} / \mathrm{kg}$ Indole-3-carbinol by Gavage for Three Months

\begin{tabular}{lcccc}
\hline Disease or Function Annotation & P Value & $\begin{array}{c}\text { Predicted Activation } \\
\text { State }\end{array}$ & $\begin{array}{c}\text { Activation } \\
\text { Z-score }\end{array}$ & $\begin{array}{c}\text { Number of } \\
\text { Molecules }\end{array}$ \\
\hline Metabolism of terpenoid & $1.03 \mathrm{E}-08$ & Increased & 2.726 & 19 \\
Conjugation of glutathione & $1.08 \mathrm{E}-09$ & Increased & 2.605 & 7 \\
Steroidogenesis of hormone & $3.00 \mathrm{E}-03$ & Increased & 2.425 & 6 \\
Metabolism of hormone & $2.77 \mathrm{E}-03$ & Increased & 2.404 & 8 \\
Steroid metabolism & $3.98 \mathrm{E}-09$ & Increased & 2.372 & 18 \\
Joining of glutathione & $2.99 \mathrm{E}-11$ & Increased & 2.320 & 8 \\
Metabolism of retinoid & $4.32 \mathrm{E}-05$ & Increased & 2.204 & 6 \\
Hydroxylation of hormone & $1.42 \mathrm{E}-07$ & Increased & 2.189 & 5 \\
Binding of DNA & $1.27 \mathrm{E}-06$ & Increased & 2.188 & 23 \\
Oxidation of lipid & $2.14 \mathrm{E}-04$ & Increased & 2.063 & 10 \\
Synthesis of fatty acid & $2.04 \mathrm{E}-03$ & Decreased & -2.138 & 11 \\
Quantity of vitamin & $3.14 \mathrm{E}-06$ & Decreased & -2.183 & 8 \\
Quantity of GPT in blood & $4.54 \mathrm{E}-04$ & Decreased & -2.200 & 5 \\
Tumorigenesis of epithelial tumor & $2.93 \mathrm{E}-04$ & Decreased & -2.348 & 11 \\
Organismal death & $6.47 \mathrm{E}-04$ & Decreased & -2.371 & 54 \\
Quantity of enzyme & $1.41 \mathrm{E}-03$ & Decreased & -2.420 & 6 \\
Inflammation of organ & $4.83 \mathrm{E}-03$ & Decreased & -2.679 & 28 \\
Hyperplasia & $8.43 \mathrm{E}-03$ & Decreased & -2.928 & 15 \\
\hline & & & & \\
\hline
\end{tabular}

Table L-3. Enriched Ingenuity Pathway Analysis ${ }^{\mathrm{TM}}$ Toxicity Functions that Exhibit Activation/inhibition in the Liver of Female Sprague Dawley Rats Administered Daily Doses of $300 \mathrm{mg} / \mathrm{kg}$ Indole-3-carbinol by Gavage for Three Months

\begin{tabular}{ccccc}
\hline $\begin{array}{c}\text { Disease or Function } \\
\text { Annotation }\end{array}$ & P Value & $\begin{array}{c}\text { Predicted Activation } \\
\text { State }\end{array}$ & $\begin{array}{c}\text { Activation } \\
\text { Z-score }\end{array}$ & $\begin{array}{c}\text { Number of } \\
\text { Molecules }\end{array}$ \\
\hline Conjugation of glutathione & $1.08 \mathrm{E}-09$ & Increased & 2.605 & 7 \\
\hline
\end{tabular}


Table L-4. Ingenuity Pathway Analysis ${ }^{\mathrm{TM}}$ Canonical Pathways Exhibiting Significant $(\mathbf{P}<\mathbf{0 . 0 5})$ Enrichment in the Liver of Female Sprague Dawley Rats Administered Daily Doses of $300 \mathrm{mg} / \mathrm{kg}$ Indole-3-carbinol by Gavage for Three Months

\begin{tabular}{|c|c|}
\hline Ingenuity Canonical Pathways & $-\log (P$ Value $)$ \\
\hline Xenobiotic metabolism signaling & 16.1 \\
\hline Nrf2-mediated oxidative stress response & 12.6 \\
\hline Aryl hydrocarbon receptor signaling & 12.3 \\
\hline Glutathione-mediated detoxification & 10.8 \\
\hline LPS/IL-1 mediated inhibition of RXR function & 10 \\
\hline Nicotine degradation II & 8.75 \\
\hline Nicotine degradation III & 8.15 \\
\hline Melatonin degradation I & 7.93 \\
\hline Superpathway of melatonin degradation & 7.58 \\
\hline Pxr/Rxr activation & 7.15 \\
\hline Bupropion degradation & 6.39 \\
\hline Acetone degradation I (to methylglyoxal) & 6.28 \\
\hline Estrogen biosynthesis & 5.36 \\
\hline Asparagine degradation I & 3.88 \\
\hline p53 signaling & 3.86 \\
\hline Glutathione redox reactions II & 3.41 \\
\hline Serine biosynthesis & 3.11 \\
\hline Gadd45 signaling & 2.83 \\
\hline Cell cycle: G2/M DNA damage checkpoint regulation & 2.79 \\
\hline Superpathway of serine and glycine biosynthesis I & 2.72 \\
\hline Atm signaling & 2.3 \\
\hline Serotonin degradation & 2.27 \\
\hline Hepatic cholestasis & 2.26 \\
\hline Thyroid hormone metabolism II (via conjugation and/or degradation) & 2.24 \\
\hline Antigen presentation pathway & 2.06 \\
\hline Vitamin-C transport & 1.96 \\
\hline Asparagine biosynthesis I & 1.94 \\
\hline 4-Hydroxybenzoate biosynthesis & 1.94 \\
\hline 4-Hydroxyphenylpyruvate biosynthesis & 1.94 \\
\hline Methylglyoxal degradation III & 1.9 \\
\hline Prostate cancer signaling & 1.81 \\
\hline Glutathione redox reactions I & 1.8 \\
\hline Fxr/Rxr activation & 1.76 \\
\hline Bladder cancer signaling & 1.71 \\
\hline
\end{tabular}


Indole-3-carbinol, NTP TR 584

\begin{tabular}{lc}
\hline \multicolumn{1}{c}{ Ingenuity Canonical Pathways } & $-\log (\mathbf{P}$ Value $)$ \\
\hline Glutamine biosynthesis I & 1.64 \\
Glutamine degradation I & 1.64 \\
Ppar signaling & 1.63 \\
Antioxidant action of vitamin C & 1.59 \\
Protein ubiquitination pathway & 1.55 \\
Phospholipases & 1.54 \\
Coenzyme A biosynthesis & 1.47 \\
Ascorbate recycling (cytosolic) & 1.47 \\
Hypoxia signaling in the cardiovascular system & 1.39 \\
Hereditary breast cancer signaling & 1.35 \\
1,25-Dihydroxyvitamin D3 biosynthesis & 1.34 \\
\hline
\end{tabular}

Table L-5. Enriched Ingenuity Pathway Analysis'M Transcription Factors that Exhibit Activation/inhibition in the Liver of Female Sprague Dawley Rats Administered Daily Doses of $300 \mathrm{mg} / \mathrm{kg}$ Indole-3-carbinol by Gavage for Three Months

\begin{tabular}{|c|c|c|c|c|c|}
\hline $\begin{array}{l}\text { Upstream } \\
\text { Regulator }\end{array}$ & Fold Change $^{a}$ & Molecule Type & $\begin{array}{c}\text { Predicted } \\
\text { Activation } \\
\text { State }\end{array}$ & $\begin{array}{c}\text { Activation } \\
\text { Z-score }\end{array}$ & $\begin{array}{l}P \text { Value of } \\
\text { Overlap }\end{array}$ \\
\hline Nfe212 & 2.075 & Transcription regulator & Activated & 4.399 & $2.31 \mathrm{E}-23$ \\
\hline Nrli3 & 2.492 & Ligand-dependent nuclear receptor & Activated & 4.155 & $2.60 \mathrm{E}-20$ \\
\hline Atf4 & 1.684 & Transcription regulator & Activated & 3.085 & $3.20 \mathrm{E}-07$ \\
\hline Tp63 & - & Transcription regulator & Activated & 2.743 & $2.46 \mathrm{E}-04$ \\
\hline Nr1i2 & - & Ligand-dependent nuclear receptor & Activated & 2.366 & $5.49 \mathrm{E}-16$ \\
\hline Brca1 & - & Transcription regulator & Activated & 2.359 & $1.35 \mathrm{E}-07$ \\
\hline Nupr1 & - & Transcription regulator & Activated & 2.309 & $5.10 \mathrm{E}-03$ \\
\hline Xbp1 & - & Transcription regulator & Activated & 2.207 & $1.70 \mathrm{E}-02$ \\
\hline Arnt & - & Transcription regulator & Activated & 2.165 & $8.36 \mathrm{E}-10$ \\
\hline AhR & 2.026 & Ligand-dependent nuclear receptor & Activated & 2.062 & $1.43 \mathrm{E}-11$ \\
\hline Trim24 & - & Transcription regulator & Activated & 2.000 & $1.75 \mathrm{E}-02$ \\
\hline
\end{tabular}


Indole-3-carbinol, NTP TR 584

Table L-6. Enriched Ingenuity Pathway Analysis ${ }^{\mathrm{TM}}$ Chemical Signaling Patterns that Exhibit Activation/inhibition in the Liver of Female Sprague Dawley Rats Administered Daily Doses of $300 \mathrm{mg} / \mathrm{kg}$ Indole-3-carbinol by Gavage for Three Months

\begin{tabular}{|c|c|c|c|c|}
\hline Upstream Regulator & Molecule Type & $\begin{array}{c}\text { Predicted } \\
\text { Activation } \\
\text { State }\end{array}$ & $\begin{array}{c}\text { Activation } \\
\text { Z-score }\end{array}$ & $\begin{array}{l}\text { P Value of } \\
\text { Overlap }\end{array}$ \\
\hline 1,2-Dithiol-3-thione & Chemical reagent & Activated & 4.182 & $1.12 \mathrm{E}-19$ \\
\hline Tetrachlorodibenzodioxin & Chemical toxicant & Activated & 3.728 & $3.66 \mathrm{E}-14$ \\
\hline Benzo(a)pyrene & Chemical toxicant & Activated & 3.722 & $5.51 \mathrm{E}-16$ \\
\hline Phenobarbital & Chemical drug & Activated & 3.640 & $7.04 \mathrm{E}-18$ \\
\hline 3,4,5,3',4'-Pentachlorobiphenyl & Chemical toxicant & Activated & 3.356 & $3.86 \mathrm{E}-15$ \\
\hline 2,3,4,7,8-Pentachlorodibenzofuran & Chemical toxicant & Activated & 3.080 & $4.15 \mathrm{E}-15$ \\
\hline 1,4-Bis[2-(3,5-dichloropyridyloxy)]benzene & Chemical toxicant & Activated & 3.064 & $2.41 \mathrm{E}-12$ \\
\hline Pirinixic acid & Chemical toxicant & Activated & 3.056 & $5.26 \mathrm{E}-13$ \\
\hline 3-Methylcholanthrene & Chemical toxicant & Activated & 3.009 & $9.62 \mathrm{E}-16$ \\
\hline Tert-butyl-hydroquinone & Chemical reagent & Activated & 2.908 & $3.16 \mathrm{E}-10$ \\
\hline Tunicamycin & $\begin{array}{l}\text { Chemical—endogenous non- } \\
\text { mammalian }\end{array}$ & Activated & 2.782 & $1.35 \mathrm{E}-07$ \\
\hline Thapsigargin & Chemical toxicant & Activated & 2.781 & $4.06 \mathrm{E}-06$ \\
\hline Ethoxyquin & Chemical toxicant & Activated & 2.779 & $2.34 \mathrm{E}-12$ \\
\hline Beta-naphthoflavone & Chemical toxicant & Activated & 2.749 & $8.28 \mathrm{E}-14$ \\
\hline Troglitazone & Chemical drug & Activated & 2.734 & $5.67 \mathrm{E}-04$ \\
\hline Bardoxolone & Chemical drug & Activated & 2.621 & $1.36 \mathrm{E}-08$ \\
\hline Tosedostat & Chemical drug & Activated & 2.619 & $1.76 \mathrm{E}-08$ \\
\hline Pxr ligand-Pxr-Retinoic Acid-Rxr $\alpha$ & Complex & Activated & 2.593 & $1.76 \mathrm{E}-07$ \\
\hline Oltipraz & Chemical drug & Activated & 2.576 & 4.33E-09 \\
\hline Arsenic trioxide & Chemical drug & Activated & 2.504 & $9.92 \mathrm{E}-07$ \\
\hline Diallyl disulfide & $\begin{array}{l}\text { Chemical_endogenous non- } \\
\text { mammalian }\end{array}$ & Activated & 2.425 & $3.15 \mathrm{E}-09$ \\
\hline 8-Bromo-camp & Chemical reagent & Activated & 2.407 & $7.91 \mathrm{E}-03$ \\
\hline Allyl sulfide & Chemical drug & Activated & 2.388 & $7.79 \mathrm{E}-09$ \\
\hline Cholic acid & $\begin{array}{l}\text { Chemical—endogenous } \\
\text { mammalian }\end{array}$ & Activated & 2.359 & $1.28 \mathrm{E}-04$ \\
\hline Bilirubin & $\begin{array}{l}\text { Chemical_endogenous } \\
\text { mammalian }\end{array}$ & Activated & 2.236 & $8.16 \mathrm{E}-07$ \\
\hline Sulforafan & Chemical drug & Activated & 2.212 & $2.12 \mathrm{E}-14$ \\
\hline Pregnenolone carbonitrile & Chemical drug & Activated & 2.193 & $2.23 \mathrm{E}-06$ \\
\hline Polycyclic aromatic hydrocarbons & Chemical toxicant & Activated & 2.188 & $7.93 \mathrm{E}-08$ \\
\hline Benz[a]anthracene & Chemical toxicant & Activated & 2.156 & $6.31 \mathrm{E}-10$ \\
\hline Cigarette smoke & Chemical toxicant & Activated & 2.154 & $7.73 \mathrm{E}-13$ \\
\hline 15-Deoxy-delta-12,14 -PGJ 2 & $\begin{array}{l}\text { Chemical—endogenous non- } \\
\text { mammalian }\end{array}$ & Activated & 2.136 & $5.22 \mathrm{E}-04$ \\
\hline Butylated hydroxyanisole & Chemical toxicant & Activated & 2.050 & $1.96 \mathrm{E}-10$ \\
\hline Cephaloridine & Chemical drug & Activated & 2.025 & $1.04 \mathrm{E}-07$ \\
\hline Capsaicin & Chemical drug & Activated & 2.000 & $1.95 \mathrm{E}-03$ \\
\hline
\end{tabular}


Indole-3-carbinol, NTP TR 584

\section{Appendix M. Summary of Peer Review Panel Comments}

On May 22, 2014, the draft Technical Report on the toxicology and carcinogenesis studies of indole-3-carbinol received public review by the National Toxicology Program’s Technical Reports Peer Review Panel. The review meeting was held at the National Institute of Environmental Health Sciences, Research Triangle Park, NC.

Dr. M.E. Wyde, NIEHS, introduced the toxicology and carcinogenesis studies of indole-3carbinol, a commercially available dietary supplement and natural product by discussing the uses of the chemical and the rationale for study, describing the experimental design, reporting on survival and body weight effects, and commenting on compound-related neoplastic and nonneoplastic lesions in rats and mice. The proposed conclusions for the 2-year studies were no evidence of carcinogenic activity in male Sprague Dawley rats, some evidence of carcinogenic activity in female Sprague Dawley rats, clear evidence of carcinogenic activity in male B6C3F1/N mice, and no evidence of carcinogenic activity in female B6C3F1/N mice.

Dr. Carpenter noted that there were no written or oral public comments concerning this report.

Dr. Perdew, the first primary reviewer, noted the study was well done. He questioned if high concentrations of indole-3-carbinol were insoluble in corn oil, and if the insolubility might affect the results. He asked whether any inflammatory markers were examined in tissues or serum. Dr. Wyde said all doses used in the studies were suspensions, and no inflammatory markers were looked at in tissue or serum. Dr. Perdew asked if it was standard practice to not look at inflammatory markers, and Dr. Wyde replied it was. Dr. Perdew questioned a statement in the report regarding comparative affinity for the aryl hydrocarbon receptor (AhR). Dr. Wyde said he could adjust the language accordingly. Dr. Perdew recommended more careful wording for the discussion concerning the mechanism of toxicity for indole-3-carbinol and 2,3,7,8tetrachlorodibenzo-p-dioxin. Dr. Wyde said National Toxicology Program staff had discussed how much to write about the AhR and dioxins, versus just the mechanism associated with indole3-carbinol.

Dr. Regan, the second primary reviewer, said the standard and extended uterine examinations should be presented together, as that is the basis of the carcinogenicity conclusion. She suggested less emphasis on the findings from the standard and extended examinations alone in the report, focusing instead on positive trends and statistical significance seen when the two are combined. She noted there was no observable increase in atypical endometrial hyperplasia in the uterine neoplasms. She suggested clarifying the estrous cycle data in cycling F344/N rats. Regarding nose inflammation, she said the animals with foreign material present in the nasal cavity should be shown separately in the incidence table, as inflammation could be attributed to the foreign material. She asked if assays for cytochrome P450 (CYP) induction were conducted on the nose.

Dr. Wyde, responding to Dr. Regan's comments, said the carcinogenicity call in female rats was based on the combined data, both the standard and extended examinations of the uterus. Dr.

Regan recommended limiting discussion in the text to the combined incidences. Dr. J.R. Bucher, NIEHS, noted this was presented based on a historical precedent, with substantial historical control information available. Because there was no substantial historical database in this study, he understood Dr. Regan's suggestion. Dr. Wyde said he could clarify the estrous cycle characterization data for female F344/N rats. He stated that NTP staff had not conducted assays 
for CYP induction in the nose. Dr. R.A. Herbert, the NIEHS study pathologist, said foreign bodies are often diagnosed in the nose and often associated with inflammation. NTP diagnoses the foreign body, not the inflammatory change, because the inflammatory change is considered secondary to the foreign body. He described how this issue was approached in the indole-3carbinol studies. He said in female mice there was only one diagnosis of foreign body in the mid dose group with no associated evidence of inflammation and two diagnoses in the high dose group, both of which were in animals that had a treatment-related diagnosis of inflammation. Dr. Regan suggested adding more information in the report reflective of Dr. Herbert's statement.

Dr. Mirsalis, the third primary reviewer, said lower doses may not impact the overall conclusions, but it would be good to see if there was a threshold by using lower doses. He said the increases in liver weights were equally striking in rats and mice and recommended bringing the table for mice into the main body of the text from the appendix. The report has an extended discussion about how indole-3-carbinol is an AhR agonist, and compares the responses to those seen with dioxin. The increases in mouse liver tumors could be explained by induction of CYPs resulting in increased liver size, which has been shown to be a significant risk factor for mouse liver tumors; therefore, he recommended removing the dioxin discussion.

Dr. Perdew asked Dr. Mirsalis to clarify his point, because the CYP induction is through the AhR in the liver. Dr. Mirsalis said the response in the mice is different than that seen with dioxin, and noted that in the report, after extensive discussion about dioxin, the conclusion was that the response was not similar to dioxin. Dr. Bucher noted the nomination of this substance was related to the question about how there could be an AhR agonist that was unlike dioxin, and perhaps there was overemphasis in the discussion about dioxin. Dr. Wyde said it would be remiss not to mention the dioxin comparison at all. Dr. Fanucchi recommended adding language to the discussion related to Dr. Bucher's comments. Dr. Wyde agreed with Dr. Mirsalis that the liver weight data in mice could be brought forward in the report.

Dr. Mirsalis moved to accept the proposed conclusions as written. Dr. Perdew seconded the motion. Dr. Regan recommended changing the conclusion in the female rats from some evidence to equivocal evidence because there was no dose-related increase in malignant uterine neoplasms at the high dose or positive trend test, and because no preneoplastic changes were noted in the uterus. Dr. N.J.Walker, NIEHS, explained that in the level of evidence criteria, some evidence is used when there are treatment-related increases; in this case, there was a pairwise significance against the concurrent vehicle control. Dr. Conner disagreed with mentioning an increase that did not reach statistical significance; thus, he agreed that the call should be equivocal evidence rather than some evidence. Dr. Mirsalis said with the multiple adenocarcinomas involved, the some evidence call was appropriate. Dr. G.E. Kissling, NIEHS, stated statistical significance is only one piece of evidence used to make calls, and that there are cases where a biological increase is seen without reaching statistical significance. Dr. D.E. Malarkey, NIEHS, said it could be difficult to interpret preneoplastic lesions. Dr. Regan said even if the preneoplastic lesions were added in this case, it would not change the numbers. Dr. Carpenter called for a vote on the motion. The panel voted 4 to 2 in favor of the motion, so the conclusions were accepted as written. Drs. Regan and Connor cited their previous comments regarding the uterine neoplasms in rats as their reasons for voting against the motion. 


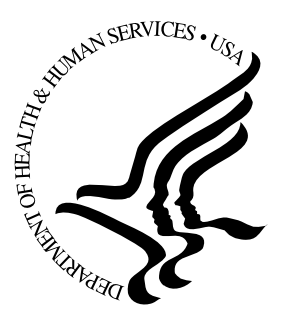

\title{
National Toxicology Program
}

\author{
NTP Central Data Management, MD EC-03
}

National Institute of Environmental Health Sciences

P.O. Box 12233

Research Triangle Park, NC 27709

http://ntp.niehs.nih.gov 